\title{
INVESTIGATIONS OF SUPPORTED
}

\author{
BI- AND TRI-METALLIC nZVI:
}

\section{NITRATE REDUCTION AND}

\section{HEAVY METAL REMOVAL}

BY

TRAN THI LOC

A thesis

submitted to the Victoria University of Wellington

in fulfilment of the requirements for the degree of

Doctor of Philosophy

Victoria University of Wellington 



\section{Attestation of Authorship}

I hereby declare that this thesis entitled

INVESTIGATIONS OF SUPPORTED BI- AND TRI-METALLIC nZVI: NITRATE REDUCTION AND

HEAVY METAL REMOVAL

is my own work and that, to the best of my knowledge and belief. It contains no materials previously published by another person except that which appears in the references and acknowledgements. I further declare that this thesis has not been submitted for any degree, diploma, or other qualification in any other institution.

Signed:

Date:

Tran Thi Loc

Victoria University of Wellington 


\begin{abstract}
$\mathrm{nZVI}$ has been well documented as an effective reagent to remove contaminants, including organic and inorganic substances. However, the drawbacks of nZVI are agglomeration and bioaccumulation due to its magnetic property and nanosize. One of the solutions for these problems is putting nZVI on non-toxic support. Microsilicate600 (Misi), a silicate from a geothermal deposit in Rotorua, New Zealand, has been established to be useful in adsorbing $\mathrm{nZVI}$. Doping $\mathrm{nZVI}$ with other metals to generate bimetallic and trimetallic materials is a method to increase its reactivity. These dopant metals function as catalysts in enhancing the electron transfer from $\mathrm{Fe}(0)$ core to contaminants. In this research project, the effect of doping metals on $n Z V I$ to generate bimetallic or trimetallic $n Z V I$ particles and effect of adsorbing these resulting particles on Misi were investigated. The reactivity of these materials towards the removal of different contaminants such as nitrate and toxic heavy metals was examined.
\end{abstract}

Supported bimetallic nZVI@Misi were synthesised using different dopant metals, including $\mathrm{Ni}, \mathrm{Cu}, \mathrm{Zn}$, and Pd. Supported trimetallic nZVI@Misi was synthesised by doping Pd and Cu on the surface of nZVI@Misi. Both supported bimetallic and trimetallic materials were prepared using different Misi preparations, contents of dopants, and synthetic methods. All these doped materials had higher reactivity than non-doped nZVI@Misi in removing nitrate. Misi that was calcined and $\mathrm{FeOOH}$-coated is the most optimised-prepared support. Moreover, materials prepared via deposition method had higher reactivity in reducing nitrate than those prepared via co-reduction method. This is due to the distribution of dopant metals on $\mathrm{nZVI}$, which was observed in TEM analysis. Of these materials, Ni-doped, Cu-doped, and Pd-Cudoped nZVI@Misi were the most promising materials. They not only reduced nitrate effectively but also were not significantly affected by naturally occurring factors such as oxygen, chloride, sulfate, carbonate, and phosphate. During nitrate reduction, the generation of metal by-products was minimal. Interestingly, their reactivity in natural water was relatively similar to that in lab-prepared samples.

The representative materials including supported $\mathrm{Ni}$-doped, Cu-doped, and non-doped nZVI@Misi, were used for heavy metal removal. These materials effectively removed cadmium, lead, and chromium in both lab-prepared samples and natural water. 


\section{Acknowledgements}

There are a great many people who I would like to acknowledge for their help and support. This thesis would not have been far from completion without their valuable contributions.

Above all else, I would like to express my thankfulness to my primary supervisor Associate Professor Robin Fulton throughout this research project. Her guidance and support have been incredibly valuable. I am very grateful for her dedicated supervision, generosity, patience, and understanding.

I am extremely grateful to Dr. Robert Keyzers, my secondary supervisor. He has given me advice and encouragement.

Similarly, my deepest gratitude goes to Dr. Putri Fraser. Her knowledge of nZVI materials and willingness to answer questions was incredibly helpful in the early stages of my research on nanomaterials.

I specially thank to Vietnam International Education Development (VIED) and Victoria University of Wellington for providing me grant throughout my doctoral study. Without their kindness assistance, this thesis would not have been done.

Thanks also to everyone in the School of Chemical and Physical Sciences. Specific mention must go to David Flynn for his tireless help for training me on the electron microscopes. This thesis would have been impossible without his contribution. I am extremely grateful to Dr. Mathew Cairns for training me AAS. Without his significant support, it would have been challenging for me to come to the end of my PhD journey. Thank you to all staffs at school for their support and encouragement.

I would like to thank Water Research Group and Fulton/Coles group, especially Professor Martyn Coles who has given insightful feedback throughout my project. Many thanks to people in the group for all the help and assistance.

Thank you to my officemates who supported and encouraged me a lot.

A big thank you to my friends who helped me in writing and grammar and friends who encouraged me during my journey. Without their friendship and support, I would have been lonelier.

I am so grateful to all my family members. I am deeply appreciative of their love, encouragement and constant loving support. None of this would have been possible without you. This thesis is the only valuable present for my Mum.

Last but not least, I really appreciate members in the examination committee including Professor Jim Johnston (Victoria University of Wellington - New Zealand), Dr Adam Hartland (University of Waikato - New Zealand), Professor Subhasis Ghoshal (McGill University Canada) for their feedback and recommendations.

To you all, my love and thankfulness. 


\section{Table of Contents}

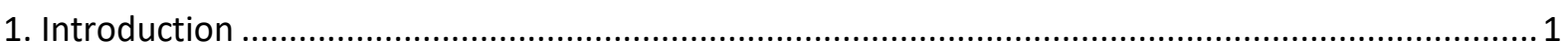

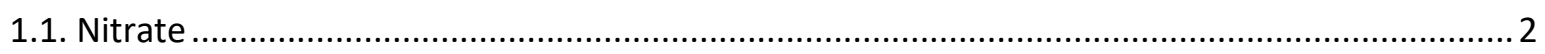

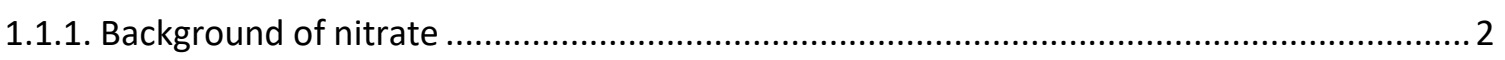

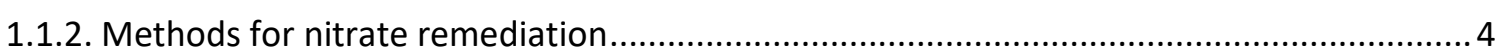

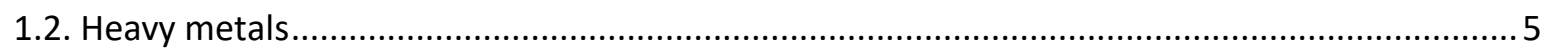

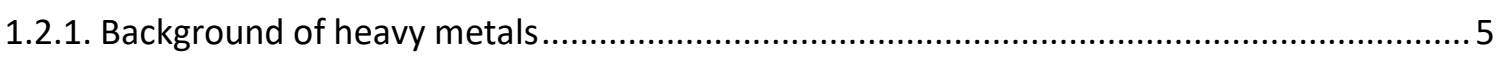

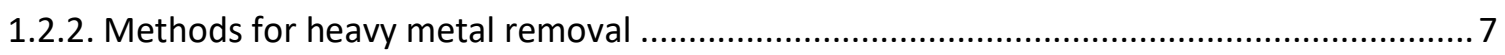

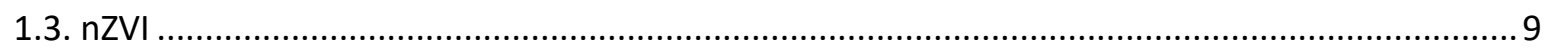

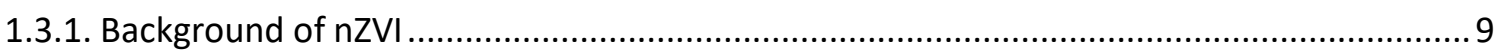

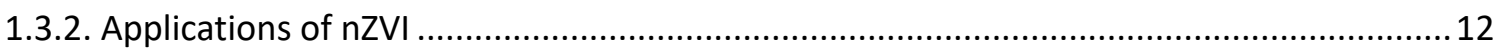

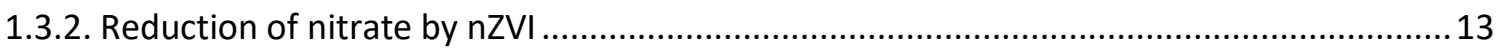

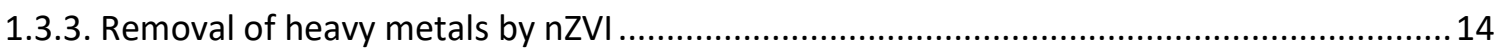

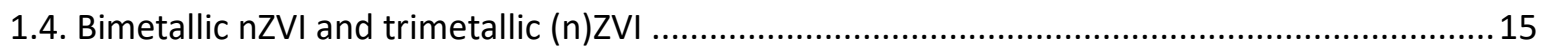

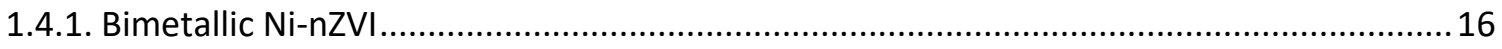

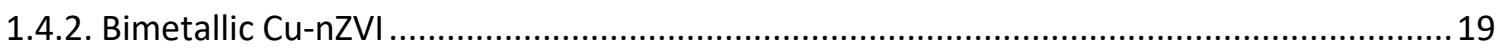

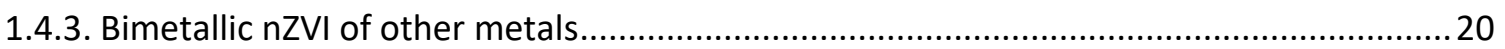

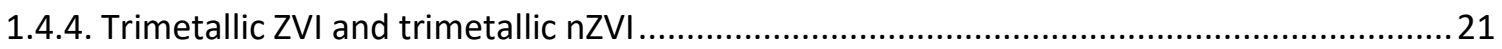

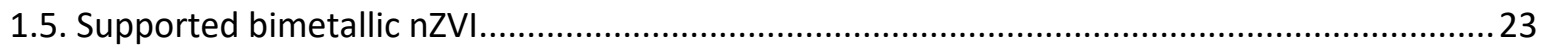

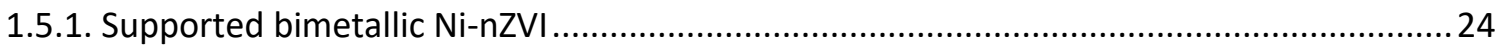

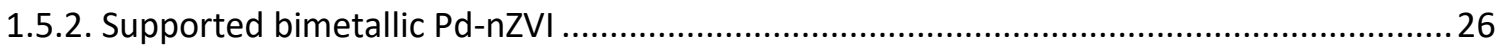

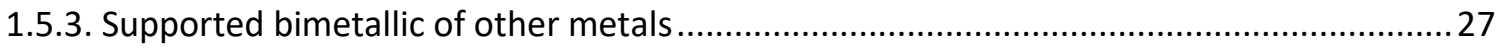

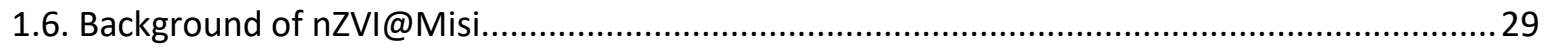

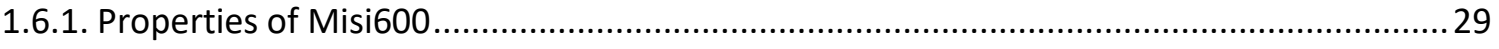

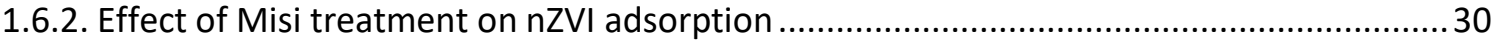

1.6.3. Effect of Misi treatment on $\mathrm{nZVI}$ reactivity towards nitrate reduction ............................... 31

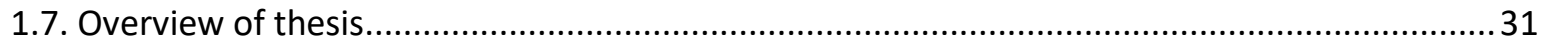

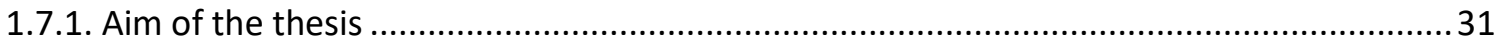

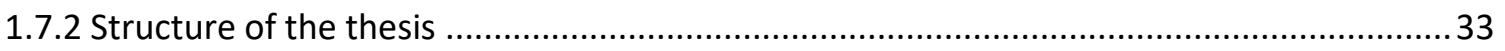

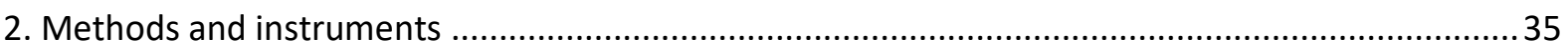

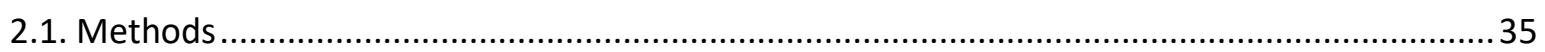

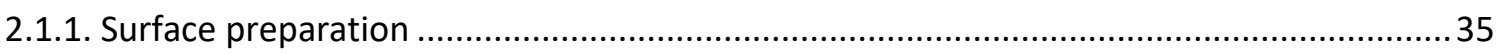

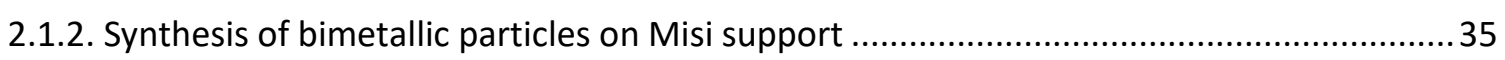

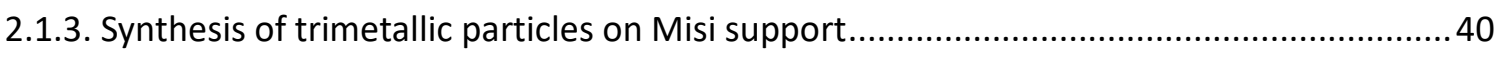

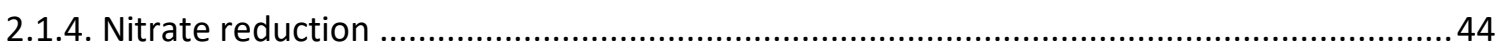


2.1.5. Elemental composition in materials

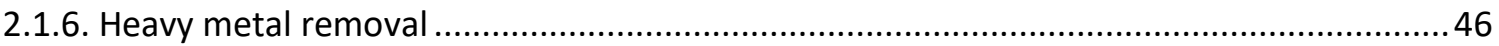

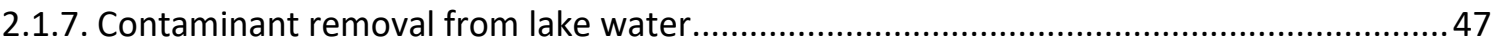

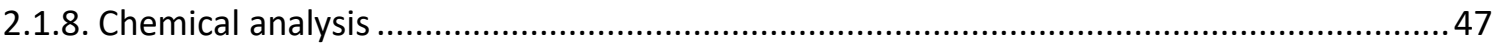

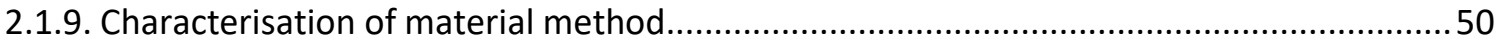

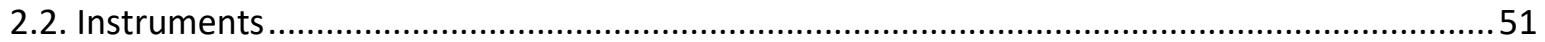

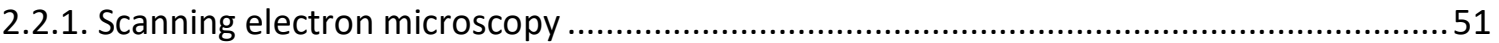

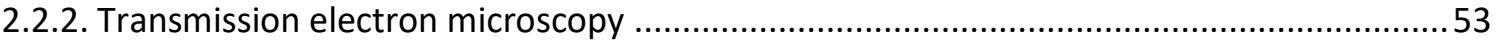

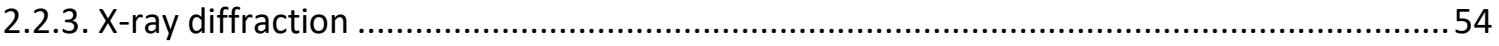

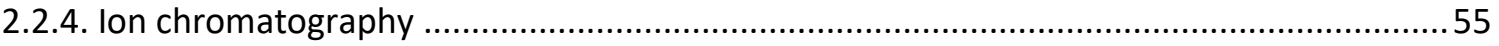

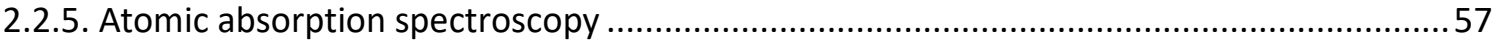

3. Determining the optimal dopant metals on nZVI@Misi in nitrate reduction ..................................59

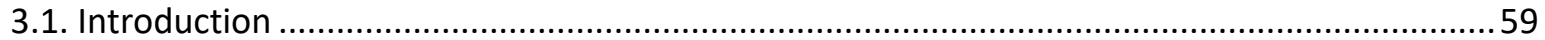

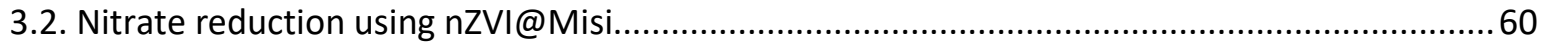

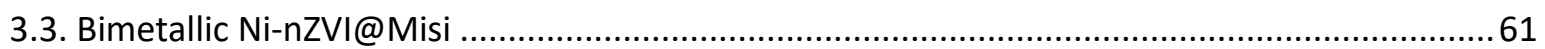

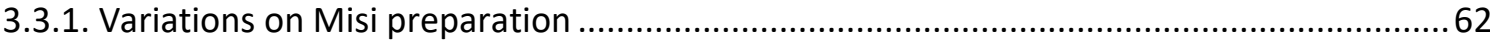

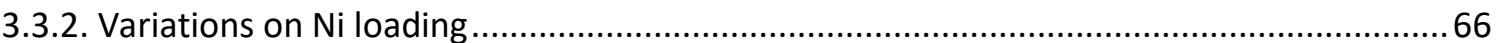

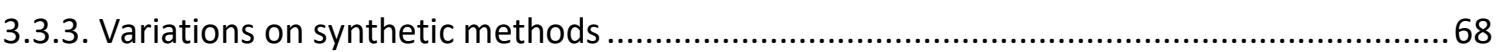

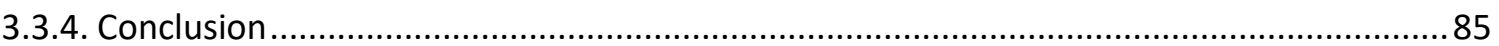

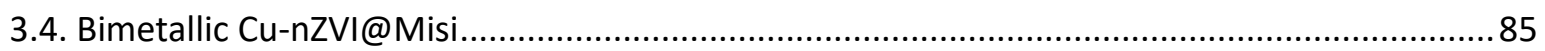

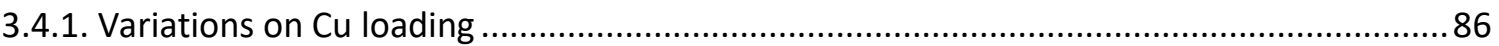

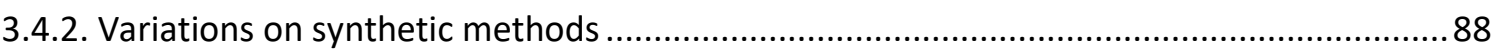

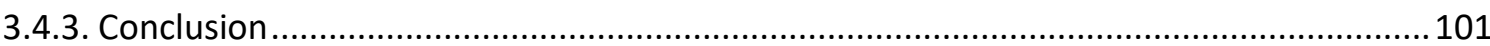

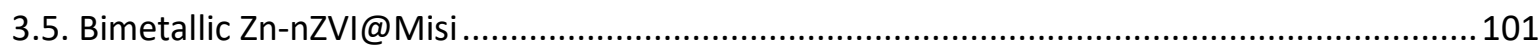

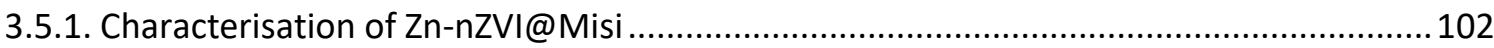

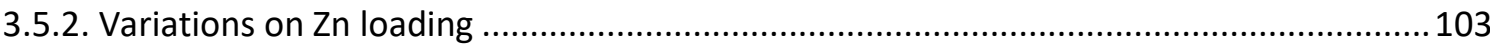

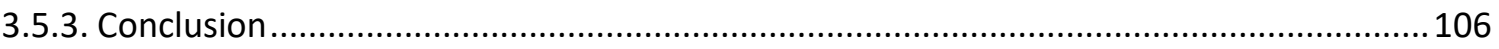

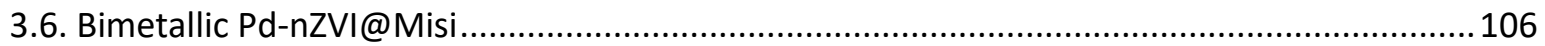

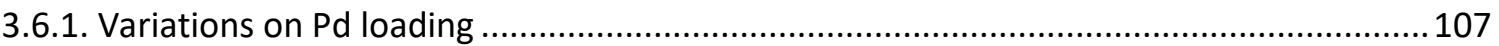

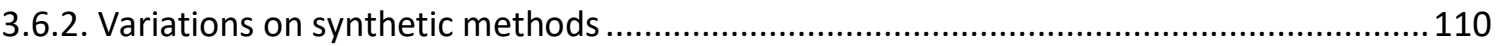

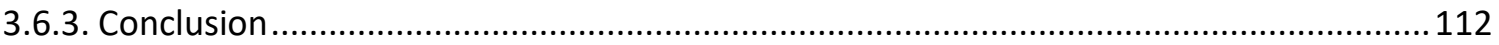

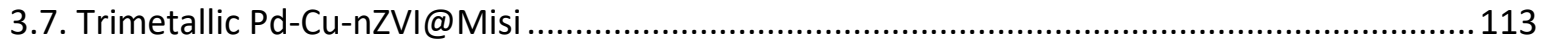

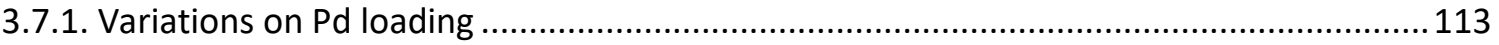

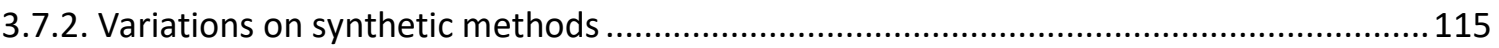

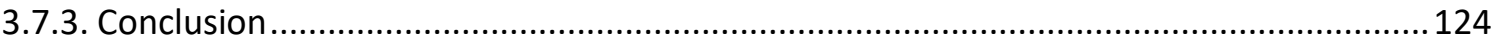

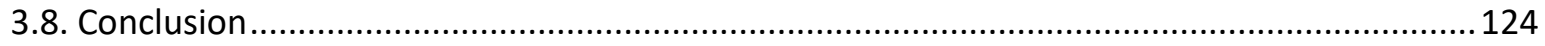


4. Nitrate reduction with optimised-doped nZVI@Misi 125

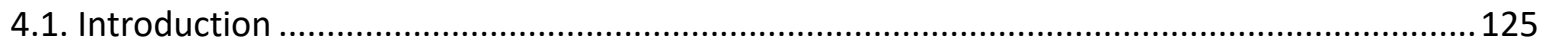

4.2. Nitrate reduction with bimetallic Ni-nZVI@Misi under anaerobic conditions .........................128

4.3. Nitrate reduction with bimetallic Cu-nZVI@Misi under anaerobic conditions ........................148

4.4. Nitrate reduction with trimetallic Pd-CU-nZVI@Misi under anaerobic conditions ..................158

4.5. Effect of reaction time and initial nitrate amount on nitrate reduction under anaerobic conditions. 166

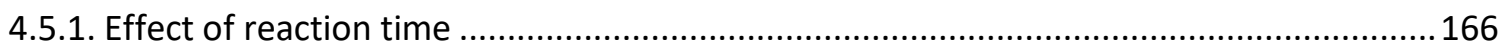

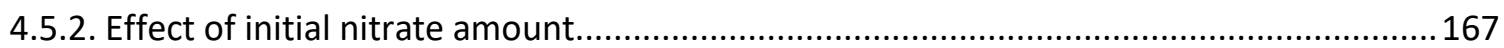

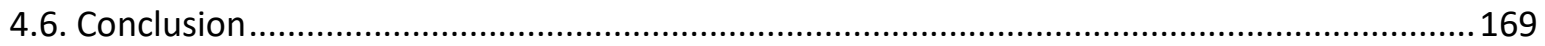

5. Effect of reaction conditions on nitrate reduction ................................................................... 170

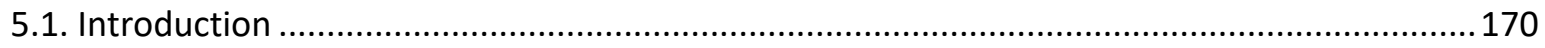

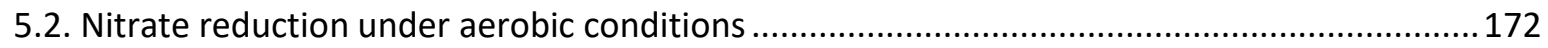

5.3. Comparison of the reactivity of materials under anaerobic and aerobic conditions .............. 175

5.4. Determination of soluble metals during nitrate reduction ................................................ 179

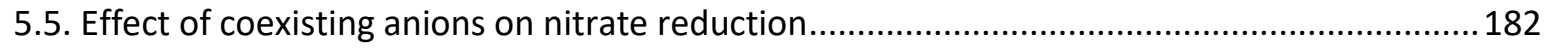

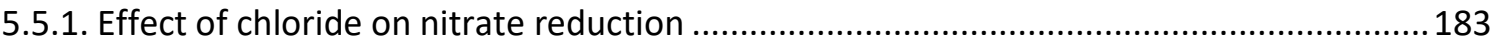

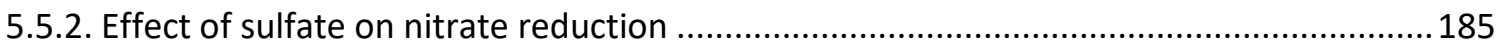

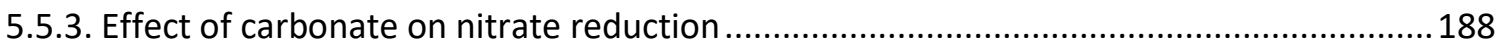

5.5.4. Effect of phosphate on nitrate reduction ..................................................................... 191

5.5.5. Simultaneous effect of coexisting anions on nitrate reduction ....................................... 194

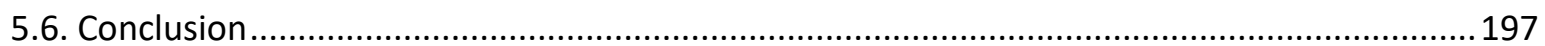

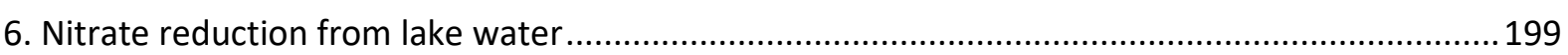

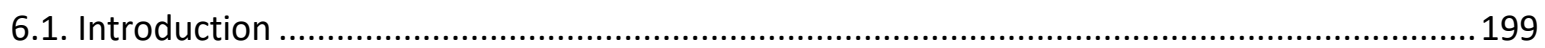

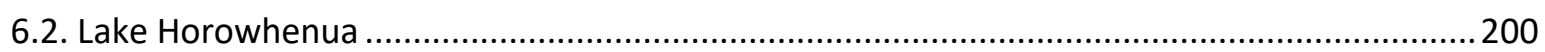

6.3. Preliminary results of nitrate reduction from lake water...................................................... 201

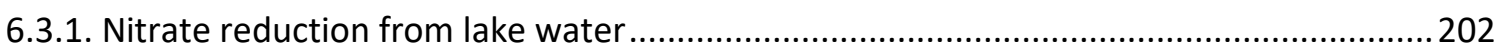

6.3.2. Nitrate reduction from lake water spiked with $10 \mathrm{ppm}$ nitrate- $\mathrm{N}$..................................2203

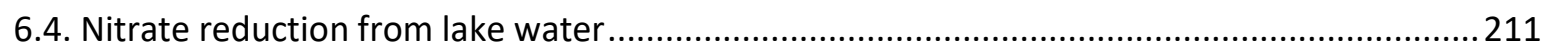

6.4.1. Nitrate reduction from lake water spiked with 10 ppm nitrate-N .................................211

6.4.2. Nitrate reduction from lake water spiked with 50 ppm nitrate-N .................................215

6.5. Determination of soluble iron during nitrate reduction from lake water ..............................219

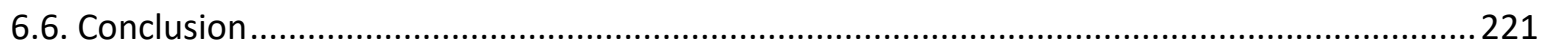

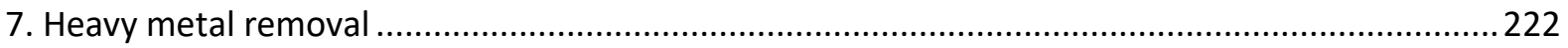

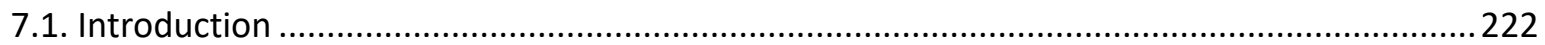

7.2. Cadmium removal by doped and non-doped nZVI@Misi ......................................................224 
7.2.1. Reactivity of doped and non-doped nZVI@Misi towards cadmium removal

7.2.2. Determination of soluble metals in cadmium removal ..................................................227

7.2.3. Elemental distribution of material after cadmium removal .............................................229

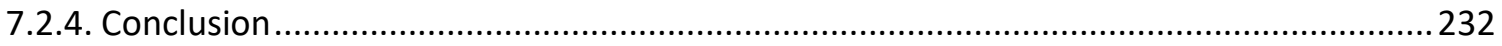

7.3. Lead removal by doped and non-doped nZVI@Misi ................................................................232

7.3.1. Reactivity of doped and non-doped nZVI@Misi towards lead removal...........................2232

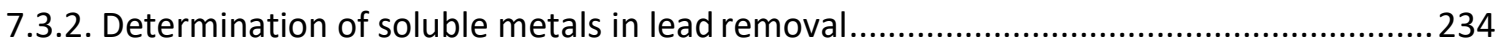

7.3.3. Elemental distribution of nZVI@MisiCFeOOH after lead removal ...................................237

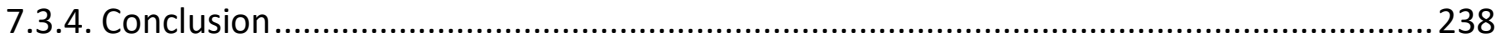

7.4. Chromium removal by doped and non-doped nZVI supported on Misi .................................238

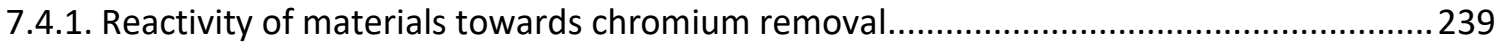

7.4.2. Determination of soluble metals in chromium removal..................................................241

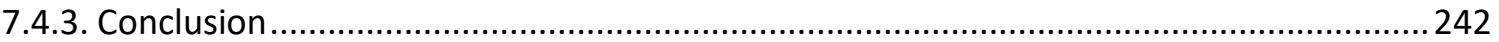

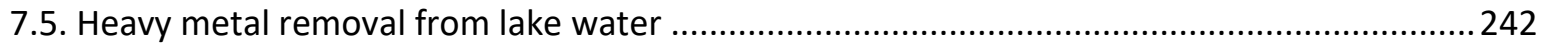

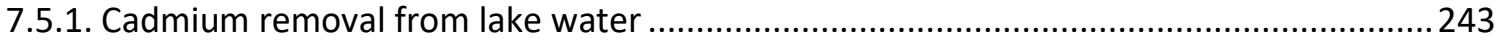

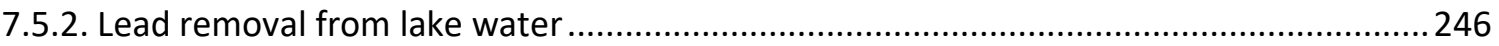

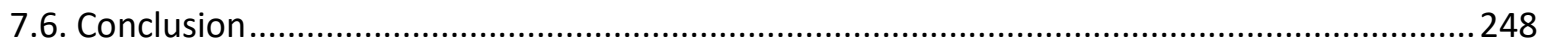

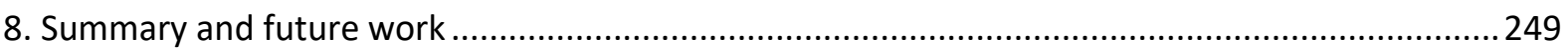

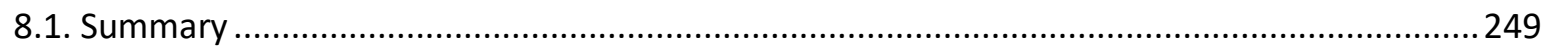

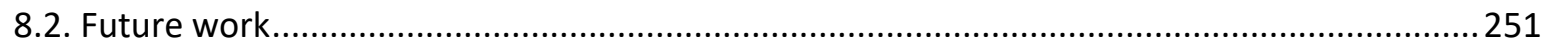

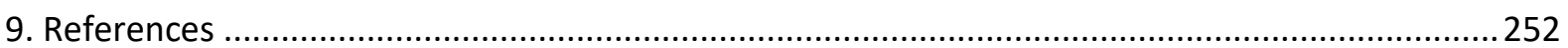




\section{List of Tables}

Table 1.1. Redox couples and $E^{0}$ of nitrogen compounds in acidic and basic conditions 14

Table 1.2. Ni-nZVI systems reported 19

Table 1.3. Cu-nZVI systems reported 20

Table 1.4. Other systems reported $\quad 21$

Table 1.5. Trimetallic ZVI and nZVI systems reported 23

Table 1.6. Supported Ni-nZVI reported 26

Table 1.7. Supported Pd-nZVI systems 27

Table 1.8. Supported bimetallic nZVI of other metals 28

Table 1.9. Chemical analysis of Misi 29

Table 1.10. Particle size analysis 29

Table 2.1. Material names and corresponding amount of metals salts added in M-nZVI@Misi

Table 2.2. Material names and corresponding amount of metals salts added in $\mathbf{M}_{1}-\mathrm{M}_{2}-$ nZVI@Misi 40

Table 2.3. Nitrate reduction under anaerobic and aerobic conditions $\quad 44$

Table 2.4. Nitrate reduction in the presence of other ions 46

Table 2.5. Heavy metal removal $\quad 47$

Table 3.1. List of Ni-nZVI@Misi systems synthesised 62

Table 3.2. Analysis of Ni-doped materials 80

Table 3.3. List of Cu-nZVI@Misi systems synthesised 86

Table 3.4. Analysis of Cu-doped materials 96

Table 3.5. List of Zn-nZVI@Misi systems synthesised 102

Table 3.6. List of Pd-nZVI@Misi systems synthesised 107

Table 3.7. List of Pd-nZVI@Misi systems synthesised 113

Table 3.8. Analysis of Pd-Cu-doped materials 119

Table 5.1. Phosphate concentrations during nitrate reduction in the presence of phosphate

Table 5.2. Phosphate concentrations during nitrate reduction in the presence of coexisting anions

Table 6.1. The components of the sample collected from Lake Horowhenua 
Table. 6.2. Nitrite accumulation in nitrate reduction from lake water spiked with $10 \mathrm{ppm}$ nitrate-N under anaerobic conditions

Table. 6.3. Nitrite accumulation in nitrate reduction from lake water spiked with $10 \mathrm{ppm}$ nitrate- $\mathrm{N}$ under aerobic conditions

Table. 6.4. Nitrite accumulation in nitrate reduction from lake water spiked with $50 \mathrm{ppm}$ nitrate-N under anaerobic conditions

Table. 6.5. Nitrite accumulation in nitrate reduction from lake water spiked with $50 \mathrm{ppm}$ nitrate-N under aerobic conditions

Table 7.1. Nickel concentration in Cd(II) removal

Table 7.2. Lead concentration using $\mathrm{MisiCFeOOH}$

Table 7.3. Nickel concentration in the removal of $\mathrm{Pb}(\mathrm{II}) 20 \mathrm{ppm}$

Table 7.4. Nickel concentration in the removal of $\mathrm{Pb}(\mathrm{II}) 40 \mathrm{ppm}$

Table 7.5. Chromium concentration using MisiCFeOOH

Table 7.6. Nickel concentration in $\mathrm{Cr}(\mathrm{VI})$ removal 


\section{List of Figures}

Figure 1.1. The structure of nZVI 11

Figure 1.2. Pathway of heavy metal removal by nZVI 15

Figure 2.1. Diagram showing the distribution of signals in SEM 52

Figure 2.2. Diagram showing the signals used for TEM 54

Figure 2.3. Diagram showing the interaction of X-rays with layers of atoms in a crystal 55

Figure 2.4. Diagram showing suppressed anion chromatography 56

Figure 2.5. Diagram showing atomic absorption experiment 58

Figure 3.1. Reduction characterisation of nZVI@Misi towards nitrate-N 10 ppm 61

Figure 3.2. COMPO images of 5\%Ni-nZVI on: (a) MisiU, (b) MisiC, (c) MisiC-B and (d)

MisiCFeOOH synthesised via co-reduction method (MA) 63-64

Figure 3.3. COMPO images of (a) 5\%Ni-nZVI and (b) nZVI 65

Figure 3.4. Reduction characterisation of Ni-nZVI on different preparations of Misi 66

Figure 3.5. COMPO images of (a) 2\%Ni-nZVI@MisiC-MA and (b) 5\%Ni-nZVI@MisiC-MA 67

Figure 3.6. Reduction characterisation of Ni-nZVI@MisiC on different Ni concentrations 68

Figure 3.7.COMPO images of (a) 5\%Ni-nZVI@MisiC-MA, (b) 5\%Ni-nZVI@MisiCFeOOH-MA, (c)

5\%Ni-nZVI@MisiC-MB1, (d) 5\%Ni-nZVI@MisiCFeOOH-MB1, (e) 5\%Ni-nZVI@MisiC-MB2, (f)

5\%Ni-nZVI@MisiCFeOOH-MB2 70

Figure 3.8. SEM-EDS mapping of 5\%Ni-nZVI@MisiCFeOOH-MA 71

Figure 3.9. SEM-EDS mapping of nZVI@MisiCFeOOH 71

Figure 3.10. XRD spectrum of 5\%Ni-nZVI@MisiCFeOOH-MA 72

Figure 3.11. TEM images in different magnifications of (a) and (d) 5\%Ni-nZVI@MisiCFeOOHMA; (b) and (e) 5\%Ni-nZVI@MisiCFeOOH-MB1; (c) and (f) 5\%Ni-nZVI@MisiCFeOOH-MB2 73

Figure. 3.12.TEM-EDS spectrum of 5\%Ni-nZVI@MisiCFeOOH-MA 74

Figure 3.13. TEM-EDS spectrum of 5\%Ni-nZVI@MisiCFeOOH-MB1 74

Figure 3.14. TEM-EDS spectrum of 5\%Ni-nZVI@MisiCFeOOH-MB2 75

Figure 3.15. TEM-EDS mapping of 5\%Ni-nZVI@MisiCFeOOH-MA 76

Figure 3.16. TEM-EDS mapping of 5\%Ni-nZVI@MisiCFeOOH MB1 77-78

Figure 3.17. TEM-EDS mapping of 5\%Ni-nZVI@MisiCFeOOH-MB2 79 
Figure 3.18. Reduction characterisation of 5\%Ni-nZVI@MisiCFeOOH prepared via different methods

Figure 3.19. Reduction characterisation of 5\%Ni-nZVI@MisiC prepared via different methods

Figure 3.20. Nitrate reduction, ammonium production and mass balance by 5\%Ni-nZVI@Misi

Figure 3.21. Nitrate reduction on different $\mathrm{Cu}$ loading contents

Figure 3.22. COMPO images of (a) 5\%Cu-nZVI@MisiC-MA, (b) 5\%Cu-nZVI@MisiCFeOOH-MA, (c) 5\%Cu-nZVI@MisiC-MB1, (d) 5\%Cu-nZVI@MisiCFeOOH-MB1

Figure 3.23. TEM-EDS spectra of 5\%Cu-nZVI@MisiCFeOOH-MA in different areas 90-91

Figure 3.24. TEM-EDS mapping of 5\%Cu-nZVI@MisiCFeOOH-MA with low magnification 92 Figure 3.25. TEM-EDS mapping of 5\%Cu-nZVI@MisiCFeOOH-MA with high magnification 93 Figure 3.26. TEM-EDS spectrum of 5\%Cu-nZVI@MisiCFeOOH-MB1 94

Figure 3.27. TEM-EDS mapping of 5\%Cu-nZVI@MisiCFeOOH-MB1 95

Figure 3.28. Reduction characterisation of 5\%Cu-nZVI@MisiC prepared via different methods

Figure 3.29. Reduction characterisation of 5\%Cu-nZVI@MisiCFeOOH prepared via different methods

Figure 3.30. Nitrate reduction, ammonium production and mass balance by 5\%Cu-nZVI@Misi

Figure 3.31. COMPO images of (a) 5\% Zn-nZVI@MisiC and (b) 5\% Zn-nZVI@MisiCFeOOH 103 Figure 3.32. Reduction characterisation of 5\%Zn-nZVI@Misi on different Zn loadings 104 Figure 3.33. Nitrate reduction, ammonium production and mass balance by Zn-nZVI@Misi

Figure 3.34. COMPO images of (a) 0.5\%Pd-nZVI@MisiCFeOOH-MA, (b) 1\%PdnZVI@MisiCFeOOH-MA, (c) 0.5\%Pd-nZVI@MisiCFeOOH-MB1, (d) 1\%Pd-nZVI@MisiCFeOOHMB1

Figure 3.35. Reduction characterisation of Pd-nZVI@MisiC-MA on different Pd contents 109 Figure 3.36. Reduction characterisation of Pd-nZVI@MisiCFeOOH-MA on different Pd contents

Figure 3.37. Reduction characterisation of Pd-nZVI@Misi prepared via different methods 
Figure 3.38. Nitrate reduction, ammonium production and mass balance by 1\%Pd-nZVI@Misi

Figure 3.39. COMPO images of (a) 0.5\%Pd-nZVI@MisiCFeOOH-MC1 and (b) 1\%PdnZVI@MisiCFeOOH-MC1

Figure 3.40. Reduction characterisation of Pd-Cu-nZVI@MisiC-MC1 on different Pd loadings

Figure 3.41. COMPO images of 0.5\%Pd-5\%Cu-nZVI@MisiCFeOOH synthesised via different methods (a) $M A_{\text {tri, }}$ (b) MC1, (c) MC2 and (d) MD

Figure 3.42. TEM-EDS spectrum of 0.5\%Pd-5\%Cu-nZVI@MisiCFeOOH-MC1

Figure 3.43. TEM-EDS mapping of 0.5\%Pd-5\%Cu-nZVI@MisiCFeOOH-MC1

Figure 3.44. Reduction characterisation of Pd-Cu-nZVI@MisiC prepared via different methods

Figure 3.45. Reduction characterisation of Pd-Cu-nZVI@MisiCFeOOH prepared via different methods

Figure 3.46. Nitrate reduction, ammonium production and mass balance by $0.5 \% \mathrm{Pd}-5 \% \mathrm{Cu}-$ nZVI@MisiC

Figure 4.1. Reduction characterisation of 5\%Ni-nZVI@Misi towards nitrate- N 10 ppm under anaerobic conditions

Figure 4.2. Reduction characterisation of 5\%Ni-nZVI@Misi towards nitrate-N 10 ppm under anaerobic conditions with high uncertainty after 5 minutes

Figure 4.3. Nitrite accumulation using 5\%Ni-nZVI@Misi towards nitrate-N 10 ppm reduction under anaerobic conditions

Figure 4.4. Nitrite accumulation using 5\%Ni-nZVI@Misi towards nitrate-N 10 ppm reduction under anaerobic conditions with high uncertainty after 5 minutes

Figure 4.5. Reduction characterisation of 5\%Ni-nZVI@Misi towards nitrate-N 50 ppm under anaerobic conditions

Figure 4.6. Nitrite accumulation using 5\%Ni-nZVI@Misi towards nitrate-N reduction of 50 ppm under anaerobic conditions

Figure 4.7. Kinetics of nitrate reduction using 5\%Ni-nZVI@MisiCFeOOH-MB1 under anaerobic conditions

Figure 4.8. Kinetics of nitrate reduction using 5\%Ni-nZVI@MisiCFeOOH-MB1 under anaerobic conditions in different stages 
Figure 4.9. COMPO images of 5\%Ni-nZVI@MisiCFeOOH-MB1 after nitrate-N reduction of: (a) $10 \mathrm{ppm}$ and (b) $50 \mathrm{ppm}$

Figure 4.10. TEM images of 5\%Ni-nZVI@MisiCFeOOH-MB1 after nitrate-N reduction of (a), (b) 10 ppm for 15 minutes and (c), (d) 50 ppm for 120 minutes

Figure 4.11. TEM-EDS spectrum of 5\%Ni-nZVI@MisiCFeOOH-MB1 after reduction of 10 ppm nitrate- $\mathrm{N}$

Figure 4.12. TEM-EDS mapping of 5\%Ni-nZVI@MisiCFeOOH-MB1 after reduction of 10 ppm nitrate- $\mathrm{N}$

Figure 4.13. TEM-EDS spectrum of 5\%Ni-nZVI@MisiCFeOOH-MB1 after reduction of 50 ppm nitrate- $\mathrm{N}$

Figure 4.14. TEM-EDS mapping of 5\%Ni-nZVI@MisiCFeOOH-MB1 after reduction of 50 ppm nitrate- $\mathrm{N}$

Figure 4.15. Reduction characterisation of 5\%Cu-nZVI@Misi towards nitrate-N 10 ppm under anaerobic conditions

Figure 4.16. Reduction characterisation of 5\%Cu-nZVI@Misi towards nitrate-N 10 ppm under anaerobic conditions with high uncertainty after 5 minutes

Figure 4.17. Nitrite accumulation using 5\%CU-nZVI@Misi towards nitrate-N 50 ppm reduction under anaerobic conditions

Figure 4.18. Nitrite accumulation using 5\%CU-nZVI@Misi towards nitrate-N 10 ppm reduction under anaerobic conditions with high uncertainty

Figure 4.19. Reduction characterisation of 5\%Cu-nZVI@Misi towards nitrate-N 50 ppm under anaerobic conditions

Figure 4.20 Nitrite accumulation using 5\%Cu-nZVI@Misi towards nitrate-N 50 ppm reduction under anaerobic conditions

Figure 4.21. COMPO images of 5\%Cu-nZVI@MisiCFeOOH-MB1 after nitrate reduction of (a) $10 \mathrm{ppm}$ and (b) $50 \mathrm{ppm}$

Figure 4.22. Reduction characterisation of 0.5\%Pd-5\%Cu-nZVI@Misi towards nitrate-N 10 ppm under anaerobic conditions with high uncertainty after 5 minutes

Figure 4.23. Nitrite accumulation using 0.5\%Pd-5\%Cu-nZVI@Misi towards nitrate-N 10 ppm reduction under anaerobic conditions

Figure 4.24. Reduction characterisation of 0.5\%Pd-5\%Cu-nZVI@Misi towards nitrate-N 50 ppm under anaerobic conditions 
Figure 4.25. Nitrite accumulation using 0.5\%Pd-5\%Cu-nZVI@Misi towards nitrate-N 50 ppm reduction under anaerobic conditions

Figure 4.26. Reduction characterisation of 5\%Cu-nZVI@MisiCFeOOH-MB1 and 0.5\%Pd-5\%CunZVI@MisiCFeOOH-MC1 towards nitrite-N 20 ppm under anaerobic conditions

Figure 4.27. COMPO images of 0.5\%Pd-5\%Cu-nZVI@MisiCFeOOH-MC1 after nitrate-N reduction of (a) $10 \mathrm{ppm}$ and (b) $50 \mathrm{ppm}$

Figure 4.28. Effect of reaction time on nitrate reduction under anaerobic conditions

Figure 4.29. Reduction characterisation of representative materials towards nitrate- $\mathrm{N} 100$ ppm under anaerobic conditions

Figure 4.30. Nitrite accumulation using representative materials towards nitrate-N 100 ppm reduction under anaerobic conditions

Figure 5.1. Nitrate reduction of $10 \mathrm{ppm}$ nitrate-N under aerobic conditions

Figure 5.2. Nitrate reduction of $50 \mathrm{ppm}$ nitrate-N under aerobic conditions

Figure 5.3. Nitrite accumulation in the reduction of $50 \mathrm{ppm}$ nitrate- $\mathrm{N}$ under aerobic conditions

Figure 5.4. Nitrate reduction of $50 \mathrm{ppm}$ nitrate-N under anaerobic and aerobic conditions

Figure 5.5. Nitrite accumulation in the reduction of $50 \mathrm{ppm}$ nitrate- $\mathrm{N}$ under anaerobic and aerobic conditions

Figure 5.6. Iron concentration in nitrate reduction of $10 \mathrm{ppm}$ nitrate- $\mathrm{N}$ under aerobic conditions

Figure 5.7. Iron concentration in nitrate reduction of $50 \mathrm{ppm}$ nitrate-N under aerobic conditions

Figure 5.8. Corrosion of materials under aerobic conditions

Figure 5.9. Effect of chloride on nitrate reduction and nitrate reduction in the absence of ions under anaerobic conditions

Figure 5.10. Effect of chloride on nitrate reduction and nitrate reduction in the absence of ions under aerobic conditions

Figure 5.11. Effect of sulfate on nitrate reduction and nitrate reduction in the absence of ions under anaerobic conditions

Figure 5.12. Effect of sulfate on nitrate reduction and nitrate reduction in the absence of ions under aerobic conditions 
Figure 5.13. Effect of carbonate on nitrate reduction and nitrate reduction in the absence of ions under anaerobic conditions

Figure 5.14. Effect of carbonate on nitrate reduction and nitrate reduction in the absence of ions under aerobic conditions

Figure 5.15. Effect of phosphate on nitrate reduction and nitrate reduction in the absence of ions under anaerobic conditions

Figure 5.16. Effect of phosphate on nitrate reduction and nitrate reduction in the absence of ions under aerobic conditions

Figure 5.17. Effect of coexisting anions on nitrate reduction and nitrate reduction in the absence of ions under anaerobic conditions

Figure 5.18. Effect of coexisting anions on nitrate reduction and nitrate reduction in the absence of ions under aerobic conditions

Figure 6.1. Water sample in Lake Horowhenua

201

Figure 6.2. Nitrate reduction in lake water under anaerobic conditions

Figure 6.3. Nitrate reduction in lake water under aerobic conditions

Figure 6.4. Nitrate reduction in lake water spiked with $10 \mathrm{ppm}$ nitrate- $\mathrm{N}$ without $\mathrm{pH}$ adjustment under anaerobic conditions

Figure 6.5. Nitrite accumulation in nitrate reduction from lake water spiked with $10 \mathrm{ppm}$ without $\mathrm{pH}$ adjustment under anaerobic conditions

Figure 6.6. $\mathrm{pH}$ change in nitrate reduction from lake water spiked with $10 \mathrm{ppm}$ without $\mathrm{pH}$ adjustment under anaerobic conditions 206

Figure 6.7. Nitrate reduction in lake water spiked with $10 \mathrm{ppm}$ nitrate-N without pH adjustment under aerobic conditions

Figure 6.8. Nitrite accumulation in nitrate reduction from lake water spiked with $10 \mathrm{ppm}$ without $\mathrm{pH}$ adjustment under aerobic conditions

Figure 6.9. $\mathrm{pH}$ change in nitrate reduction from lake water spiked with $10 \mathrm{ppm}$ without $\mathrm{pH}$ adjustment under aerobic conditions 208

Figure 6.10. Nitrate reduction in lake water spiked with $10 \mathrm{ppm}$ at initial $\mathrm{pH} 3$ under anaerobic conditions 209

Figure 6.11. Nitrate reduction in lake water spiked with $10 \mathrm{ppm}$ at initial pH 3 under aerobic conditions 
Figure 6.12. pH change in nitrate reduction from lake water spiked with $10 \mathrm{ppm}$ at initial pH 3 under anaerobic conditions

Figure 6.13. $\mathrm{pH}$ change in nitrate reduction from lake water spiked with $10 \mathrm{ppm}$ at initial pH 3 under aerobic conditions

Figure 6.14. Nitrate reduction in lake water spiked with $10 \mathrm{ppm}$ under anaerobic conditions

Figure 6.15. Nitrate reduction in lake water spiked with $10 \mathrm{ppm}$ under aerobic conditions

Figure 6.16. Nitrate reduction in lake water spiked with $50 \mathrm{ppm}$ under anaerobic conditions

Figure 6.17. Nitrate reduction in lake water spiked with $50 \mathrm{ppm}$ under aerobic conditions

Figure 6.18. Iron concentration in nitrate reduction from lake water spiked with $10 \mathrm{ppm}$ under aerobic conditions

Figure 6.19. Iron concentration in nitrate reduction from lake water spiked with $50 \mathrm{ppm}$ under aerobic conditions 221

Figure 7.1. Removal characterisation towards $\mathrm{Cd}(\mathrm{II})$

Figure 7.2. Iron concentration in the removal of $\mathrm{Cd}(\mathrm{II}) 20 \mathrm{ppm}$

Figure 7.3. Cadmium and iron concentrations using 5\%Ni-nZVI@MisiCFeOOH-MA

Figure 7.4. TEM-EDS spectrum of nZVI@MisiCFeOOH after Cd(II) removal

Figure 7.5. TEM-EDS mapping of nZVI@MisiCFeOOH after Cd(II) removal

Figure 7.6. Iron concentration in the removal of 20 ppm Pb(II)

Figure 7.7. Iron concentration in the removal of $40 \mathrm{ppm} \mathrm{Pb(II)}$

Figure 7.8. TEM-EDS spectrum of nZVI@MisiCFeOOH after Pb(II) removal

Figure 7.9. TEM-EDS mapping of nZVI@MisiCFeOOH after Pb(II) removal.

Figure 7.10. Removal characterisation towards $\mathrm{Cr}(\mathrm{VI})$

Figure 7.11. Cadmium concentration in the removal of $\mathrm{Cd}(\mathrm{II})$ in lake water spiked with $20 \mathrm{ppm}$ under anaerobic conditions

Figure 7.12. Iron concentration in the removal of $\mathrm{Cd}(\mathrm{II})$ in lake water spiked $20 \mathrm{ppm}$ under anaerobic conditions

Figure 7.13. Cadmium concentration in the removal of $\mathrm{Cd}(\mathrm{II})$ in lake water spiked with $20 \mathrm{ppm}$ under aerobic conditions 
Figure 7.14. Iron concentration in the removal of $\mathrm{Cd}(\mathrm{II})$ in lake water spiked $20 \mathrm{ppm}$ under aerobic conditions

Figure 7.15. Iron concentration in the removal of $\mathrm{Pb}(\mathrm{II})$ in lake water spiked with $20 \mathrm{ppm}$ under anaerobic conditions

Figure 7.16. Iron concentration in the removal of $\mathrm{Pb}(\mathrm{II})$ in lake water spiked with $20 \mathrm{ppm}$ under aerobic conditions 


\section{List of Abbreviations}

AAS

ADF

BF

COMPO

EDS

EPA

HEPES

IC

Misi

MisiC

$\mathrm{MisiCFeOOH}$

MO

ND

$\mathrm{nZVI}$

PCBs

ppm

SEM

SS

STEM

TCE

TEM

UV-Vis

WHO

$w / w$

XPS

XRD

ZVI
Atomic Absorption Spectroscopy

Annular Dark-Field

Bright-Field

Composite image

Energy Dispersive X-ray Spectroscopy

Environmental Protection Agency

4-(2-hydroxyethyl)-1-piperazineethanesulfonic acid

Ion Chromatography

Microsilicate600

Calcined Misi

Calcined/FeOOH-coated Misi

Methyl Orange

No detection

Nano Zero-Valent Iron

Polychlorinated biphenyls

parts per million

Scanning Electron Microscope

Starch and Sodium citrate

Scanning Transmission Electron Microscope

Trichloroethylene

Transmission Electron Microscope

Ultraviolet/Visible Spectroscopy

World Health Organisation

weight/weight

X-ray photoelectron spectrometer

X-ray diffraction

Zero-valent iron 


\section{Introduction}

The pollution of nitrate and heavy metals is a significant concern. Due to their toxicity, heavy metals and high nitrate concentration damage human health, organisms, and ecosystems. As such, several methods have been developed to remediate these compounds from the environment. Each method has both advantages and disadvantages, depending on the concentrations of contaminants and reaction conditions.

Nano zero-valent iron ( $\mathrm{nZVI}$ ) has been considered an effective agent to reduce nitrate and heavy metals. nZVI provides more active sites and high reactivity than bulk or micro size materials. Unfortunately, due to its nano size, nZVI causes bio-accumulation and agglomeration. In order to minimise the environmental issue and maximise the activity of $\mathrm{nZVI}$, it is crucial to adsorb nZVI onto non-toxic supports. These non-toxic supports optimise the distribution of nZVI particles, which helps maintain a large surface area, and minimise bioaccumulation. One of the best-documented supports is silicates which are low-cost and environmentally friendly. Research in the Fulton group has shown that microsilicate600 (Misi), a by-product of geothermal generation, can be used to support nZVI. ${ }^{1}$ This material is capable of removing nitrate from water.

An efficient way to increase the reactivity of $n Z V I$ is doping it with other metals to generate bimetallic ${ }^{2-3}$ and trimetallic materials. ${ }^{4-5}$ These materials are more reactive due to the catalytic role of dopant metals. ${ }^{2,} 6$ In the current research project, a variety of metals were used to dope on nZVI@Misi, forming supported bimetallic (M-nZVI@Misi) and trimetallic (Pd-CunZVI@Misi) materials. Variables in these studies included different dopant metals, dopant concentrations, and synthetic methods. The morphology, shape, composition, and elemental distribution of the resulting materials were examined. Additionally, the capacity of these materials towards nitrate reduction and heavy metal removal was investigated. 


\subsection{Nitrate}

\subsubsection{Background of nitrate}

Nitrate is one of the most common inorganic ions in groundwaters and surface waters. In groundwaters, nitrate persists for decades, increasing in concentration due to the increasing application of nitrogen on land. ${ }^{7}$ Due largely to its high solubility, nitrate is easily transported into groundwaters. Nitrate comes from a number of different sources. These include agricultural activities, ${ }^{8}$ industrial wastewater, food processing, decomposition of dairy and meat products, ${ }^{9}$ septic tanks, ${ }^{9-10}$ refuse dump, erosion of natural deposits, ${ }^{11}$ discharge from treatment systems of domestic sewage, ${ }^{12}$ and decomposition of organic matter. ${ }^{9,}{ }^{12}$ Of these, the main source of nitrate is from agriculture such as irrigation and runoffs from farmlands. The abundant use of chemical fertilizers, uncontrolled discharge of wastewater, concentrated animal feeding, and animal waste is a major contributor of residual nitrate in the environment. For example, in a report about nitrate pollution in California's agricultural areas (2012), $96 \%$ of nitrate is from agriculture, only $4 \%$ of nitrate comes from other sources such as water treatment plants, food processing, and septic systems. ${ }^{13}$

Nitrate can also be produced by the process of decomposition of wastes from the food industry or household use which contain inorganic and organic nitrogen substances. In addition, as an essential part of the natural nitrogen cycle, nitrate is always found in soil and water as the final product of nitrification processes. ${ }^{14}$ Although fertilisers might not always be nitrate-based, ammonia/ammonium is readily converted into nitrite and then nitrate by nitrosomas bacteria and nitrobactor bacteria in the ground. ${ }^{15}$

As far as human health is concerned, nitrate at elevated concentrations causes skin rashes and hair loss. ${ }^{13}$ It can also cause abdominal pains, diarrhea and vomiting. ${ }^{12}$ High levels of nitrate compete with iodine uptake, leading to an increase in thyroid volume and the possibility of thyroid disorders. ${ }^{16-18}$ In the human body, oral bacteria convert nitrate into nitrite, which causes more severe health problems. For instance, nitrite reacts with $\mathrm{Fe}(\mathrm{II})$ in hemoglobin to form methhemoglobin which cannot bind to oxygen. This leads to low oxygen levels in the blood and causes methemoglobinemia (blue baby syndrome), especially in infants under six months. ${ }^{11}$ Nitrite also reacts with stomach acid and amine/amide functional groups in protein to form nitrosamine compounds that are known to be carcinogenic, 
teratogenic, and mutagenic. ${ }^{9}$ Thereby, nitrate is one of the health concerns that need to be remediated to the guideline value of safe waters proposed by the United States Environmental Protection Agency (U.S. EPA) and the World Health Organization (WHO). According to U.S. EPA, the maximum contaminant level of nitrate for drinking water is $45 \mathrm{mg} / \mathrm{L}$ $\mathrm{NO}_{3}{ }^{-}(10 \mathrm{ppm}$ nitrate- $\mathrm{N}){ }^{11}$ This amount is $50 \mathrm{mg} / \mathrm{LNO}_{3}{ }^{-}$(11.3 ppm nitrate- $\left.\mathrm{N}\right)$, as set by WHO. ${ }^{19}$

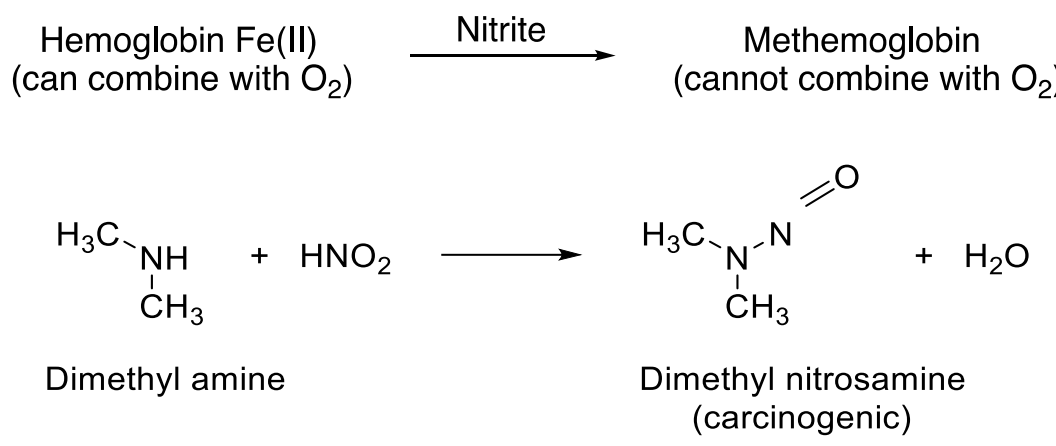

Nitrate and phosphate are necessary nutrients for algae. However, if the amount of these two substances is high, it causes eutrophication, endangering aquatic life. ${ }^{20-21}$ The eutrophication accompanied by extensive algal blooms causes the deterioration of the water quality which represents a serious threat to the ecosystems. ${ }^{22-23}$ Algal blooms not only limit light penetration but also increase the concentration of suspended particles. The lack of sunlight decreases the quality of photosynthetic processes, along with the destruction of benthic habitat. When the algae die, the decomposition of dead algae consumes oxygen, thus reducing the amount of dissolved oxygen in water. The water is then unable to support fish and shellfish. In addition, some algal blooms have toxins or harmful metabolites that may poison and/or kill aqueous wildlife, and other animals drinking these water sources. ${ }^{23}$

Nitrate pollution has become an environmental problem in many countries given that it is a widespread contaminant to both groundwaters and surface waters. In Europe, the water in some regions does not meet the Europe standards of nitrate despite the implementation of legislation to prevent nitrates leaching from agriculture. For example, a report in 2010 shows that $15 \%$ of groundwater monitoring stations and $3 \%$ of surface stations still had nitrate concentrations above the WHO limit for the drinking water quality standard. ${ }^{24}$ In Asian countries, the problem of nitrate pollution in Asia is even worse. In the Philippines and Thailand, $30 \%$ of the groundwater wells were reported to have higher nitrate levels than the WHO nitrate standard for drinking water, and up to three times greater in some areas. ${ }^{25}$ 
New Zealand is an agricultural country in which forestry, produce, and cattle and poultry products are the most popular. Thus, fertilizers and livestock waste are the primary sources of nitrogen leaching. From 1990 to 2012, the amount of nitrogen leached from agriculture has increased by $28.6 \%{ }^{26}$ In 1990 , sheep farming accounted for more than $50 \%$ of nitrogen leaching, followed by dairy cattle (24\%), beef cattle (21\%), and fertilizers (4\%). By 2012, dairy cattle were the main source of nitrogen leaching with a contribution of $36 \%$, followed by sheep (27\%), fertilizers (19\%), and beef cattle (15\%). In a report (2019) about nitrate-N leached from livestock, from 1990 to 2017, the amount of nitrate- $N$ leaching increased by 10,000 tonnes (5.3\%). ${ }^{27}$ By 2017 , dairy cattle contributed $65 \%$ nitrate-N leaching, followed by beef (19\%) and sheep (15\%). Fortunately, although there has been an increase in algae blooms across New Zealand, such nitrogen leaching has limited effect on New Zealand drinking water as a separate report (2017) showed that only $13 \%$ of the sites monitored have nitrate levels above the WHO limit for drinking water standard. ${ }^{28}$

\subsubsection{Methods for nitrate remediation}

Given the problem of excess nitrate on the environment and living creatures, several common techniques have been developed to remove nitrate from soil and waters. These include ion exchange, reverse osmosis, adsorption, biological denitrification, and chemical reduction. In ion exchange, a column that is modified from macro-porous structure resin is used to exchange nitrate. ${ }^{9,} 29-30$ Nitrate is absorbed on the column and then $\mathrm{NaCl}$ solution as the regenerant is applied to elute nitrate from the column. Ion exchange is technically simple and cost-effective. ${ }^{31}$ However, this method produces water with high total dissolved solids and the treatment of excess regenerant is potentially necessary.

In reverse osmosis, nitrate is removed by reversing the normal water flow under pressure through membranes. ${ }^{32}$ This method is effective to apply for water desalination. However, one particular drawback of this method is that the membranes used in reverse osmosis easily get damaged by organic matter, both suspended and colloidal particles in samples. ${ }^{33}$ Thus, pretreatment of samples is necessary prior to nitrate removal.

In the adsorption method, nitrate is removed by adsorbents that are derived from biobamboo powder charcoal or activated carbon in the form of micrometer scale. ${ }^{34}$ The removal efficiency can be increased by heating the adsorbent materials before use and conducting 
process at low temperature. The adsorption method is cost-effective, convenient, ${ }^{35}$ and feasible to apply for in situ treatment of underground and surface waters. ${ }^{34}$

In biological denitrification method, carbon sources are used to supply energy for bacteria to convert nitrate into final product of nitrogen gas. This process may undergo four steps, during which intermediates are generated. These intermediates can be nitrite, nitric oxide, and nitrous oxide or a combination of more than one. There have been numerous studies on biodenitrification using different carbon supplying sources such as woodchip, ${ }^{36}$ natural organic solid substrates, ${ }^{37-38}$ newspaper, ${ }^{39}$ and sugar, or glucose syrup. ${ }^{40}$ The major benefit of the denitrification method is that under optimal conditions, the final product is non-toxic nitrogen gas. $^{36,39}$ Unfortunately, bacteria and organic sources remaining during this removal process are dangerous to drinking water. ${ }^{41}$ It is also difficult to maintain the biological process at optimum conditions. ${ }^{34}$ Another drawback of the denitrification method is that it can be sensitive to temperature and oxygen levels. ${ }^{36}$

In chemical reduction method, zero valent metals have been used as reducing reagents, such as bulk Fe(0), ${ }^{42}$ microsized Fe $(\mathrm{ZVI}),{ }^{10,43-45} \mathrm{Cd}(0),{ }^{46}$ and $\mathrm{Mg}(0)^{47}$. The introduction of nano-sized particles increases the efficiency significantly due to the increase in surface area; as the particle size decreases, the ratio of surface to volume increases. ${ }^{48}$ One of the most prominent examples with considerable potential benefits due to more active sites and higher reactivity is nanoscale zero-valent iron (nZVI). ${ }^{49-50}$ This will be discussed in detail in 1.3.

\subsection{Heavy metals}

\subsubsection{Background of heavy metals}

There is no chemically complete definition of heavy metals. Heavy metals are typically defined as any elements with a density of greater than $5 \mathrm{~g} / \mathrm{cm}^{3}$, although this is a rough guideline. ${ }^{51}$ Heavy metals include toxic heavy elements such as cadmium, lead and mercury, but also biologically important metals such as zinc. Heavy metal contamination can be from either natural sources or industrial and agricultural waste sources. The erosion of natural deposits release heavy metals. ${ }^{11}$ In industry, heavy metals are released from factories that manufacture metal products, glass, electronic products, batteries, and paints, etc. ${ }^{11}$ In agriculture, heavy metals are from runoffs from landfills and croplands as well as the use of commercial fertilizers. ${ }^{52}$ In New Zealand, the application of superphosphate, which contains 
cadmium impurities, has resulted in increasing cadmium levels in soils. ${ }^{53}$ Every $20 \mathrm{~kg}$ phosphorus/ha is applied, an application of $8.87 \mathrm{~g}$ cadmium/ha is assumed in the top of 2.5 $\mathrm{cm}$ soil. This would result that the concentration of cadmium increases by $0.04 \mathrm{ppm} /$ year. Heavy metals, particularly lead, can also come from the corrosion of household plumbing systems. ${ }^{11}$ In New Zealand, the use of lead in household paints was only started to be phased out in the 1960's. ${ }^{54}$

Heavy metals are toxic and persistent in the environment. ${ }^{55}$ In contrast to organic substances or other inorganic ions, heavy metals are non-biodegradable to form harmless end products. In living organisms, heavy metals are unlikely to be completely consumed or removed by any biological processes in the organisms. Thereby, heavy metals accumulate up to the food chain. ${ }^{56}$

Some heavy metals are crucial for biological functions. A number of enzymes or bioactive complexes contain heavy metals such as zinc, manganese, nickel, copper, and iron, and thus the presence of heavy metals is important in maintaining biochemical processes. ${ }^{51}$ For example, zinc is found in several enzymes, including carbonic anhydrase which is responsible for the interconversion of carbon dioxide and biocarbonate. ${ }^{57}$ Copper is found in redox proteins which are responsible for transportation of electron or oxygen or in enzymes which act as oxidases to catalyse the reactions of oxidation-reduction. ${ }^{58}$ Iron plays a role of carrying oxygen to the tissues from the lungs. ${ }^{59}$ Many of these biologically important heavy metals cause problems at higher concentrations. ${ }^{55}$ For instance, a high concentration of zinc results in some health issues such as stomach problems, skin irritations, vomiting, anaemia, the disorder of metabolism, depression, and malaise. ${ }^{60}$ Too much copper results in accumulation in the kidneys, brains, and eyes. Wilson disease is a condition in which the body cannot regulate copper; as such, it results in a build-up of copper. ${ }^{11}$

In contrast to zinc, iron, and copper, lead does not play any functions in biological systems. ${ }^{51}$ It accumulates progressively and has multi-toxicity pathways. It can damage the nervous and reproductive systems and kidney. ${ }^{61-62}$ It can also cause many problems to other organs such as liver and brain. ${ }^{62}$ One of the molecular targets of lead is the Amino Levulinic Acid Dehydratase (ALAD) protein, which is involved in the biosynthetic pathway of hemoglobin; ${ }^{63}$ hence, anaemia is one of the symptoms of lead poisoning. In addition, the presence of lead limits the amount of antioxidants, damaging living cells and causing oxidative stress. ${ }^{55}$ 
Cadmium is another toxic heavy metal, although the targets of cadmium are different to that of lead. Exposure to cadmium results in damage to kidneys, ${ }^{11,60}$ livers, ${ }^{60-61}$ and lungs ${ }^{60}$ as well as bone degradation and renal dysfunction. ${ }^{61}$

With regards to chromium, the oxidation state is important. At low levels, $\mathrm{Cr}$ (III) is an essential nutrient, potentiating the action of insulin. ${ }^{64}$ In contrast, $\mathrm{Cr}(\mathrm{VI})$ is highly toxic to humans and animals and known as a potential carcinogenic factor. ${ }^{65-66} \mathrm{Cr}(\mathrm{VI})$ also causes allergic dermatitis. ${ }^{11}$

Heavy metals can damage human and livestock drinking water and irrigation water. ${ }^{67}$ In addition, heavy metals in polluted irrigation water can be transferred to agricultural fields and then accumulate in the soil. Due to their non-degradability, mobility and toxicity, heavy metals need addressing urgently by using methods with high efficiency and environmental friendliness.

\subsubsection{Methods for heavy metal removal}

Numerous investigations on heavy metal removal have been reported. They include chemical precipitation, ion exchange, adsorption, membrane filtration, and nanotechnology. Of these, chemical precipitation is the method that has been most commonly used in industry due to its effectiveness and low-cost operation. ${ }^{68}$ In this method, heavy metals react with chemicals to form insoluble substances. The resulting precipitates are then discarded from the solutions by sedimentation or filtration processes. Typically, the chemicals used include alkaline solutions such as $\mathrm{Ca}(\mathrm{OH})_{2}{ }^{69-70}, \mathrm{NaOH},{ }^{70}$ and sulfide solutions. ${ }^{71}$ However, the use of $\mathrm{Ca}(\mathrm{OH})_{2}$ causes either numerous secondary precipitates or stable complexes, which leads to a significant de-stabilisation of the primary heavy metal precipitates. ${ }^{69}$ In order to enhance the precipitation, fly ash is sometimes used as seed material to increase the size of hydroxide precipitates. ${ }^{72}$ Another way to enhance the precipitation is using coagulants such as aluminum sulfate, polyalumium chloride, and magnesium chloride. ${ }^{73}$ Heavy metals are also removed by chelating precipitation. Many chelates, including dipropyl dithiophosphate ${ }^{74}$ and potassium ethyl xanthate, ${ }^{75}$ were used to form insoluble chelating complexes. This method is simple and inexpensive, but it is only suitable for the removal of high concentrations of heavy metals such as lead, cadmium, copper, and mercury. 
In the ion exchange method, cations in the resin exchange with heavy metals in wastewaters. The cation exchange resin is made of synthetic or natural solid. The synthetic cation exchange resins typically contain a sulfonic acid group that can exchange protons and metals. ${ }^{76-78} \mathrm{~A}$ common natural resin is a zeolite which can exchange cations such as $\mathrm{Na}(\mathrm{I}), \mathrm{K}(\mathrm{I})$ and $\mathrm{Ca}(\mathrm{II})$ and heavy metals. ${ }^{79-81}$ Ion exchange is selective given that using this method, all ions can be removed or substances are separated. ${ }^{82}$ However, ion exchange has drawbacks such as high operational cost and the disposal of the residual metal sludge. ${ }^{83}$

In the adsorption method, carbon is usually used as an adsorbent to remove heavy metals. Adsorption is considered an effective and low-cost method. ${ }^{84}$ It can operate with flexibility in design and adsorbents can be regenerated. Yet, this method is only effective when the concentrations of heavy metals in wastewater are low. In this method, carbon materials such as activated carbon, ${ }^{85-86}$ or carbon nanotubes ${ }^{87-88}$ can be used as adsorbents. Due to the high cost of activated carbon, natural substances, and metallurgical waste have alternatively been used as adsorbents such as kaolin, ${ }^{89}$ kaolinite ${ }^{90-92}$ montmorillonite, goethite, humic acid, ${ }^{92-94}$ bentonite, ${ }^{95}$ bauxite, ${ }^{96}$ fly ash, ${ }^{97}$ diatomite ${ }^{98}$, and zeolite. ${ }^{99}$ Bio-adsorbents have also been considered promising materials. These can be derived from algal biomass ${ }^{100-101}$ or microbial biomass such as bacteria ${ }^{102-103}$ and fungi. ${ }^{104-105}$ Theoretically, using bio-sorbents is a costeffective and rapid way to treat heavy metals, but it is difficult to remove bio-sorbents and desorb heavy metals afterward.

Membrane filtration is a method in which heavy metals are removed by the formation of metal-polymer complexes directly in the membrane. The polymers in the membranes are made of high-molecular-weight substances with carboxylic or amine groups, which bind to metal ions. ${ }^{106}$ These polymers can be used in several different processes including ultrafiltration, ${ }^{106}$ reverse osmosis, ${ }^{107}$ and nanofiltration. ${ }^{108-110}$ The membrane filtration is effective, but expensive and complicated to operate.

$\mathrm{ZVI}$ is a versatile substance that can act in different roles. ${ }^{111}$ It can be a reductant via $\mathrm{Fe}(0)$ and $\mathrm{Fe}(\mathrm{II})$ on the surface. It can be an adsorbent via FeOOH layer or a coagulant via the dissolution of $\mathrm{Fe}(0)$ on the surface to form Fe(II). Therefore, ZVI has been used to remove heavy metals. ${ }^{65 \text {, }}$ ${ }^{112-113}$ Although using ZVI is an effective way, the removal of ZVI is significantly improved when it is in nano scale. For example, when the removal of $\mathrm{Pb}(\mathrm{II})$ was carried out in the same conditions, $\mathrm{nZVI}$ removed $\mathrm{Pb}(\mathrm{II}) 12$ times faster than macro size $\mathrm{Fe}(0) .{ }^{114}$ 
Nanotechnology has been used to remove heavy metals. In this method, nanosized substances are used as the adsorbent or reductant to remove heavy metals. This includes nanostructured graphite oxide, silica/graphite oxide composites, and silica nanoparticles. ${ }^{115}$ Zero-valent iron nanoparticles (nZVI) are widely used as reductants. ${ }^{116-117}$

\section{3. nZVI}

\subsubsection{Background of nZVI}

Zerovalent iron $\mathrm{Fe}(0)$ is a mild reducing agent that can reduce oxidative contaminants effectively. The surface area of $\mathrm{Fe}(0)$ particles has an important effect on their reactivity because the higher the surface area is, the more reactive these particles get. ${ }^{14,}{ }^{118}$ For example, when the surface area of Fe increased from $1.8 \mathrm{~m}^{2} / \mathrm{g}$ to $4.9 \mathrm{~m}^{2} / \mathrm{g}$, the removal capacity of Fe towards trichloroethylene (TCE) increased from $0.311 \mathrm{mg} / \mathrm{g}$ to $0.887 \mathrm{mg} / \mathrm{g} .{ }^{14} \mathrm{In}$ order to increase the number of the active site of $\mathrm{Fe}$, several methods have been used, including hydrogen reduction, ${ }^{14,119}$ acid washing, ${ }^{14,120}$ and ultrasound. ${ }^{121}$ The goal of these methods is to destroy the deactivated layers and prevent the formation of oxide layers on the surface of ZVI. For example, at the same reaction conditions, the nitrate reduction increased from $45 \%$ to $99 \%$ when the ultrasonic bath was used. ${ }^{121}$ However, even though one or more than one of these methods were applied to treat ZVI, the surface area of ZVI ( $\left.<1 \mathrm{~m}^{2} / \mathrm{g}\right)$ was significantly smaller than that of commercially available nanosized Fe $\left(7-60 \mathrm{~m}^{2} / \mathrm{g}\right) .{ }^{122}$

Over the past decades, nanosize $\mathrm{Fe}(0)$, referred to as nano zero valent iron (nZVI), has gained much attention due to its promising approach in environmental remediation. nZVI was firstly introduced in contaminant remediation in the late $20^{\text {th }}$ century. $.^{49}, 123$ It has widely been used to remove both organic and inorganic substances. The increasing application of $\mathrm{nZVI}$ is due to the nanoscale properties such as size and surface area that contribute to higher adsorption capacity and reactivity. The majority of nZVI particles are in the size $50-100 \mathrm{~nm} .{ }^{124}$ Compared to the micro size $\mathrm{Fe}(0), \mathrm{nZVI}$ maximises surface area and provides more active sites for contaminants adsorbing or forming complexes. ${ }^{41}$ This results in increasing the reaction rate of contaminant removal. ${ }^{49}, 124-125$ For example, the surface area of nZVI and commercial iron powder was $33.5 \mathrm{~m}^{2} / \mathrm{g}$ and $0.9 \mathrm{~m}^{2} / \mathrm{g}$; thus, the rate constant of TCE removal or polychlorinated biphenyls (PCBs) using nZVI was 10 - 100 times greater than using Fe powder. ${ }^{49}$ In another 
study, the surface area for $n Z V I$ and $Z V I$ was $16.67 \mathrm{~m}^{2} / g$ and $0.56 \mathrm{~m}^{2} / \mathrm{g}$, respectively; therefore, $\mathrm{nZVI}$ reduced nitrate 40 times faster than ZVI. ${ }^{126}$

Typically, nZVI has a spherical shape and tends to aggregate to form clusters due to its magnetic nature. ${ }^{127} \mathrm{nZVI}$ has a core-shell structure with a $\mathrm{Fe}(0)$ core surrounded by an iron oxide/hydroxide shell (Figure 1). ${ }^{48,124,128}$ The core acts as the electron donor and provides reducing power for reactions while the shell maintains sites for the adsorption of contaminants. Reported literature on the composition of the shell has been complicated. Li et al. reported that the shell consisted of the mixed valence iron oxides and that the shell composition depends on the fabrication processes and surrounding media. ${ }^{48}$ For example, when $\mathrm{nZVI}$ was produced by supersaturated metal vapour in a hollow cathode, the nanocrystalline oxide shell consisted of either maghaemite $\left(\nu-\mathrm{Fe}_{2} \mathrm{O}_{3}\right)$ or magnetite $\left(\mathrm{Fe}_{3} \mathrm{O}_{4}\right) .{ }^{129}$ When nZVI was generated using the hydrogen reduction of goethite, only magnetite $\left(\mathrm{Fe}_{3} \mathrm{O}_{4}\right)$ was observed in the shell. ${ }^{130}$ For the $\mathrm{nZVI}$ prepared using the reduction of sodium borohydride with iron ions, there have been several investigations of the composition of the shell. For example, in Liu et al.'s study, the shell contained magnetite $\left(\mathrm{Fe}_{3} \mathrm{O}_{4}\right) \cdot{ }^{131}$ However, Sun et al. reported that wustite $(\mathrm{FeO})$ was the component in the shell. ${ }^{132}$ In other studies, the shell was the combination of iron oxide and hydroxide. ${ }^{124,128}$ As such, the nature of the shell is still not well-understood.

A study by Kim et al., investigated how modified nZVI ages when it was exposed to air and water. ${ }^{133}$ In this study, modified nZVI was initially prepared by exposing nZVI to air (4 - 50 $\mathrm{ml} /$ minute) for 24 hours or to the atmosphere in 5 minutes to form shell-modified $\mathrm{nZVI}$ and rapid-oxidised $\mathrm{nZVI}$. Shell-modified $\mathrm{nZVI}$ comprised around $60 \% \mathrm{Fe}(0)$ and around $40 \%$ magnetite $\left(\mathrm{Fe}_{3} \mathrm{O}_{4}\right)$. A small amount of goethite $(1-3 \%)$ was observed when shell-modified $\mathrm{nZVI}$ was produced at high contact rate of air (from $20 \mathrm{~mL} /$ minute). However, rapid-oxidised $\mathrm{nZVI}$ had lower $\mathrm{Fe}(0)$ and there were three iron oxides, consisting of $20 \% \mathrm{Fe}(0), 21 \%$ wustite (FeO), 20\% hematite $\left(\alpha-\mathrm{Fe}_{2} \mathrm{O}_{3}\right)$, and $19 \%$ maghaemite $\left(\gamma-\mathrm{Fe}_{2} \mathrm{O}_{3}\right)$. When shell-modified $\mathrm{nZVI}$ was aged in water (one day), goethite $(\alpha-\mathrm{FeOOH})$ was newly formed. When shell-modified $n Z V I$ was aged in ambient air (one month), both hematite $\left(\alpha-\mathrm{Fe}_{2} \mathrm{O}_{3}\right)$ and maghemite $\left(\gamma-\mathrm{Fe}_{2} \mathrm{O}_{3}\right)$ were newly formed. 


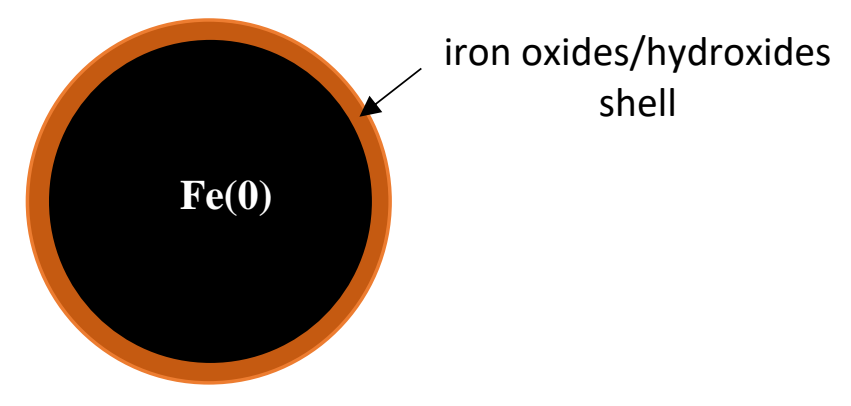

Figure 1.1. The structure of $n Z V I .{ }^{48,134}$

The oxide/hydroxide shell of nZVI has both advantages and disadvantages. The function of the shell was reported although the mechanism of the interaction between the shell and contaminants was not clear. In the removal of contaminants via adsorption, the shell supplies the reactive sites for contaminants to adsorb on its surface. ${ }^{135}$ 136-137 In the removal of contaminants via reduction, the shell functions as the sites for the initial adsorption and then contaminants are reduced by the $\mathrm{Fe}(0)$ core ${ }^{134}$ In contrast, the surface of the shell is a layer that can interfere with electron transfer as the shell encapsulates nZVI core. ${ }^{134}$ Among compositions of the shell, magnetite $\left(\mathrm{Fe}_{3} \mathrm{O}_{4}\right)$ did not affect electron migration due to its conductive property whereas lepidocrocite $\left(\gamma\right.$-FeOOH) limited the electron transfer. ${ }^{138}$ However, in the presence of $\mathrm{Fe}(\mathrm{II})$, lepidocrocite $(\gamma$-FeOOH) can be transformed to magnetite $\left(\mathrm{Fe}_{3} \mathrm{O}_{4}\right)$ (eq. 1.1).

$$
2 \gamma-\mathrm{FeOOH}+\mathrm{Fe}^{2+} \longrightarrow \mathrm{Fe}_{3} \mathrm{O}_{4}+2 \mathrm{H}^{+} \quad \text { (eq. 1.1) }
$$

There are two major routes to prepare nZVI. The first route is to transform Fe substances into $\mathrm{nZVI}$ using hydrogen reduction, electrochemical method, and arc discharge. In the hydrogen reduction method, a stable flow of hydrogen is used to reduce goethite (FeOOH) at high temperature $\left(250-650^{\circ} \mathrm{C}\right)$ for 6 hours. ${ }^{139}$ The electrochemical method is a combination of electrochemistry and ultrasonic methods in which $\mathrm{nZVI}$ is formed by the reduction of Fe(III) in the cathode with the support of the stabiliser (platinum). ${ }^{140}$ In the arc discharge method, a current of electricity goes through water-submerged iron electrodes and then the electrodes are evaporated. ${ }^{141}$ The cooled metal vapor forms nZVI.

In the second route, iron ions are reduced using chemical reagents. In the green synthetic method, nZVI is formed by polyphenolic extracts from leaves reacting with iron ions. ${ }^{142} \mathrm{~A}$ 
common way that has been used to synthesise $\mathrm{nZVI}$ in many laboratories involves the reduction of iron ions with borohydride salts. ${ }^{49}, 143$ Although borohydride compounds are costly, their use for synthesising nZVI is less time-consuming. Under an atmosphere of nitrogen gas or argon, ferrous or ferric are typically reduced by either sodium borohydride or potassium borohydride to form nZVI, boric acid and hydrogen gas (eq. 1.2 and eq. 1.3). ${ }^{144-146}$ The reaction can be carried out in deionised water or a mixture of ethanol and deionised water.

$$
\begin{aligned}
& \mathrm{FeCl}_{3}+3 \mathrm{NaBH}_{3}+9 \mathrm{H}_{2} \mathrm{O} \longrightarrow \mathrm{Fe}_{(\mathrm{nZVI})}+3 \mathrm{~B}(\mathrm{OH})_{3}+10.5 \mathrm{H}_{2}+3 \mathrm{NaCl} \\
& \mathrm{FeSO}_{4}+2 \mathrm{NaBH}_{3}+6 \mathrm{H}_{2} \mathrm{O} \longrightarrow \mathrm{Fe}_{(\mathrm{nZVl})}+2 \mathrm{~B}(\mathrm{OH})_{3}+7 \mathrm{H}_{2}+\mathrm{Na}_{2} \mathrm{SO}_{4} \text { (eq. 1.2) }
\end{aligned}
$$

The size and surface area of nZVI particles synthesised is strongly influenced by many factors such as precursor concentrations of iron solutions, solvents and dispersion agent in iron solutions, and the addition rate of reducing agents. Low concentrations of iron solutions produce small nZVI. For instance, when the same amount of $\mathrm{FeCl}_{3}$ in different concentrations of $0.01 \mathrm{M}$ or $0.5 \mathrm{M}$ were used, the average size of $\mathrm{nZVI}$ was $9.5 \mathrm{~nm}$ and $45 \mathrm{~nm}$, respectively. ${ }^{145}$ The surface area of $\mathrm{nZVI}$ is also affected by solvent and dispersion agent. The presence of ethanol as a co-solvent and polyethylene glycol (PEG) as a dispersant reduced the agglomeration ${ }^{147-148}$ and produced particles with uniform size and shape distribution. ${ }^{148}$ Hwang et al. reported that an increase in the addition rate of $\mathrm{BH}_{4}^{-}$produces smaller nanoparticles. For example, when the reductant delivery rates went from $2 \mathrm{~mL} /$ minute and $20 \mathrm{~mL} /$ minute, the particle size decreased from $87.4 \mathrm{~nm}$ to $9.5 \mathrm{~nm} \cdot{ }^{149}$ This was explained that in case of high injection of $\mathrm{BH}_{4}^{-}, \mathrm{nZVI}$ nuclei had fewer opportunities to form and grow due to the fast reduction. The rate of reductant addition also affects the shape of nZVI. For example, spherical particles were formed under slow injection of the reductant $(2 \mathrm{~mL} /$ minute) while particles were non-spherical and scattered under fast injection (20 mL/minute). ${ }^{149}$

\subsubsection{Applications of $\mathrm{nZVI}$}

Over the past 20 years, $\mathrm{nZVI}$ has been considered as a new tool for treating contaminants in water and soil. Given that $\mathrm{nZVI}$ is available, easy to synthesise, and environmentally friendly, it can be applied to remove both organic and inorganic substances. Typically, nZVI has been used for dechlorination of substances such as TCE, 49,150 PCBs, ${ }^{49}, 151$ polybrominated diphenyl ethers, ${ }^{151}$ tetrachloroethene, ${ }^{152}$ and chlorinated methanes. ${ }^{153}$ In addition, nZVI has been also used for heavy metal remediation. ${ }^{154-155}$ The first report of nZVI for nitrate reduction was 
published in $2000 .{ }^{156}$ Since then, several more studies on the reactivity, kinetics, and pathways of nitrate reduction using nZVI have been carried out. ${ }^{41,157-158}$

Despite its numerous applications, nZVI has potential drawbacks. nZVI can be hazardous to organisms, ${ }^{159}$ and might cause environmental risks. The cytotoxicity of nZVI with organisms proceeds via two pathways, including physical damage and biochemical destruction. ${ }^{160}$ The former includes the destruction of the cell membrane integrity and enhancement of the permeability of membranes. The latter consists of the decomposition of protein functional groups, competition with respiration as well as damage of DNA and protein due to the production of reactive oxygen species from the nZVI corrosion. Moreover, when $\mathrm{nZVI}$ adsorbs and accumulates on the root surface of plants, it can influence the water and nutrient uptake, inhibiting the growth of plants. ${ }^{161} \mathrm{nZVI}$ can persist in biological systems and cause long-term effect due to its low solubility. ${ }^{122}$ Other concerns of $\mathrm{nZVI}$ are its stability and storability. Due to the magnetic and electrostatic forces of $n Z \mathrm{VI}$ particles, they tend to agglomerate to forms larger particles. ${ }^{127,162}$ This results in a decrease in surface areas (active sites) for contaminant adsorption and reduction. In addition, given its high reactivity, nZVI is also oxidised by nontarget compounds to form a thicker layer of oxides and hydroxides on the surface. ${ }^{134}$

\subsubsection{Reduction of nitrate by $n Z V I$}

The standard reduction potential of iron $(\mathrm{Fe}(\mathrm{II}) / \mathrm{Fe})$ is $\mathrm{E}^{0}=-0.44 \mathrm{~V} .{ }^{48}$ The reduction potential values of nitrate/N-products are different and more significantly positive than the reduction potential of $\mathrm{Fe}(0)$ (Table 1.1). ${ }^{163-164} \mathrm{Fe}(0)$ can reduce nitrate to potentially form a variety of products, depending on reaction conditions. Of these, four main $\mathrm{N}$-containing products, including nitrite $\left(\mathrm{NO}_{2}{ }^{-}\right)$, nitrous oxide gas $\left(\mathrm{N}_{2} \mathrm{O}\right)$, nitrogen gas $\left(\mathrm{N}_{2}\right)$, and ammonium $\left(\mathrm{NH}_{4}{ }^{+}\right)$can be formed (Scheme 1.1). Nitrite is toxic to humans while nitrous oxide is a greenhouse gas which contributes to global warming. Dinitrogen gas is non-toxic and the ideal product. Ammonium is less mobile than nitrate due to its adsorption capacity in the soil but it can undergo nitrification back to nitrite and then nitrate. As such, if ammonium is not trapped, its formation only temporarily removes nitrogen from the water. Typically, ammonium is reported as the predominant final product in the $\mathrm{nZVI}$ reduction of nitrate. ${ }^{145,165-167}$ 


$\begin{array}{cccccc}\mathrm{NO}_{3}^{-} \longrightarrow & \mathrm{NO}_{2}^{-} \longrightarrow & \mathrm{N}_{2} \mathrm{O} & \mathrm{N}_{2} \longrightarrow & \mathrm{NH}_{4}^{+} \\ +5 & +4 & +1 & 0 & -3 \\ \text { nitrate } & \text { nitrite } & \begin{array}{l}\text { nitrous } \\ \text { oxide }\end{array} & \text { nitrogen } & \text { ammonium }\end{array}$

Scheme 1.1. Common nitrate reduction products and their oxidation states.

Table 1.1. Redox couples and $\mathrm{E}^{0}$ of nitrogen compounds in acidic and basic conditions.

\begin{tabular}{|c|c|c|}
\hline Redox couple & Condition & $E^{0}(\mathrm{~V})$ \\
\hline $\mathrm{NO}_{3}{ }^{-} / \mathrm{NO}_{2}{ }^{-}$ & acidic & +0.49 \\
\hline $\mathrm{NO}_{3}{ }^{-} / \mathrm{N}_{2}$ & acidic & +1.25 \\
\hline $\mathrm{NO}_{3}{ }^{-} / \mathrm{NH}_{4}{ }^{+}$ & acidic & +0.88 \\
\hline $\mathrm{NO}_{3}{ }^{-} / \mathrm{N}_{2}$ & basic & +0.43 \\
\hline $\mathrm{NO}_{3}{ }^{-} / \mathrm{NH}_{3}$ & basic & -0.01 \\
\hline
\end{tabular}

\subsubsection{Removal of heavy metals by nZVI}

$\mathrm{nZVI}$ removes heavy metals via different pathways, depending on their reduction potential. These pathways include adsorption, complex formation, reduction, or a combination of these (Figure 1.2). ${ }^{116}$ Typically, metals that have reduction potential close to that of iron adsorb on the surface of the iron or form complexes with iron. In aquatic systems, the adsorption depends on solution chemistry such as $\mathrm{pH}$ due to its effect on the charge of iron oxides. For example, at high $\mathrm{pH}$, iron oxides are negatively charged, resulting in complexes with cations such as $\mathrm{Zn}(\mathrm{II})$ and $\mathrm{Cd}(\mathrm{II}) .{ }^{116,154} \mathrm{nZVI}$ is also used to remove $\mathrm{AsO}_{2}{ }^{-}$via the adsorption even though the reduction potential of $\mathrm{HAsO}_{2} / \mathrm{As}\left(\mathrm{E}^{0}=+0.24 \mathrm{~V}\right)$ is more positive than that of $\mathrm{Fe} .{ }^{168}$ Metals that have considerably more positive reduction potential than Fe such as $\mathrm{Ag}(\mathrm{I}) / \mathrm{Ag}\left(\mathrm{E}^{0}\right.$ $=+0.80 \mathrm{~V}), \mathrm{Cu}(\mathrm{II}) / \mathrm{Cu}\left(\mathrm{E}^{0}=+0.34 \mathrm{~V}\right), \mathrm{Hg}(\mathrm{II}) / \mathrm{Hg}\left(\mathrm{E}^{0}=+0.85 \mathrm{~V}\right)$ are reduced by $\mathrm{Fe}(0) .{ }^{116}$ Metals with slightly more positive reduction potential can be removed by reduction or a combination of reduction, adsorption and co-precipitation. For example, $\mathrm{Ni}(\mathrm{II}) / \mathrm{Ni}\left(\mathrm{E}^{0}=-0.23 \mathrm{~V}\right)$ was removed by $\mathrm{nZVI}$ via both reduction and adsorption. ${ }^{116}$ Although the reduction potential of $\mathrm{Pb}(\mathrm{II}) / \mathrm{Pb}\left(\mathrm{E}^{0}=-0.13 \mathrm{~V}\right)$ is more positive than that of $\mathrm{Fe}(\mathrm{II}) / \mathrm{Fe}, \mathrm{Pb}(\mathrm{II})$ is removed via different pathways such as reduction, ${ }^{117}$ both reduction and adsorption, ${ }^{116,154}$ or a combination of 
reduction, adsorption and co-precipitation. ${ }^{169}$ Antimony, $\mathrm{SbC}_{4} \mathrm{H}_{4} \mathrm{O}_{7}-$ and $\mathrm{H}_{2} \mathrm{Sb}_{2} \mathrm{O}_{7}{ }^{2-}$, is removed via co-precipitation ${ }^{170}$ and $\mathrm{Cr}_{2} \mathrm{O}_{7}{ }^{2-}$ is removed via reduction ${ }^{117}$ or a combination of reduction and precipitation. ${ }^{171}$

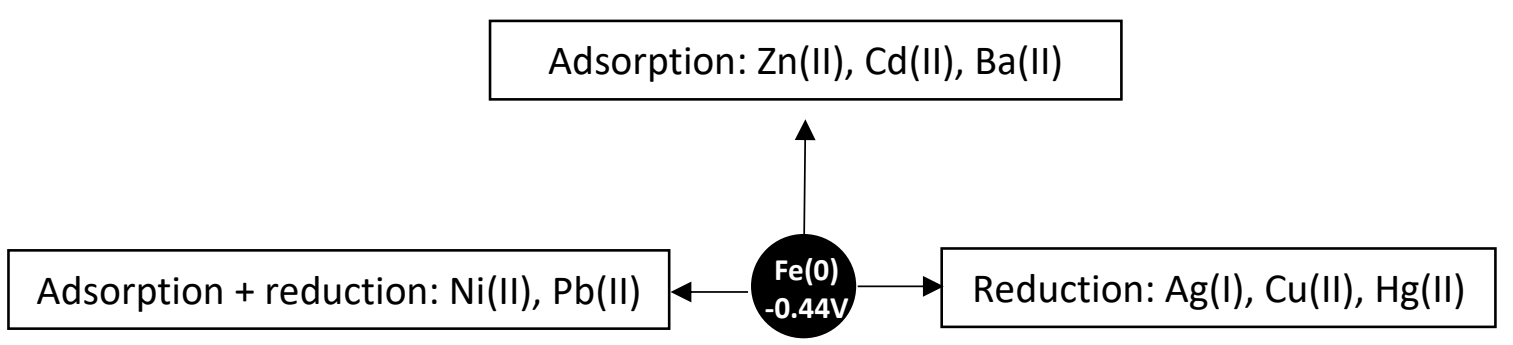

Figure 1.2. Pathway of heavy metal removal by $n Z V I .{ }^{116-117,154}$

\subsection{Bimetallic $n Z V I$ and trimetallic $(n) Z V I$}

In order to increase the chemical reactivity of $n Z \mathrm{VI}$, a secondary metal $(\mathrm{M})$ can be added to generate bimetallic $\mathrm{nZVI}$ systems (M-nZVI). The secondary metal acts as a catalyst for contaminant reduction by lowering the activation barrier for this reaction. ${ }^{2-3,6}$ Additionally, the introduction of secondary metal also slows down the corrosion of iron on the surface of $\mathrm{nZVI}$, which leads to remain the number of nZVI active sites. ${ }^{172-173}$ Thus, bimetallic nZVI can enhance the reaction rate and degradation yield.

Several studies on bimetallic nZVI (M-nZVI) with different secondary metals have been reported. nZVI was formed from the precursor salts of either $\mathrm{FeSO}_{4}$ or $\mathrm{FeCl}_{3}$. The secondary metals $(\mathrm{M})$ are $\mathrm{Ni}, \mathrm{Cu}, \mathrm{Pd}, \mathrm{Ag}, \mathrm{Au}, \mathrm{Bi}$, and $\mathrm{Pt}$ that are from their salts of chloride, sulfate and acetate. Bimetallic M-nZVI can be synthesised by two different methods. ${ }^{2,126,167,174-175}$ The co-reduction method (MA) is a one-step procedure in which both iron and the dopant ions are simultaneously reduced (Scheme 1.2). ${ }^{2}$ The deposition method (MB) is a two-step process in which $\mathrm{nZVI}$ is initially synthesised, followed by the secondary metal being deposited on the surface of nZVI (Scheme 1.3). 126, 167, 174-175

$$
\underset{\mathrm{M}^{\mathrm{m}+}}{\mathrm{Fe}^{\mathrm{n}+}} \stackrel{\mathrm{NaBH}_{4}}{\longrightarrow} \mathrm{M-nZVI}
$$

$n$ : oxidation state of iron $(n=2,3) ; m$ : oxidation state of secondary metals ( $m=1,2$, and 3 )

Scheme 1.2. Bimetallic M-nZVI@Misi synthesised using co-reduction method (MA). 


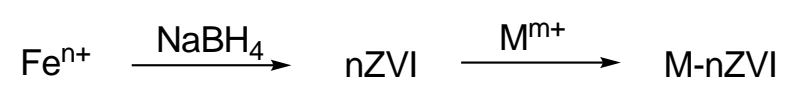

$n$ : oxidation state of iron $(n=2,3) ; m$ : oxidation state of secondary metals ( $m=1,2$, and 3 )

Scheme 1.3. Bimetallic M-nZVI@Misi synthesised using deposition method (MB).

\subsubsection{Bimetallic Ni-nZVI}

One of the common bimetallic systems is Ni-nZVI, which is synthesised from a variety of precursor salts, such as $\mathrm{NiSO}_{4}, \mathrm{NiCl}_{2}$, and $\mathrm{Ni}\left(\mathrm{CH}_{3} \mathrm{COO}\right)_{2},{ }^{176}$ and a range of $\mathrm{Ni}$ :Fe ratios. The mass

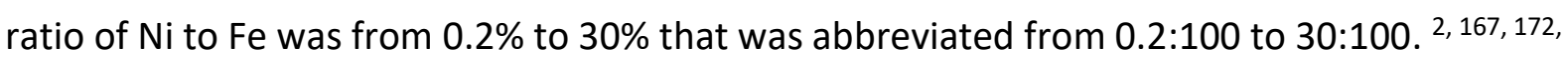
175-177 For example, $30 \% \mathrm{Ni}-\mathrm{nZVI}$ means that the mass ratio of $\mathrm{Ni}$ to $\mathrm{Fe}$ is $30: 100$. These materials were synthesised using both co-reduction (MA) and deposition methods (MB). The resulting Ni-nZVI systems were used for removing organic and inorganic substances. The addition of $\mathrm{Ni}$ has been reported to enhance the reduction rate of contaminants.

A study by Wang et al. showed that $20 \% \mathrm{Ni}-\mathrm{nZVI}$ synthesised using the deposition method (MB) effectively reduced trichloroethylene (TCE). After 36 hours, this Ni-nZVI system removed TCE faster than non-doped nZVI. However, after 504 hours, the same yield was achieved with or without the presence of $\mathrm{Ni}^{3}{ }^{3}$ This indicated that dopant $\mathrm{Ni}$ only had function in increasing the reaction rate but did not have higher yield when the reaction was examined in prolonged time.

$\mathrm{Ni}-\mathrm{nZVI}$ systems have received a lot attention in nitrate reduction. Several studies on the reactivity of $\mathrm{Ni}-\mathrm{nZVI}$ towards nitrate reduction in terms of $\mathrm{Ni}$ contents, synthetic methods, and reaction conditions were carried out. For instance, Kang et al. examined Ni-nZVI systems with various $\mathrm{Ni}$ amounts $(1 \%, 5 \%, 10 \%, 20 \%) .{ }^{2}$ The reactivity of materials was a function of the amount of $\mathrm{Ni}$, synthetic method, and solution $\mathrm{pH}$. Of these materials, $5 \% \mathrm{Ni}-\mathrm{nZVI}$ was the most effective material because this optimal $\mathrm{Ni}$ amount was ideal for maximising the number of active sites. In addition, Ni-nZVI materials prepared via the co-reduction method (MA) had higher reactivity than those prepared via the deposition method (MB). When the initial concentration was 80 ppm nitrate-N and nZVI dosage was $1.5 \mathrm{~g} / \mathrm{L}$, all nitrate was reduced using materials prepared via the co-reduction method (MA) after 40 minutes. Approximately $70 \%$ of nitrate was reduced using materials prepared via the deposition method (MB) after 120 minutes. Furthermore, at a neutral pH of $6.5, \mathrm{Ni}-\mathrm{nZVI}$ reduced nitrate more quickly than under either acidic $(\mathrm{pH} 2.0$ and 4.0) or basic $(\mathrm{pH} \mathrm{9.0)} \mathrm{conditions.} \mathrm{The} \mathrm{nitrate} \mathrm{removal} \mathrm{was}$ 
$100 \%, 91 \%$, and $50 \%$ at $\mathrm{pH} 6.5, \mathrm{pH} 4$, and $\mathrm{pH} 9$, respectively. In contrast, another study on $\mathrm{Ni}-$ $\mathrm{nZVI}$ with the Ni content of $5 \%$ to $30 \%$ showed that $10 \% \mathrm{Ni}-\mathrm{nZVI}$ was the highest performing material when these materials were prepared via the deposition method (MB). ${ }^{175}$ The reactivity of $\mathrm{Ni}-\mathrm{nZVI}$ was also affected by $\mathrm{pH}$, but acidic conditions $(\mathrm{pH} \leq 3)$ were more favourable for nitrate reduction. When the nitrate concentration of 100 ppm (22.6 ppm nitrate- $\mathrm{N}), \mathrm{pH} 2$, and $0.4 \mathrm{~g} / \mathrm{L}$ of particles, all nitrate was removed after 20 minutes.

In a separate study, three types of $0.2 \% \mathrm{Ni}-\mathrm{nZVI}$ synthesised via the deposition method (MB) were examined towards nitrate reduction, including fresh (F-Ni-nZVI), dried (D-Ni-nZVI) and dried-sonicated (DS-Ni-nZVI). The material dosage was $10 \mathrm{~g} / \mathrm{L}$ and the initial nitrate concentrations were from $10 \mathrm{ppm}$ to $10,000 \mathrm{ppm}$. The highest performing material was $\mathrm{F}-\mathrm{Ni}$ $\mathrm{nZVI}$ while the lowest performing material was D-Ni-nZVI. ${ }^{167}$ The difference in reactivity was attributed to the levels in agglomeration of materials. This is because the fresh material had the lowest level of agglomeration while more aggregates were formed during the drying process. Sonication broke large aggregates in dried material into smaller ones. D-Ni-nZVI and DS-Ni-nZVI reduced nitrate faster than D-nZVI and DS-nZVI, respectively due to the presence of Ni catalyst. In contrast, F-Ni-nZVI and F-nZVI had the same nitrate reduction rate even though the initial nitrate concentration was up to $10,000 \mathrm{ppm}$. Ryu et al. postulated that the influence of agglomeration was more significant than that of catalyst $\mathrm{Ni}$ and that the role of the catalyst was minimal when the level of the agglomeration was low.

Ni-nZVI materials were also used for heavy metal removal. They were prepared using both co-reduction (MA) and deposition methods (MB) with the mass concentrations of Ni of $20 \%$ 30\%. ${ }^{172,} 177$ The heavy metals examined concluded $\mathrm{Pb}(\mathrm{II})$ and $\mathrm{Cr}(\mathrm{VI})$. The removal of heavy metals was investigated against variables such as the concentration of heavy metals, solution $\mathrm{pH}$, and material dosage. In Saberi's study, Ni-nZVI with the molar ratio of Ni to Fe was 1:3.5 (mass ratio 0.3:1) prepared via the co-reduction method (MA) was examined towards $\mathrm{Pb}(\mathrm{II})$ removal. ${ }^{177}$ With an increase of the initial $\mathrm{pH}$ of the solution $(1-5)$, particles dosage and contact time, the $\mathrm{Pb}(\mathrm{II})$ removal increased using both either $\mathrm{Ni}-\mathrm{nZVI}$ and $\mathrm{nZVI}$. In contrast, when initial $\mathrm{Pb}(\mathrm{II})$ concentration increased, the $\mathrm{Pb}(\mathrm{II})$ removal decreased. The maximum removal efficiency (100\%) was achieved at a $\mathrm{pH}$ of $5, \mathrm{~Pb}(\mathrm{II})$ concentration at $500 \mathrm{ppm}$ and material dosage at $3.75 \mathrm{~g} / \mathrm{L}$ in 4 hours. Interestingly, more Ni-nZVI particles than $\mathrm{nZVI}$ was 
required in the same volume of $\mathrm{Pb}$ (II) solution. The mechanism of $\mathrm{Pb}$ (II) removal was adsorption, in which both Ni-nZVI and nZVI were used as the adsorbents.

Bimetallic Ni-nZVI was also used for $\mathrm{Cr}(\mathrm{VI})$ removal. In this study, Ni-nZVI with the mass ratio of $\mathrm{Ni}$ to Fe of 0.2:1 was prepared via the deposition method (MB). ${ }^{172}$ The removal of $\mathrm{Cr}(\mathrm{VI})$ depended on the solution $\mathrm{pH}$. When the initial $\mathrm{Cr}(\mathrm{VI})$ concentration was $25 \mathrm{ppm}$ and material dosage was $1 \mathrm{~g} / \mathrm{L}, \mathrm{Cr}(\mathrm{VI})$ was removed faster at $\mathrm{pH} 2$ (96.33\%) than at $\mathrm{pH} 7$ (60.31\%). Ni-nZVI removed $\mathrm{Cr}(\mathrm{VI})$ faster than $\mathrm{nZVI}$, regardless of solution $\mathrm{pH}$. The mechanism of $\mathrm{Cr}(\mathrm{VI})$ removal was proposed via both adsorption and reduction. $\mathrm{Cr}(\mathrm{VI})$ adsorbed on the surface of $\mathrm{nZVI}$ and then was reduced by $\mathrm{nZVI}$ with the catalysis of $\mathrm{Ni}$ to form $\mathrm{Cr}$ (III). $\mathrm{Cr}$ (III) was then captured on the surface of $n Z \mathrm{VI}$ via adsorption, precipitation of $\mathrm{Cr}(\mathrm{OH})_{3}$, and co-precipitation of $\mathrm{Cr}_{x} \mathrm{Fe}_{1}$ ${ }_{x}(\mathrm{OH})_{3}$.

In summary, the best Ni content in Ni-nZVI systems was not clear, depending on the reaction conditions. In many cases, the $\mathrm{Ni}$ concentration chosen was not explained. In the nitrate reduction, there was an argument in the effect of the solution $\mathrm{pH}$. The material dosages were different, from $0.4 \mathrm{~g} / \mathrm{L}$ to $10 \mathrm{~g} / \mathrm{L}$ and different initial nitrate concentrations were used, from $10 \mathrm{ppm}$ to $10,000 \mathrm{ppm}$. Even though $\mathrm{Ni}$ was used in different reaction conditions, the introduction of $\mathrm{Ni}$ was effective at increasing reaction rate in most of the cases compared to nZVI. In the removal of heavy metals, the material dosage was from $1 \mathrm{~g} / \mathrm{L}$ and $3.75 \mathrm{~g} / \mathrm{L}$. The acid media was favourable for removing heavy metals, regardless of the mechanism of metal removal. 
Table 1.2. Ni-nZVI systems reported. Note: $0.2 \% \mathrm{Ni}$ means the mass ratio of $\mathrm{Ni}$ to $\mathrm{Fe}$ is $0.2: 100$.

\begin{tabular}{|c|c|c|c|}
\hline \multirow{2}{*}{ Ni content } & \multicolumn{2}{|c|}{ Method } & \multirow{2}{*}{ Contaminant } \\
\cline { 2 - 4 } & co-reduction & deposition & \\
\hline $0.2 \%$ & & $X$ & nitrate \\
\hline $1 \%$ & $\mathrm{X}$ & $\mathrm{X}$ & nitrate \\
\hline $2 \%$ & $\mathrm{X}$ & $\mathrm{X}$ & nitrate \\
\hline $5 \%$ & $\mathrm{X}$ & $\mathrm{X}$ & nitrate \\
\hline $10 \%$ & $\mathrm{X}$ & $\mathrm{X}$ & nitrate \\
\hline $20 \%$ & $\mathrm{X}$ & $\mathrm{X}$ & $\mathrm{TCE}, \mathrm{Pb}(\mathrm{II})$ and $\mathrm{Cr}(\mathrm{VI})$ \\
\hline $30 \%$ & $\mathrm{X}$ & $\mathrm{Pb}(\mathrm{II})$ and $\mathrm{Cr}(\mathrm{VI})$ \\
\hline
\end{tabular}

\subsubsection{Bimetallic Cu-nZVI}

Numerous studies have investigated bimetallic $\mathrm{CU}-\mathrm{nZVI}$ systems with the $\mathrm{Cu}$ concentration from $1 \%$ to $20 \%$. Similar to $\mathrm{Ni}-\mathrm{nZVI}$ systems, $1 \% \mathrm{Cu}-\mathrm{nZVI}$ means the mass ratio of $\mathrm{Cu}$ to $\mathrm{nZVI}$ was 1:100. All of the materials were synthesised using the deposition method (MB) with either $\mathrm{CuCl}_{2}$ or $\mathrm{CuSO}_{4}$ as the $\mathrm{Cu}$ source. ${ }^{41,178-181}$ The resulting $\mathrm{Cu}-\mathrm{nZVI}$ was used for nitrate reduction under neutral and acidic conditions. The introduction of $\mathrm{Cu}$ enhanced the reactivity of $\mathrm{nZVI}$, leading to a higher amount of nitrate reduced. For example, the reactivity of materials depended on Cu levels (1\% - 20\%), with 5\%Cu-nZVI found to be the highest efficiency material in increasing the reaction rate. ${ }^{179}$ When the initial nitrate concentration was 80 ppm (18 ppm nitrate- $\mathrm{N}$ ) and the bimetallic dosage was $1.4 \mathrm{~g} / \mathrm{L}$, all nitrate was reduced within 20 minutes under neutral pH's. Muradova et al. also investigated nitrate reduction of 57.5 ppm (13 ppm of nitrate-N) and the mass ratio of Fe to nitrate of 15:1. ${ }^{182}$ All nitrate was reduced after 60 minutes using $5 \% \mathrm{Cu}$-nZVI while $98 \%$ of nitrate was removed after 150 minutes using nZVI. The $\mathrm{pH}$ increased from 5 to a level between 8 and 9 as the final $\mathrm{pH}$ values. In a separate study, Krasae et al.'s study showed that $2.5 \% \mathrm{Cu}-\mathrm{nZVI}$ reduced nitrate under either acidic or neutral conditions. ${ }^{178}$ After 30 minutes, when $10 \mathrm{ppm}$ nitrate- $\mathrm{N}$ was used, the reduction yields were $50 \%$ and $42 \%$ at $\mathrm{pH} 3$ and $\mathrm{pH} \mathrm{7,} \mathrm{respectively.} \mathrm{In} \mathrm{contrast,} \mathrm{the} \mathrm{nitrate} \mathrm{removal} \mathrm{was} 14 \%$ and $11 \%$ when nZVI was used, respectively. 
In summary, CU-nZVI has been examined; however, the investigation of different methods to synthesise CU-nZVI systems and the optimal Cu concentration was not examined in detail. In comparison with $\mathrm{nZVI}$, the presence of $\mathrm{Cu}$ enhanced reactivity but this difference was not much. ${ }^{181}$ The generation of nitrite during nitrate reduction has not monitored. Hence, more studies need to be done to examine the effectiveness of $\mathrm{Cu}$ as well as to optimise the $\mathrm{Cu}$ concentration.

Table 1.3. Cu-nZVI systems reported. Note: all systems were synthesised using the deposition method and 1\%Cu-nZVI means the mass ratio of $\mathrm{Cu}$ to Fe is 1:100.

\begin{tabular}{|c|c|}
\hline Material & Contaminant \\
\hline $1 \% \mathrm{Cu}-\mathrm{nZVI}$ & nitrate \\
\hline $2.5 \% \mathrm{Cu}-\mathrm{nZVI}$ & nitrate \\
\hline $5 \% \mathrm{Cu}-\mathrm{nZVI}$ & nitrate \\
\hline $20 \% \mathrm{Cu}-\mathrm{nZVI}$ & nitrate \\
\hline
\end{tabular}

\subsubsection{Bimetallic $n Z V I$ of other metals}

Studies have also investigated the degradation of contaminants using Pd-nZVI systems prepared via the deposition method (MB). The Pd contents were examined from $0.054 \%$ to $50 \%$ with either $\mathrm{Pd}\left(\mathrm{CH}_{3} \mathrm{COO}\right)_{2}$ or $\mathrm{K}_{2} \mathrm{PdCl}_{6}$ as the palladium source. ${ }^{49}, 183-186 \mathrm{Pd}-\mathrm{nZVI}$ systems were commonly used for reducing halogenated organic compounds. A Pd-nZVI system (unpublished ratio) was used to reduce TCE and PCBs. ${ }^{49}$ Separate studies also examined the reactivity of TCE using either 5\%Pd-nZVI or Pd-nZVI (1\% - 50\%). ${ }^{183}$ Other studies used $0.054 \%$ Pd-nZVI for reducing PCP in the presence of some ions such as sulfate, $\mathrm{Cu}(\mathrm{II}), \mathrm{Ni}(\mathrm{II})$ and $\mathrm{Fe}(\mathrm{III}),{ }^{184} 0.2 \% \mathrm{Pd}-\mathrm{nZVI}$ for removing chlorinated methanes, ${ }^{185}$ and $0.5 \% \mathrm{Pd}-\mathrm{nZVI}$ for dechlorinating chlorophenols. ${ }^{186}$ In addition, Bi-nZVI systems with different Bi:Fe ratios $(0 \%<$ $\mathrm{Bi} / \mathrm{Fe} \leq 7 \%)$ were prepared via the co-reduction method $(\mathrm{MA})$ to reduce hexahydro-1,3,5trinitro-1,3,5-triazine (RDX). ${ }^{187}$ Degradation of RDX was significantly dependent on the $\mathrm{Bi}$ concentrations and the highest degradation rate was achieved at 4\%Bi-nZVI. All Pd-nZVI and $\mathrm{Bi}-\mathrm{nZVI}$ were reported to only remove organic substances via reduction. 
$\mathrm{nZVI}$ systems doped with $1 \% \mathrm{Ag}$ or $1 \% \mathrm{Au}$ prepared via the deposition method $(\mathrm{MB})$ were examined for simultaneously removing $\mathrm{Cd}(\mathrm{II})$ and nitrate. ${ }^{188}$ The rates of nitrate reduction using these systems were higher than that of nondoped nZVI. Au-nZVI exhibited the highest rate and lowest nitrite build-up. When the initial nitrate- $\mathrm{N}$ concentration was $45 \mathrm{ppm}$, over $77 \%$ nitrate was reduced and only $6 \%$ nitrite accumulation was observed in the presence of $30 \mathrm{ppm}$ of $\mathrm{Cd}(\mathrm{II})$. Bimetallic Pt-nZVI using $\mathrm{PtCl}_{4}$ as the Pt source synthesised via the coreduction method (MA) was also examined. ${ }^{189}$ In this reported system, the molar ratio of Pt to Fe was 1:1. When the initial nitrate concentration was $200 \mathrm{ppm}$ (45 ppm nitrate-N) and nanoparticle dosage was $0.25 \mathrm{~g} / \mathrm{L}, 97 \%$ of nitrate was reduced at $\mathrm{pH} 7$ after 120 minutes. This Pt-nZVI was dramatically reactive compared to using anion exchange resins. However, due to the very high concentration of Pt (molar ratio of Pt to Fe was 1:1) and the cost of Pt, this material will not be applicable in nitrate reduction on a large scale.

Table 1.4. Other systems reported.

\begin{tabular}{|c|c|c|c|}
\hline \multirow{2}{*}{ Material } & \multicolumn{2}{|c|}{ Method } & \multirow{2}{*}{ Contaminant } \\
\cline { 2 - 4 } & Co-reduction & Deposition & \\
\hline $0.054 \%$ Pd-nZVI & & $X$ & PCP \\
\hline $0.2 \% \mathrm{Pd}-\mathrm{nZVI}$ & & $\mathrm{X}$ & chlorinated methanes \\
\hline $0.5 \% \mathrm{Pd}-\mathrm{nZVI}$ & & $\mathrm{X}$ & TCE \\
\hline $\begin{array}{c}\text { Pd-nZVI } \\
(1 \%-50 \%)\end{array}$ & & & RDX \\
\hline $\begin{array}{c}\mathrm{Bi}-\mathrm{nZVI} \\
0 \%<\mathrm{Bi} / \mathrm{Fe} \leq 7 \%\end{array}$ & $\mathrm{X}$ & & \\
\hline
\end{tabular}

\subsubsection{Trimetallic $\mathrm{ZVI}$ and trimetallic $\mathrm{nZVI}$}

Trimetallic ZVI (micro size) has been synthesised by doping bimetallic on the surface of ZVI particles via the co-reduction $\left(\mathrm{MA}_{\text {tri }}\right)$ and the deposition method $(\mathrm{MC}) .{ }^{190}$ The co-reduction process is a one-step procedure in which iron and two dopants are simultaneously reduced (Scheme 1.4). The deposition method is a three-step process in which ZVI is synthesised and then successively deposited of $M_{2}$ and $M_{1}$ on its surface (Scheme 1.5). 


$$
\text { ZVI } \stackrel{M_{1} X_{2}+M_{2} X_{2}}{\longrightarrow} M_{1}-M_{2}-Z V I
$$

Scheme 1.4. Trimetallic $M_{1}-M_{2}-Z V I @ M i s i$ synthesised using co-reduction method ( $\left.M A_{\text {tri }}\right)$.

$$
\mathrm{ZVI} \stackrel{\mathrm{M}_{2} \mathrm{X}_{2}}{\longrightarrow} \mathrm{M}_{2}-\mathrm{ZVI} \stackrel{\mathrm{M}_{1} \mathrm{X}_{2}}{\longrightarrow} \mathrm{M}_{1}-\mathrm{M}_{2}-\mathrm{ZVI}
$$

Scheme 1.5. Trimetallic $M_{1}-M_{2}-Z V I @ M i s i$ synthesised using deposition method (MC).

In Liou et al.'s study, the reactivity of microsized Pd-Cu-ZVI, Pt-Cu-ZVI, and Au-Cu-ZVI in reducing $40 \mathrm{ppm}$ nitrate- $\mathrm{N}$ in 4-(2-hydroxyethyl)-1-piperazineethanesulfonic acid (HEPES) buffered solution was examined. ${ }^{190}$ These materials were prepared via the co-reduction method $\left(\mathrm{MA}_{\text {tri }}\right)$ and the deposition method $(\mathrm{MC})$. The results showed that Pt-Cu-ZVI or Au-CuZVI both had higher reactivity than $\mathrm{Pd}-\mathrm{Cu}-\mathrm{ZVI}$ and that the presence of $\mathrm{Pd}$ increased $\mathrm{N}_{2}$ selectivity. Additionally, the $0.3 \% \mathrm{Pd}-0.5 \% \mathrm{Cu}-\mathrm{ZVI}$ prepared via the deposition method (MC) reduced nitrate faster and produced more nitrogen gas than that prepared via the coreduction method $\left(\mathrm{MA}_{\text {tri }}\right.$ ). After 360 minutes, when the optimal conditions of $\mathrm{pH}$ were 8 - 8.5, the nitrate conversion and nitrogen gas selectivity of the material prepared via the deposition method (MC) were $91 \%$ and $30 \%$, respectively. However, for the materials prepared via the co-reduction method $\left(\mathrm{MA}_{\text {tri }}\right)$, the nitrate conversion and nitrogen gas selectivity were $50 \%$ and $8.8 \%$, respectively.

Other studies have also investigated nitrate reduction using two types of trimetallic nZVI, including Cu-Pd-nZVI and Pd-Cu-nZVI prepared via the deposition method (MC). ${ }^{4-5}$ Even though trimetallic systems had a slightly lower reduction rate than bimetallic and monometallic systems, they resulted in higher nitrogen gas selectivity. For instance, Lubphoo et al.'s study showed that nitrate reduction using Pd-Cu-nZVI depended on initial nitrate concentrations, $\mathrm{pH}$, temperature, and the presence of hydrogen gas. ${ }^{4}$ In this study, the initial nitrate concentration was $100 \mathrm{ppm}\left(22.6 \mathrm{ppm}\right.$ of nitrate- $\mathrm{N}$ ), the solution $\mathrm{pH}$ was 7 at $50^{\circ} \mathrm{C}$ and in the presence of hydrogen gas. After 60 minutes, $90 \%$ of nitrate was removed and nitrogen gas selectivity was highest at $60.5 \%$. Lubphoo et al.'s also investigated the effect of Pd and Cu contents and the order of the deposition on nitrate reduction. ${ }^{5} \mathrm{Pd}-\mathrm{Cu}-\mathrm{nZVI}$ which contains $10 \%$ of deposited metals and a mass ratio of $\mathrm{Pd}$ to $\mathrm{Cu}$ at 2:1 had the highest reactivity and nitrogen gas selectivity. When the initial nitrate concentration was 100 ppm (22.6 ppm of 
nitrate- $\mathrm{N}$ ), the particles dosage was $0.25 \mathrm{~g} / \mathrm{L}$, the nitrate removal was $63 \%$ and the ratio of nitrogen gas to ammonium was 41:51 after 60 minutes.

In summary, trimetallic materials of $\mathrm{ZVI}$ and $\mathrm{nZVI}$ have been reported to promote the production of nitrogen gas. However, only unsupported trimetallic was investigated. In addition, the difference in the reactivity of these materials prepared using different methods as well as different versions of the deposition methods has not been investigated.

Table 1.5. Trimetallic ZVI and nZVI systems reported. Note: all systems were used for nitrate reduction.

\begin{tabular}{|c|c|c|}
\hline \multirow{2}{*}{ Material } & \multicolumn{2}{|c|}{ Method } \\
\cline { 2 - 3 } & Co-reduction & Deposition \\
\hline $0.3 \% \mathrm{Pd}-0.5 \% \mathrm{Cu}-\mathrm{ZVI}$ & $\mathrm{X}$ & $\mathrm{X}$ \\
\hline $0.3 \% \mathrm{Pt}-0.5 \% \mathrm{Cu}-\mathrm{ZVI}$ & $\mathrm{X}$ & $\mathrm{X}$ \\
\hline $0.3 \% \mathrm{Au}-0.5 \% \mathrm{Cu}-\mathrm{ZVI}$ & & $\mathrm{X}$ \\
\hline $\mathrm{Cu}-\mathrm{Pd}-\mathrm{nZVI}$ & & $\mathrm{X}$ \\
\hline $\mathrm{Pd}: \mathrm{Cu}=2: 1 ;(\mathrm{Pd}+\mathrm{Cu})=10 \%$ & & \\
\hline $\mathrm{Pd}-\mathrm{Cu}-\mathrm{nZVI}$ & & \\
\hline $\mathrm{Pd}: \mathrm{Cu}=2: 1 ;(\mathrm{Pd}+\mathrm{Cu})=10 \%$ & & \\
\hline
\end{tabular}

\subsection{Supported bimetallic nZVI}

The addition of metal(s) to nZVI enhanced the reactivity of bimetallic and trimetallic materials. However, these systems still have agglomeration problem. ${ }^{191}$ One possible solution is to place the nanoparticles onto some types of support that will increase the distribution of particles and minimise agglomeration. ${ }^{191-192}$ In addition, depositing nanoparticles on a support slows down their oxidation with surroundings. ${ }^{191,} 193$ These supported materials have been postulated to enhance their surface area ${ }^{193-194}$ and reactivity. ${ }^{191-195}$ Typically, silica, silicates, and activated carbon are used as supports. The resulting materials have been used to examine the property and reactivity in removing contaminants. 


\subsubsection{Supported bimetallic Ni-nZVI}

Several studies examined supported bimetallic Ni-nZVI materials. They were prepared by doping Ni-nZVI on different supports using both the co-reduction method (MA) and deposition method $(\mathrm{MB})$. The resulting materials were used for removing organic and inorganic substances. For example, bimetallic Ni-nZVI on cellulose acetate was synthesised by initially preparing Ni-nZVI using either the co-reduction method (MA) or the deposition method (MB). ${ }^{196}$ Then, the Ni-nZVI was mixed with cellulose acetate in the solvent to form $\mathrm{Ni}$ nZVI@cellulose acetate. The reactivity of these systems towards TCE removal was examined. The highest TCE removal rate was achieved when the Ni content was between $14.3 \%$ and 21.4\%. Materials prepared using Ni-nZVI synthesised via the deposition method (MB) had higher reactivity than those prepared using Ni-nZVI synthesised via the co-reduction method (MA). The mechanism for the TCE removal was sorption of TCE on cellulose acetate and reduction of TCE by nanoparticles in which Ni was a catalyst and Fe was a reducing agent.

In addition, Ni-nZVI on silica/silicates systems have been reported in nitrate reduction. They were also synthesised using either the co-reduction method (MA) or the deposition method (MB). Typically, natural silicates such as kaolinite and zeolite have been used. He et al.'s study examined Ni-nZVI@zeolite prepared via the co-reduction method (MA) with the mass ratio of Ni:nZVI:zeolite at 1:10:20. ${ }^{193}$ The reactivity of Ni-nZVI@zeolite was slightly sensitive to the initial solution $\mathrm{pH}$. When the solution $\mathrm{pH}$ increased from 3 to 8 , the nitrate removal decreased from $85 \%$ to $72.5 \%$, respectively using the initial nitrate- $\mathrm{N}$ concentration of $20 \mathrm{ppm}$, the $\mathrm{Ni}$ $\mathrm{nZVI}$ dosage of $0.5 \mathrm{~g} / \mathrm{L}$, and reaction time of 4 hours. He et al. also reported that the presence of phosphate diminished the nitrate reduction while nitrate enhanced phosphate removal.

Based on this study, supported Ni-nZVI was effective in nitrate reduction. However, the reaction time was long, up to 4 hours and solution $\mathrm{pH}$ was adjusted. As such, the aim of the investigation of supported Ni-nZVI@Misi in this study is to experience reactions without pH adjustment in shorter reaction time but a higher nZVI dosage of $0.6 \mathrm{~g}$. After that, the effectiveness of Ni-nZVI@Misi will be compared and evaluated.

Supported Ni-nZVI systems have also been used for heavy metal removal, usually $\mathrm{Cr}(\mathrm{VI})$ removal. Lu et al. examined Ni-nZVI on montmorillonite prepared via the co-reduction method (MA). ${ }^{197} \mathrm{Ni}-\mathrm{nZVI@montmorillonite} \mathrm{with} \mathrm{the} \mathrm{mass} \mathrm{ratio} \mathrm{of} \mathrm{Ni} \mathrm{to} \mathrm{nZVI} \mathrm{at} \mathrm{1:10} \mathrm{was} \mathrm{used}$ to remove $\mathrm{Cr}(\mathrm{VI})$. The removal increased when the dosage of the material increased from 0.4 
$\mathrm{g} / \mathrm{L}$ to $0.8 \mathrm{~g} / \mathrm{L}$. At the optimum dosage of $0.8 \mathrm{~g} / \mathrm{L}, \mathrm{pH}$ of 3, and $\mathrm{Cr}(\mathrm{VI})$ concentration of $50 \mathrm{ppm}$, all $\mathrm{Cr}(\mathrm{VI})$ was removed after 180 minutes. In a separate study, Wang et al. doped Ni-nZVI on starch and sodium citrate (SS) using the co-reduction method (MA). ${ }^{198}$ The removal of $\mathrm{Cr}(\mathrm{VI})$ decreased when the solution $\mathrm{pH}$ increased. When the initial $\mathrm{Cr}(\mathrm{VI})$ concentration was $1 \mathrm{ppm}$, the solution $\mathrm{pH}$ was 2 and material dosage was $10 \mathrm{~g} / \mathrm{L}, 95.70 \%$ of $\mathrm{Cr}(\mathrm{VI})$ was removed after 120 minutes. Wang et al. also concluded that $\mathrm{Cr}(\mathrm{VI})$ was removed via two different pathways, adsorption and reduction. This is consistent with the results examining the removal of $\mathrm{Cr}(\mathrm{VI})$ using Ni-nZVI reported by Zhou et al.. ${ }^{172}$ However, in the reduction pathway, both Fe and $\mathrm{Ni}$ served as reductants to convert $\mathrm{Cr}(\mathrm{VI})$ into $\mathrm{Cr}(\mathrm{III})$. After the reaction, an oxide/hydroxide alloy containing Fe(II), Fe(III), Ni(II), and Cr(III) was formed. These supported Ni-nZVI materials were reactive in $\mathrm{Cr}(\mathrm{VI})$ removal; however, the material dosages were large and all these reactions were in acidic conditions. The attempt using lower dosage, $\mathrm{pH}$ without adjustment and reducing reaction time will be examined to test Ni-nZVI@Misi.

Supported Ni-nZVI materials have the ability to simultaneously remove nitrate and heavy metals. In these studies, Ni:Fe:kaolinite was prepared via the co-reduction method (MA) and the mass ratio of Ni:Fe:kaolinite was 1:10:20. ${ }^{192,199} \mathrm{Ni}$-nZVI@kaolinite was used for reducing the solutions containing both nitrate $(180 \mathrm{ppm})$ and $\mathrm{Pb}(\mathrm{II})(300 \mathrm{ppm}) .{ }^{192}$ When the $\mathrm{nZVI}$ dosage was $0.5 \mathrm{~g} / \mathrm{L}, \mathrm{pH}$ was at 4 and temperature was $35^{\circ} \mathrm{C}$, the nitrate and $\mathrm{Pb}(\mathrm{II})$ removal of Ni-nZVI@kaolinite after 16 minutes was $86.3 \%$ and 79.0\%, respectively. In contrast, under the same conditions, the nitrate and Pb(II) removal of nZVI@kaolinite was 73.6\% and 67.9\%, respectively. This indicated that Ni promoted the removal of both nitrate and $\mathrm{Pb}(\mathrm{II})$. Nitrate was removed via the reduction while $\mathrm{Pb}(\mathrm{II})$ was removed via the combination of adsorption, reduction, and co-precipitation.

In Cai et al.'s study, Ni-nZVI@kaolinite was used to simultaneously remove nitrate and $\mathrm{CU}(\mathrm{II}){ }^{199}$ The presence of $\mathrm{CU}(\mathrm{II})$ significantly increased nitrate reduction while nitrate slightly inhibited $\mathrm{Cu}(\mathrm{II})$ removal. Specifically, when the initial nitrate concentration was $40 \mathrm{ppm}$ and the nZVI dosage was $2.48 \mathrm{~g} / \mathrm{L}$, the nitrate removal was $26.9 \%$ and $40.4 \%$ in the absence and presence of $\mathrm{Cu}(\mathrm{II})$ (200 ppm), respectively. When the initial Cu(II) concentration was 200 ppm, the $\mathrm{Cu}(\mathrm{II})$ removal was $99.7 \%$ and $96.5 \%$ in the absence and the presence of nitrate (100 ppm). Cai et al. explained that during the reaction, $\mathrm{Cu}(\mathrm{II})$ was reduced to form $\mathrm{Cu}(0)$ on the surface of bimetallic material. In this form, $\mathrm{Cu}(0)$ was considered as an addition catalyst that helped 
increase the rate of nitrate reduction. In contrast, the reduction of nitrate produced hydroxide ions, which prevents electron transfer from Fe core to $\mathrm{Cu}(\mathrm{II})$. As such, the reduction of $\mathrm{Cu}(\mathrm{II})$ by $\mathrm{nZVI}$ decreased. This is inconsistent with the result examining the simultaneous removal of nitrate and $\mathrm{Pb}(\mathrm{II}){ }^{192}$

Table 1.6. Supported Ni-nZVI reported.

\begin{tabular}{|c|c|c|c|}
\hline \multirow{2}{*}{ Material } & \multicolumn{2}{|c|}{ Method } & \multirow{2}{*}{ Contaminant } \\
\cline { 2 - 4 } & co-reduction & deposition & TCE \\
\hline $\begin{array}{c}\text { Ni-nZVI@cellulose acetate } \\
0 \%<\text { Ni } \leq 21.4 \%\end{array}$ & $X$ & $X$ & nitrate \\
\hline Ni-nZVI@zeolite & $X$ & $X$ & Cr(VI) \\
\hline 1:10:20 & $X$ & & Cr(VI) \\
\hline Ni-nZVI@montmorillonite & $X$ & & Both nitrate and Pb(II) \\
\hline $\begin{array}{c}\text { Ni-nZVI on starch and } \\
\text { sodium citrate (SS) }\end{array}$ & $X$ & & Both nitrate and Cu(II) \\
\hline Ni-nZVI@kaolinite \\
1:10:20
\end{tabular}

\subsubsection{Supported bimetallic Pd-nZVI}

Supported Pd-nZVI systems have been investigated and their reactivity was examined towards organic substance removal. These supports included synthetic silica, natural silicate, activated carbon, and green agents. All these supported Pd-nZVI systems were prepared via the deposition method (MB). The mass ratio of Fe to support ranged from $10 \%$ to $50 \%$ and the mass ratio of Pd to Fe was from $0.1 \%$ to $75 \%{ }^{151,191,200-202}$ For instance, Doong et al. examined Pd-nZVI@mesoporous silica systems in which Fe content was from 10\% to 50\% and Pd content was from $0.1 \%$ to $3 \%{ }^{200}$ The results showed that materials with the ratio of Fe to silica at $30 \%$ and $\mathrm{Pd}$ ranging from $0.5 \%$ to $3 \%$ had the highest reactivity in the dechlorination of tetrachloroethylene. In a separate study, Pd-nZVI@bentonite with the ratio of Pd:Fe:bentonite at 0.75:1:1 was used to remove organic substances such as methyl orange (MO) ${ }^{191}$ and $p$-chlorophenol. ${ }^{201}$ In addition, supported bimetallic Pd-nZVI on granular 
activated carbon was used for removing polybrominated diphenyl ethers (PBDEs) and PCBs. ${ }^{151}$ In Wang et al.'s study, supported bimetallic Pd-nZVI on green agents such as polyethylene glycol, starch, and guar gum was examined. ${ }^{202}$ These materials reduced 2,4-dichlorophenol effectively and their removal was highest with the addition of $0.1 \% \mathrm{Pd}$. In all these studies, organic substances were removed via reduction in which $\mathrm{nZVI}$ was a reductant and Pd served as a catalyst promoting the electron flow from $\mathrm{nZVI}$.

Table 1.7. Supported Pd-nZVI systems. Note: all these systems were synthesised using the deposition method.

\begin{tabular}{|c|c|}
\hline Material & Contaminant \\
\hline Pd-nZVI@mesoporous silica & \\
Fe 10\%-50\% & tetrachloroethylene \\
Pd 0.1\%-3\% & methyl orange \\
\hline Pd-nZVI@bentonite & $p$-chlorophenol \\
\hline P.75:1:1 & PBDEs and PCBs \\
\hline Pd-nZVI@ polyethylene glycol & 2,4-dichlorophenol \\
\hline Pd-nZVI@starch & 2,4-dichlorophenol \\
\hline Pd-nZVI@ guar gum & 2,4-dichlorophenol \\
\hline
\end{tabular}

\subsubsection{Supported bimetallic of other metals}

Supported $\mathrm{nZVI}$ doped with other metals such as $\mathrm{Co}, \mathrm{Cu}$, and $\mathrm{Mn}$ was also examined. For example, Bragança et al. used two types of synthetic silica, hexagonal mesoporous silica and Santa Barbara Amorphous silica to synthesise Co-nZVI@silica materials. ${ }^{203}$ In these materials, the total content of $\mathrm{Co}$ and Fe was $25 \%$. Although their surface area, volume, and diameter of these materials were examined, their reactivity was not examined in this study.

Zeng et al. investigated the reactivity of bimetallic Cu-nZVI and Mn-nZVI on zeolite in nitrate reduction. ${ }^{194}$ Two these systems were synthesised using the deposition method (MB). The mass ratio of total metals to zeolite was $1: 1$. The mass percentage of $\mathrm{Cu}$ was $20 \%$ in $\mathrm{Cu}$ nZVI@zeolite and Mn was 4\% in Mn-nZVI@zeolite. Both Cu-doped and Mn-doped materials 
had higher surface area than unsupported nZVI, leading to higher reactivity in nitrate reduction. Additionally, when the reduction was carried out in $\mathrm{pH} 3-9$, the supported bimetallic materials and nZVI@zeolite were not affected by the initial pH. In contrast, unsupported nZVI was strongly dependent on $\mathrm{pH}$. The initial nitrate-N concentration was 22.6 ppm (100 ppm nitrate), material dosage was $4 \mathrm{~g} / \mathrm{L}$ and $\mathrm{pH}$ was 9 . In this reported system, after 6 hours, more than 94\% of nitrate was removed using either Cu-doped, Mn-doped or non-doped nZVI@zeolite while nitrate removal was 70\% using unsupported nZVI.

There were also supported bimetallic M-nZVI being used for heavy metal removal. For instance, Kakavandi et al. used Ag-nZVI on activated carbon powder prepared via the deposition method $(\mathrm{MB})$ to examine $\mathrm{Cr}(\mathrm{VI})$ removal. ${ }^{204}$ The predominant mechanism of $\mathrm{Cr}(\mathrm{VI})$ removal was adsorption. The solution $\mathrm{pH}$ also strongly affected $\mathrm{Cr}(\mathrm{VI})$ removal. The reactivity of Ag-nZVI@activated carbon powder in acidic conditions was higher than that observed in more basic conditions. When the initial $\mathrm{Cr}(\mathrm{VI})$ concentration was $4 \mathrm{ppm}$ and the dosage of the material was $0.3 \mathrm{~g} / \mathrm{L}$ ( $\mathrm{nZVI}$ dosage of $0.06 \mathrm{~g} / \mathrm{L}$ ), 91.95\% of $\mathrm{Cr}(\mathrm{VI})$ was removed at $\mathrm{pH} 3$ after 60 minutes. Kakavandi et al. explained that at low $\mathrm{pH}$, the surface of Fe was positively charged and $\mathrm{Fe}(0)$ was easily oxidised to form $\mathrm{Fe}(\mathrm{II})$, which promoted the adsorption of $\mathrm{Cr}(\mathrm{VI})$.

Table 1.8. Supported bimetallic nZVI of other metals.

\begin{tabular}{|c|c|c|c|}
\hline \multirow{2}{*}{ Material } & \multicolumn{2}{|c|}{ Method } & \multirow{2}{*}{ Contaminant } \\
\hline & Co-reduction & Deposition & \\
\hline $\begin{array}{l}\text { Co-nZVI@silica } \\
\text { (Co + Fe)=25\% }\end{array}$ & $x$ & & Not examine \\
\hline $\begin{array}{c}\text { Cu-nZVI@zeolite } \\
\% \text { Cu = 20\% } \\
\text { Metals:zeolite = 1:1 }\end{array}$ & & $x$ & nitrate \\
\hline $\begin{array}{c}\text { Mn-nZVI@zeolite } \\
\quad \% M n=4 \% \\
\text { Metals:zeolite = 1:1 }\end{array}$ & & $x$ & nitrate \\
\hline Ag-nZVI@activated carbon powder & & $x$ & $\mathrm{Cr}(\mathrm{VI})$ \\
\hline
\end{tabular}




\subsection{Background of nZVI@Misi}

Research in Fulton group investigated the effectiveness of different silicates such as Misi600 (referred to as Misi), rice husk ash, Western Australia silica fume, and fly ash, on supporting nZVI. Of these, Misi was considered the most effective support. In addition, different pretreatments of Misi prior adsorption of $\mathrm{nZVI}$ were examined. In this section, an overview of Misi and a summary of the results of this study will be included.

\subsubsection{Properties of Misi600}

Misi600 (referred to as Misi) is an amorphous silica from a geothermal deposit. ${ }^{205}$ It was supplied by Golden Bay Cement, a company based in Rotorua, New Zealand. Misi is produced from ignimbrite and pumice breccia during the hydrothermal alterations over 10,000 years. ${ }^{206}$ Although this alteration forms a variety of silicification, Misi in the Rotorua area is unique because it is formed due to the migration of hydrothermal fluids with rich silica under pressure. Despite the majority of silica in Misi, it consists of impurities such as clay, ironsand, alunite, and sulfur. Misi is a natural highly-reactive pozzolan. ${ }^{205}$ Due to its pozzolan property, Misi reacts with $\mathrm{Ca}(\mathrm{OH})_{2}$ solution to form hydrates of calcium silicate or calcium silicate aluminate known as cementitious hydration products. ${ }^{207}$ According to Golden Bay Cement, the main composition of Misi is $\mathrm{Si}$ with $87.99 \%$ of $\mathrm{SiO}_{2}$, followed by $\mathrm{Al}$ with $4.31 \%$ of $\mathrm{Al}_{2} \mathrm{O}_{3}$ (Table 1.9). ${ }^{205}$ Misi also has a small amount of Ti, $\mathrm{Fe}, \mathrm{K}, \mathrm{Ca}, \mathrm{Na}, \mathrm{S}$, and so on. All Misi particles have the size below $100 \mu \mathrm{m}$, in which more than $90 \%$ of particles are below $5 \mu \mathrm{m}$ (Table 1.10).

Table 1.9. Chemical analysis of Misi.

\begin{tabular}{|c|c|c|c|c|c|c|c|c|c|c|c|}
\hline Composition & $\mathrm{SiO}_{2}$ & $\mathrm{Al}_{2} \mathrm{O}_{3}$ & $\mathrm{TiO}_{2}$ & $\mathrm{Fe}_{2} \mathrm{O}_{3}$ & $\mathrm{~K}_{2} \mathrm{O}$ & $\mathrm{CaO}$ & $\mathrm{Na}_{2} \mathrm{O}$ & $\mathrm{SO}_{3}$ & $\mathrm{P}_{2} \mathrm{O}_{2}$ & $\mathrm{MnO}$ & $\mathrm{MgO}$ \\
\hline Mass (\%) & 87.99 & 4.31 & 1.16 & 0.59 & 0.49 & 0.32 & 0.14 & 0.13 & 0.05 & 0.03 & $<0.02$ \\
\hline
\end{tabular}

Table 1.10. Particle size analysis.

\begin{tabular}{|c|c|c|c|c|c|c|c|c|}
\hline$\mu \mathrm{m}$ & 100 & 50 & 20 & 10 & 5 & 2 & 1 & 0.4 \\
\hline Passing (\%) & 100 & 99.6 & 97.9 & 94.5 & 84.6 & 55.6 & 35.0 & 12.2 \\
\hline
\end{tabular}

Fraser investigated the morphology, elemental distribution, surface structure, surface area and zeta potential of Misi. ${ }^{1}$ Scanning Electron Microscope (SEM) images showed that both the size and shape of Misi were heterogeneous. In SEM-EDS mapping, there was an even 
distribution of Si and Al. Ti was observed and it unevenly dispersed on Si. Other elements such as Fe, S were also observed but the signals corresponding to these elements are weak. X-ray diffraction (XRD) analysis was used to examine the elemental distribution. The peaks corresponding to crystalline silica such as cristobalite $\left(2 \theta=22^{\circ}\right)$ and quartz $\left(2 \theta=27^{\circ}\right)$ were observed. Some peaks with $2 \theta$ in a range of $20^{\circ}-30^{\circ}$ corresponding to the amorphous silicate phase were also observed. Numerous unassigned peaks were also present due to the natural origin of Misi composite. The infrared spectrum of Misi600 showed a very strong peak at 1096 $\mathrm{cm}^{-1}$ corresponding to siloxane Si-O-Si. As this peak overlaps with Si-O-metal stretches $(900$ $\left.950 \mathrm{~cm}^{-1}\right)$, thus the presence of Si-O-metal was not confirmed. A band at $791-698 \mathrm{~cm}^{-1}$ was observed, which was consistent with the Si-O band of cristobalite. In addition, there was also a broadband at $3000-3750 \mathrm{~cm}^{-1}$, corresponding to hydroxyl groups. Fraser also used Brunauer-Emmett-Teller to determine the surface area $\left(17.53 \pm 0.04 \mathrm{~m}^{2} / \mathrm{g}\right)$ and pore size of Misi (161 Å). The $\mathrm{pH}$ of the dispersion of Misi in deionised water was 6.27.

\subsubsection{Effect of Misi treatment on $\mathrm{nZVI}$ adsorption}

Misi was subjected to numerous treatments before coating with nZVI. ${ }^{1}$ These included calcination, acid washing, boiling, and $\mathrm{FeOOH}$ coating. In the calcination method, two different temperatures, $400{ }^{\circ} \mathrm{C}$ and $600{ }^{\circ} \mathrm{C}$, were trialled. In the acid washing method, different concentrations of $\mathrm{HCl}(5.6 \mathrm{M}, 2.8 \mathrm{M}$, and $1.1 \mathrm{M})$ were applied. The order of calcination and acid washing was also examined. The morphology of the resulting materials was examined. The results from SEM image analysis showed that calcination was the most important step in improving the dispersion of nZVI on the surface of Misi, followed by acid washing. As acid washing only was used, the higher the acid concentration was, the more Misi's surfaces were promoted, enhancing nZVI adsorption. The difference in calcination temperature did not affect nZVI adsorption.

In order to deposit FeOOH on the surface of Misi, Misi was soaked in $\mathrm{FeSO}_{4}$ and then the resulting Misi was dried in air. ${ }^{208-209}$ In Fraser's thesis, $0.5 \mathrm{~g}$ of $\mathrm{FeSO}_{4} .7 \mathrm{H}_{2} \mathrm{O}$ was added to $1 \mathrm{~g}$ of Misi $\left(\mathrm{FeSO}_{4} .7 \mathrm{H}_{2} \mathrm{O} / \mathrm{Misi}=1: 2 \mathrm{w} / \mathrm{w}\right) .{ }^{1}$ This was equivalent to the ratio of iron added to Misi of 10:100. After MisiCFeOOH generation, the material was digested, and the amount of iron was measured using Atomic Absorption Spectroscopy (AAS). The results showed that the final ratio of iron to MisiCFeOOH was $0.7: 100$ and $7 \%$ of iron in solution was adsorbed on the surface of Misi. In contrast, only $0.06 \%$ of iron was found in MisiC. However, the presence of 
the crystalline material of $\mathrm{FeOOH}$ was not ascertained due to the small percentage of iron in $\mathrm{MisiCFeOOH}$ and the heterogeneity of Misi. For example, there was no additional crystalline peak in MisiCFeOOH compared to MisiC when the XRD spectra of these materials were examined. Moreover, the peak confirming iron as goethite $(\alpha-\mathrm{FeOOH})$ was not found in all samples examined when Raman spectroscopy was used. In terms of nZVI adsorption, the effect of FeOOH coating was not clear.

\subsubsection{Effect of Misi treatment on $\mathrm{nZVI}$ reactivity towards nitrate reduction}

The calcination temperature of Misi affected the reactivity of nZVI@Misi. ${ }^{1}$ Specifically, nZVI@Misi prepared using Misi calcined at $600{ }^{\circ} \mathrm{C}$ reduced nitrate faster than that prepared using Misi calcined at $400{ }^{\circ} \mathrm{C}$. When nitrate- $\mathrm{N}$ concentration was $10 \mathrm{ppm}$, the molar ratio of Fe to nitrate was $50: 1$, nitrate removal was $39 \pm 5 \%$ and $62 \pm 9 \%$ after 60 minutes, respectively. Although coating with $\mathrm{FeOOH}$ did not change nZVI adsorption, it significantly enhanced nitrate reduction. For example, when either 5-C600-AnF-nZVI (Misi which was acid washed, calcined, and boiled) or 5-C600-AF-nZVI (Misi which was acid washed, calcined, boiled, and coated with $\mathrm{FeOOH}$ ) was used to reduce nitrate, nitrate removal was $65 \%$ and $90 \%$ after 60 minutes, respectively. In contrast, although acid washing increased nZVI adsorption, it did not significantly affect nitrate reduction. For example, when either 5-C600-nAF-nZVI (Misi which was acid washed, calcined, and coated with FeOOH) or C600-nAF-nZVI (Misi which was calcined and coated with $\mathrm{FeOOH}$ ) was used to reduced nitrate, nitrate removal was $74 \%$ and $70 \%$, respectively.

\subsection{Overview of thesis}

\subsubsection{Aim of the thesis}

In previous research within the Fulton group, numerous systems of non-doped nZVI@Misi with different preparations of Misi were examined. However, the maximum nitrate removal was only $70 \%$ after 30 minutes using $10 \mathrm{ppm}$ of nitrate- $\mathrm{N}$ and the molar ratio of iron to nitrate of $25: 1$. Therefore, the research reported in this thesis was undertaken with the objective of making more effective materials that are capacity of removing nitrate faster than nZVI@Misi by doping nZVI@Misi with a variety of different metals. In addition, we wanted to expand the chemistry of our doped nZVI@Misi to the removal of other contaminants. The aims are listed below. 
Firstly, doping metal(s) on unsupported nZVI lowered activation barrier and increased the reactivity of the resulting materials. ${ }^{2}$ In addition, dopant in bimetallic M-nZVI could slow down the oxidation of nZVI. ${ }^{172}$ Therefore, we postulated that the addition of other metals on supported nZVI@Misi can increase the nitrate removal rate and stability of the materials.

Secondly, the introduction of two simultaneous dopants not only increased nitrate reduction but also supported the conversion of nitrate into nitrogen gas. Consequently, the selectivity of nitrogen gas in trimetallic systems was higher than in non-doped nZVI. ${ }^{5}$ In this study, the effect of dopant(s) in supported bimetallic and trimetallic nZVI@Misi on the formation of final nitrogen products is examined.

Thirdly, most reported systems have only focused on examining their reactivity in removing nitrate from lab-prepared water under anaerobic conditions such as nitrogen or argon atmospheres. In this study, the synthesised materials are used for removing nitrate from labprepared water in the presence of oxygen and other co-existing ions. Moreover, the reactivity of these materials is examined in nitrate removal from "real water", for example, lake water. Finally, heavy metals were usually removed using adsorbents such as silicates ${ }^{90-92}$ and activated carbon. ${ }^{85-86}$ They were also removed using doped $\mathrm{nZVI}{ }^{172}$ and doped $\mathrm{nZVI}$ coated on supports. ${ }^{197-198}$ However, in reported systems, the high dosage of materials were used and the reaction conditions were monitored; for example, in $\mathrm{pH}$ control. In this study, the reactivity of these materials towards heavy metal removal of using supported non-doped nZVI@Misi and doped nZVI@Misi is carried out in neutral conditions.

From the above aims, in this current research, supported bimetallic and trimetallic nZVI on Misi systems are investigated. Bimetallic systems include Ni-nZVI@Misi, Cu-nZVI@Misi, ZnnZVI@Misi, and Pd-nZVI@Misi. The trimetallic system is Pd-Cu-nZVI@Misi. In all systems, the three variables examined are Misi preparation, dopant metal concentration, and synthetic method. Four different preparations of Misi, including uncalcined Misi, calcined Misi, calcined-boiled Misi, and calcined/FeOOH-coated Misi, were used. Different concentrations of dopant metals, from $0.1 \%$ to $10 \%$, were also tested. Moreover, different synthetic methods, co-reduction and deposition with various versions, were also examined.

After screening the reactivity of these systems towards nitrate reduction, the most promising materials were used to investigate further in their ability to both reduce nitrate, along with 
their activity in the presence of oxygen and coexisting ions. The best optimal materials were also used to reduce nitrate in natural water taken from Lake Horowhenua. Additionally, the reactivity of some representative materials towards heavy metals ( $\mathrm{Cd}(\mathrm{II}), \mathrm{Pb}(\mathrm{II})$, and $\mathrm{Cr}(\mathrm{VI}))$ was examined.

\subsubsection{Structure of the thesis}

The thesis includes 8 chapters. Chapter 1 has generally introduced the topic and addresses previous studies. This includes the scope of nitrate, heavy metals, and methods for the removal of these contaminants. This also consists of the scope of non-doped, bimetallic and trimetallic $\mathrm{nZVI}$ as well as supported bimetallic $\mathrm{nZVI}$ in removing contaminants.

Chapter 2 describes methods and instruments used to conduct the current research. It describes the methods for preparing Misi support, synthesising supported bimetallic and trimetallic nZVI@Misi materials. It then describes the methods for characterising and analysing materials. Additionally, the methods for nitrate reduction, heavy metals removal, and determination of soluble by-products are also discussed in this chapter. Finally, it describes the instruments used to characterise the materials and to examine their reactivity.

Chapter 3 determines the most promising doped nZVI@Misi towards nitrate reduction. In this chapter, all the materials were characterised and their reactivity towards nitrate reduction was screened. Materials included supported bimetallic, Ni-nZVI@Misi, Cu-nZVI@Misi, ZnnZVI@Misi, and Pd-nZVI@Misi, as well as trimetallic Pd-Cu-nZVI@Misi. The morphology, composition and elemental distribution of each system towards several variables including Misi preparation, dopant concentration, and synthetic method, were examined.

Chapter 4 focusses in greater detail on the most promising materials described in Chapter 3. The efficiency of these materials was examined through nitrate conversion and nitrite accumulation. After nitrate reduction, the change in the morphology and elemental distribution of these materials was also investigated.

Chapter 5 examines the effect of reaction conditions on nitrate reduction. The reactivity of the most promising materials in the presence of oxygen and coexisting ions towards nitrate reduction was examined. During nitrate reduction, the generation of soluble metal byproducts such as iron, nickel, and copper was also determined. 
Chapter 6 investigates the reactivity of the most promising materials in nitrate reduction from lake water taken from Lake Horowhenua. It included the investigations of these materials towards nitrate reduction from lake water and lake water spiked. The effect of $\mathrm{pH}$ adjustment in nitrate reduction from lake water was also investigated. During the reaction, the concentrations of soluble iron were monitored.

Chapter 7 explores the reactivity of the representative materials towards heavy metal removal. It included the investigations of their reactivity in removing $\mathrm{Cd}(\mathrm{II}), \mathrm{Pb}(\mathrm{II})$, and $\mathrm{Cr}(\mathrm{VI})$. During heavy metal removal, the determination of soluble iron, nickel, and copper as byproducts was also monitored. Additionally, the elemental distribution of the resultant material was examined.

Chapter 8 summaries all the findings and suggests future work. 


\section{Methods and instruments}

In this research project, $\mathrm{nZVI}$ was generated in an inert atmosphere utilising Schlenk techniques. Misi was generously donated by Golden Bay Cement in 2015. Prior to use, Misi was kept in a black plastic bag away from moisture. Unless noted, all water used was deionised (18 $\mu \Omega$, Ultrapure (Type1) water). A complete list of chemical sources can be found in the list of chemicals used.

\subsection{Methods}

\subsubsection{Surface preparation}

The ability of micro silicate (Misi) in supporting nanoparticles is influenced by pre-treatment methods. Four different preparations of Misi were generated based on previous results in our group: uncalcined Misi (MisiU), calcined Misi (MisiC), calcined and boiled Misi (MisiC-B) and calcined/FeOOH-coated Misi (MisiCFeOOH). ${ }^{1}$ MisiU was prepared by heating Misi at $120{ }^{\circ} \mathrm{C}$ for 5 hours. MisiC was prepared by heating Misi in the oven at $600^{\circ} \mathrm{C}$ for 6 hours. MisiC-B was produced by boiling MisiC in deionised water for 2 hours and then separated from the liquid using a centrifuge $(5,000 \mathrm{rpm}, 5$ minutes $)$ and left in the oven $\left(120^{\circ} \mathrm{C}\right)$ to dry for 5 hours. For MisiCFeOOH, $\mathrm{FeSO}_{4} .7 \mathrm{H}_{2} \mathrm{O}$ was added to MisiC (Fe: MisiC ratio of 1:2 w/w) in $200 \mathrm{~mL}$ deionised water and boiled for 2 hours. The solid was separated by centrifugation (5,000 rpm, 5 minutes) and then sonicated in ethanol. The solid was again separated by centrifugation $(5,000 \mathrm{rpm}, 5$ minutes $)$ and left in the oven $\left(120^{\circ} \mathrm{C}\right)$ to dry for 5 hours.

\subsubsection{Synthesis of bimetallic particles on Misi support}

Several different methods were investigated to coat the silicate. For consistency, the names of the resulting materials were listed in Table 2.1 and 2.2. These tables detail the amount of added dopants, both in terms of total metal salt added as well as the mass of added dopant compared to Misi and iron. Note: for all experiments, the amount of Misi used was $1.00 \mathrm{~g}$ and the amount of $\mathrm{FeSO}_{4} .7 \mathrm{H}_{2} \mathrm{O}$ added was $0.500 \mathrm{~g}$ or $0.100 \mathrm{~g}$ iron.

Two chemical methods were employed to adsorb nanoparticles (M-nZVI) onto the Misi surface. These methods were a one-step co-reduction method (MA) and a two-step deposition method (MB) that was modified from Kang et al. for synthesising unsupported Ni$\mathrm{nZVI}^{2}{ }^{2}$ The amount of chemicals used for the synthesis is listed in Table 2.1. Due to the use of 
small amount of dopants including nickel, copper, zinc, and palladium in the material, solutions containing these elements were prepared. The solution containing $2.5 \times 10^{-3} \mathrm{~g}$ nickel/mL by adding $0.5520 \mathrm{~g} \mathrm{NiCl}_{2}$ to deionised water to make up $100.0 \mathrm{~mL}$ solution. The solution containing $2.5 \times 10^{-3} \mathrm{~g}$ copper/mL by adding $0.5290 \mathrm{~g} \mathrm{CuCl}_{2}$ to deionised water to make up $100.0 \mathrm{~mL}$ solution. The solution containing $2.5 \times 10^{-3} \mathrm{~g}$ zinc/ $\mathrm{mL}$ by adding $0.5211 \mathrm{~g}$ $\mathrm{ZnCl}_{2}$ to deionised water to make up $100.0 \mathrm{~mL}$ solution. The solution containing $2 \times 10^{-4} \mathrm{~g}$ palladium $/ \mathrm{mL}$ was prepared by adding $0.0422 \mathrm{~g} \mathrm{Pd}\left(\mathrm{CH}_{3} \mathrm{COO}\right)_{2}$ to deionised water to make up $100.0 \mathrm{~mL}$ solution. The name used for each material corresponds to the relative amount added and is not necessarily a reflection of the exact composition of the material. 
Table 2.1. Material names and corresponding amount of metals salts added in MnZVI@Misi. Note, for all examples, the amount of Misi is $1.00 \mathrm{~g}$ and the amount of $\mathrm{FeSO}_{4} .5 \mathrm{H}_{2} \mathrm{O}$ added is $0.500 \mathrm{~g}$, or $0.100 \mathrm{~g}$ iron.

\begin{tabular}{|c|c|c|c|c|}
\hline Name & $\begin{array}{c}\mathrm{NiCl}_{2} \\
2.5 \times 10^{-3} \mathrm{~g} \\
\text { nickel/mL } \\
(\mathrm{mL}) \\
\text { nickel (g) }\end{array}$ & $\begin{array}{c}\mathrm{CuCl}_{2} \\
2.5 \times 10^{-3} \mathrm{~g} \\
\text { copper } / \mathrm{mL} \\
(\mathrm{mL}) \\
\text { copper }(\mathrm{g})\end{array}$ & $\begin{array}{c}\mathrm{ZnCl}_{2} \\
2.5 \times 10^{-3} \mathrm{~g} \\
\text { zinc/mL } \\
(\mathrm{mL}) \\
\text { zinc }(\mathrm{g})\end{array}$ & $\begin{array}{l}\mathrm{Pd}\left(\mathrm{CH}_{3} \mathrm{COO}\right)_{2} \\
2 \times 10^{-4} \mathrm{~g} \\
\text { palladium } / \mathrm{mL} \\
(\mathrm{mL}) \\
\text { palladium (g) }\end{array}$ \\
\hline 2\%Ni-nZVI@Misi & $\begin{array}{c}0.8 \mathrm{~mL} \\
2.0 \times 10^{-3} \mathrm{~g}\end{array}$ & & & \\
\hline 5\%Ni-nZVI@Misi & $\begin{array}{c}2.0 \mathrm{~mL} \\
5.0 \times 10^{-3} \mathrm{~g}\end{array}$ & & & \\
\hline 10\%Ni-nZVI@Misi & $\begin{array}{c}4.0 \mathrm{~mL} \\
1.0 \times 10^{-2} \mathrm{~g}\end{array}$ & & & \\
\hline 2\%Cu-nZVI@Misi & & $\begin{array}{c}0.8 \mathrm{~mL} \\
2.0 \times 10^{-3} \mathrm{~g}\end{array}$ & & \\
\hline 5\%Cu-nZVI@Misi & & $\begin{array}{c}2.0 \mathrm{~mL} \\
5.0 \times 10^{-3} \mathrm{~g}\end{array}$ & & \\
\hline 10\%Cu-nZVI@Misi & & $\begin{array}{c}4.0 \mathrm{~mL} \\
1.0 \times 10^{-2} \mathrm{~g}\end{array}$ & & \\
\hline 5\%Zn-nZVI@Misi & & & $\begin{array}{c}2.0 \mathrm{~mL} \\
5.0 \times 10^{-3} \mathrm{~g}\end{array}$ & \\
\hline 10\%Zn-nZVI@Misi & & & $\begin{array}{c}4.0 \mathrm{~mL} \\
1.0 \times 10^{-2} \mathrm{~g}\end{array}$ & \\
\hline 0.1\%Pd-nZVI@Misi & & & & $\begin{array}{c}0.5 \mathrm{~mL} \\
1.0 \times 10^{-4} \mathrm{~g}\end{array}$ \\
\hline 0.5\%Pd-nZVI@Misi & & & & $\begin{array}{c}2.5 \mathrm{~mL} \\
5.0 \times 10^{-4} \mathrm{~g}\end{array}$ \\
\hline 1\%Pd-nZVI@Misi & & & & $\begin{array}{c}5.0 \mathrm{~mL} \\
1.0 \times 10^{-3} \mathrm{~g}\end{array}$ \\
\hline
\end{tabular}




\subsubsection{Co-reduction method (MA)}

The co-reduction method involved the simultaneous reduction of both iron and dopant metal in the presence of Misi (Scheme 2.1). The reduction potential of both iron $\left(E^{0}=-0.44 \mathrm{~V}\right.$ ) and dopant $(\mathrm{M})$ is more positive than that of borohydride $\left(E^{0}=-2.23 \mathrm{~V}\right)$. Therefore, iron and dopant are reduced by borohydride (eq. 2.1 and eq. 2.2).

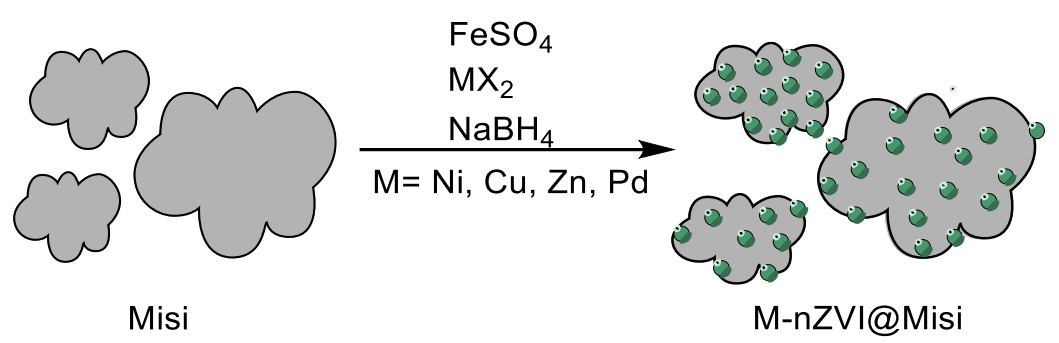

Scheme 2.1.M-nZVI@Misi synthesised using co-reduction method (MA). Green dots attribute to bimetallic $n Z V I$ particles.

$$
\begin{gathered}
\mathrm{Fe}^{2+}(\mathrm{aq})+2 \mathrm{BH}_{4(\mathrm{aq})}^{-}+6 \mathrm{H}_{2} \mathrm{O}_{(\mathrm{aq})} \longrightarrow \mathrm{Fe}_{(\mathrm{s})}+2 \mathrm{~B}(\mathrm{OH})_{3(\mathrm{aq})}+7 \mathrm{H}_{2(\mathrm{~g})} \mathrm{E}=+1.79 \mathrm{~V} \text { (eq. 2.1) } \\
\mathrm{M}^{2+}{ }_{(\mathrm{aq})}+2 \mathrm{BH}_{4(\mathrm{aq})}^{-}+6 \mathrm{H}_{2} \mathrm{O}_{(\mathrm{aq})} \longrightarrow \mathrm{M}_{(\mathrm{s})}+2 \mathrm{~B}(\mathrm{OH})_{3(\mathrm{aq})}+7 \mathrm{H}_{2(\mathrm{~g})} \\
\mathrm{M}=\mathrm{Ni}\left(\mathrm{E}^{0}=-0.26 \mathrm{~V}\right) ; \mathrm{Cu}\left(\mathrm{E}^{0}=+0.34 \mathrm{~V}\right) ; \mathrm{Pd}\left(\mathrm{E}^{0}=+0.92 \mathrm{~V}\right) ; \mathrm{Zn}\left(\mathrm{E}^{0}=-0.76 \mathrm{~V}\right) .
\end{gathered}
$$

In a typical experiment, a Schlenk flask was charged with $1.00 \mathrm{~g}$ Misi, $0.5000 \mathrm{~g}\left(1.8 \times 10^{-3} \mathrm{~mol}\right)$ of $\mathrm{FeSO}_{4} .7 \mathrm{H}_{2} \mathrm{O}$ and doping metal (e.g. $2.0 \mathrm{~mL}$ of $2.5 \times 10^{-3} \mathrm{~g}$ nickel $/ \mathrm{mL} \mathrm{NiCl}_{2}$ solution, $8.5 \times 10^{-}$ ${ }^{5} \mathrm{~mol}_{\text {of }} \mathrm{NiCl}_{2}$ ) in $30 \mathrm{~mL}$ of deoxygenated deionised water under nitrogen atmosphere. The resulting suspension was vigorously stirred with a glass-coated magnetic stir bar for 15 minutes. To this suspension, $0.37 \mathrm{~g}\left(9.8 \times 10^{-3} \mathrm{~mol}\right) \mathrm{NaBH}_{4}$ in $30 \mathrm{~mL}$ deoxygenated deionised water was added dropwise $(0.5 \mathrm{~mL} / \mathrm{min})$ via a syringe pump. The suspension was kept stirring until gas evolution stopped (approximately 90 minutes). The resulting particles were isolated with a strong magnet and the solvent was removed via a cannula. The material was washed with $2 \times 30 \mathrm{~mL}$ deoxygenated deionised water, followed by $2 \times 30 \mathrm{~mL}$ deoxygenated ethanol. The particles were then isolated and dried at $60^{\circ} \mathrm{C}$ under vacuum for 15 hours.

\subsubsection{Deposition method}

The deposition method was a two-step sequential procedure (Scheme 2.2). nZVI on Misi particles were synthesised (eq. 2.1). After that, due to the more negative reduction potential 
of $n Z V I$, it oxidises dopant ion (M(II)) to form $M$ on the surface of $n Z V I)$ (eq. 2.3). In method MB1, wet nZVI@Misi particles were used while drynZVI@Misi particles were used in method MB2.

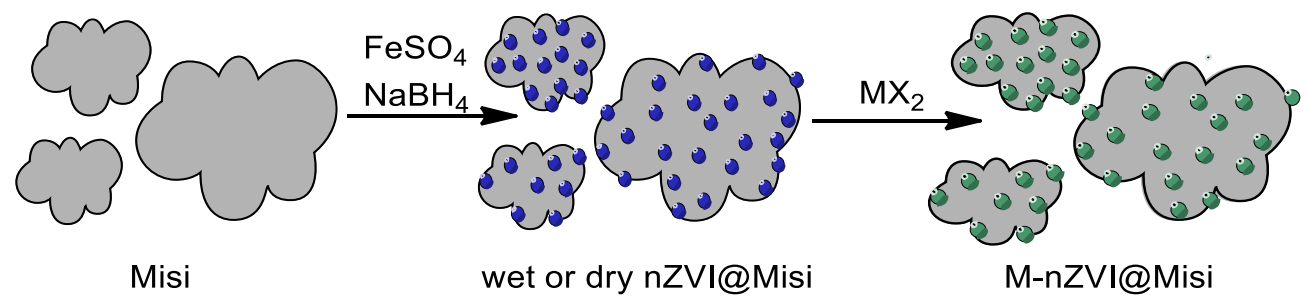

Scheme 2.2. M-nZVI@Misi synthesised using deposition method (MB). Blue dots attribute to $n Z V I$ particles and green dots attribute to bimetallic nZVI particles.

$$
\begin{aligned}
& \mathrm{Fe}_{(\mathrm{s})}+\mathrm{M}^{2+}{ }_{(\mathrm{aq})} \longrightarrow \mathrm{Fe}^{2+}{ }_{(\mathrm{aq})}+\mathrm{M}_{(\mathrm{s})} \quad \text { (eq. 2.3) } \\
& \mathrm{M}=\mathrm{Ni}\left(\mathrm{E}^{0}=-0.26 \mathrm{~V}\right) ; \mathrm{Cu}\left(\mathrm{E}^{0}=+0.34 \mathrm{~V}\right) ; \mathrm{Pd}\left(\mathrm{E}^{0}=+0.92 \mathrm{~V}\right)
\end{aligned}
$$

\section{a. Method MB1}

nZVI@Misi synthesis: Under nitrogen atmosphere, a Schlenk flask was charged with $1.00 \mathrm{~g}$ of Misi and $0.500 \mathrm{~g}\left(1.8 \times 10^{-3} \mathrm{~mol}\right)$ of $\mathrm{FeSO}_{4} .7 \mathrm{H}_{2} \mathrm{O}$ in $30 \mathrm{~mL}$ of deoxygenated deionised water. The resulting suspension was vigorously stirred with a glass-coated magnetic stir bar for 15 minutes. To this suspension, $0.37 \mathrm{~g}\left(9.8 \times 10^{-3} \mathrm{~mol}\right)$ of $\mathrm{NaBH}_{4}$ in $30 \mathrm{~mL}$ deoxygenated deionised water was added dropwise $(0.5 \mathrm{~mL} / \mathrm{min})$ via a syringe pump to generate nZVI@Misi. The mixture was kept stirring until gas evolution stopped (approximately 90 minutes). The nZVI@Misi particles were isolated by a strong magnet and the solvent was removed via a cannula.The nZVI@Misi particles were washed with 2 ×30 mL deoxygenated deionised water.

Doping metal: A solution containing dopant metal M (e.g. $2.0 \mathrm{~mL}$ of $2.5 \times 10^{-3} \mathrm{~g}$ nickel $/ \mathrm{mL} \mathrm{NiCl}$ solution, $8.5 \times 10^{-5} \mathrm{~mol}$ of $\mathrm{NiCl}_{2}$ ) in $30 \mathrm{~mL}$ of deoxygenated deionised water was added to nZVI@Misi via cannula. The mixture was stirred continuously for 30 minutes so that nZVI reacts with M(II) to form M-nZVI@Misi. After that, the generated M-nZVI@Misi particles were isolated with a strong magnet and solvent was removed via a cannula. The particles were isolated and washed with $2 \times 30 \mathrm{~mL}$ deoxygenated deionised water and $2 \times 30 \mathrm{~mL}$ deoxygenated ethanol. The particles were then dried at $60{ }^{\circ} \mathrm{C}$ under vacuum for 15 hours. 


\section{b. Method MB2}

After synthesising nZVI@Misi, the particles were washed with $2 \times 30 \mathrm{~mL}$ deoxygenated deionised water, followed by $2 \times 30 \mathrm{~mL}$ deoxygenated ethanol, and then dried at $60{ }^{\circ} \mathrm{C}$ under vacuum for 15 hours. Dry nZVI@Misi was used for depositing secondary metal. The procedure for doping metal was similar to method MB1.

\subsubsection{Synthesis of trimetallic particles on Misi support}

Two methods were employed to adsorb nanoparticles $\left(\mathrm{M}_{1}-\mathrm{M}_{2}-\mathrm{nZVI}\right)$ on Misi. These methods include co-reduction ( $M A_{\text {tri }}$ ) and deposition that have three versions: $M C 1, M C 2$, and MD.

Table 2.2. Material names and corresponding amount of metals salts added in $M_{1}-M_{2}-$ nZVI@Misi. Note: for all examples, the amount of Misi is $1.00 \mathrm{~g}$ and the amount of

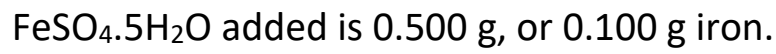

\begin{tabular}{|c|c|c|}
\hline Name & $\begin{array}{c}\mathrm{CuCl}_{2} \\
2.5 \times 10^{-3} \mathrm{~g} \text { copper } / \mathrm{mL} \\
(\mathrm{mL}) \\
\text { copper }(\mathrm{g})\end{array}$ & $\begin{array}{c}\mathrm{Pd}\left(\mathrm{CH}_{3} \mathrm{COO}\right)_{2} \\
2 \times 10^{-4} \mathrm{~g} \text { palladium } / \mathrm{mL} \\
(\mathrm{mL}) \\
\text { palladium }(\mathrm{g})\end{array}$ \\
\hline 0.5\%Pd-5\%Cu-nZVI@Misi & $\begin{array}{c}2.0 \mathrm{~mL} \\
5.0 \times 10^{-3} \mathrm{~g}\end{array}$ & $\begin{array}{c}2.5 \mathrm{~mL} \\
5.0 \times 10^{-4} \mathrm{~g}\end{array}$ \\
\hline 1\%Pd-5\%Cu-nZVI@Misi & $\begin{array}{c}2.0 \mathrm{~mL} \\
5.0 \times 10^{-3} \mathrm{~g}\end{array}$ & $\begin{array}{c}5.0 \mathrm{~mL} \\
1.0 \times 10^{-3} \mathrm{~g}\end{array}$ \\
\hline 2\%Pd-5\%Cu-nZVI@Misi & $\begin{array}{c}2.0 \mathrm{~mL} \\
5.0 \times 10^{-3} \mathrm{~g}\end{array}$ & $\begin{array}{c}10.0 \mathrm{~mL} \\
2.0 \times 10^{-3} \mathrm{~g}\end{array}$ \\
\hline
\end{tabular}

\subsubsection{Co-reduction method ( $\left.M A_{t r i}\right)$}

The co-reduction method used to generate trimetallic particles is similar to that used to generate bimetallic particles except for two dopant metals were used instead of one. In this method, iron and the two dopant metals are reduced simultaneously in the presence of Misi (Scheme 3.3). 


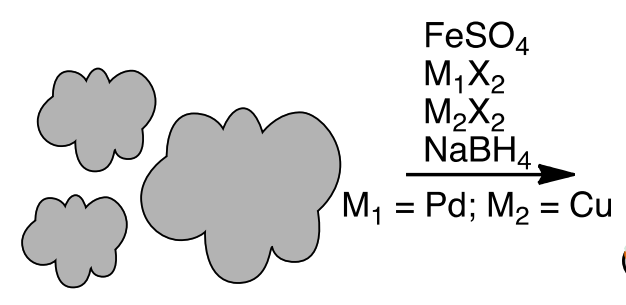

Misi

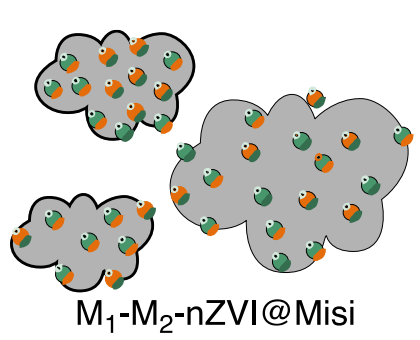

Scheme 2.3. M1-M2-nZVI@Misi synthesised using co-reduction method (MAtri). Green dots with orange attribute to trimetallic nZVI particles.

Under nitrogen atmosphere, a Schlenk flask was charged with $1.00 \mathrm{~g}$ of Misi, $0.500 \mathrm{~g}(1.8 \mathrm{x}$ $10^{-3} \mathrm{~mol}$ ) of $\mathrm{FeSO}_{4} .7 \mathrm{H}_{2} \mathrm{O}$ and two dopants $\left(\mathrm{M}_{1}, \mathrm{M}_{2}\right)$ (eg. $2.0 \mathrm{~mL}$ of $2.5 \times 10^{-3} \mathrm{~g}$ copper $/ \mathrm{mL} \mathrm{CuCl}$ solution, $8.1 \times 10^{-5} \mathrm{~mol}$ of $\mathrm{CuCl}_{2}$ and $2.5 \mathrm{~mL}$ of $2.0 \times 10^{-4} \mathrm{~g}$ palladium $/ \mathrm{mL} \mathrm{Pd}\left(\mathrm{CH}_{3} \mathrm{COO}\right)_{2}$ solution $\left(4.7 \times 10^{-6} \mathrm{~mol}\right.$ of $\left.\mathrm{Pd}\left(\mathrm{CH}_{3} \mathrm{COO}\right)_{2}\right)$ in $30 \mathrm{~mL}$ of deoxygenated deionised water. The resulting suspension was vigorously stirred with a glass-coated magnetic stir bar for 15 minutes. To this suspension, $0.37 \mathrm{~g}\left(9.8 \times 10^{-3} \mathrm{~mol}\right)$ of $\mathrm{NaBH}_{4}$ in $30 \mathrm{~mL}$ deoxygenated deionised water was added dropwise $(0.5 \mathrm{~mL} / \mathrm{min})$ via a syringe pump and stirred continuously until gas evolution stopped (approximately 30 minutes) (eq. 2.1, eq. 2.4 and eq. 2.5). The $M_{1}-M_{2}-n Z V I @ M i s i$ particles were isolated with a strong magnet and the solvent was removed via a cannula. The material was washed with $2 \times 30 \mathrm{~mL}$ deoxygenated deionised water, followed by $2 \times 30 \mathrm{~mL}$ deoxygenated ethanol. The particles were then isolated and dried at $60{ }^{\circ} \mathrm{C}$ under vacuum for 15 hours.

$$
\begin{aligned}
& \mathrm{Cu}^{2+}{ }_{(\mathrm{aq})}+2 \mathrm{BH}_{4(\mathrm{aq})}^{-}+6 \mathrm{H}_{2} \mathrm{O}_{(\mathrm{aq})} \longrightarrow \mathrm{Cu}_{(\mathrm{s})}+2 \mathrm{~B}(\mathrm{OH})_{3(\mathrm{aq})}+7 \mathrm{H}_{2(\mathrm{~g})} \mathrm{E}=+2.57 \mathrm{~V} \text { (eq. 2.4) } \\
& \mathrm{Pd}^{2+}{ }_{(\mathrm{aq})}+2 \mathrm{BH}_{4(\mathrm{aq})}^{-}+6 \mathrm{H}_{2} \mathrm{O}_{(\mathrm{aq})} \longrightarrow \mathrm{Pd}_{(\mathrm{s})}+2 \mathrm{~B}(\mathrm{OH})_{3(\mathrm{aq})}+7 \mathrm{H}_{2(\mathrm{~g})} \mathrm{E}=+3.15 \mathrm{~V} \text { (eq. 2.5) }
\end{aligned}
$$

\subsubsection{Deposition method}

In the deposition method, nZVI@Misi particles are initially synthesised and then two dopants $\left(M_{1}, M_{2}\right)$ were sequentially deposited on nZVI@Misi in three different methods: $M C 1, M C 2$, and MD.

\section{a. Method MC1}

Method MC1 was a three-step procedure, similar to method MB1 (Section 2.1.2.2). In MC1, each dopant was sequentially deposited on nZVI@Misi and only $\mathrm{FeSO}_{4}$ was subjected to $\mathrm{NaBH}_{4}$ (Scheme 2.4). ${ }^{4}$ 


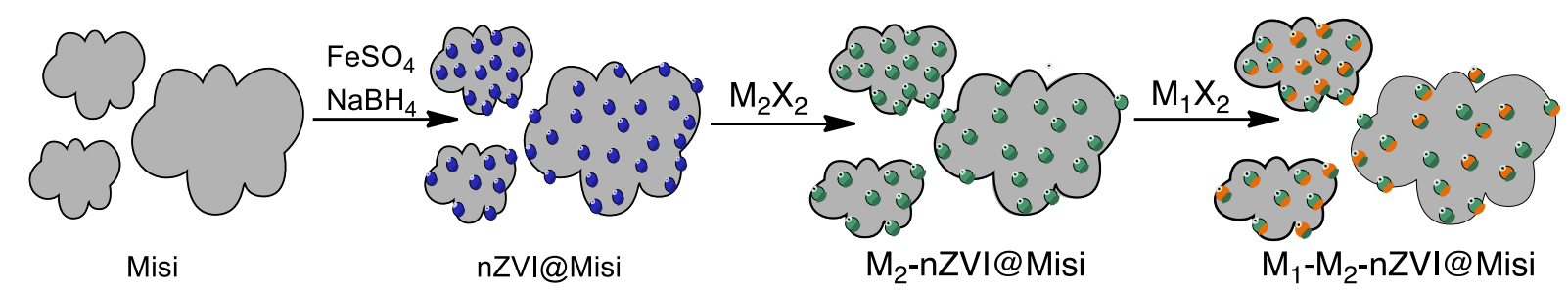

Scheme 2.4. M1-M2-nZVI@Misi synthesised using deposition method (MC1). Blue dots attribute to $n Z V I$ particles, green dots attribute to bimetallic $n Z V I$ particles and green dots with orange attribute to trimetallic nZVI particles.

nZVI@Misi was synthesised by the reduction of $\mathrm{FeSO}_{4}$ by $\mathrm{NaBH}_{4}$ (Section 2.1.2.2). nZVI particles were washed with $2 \times 30 \mathrm{~mL}$ deoxygenated deionised water. A solution containing dopant metal $\mathrm{M}_{2}$ (e.g, $2.0 \mathrm{~mL}$ of $2.5 \times 10^{-3} \mathrm{~g}$ copper $/ \mathrm{mL} \mathrm{CuCl}_{2}$ solution, $8.1 \times 10^{-5} \mathrm{~mol}$ of $\mathrm{CuCl}_{2}$ ) in $30 \mathrm{~mL}$ of deoxygenated deionised water was added to these particles via cannula. The resulting suspension was stirred continuously for 30 minutes to generate bimetallic $\mathrm{M}_{2}$ nZVI@Misi (Cu-nZVI@Misi) (eq. 2.6).M2-nZVI@Misi was washed with 2 x 30 mL deoxygenated deionised water. A solution containing dopant metal $M_{1}$, (e.g, $2.5 \mathrm{~mL}$ of $2.0 \times 10^{-4} \mathrm{~g}$ palladium $/ \mathrm{mL} \mathrm{Pd}\left(\mathrm{CH}_{3} \mathrm{COO}\right)_{2}$ solution, $\left.4.7 \times 10^{-6} \mathrm{~mol} \mathrm{Pd}\left(\mathrm{CH}_{3} \mathrm{COO}\right)_{2}\right)$ in $30 \mathrm{~mL}$ of deoxygenated deionised water was added to these particles. The suspension was stirred continuously for 30 minutes to generate trimetallic M1-M2-nZVI@Misi (Pd-Cu-nZVI@Misi) (eq. 2.7 and possibly eq. 2.8). $M_{1}-M_{2}-n Z V I @ M i s i$ was washed with $2 \times 30 \mathrm{~mL}$ deoxygenated deionised water, followed by $2 \times 30 \mathrm{~mL}$ deoxygenated ethanol. The particles were then isolated and dried at $60{ }^{\circ} \mathrm{C}$ under vacuum for 15 hours.

$$
\begin{array}{lllll}
\mathrm{Fe}_{(\mathrm{s})}+\mathrm{Cu}^{2+}{ }_{(\mathrm{aq})} \longrightarrow \mathrm{Fe}^{2+}{ }_{(\mathrm{aq})}+\mathrm{Cu}_{(\mathrm{s})} & \mathrm{E}=+0.78 \\
\mathrm{Fe}_{(\mathrm{s})}+\mathrm{Pd}^{2+}{ }_{(\mathrm{aq})} \longrightarrow \mathrm{Fe}^{2+}{ }_{(\mathrm{aq})}+\mathrm{Pd}_{(\mathrm{s})} & \mathrm{E}=+1.36 \\
\mathrm{Cu}_{(\mathrm{s})}+\mathrm{Pd}^{2+}{ }_{(\mathrm{aq})} \longrightarrow \mathrm{Cu}^{2+}{ }_{(\mathrm{aq})}+\mathrm{Pd}_{(\mathrm{s})} & \mathrm{E}=+0.58 & \text { (eq. 2.7) }
\end{array}
$$

\section{b. Method MC2}

Method MC2 was a three-step procedure, similar to method MC1. However, after each addition of doping metal and mixing, a solution of $\mathrm{NaBH}_{4}$ was added to the suspension. This was modified from the method for synthesising unsupported Pd-Cu-nZVI and Cu-Pd-nZVI reported by Lubphoo et al. ${ }^{5}$ (Scheme 2.5 ). 


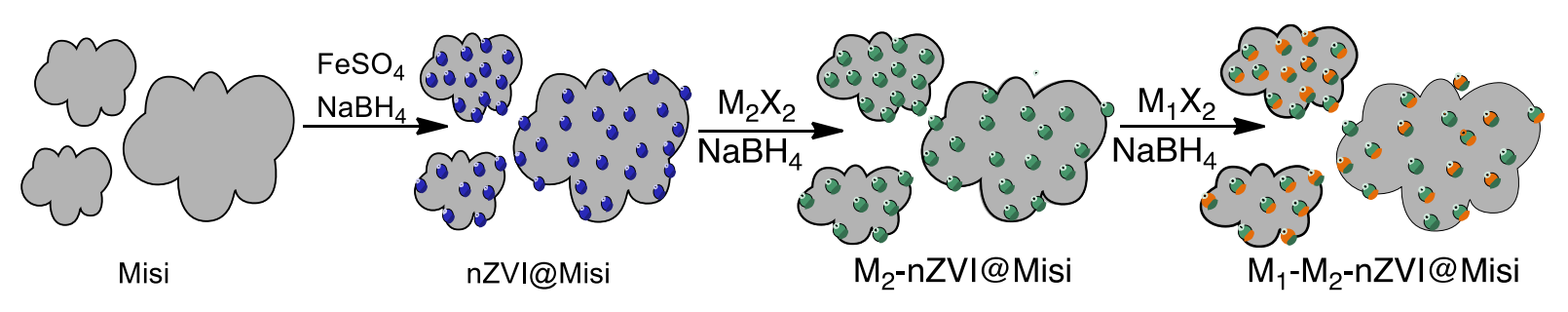

Scheme 2.5. M1-M2-nZVI@Misi synthesised using deposition method (MC2). Blue dots attribute to $n Z V I$ particles, green dots attribute to bimetallic $n Z V I$ particles and green dots with orange attribute to trimetallic $n Z V I$ particles.

nZVI@Misi was synthesised by the reduction of $\mathrm{FeSO}_{4}$ by $\mathrm{NaBH}_{4}$ (Section 2.2.2.2). A solution containing $\mathrm{M}_{2}$ (e.g, $2.0 \mathrm{~mL}$ of $2.5 \times 10^{-3} \mathrm{~g}$ copper $/ \mathrm{mL} \mathrm{CuCl}_{2}$ solution, $8.1 \times 10^{-5} \mathrm{~mol}$ of $\mathrm{CuCl}_{2}$ ) in $30 \mathrm{~mL}$ of deoxygenated deionised water was added to these particles and the suspension was stirred in 10 minutes. To this suspension, a solution of $0.10 \mathrm{~g}\left(2.6 \times 10^{-3} \mathrm{~mol}\right)$ of $\mathrm{NaBH}_{4}$ in 10 $\mathrm{mL}$ deoxygenated deionised water was added dropwise $(0.5 \mathrm{~mL} / \mathrm{min})$ and the suspension was vigorously stirred until no gas bubbles evolved (approximately 20 minutes). The $\mathrm{M}_{2}-$ nZVI@Misi particles were separated and washed with 2 x $30 \mathrm{~mL}$ deoxygenated deionised water. A solution containing $\mathrm{M}_{1}$ (e.g, $2.5 \mathrm{~mL}$ of $2.0 \times 10^{-4} \mathrm{~g}$ palladium $/ \mathrm{mL} \mathrm{Pd}\left(\mathrm{CH}_{3} \mathrm{COO}\right)_{2}$ solution, $\left.4.7 \times 10^{-6} \mathrm{~mol} \mathrm{Pd}\left(\mathrm{CH}_{3} \mathrm{COO}\right)_{2}\right)$ in $30 \mathrm{~mL}$ deoxygenated deionised water was added to these particles and the suspension was stirred in 10 minutes. A solution of $0.10 \mathrm{~g}\left(2.6 \times 10^{-3}\right.$ $\mathrm{mol}$ ) of $\mathrm{NaBH}_{4}$ in $10 \mathrm{~mL}$ deoxygenated deionised water was added dropwise $(0.5 \mathrm{~mL} / \mathrm{min})$ and the suspension was vigorously stirred until no gas bubbles evolved (approximately 20 minutes), resulting in trimetallic $\mathrm{M}_{1}-\mathrm{M}_{2}-\mathrm{nZVI@Misi.} \mathrm{These} \mathrm{particles} \mathrm{were} \mathrm{isolated,} \mathrm{washed}$ with $2 \times 30 \mathrm{~mL}$ deoxygenated deionised water, followed by $2 \times 30 \mathrm{~mL}$ deoxygenated ethanol. The particles were isolated and dried at $60{ }^{\circ} \mathrm{C}$ under vacuum for 15 hours.

\section{c. Method MD}

Method D was a two-step procedure in which two dopant metals were simultaneously deposited on nZVI@Misi.nZVI@Misi was initially synthesised by the reduction of $\mathrm{FeSO}_{4}$ by $\mathrm{NaBH}_{4}$ (Section 2.2.2.2). A solution containing both dopant $\mathrm{M}_{1}$ and dopant $\mathrm{M}_{2}$ (e.g, $2.0 \mathrm{~mL}$ of $2.5 \times 10^{-3} \mathrm{~g}$ copper/mL $\mathrm{CuCl}_{2}$ solution, $8.1 \times 10^{-5} \mathrm{~mol}$ of $\mathrm{CuCl}_{2}$ and $2.5 \mathrm{~mL}$ of $2.0 \times 10^{-4} \mathrm{~g}$ palladium $/ \mathrm{mL} \mathrm{Pd}\left(\mathrm{CH}_{3} \mathrm{COO}\right)_{2}$ solution, $4.7 \times 10^{-6} \mathrm{~mol}$ of $\left.\mathrm{Pd}\left(\mathrm{CH}_{3} \mathrm{COO}\right)_{2}\right)$ in $30 \mathrm{~mL}$ deoxygenated deionised water was added to the particles. The suspension was vigorously stirred in 30 minutes to generate trimetallic $\mathrm{M}_{1}-\mathrm{M}_{2}-\mathrm{nZVI} @$ Misi (Scheme 2.6). The particles were isolated 
and washed with $2 \times 30 \mathrm{~mL}$ deoxygenated deionised water and $2 \times 30 \mathrm{~mL}$ deoxygenated ethanol. The particles were then isolated and dried at $60{ }^{\circ} \mathrm{C}$ under vacuum for 15 hours.

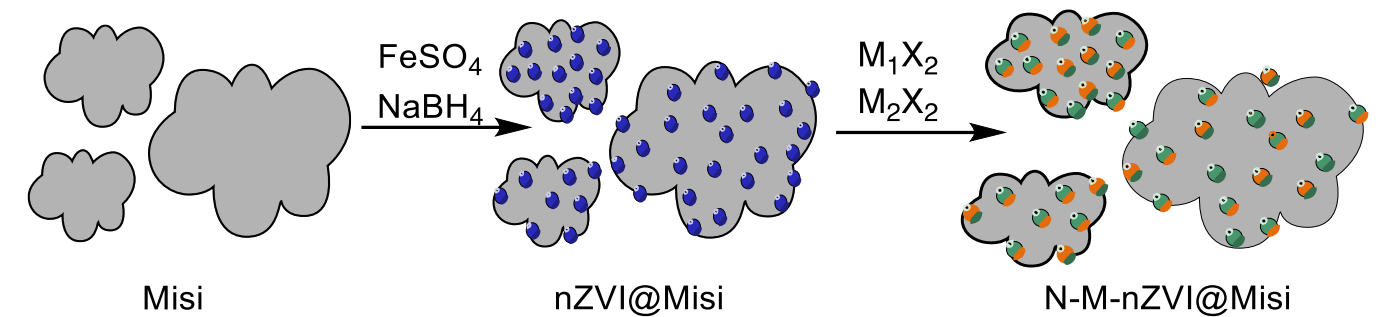

Scheme 2.6. M1-M2-nZVI@Misi synthesised using deposition method (MD). Blue dots attribute to $\mathrm{nZVI}$ particles and green dots with orange attribute to trimetallic $n Z V I$ particles.

\subsubsection{Nitrate reduction}

Nitrate reduction by doped and non-doped nZVI@Misi particles was examined under both anaerobic and aerobic conditions. The effect of other coexisting ions was also examined. Additionally, the reactivity of doped and non-doped nZVI@Misi towards nitrate reduction from lake water was investigated.

A stock solution of $1,000 \mathrm{ppm}$ nitrate- $\mathrm{N}$ was prepared from dehydrated $\mathrm{KNO}_{3}\left(105{ }^{\circ} \mathrm{C}, 24\right.$ hours) in deionised water ( $0.7218 \mathrm{~g} \mathrm{KNO}_{3}$ in $\left.1.00 \mathrm{~L}\right)$. Working nitrate- $\mathrm{N}$ solutions, including 10 ppm, $50 \mathrm{ppm}$ and $100 \mathrm{ppm}$ were diluted from the stock solution with deionised water. For each experiment, $0.150 \mathrm{~g}$ of material was added to $25.0 \mathrm{~mL}$ of nitrate solution. Under anaerobic conditions, nitrate concentrations of 10 ppm, 50 ppm, and 100 ppm were used. Under aerobic conditions, nitrate concentrations of $10 \mathrm{ppm}$ and $50 \mathrm{ppm}$ were used (Table 2.3).

Table 2.3. Nitrate reduction under anaerobic and aerobic conditions.

\begin{tabular}{|c|c|c|c|c|}
\hline Condition & Mass of material (g) & \multicolumn{3}{|c|}{ Nitrate-N concentration (ppm) } \\
\hline Anaerobic conditions & 0.150 & 10 & 50 & 100 \\
\hline Aerobic conditions & 0.150 & 10 & 50 & \\
\hline
\end{tabular}

\subsubsection{Anaerobic conditions}

Prior to reduction, $25.0 \mathrm{~mL}$ of nitrate solution in a Schlenk flask was bubbled with nitrogen gas for at least 20 minutes. Supported nanoparticles $(0.150 \mathrm{~g})$ were quickly added to this 
solution and the suspension was stirred vigorously with a glass-coated magnetic stir bar. Aliquots of $3-5 \mathrm{~mL}$ samples were withdrawn using a syringe at set time intervals such as 5 minutes, 15 minutes, 20 minutes, and 30 minutes and filtered through $0.22 \mu \mathrm{m}$ cellulose acetate syringe filter.

\subsubsection{Aerobic conditions}

In the aerobic conditions, $0.150 \mathrm{~g}$ of material was quickly added to $25.0 \mathrm{~mL}$ of nitrate solution. The suspension was stirred vigorously with a glass-coated magnetic stir bar. Aliquots $(5 \mathrm{~mL})$ were withdrawn using a syringe at set time intervals ( 5 minutes, 15 minutes, and 30 minutes) and filtered through $0.22 \mu \mathrm{m}$ cellulose acetate syringe filter.

\subsubsection{Interfering ions}

Four different ions (chloride, sulfate, carbonate, and phosphate) were used in the investigation. Stock solutions of coexisting ions including chloride $(1,000 \mathrm{ppm})$, sulfate-S $(1,000 \mathrm{ppm})$, carbonate-C (100 ppm) and phosphate-P (100 ppm) were prepared separately by adding $\mathrm{KCl}(2.110 \mathrm{~g}), \mathrm{K}_{2} \mathrm{SO}_{4}(5.440 \mathrm{~g}), \mathrm{K}_{2} \mathrm{CO}_{3}(1.150 \mathrm{~g})$, or $\mathrm{KH}_{2} \mathrm{PO}_{4}(0.440 \mathrm{~g})$ to a $1.00 \mathrm{~L}$ volumetric flask and adding deionised water to make up a $1.00 \mathrm{~L}$ solution.

The procedures for investigating the effect of coexisting ions on nitrate reduction were similar to that of nitrate reduction. However, materials were added to the solution containing nitrate and these ions. The influence of these ions on nitrate reduction was examined under both anaerobic and aerobic conditions (Table 2.4). For example, in the experiment of chloride influence, a $25 \mathrm{~mL}$ solution containing $10 \mathrm{ppm}$ nitrate- $\mathrm{N}$ and $100 \mathrm{ppm}$ chloride was prepared by adding $2.5 \mathrm{~mL}$ of nitrate- $\mathrm{N}$ solution of $100 \mathrm{ppm}$ and $2.5 \mathrm{~mL}$ of chloride solution of 1,000 ppm to deionised water to make up a $25.0 \mathrm{~mL}$ solution. 
Table 2.4. Nitrate reduction in the presence of other ions. Note: for all experiments, the amount of materials is $0.150 \mathrm{~g}, 2.5 \mathrm{~mL}$ of $100 \mathrm{ppm}$ nitrate- $\mathrm{N}$ solution and the volume of other ions are added to deionised water to make up a $25.0 \mathrm{~mL}$ solution.

\begin{tabular}{|c|c|c|c|c|}
\hline $\begin{array}{c}\text { Chloride 1,000 } \\
\mathrm{ppm}(\mathrm{mL})\end{array}$ & $\begin{array}{c}\text { Sulfate-S 1,000 } \\
\mathrm{ppm}(\mathrm{mL})\end{array}$ & $\begin{array}{c}\text { Carbonate-C } \\
100 \mathrm{ppm}(\mathrm{mL})\end{array}$ & $\begin{array}{c}\text { Phosphate-P } \\
100 \mathrm{ppm}(\mathrm{mL})\end{array}$ & $\begin{array}{c}\text { Deionised } \\
\text { water }(\mathrm{mL})\end{array}$ \\
\hline 2.5 & 2.5 & 5.0 & 2.5 & up to $25.0 \mathrm{~mL}$ \\
\hline
\end{tabular}

\subsubsection{Elemental composition in materials}

In order to quantify their elemental composition, materials were digested using strong and oxidising acid. Concentrated $\mathrm{HNO}_{3}(68-70 \%, 5.0 \mathrm{~mL})$ was added in a plastic centrifuge tube containing 0.250 g each material. Materials investigated were 5\%Ni-nZVI@MisiCFeOOH-MA, 5\%Ni-nZVI@MisiCFeOOH-MB1, 5\%Cu-nZVI@MisiCFeOOH-MA, 5\%Cu-nZVI@MisiCFeOOHMB1，0.5\%Pd-5\%Cu-nZVI@MisiCFeOOH-MAtri, 0.5\%Pd-5\%Cu-nZVI@MisiCFeOOH-MC1, nZVI@MisiCFeOOH and MisiCFeOOH. These suspensions were shaken, sonicated until no brown gas $\left(\mathrm{NO}_{2}\right)$ released. Deionised water $(40 \mathrm{~mL})$ was then added to this suspension. These resulting suspensions were sonicated, centrifuged and separated to remove solid and diluted to the final volume of $50.0 \mathrm{~mL}$. The concentrations of metal loading were measured using Atomic Absorption Spectroscopy (AAS).

\subsubsection{Heavy metal removal}

Stock solutions of cadmium (1,000 ppm), lead (1,000 ppm), and chromium (VI) (1,000 ppm) were prepared by adding $\mathrm{CdCl}_{2}(0.163 \mathrm{~g}), \mathrm{Pb}\left(\mathrm{NO}_{3}\right)_{2}(0.160 \mathrm{~g})$, and $\mathrm{K}_{2} \mathrm{Cr}_{2} \mathrm{O}_{7}(0.283 \mathrm{~g})$ to a 100.0 $\mathrm{mL}$ volumetric flask and adding deionised water to make up a $100.0 \mathrm{~mL}$ solution, respectively. Due to the low solubility of $\mathrm{PbCl}_{2}$, stock solution of $\mathrm{Pb}(200 \mathrm{ppm})$ was prepared by adding $\mathrm{PbCl}_{2}(0.027 \mathrm{~g})$ to a $100.0 \mathrm{~mL}$ volumetric flask and adding deionised water to make up a 100.0 $\mathrm{mL}$ solution. $\mathrm{Pb}\left(\mathrm{NO}_{3}\right)_{2}, \mathrm{PbCl}_{2}, \mathrm{~K}_{2} \mathrm{Cr}_{2} \mathrm{O}_{7}$, and $\mathrm{CdCl}_{2}$ were provided by Sigma-Aldrich. $\mathrm{CdCl}_{2}$ was stored and weighed in an inert atmosphere glove box to minimise hydrolysis. The working solutions of heavy metals ( $20 \mathrm{ppm}$ and $40 \mathrm{ppm}$ ) were prepared from these stock solutions.

Prior to reaction, $25.0 \mathrm{~mL}$ of heavy metal solution was bubbled with nitrogen gas in a Schlenk flask for at least 20 minutes. Supported nanoparticles $(0.100 \mathrm{~g})$ were quickly added to this solution (e.g, $25.0 \mathrm{~mL}$ of cadmium $20 \mathrm{ppm}$ ) and the suspension was stirred vigorously with a 
glass-coated magnetic stir bar. Aliquots $(7 \mathrm{~mL})$ were withdrawn using a syringe at set time intervals ( 5 minutes, 15 minutes, and 30 minutes) and filtered through $0.22 \mu \mathrm{m}$ cellulose acetate syringe filter into a polyethylene test tube and then loaded into autosampler of the atomic absorption spectroscopy (AAS). Sets of known standards with appropriate concentrations were conducted for each metal measurement.

Table 2.5. Heavy metal removal. Note: for all experiments, the amount of materials is 0.100 $\mathrm{g}$ and $25.0 \mathrm{~mL}$ of heavy metal solutions with concentrations as listed.

\begin{tabular}{|c|c|c|c|}
\hline $\begin{array}{c}\text { Cadmium 20 ppm } \\
(\mathrm{mL})\end{array}$ & $\begin{array}{c}\text { Lead 20 ppm } \\
(\mathrm{mL})\end{array}$ & $\begin{array}{c}\text { Lead 40 ppm } \\
(\mathrm{mL})\end{array}$ & $\begin{array}{c}\text { Chromium 20 ppm } \\
(\mathrm{mL})\end{array}$ \\
\hline 25.0 & 25.0 & 25.0 & 25.0 \\
\hline
\end{tabular}

\subsubsection{Contaminant removal from lake water}

Natural water samples were from Lake Horowhenua, North Island, New Zealand. Samples were taken near the lake's edge and contained in clean polyethylene bottles. Samples were collected on April 27, 2017 and on April 23, 2018. Samples were transported to the laboratory and kept at $4{ }^{\circ} \mathrm{C}$.

\subsubsection{Nitrate reduction from lake water}

Nitrate reduction from lake water samples was carried out under anaerobic (Section 2.1.4.1) and aerobic conditions (Section 2.1.4.2). For the investigation on lake water spiked, nitrate solution was added to the samples prior to use. Lake water spiked with $10 \mathrm{ppm}$ of nitrate-N was prepared by adding $10.0 \mathrm{~mL}$ of $1,000 \mathrm{ppm}$ nitrate- $\mathrm{N}$ to a $1.00 \mathrm{~L}$ volumetric flask and adding lake water to make up a $1.00 \mathrm{~L}$ solution. Lake water spiked with $50 \mathrm{ppm}$ of nitrate-N was prepared by adding $50.0 \mathrm{~mL}$ of $1,000 \mathrm{ppm}$ nitrate- $\mathrm{N}$ to a $1.00 \mathrm{~L}$ volumetric flask and adding lake water to make up a $1.00 \mathrm{~L}$ solution.

\subsubsection{Heavy metal removal from lake water}

Prior to use, lake water spiked with $20 \mathrm{ppm}$ heavy metal was prepared by adding $20.00 \mathrm{~mL}$ of $1,000 \mathrm{ppm}$ heavy metal to a $1.00 \mathrm{~L}$ volumetric flask and adding lake water to make up a 1.00 L solution. Heavy metal removal from lake water spiked under anaerobic was carried out as mentioned in Section 2.1.6. Heavy metal removal from lake water spiked under aerobic 
conditions was carried out as mentioned in Section 2.1.6; however, neither conduction under nitrogen gas nor deoxygenation was needed.

\subsubsection{Chemical analysis}

\subsubsection{Determination of nitrate}

In this research, two methods were used to determine nitrate concentrations: ultravioletvisible (UV-Vis) spectrophotometry ${ }^{210}$ and ion chromatography (IC). ${ }^{211}$

\section{a. Ultraviolet-visible spectroscopy}

Nitrate concentrations were quantified at $220 \mathrm{~nm}$ using a Carry Bio-50 spectrophotometer. Approximately $3 \mathrm{~mL}$ of sample in a quartz cuvette was placed in the sample holder. The nitrate- $\mathrm{N}$ concentration was calculated from a known standard curve from $0.2 \mathrm{ppm}$ to $10 \mathrm{ppm}$ nitrate-N.

\section{b. Ion chromatography}

Nitrate, nitrite, and phosphate standard solutions were prepared from commercial ion chromatography (IC) standards (1,000 ppm, Sigma Aldrich and Fluka). The standards for chloride $(1,000 \mathrm{ppm})$ and sulfate $(1,000 \mathrm{ppm})$ were prepared by adding $\mathrm{KCl}(2.110 \mathrm{~g})$ and $\mathrm{K}_{2} \mathrm{SO}_{4}(1.812 \mathrm{~g})$ provided by Sigma-Aldrich to a $1.00 \mathrm{~L}$ volumetric flask and adding lake water to make up a 1.00 L solution, respectively.

A PolyVial ${ }^{\mathrm{TM}}$ was filled with approximately $5 \mathrm{~mL}$ of sample and capped. The samples were loaded into an autosampler with at least 5 known standard solutions. These standards were in different nitrate concentration ranges, depending on the estimated nitrate concentrations of the samples. In this study, the standard curves were from 0.5 - 50 ppm nitrate and $25-450$ ppm nitrate. Concentrations of nitrate, nitrite, chloride, sulfate, and phosphate were determined using the Thermofisher IC 1100 with Dionex IonPac AS14 column, Dionex IonPac AG 14 column guard and Dionex AERS 500 suppressor. The IC mobile phase was a mixture of $\mathrm{Na}_{2} \mathrm{CO}_{3} 4.5 \mathrm{mM}$ and $\mathrm{NaHCO}_{3} 1.4 \mathrm{mM}$ that was diluted from Dionex AS23 Eluent concentrated in deionised water. The flow rate was $1.2 \mathrm{~mL} / \mathrm{min}$ and the injection volume was $25 \mu \mathrm{L}$.

\subsubsection{Determination of ammonium}

Ammonium was measured using the Nessler method. The Nessler reagent was prepared as followed: in a beaker, $\mathrm{HgI}_{2}(10.00 \mathrm{~g})$ and $\mathrm{KI}(7.00 \mathrm{~g})$ were dissolved in deionised water (40.0 
$\mathrm{mL}$ ) (solution A). In another beaker, $\mathrm{NaOH}(16.00 \mathrm{~g})$ was dissolved in deionised water (40.0 $\mathrm{mL}$ ) (solution B). Solution A was added to solution B. The resulting solution was stirred continuously and then diluted with deionised water to a final volume of $100.0 \mathrm{~mL}$ of Nessler reagent. A stock ammonium- $\mathrm{N}$ solution (100 ppm) was prepared by adding $\mathrm{NH}_{4} \mathrm{Cl}(0.382 \mathrm{~g})$ to a $1.00 \mathrm{~L}$ volumetric flask and adding deionised water to make up a $1.00 \mathrm{~L}$ solution. Standards and samples of ammonium were diluted from stock solutions with deionised water. $\left.\mathrm{Hg}\right|_{2}, \mathrm{KI}$ and $\mathrm{NH}_{4} \mathrm{Cl}$ were provided Sigma-Aldrich. $\mathrm{NaOH}$ was provided by Vickers laboratories limited. To determine the amount of ammonium, $0.1 \mathrm{~mL}$ of Nessler reagent was added to a $5.0 \mathrm{~mL}$ volumetric flask and enough ammonia solution was added to the line. The resulting solution was left in 20 minutes to form maximum complex. The ammonium concentrations were measured using the Carry Bio-50 spectrophotometer at $425 \mathrm{~nm}$.

\subsubsection{Determination of dopant metal and iron after nitrate reduction}

Working standards of elements including iron, nickel, and copper were prepared by diluting their standards for the AAS (1,000 ppm) provided by Sigma-Aldrich. The standard curves were from 0.1 - 5 ppm for both iron and nickel and from 0.5 - 5 ppm for copper.

After nitrate reduction, the remaining solutions were filtered via $0.22 \mu \mathrm{m}$ cellulose acetate syringe filter into polyethylene test tubes and then loaded into the AAS autosampler. The absorption signals of iron, nickel, and copper were measured with the ThermoFisher iCE 3500 with SOLAAR ${ }^{\mathrm{TM}}$ software using an acetylene and air flame. The concentrations of these ions were then calculated from the standard curves consisting of five known standards.

\subsubsection{Determination of element composition in materials}

For nickel and copper determination, the digested solutions were adjusted with an alkaline solution ( $\mathrm{NaOH} 10 \mathrm{~N}$ ) to achieve $\mathrm{pH}$ from 1 to 2 . For iron determination, the digested solutions were quantitatively diluted 100 times with deionised water. The absorption signals of iron, nickel, and copper were measured with the ThermoFisher iCE 3500 with SOLAAR ${ }^{\mathrm{TM}}$ software using an acetylene and air flame. The metal concentrations were quantified from the calibration curve consisting of five known standards. The standard curves were from 0.1 - 5 ppm for either iron or nickel and from 0.5 - 5 ppm for copper. 


\subsubsection{Determination of heavy metal concentration}

Standard solutions of heavy metals were prepared by diluting AAS standards $(1,000 \mathrm{ppm}$, Sigma-Aldrich) with deionised water to appropriate concentrations. The standard curves of cadmium, lead, and chromium was from 0.1 - 5 ppm.

Approximately $7 \mathrm{~mL}$ of sample was filtered into a polyethylene test tube and then loaded into autosampler of the AAS. The absorption signals of cadmium, lead, and chromium as well as metals after heavy metal removal such as iron, nickel, and copper were measured by the ThermoFisher iCE 3500 with SOLAAR ${ }^{\mathrm{TM}}$ software using an acetylene and air flame except for chromium where nitrous oxide and acetylene flame were used. The concentrations of these ions were then calculated from the standard curves.

\subsubsection{Determination of detection limit}

The detection limit of analytes was determined using the standard deviation of the measurement and the slope of the linear calibration curve. ${ }^{212}$ The results showed that the detection limit of nitrate- $\mathrm{N}$ and nitrite- $\mathrm{N}$ using IC are $0.06 \mathrm{ppm}$ and $0.04 \mathrm{ppm}$, respectively. The detection limits of metals using AAS were also calculated, iron (0.09 ppm), nickel (0.04 ppm), copper (0.66 ppm), and lead (0.05 ppm).

\subsubsection{Characterisation of material method}

The morphology of materials was characterised using a scanning electron microscope (SEM) and transmission electron microscope (TEM). ${ }^{161}$ The elemental composition was examined using energy dispersive X-ray spectroscopy in SEM (SEM-EDS) and in TEM (TEM-EDS). The crystalline composition of materials was examined using X-ray diffraction (XRD).

\subsubsection{Method for scanning electron microscope}

Samples were loaded on a carbon tape that had been placed on a $1 \mathrm{~cm}$ Al sample holder. Samples were then coated with a 7 - $9 \mathrm{~nm}$ carbon layer using a Quorum Q150T E carbon coater and dried under vacuum for at least 6 hours prior to use. All SEM images were taken using a JEOL 6500F SEM instrument. The probe current was $13 \mathrm{kV}, 15 \mathrm{kV}$, or $20 \mathrm{kV}$. The working distance was from $8.3 \mathrm{~mm}$ to $10.3 \mathrm{~mm}$. 


\subsubsection{Method for transmission electron microscope}

Samples were dispersed in ethanol. A drop of dispersion was loaded on a grid and dried. The Cu-containing materials were loaded on a gold grid while others were loaded on copper grids. All TEM images and TEM-EDS information were generated using a JEOL TEM 2100F instrument with $200 \mathrm{kV}$ probe current.

\subsubsection{Method for X-ray diffraction}

Samples were loaded on a stainless-steel holder and compacted to achieve a smooth surface. All XRD patterns were taken using PANalytical X'Pert Pro diffractometer. The X-ray source is a CuK $\alpha$ tube in which the current is $40 \mathrm{~mA}$ and the voltage was $45 \mathrm{kV}$. Scans were determined in the region from $5-90^{\circ}$ with $0.013^{\circ}$ step.

\subsection{Instruments}

\subsubsection{Scanning electron microscopy}

Scanning electron microscopy is the technique that is used for observing microscopic objects in a stereographic image with high magnifications. ${ }^{213}$ Objects can be magnified in a wide range, from 10 to 1,000,000 times. In SEM, when an electron beam irradiates the surface of the sample, the beam interacts with atoms in different areas or depths of the sample. Consequently, a variety of information is produced, depending on the areas that are analysed (Figure 2.1).

There are three prevalent types of signals generated by the interaction of electrons with samples, including secondary electrons, backscattered electrons, and X-rays. Secondary electrons are used for morphological observation. When an electron beam irradiates a sample, secondary electrons of the surface atoms are scattered. The number of electrons emitted is a function of the incident angle of the electron beam to the sample. Due to the low energy of secondary electrons, they only escape from a thin layer on the surface of the sample, so their signals are suitable for the investigation of the morphology of the surface. Images collected from secondary electrons is called Secondary Electron Images (SEI).

Backscattered electrons give information about the elemental distribution. Backscattered electrons are scattered at deeper areas of the sample's surface. The number and scattering direction of backscattered electrons depend on the elemental composition of the sample and 
the incident angle of the electron beam to the sample. The combination of surface morphology and backscattered electrons gives a composition image (COMPO) when a backscattered electron detector is used. In the COMPO image, there is a contrast in which heavier elements have a brighter color than lighter elements.

$\mathrm{X}$-rays analysis provides both qualitative and quantitative information about the sample. It can identify the elemental composition of the sample and the weight concentration of elements at the specified areas. When the incident beam irradiates the sample to create secondary electrons, it leaves holes in the electron shells. If these holes are in inner electronic shells, electrons from the outer shell will transfer into the inner shell to stabilise the atom. The excess energy of this movement generates X-rays. There are two methods to analyse characteristic X-rays, including wavelength dispersive X-ray spectroscopy (WDS) and energy dispersive X-ray spectroscopy (EDS) but EDS is more common. EDS is a method in which a Si (Li) semiconductor detector is used to detect the energy of the characteristic X-rays. In EDS, the location of peaks attributed to the energy of identified elements and the intensities or areas of peaks are proportional with the percentage mass of elements.

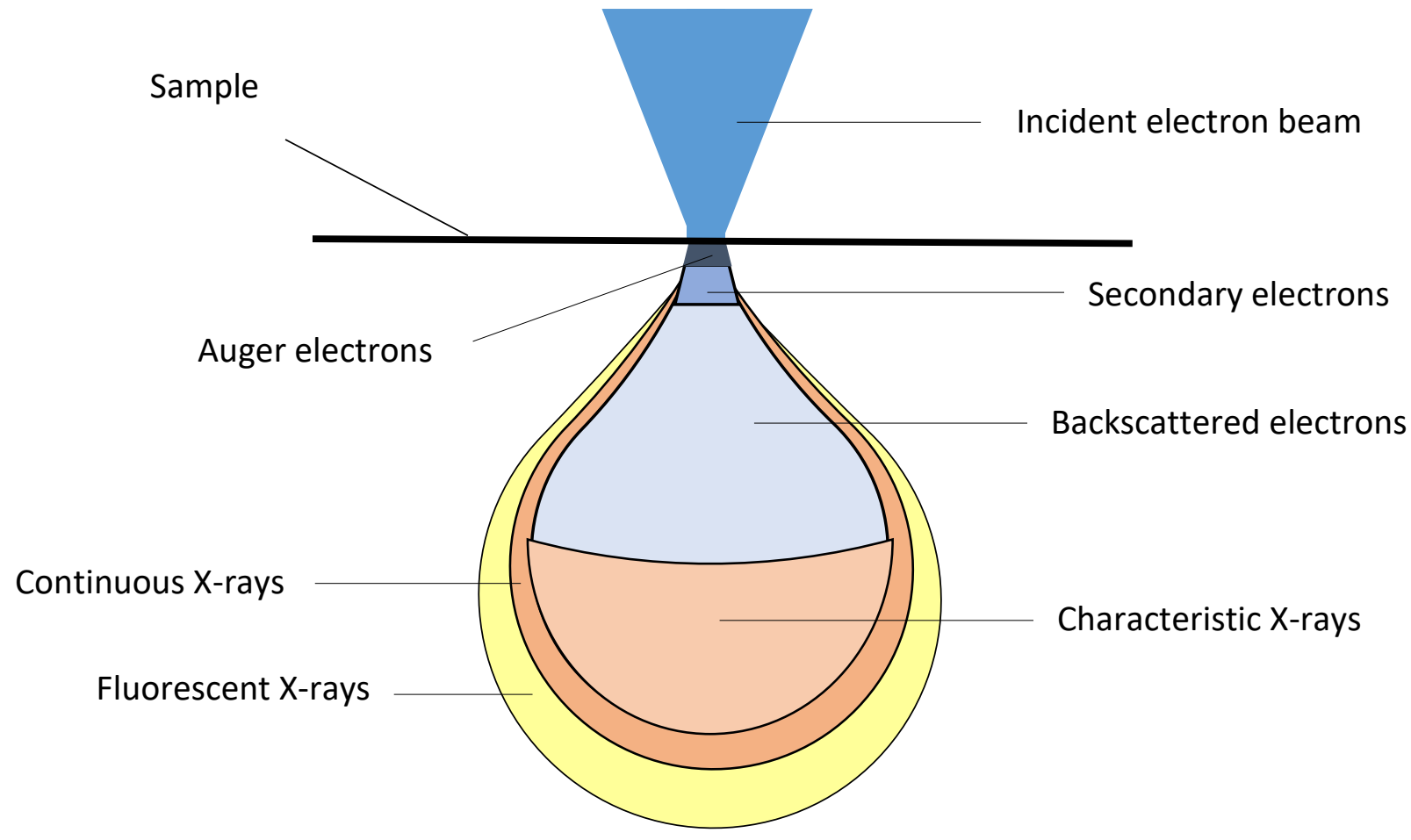

Figure 2.1. Diagram showing the distribution of signals in SEM. ${ }^{213}$ 


\subsubsection{Transmission electron microscopy}

Transmission electron microscopy is a technique in which an electron beam interacts with a specimen to provide information about morphology, composition, and crystallography. ${ }^{214}$ The sample can be magnified to 5,000,000 times. In TEM, when an electron beam enters a thin sample (less than $100 \mathrm{~nm}$ ), electrons can go through the sample to form a transmitted beam. The interactions between high energy electrons and atoms of the sample produce a variety of signals (Figure 2.2).

Two common signals are TEM imaging and scanning transmission electron microscope (STEM). TEM image contrast is formed due to the interaction of the incident electron beam with the sample. Electrons go through the sample as a wave, yielding a change in amplitude and phase. The contrast of amplitude and phase results in TEM imaging. In TEM images, the darker areas response regions with greater absorption of the electron while regions with fewer absorbed electrons are represented in lighter areas. Compared to SEM images, TEM images give higher magnification and resolution.

STEM is formed when the electron beam scans over the sample in a raster. There are two different detectors to collect the transmitted beam of scattered electrons. Scattered electrons with low-angle ( $<10 \mathrm{mrad}$ ) are collected using either bright-field (BF) detection to form STEM-BF image while scattered electrons with the angle less than 50 mrad are collected using annular dark-field (ADF) detector to form STEM-ADF image. In this study, the STEM-BF image was used.

When a sample interacts with an electron beam, some of the electrons scatter inelastically and lose energy. The lost energy is transferred to atoms and generates characteristic X-rays. The number and the energy of characteristic X-rays are recorded when an energy-dispersive $\mathrm{X}$-ray spectrometer (EDS) is used. EDS provides elemental information of the sample. 
Incident electron beam

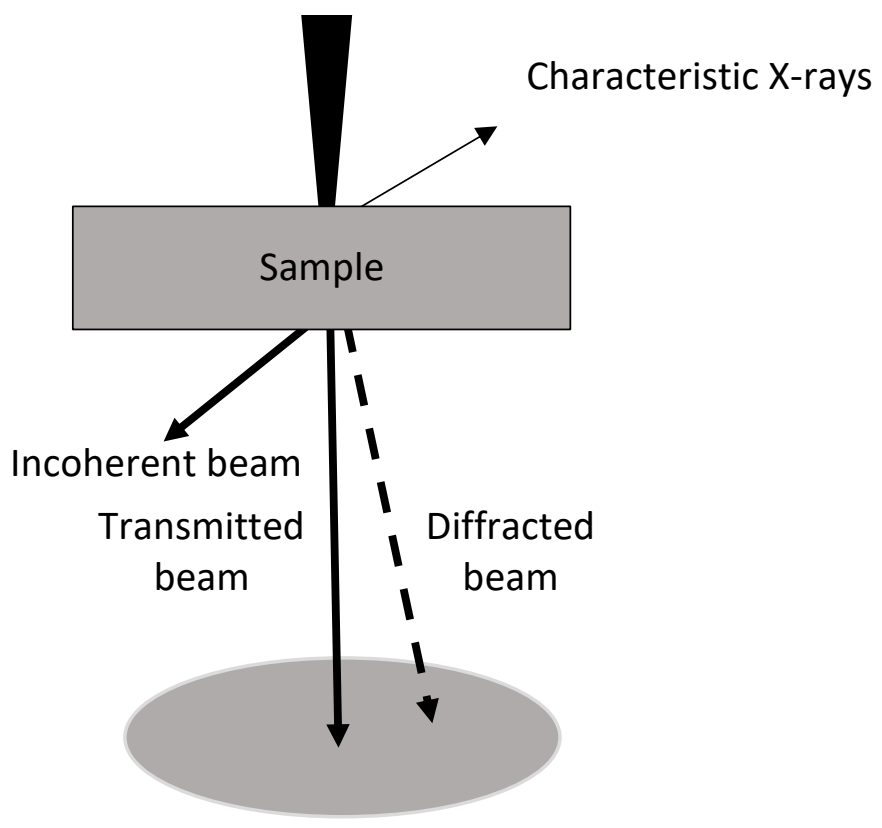

Figure 2.2. Diagram showing the signals used for TEM. ${ }^{214}$

\subsubsection{X-ray diffraction}

X-ray diffraction (XRD) analysis uses X-ray sources to study the arrangement of atoms in crystalline or polycrystalline materials. ${ }^{215}$ XRD is based on diffraction of monochromatic Xrays and atoms within a sample. The type and arrangement of atoms in materials are obtained via diffraction patterns, including the scattered intensity and the diffraction angle. There are two standard uses of XRD: single crystal XRD and X-ray powder diffraction. The latter was utilised in this research.

X-ray powder diffraction spectroscopy analyses polycrystalline powder samples. It gives information about phase identification of crystalline materials and the average composition of materials. An X-ray powder diffractometer consists of a goniometer, an X-ray tube, incident beam optics, diffracted beam optics, and a detector. ${ }^{216-217}$ Monochromatic X-ray radiation irradiates sample and the $\mathrm{X}$-rays are scattered by the electrons of atoms in the lattice planes. As the sample and the detector are rotated, the intensity of the reflected X-rays is recorded. The sample rotates in the path of the X-ray beam at an angle $\theta$ while the X-ray detector collects the diffracted $\mathrm{X}$-rays and rotates at an angle of $2 \theta$ (Figure 2.3). The diffraction angle 
can reveal information about the composition of the material. For instance, the diffraction angle of $\mathrm{Fe}(0) 2 \theta$ is $45^{\circ} .{ }^{218}$

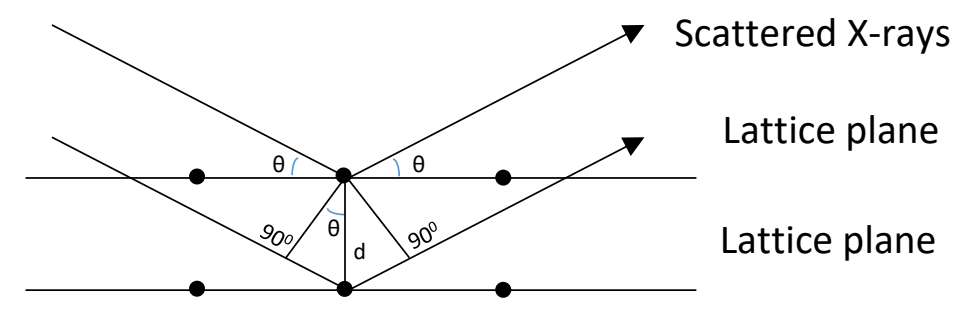

Figure 2.3. Diagram showing the interaction of X-rays with layers of atoms in a crystal. ${ }^{215}$

\subsubsection{Ion chromatography}

Ion chromatography (IC) is a form of liquid chromatography. It is commonly used in the environmental, clinical, and industrial analysis. IC is considered a reliable and sensitive method to analyse inorganic ions. ${ }^{219}$ It is also used for the analysis of charged organic substances, such as acids, bases, amino acids, and carbohydrates.

In IC, there is a stationary phase and a mobile phase that both play a role in separating ions. A stationary phase is a solid support coated with different functional groups, depending on types of ions undergoing separation. In cation separation, the cation-exchange column is coated with sulfonic or carboxylic acid groups. In anion separation, the anion-exchange column is modified with quaternary ammonium functional groups. The mobile phase must contain ionic eluents in both cation and anion analysis. Ions are measured by conductivity or UV detectors. ${ }^{220}$

The procedure of the ion analysis by using IC consists of equilibration, adsorption, elution and desorption. ${ }^{221}$ Analytes are injected and adsorbed on the active sites of the separator column. Ions in eluent solution compete with analytes and then analytes are eluted. Analytes are detected and quantified by the detector.

The ion identity is determined by its retention time. The areas of peaks correspond to the concentration of analyte ions. The retention time is influenced by the nature of ions such as valence, size, and polarization of ions, concentration of the mobile phase, and temperature of the separator column. ${ }^{212} \mathrm{~A}$ high concentration of the eluent decreases retention time but also yields poor separation. A higher column temperature decreases the viscosity of the mobile phase, leading to an increase in chromatography efficiency. ${ }^{220}$ 
There are two kinds of IC: suppressed and non-suppressed. Suppressed IC is used widely to determine common inorganic ions, such as iodide, nitrate, or bromide in water ${ }^{222}$ as well as pharmaceuticals. ${ }^{223}$ The sample separated from the column contains analyte ions and an eluent. This eluent has high conductivity, leading to a low signal to noise ratio. To deal with this drawback, the suppressor plays a role in replacing cations from the separated solution with hydronium ions for anion analysis and hydroxide ions for cation analysis by using an ion exchange membrane. It decreases eluent conductivity to nearly zero and increases the selectivity, performance, and resolution of the method. ${ }^{212}$ Bicarbonate, a mixture of bicarbonate and carbonate, hydroxide ${ }^{219}$ or borate ${ }^{224}$ are often used as eluents in suppressed IC (Figure 2.4).

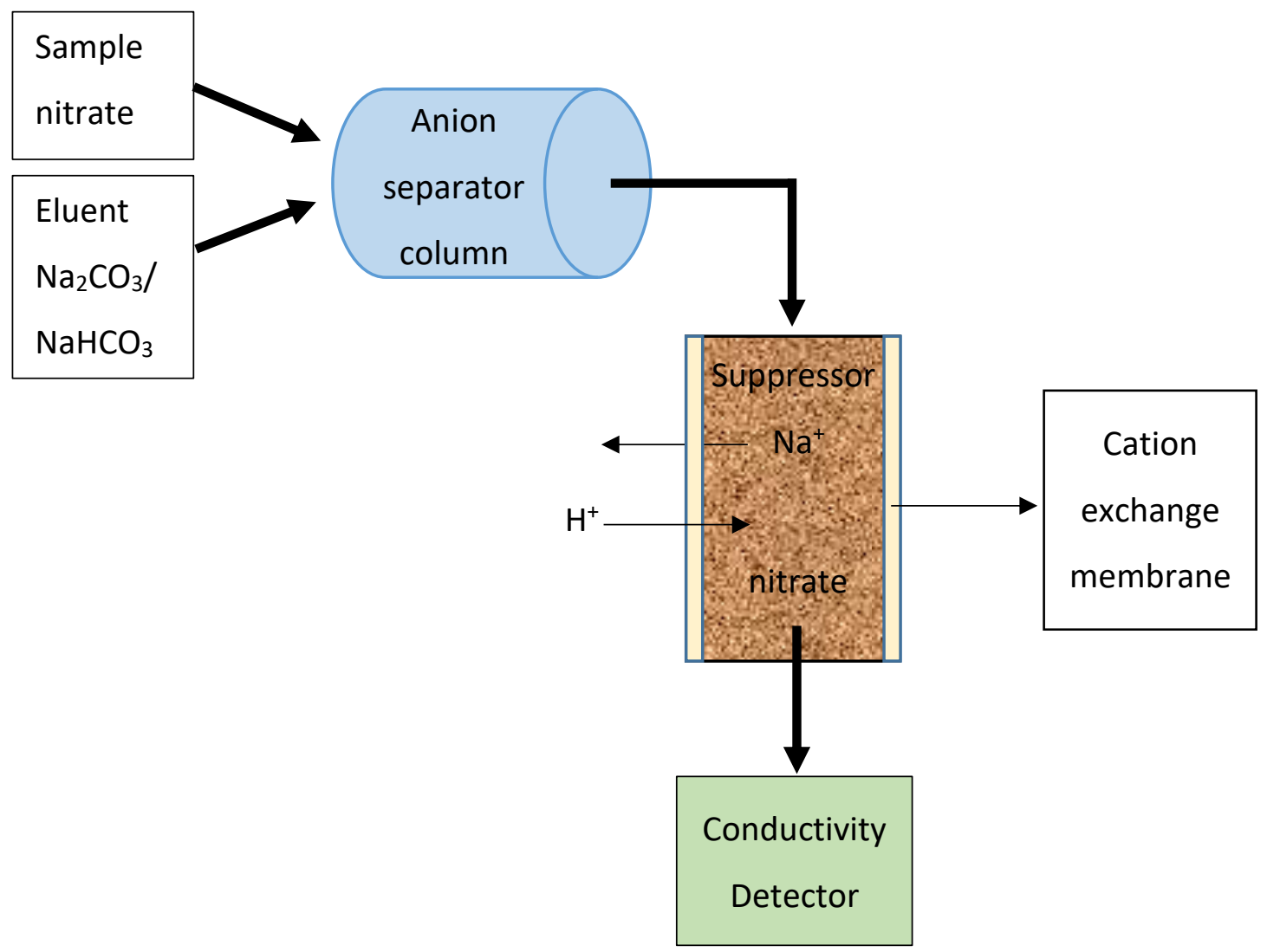

Figure 2.4. Diagram showing suppressed anion chromatography. ${ }^{212}$

In the non-suppressed IC, the separator column is a resin which has a low ion-exchange capacity. Eluents are dilute solutions, such as $\mathrm{KOH}$ for anion separation and $\mathrm{HCl}$ for cation separation. The matrix of the sample does not affect the signal of analyses. The conductivity increases when analytes emerge from the column. Ions are characterized by the difference between the conductivity of the analyte and eluent. ${ }^{212}$ Nitrate can be determined by IC using 
a conductivity detector ${ }^{225}$ or a UV detector ${ }^{226-229}$ at $225 \mathrm{~nm}$ or $210 \mathrm{~nm}$. In this project, suppressed anion chromatography in which the mixture solution of $\mathrm{Na}_{2} \mathrm{CO}_{3}$ and $\mathrm{NaHCO}_{3}$ was the eluent and a conductivity detector was used.

\subsubsection{Atomic absorption spectroscopy}

Atomic spectroscopy is a spectroscopic method in which the absorption of ultraviolet and visible radiation depends on transitions between electronic energy levels. ${ }^{230}$ When an atom absorbs energy, an electron is promoted from the ground state to the excited state in higher orbitals. The atomic spectroscopy method has high sensitivity and hence, it is possible to distinguish and measure one element from other elements in a complex matrix with high reliability. It is used to measure analytes at ppm or ppb level.

There are two popular types of atomic spectrometry, including atomic emission spectrometry (AES) and atomic absorption spectrometry (AAS). Both rely on the atomising process that ions in solution are vaporised and atomised to form gaseous ions by heat from the flame or furnace. The intensity of emission or absorption at a particular wavelength is proportional to the number of atoms. Thus, it is utilised to measure the concentrations of ions in solution. In this study, AAS as a method for measuring the concentrations of metal ions was used.

Under thermal excitation, atoms absorb energy as photons to promote electrons from the ground state to the excited state. The sample is only irradiated with the wavelengths that match the transitions between the energy levels of elements to escape the ground state. Each element absorbs at specific wavelengths; as such, a specific radiation source is used for a particular element. The radiation source is a hollow cathode lamp.

In flame AAS, depending on the elements analysed, either acetylene/air or acetylene/nitrous oxide flame is used. The combustion of acetylene and air is commonly used as it typically produces a flame temperature of $2,400-2,700{ }^{\circ} \mathrm{K}$ (e.g, iron, nickel, copper, cadmium, and lead analysis in this study). When a hotter flame is required, acetylene and nitrous oxide are used (e.g, chromium measurement.) 


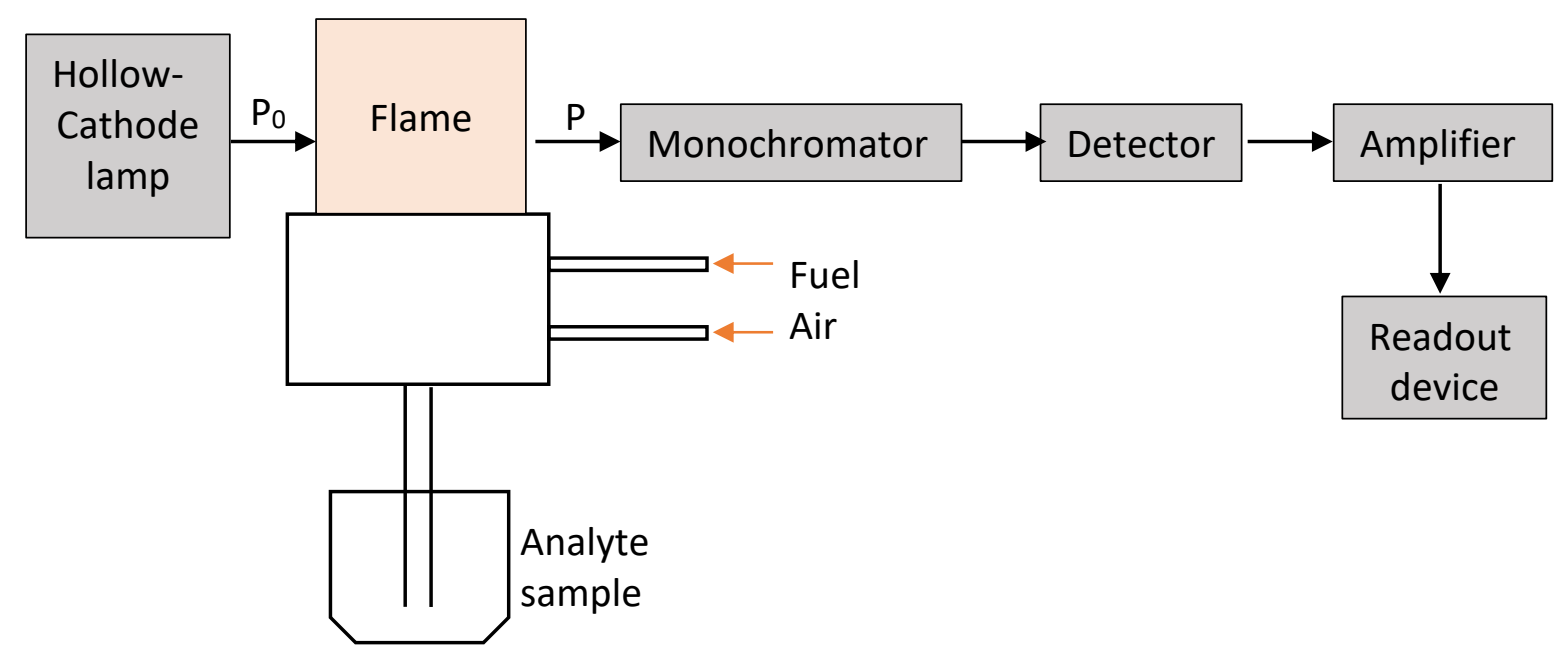

Figure 2.5. Diagram showing atomic absorption experiment. ${ }^{212}$ 


\section{Determining the optimal dopant metals on nZVI@Misi in nitrate}

reduction

\subsection{Introduction}

In this chapter, investigations into different types of supported bimetallic and trimetallic nZVI are discussed. The goal is to determine the best nZVI dopant in terms of both adsorbing onto Misi and reactivity towards nitrate reduction. These studies included investigations on the types of doping metals, Misi preparations and different synthetic methods for adding the dopant. The doped metals to generate supported bimetallic $n Z V I$ and trimetallic $n Z V I$ were $\mathrm{Ni}, \mathrm{Cu}, \mathrm{Zn}$, and Pd. Bimetallic and trimetallic nZVI on Misi were synthesised using different methods including co-reduction (MA) and deposition (MB). Four different preparations of Misi were used to examine the effect of Misi pre-treatment on absorption and reactivity. For each material synthesised, the morphology, composition and elemental distribution were characterised and the reactivity towards nitrate reduction was examined.

The Misi silicate was prepared using procedures to that has been reported previously by our group. ${ }^{1}$ Initially, four different preparations of Misi were examined. This included heating raw Misi to $120^{\circ} \mathrm{C}$ (MisiU), calcining Misi at $600^{\circ} \mathrm{C}$ for 6 hours (MisiC), boiling MisiC in deionised water for 2 hours (MisiC-B) and coating MisiC with iron oxyhydroxide (MisiCFeOOH). These procedures were shown to affect not only the amount of nZVI adsorbed onto the silicate surface but also the reactivity of supported nZVI towards nitrate reduction. Misi that had undergone calcination (MisiC) exhibited better dispersion of adsorbed nZVI particles and the MisiCFeOOH supported $\mathrm{nZVI}$ reduced nitrate faster than other materials.

Three different synthetic methods were trialled to generate supported bimetallic $\mathrm{M}$ nZVI@Misi, named method A (MA), method B1 (MB1) and method B2 (MB2). Four different synthetic methods were used to generate supported trimetallic M-nZVI@Misi, named method $A$ ( $\left.M A_{\text {tri }}\right), M C 1, M C 2$, and MD. The morphology of the resulting materials was examined using SEM and TEM. The elemental composition was examined using SEM-EDS and TEM-EDS. It was also determined by subjecting to nitric acid and the concentration of metals in the resultant solutions was measured using AAS. Additionally, the crystalline composition was investigated using XRD. 
In Fraser's study, when the molar ratio of iron to nitrate of 25:1 was used, the maximum of 70\% nitrate was reduced using non-doped nZVI@Misi under anaerobic conditions. ${ }^{1}$ However, when this ratio was applied using doped systems, no difference in the reactivity of these materials was found due to the quick nitrate reduction. Therefore, in order to compare the reactivity of doped $\mathrm{nZVI}$ materials, the nitrate reduction was conducted with the molar ratio of iron to nitrate at 15:1 (10 ppm nitrate-N). All of the reactivity studies reported in Chapter 3 were carried out without $\mathrm{pH}$ control. The ability of each of the materials towards nitrate reduction was screened using UV-Vis to monitor the disappearance of nitrate. The uncertainties of the results in this chapter were calculated using linear least-squares analysis. ${ }^{212}$ The average and standard deviation of nitrate concentrations were calculated based on standard curve. The most efficient systems are examined in greater detail in Chapter 4, including IC analysis of the reaction mixtures as well as determining the reproducibility of each system.

The Nessler method was utilised to measure the ammonium concentration in solutions after nitrate reduction. ${ }^{231}$ In this method, ammonium reacts with $\mathrm{Hgl}_{2}$ and $\mathrm{KI}$ in an alkaline condition to form a yellow complex. The concentrations of this complex were measured using UV-Vis. Other potential $\mathrm{N}$-containing products such as $\mathrm{N}_{2}, \mathrm{~N}_{2} \mathrm{O}$, and $\mathrm{NO}_{2}$ were not measured.

Name of materials: In the synthesis of the doped materials, the ratio of dopant to iron was always tightly controlled; however, as we discovered, the ratio of the dopant to iron did not always exactly reflect the ratio of species in the reaction mixture. However, due to brevity and consistency throughout the thesis as well as with other reported bimetallic and trimetallic $\mathrm{nZVI}$, the materials will be named by the initial amounts used in full recognition that the absolute ratio might be slightly different. For example,5\%Ni-nZVI@Misi means that the mass ratio of $\mathrm{Ni}$ to $\mathrm{Fe}$ is $5: 100$ and the mass ratio of Fe to Misi is 10:100. In another way, the ratio of Ni:Fe:Misi is 0.5:10:100.

\subsection{Nitrate reduction using nZVI@Misi}

As the control materials, the reactivity of nZVI@Misi that was synthesised using the same amount of Fe and Misi with supported bimetallic and trimetallic nZVI@Misi was examined. Two different materials, nZVI on calcined Misi (nZVI@MisiC) and nZVI on calcined/FeOOHcoated Misi (nZVI@MisiCFeOOH), were used. The molar ratio of iron to nitrate was 15:1 (10 
ppm nitrate-N). The results showed that nZVI@MisiCFeOOH has a slightly higher reactivity than nZVI@MisiC but the difference was minimal (Figure 3.1). After 5 minutes, nitrate-N concentrations were more than 9 ppm for either material. After 30 minutes, nitrate-N concentrations were $5.5 \pm 0.6 \mathrm{ppm}$ ( $45 \%$ nitrate conversion) and $4.5 \pm 0.6 \mathrm{ppm}$ ( $55 \%$ nitrate conversion) when either nZVI@MisiC or nZVI@MisiCFeOOH was used, respectively.

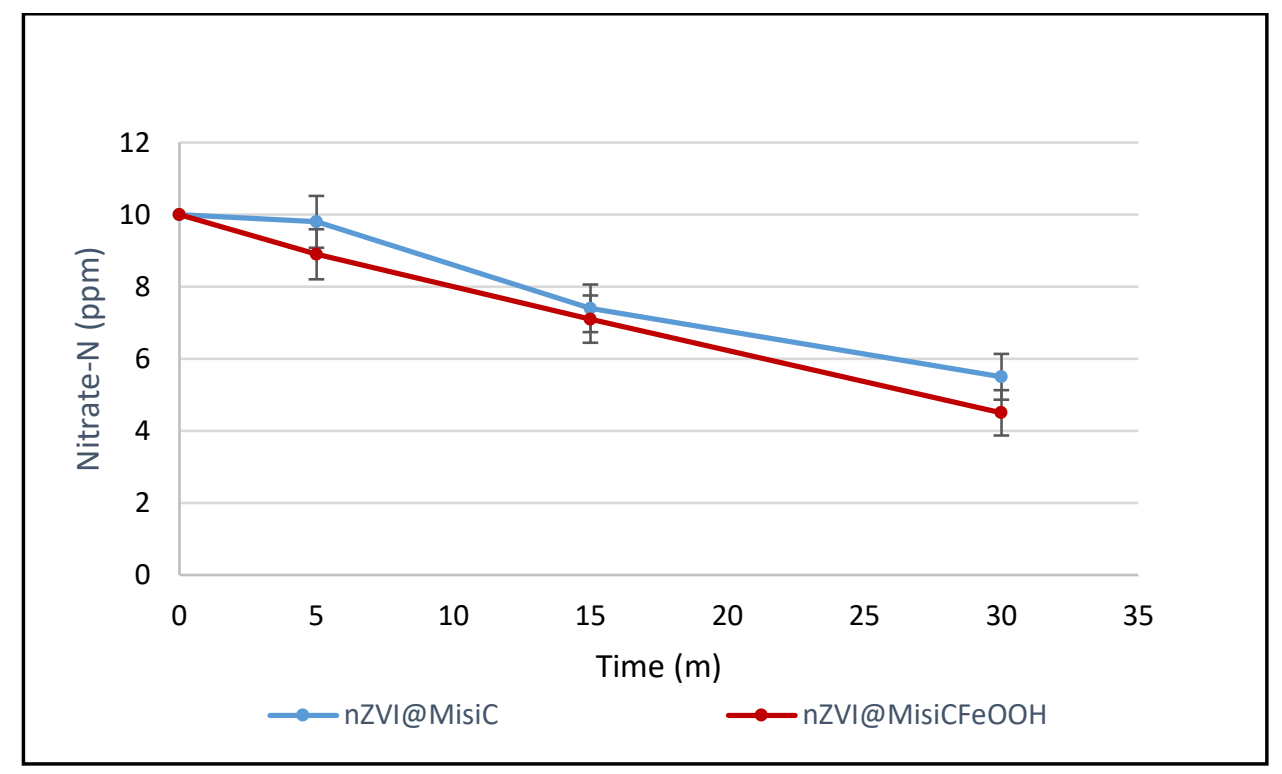

Figure 3.1. Reduction characterisation of nZVI@Misi towards nitrate-N 10 ppm $(0.150 \mathrm{~g}$ of material, $25.0 \mathrm{~mL}$ of solution, under anaerobic conditions).

\subsection{Bimetallic Ni-nZVI@Misi}

Several studies on doping $\mathrm{Ni}$ on the surface of nZVI were published. In unsupported Ni-nZVI systems, the effect of $\mathrm{Ni}$ concentrations (from $0.2 \%$ to $30 \%$ ) on the reactivity of materials was examined. $^{2}$ 167, 175 However, in supported Ni-nZVI, for example Ni-nZVI@zeolite, ${ }^{193} \mathrm{Ni}$ nZVI@kaolinite, ${ }^{192,199}$ systems with the ratio of Ni:Fe:support (0.1:1:2) were reported. In this section, the synthesis, characterisation, and reactivity towards nitrate reduction of bimetallic Ni-nZVI@Misi systems were investigated. Ni-nZVI@Misi was synthesised using all three synthetic methods, MA, MB1, and MB2. In these experiments, the mass ratio of Fe to Misi was $1: 10$ and the mass ratios of $\mathrm{Ni}$ to Fe were varied from $2: 100$ to $10: 100$ with $\mathrm{NiCl}_{2}$ as the nickel source. Since then, three different variables were examined. They included variations on Misi preparations (MisiU, MisiC, MisiC-B, and MisiC-FeOOH) using 5\%Ni and co-reduction method (MA), variations on Ni loading using MisiC and co-reduction method (MA) and variations regarding dopant addition, including co-reduction method (MA) and deposition 
method (MB1 and MB2). The systems investigated are listed in Table 3.1. They include 2\%NinZVI coated on MisiC using co-reduction method (MA), 5\%Ni-nZVI coated on four different types of Misi using the co-reduction method (MA), 5\%Ni-nZVI coated both MisiC and MisiCFeOOH using the deposition method (MB1 and MB2), and 10\%Ni-nZVI coated on MisiC using the co-reduction method (MA).

Table 3.1. List of Ni-nZVI@Misi systems synthesised.

\begin{tabular}{|c|c|c|c|c|c|}
\hline \multirow{2}{*}{ Materials } & \multicolumn{4}{|c|}{ Misi preparation } & \multirow{2}{*}{ Method } \\
\hline & MisiU & MisiC & MisiC-B & MisiCFeOOH & \\
\hline 2\%Ni-nZVI@ & & $x$ & & & Co-reduction (MA) \\
\hline \multirow{3}{*}{ 5\% Ni-nZVI@ } & $\mathrm{X}$ & $\mathrm{X}$ & $\mathrm{X}$ & $\mathrm{X}$ & Co-reduction (MA) \\
\hline & & $\mathrm{X}$ & & $\mathrm{X}$ & Deposition (MB1) \\
\hline & & $x$ & & $x$ & Deposition (MB2) \\
\hline 10\% Ni-nZVI@ & & $\mathrm{X}$ & & & Co-reduction (MA) \\
\hline
\end{tabular}

\subsubsection{Variations on Misi preparation}

The importance of silicate preparation on supporting Ni-nZVI on Misi was examined by characterising the morphology of the materials with SEM (Figure 3.2). In the COMPO images, higher atomic number (higher density) elements are shown as a brighter signal, thereby, the $\mathrm{Ni}-\mathrm{nZVI}$ is observed as the lighter components and the silicate is grey. The COMPO images revealed an uneven dispersion of nanoparticles on the surface of the Misi particles. In some areas (red circle), good adsorption is observed. However, in other areas (blue circle), the nanoparticles are observed in the form of chains, indicating agglomeration due to magnetic and electrostatic forces of Fe. ${ }^{162}$ The disordered distribution of nanoparticles on support Misi is similar to that observed by Shi et al. ${ }^{192}$ However, in their SEM images, a higher degree of agglomeration was observed on kaolinite, potentially due to the higher ratio of Fe to silicate (1:2).

Agglomeration was visibly observed to be more significant on Misi that had not undergone calcination (MisiU) (Figure 3.2 a) than that of any other preparations (Figure 3.2 b, c, d). This is consistent with previous investigations in our group with non-doped nZVI on Misi. 
Calcination was concluded to be an important step in Misi treatment for adsorbing nanoparticles. ${ }^{1}$ In materials from calcined Misi, including MisiC, MisiC-B, and MisiCFeOOH, the difference in the dispersion of Ni-nZVI was not visibly observed in SEM.
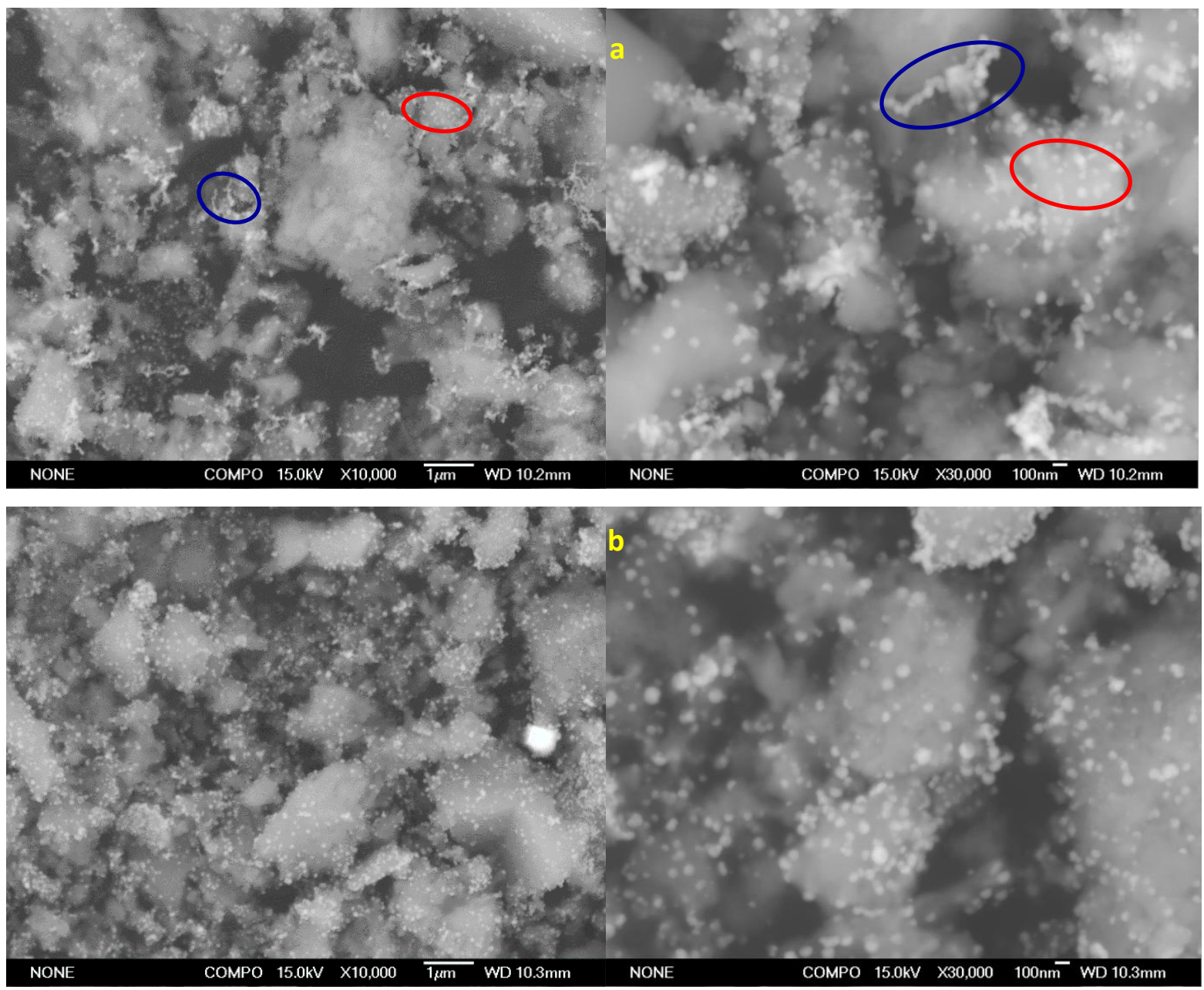

Figure 3.2. COMPO images of 5\%Ni-nZVI on: (a) MisiU, (b) MisiC, (c) MisiC-B and (d) MisiCFeOOH synthesised via co-reduction method (MA) in two different magnifications. The lighter component is attributed to Ni-nZVI and silicate is grey. Figure $c$ and $d$ is in the next page. 

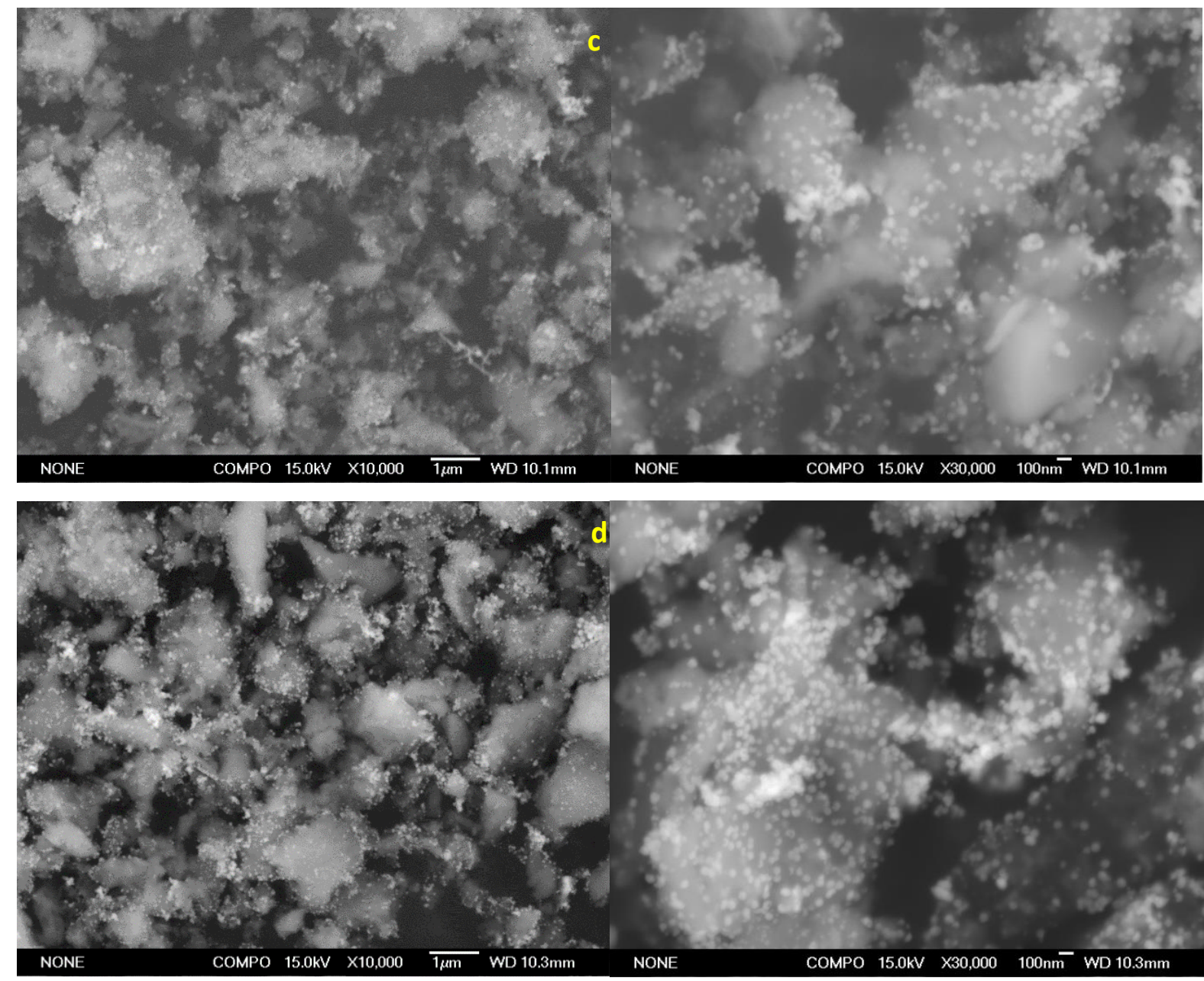

Figure 3.2. COMPO images of 5\%Ni-nZVI on: (a) MisiU, (b) MisiC, (c) MisiC-B and (d) MisiCFeOOH synthesised via co-reduction method (MA) in two different magnifications. The lighter component is attributed to Ni-nZVI and silicate is grey. Figure $a$ and $b$ are in the previous page.

The morphology of Ni-nZVI@Misi was compared to that of unsupported materials. COMPO images showed that unsupported Ni-nZVI and nZVI particles are more aggregated and stacked together to form long chains (Figure 3.3). In contrast, in the presence of Misi, the electromagnetic attraction of $\mathrm{nZVI}$ particles is reduced, preventing nanoparticles from clustering. ${ }^{191}$ Therefore, using Misi as the support is one of the key requirements to spread out nanoparticles. 


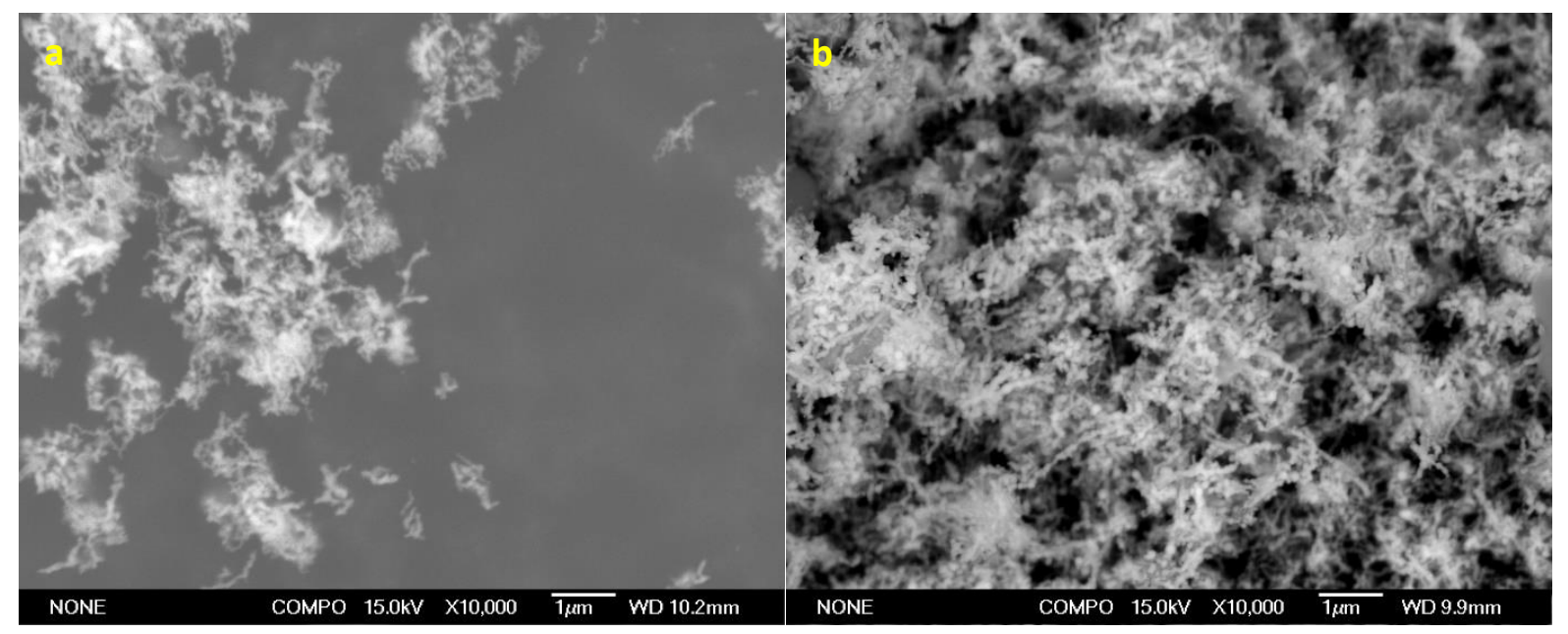

Figure 3.3. COMPO images of (a) 5\%Ni-nZVI and (b) nZVI.

In studies on non-doped nZVI@Misi, the adsorption of nZVI on Misi was quantified using Picasa and FIJI software. ${ }^{1}$ As discussed by Fraser, both particle size of nZVI and the distribution of nanoparticles on Misi was difficult to determine. These variables were not reproducible due to the heterogeneity of Misi when different bathes of materials were used. Therefore, in this current study, the size and distribution of bimetallic particles have not been investigated.

Nitrate reduction of the Ni-nZVI on different preparations of Misi was examined. In these studies, four materials, including 5\%Ni-nZVI@MisiU,5\%Ni-nZVI@MisiC,5\%Ni-nZVI@MisiC-B and 5\%Ni-nZVI@MisiCFeOOH prepared via co-reduction method (MA) were used. The molar ratio of Fe to nitrate was 15:1. Nitrate was reduced more slowly using 5\%Ni-nZVI@MisiU than that of any materials on Misi that involved a calcination step. After 5 minutes, nitrate-N concentrations were 9.9 ppm (5\%Ni-nZVI@MisiU) and less than 8 ppm (either 5\% NinZVI@MisiC, 5\%Ni-nZVI@MisiC-B or 5\%Ni-nZVI@MisiCFeOOH). After 20 minutes, the nitrate$\mathrm{N}$ level was $4 \mathrm{ppm}$ and above $1 \mathrm{ppm}$, respectively (Figure 3.4). A minimal difference in the nitrate reduction of 5\%Ni-nZVI@MisiC,5\%Ni-nZVI@MisiC-B and 5\%Ni-nZVI@MisiC-FeOOH was observed. Based on the distribution of nanoparticles on Misi, there appears to be a correlation between the dispersion and the reactivity. The better the particles adsorbed on Misi, the faster the materials reduced nitrate.

Accordingly, two preparations of Misi, including calcined Misi (MisiC) and calcined/FeOOHcoated Misi (MisiCFeOOH) were used for future work because of three reasons. Calcination is useful for adsorbing nanoparticles on the surface of silicate, resulting in higher nitrate removal. Generating MisiC is more timesaving than MisiC-B and their resulting materials had 
similar reactivity towards nitrate reduction. Furthermore, preliminary results showed that materials with the FeOOH coating layer are less impacted by potentially inhibitory ions such as carbonate and phosphate.

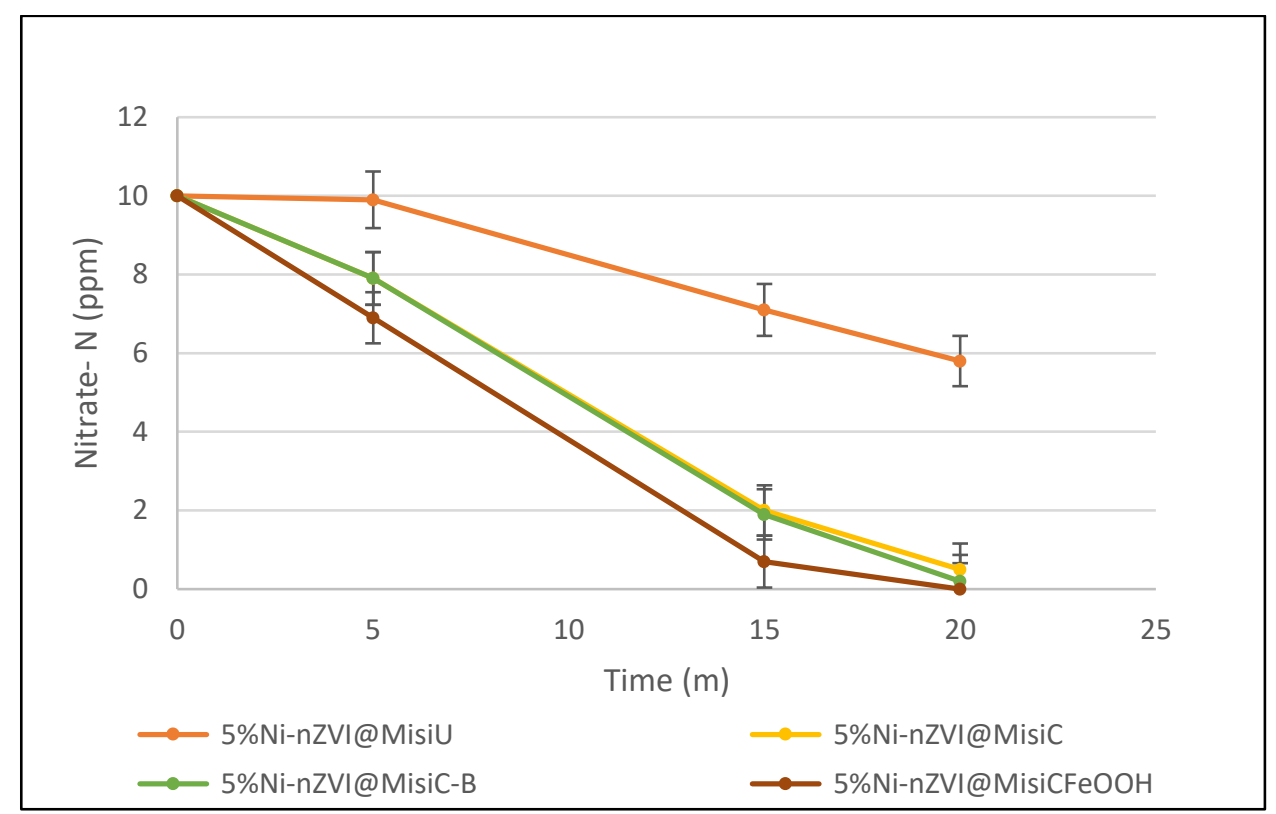

Figure 3.4. Reduction characterisation of Ni-nZVI on different preparations of Misi $(0.150 \mathrm{~g}$ of materials synthesised by co-reduction method (MA), $25.0 \mathrm{~mL}$ of $10 \mathrm{ppm}$ nitrate- $\mathrm{N}$ solution, under anaerobic conditions).

\subsubsection{Variations on Ni loading}

In the investigations into the effect of Ni loading on the nitrate reducing capacity of Ni-nZVI, other authors reported that $5 \% \mathrm{Ni}^{2}$ or $10 \% \mathrm{Ni}^{175}$ in $\mathrm{Ni}-\mathrm{nZVI}$ contributed to the highest reactivity. In addition, in studies on Ni-nZVI@support, 10\%Ni was chosen to test. ${ }^{192-193,199}$ Therefore, in this study, three different Ni concentrations, $2 \%, 5 \%$, and $10 \%$, were used to generate Ni-nZVI on calcined Misi (MisiC) via co-reduction method (MA). The effect of $\mathrm{Ni}$ loading on both the adsorption of Ni-nZVI onto MisiC and the subsequent reactivity towards nitrate were examined.

To investigate the effect of $\mathrm{Ni}$ loading on the adsorption, the morphology of two representative materials including 2\%Ni-nZVI@MisiC and 5\%Ni-nZVI@MisiC was characterised via SEM. A good dispersion of nanoparticles across the silicate support is observed for both dopant contents, although some agglomeration is observed (Figure 3.5). No difference in the dispersion of Ni-nZVI with different Ni contents ( $2 \%$ and $5 \%$ ) on MisiC is 
visibly observed. Therefore, other supported doped nZVI@Misi systems do not need to be examined their morphology. Although the effect of Ni loading on nZVI towards contaminant reduction was examined by other authors, the difference in the morphology of these reported materials was not examined. ${ }^{2,175}$

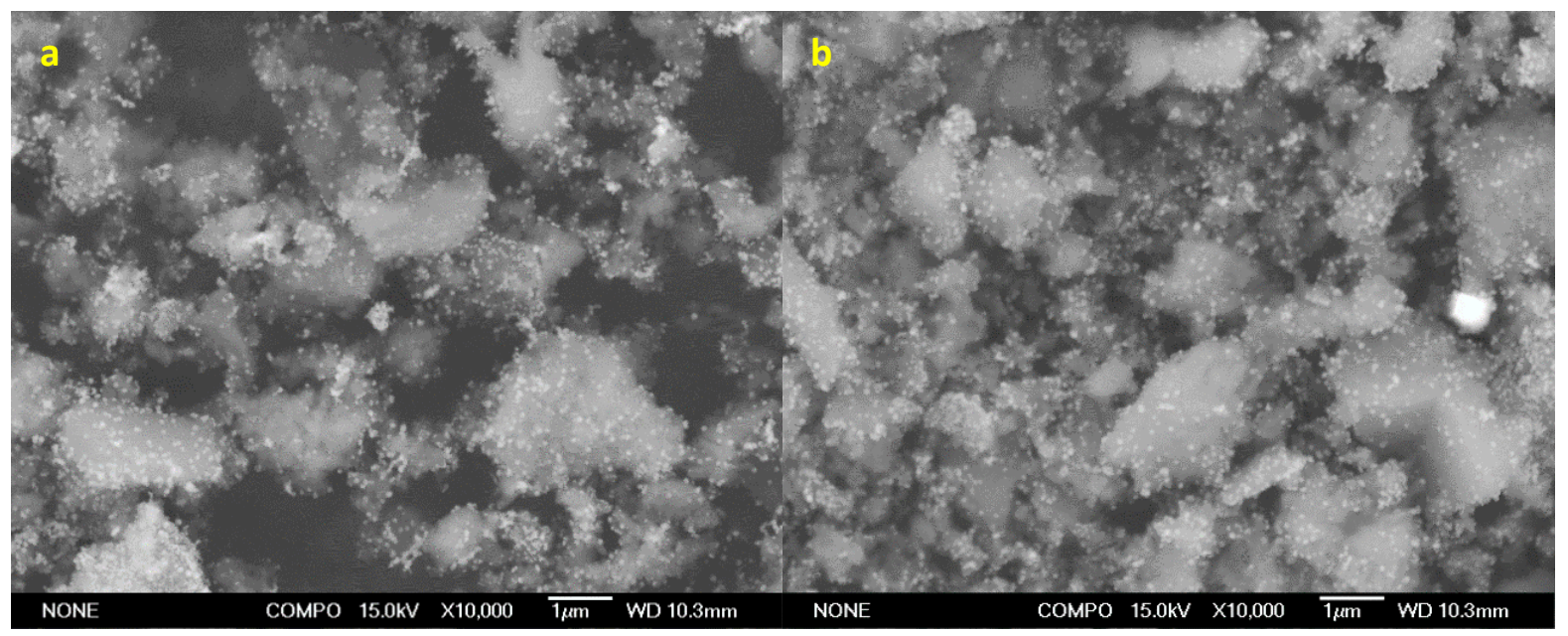

Figure 3.5. COMPO images of (a) 2\%Ni-nZVI@MisiC-MA and (b) 5\%Ni-nZVI@MisiC-MA. The lighter component is attributed to Ni-nZVI and silicate is grey.

The relative reactivity of materials as a function of $\mathrm{Ni}$ loading was investigated. In these studies, three materials, 2\%Ni-nZVI@MisiC, 5\%Ni-nZVI@MisiC and 10\%Ni-nZVI@MisiC prepared via co-reduction method (MA), were used. The molar ratio of Fe to nitrate was 15:1. The results showed that these materials reduced nitrate faster than non-doped nZVI@MisiC (Section 3.2). Nitrate removal increased with increasing Ni concentration. The material with 2\%Ni had the lowest reactivity while 10\%Ni-nZVI@MisiC was the best performing material. After 5 minutes, nitrate- $\mathrm{N}$ concentrations were 9.0 ppm, 7.9 ppm, and 6.9 ppm when either 2\%Ni-nZVI@MisiC, 5\%Ni-nZVI@MisiC or 10\%Ni-nZVI@MisiC was used, respectively (Figure 3.6). Nevertheless, after 20 minutes, the nitrate-N level was 1.7 ppm using 2\%Ni-nZVI@MisiC while the nitrate-N levels were below 1 ppm for either 5\% Ni-nZVI@MisiC or 10\%NinZVI@MisiC, indicating a minimal difference in the reactivity of $5 \%$ and $10 \% \mathrm{Ni}$.

In Kang et al.'s study, 5\% Ni-nZVI showed the fastest reduction rate while the reactivity with $1 \% \mathrm{Ni}-\mathrm{nZVI}, 10 \% \mathrm{Ni}$-nZVI or $20 \% \mathrm{Ni}-\mathrm{nZVI}$ was significantly slower in reducing nitrate. ${ }^{2}$ In another study, Tehrani et al. reported that $10 \% \mathrm{Ni}-\mathrm{nZVI}$ exhibited the fastest nitrate conversion compared to $\mathrm{Ni}-\mathrm{nZVI}$ with $\mathrm{Ni}$ concentration from $5 \%$ to $30 \% .{ }^{175}$ In the different understanding, the reactivity of unsupported $\mathrm{Ni}-\mathrm{nZVI}$ increased to reach the peak and then decreased when 
the Ni content increased. Kang et al. postulated that the highest reactivity was due to the optimum level of doped metal, resulting in the greatest number of nZVI active sites. When there was too much secondary metal (Ni) on the surface of $\mathrm{nZVI}$, the electron transfer from the Fe core to contaminants is hindered, leading to a slower reaction rate. In our work, Fe adsorbed on the surface of Misi with the Fe:Misi ratio of 10:100. This caused much better dispersion of $\mathrm{Fe}$, therefore, the increase in Ni concentration to $10 \%$ ( $\mathrm{Ni}: \mathrm{Fe}=10: 100$ ) could not be enough to deactivate the active sites of Fe particles. However, as there was a minimal difference in the reactivity of 5\% Ni and 10\%Ni on nZVI@Misi, the 5\%Ni-nZVI@Misi was chosen for further work to minimise the amount of nickel used while still maintaining high activity.

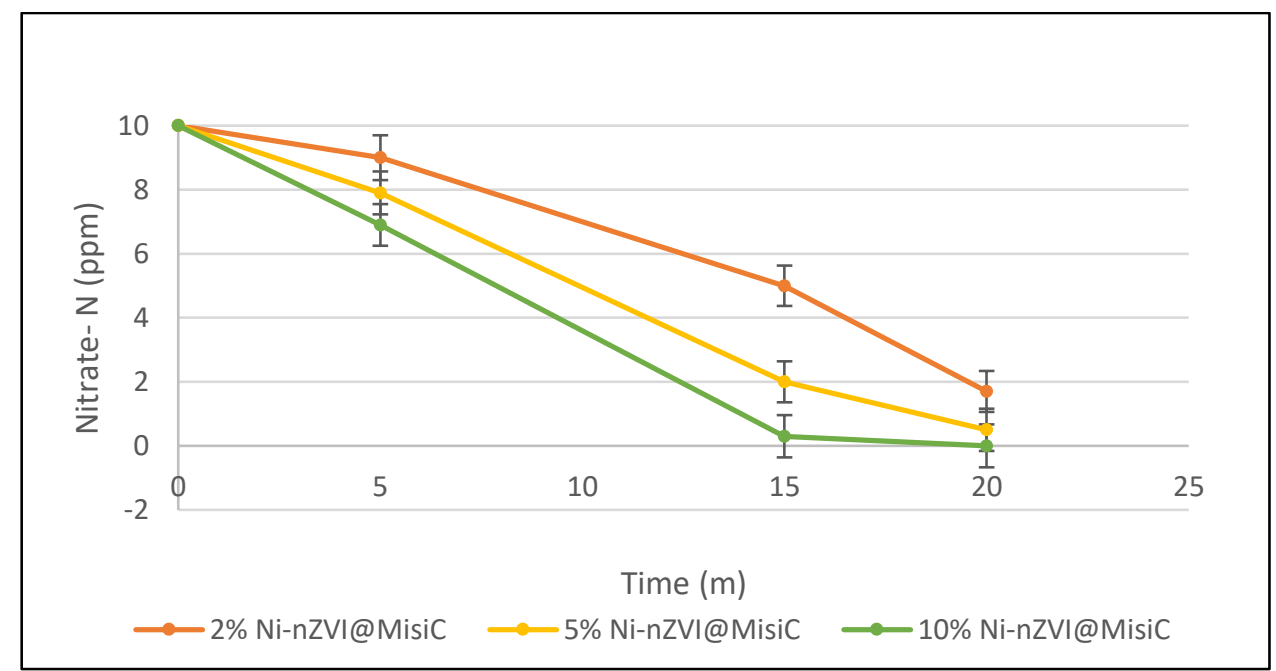

Figure 3.6. Reduction characterisation of Ni-nZVI@MisiC on different Ni concentrations $(0.150 \mathrm{~g}$ of materials synthesised via co-reduction method (MA), $25.0 \mathrm{~mL}$ of $10 \mathrm{ppm}$ nitrate$N$ solution, under anaerobic conditions).

\subsubsection{Variations on synthetic methods}

In previously reported studies, Ni-nZVI was synthesised using both co-reduction (MA) ${ }^{2}$ and deposition methods (MB), ${ }^{2,167,175}$ although all of the Ni-nZVI@support systems including NinZVI@kaolinite, Ni-nZVI@zeolite and Ni-nZVI@cellulose acetate reported were synthesised via co-reduction (MA). ${ }^{192-193,199}$ Regarding to deposition method, wet nZVI was typically used for the deposition step. In our study, the effect of different methodologies for doping nZVI@Misi with Ni was examined. For this, 5\%Ni-nZVI on two preparations of Misi (calcined Misi (MisiC) as well as calcined/FeOOH-coated Misi (MisiCFeOOH)), were synthesised using 
three different methods (MA, MB1, and MB2). The morphology of these resulting materials was examined using SEM, TEM, and XRD. The reactivity of these materials towards nitrate reduction was also examined.

SEM images revealed a good dispersion of Ni-nZVI on Misi for each material (Figure 3.7). In most of the areas, nanoparticles evenly adsorb on the surface of Misi although a low degree of agglomeration is observed in some areas. The appearance of 5\%Ni-nZVI@Misi synthesised using these different methods are similar and are not visibly distinguished via SEM. 

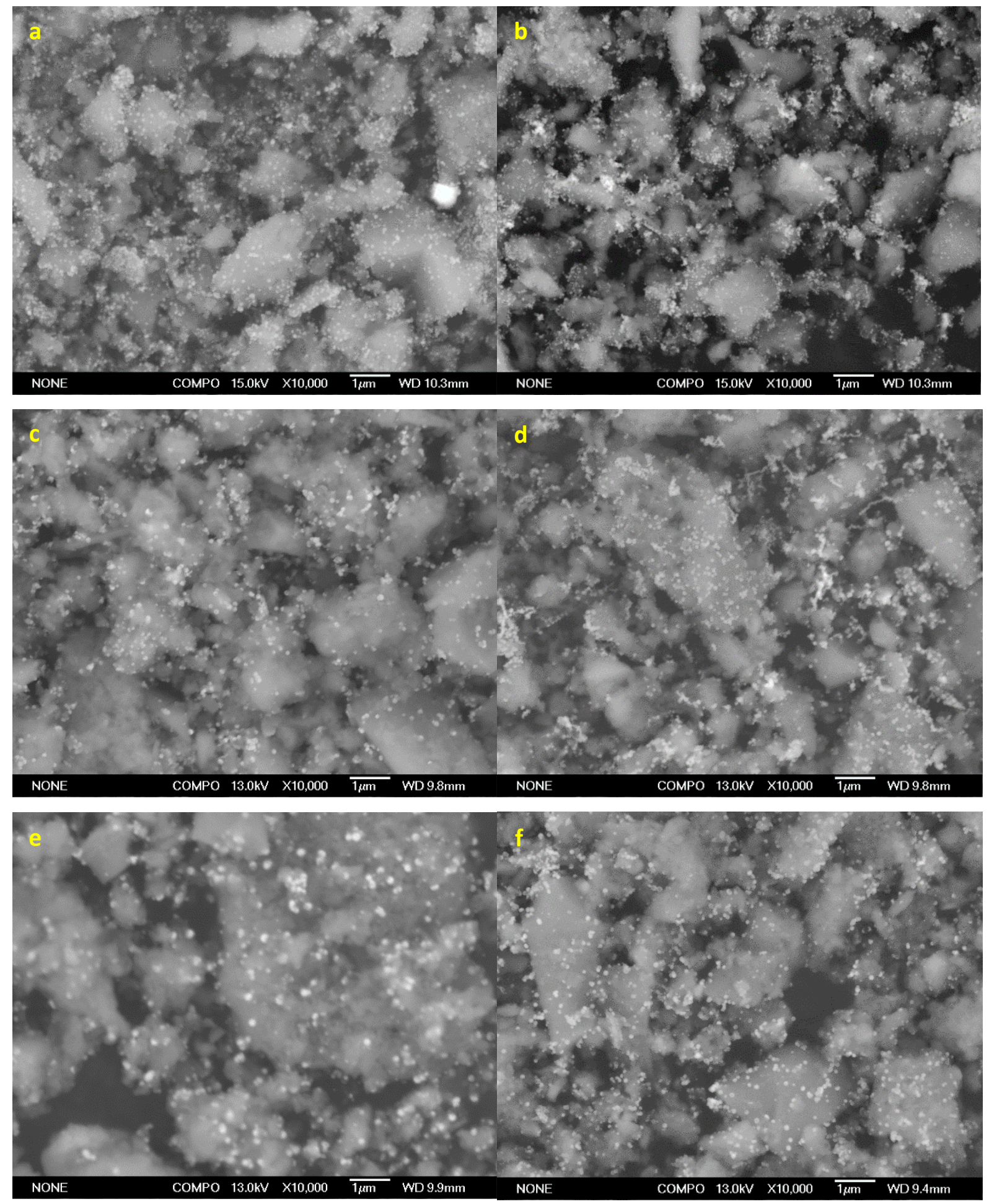

Figure 3.7. COMPO images of (a) 5\%Ni-nZVI@MisiC-MA, (b) 5\%Ni-nZVI@MisiCFeOOH-MA, (c) 5\%Ni-nZVI@MisiC-MB1, (d) 5\%Ni-nZVI@MisiCFeOOH-MB1, (e) 5\%Ni-nZVI@MisiC-MB2, (f) 5\%Ni-nZVI@MisiCFeOOH-MB2. The lighter component is attributed to Ni-nZVI and silicate is grey. 
The elemental distribution of 5\%Ni-nZVI@MisiCFeOOH-MA was examined by using SEM- EDS (Figure 3.8). The signals corresponding to $\mathrm{Si}, \mathrm{O}$, and $\mathrm{Al}$ are very strong, indicating the main components of silicate (Misi). Signals corresponding to $\mathrm{S}$ and $\mathrm{K}$ are also detected with low density. This is due to impurities within the naturally occurring silicate. The signal corresponding to Fe is observed throughout and several high-density regions are observed, presumably corresponding to the nanoparticles. Signals corresponding to $\mathrm{Ni}$ observed in 5\%Ni-nZVI@MisiCFeOOH-MA is not clear and relatively similar to Ni signal examined in nondoped nZVI@MisiCFeOOH (Figure 3.9). There looks to be some correlation between $\mathrm{Ni}$ and Fe in 5\%Ni-nZVI@MisiCFeOOH-MA but mainly, Ni signal does not follow Fe distribution. This is due to the low resolution of the Ni signal; as such, the technique with higher resolution will be needed, for example, TEM-EDS.

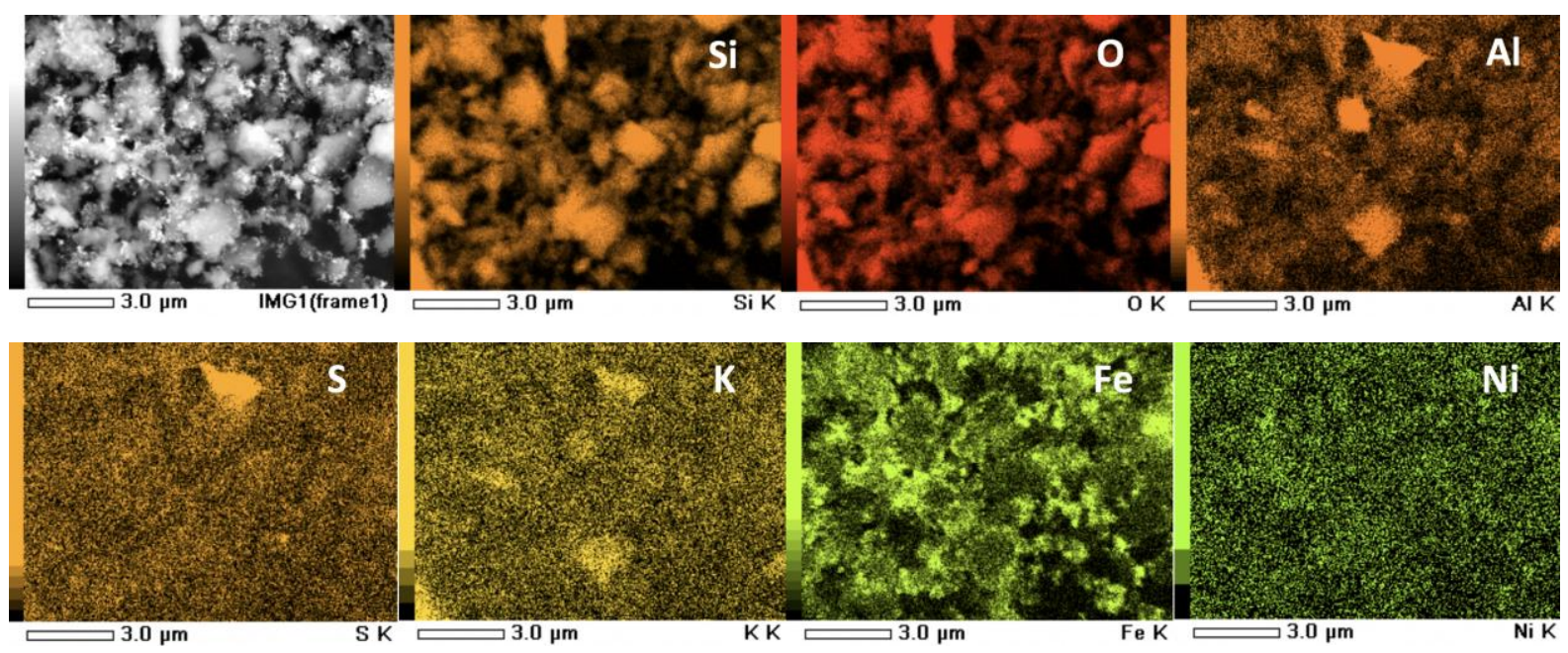

Figure 3.8. SEM-EDS mapping of 5\%Ni-nZVI@MisiCFeOOH-MA.
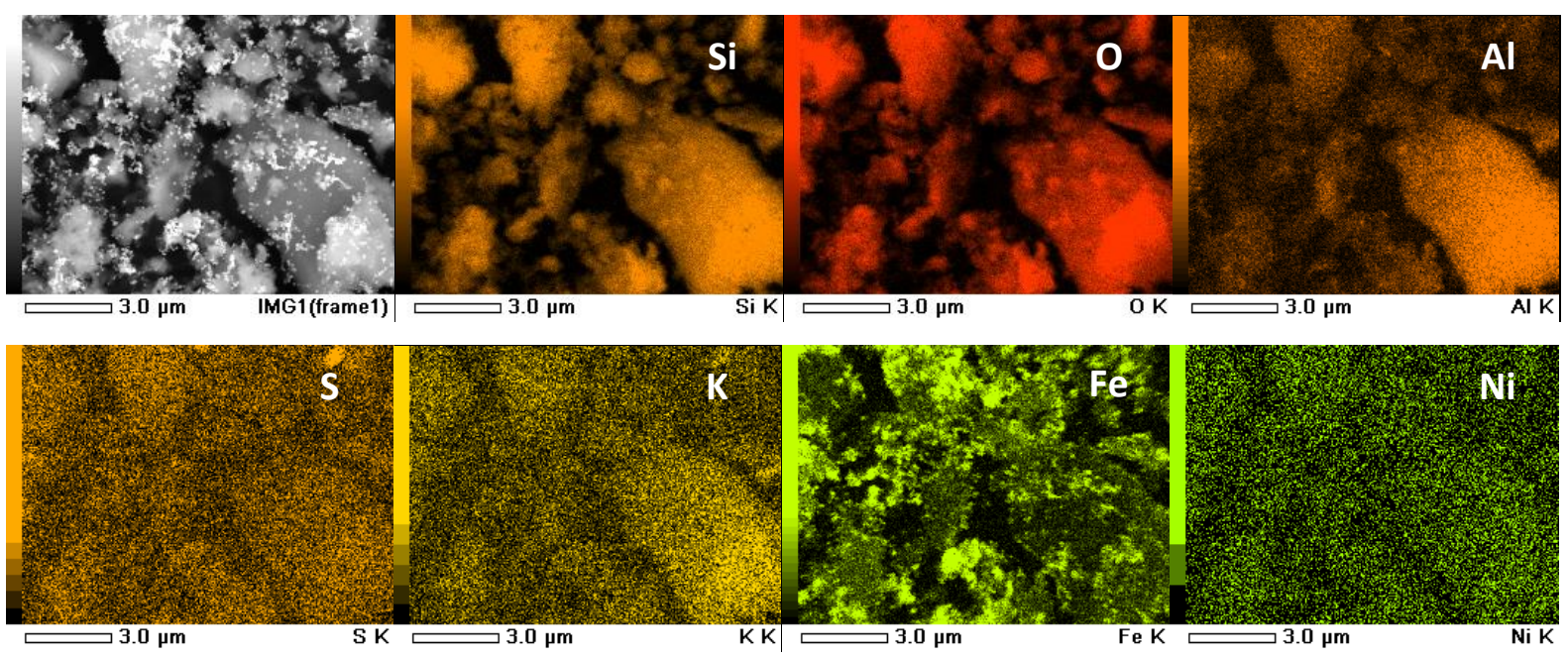

Figure 3.9. SEM-EDS mapping of nZVI@MisiCFeOOH. 
The crystalline composition of 5\%Ni-nZVI@MisiCFeOOH-MA was examined using XRD analysis (Figures 3.10). The main peaks are attributed to silicate, corresponding to cristobalite $\left(2 \theta=22^{\circ}\right)$ and quartz $\left(2 \theta=27^{\circ}\right) \cdot 232$ The broad peak at $2 \theta=45^{\circ}$ can tentatively be assigned to $\mathrm{Fe}(0)$. In studies on M-nZVI on other silicates such as kaolinite and bentonite, a strong peak corresponding to $\mathrm{Fe}(0)$ was found, indicating the presence of nZVI. ${ }^{192,} 233$ In these studies, a higher mass ratio of Fe to silicate was reported, 1:2 and 1:1 for kaolinite and bentonite materials, respectively, in contrast to our 1:10 ratio. In contrast, $\mathrm{Fe}(0)$ was never confirmed in previous studies on non-doped nZVI@MisiCFeOOH. ${ }^{1}$ As such, the XRD analysis of other materials will be in appendix and no further discussion on XRD will be done.

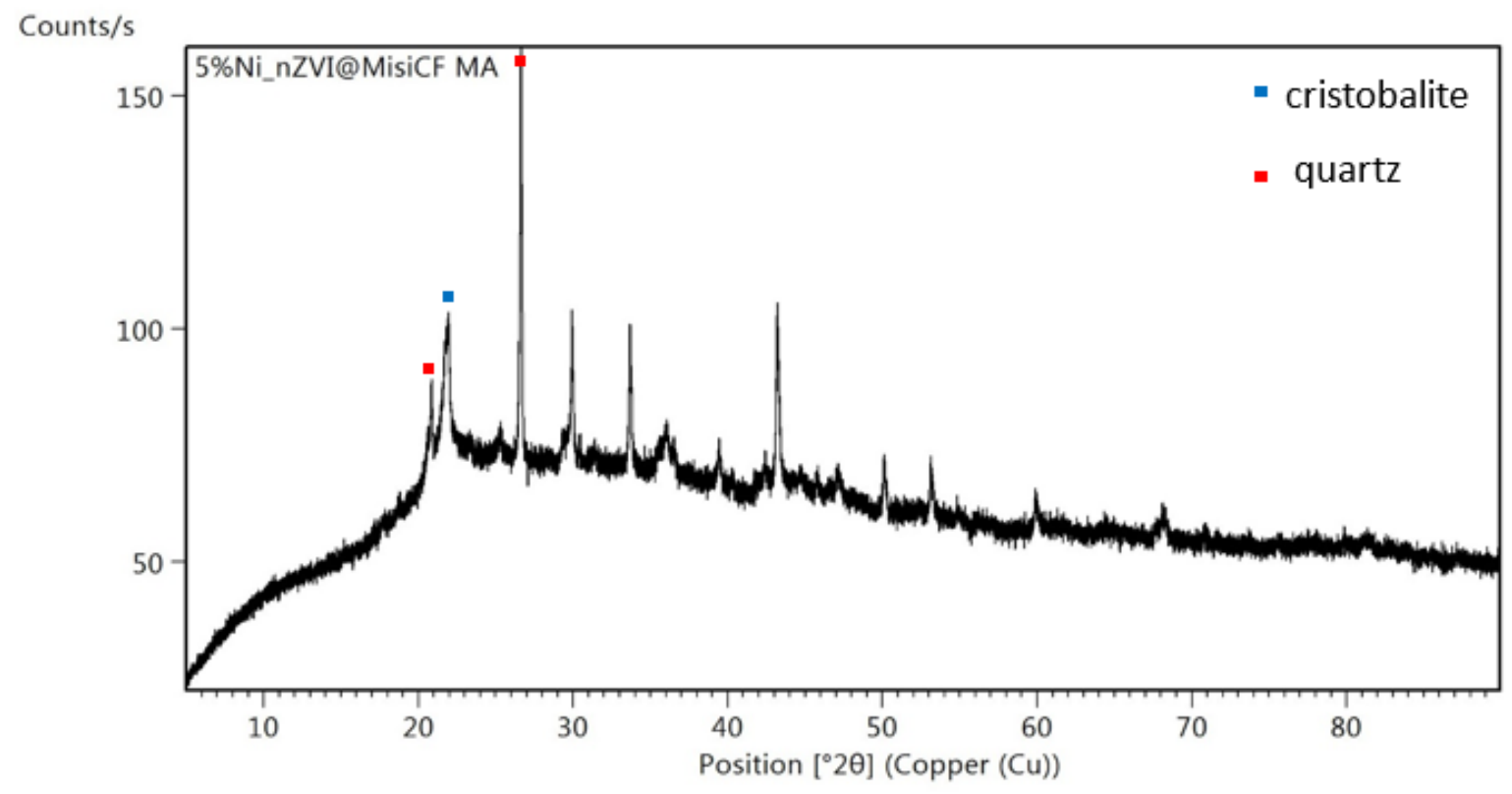

Figure 3.10. XRD spectrum of 5\%Ni-nZVI@MisiCFeOOH-MA.

The shape of Ni-nZVI particles in 5\%Ni-nZVI@MisiCFeOOH synthesised via co-reduction method (MA) and deposition (MB1 and MB2) was examined by TEM (Figure 3.11). The images revealed that the nanoparticles have core-shell structure and are in the form of agglomerates. The core is presumably Fe or Fe-Ni alloy and the shell is presumably iron oxides or iron-nickel oxides. The structure of $\mathrm{Ni}-\mathrm{nZVI}$ on MisiCFeOOH is similar to that of non-doped $\mathrm{nZVI}$ reported in the previous paper. ${ }^{143} \mathrm{In} \mathrm{nZVI}$ particles, the core is $\mathrm{Fe}(0)$ and the shell is an iron oxide layer coming from the oxidation of nZVI during synthesis. 

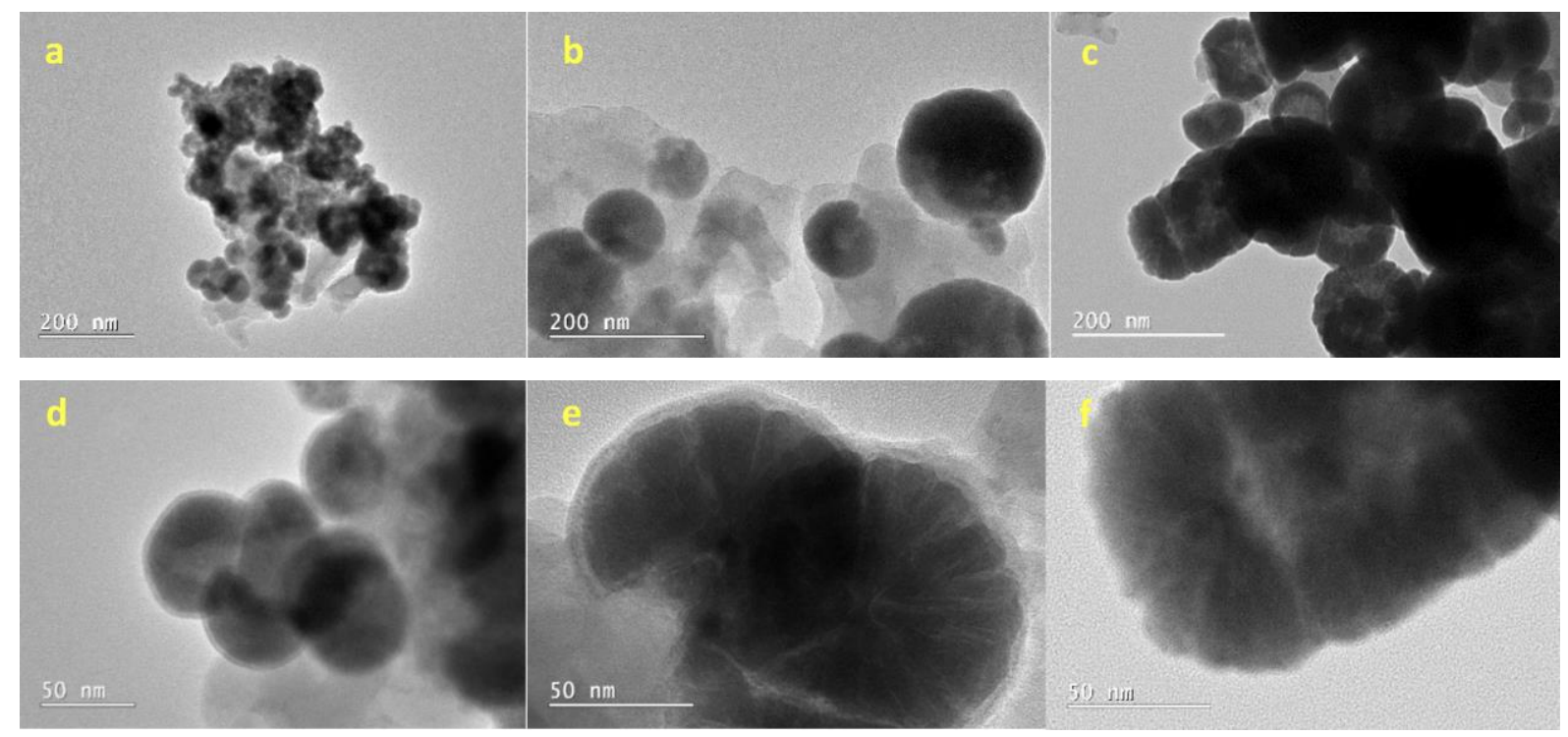

Figure 3.11. TEM images in different magnifications of (a) and (d) 5\%Ni-nZVI@MisiCFeOOHMA; (b) and (e) 5\%Ni-nZVI@MisiCFeOOH-MB1; (c) and (f) 5\%Ni-nZVI@MisiCFeOOH-MB2.

The composition of $\mathrm{Ni}-\mathrm{nZVI}$ supported on MisiCFeOOH prepared via co-reduction method (MA) and deposition method (MB1 and MB2) was analysed. TEM-EDS was taken in different areas and magnifications in each sample. However, in order to examine the elemental composition of nanoparticles and compare the difference in the elemental distribution of particles that were synthesised via different methods, TEM-EDS in small specific areas was chosen to discuss (Figures 3.12, 3.13 and 3.14). High-intensity peaks corresponding to $\mathrm{O}, \mathrm{Si}$, $\mathrm{Al}$, and $\mathrm{S}$ are observed in all three samples, which are attributed to the natural silicate. A peak corresponding to Fe is strong while a peak corresponding to $\mathrm{Ni}$ is weak, consistent with the percentage of these elements in the samples. Based on the quantitative results of elements in TEM-EDS spectra, the percentages of these two elements in examined areas each sample are different. In 5\%Ni-nZVI@MisiCFeOOH-MA, the mass percentage for Fe and Ni was 26.0\% and $0.8 \%$ and the atom percentage was $10.4 \%$ and $0.3 \%$, respectively. In $5 \% \mathrm{Ni}$ nZVI@MisiCFeOOH-MB1, the mass percentage for Fe and Ni was $40.4 \%$ and $1.8 \%$ and the atom percentage was $16.7 \%$ and $0.7 \%$, respectively. In 5\%Ni-nZVI@MisiCFeOOH-MB2, the mass percentage for $\mathrm{Fe}$ and $\mathrm{Ni}$ was $69.4 \%$ and $3.2 \%$ and the atom percentage was $38.9 \%$ and $1.7 \%$, respectively. A strong peak corresponding to $\mathrm{Cu}$ (at $8 \mathrm{keV}$ ) is also observed due to the Cu grid that supports the samples during TEM analysis. 


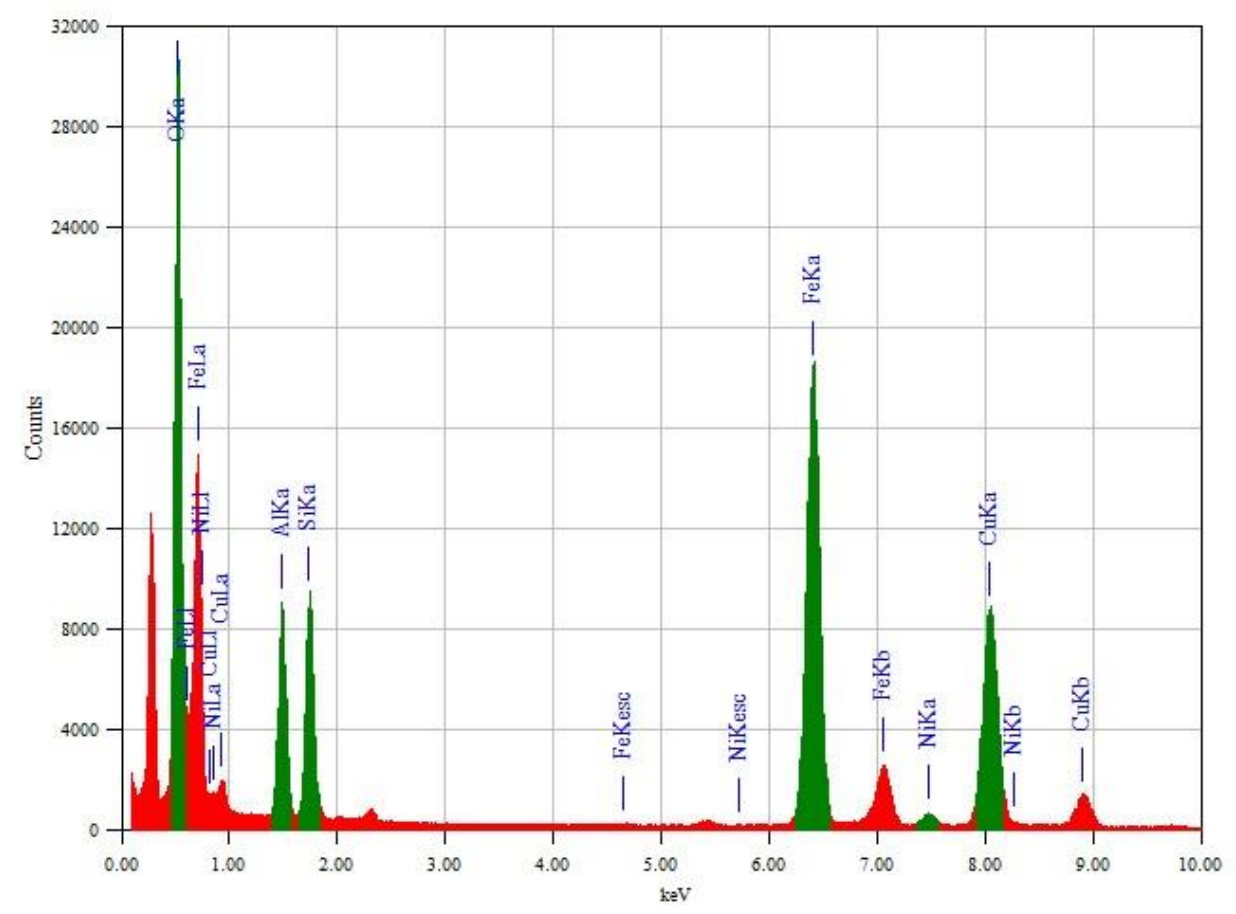

Figure 3.12. TEM-EDS spectrum of 5\%Ni-nZVI@MisiCFeOOH-MA. Note: labels have been modified to highlight peaks relevant to this research. Please see appendix B.1 for complete spectrum.

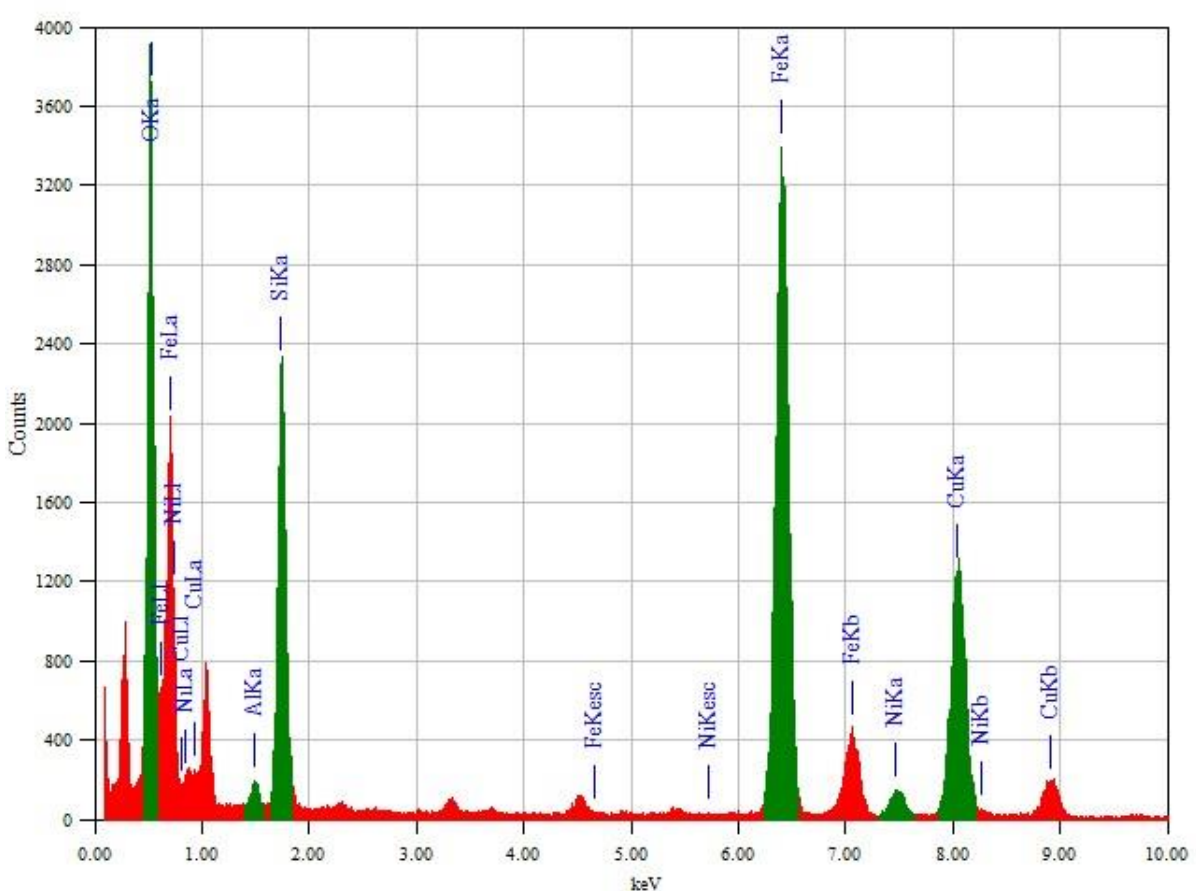

Figure 3.13. TEM-EDS spectrum of 5\%Ni-nZVI@MisiCFeOOH-MB1. Note: labels have been modified to highlight peaks relevant to this research. Please see appendix B.2 for complete spectrum. 


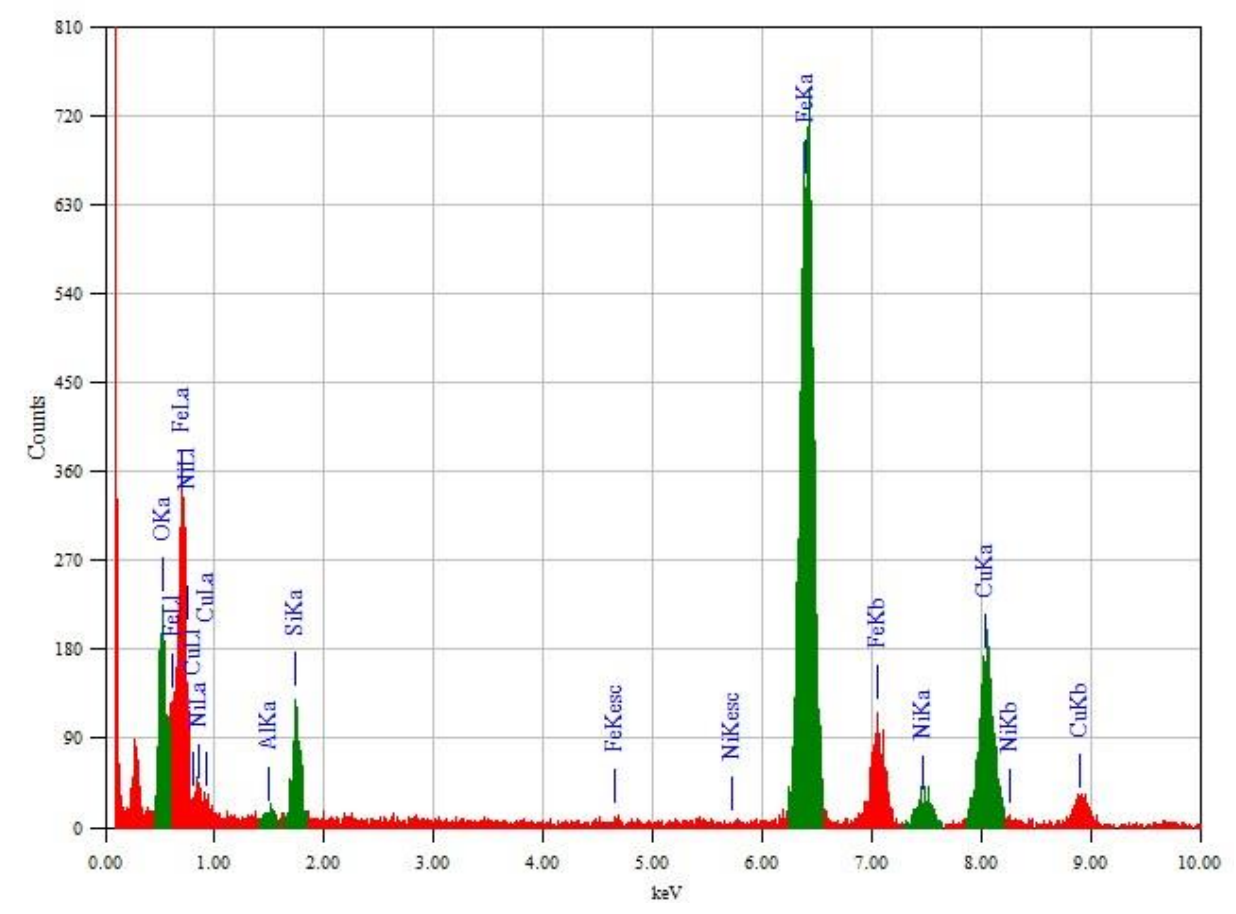

Figure 3.14. TEM-EDS spectrum of 5\%Ni-nZVI@MisiCFeOOH-MB2. Note: labels have been modified to highlight peaks relevant to this research. Please see appendix B.3 for complete spectrum.

In the TEM-EDS mapping of 5\%Ni-nZVI@MisiCFeOOH-MA, signals corresponding to $\mathrm{Si}, \mathrm{O}$ and Al are strong, consistent with the presence of Misi. In addition, signal corresponding to $\mathrm{S}$ was also found due to the property of the geothermal waste (Figure 3.15). The signals of these elements in Misi are in correlation. A signal corresponding to Fe is also strong, indicating the Fe in bimetallic Ni-nZVI. A signal corresponding to $\mathrm{Ni}$ is also observed although the Ni peak is relatively weak in the TEM-EDS spectrum. When the signals of Fe and $\mathrm{Ni}$ are overlaid, there is a good overlap observed. In addition, there is also Ni signal on the surface of Fe, but its density is weak (Figure 3.15a). The elemental distribution is due to the formation of a Fe-Ni alloy that was generated by the simultaneous reduction of $\mathrm{Fe}(\mathrm{II})$ and $\mathrm{Ni}(\mathrm{II})$ by using $\mathrm{NaBH}_{4}$. In addition, the signals of $\mathrm{O}$ and Fe do not significantly overlap each other (Figure 3.15b). Similarly, $\mathrm{O}$ and Ni signals do not overlap (Figure 3.15c). This data is also consistent with an Fe-Ni alloy. 

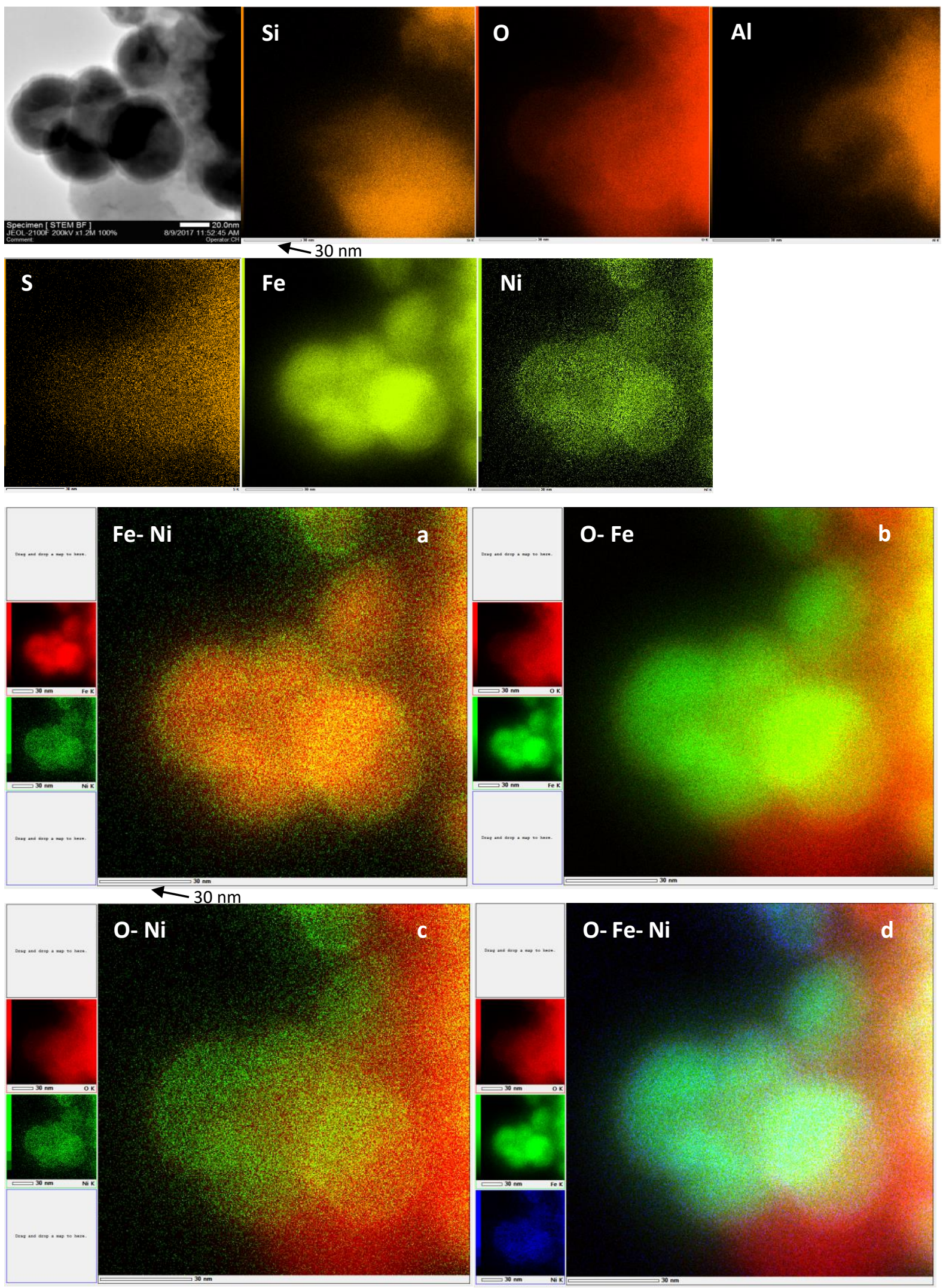

Figure 3.15. TEM-EDS mapping of 5\%Ni-nZVI@MisiCFeOOH-MA. 
In TEM-EDS mapping of 5\%Ni-nZVI@MisiCFeOOH-MB1 (Figure 3.16), there are strong signals corresponding to $\mathrm{Si}, \mathrm{O}, \mathrm{Al}$, and $\mathrm{S}$, confirming the presence of Misi. In addition to common elements, the signals corresponding to other elements, including $\mathrm{Cl}, \mathrm{Ti}, \mathrm{Na}$, and $\mathrm{Mg}$ are also observed. This can be explained due to the heterogeneity of Misi as these elements are in the region corresponding to Misi.

The signals corresponding to $\mathrm{Fe}$ and $\mathrm{Ni}$ are strong and there is a good correlation of these two elements. Interestingly, a significant amount of $\mathrm{Ni}$ is observed on the surface of Fe (Figure 3.16a). This can be explained by the sequential method to synthesise this material. In this method, $\mathrm{Ni}$ is deposited on the surface of $\mathrm{nZVI}$ by the reduction of $\mathrm{Ni}(\mathrm{II})$ by $\mathrm{nZVI}$. This leads to the adsorption of $\mathrm{Ni}(0)$ on the surface of $\mathrm{nZVI}$. The signal of $\mathrm{O}$ correlates with the signals of $\mathrm{Si}$, $\mathrm{Fe}$ and $\mathrm{Ni}$ with higher density in the region where $\mathrm{Si}$ is. When the signals of $\mathrm{O}$ and Fe were overlaid, there is an overlap and an O layer is observed on the surface of Fe (Figure 3.16b). Moreover, when the signals of $\mathrm{O}$ and $\mathrm{Ni}$ were overlaid, there is an overlap of $\mathrm{Ni}$ and $\mathrm{O}$, although the signal for $\mathrm{Ni}$ is significantly stronger than that of $\mathrm{O}$ (Figure 3.16c). These results suggested that bimetallic Ni-nZVI particles in 5\%Ni-nZVI@MisiCFeOOH-MB1 have a spherical shape with a core of $\mathrm{Fe}(0)$ and an outer layer, consisting of $\mathrm{Ni}(0)$ and potentially nickel oxides and iron oxides (Figure 3.16d). This finding is consistent with that reported when unsupported $2 \% \mathrm{Ni}-\mathrm{nZVI}$ prepared via deposition method (MB1) was examined via XPS analysis. ${ }^{167}$ In this reported material, $\mathrm{Ni}$ was found in the form of both $\mathrm{Ni}(0)$ and $\mathrm{Ni}(\mathrm{II})$ in $\mathrm{Ni}(\mathrm{OH})_{2}$ on the surface of $\mathrm{nZVI}$ as determined by their binding energy.

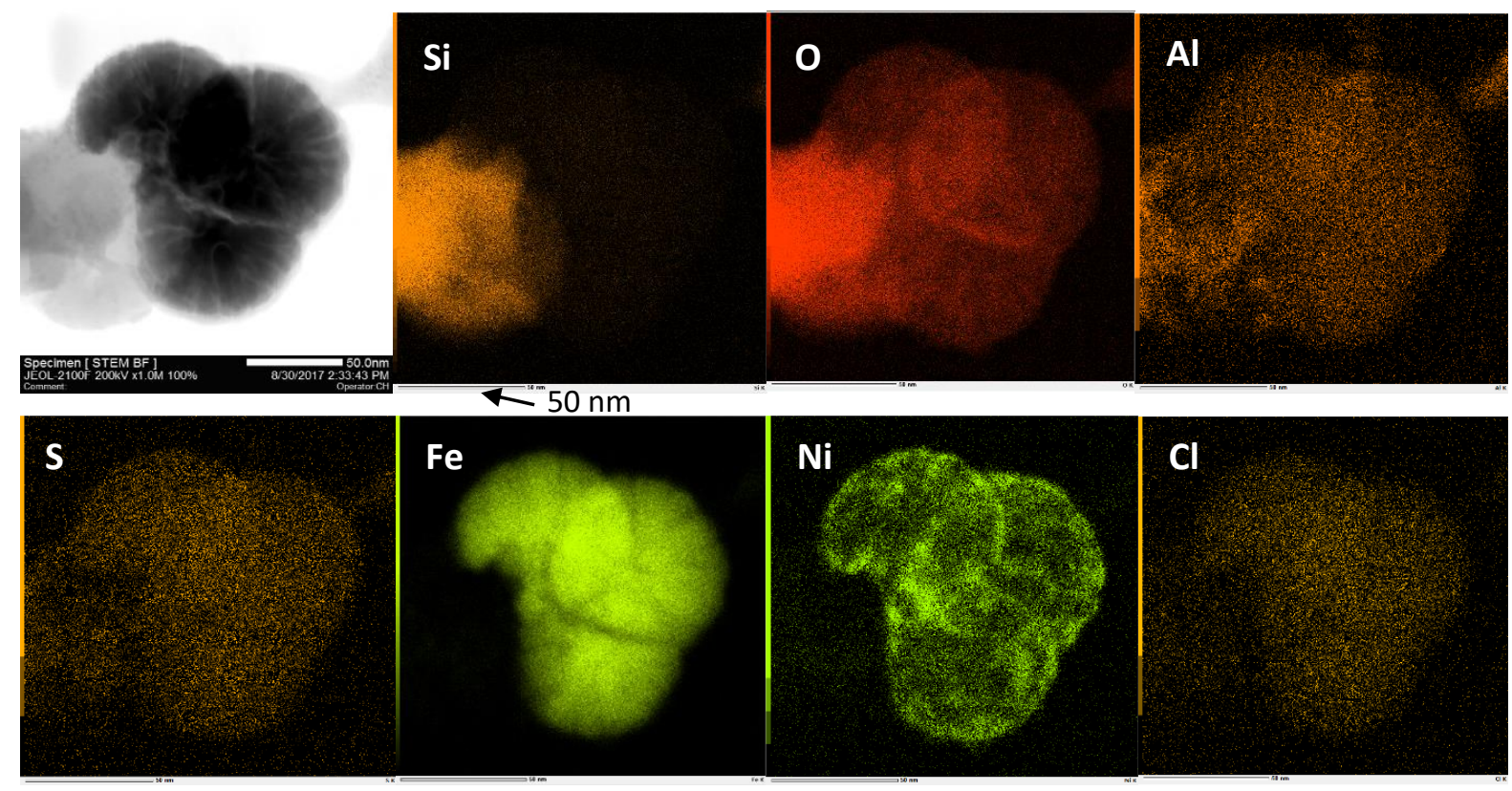




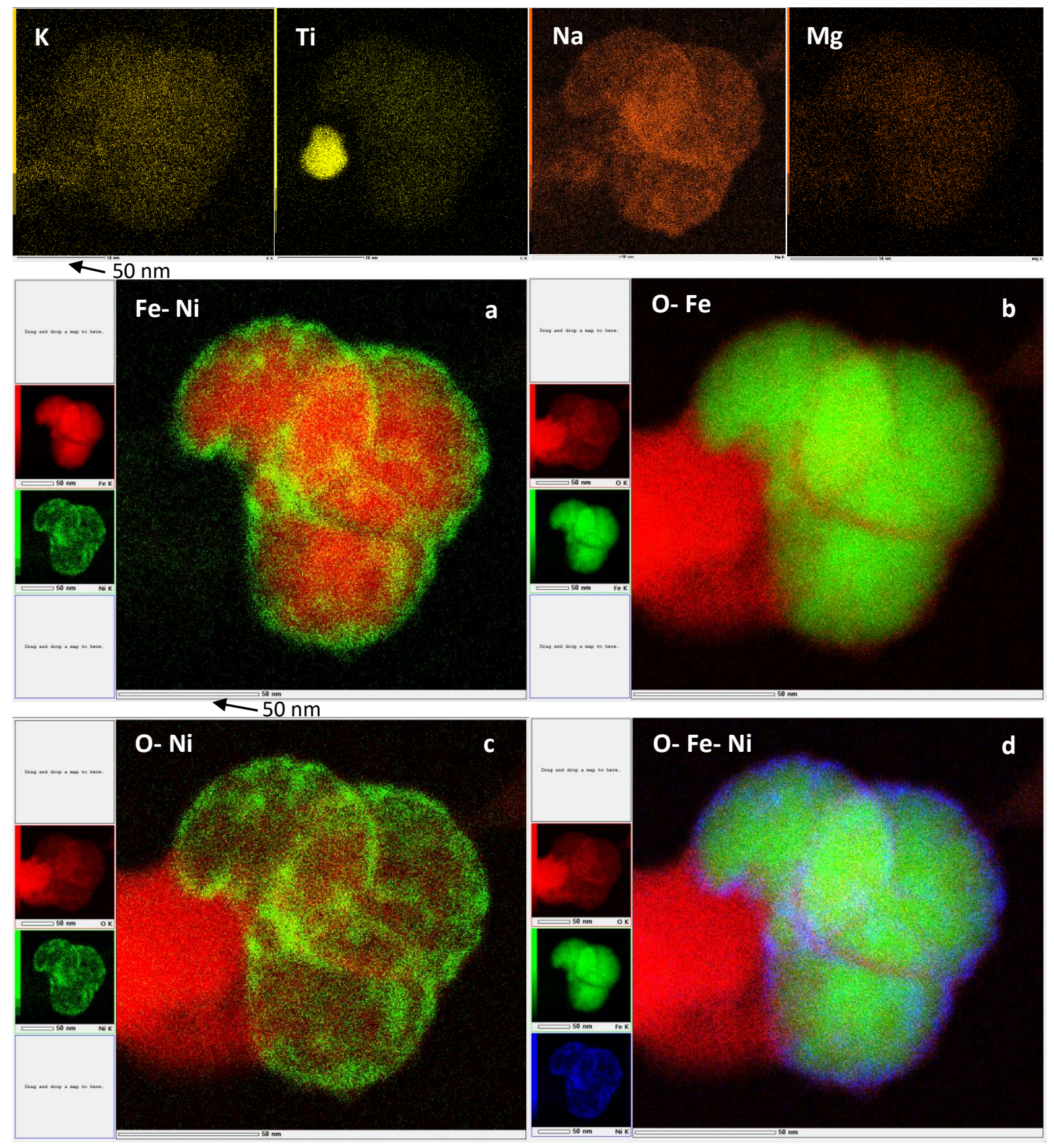

Figure 3.16. TEM-EDS mapping of 5\%Ni-nZVI@MisiCFeOOH-MB1.

The TEM-EDS mapping of 5\%Ni-nZVI@MisiCFeOOH-MB2 was examined. Signals corresponding to $\mathrm{Si}$ and $\mathrm{O}$ are observed, consistent with the presence of Misi in the material (Figure 3.17). Signals corresponding to both $\mathrm{Fe}$ and $\mathrm{Ni}$ are strong. When signals of Fe and $\mathrm{Ni}$ were overlaid, a denser layer of $\mathrm{Ni}$ surrounding the surface of Fe is observed (Figure 3.17a). The similarity is also observed when signals of Fe and O were overlaid, an O layer covers the surface of Fe (Figure 3.17b). Additionally, there is an overlap when signals of $\mathrm{Ni}$ and $\mathrm{O}$ were overlaid (Figure 3.17c). Based on these results, 5\%Ni-nZVI@MisiCFeOOH-MB2 particles have 
spherical shape with an $\mathrm{Fe}(0)$ core covered by a layer consisting of $\mathrm{Ni}(0)$ and potentially nickel oxides and iron oxides (Figure $3.17 \mathrm{~d}$ ). This is similar to the structure of $5 \% \mathrm{Ni}$ nZVI@MisiCFeOOH-MB1.
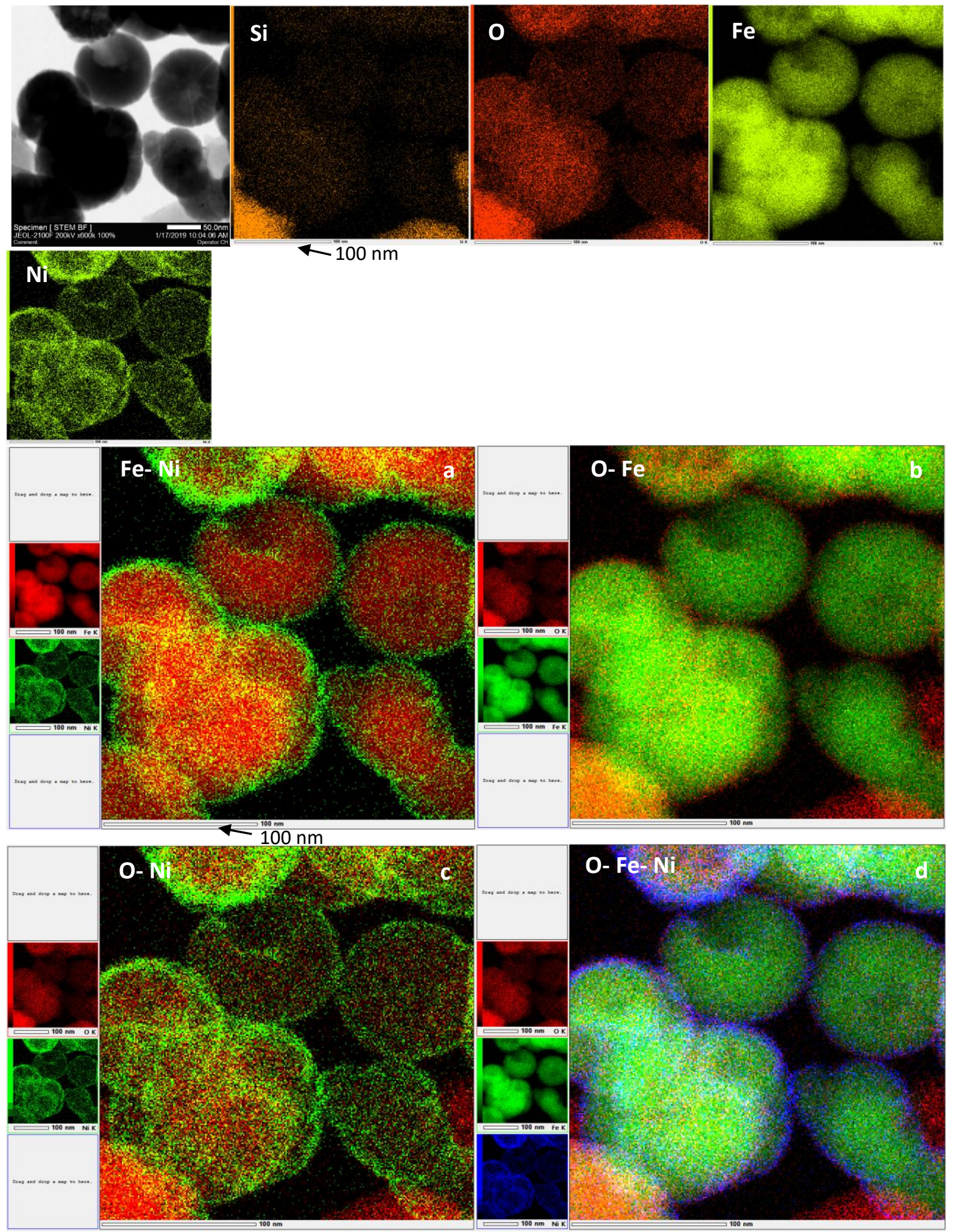

Figure 3.17. TEM-EDS mapping of 5\%Ni-nZVI@MisiCFeOOH-MB2. 
There have been investigations on the structure of bimetallic Ni-nZVI. However, no elemental distribution of secondary metal on nZVI was characterised by TEM-EDS. Thus, these new findings may provide reasonable reasons to explain the facile reactivity of bimetallic nZVI.

The elemental composition of bimetallic Ni-nZVI in supported bimetallic nZVI@Misi was quantified by AAS. For this, $5 \% \mathrm{Ni}-\mathrm{nZVI}$ on calcined/FeOOH-coated Misi (MisiCFeOOH) prepared via co-reduction method (MA) and deposition method (MB1) were digested in concentrated nitric acid and the resulting solutions were used to measure the concentrations of nickel and iron. The amount of nickel and iron was then calculated from their concentrations and the mass of MisiCFeOOH was subtracted from the initial amount of material with the assumption that the only digested material is iron and nickel. The result shows that the mass ratio of $\mathrm{Ni}: \mathrm{Fe}: \mathrm{MisiCFeOOH}$ is $0.56: 12.77: 100$ (molar ratio $\mathrm{Ni}: \mathrm{Fe}=1: 24.03$ ) and 0.52:11.63:100 (molar ratio Ni:Fe = 1:23.56) for 5\%Ni-nZVI@MisiCFeOOH-MA and 5\%NinZVI@MisiCFeOOH-MB1, respectively (Table 3.2).

Table 3.2. Analysis of Ni-doped materials $(0.250 \mathrm{~g}$ of material, $5.0 \mathrm{~mL}$ of concentrated $\mathrm{HNO}_{3}$ ). Note: Misi $=$ MisiCFeOOH.

\begin{tabular}{|c|c|c|c|c|}
\hline Material & Ni (mg) & Fe (mg) & Misi (mg) & $\begin{array}{c}\text { Mass ratio of } \\
\text { Ni:Fe:Misi }\end{array}$ \\
\hline $5 \%$ Ni-nZVI@MisiCFeOOH-MA & $1.233 \pm 0.005$ & $28.2 \pm 0.1$ & $220.6 \pm 0.1$ & $0.56: 12.77: 100$ \\
\hline $5 \%$ Ni-nZVI@MisiCFeOOH-MB1 & $1.154 \pm 0.005$ & $25.9 \pm 0.1$ & $222.9 \pm 0.1$ & $0.52: 11.63: 100$ \\
\hline MisiCFeOOH & 0 & 2.32 & 247.68 & $0: 0.94: 100$ \\
\hline
\end{tabular}

The ratio of Fe to Misi in both samples is higher compared to the ratio of Fe:MisiCFeOOH $(10: 100)$ used in the synthesis of these materials. A couple of reasons can be used to explained this. Firstly, in the support MisiCFeOOH, the ratio of Fe to Misi was 0.94:100, thus the expected ratio from this would be 11:100. In addition, the isolating steps to make the materials involved using a strong magnet; as such, any free Misi that did not adsorb nanoparticles was not attracted to this magnet and was discharged into the waste solutions. This leads to the loss of Misi, resulting in a higher concentration of nanoparticles. The 
$\mathrm{Ni}$ :MisiFeOOH ratio between two samples is similar; however, the real amount of $\mathrm{Fe}$ in $5 \% \mathrm{Ni}-$ nZVI@MisiCFeOOH-MA was higher than that of 5\%Ni-nZVI@MisiCFeOOH-MB1. This could be due to the loss of Fe in the deposition step that $\mathrm{nZVI}$ reduced $\mathrm{Ni}(\mathrm{II})$ to form $\mathrm{Ni}(0)$ and $\mathrm{Fe}(\mathrm{II})$. In order to investigate the influence of synthetic methods on the reactivity of bimetallic materials, nitrate reduction was examined using materials prepared via co-reduction method (MA) and deposition method (MB1 and MB2). In these studies, two different preparations of Misi, including calcined Misi (MisiC) and calcined/FeOOH-coated Misi (MisiFeOOH), were used to synthesise Ni-nZVI@Misi. The results show that all these Ni-doped materials reduced nitrate faster than non-doped nZVI@Misi (Section 3.2) and that nitrate reduction was dependent upon the synthetic method to generate materials. When MisiCFeOOH was used as the support, 5\%Ni-nZVI@MisiCFeOOH-MB1 had the highest reactivity while 5\% NinZVI@MisiCFeOOH-MB2 was the lowest performing material. The 5\%Ni-nZVI@MisiCFeOOHMA exhibited the medium performing activity. For instance, after 5 minutes, the nitrate-N concentrations were 3.8 ppm, 6.9 ppm, and 8.8 ppm when either 5\%Ni-nZVI@MisiCFeOOHMB1, 5\%Ni-nZVI@MisiCFeOOH-MA or 5\%Ni-nZVI@MisiCFeOOH-MB2 was used (Figure 3.18). In contrast, after 30 minutes, nitrate- $\mathrm{N}$ levels were not detected for all materials examined. When MisiC was used as the support, 5\%Ni-nZVI@MisiC-MB1 reduced nitrate faster than both 5\%Ni-nZVI@MisiC-MA and 5\%Ni-nZVI@MisiC-MB2 in the first 5 minutes. The nitrate-N concentration was below 1 ppm when 5\%Ni-nZVI@MisiC-MB1 was used while the nitrate-N concentrations were 2.9 ppm and 7.9 ppm when 5\%Ni-nZVI@MisiC-MB2 and 5\% NinZVI@MisiC-MA were used, respectively (Figure 3.19). However, after 15 minutes, the difference in their reactivity was not found. 


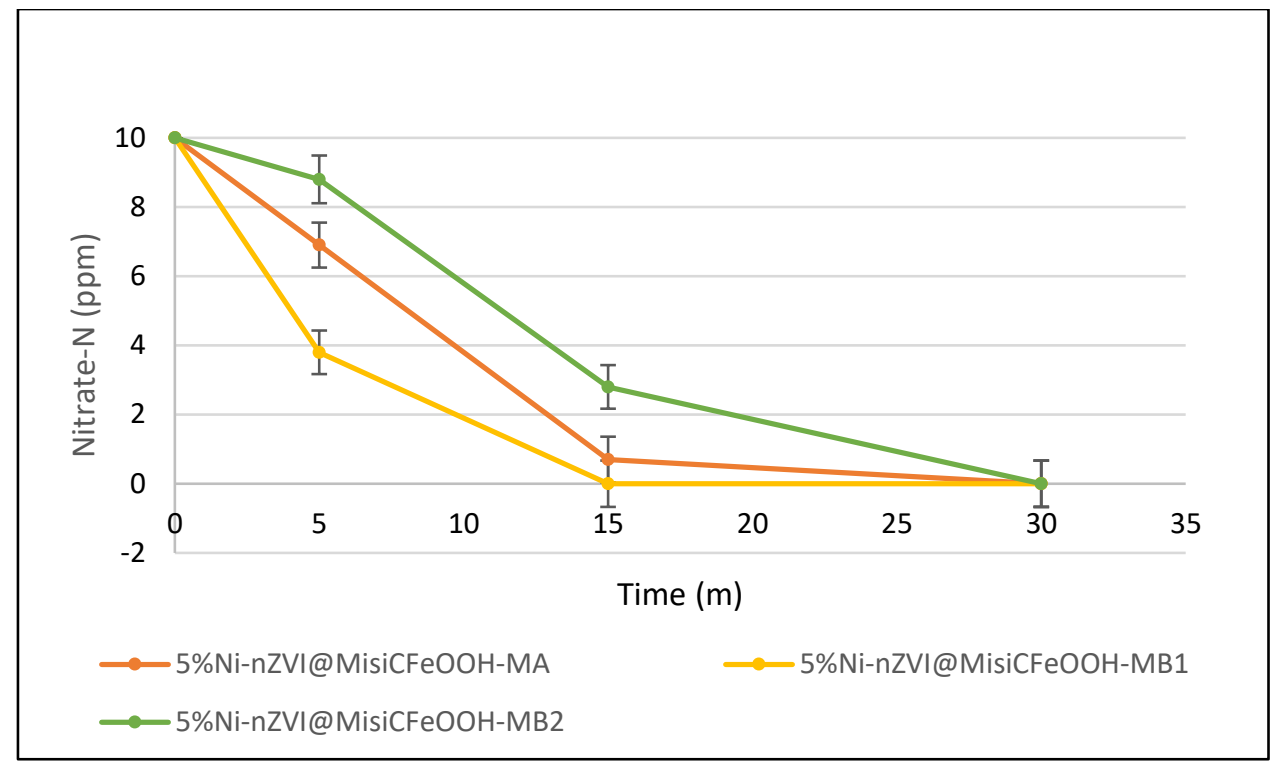

Figure 3.18. Reduction characterisation of 5\%Ni-nZVI@MsiCFeOOH prepared via different methods $(0.150 \mathrm{~g}$ of materials, $25.0 \mathrm{~mL}$ of $10 \mathrm{ppm}$ nitrate- $\mathrm{N}$ solution, under anaerobic conditions).

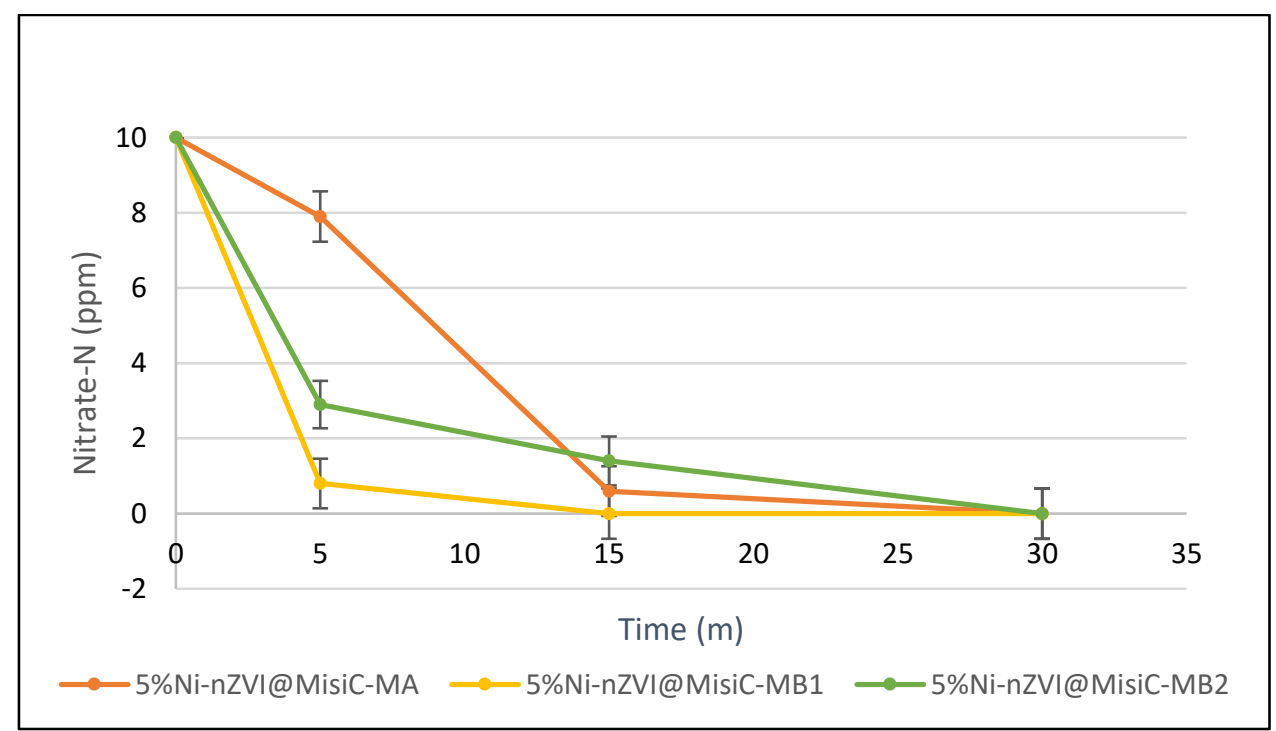

Figure 3.19. Reduction characterisation of 5\%Ni-nZVI@MisiC prepared via different methods (0.150 g of materials, $25.0 \mathrm{~mL}$ of $10 \mathrm{ppm}$ nitrate- $N$ solution, under anaerobic conditions). In the deposition method (MB), the reactivity of 5\%Ni-nZVI@Misi synthesised using MB1 was higher than that of 5\%Ni-nZVI@Misi synthesised using MB2, regardless of Misi preparation. However, the difference is minimal. Although the reason is uncertain, this can be explained due to the loss in the number of active sites of Fe prior to the deposition step. During the drying procedure of nZVI@Misi, the surface of the particles might be oxidised to form a 
protective oxide layer. This was evident by a yellow layer observed on the wall of Schlenk. This layer can block the electron transfer from nZVI to contaminants such as nitrate. In comparisons between method $\mathrm{MA}$ and $\mathrm{MB1}$, materials prepared via MB1 had higher reactivity towards nitrate reduction than those prepared via MA. This is inconsistent with a previous study on unsupported $\mathrm{Ni}-\mathrm{nZVI}$ reported by $\mathrm{H}$. Kang et al.. ${ }^{2}$ In their report, the unsupported Ni-nZVI generated by method MA reduced nitrate much faster than that prepared via method MB1. In our study, the presence of Misi might change the distribution of nanoparticles. This results in the change in the reactivity of particles prepared via different synthetic methods. However, our results are consistent with Wu et al.'s study in which NinZVI@cellulose acetate was used to remove TCE. ${ }^{196}$ Materials prepared via method MB had higher reactivity than those prepared via method MA. Based on these results, the coreduction (MA) and deposition method using wet nZVI@Misi (MB1) were chosen for future work due to their easier synthesis and slightly higher reactivity.

In order to examine final $\mathrm{N}$-containing products, nitrate reduction was carried out and ammonium concentrations in the resulting solutions were determined using the Nessler method. For this, 5\% Ni-nZVI on MisiC and MisiCFeOOH, synthesised by both co-reduction method (MA) and deposition method (MB1), were used. Ammonium was generated fast when all four materials were used (Figure 3.20). After 5 minutes, ammonium-N concentrations were more than $8.3 \mathrm{ppm}$ using all types of $\mathrm{Ni}$-doped materials except for 5\%Ni-nZVI@MisiCFeOOH-MA where the ammonium-N concentration was 5.5 ppm. After 30 minutes, ammonium- $\mathrm{N}$ concentrations were about $9 \mathrm{ppm}$ ( $90 \%$ ammonium production) when these four materials were used. Ammonium is the prominent final product in nitrate reduction. This is consistent with previously reported work. In these studies, ammonium is known as the main final product in nitrate reduction when either $\mathrm{nZVI} I^{165}$ or $\mathrm{ZVI}^{234}$ were used. In terms of nitrogen mass balance, after 5 minutes, the total nitrogen was higher than the initial nitrogen. For example, after 5 minutes, nitrate- $\mathrm{N}$, ammonium- $\mathrm{N}$ concentrations and total nitrogen were $7.9 \pm 0.7 \mathrm{ppm}, 8.3 \pm 0.2 \mathrm{ppm}$, and $16.2 \mathrm{ppm}$, respectively when $5 \% \mathrm{Ni}-$ nZVI@MisiC-MA was used. Since the samples for ammonium and nitrate determination were not run from the same experiment, the 5-minute mass balance concentration is not reliable. The most reliable nitrogen balance that can be examined is at the end (after 30 minutes) after the reaction has gone to completion. After 30 minutes, the total nitrogen was around 9 ppm 
(90\% recovery). The loss of nitrogen could be in other gas products that need to be examined in further study using the instruments for gas analysis.
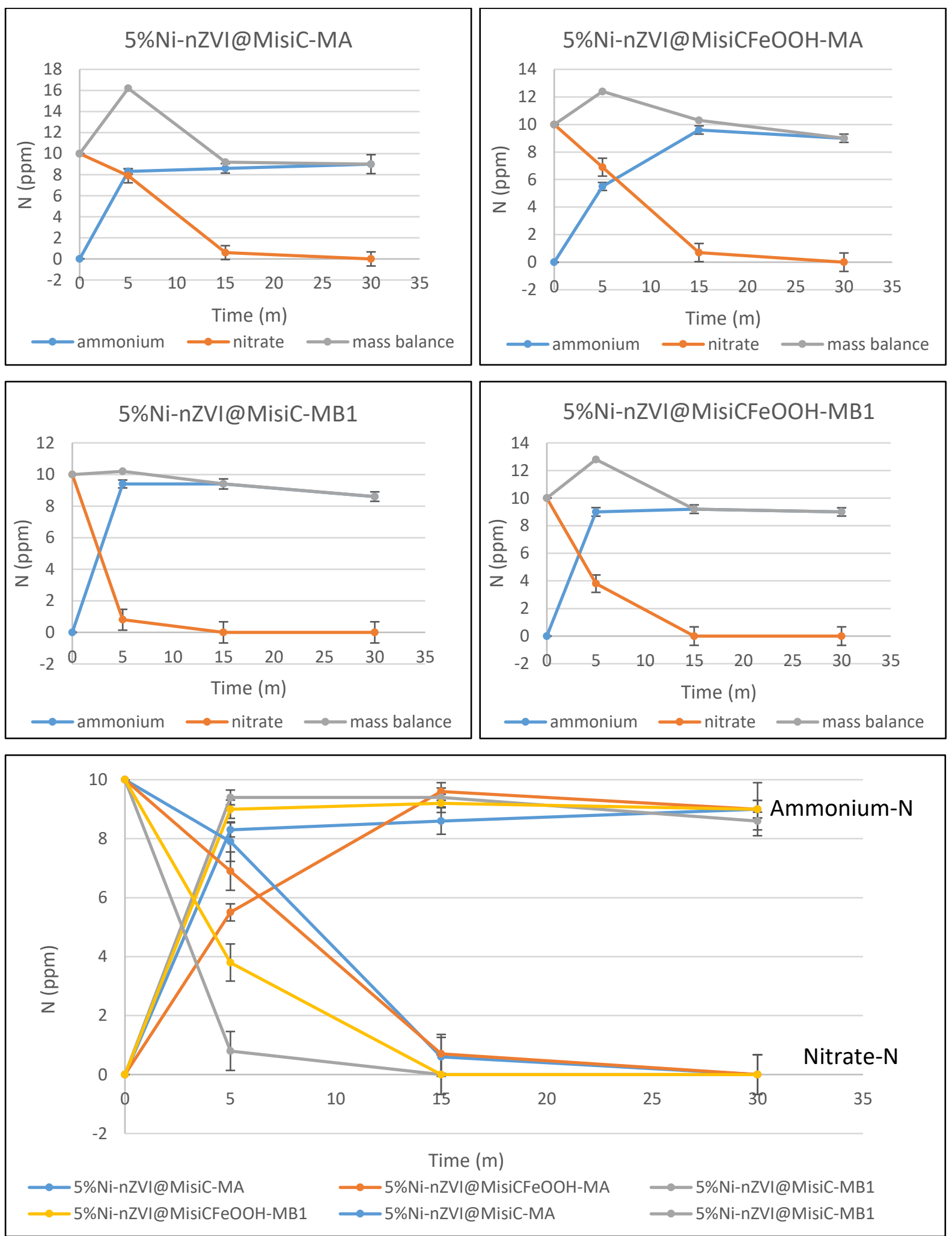

Figure 3.20. Nitrate reduction, ammonium production and mass balance by 5\%Ni-nZVI@Misi (0.150 g of materials, $25.0 \mathrm{~mL}$ of $10 \mathrm{ppm}$ nitrate- $\mathrm{N}$ solution, under anaerobic conditions). 


\subsubsection{Conclusion}

In conclusion, all systems with dopant nickel had higher reactivity than non-doped nZVI@Misi, regardless of nickel concentration or synthetic method. The presence of nickel could play the role of the catalyst. Nitrate adsorbs onto the nickel surface, which promotes the reaction of nitrate and electrons from $n Z V I$. The reduction potential of nickel $\left(E^{0}=-0.26 \mathrm{~V}\right)$ is more negative than those of nitrate to any nitrogen products including nitrite, nitrogen gas, and ammonium (Table 1.1); therefore, $\mathrm{Ni}(0)$ could be an additional reductant that produces electrons to reduce nitrate. In addition, the distribution of nickel in Ni-nZVI@Misi is dependent on synthetic methods. An alloy Ni-Fe was formed in materials prepared via the coreduction method $(\mathrm{MA})$ while nickel in form of $\mathrm{Ni}(0)$ and nickel oxides covered on the surface of $\mathrm{nZVI}$ in materials prepared via the deposition method (MB). Due to the core-shell structure of Ni-nZVI@Misi (MB), these systems were more reactive than Ni-nZVI@Misi (MA).

\subsection{Bimetallic Cu-nZVI@Misi}

In addition to $\mathrm{Ni}$, dopant $\mathrm{Cu}$ has been received a lot attention in increasing the reactivity of nZVI materials. Most of studies have examined unsupported Cu-nZVI with the $\mathrm{Cu}$ concentrations ranging from $0.5 \%$ to $20 \% .{ }^{41,126,178,181,235}$ Cu-nZVI@zeolite is the only reported supported $\mathrm{CU}-\mathrm{nZVI}$; however, the mass percentage of $\mathrm{CU}$ and Fe in material was 20\% and $41 \%$, respectively. ${ }^{194}$ In this current study, bimetallic Cu-nZVI@Misi materials were synthesised using two different synthetic methods, co-reduction (MA) and deposition (MB1) (Section 3.1). In all these experiments, the mass ratio of Fe to Misi is 1:10 and the mass ratios of $\mathrm{Cu}$ to Fe were varied from 2:100 (2\%) to 10:100 (10\%) with $\mathrm{CuCl}_{2}$ as the copper source. Table 3.3 summaries Cu-nZVI systems that were examined. They include $2 \% \mathrm{Cu}-\mathrm{nZVI}$ coated on MisiC using co-reduction method (MA), 5\%Cu-nZVI coated on both MisiC and MisiCFeOOH using both co-reduction method (MA) and deposition method (MB1), and 10\%Cu-nZVI coated on MisiC using co-reduction method (MA).

The morphology and reactivity towards nitrate reduction of these materials were investigated. A morphology comparison was done on the samples prepared using the different synthetic methods. However, due to the similarity of the morphology of these samples with the Ni-containing and the lack of information obtained when comparing the 
morphology of different $\mathrm{Ni}$ loadings, the morphology differences between the different $\mathrm{Cu}$ concentrations were not examined.

Table 3.3. List of Cu-nZVI@Misi systems synthesised.

\begin{tabular}{|c|c|c|c|}
\hline \multirow{2}{*}{ Materials } & \multicolumn{2}{|c|}{ Misi preparation } & \multirow{2}{*}{ Method } \\
\cline { 2 - 4 } & MisiC & MisiCFeOOH & \\
\hline $2 \% C u-n Z V I @$ & $X$ & & Co-reduction (MA) \\
\hline 5\%Cu-nZVI@ & $\mathrm{X}$ & $\mathrm{X}$ & Co-reduction (MA) \\
\cline { 2 - 4 } & $\mathrm{X}$ & $\mathrm{X}$ & Deposition (MB1) \\
\hline $10 \% C u-n Z V I @$ & $\mathrm{X}$ & & Co-reduction (MA) \\
\hline
\end{tabular}

\subsubsection{Variations on Cu loading}

The effect of $\mathrm{Cu}$ loading on the reactivity of Cu-nZVI@Misi towards nitrate reduction was examined. Based on previously reported papers and considered the toxicity of $\mathrm{Cu}, \mathrm{Cu}-\mathrm{nZVI}$ on calcined Misi (MisiC) synthesised via co-reduction method (MA) with different ratios of Cu to $\mathrm{Fe}$, such as $2 \%, 5 \%$, and $10 \%$ was used for nitrate reduction. In these reactions, the molar ratio of Fe to nitrate is 15:1. The results showed that $\mathrm{Cu}$-doped materials had higher reactivity than non-doped nZVI@Misi (Section 3.2), regardless of Cu concentration. The difference in the reactivity of copper systems with different copper contents was minimal. For example, in the first minutes of the reaction ( 5 minutes) and in the end of the reaction ( 30 minutes), the nitrate-N concentrations were similar using any Cu-nZVI@MisiC systems. After 15 minutes, 5\%Cu-nZVI@MisiC had slightly higher reactivity than both 2\%Cu-nZVI@MisiC and 10\%CunZVI@MisiC. The nitrate- $\mathrm{N}$ concentrations were $4.6 \pm 0.6$ ppm, $3.1 \pm 0.6$ ppm and $3.9 \pm 0.6$ ppm using either 2\%Cu-nZVI@MisiC, 5\%Cu-nZVI@MisiC or 10\%Cu-nZVI@MisiC, respectively (Figure 3.21). Based on these results, the 5\%Cu-nZVI on Misi was chosen for future work due to the negative environmental implications of copper. 


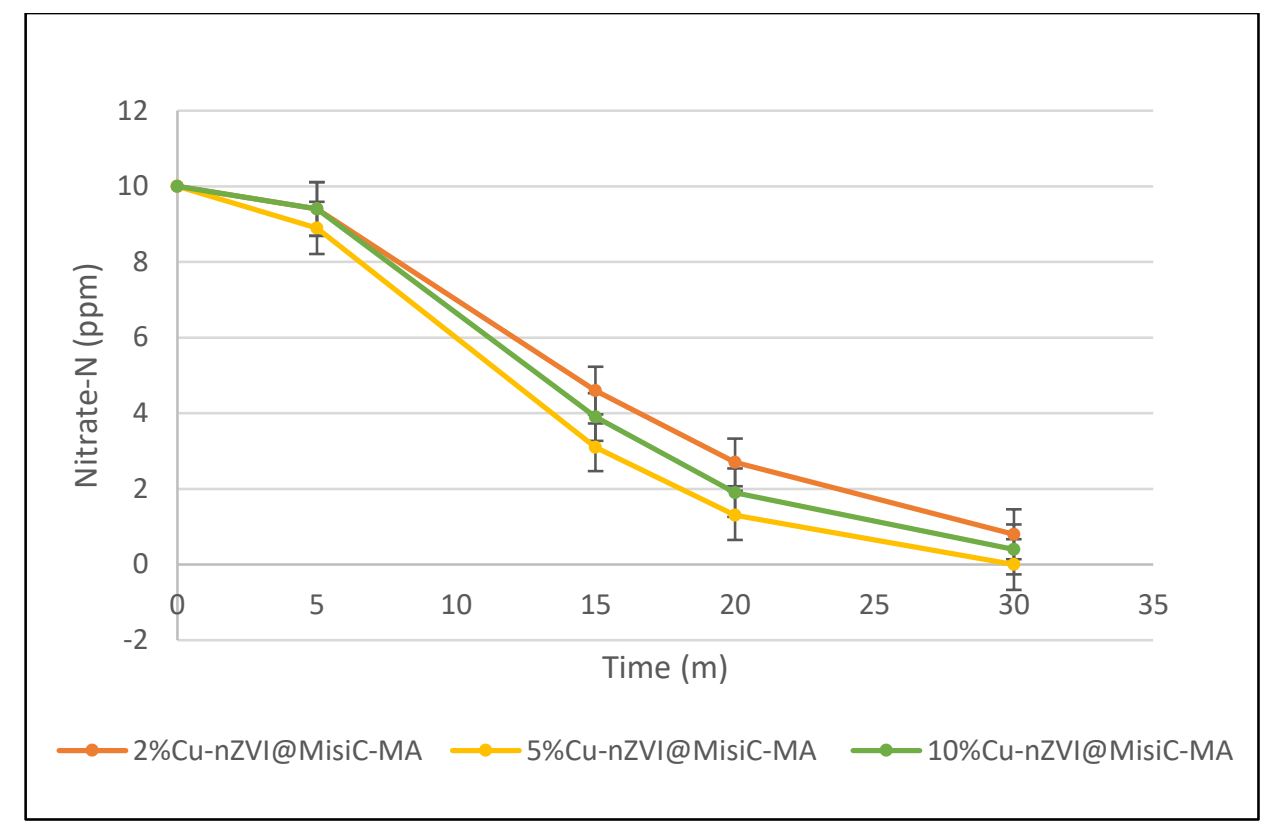

Figure 3.21. Nitrate reduction on different Cu loading contents $(0.150 \mathrm{~g}$ of materials, $25.0 \mathrm{~mL}$ of 10 ppm nitrate-N solution, under anaerobic conditions).

In several reported systems on unsupported Cu-nZVI, the dependence of the reactivity of these materials on the copper concentration was not clear. In some reports, the reactivity reached a peak at an optimum $\mathrm{Cu}$ concentration and then decreased with the increasing $\mathrm{Cu}$ concentrations. The 5\%Cu-nZVI exhibited the maximum reactivity when Cu-nZVI $(0.5 \%-20 \%)$ systems were examined towards nitrate reduction. ${ }^{126,235}$ For example, in Liou et al.'s study, Cu-nZVI $(0.5 \%$ - 20\%) systems were investigated to reduce nitrate $40 \mathrm{ppm}$ nitrate- $\mathrm{N}$ and the molar ratio of Fe to nitrate was 2.2:1. ${ }^{126}$ After 120 minutes, nitrate removal was $92 \%, 100 \%$, $86 \%$, and $78 \%$ for $0.5 \%, 5 \%, 10 \%$, and $20 \%$ Cu deposited. In Hosseini et al.'s study, Cu-nZVI (2.5\%) had the highest reactivity when nZVI was added Cu with the mass percentage of Cu to Fe from $2.5 \%$ to $10 \%{ }^{181}$ In their study, Cu-nZVI and nZVI was used to remove nitrate in a packed column. Nitrate conversion was $82 \%$ using nZVI and it increased to $89 \%$ using $2.5 \% \mathrm{Cu}$ nZVI while Cu-nZVI (5\% and 10\%) reduced nitrate more slowly than nZVI (62\% and 60\%, respectively. They explained that when the surplus amount of $\mathrm{Cu}$ was used, it has led to the agglomeration of $\mathrm{Cu}$, which inhibited the activity of nZVI. Another explanation for the decrease in the reactivity of $\mathrm{Cu}-\mathrm{ZVI}$ when $\mathrm{Cu}$ exceeds the optimum amount is that $\mathrm{Cu}$ may block the electron transfer from Fe core to nitrate. ${ }^{236}$ 


\subsubsection{Variations on synthetic methods}

All types of Cu-doped materials, unsupported CU-nZVI and supported Cu-nZVI, published were synthesised using the deposition method (MB). ${ }^{41,126,178,181,236}$ In this current study, the effect of different synthetic methods to generate Cu-nZVI@Misi on both the adsorption of CU-nZVI on Misi and the reactivity towards nitrate reduction was examined. For this, 5\%Cu-nZVI on calcined Misi (MisiC) and calcined/FeOOH-coated Misi (MisiCFeOOH) was synthesised using two different methods, MA and MB1. The morphology of resultant materials was characterised using SEM. The elemental composition of these materials was examined using TEM-EDS and their quantitative compositions were determined using AAS. The reactivity of these materials towards nitrate reduction was also examined.

COMPO images of the materials revealed that there is a good dispersion of nanoparticles on the surface of Misi although some agglomeration is observed (Figure 3.22). For examples, in some areas (red circle), iron nanoparticles evenly adsorb on Misi while there is a disordered arrangement of nanoparticles in the form of chains in other areas (blue circle). A higher level of agglomeration is visibly observed in materials prepared via co-reduction method (MA) than materials prepared via deposition method (MB1), regardless of Misi preparation but the difference is minimal.

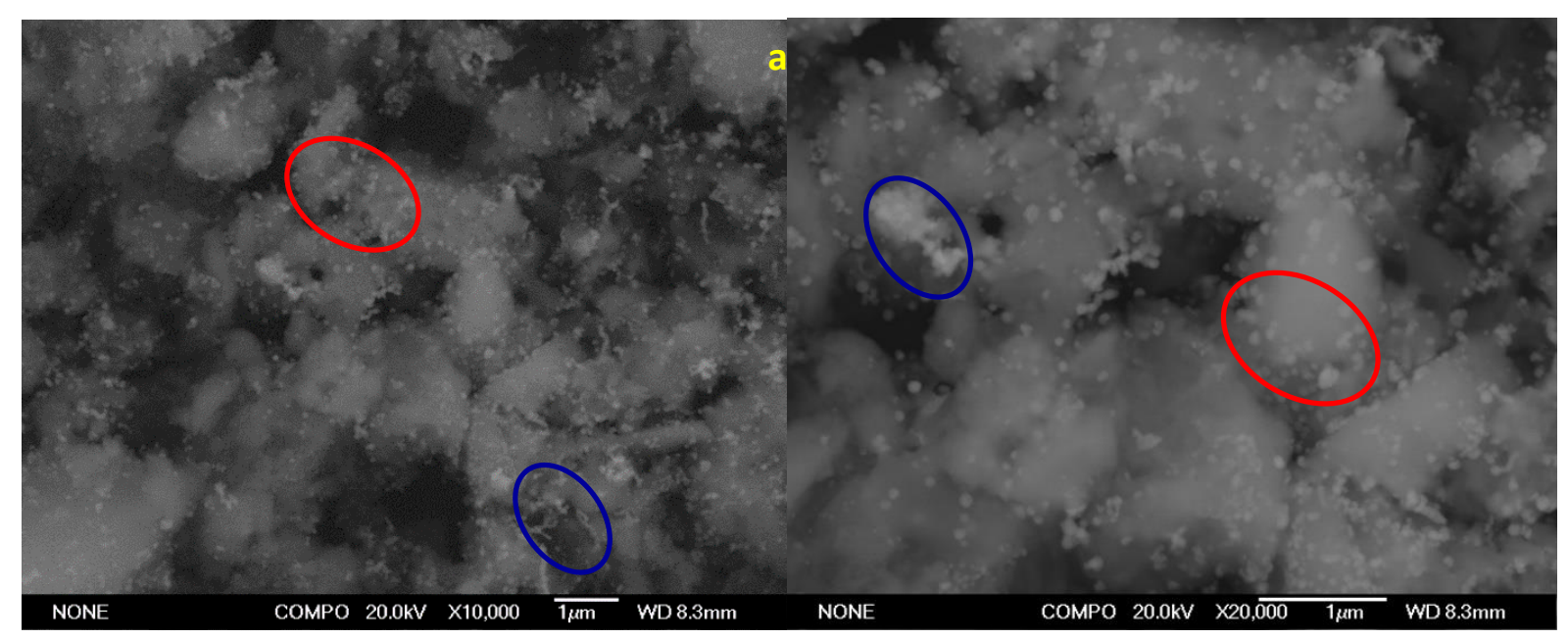

Figure 3.22. COMPO images of (a) 5\%Cu-nZVI@MisiC-MA, (b) 5\%Cu-nZVI@MisiCFeOOH-MA,

(c) 5\%CU-nZVI@MisiC-MB1, (d) 5\%Cu-nZVI@MisiCFeOOH-MB1. The lighter component is attributed to $\mathrm{Cu}-n \mathrm{ZVI}$ and silicate is grey. Figure $b, c$, and $d$ are in the next page. 

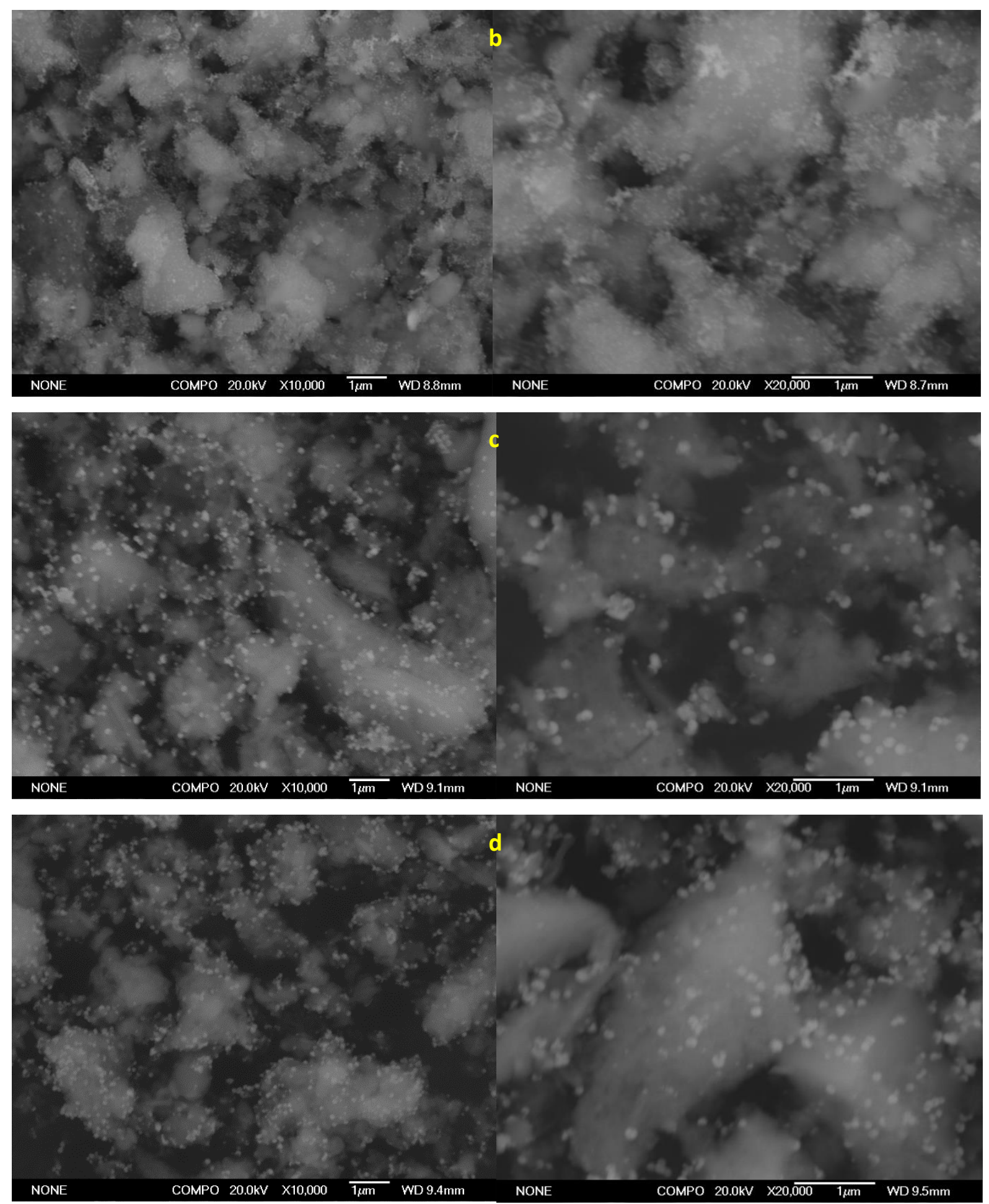

Figure 3.22. COMPO images of (a) 5\%Cu-nZVI@MisiC-MA, (b) 5\%Cu-nZVI@MisiCFeOOH-MA, (c) 5\%Cu-nZVI@MisiC-MB1, (d) 5\%Cu-nZVI@MisiCFeOOH-MB1. The lighter component is attributed to $\mathrm{Cu}-n Z \mathrm{VI}$ and silicate is grey. Figure $a$ is in the previous page.

The composition of 5\%CU-nZVI on Misi was also examined via TEM-EDS analysis. For this, 5\%Cu-nZVI@MisiCFeOOH-MA and 5\%Cu-nZVI@MisiCFeOOH-MB1 were used. In both 
samples, several peaks corresponding to $\mathrm{O}, \mathrm{Si}$, and $\mathrm{Al}$ are observed, indicating the presence of silicate (Figure 3.23 and Figure 3.26). Regarding 5\%Cu-nZVI@MisiCFeOOH-MA, an intense peak corresponding to Fe is also observed while no peak corresponding to $\mathrm{Cu}$ is observed. The absence of Cu peak is confirmed when different areas of 5\%Cu-nZVI@MisiCFeOOH-MA were examined (Figure 3.23). In the three specific areas examined, the mass percentage of $\mathrm{Cu}$ is less than $0.15 \%$. Specifically, in area 1 , the mass percentage and the atom percentage for $\mathrm{Fe}$ are $37.2 \%$ and $12.0 \%$ respectively while no Cu was quantified. In area 2 , the mass percentage for $\mathrm{Fe}$ and $\mathrm{Cu}$ was $16.0 \%$ and $0.15 \%$ and the atom percentage was $6.0 \%$ and $0.05 \%$, respectively. In area 3, the mass percentage for Fe and Cu was $7.6 \%$ and $0.15 \%$ and the atom percentage was $2.13 \%$ and $0.04 \%$, respectively. The very low concentration of $\mathrm{Cu}$ in TEM spectra was confirmed as TEM-EDS of this material was examined (Figure 3.27). A signal corresponding to $\mathrm{Cu}$ only localises in small areas while the signals corresponding to $\mathrm{Si}, \mathrm{O}, \mathrm{Al}$, $\mathrm{Ti}, \mathrm{S}$ and $\mathrm{Fe}$ are strong.

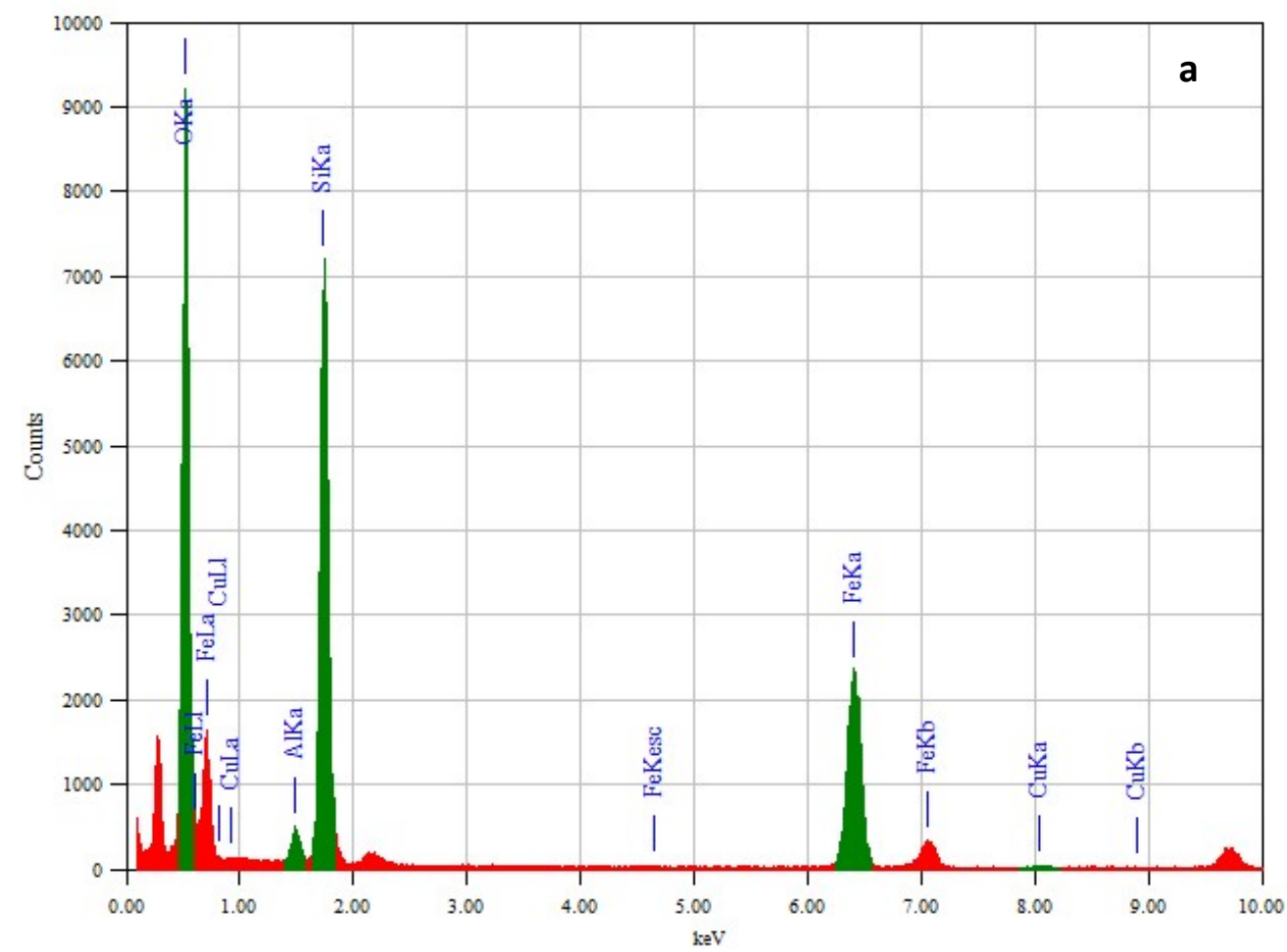

Figure 3.23. TEM-EDS spectra of 5\%CU-nZVI@MisiCFeOOH-MA in different areas $a, b$ and $c$.

Area $b$ and $c$ are in the next page. Note: labels have been modified to highlight peaks relevant to this research. Please see appendix B.4 for complete spectrum. 

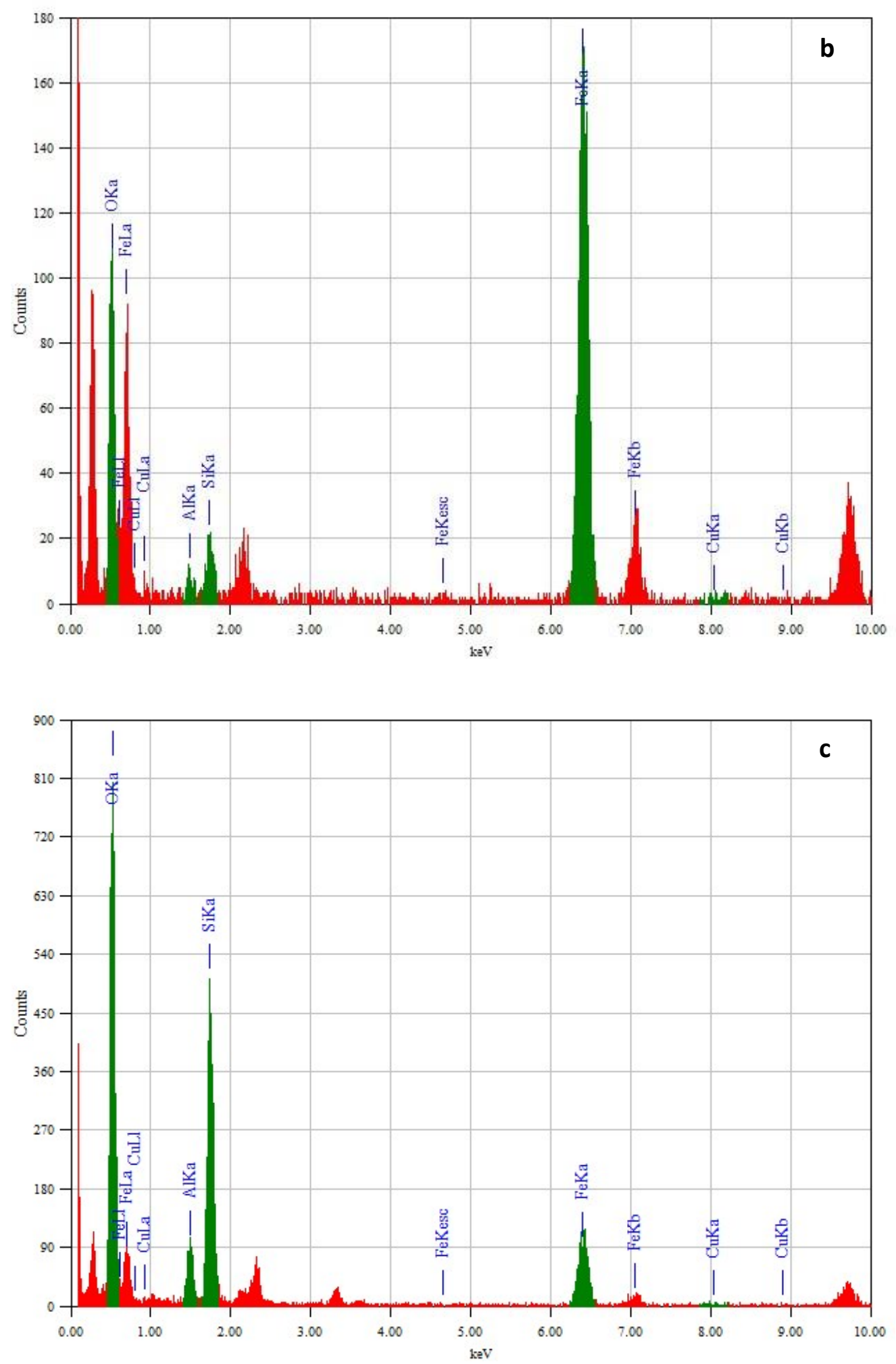

Figure 3.23. TEM-EDS spectra of 5\%CU-nZVI@MisiCFeOOH-MA in different areas $a, b$ and c. Area $a$ is in the previous page. Note: labels have been modified to highlight peaks relevant to this research. Please see appendix B.4 for complete spectrum. 


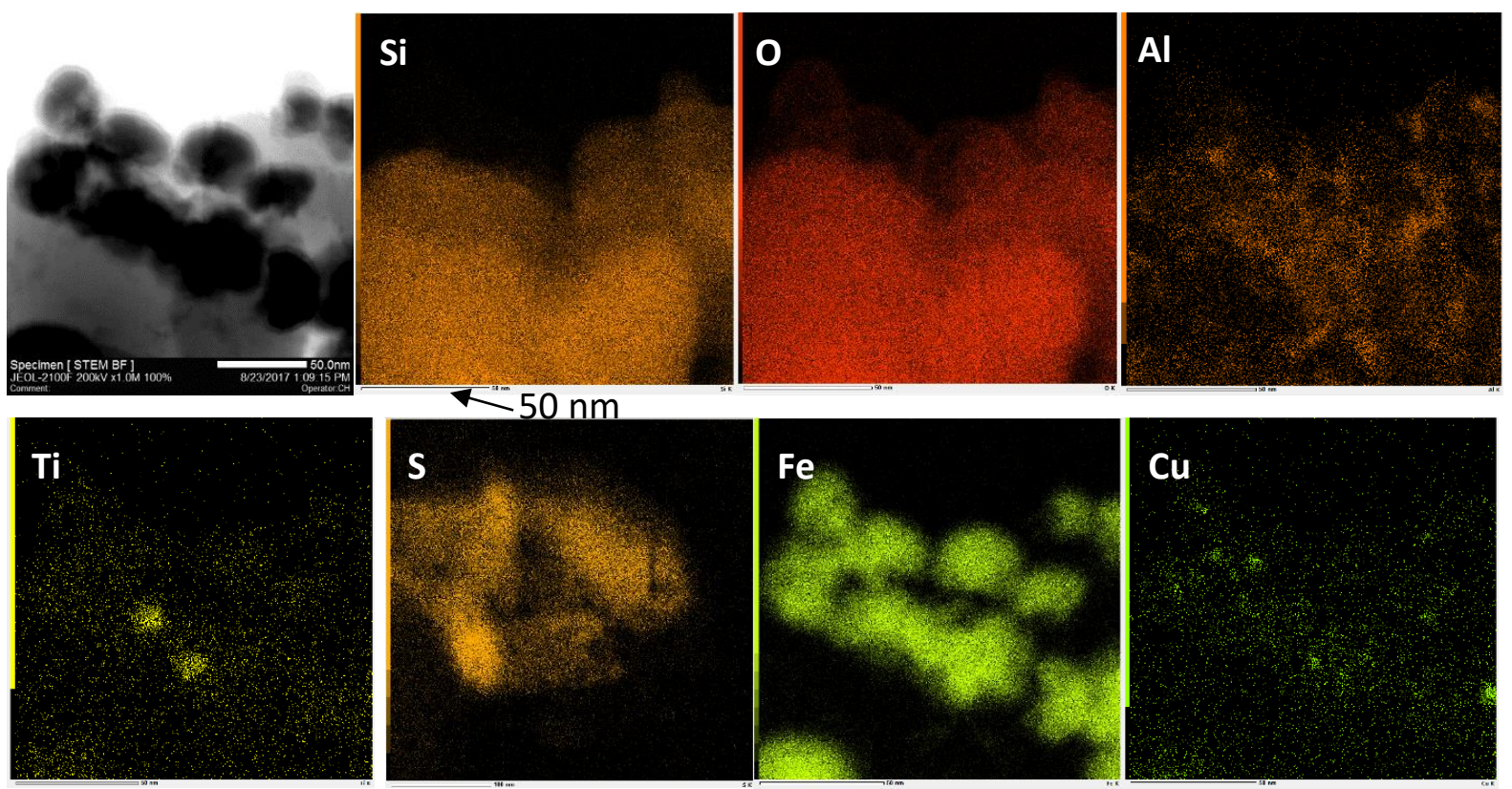

Figure 3.24. TEM-EDS mapping of 5\%CU-nZVI@MisiCFeOOH-MA with low magnification.

In order to examine the distribution of Fe and Cu in 5\%Cu-nZVI@MisiCFeOOH-MA, one small area was examined under higher magnification. In this area, the signals corresponding to $\mathrm{Si}$, $\mathrm{Al}, \mathrm{K}$, and Ti are not observed (Figure 3.25). This indicated that signals corresponding to silicate depend on the specific area examined, potentially due to the heterogeneous nature of Misi. However, the Fe signal is strong and its density in the core of the nanoparticles is greater than on the surface. The $\mathrm{O}$ signal is observed in all areas Fe presents but it is weaker than that of Fe. When signals of Fe and $\mathrm{O}$ are overlaid, there is an overlap with the denser Fe in the core (Figure 3.25b), indicating an Fe(0) core surrounded by a layer of iron oxide. Regarding $\mathrm{Cu}$, it is only present as a small island on Fe and is not distinguished from the noise in the other areas (Figure 3.25a). When the signals of $\mathrm{O}$ and $\mathrm{Cu}$ were overlaid, $\mathrm{Cu}$ is also observed in a small island on O (Figure 3.25c). 

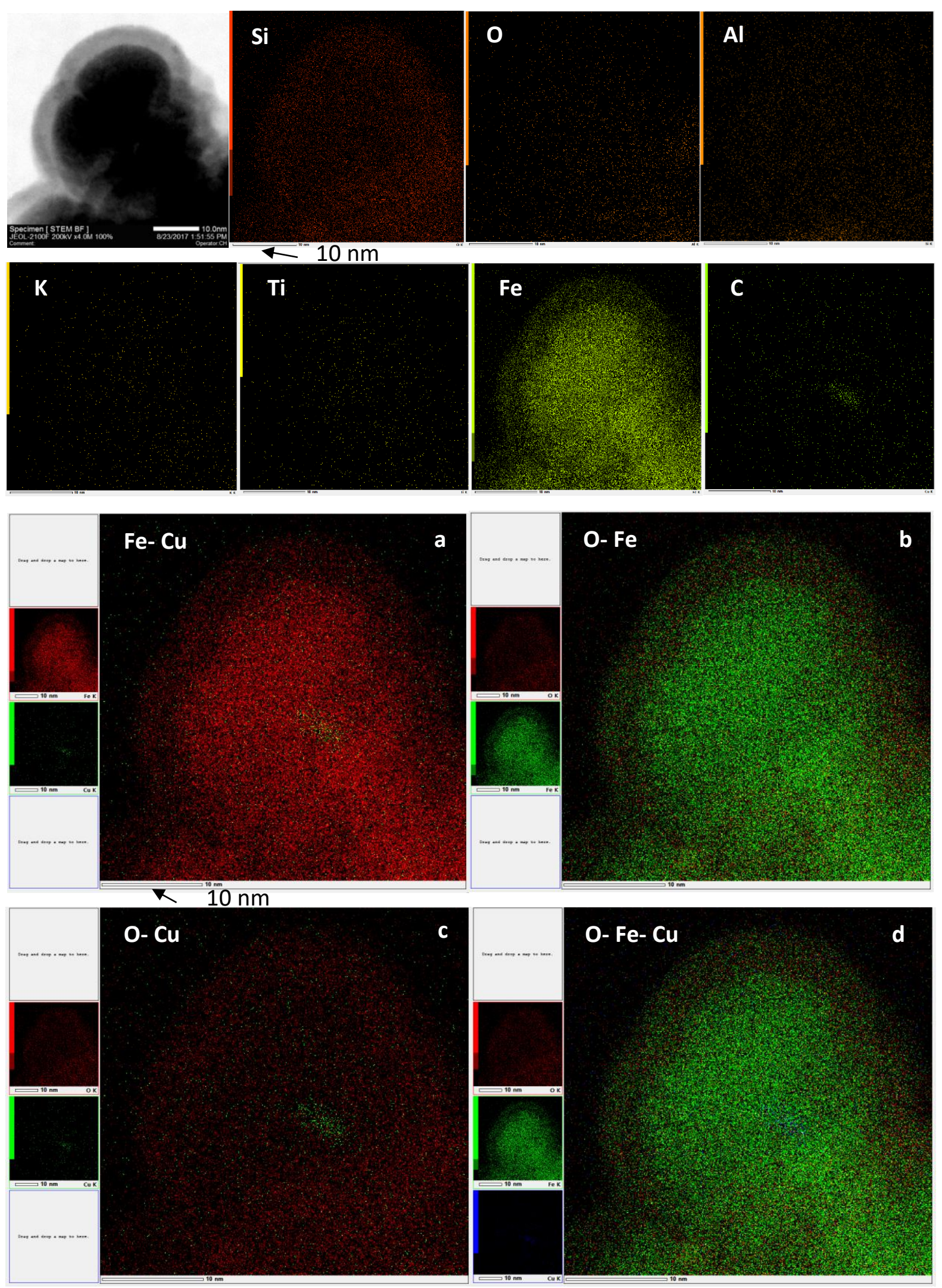

Figure 3.25. TEM-EDS mapping of 5\%CU-nZVI@MisiCFeOOH-MA with high magnification. 
In TEM spectrum of 5\%Cu-nZVI@MisiCFeOOH-MB1 (Figure 3.26), a strong peak corresponding to $\mathrm{Fe}$ is observed. A peak corresponding to $\mathrm{Cu}$ is observed, different from $\mathrm{Cu}$ signal in 5\%Cu-nZVI@MisiCFeOOH-MA. In this specific area examined, the mass percentage for $\mathrm{Fe}$ and $\mathrm{Cu}$ was $\mathbf{7 7 . 7 \%}$ and $5.1 \%$ and the atom percentage was $52.8 \%$ and $3.0 \%$, respectively.

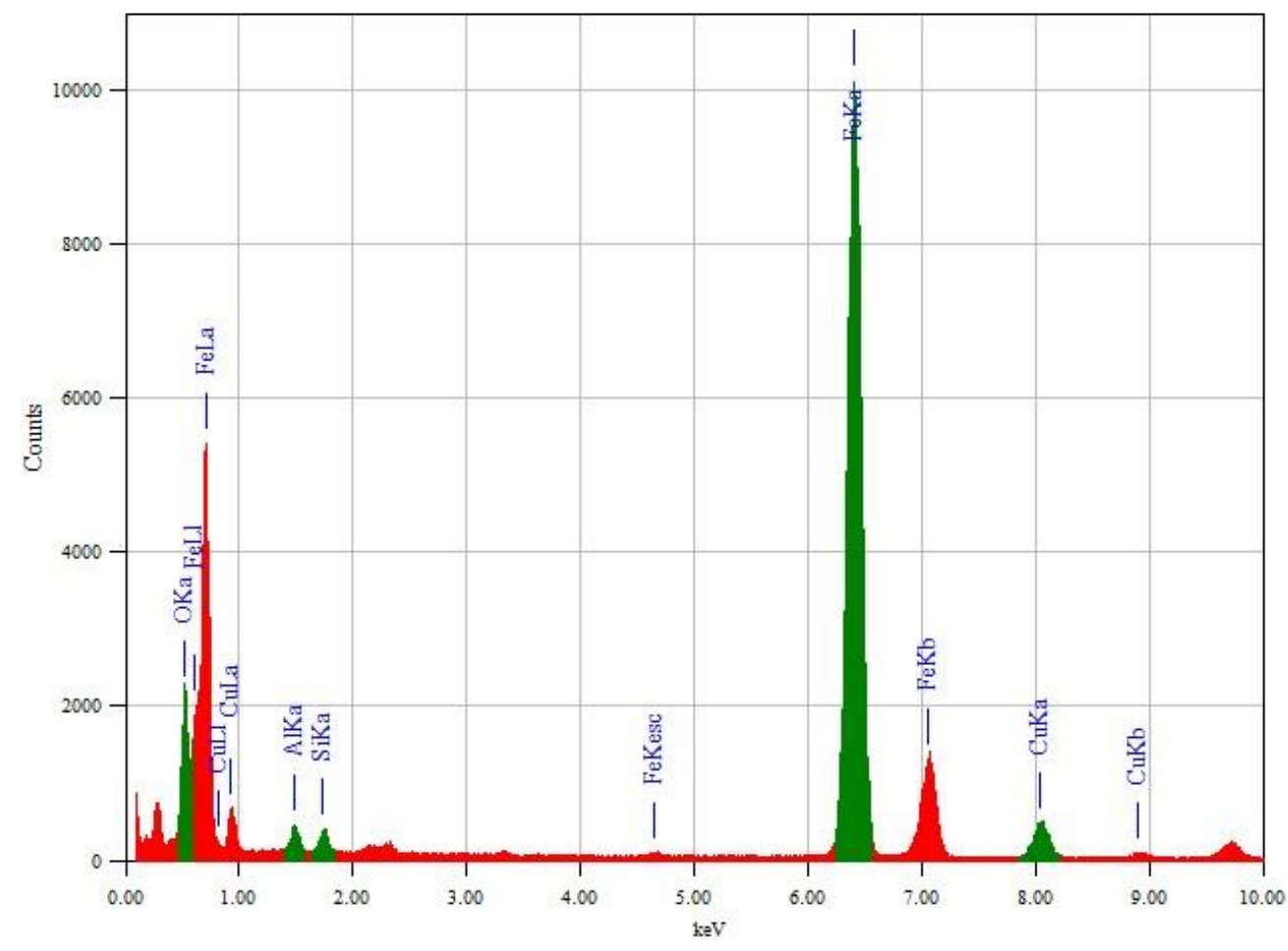

Figure 3.26. TEM-EDS spectrum of 5\%Cu-nZVI@MisiCFeOOH-MB1. Note: labels have been modified to highlight peaks relevant to this research. Please see appendix B.5 for complete spectrum.

The TEM-EDS mapping of 5\%Cu-nZVI@MisiCFeOOH-MB1 showed that the signals corresponding to $\mathrm{Si}, \mathrm{O}, \mathrm{Al}, \mathrm{S}, \mathrm{K}$, and $\mathrm{Ti}$ are strong and correlate to each other, indicating the presence of Misi (Figure 3.27). The signals corresponding to Fe and $\mathrm{Cu}$ are strong, present $\mathrm{Cu}-$ Fe nanoparticles and consistent with TEM spectrum (Figure 3.26). When the signal of Fe and $\mathrm{Cu}$ were overlaid, a significantly higher amount of $\mathrm{Cu}$ is observed on the surface of Fe than in the core (Figure 3.27a), presumably due to the deposition method to synthesise material. During this process, $\mathrm{Cu}(\mathrm{II})$ is reduced to $\mathrm{Cu}(0)$ by $\mathrm{nZVI}$ and then $\mathrm{Cu}(0)$ dopes on the surface of nZVI. When the signals of Fe and $\mathrm{O}$ are overlaid, there is an $\mathrm{O}$ layer encapsulating Fe core (Figure 3.27b). As such, Cu-nZVI particles have a core-shell structure, consisting of an $\mathrm{Fe}(0)$ core and a shell that is a combination of $\mathrm{Cu}(0)$ and potentially copper oxides (Figure $3.27 \mathrm{~d}$ ). 

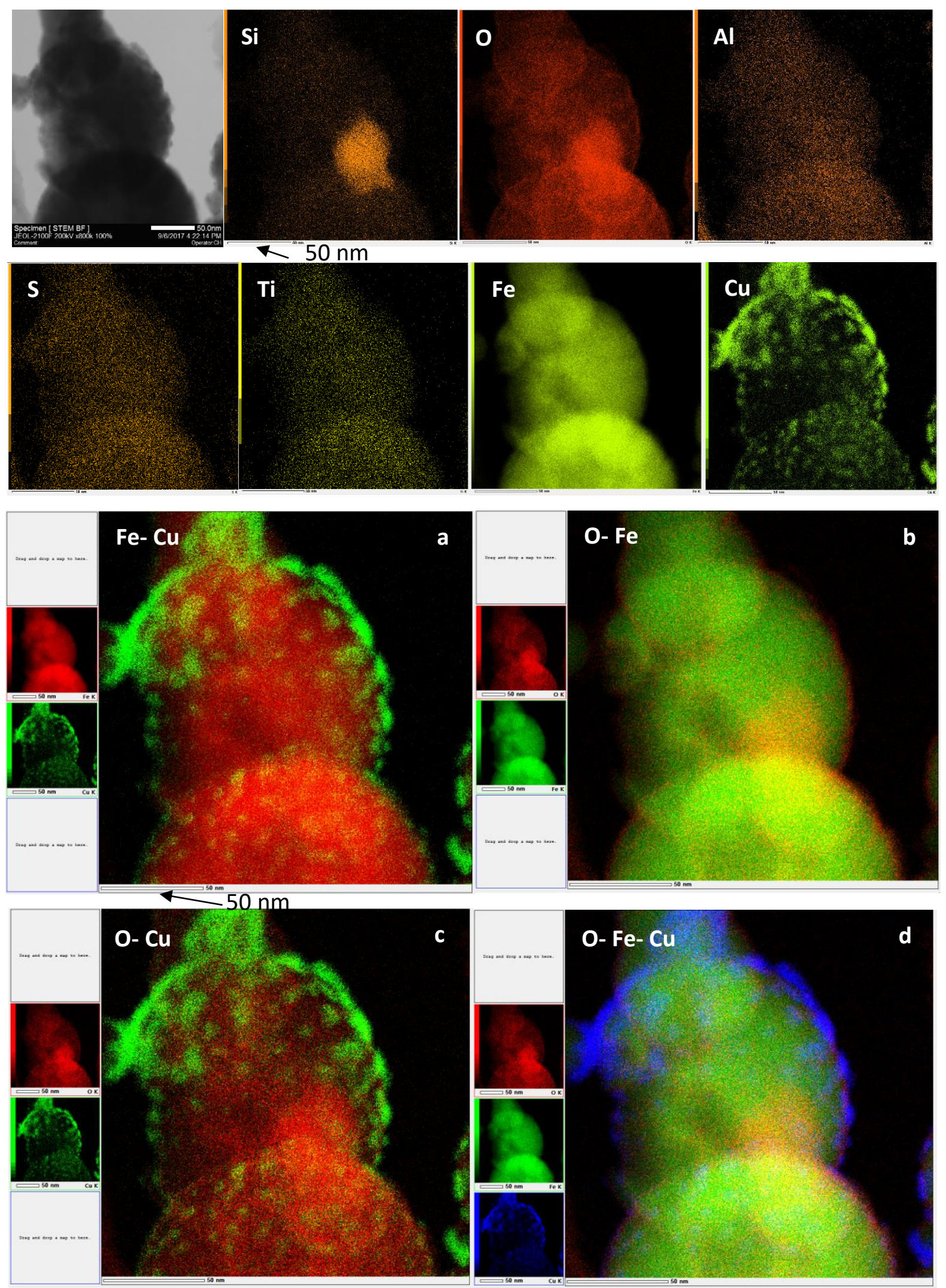

Figure 3.27. TEM-EDS mapping of 5\%Cu-nZVI@MisiCFeOOH-MB1. 
The elemental composition of 5\%Cu-nZVI@Misi was also determined using AAS. The 5\%CunZVI@MisiCFeOOH-MA and 5\%Cu-nZVI@MisiCFeOOH-MB1 were digested in concentrated nitric acid and the resulting solutions were used to measure the concentrations of iron and copper. The amount of Fe and Cu was calculated and the mass of MisiCFeOOH was subtracted from the initial amount of material. The Cu:Fe:MisiCFeOOH mass ratio is 0.52:12.01: 100 (molar ratio of $\mathrm{Cu}: \mathrm{Fe}$ is 1:26.40) and 0.55: 12.54: 100 (molar ratio of $\mathrm{Cu}: F e$ is 1:26.06) for either 5\%Cu-nZVI@MisiCFeOOH-MA or 5\%Cu-nZVI@MisiCFeOOH-MB1, respectively (Table 3.4). The ratio of Fe to MisiCFeOOH is higher than the theoretical ratio of Fe to MisiCFeOOH $(10: 100)$. This is similar to that found in Ni systems. A possible reason for the higher ratio of Fe to MisiCFeOOH is the contribution of Fe in Misi when Misi had been prepared by coating an $\mathrm{FeOOH}$ layer. Iron attributes to $0.9 \%$ in $\mathrm{MisiCFeOOH}$. The $\mathrm{Cu}: \mathrm{Fe}$ ratio in $5 \% \mathrm{Cu}$ nZVI@MisiCFeOOH-MB1 is slightly higher than that of 5\%Cu-nZVI@MisiCFeOOH-MA but the difference is minimal.

Table 3.4. Analysis of Cu-doped materials ( $0.250 \mathrm{~g}$ of material, $5.0 \mathrm{~mL}$ of concentrated $\mathrm{HNO}_{3}$ ). Note: $\mathrm{Misi}=\mathrm{MisiCFeOOH}$.

\begin{tabular}{|l|c|c|c|c|}
\hline \multicolumn{1}{|c|}{ Material } & $\mathrm{Cu}(\mathrm{mg})$ & Fe (mg) & Misi (mg) & $\begin{array}{c}\text { Mass ratio of } \\
\text { Cu:Fe:Misi }\end{array}$ \\
\hline 5\%Cu-nZVI@MisiCFeOOH-MA & $1.15 \pm 0.01$ & $26.7 \pm 0.2$ & $222.2 \pm 0.1$ & $0.52: 12.01: 100$ \\
\hline $5 \%$ Cu-nZVI@MisiCFeOOH-MB1 & $1.22 \pm 0.04$ & $27.7 \pm 0.1$ & $221.1 \pm 0.1$ & $0.55: 12.54: 100$ \\
\hline MisiCFeOOH & 0 & 2.32 & 247.68 & $0: 0.94: 100$ \\
\hline
\end{tabular}

TEM-EDS only showed traces of Cu within the samples, this must be because the $\mathrm{Cu}$ is evenly distributed through the Fe, with only small amount of $\mathrm{Cu}$ concentrated on the surface. In contrast, AAS analysis showed the concentration of elements when the material was digested. Therefore, the difference in $\mathrm{Cu}$ results in TEM-EDS and AAS of 5\%CunZVI@MisiCFeOOH-MA was observed. Regarding the real amount of Fe, the mass of Fe in 5\%Cu-nZVI@MisiFeOOH-MB1 was slightly less than that of 5\%Cu-nZVI@MisiFeOOH-MA. This could be due to the loss of $n Z V I$ in the reaction of $n Z V I$ with $\mathrm{Cu}(I I)$ in the deposition step to form $\mathrm{Cu}(0)$. 
The reactivity of $5 \% \mathrm{Cu}-\mathrm{nZVI}$ on Misi as a function of synthetic methods towards nitrate reduction was examined. To do this, 5\%Cu-nZVI was doped on either MisiC or MisiCFeOOH using both co-reduction method (MA) and deposition method (MB1). In these nitrate reactions, the molar ratio of Fe to nitrate was 15:1. Generally, these materials had higher reactivity than non-doped nZVI@Misi (Section 3.2). After 5 minutes, for MisiC-based materials, nitrate-N concentrations were $7.6 \pm 0.7 \mathrm{ppm}$ and $9.3 \pm 0.7 \mathrm{ppm}$ when either $5 \% \mathrm{Cu}$ nZVI@MisiC-MA or 5\%Cu-nZVI@MisiC-MB1 was used (Figure 3.28). Interestingly, for MisiCFeOOH-based materials, nitrate- $\mathrm{N}$ concentrations were unchanged, approximately 10 ppm for either material (Figure 3.29). This can either explained by a large time due to activation of materials or, more likely, an overlap of the UV-Vis signals of nitrate and the intermediate reduction product, nitrite. Therefore, the concentration of nitrate measured using UV-Vis could be the total concentration of nitrate and nitrite. ${ }^{237}$ This will be further addressed in Chapter 4. After 15 minutes, materials prepared via deposition method (MB1) had higher reactivity than those prepared via co-reduction method (MA). However, after 30, there was no difference in the reactivity towards nitrate reduction using materials synthesised via method MA or method MB1, regardless of Misi preparation as nitrate-N levels were below the detection limits. Based on these results, both methods, MA and MB1, were chosen for further investigation of bimetallic Cu-nZVI on Misi.

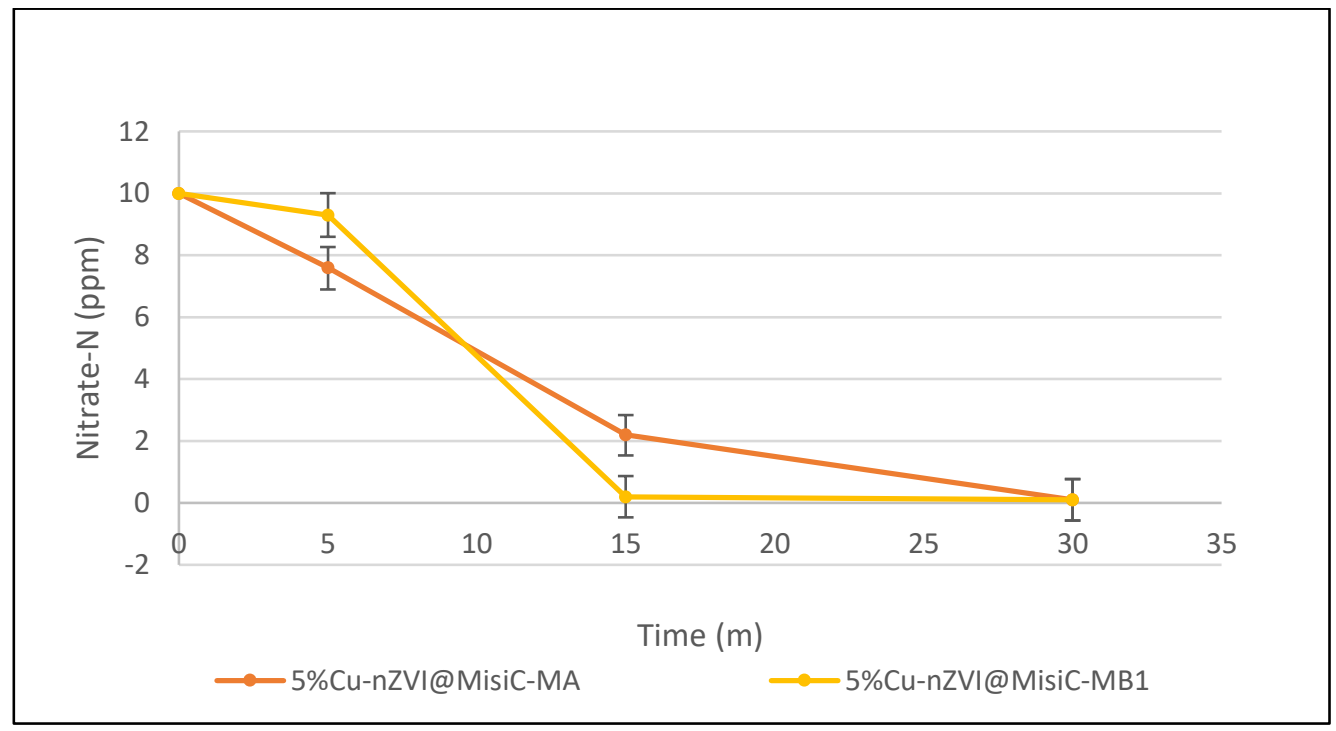

Figure 3.28. Reduction characterisation of 5\%Cu-nZVI@MisiC prepared via different methods (0.150 g of material, $25.0 \mathrm{~mL}$ of $10 \mathrm{ppm}$ nitrate-N solution, under anaerobic conditions). 


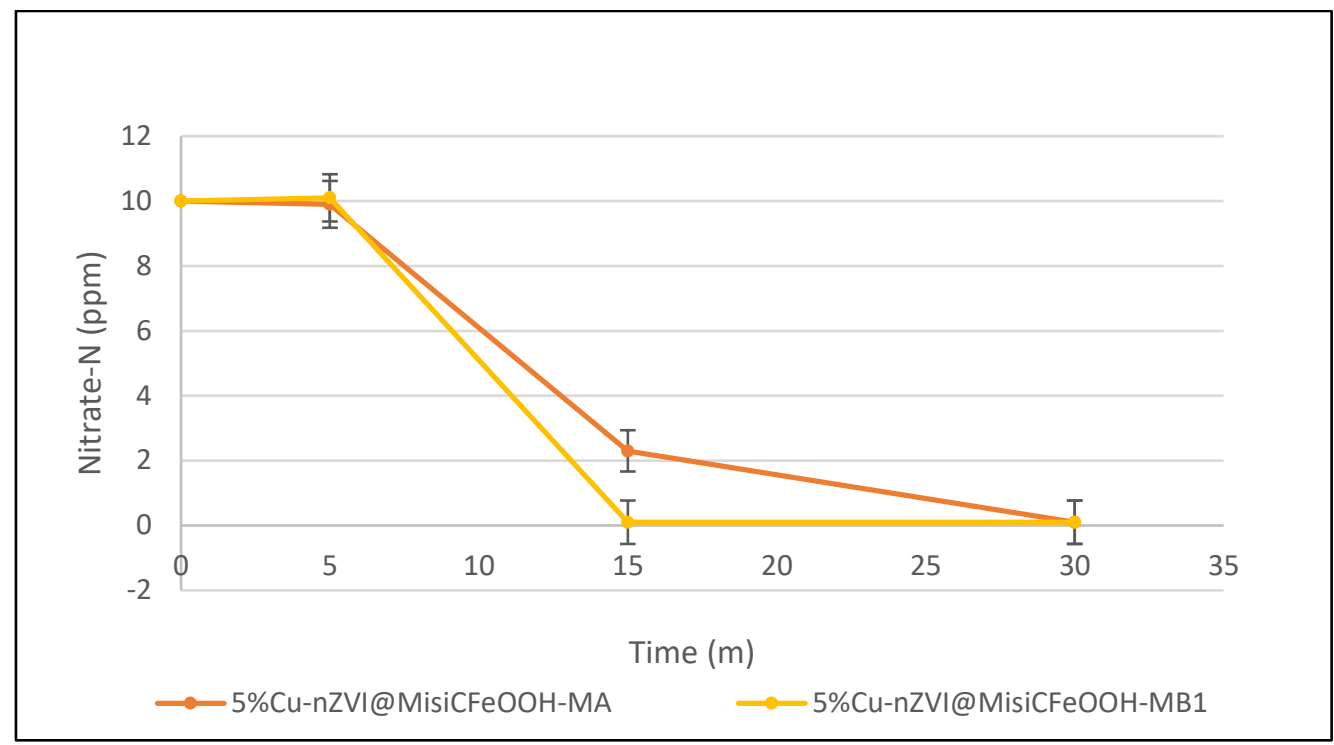

Figure 3.29. Reduction characterisation of 5\%CU-nZVI@MisiCFeOOH prepared via different methods $(0.150 \mathrm{~g}$ of material, $25.0 \mathrm{~mL}$ of $10 \mathrm{ppm}$ nitrate- $N$ solution, under anaerobic conditions).

To examine the final N-containing product, 5\%Cu-nZVI@Misi was used for nitrate reduction and ammonium concentrations in resulting solutions was determined using the Nessler method. In these experiments, 5\%Cu-nZVI on either Misi or MisiCFeOOH was prepared via coreduction method (MA) and deposition method (MB1). The conversion of nitrate to ammonium using Cu-doped systems was slower than using Ni-doped systems. After 5 minutes, ammonium-N levels were less than $3.5 \mathrm{ppm}$ using any types of $\mathrm{Cu}$-doped systems (Figure 3.30) compared to more than $5.5 \mathrm{ppm}$ ammonium- $\mathrm{N}$ was generated using any types of Ni-doped systems (Figure 3.20). In nitrate reduction using Cu-doped systems, the conversion of nitrate into nitrite was fast but the step from nitrite to ammonium was slow. Therefore, nitrite accumulation was high (more detail in Chapter 4). However, after 30 minutes, the ammonium production was greater than $86 \%$ using all types of Cu-doped materials, similar to $\mathrm{Ni}$-doped systems. In addition, ammonium levels using either material prepared via the deposition method (MB1) were slightly higher than those observed in either material prepared via co-reduction method (MA). Ammonium-N levels were 9.8 ppm (98\% ammonium production) and $8.6 \mathrm{ppm}$ ( $86 \%$ ammonium production), respectively. In summary, ammonium is also the prominent $\mathrm{N}$-containing product. This was consistent with the findings reported by Liou et al.. ${ }^{126}$ In their study, the initial nitrate-N concentration was 40 ppm and 
the molar ratio of Fe to nitrate was 2.2:1 using 5\%Cu-nZVI. However, supported CunZVI@Misi in this current study converted nitrate to ammonium faster. Around 60\% of the initial nitrate was converted into ammonium after 15 minutes compared to 120 minutes in their report.

In terms of nitrogen mass balance, after 5 minutes, the total nitrogen was higher than the initial nitrogen, especially in materials prepared via the deposition method (MB1). This is similar to the results from Ni-doped nZVI@Misi systems. For example, after 5 minutes, nitrate- $\mathrm{N}$, ammonium- $\mathrm{N}$ concentrations, and total nitrogen were $3.5 \pm 0.3 \mathrm{ppm}, 9.3 \pm 0.7 \mathrm{ppm}$, and 12.8 ppm, respectively when 5\%Cu-nZVI@MisiC-MB1 was used. However, after 30 minutes, the total nitrogen was from $8.7 \mathrm{ppm}$ to $9.0 \mathrm{ppm}$, which was more reliable. The mass balance using materials prepared via co-reduction method (MA) was lower than that of materials prepared via deposition method (MB1). It could be due to the lower reactivity of materials prepared via co-reduction method (MA) or the generation of other nitrogen products that should be examined using instruments for gas analysis. 

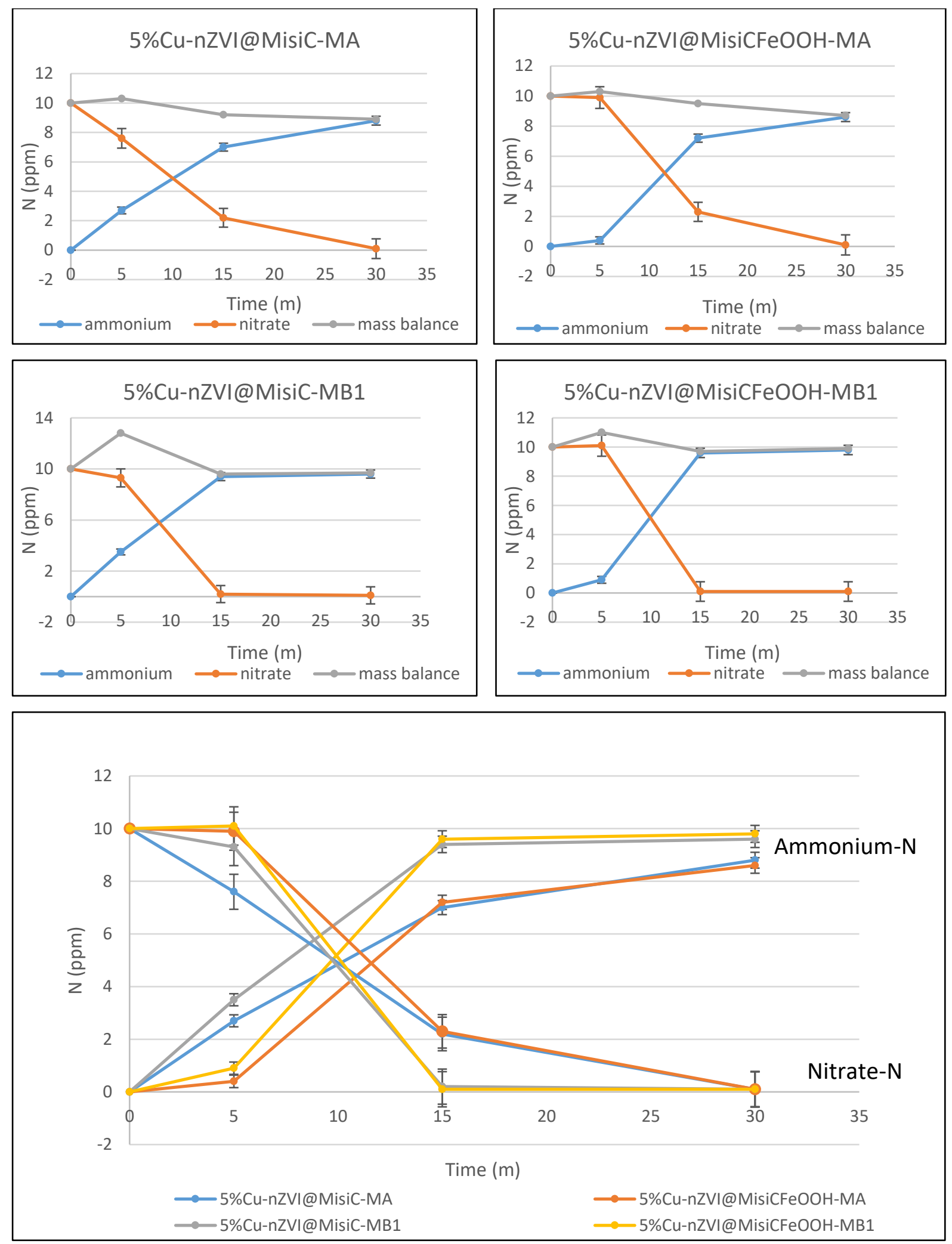

Figure 3.30. Nitrate reduction, ammonium production and mass balance by 5\%Cu-nZVI@Misi ( $0.150 \mathrm{~g}$ of materials, $25.0 \mathrm{~mL}$ of $10 \mathrm{ppm}$ nitrate- $\mathrm{N}$ solution, under anaerobic conditions). 


\subsubsection{Conclusion}

In conclusion, all systems with dopant copper had higher reactivity than non-doped nZVI@Misi, regardless of copper concentration and synthetic method. Similar to nickel, the presence of copper could play the role of a catalyst. Nitrate adsorbs on the copper surface, enhancing the reaction of nitrate and nZVI. Different from nickel, the reduction potential of copper $\left(E^{0}=+0.34 \mathrm{~V}\right)$ is slightly lower than that of nitrate/nitrite in acid condition $\left(E^{0}=+0.49\right.$ V); therefore, copper might not be a reductant that reduces nitrate to nitrite. In addition, the distribution of copper in Cu-nZVI@Misi is dependent on synthetic methods. Copper is distributed in specific areas in materials prepared via co-reduction method (MA) while copper in form of $\mathrm{Cu}(0)$ and copper oxides on the surface of $\mathrm{nZVI}$ in materials prepared via deposition method (MB). Due to the difference in the distribution of copper on iron, Cu-nZVI@Misi (MB1) systems were more reactive than Cu-nZVI@Misi (MA).

\subsection{Bimetallic Zn-nZVI@Misi}

Typically, dopant metals that have more positive reduction potential than $\mathrm{Fe}(\mathrm{II}) / \mathrm{Fe}$ have been chosen due to their catalytic properties towards nitrate reduction. In a research reported, $\mathrm{Zn}$ $\left(\mathrm{E}^{0}=-0.76 \mathrm{~V}\right)$ was used to generate $\mathrm{Zn}-\mathrm{nZVI}$ using the deposition method and the resulting material was examined towards dye removal. ${ }^{174}$ Although the physicochemical properties of $\mathrm{Zn}-\mathrm{nZVI}$ were characterised, there was no information about the presence of $\mathrm{Zn}(0)$ on $\mathrm{nZVI}$. However, in nitrate removal, dopant metal $(0)$ is essential to improve the number of electrons available for the reduction. Therefore, bimetallic Zn-nZVI on Misi was synthesised using coreduction method (MA) with an expectation of adding more reducing agent on nZVI@Misi. In the synthetic procedure, the mass ratio of Fe to Misi is 1:10. Two different preparations of Misi were used, including calcined Misi (MisiC) and calcined/FeOOH-coated Misi (MisiCFeOOH). The mass ratios of $\mathrm{Zn}$ to Fe were $5 \%$ and $10 \%$ with $\mathrm{ZnCl}_{2}$ as the zinc source. Table 3.5 summarises Zn-nZVI@Misi systems examined. They include 5\%Zn-nZVI coated on MisiC and MisiCFeOOH and 10\%Zn-nZVI coated on MisiC and MisiCFeOOH. All these systems were prepared using co-reduction method (MA). After making materials, the morphology of 5\%Zn-nZVI@Misi was characterised via SEM and the variations of Zn loading on the reactivity of Zn-nZVI@Misi towards nitrate reduction were examined. 
Table 3.5. List of Zn-nZVI@Misi systems synthesised.

\begin{tabular}{|c|c|c|c|}
\hline \multirow{2}{*}{ Materials } & \multicolumn{2}{|c|}{ Misi preparation } & \multirow{2}{*}{ Method } \\
\cline { 2 - 3 } & MisiC & MisiCFeOOH & \\
\hline $5 \%$ Zn-nZVI@ & $\mathrm{X}$ & $\mathrm{X}$ & Co-reduction (MA) \\
\hline $10 \% \mathrm{Cu}-\mathrm{nZVI@}$ & & & \\
\hline
\end{tabular}

\subsubsection{Characterisation of Zn-nZVI@Misi}

The morphology of bimetallic Zn-nZVI on different preparations of Misi, 5\%Zn-nZVI@MisiC and 5\%Zn-nZVI@MisiCMisiCFeOOH, was examined via SEM. The COMPO images revealed that there is an uneven distribution of nanoparticles on the surface of Misi in both materials even though a low degree of agglomeration is observed (Figure 3.31). In some areas, nanoparticles evenly adsorb on Misi (red circle) whereas a minimal amount of particles are observed on Misi in other areas (blue circle). The distribution of Zn-nZVI particles is not as good as that visibly observed in either Ni-nZVI (Section 3.3) or CU-nZVI particles (Section 3.4) when the morphology of these materials was characterised via SEM. Fewer Zn-nZVI particles are observed on the surface of Misi. 

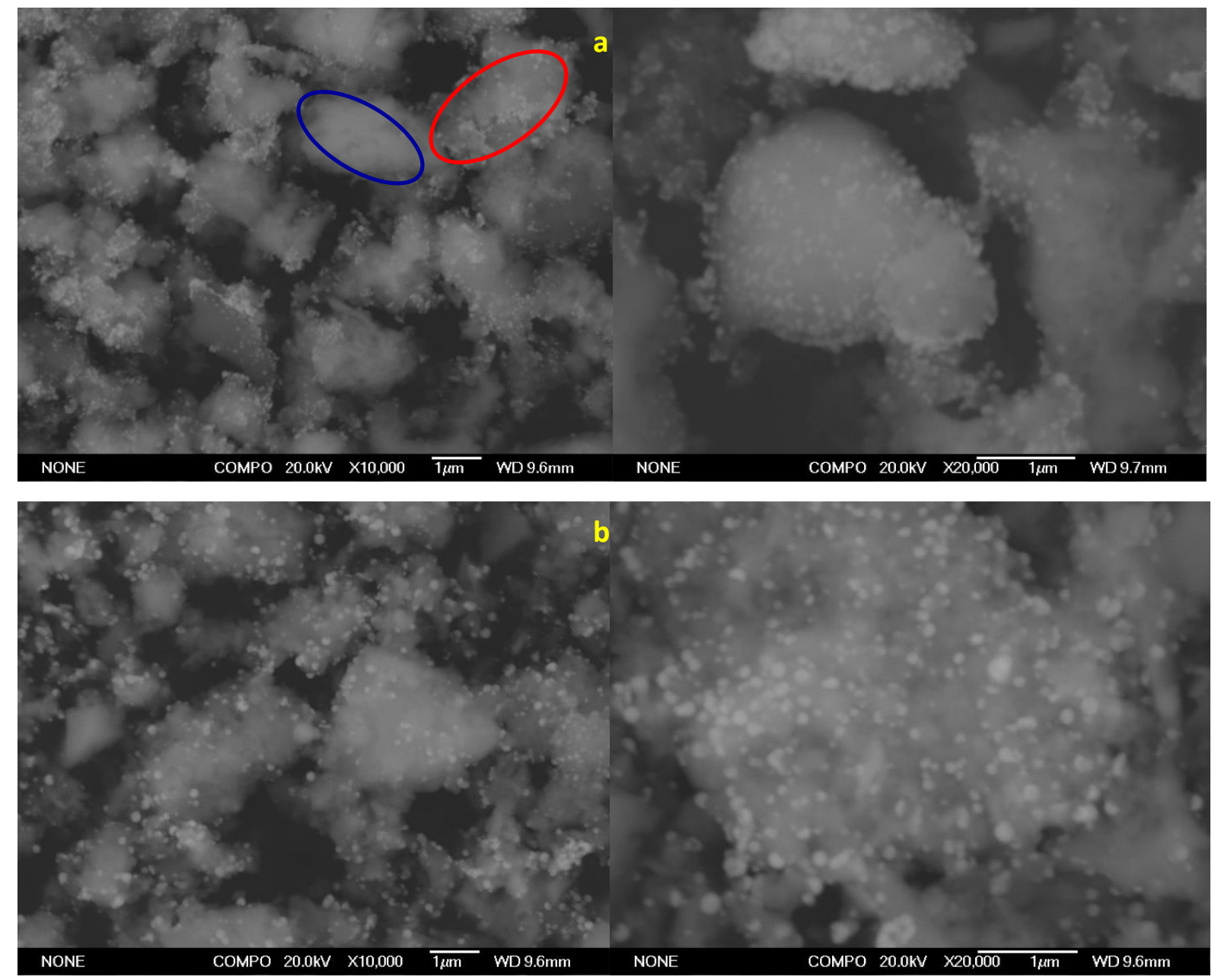

Figure 3.31. COMPO images of (a) 5\% Zn-nZVI@MisiC and (b) 5\% Zn-nZVI@MisiCFeOOH. The lighter component is attributed to Zn-nZVI and silicate is grey.

\subsubsection{Variations on Zn loading}

The effect of Zn loading on bimetallic Zn-nZVI on Misi was examined. For this, $5 \%$ and $10 \% \mathrm{Zn}$ on nZVI was synthesised on either MisiC or MisiCFeOOH. Due to the similarity of the morphology of materials with $\mathrm{Ni}$-containing as different nickel concentrations were used, the morphology difference between different $\mathrm{Zn}$ loadings was not examined. The reactivity of $\mathrm{Zn}$ doped materials as a function of $\mathrm{Zn}$ concentration towards nitrate reduction was investigated. In nitrate reduction, the molar ratio of Fe to nitrate is 15:1. All these $\mathrm{Zn}$-doped materials had higher reactivity than non-doped nZVI@Misi, except for 5\%Zn-nZVI@MisiCFeOOH. The reason for this inhibition was unclear but it could be the limitation in the number of active sites. 
After 5 minutes, the nitrate concentration was insignificantly reduced using all types of $\mathrm{Zn}$ doped materials except for 10\%Zn-nZVI@MisiCFeOOH where the nitrate- $\mathrm{N}$ concentration was 7.9 ppm (Figure 3.32). After 30 minutes, both materials with $10 \%$ of $\mathrm{Zn}$ reduced nitrate faster than materials with $5 \%$ of $\mathrm{Zn}$, regardless of Misi preparation. For MisiC-based materials, nitrate-N concentrations were $4.6 \pm 0.6 \mathrm{ppm}$ ( $54 \%$ nitrate conversion) and $2.9 \pm 0.6 \mathrm{ppm}$ (71\% nitrate conversion) using either 5\%Zn-nZVI@MisiC or 10\%Zn-nZVI@MisiC, respectively. For MisiCFeOOH-based materials, nitrate-N concentrations were $6.4 \pm 0.7 \mathrm{ppm}$ (36\% nitrate conversion) and $1.2 \pm 0.7$ ppm (88\% nitrate conversion) using either 5\%Zn-nZVI@MisiCFeOOH or 10\%Zn-nZVI@MisiCFeOOH, respectively.

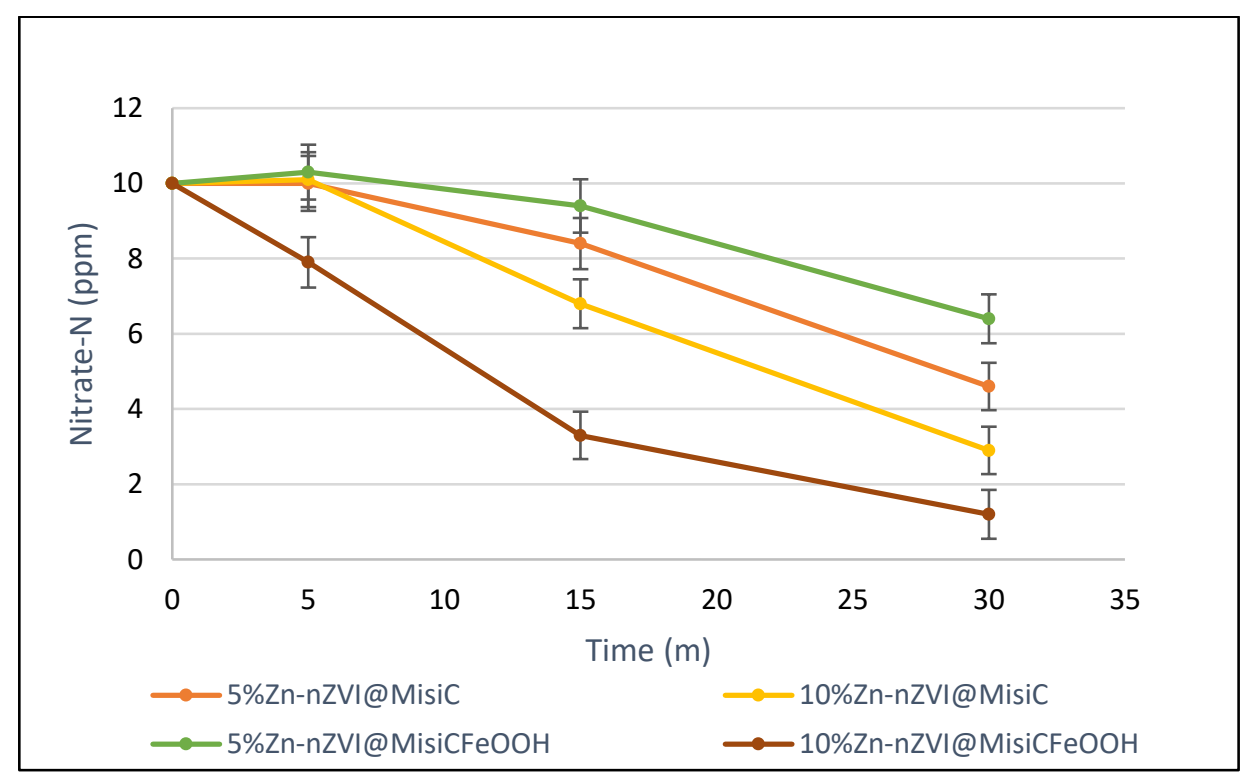

Figure 3.32. Reduction characterisation of Zn-nZVI@Misi on different Zn loadings $(0.150 \mathrm{~g}$ of Zn-nZVI@Misi, 25.0 mL of 10 ppm nitrate-N solution, under anaerobic conditions).

The ammonium concentrations were measured in the resulting solutions using the Nessler method. The ammonium concentration increased gradually over the course of 30 minutes (Figure 3.33). After 5 minutes, ammonium-N concentrations were approximately 0.8 ppm using all types of Zn-doped systems except for 10\%Zn-nZVI@MisiCFeOOH where the ammonium-N level was $3.2 \pm 0.2 \mathrm{ppm}$. After 30 minutes, ammonium- $\mathrm{N}$ concentrations were $4.8 \mathrm{ppm} \pm 0.3$ (48\% ammonium production), $3.5 \pm 0.2 \mathrm{ppm}$ ( $35 \%$ ammonium production), $6 \pm$ $0.3 \mathrm{ppm}$ (60\% ammonium production), and $8.6 \pm 0.4 \mathrm{ppm}$ ( $86 \%$ ammonium production) when either5\%Zn-nZVI@MisiC, 5\%Zn-nZVI@MisiCFeOOH, 10\%Zn-nZVI@MisiC or 10\%ZnnZVI@MisiCFeOOH was used, respectively. 

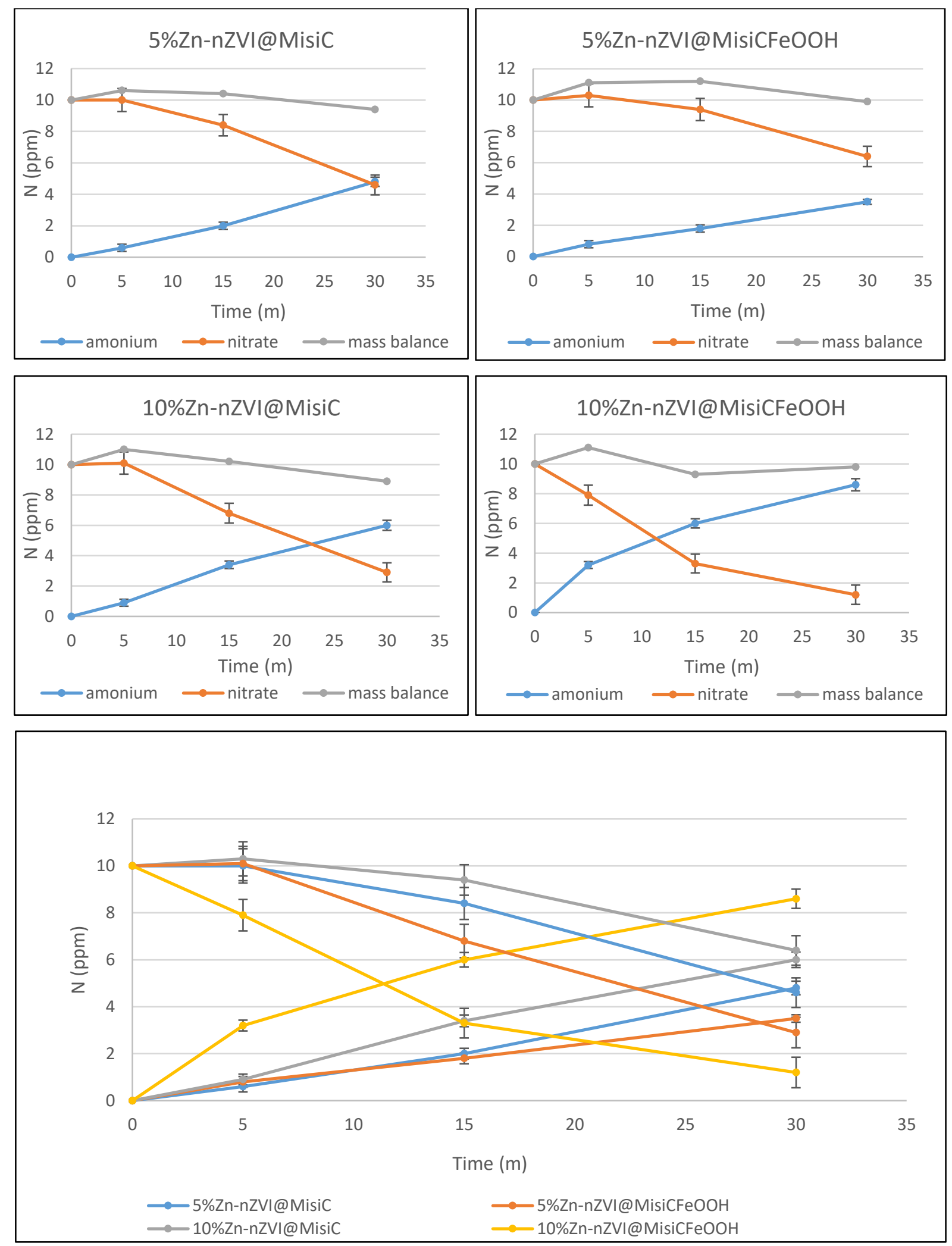

Figure 3.33. Nitrate reduction, ammonium production and mass balance by Zn-nZVI@Misi ( $0.150 \mathrm{~g}$ of materials, $25.0 \mathrm{~mL}$ of $10 \mathrm{ppm}$ nitrate- $N$ solution, under anaerobic conditions). 


\subsubsection{Conclusion}

Even though the standard reduction potential of $\mathrm{Zn}(\mathrm{II}) / \mathrm{Zn}$ is more negative than that of either $\mathrm{Fe}, \mathrm{Ni}$ or $\mathrm{Cu}, \mathrm{Zn}$-doped materials were less active than both Ni-doped nZVI@Misi and Cudoped nZVI@Misi. This can be explained by the catalytic role of $\mathrm{Ni}$ and $\mathrm{Cu}$. The presence of $\mathrm{Ni}$ and $\mathrm{Cu}$ promotes the electron transfer from nZVI to nitrate. In contrast, $\mathrm{Zn}$ only plays the role of another reducing agent accompanied with nZVI. As such, supported bimetallic ZnnZVI@Misi were not investigated in further work.

\subsection{Bimetallic Pd-nZVI@Misi}

Several studies on doping Pd on nZVI have been reported. The Pd concentration used ranged from $0.054 \%$ to $50 \% .^{3,49,183,185-186}$ In addition, Pd-nZVI on supports have also been examined. In these systems, the mass ratio of Fe to support and the mass ratio of $\mathrm{Pd}$ to Fe were 10:50 and from $0.1 \%$ to $75 \%$, respectively. ${ }^{151,191,200-202}$ However, all unsupported and supported PdnZVI were used for removing organic substances. In this study, bimetallic Pd-nZVI@Misi was synthesised using two different methods, including co-reduction method (MA) and deposition method (MB1). In all experiments, the mass ratio of Fe to Misi is 1:10 and the mass ratios of $\mathrm{Pd}$ to Fe were varied from $0.1 \%$ to $1 \%$ with $\mathrm{Pd}\left(\mathrm{CH}_{3} \mathrm{COO}\right)_{2}$ as palladium source. Two different preparations of Misi, including calcined Misi (MisiC) and calcined/FeOOH-coated Misi (MisiCFeOOH) were used as support. Table 3.6 summarises Pd-nZVI@Misi systems examined. They include $0.1 \% \mathrm{Pd}-\mathrm{nZVI}$ coated on MisiC and MisiCFeOOH using the co-reduction method (MA) and deposition method (MB1), 0.5\%Pd-nZVI coated on MisiC and MisiCFeOOH using the co-reduction method (MA) and deposition method (MB1), and 1\%Pd-nZVI coated on MisiC using the co-reduction method (MA). In these palladium studies, two different variables were examined: variations on Pd loading and variations on synthetic method. 
Table 3.6. List of Pd-nZVI@Misi systems synthesised.

\begin{tabular}{|c|c|c|c|}
\hline \multirow{2}{*}{ Materials } & \multicolumn{2}{|c|}{ Misi preparation } & \multirow{2}{*}{ Method } \\
\cline { 2 - 4 } $0.1 \% \mathrm{Pd}$ MisiC & MisiCFeOOH & \\
\hline & $\mathrm{X}$ & $\mathrm{X}$ & Co-reduction (MA) \\
\cline { 3 - 4 } & & $\mathrm{X}$ & Deposition (MB1) \\
\hline 0.5\%Pd-nZVI@ & $\mathrm{X}$ & $\mathrm{X}$ & De-reduction (MA) \\
\hline \multirow{2}{*}{ 1\%Pd-nZVI@ } & $\mathrm{X}$ & $\mathrm{X}$ & Co-reduction (MA) \\
\cline { 2 - 4 } & & $\mathrm{X}$ & Deposition (MB1) \\
\hline
\end{tabular}

\subsubsection{Variations on Pd loading}

The role of $\mathrm{Pd}$ as a function of Pd loading on both morphology and reactivity was examined. The morphology of $0.5 \% \mathrm{Pd}-\mathrm{nZVI}$ and $1 \% \mathrm{Pd}-\mathrm{nZVI}$ on MisiCFeOOH was investigated using SEM. COMPO images revealed that nanoparticles evenly adsorb on Misi with small amount of agglomeration (Figure 3.34). In the majority of areas (red circle), there is a good dispersion of $\mathrm{nZVI}$ on the surface of Misi although, in some areas (blue circle), nZVI agglomerates are observed. The difference in the degree of agglomeration in these materials was not quantified. The distribution of elements in our materials is similar to that of materials previously reported but less agglomeration was observed. In these studies, Pd-nZVI was doped on bentonite with the ratio of Fe to bentonite is $1: 191,201$ which is higher than that used in our materials (1:10). 

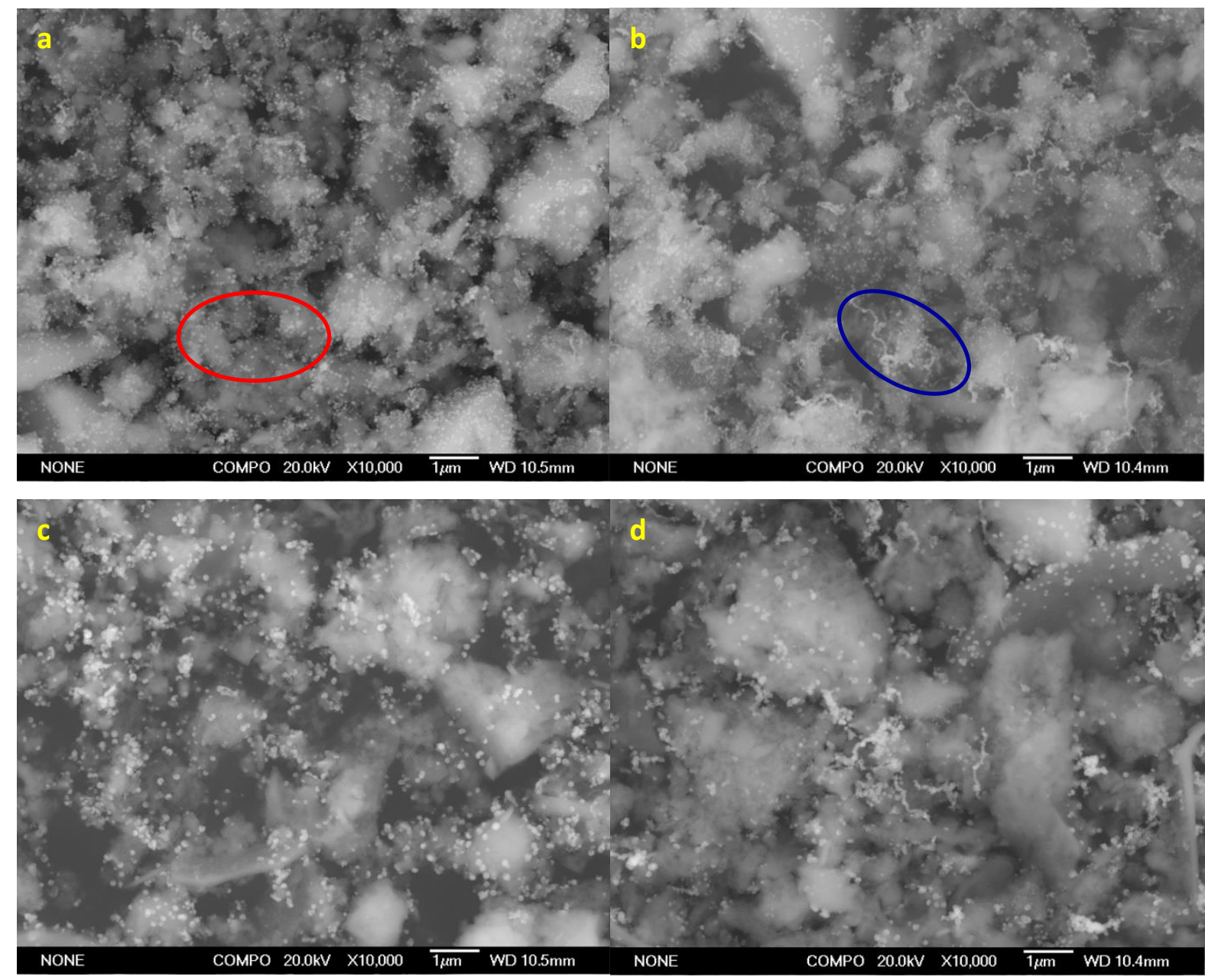

Figure 3.34. COMPO images of (a) 0.5\%Pd-nZVI@MisiCFeOOH-MA, (b) 1\%PdnZVI@MisiCFeOOH-MA, (c) 0.5\%Pd-nZVI@MisiCFeOOH-MB1, (d) 1\%Pd-nZVI@MisiCFeOOHMB1. The lighter component is attributed to Pd-nZVI and silicate is grey.

The effect of $\mathrm{Pd}$ loading on the reactivity of Pd-nZVI on Misi towards nitrate reduction was examined. These materials were produced by using co-reduction method (MA) on both MisiC and $\mathrm{MisiCFeOOH}$ and three different $\mathrm{Pd}$ contents, $0.1 \%, 0.5 \%$, and $1 \%$. In nitrate reduction, the molar ratio of Fe to nitrate is 15:1. All Pd-doped materials reduced nitrate faster than nondoped nZVI@Misi (Section 3.2), except for 0.5\%Pd-nZVI@MisiCFeOOH-MA. After 30 minutes, the nitrate- $\mathrm{N}$ concentrations were $6.5 \pm 0.7 \mathrm{ppm}$ and $4.5 \pm 0.6 \mathrm{ppm}$ when $0.5 \% \mathrm{Pd}$ nZVI@MisiCFeOOH-MA and nZVI@MisiCFeOOH were used, respectively. The higher reactivity of some of Pd-doped nZVI@Misi systems can be explained by the catalytic role of Pd. Nitrate adsorbs on the surface of $\mathrm{Pd}$, leading to catalysing the reaction of nitrate with $\mathrm{nZVI}$.

For MisiC-based materials, the nitrate reduction using 0.1\%Pd-nZVI@MisiC-MA was slower than that of both 0.5\%Pd-nZVI@MisiC-MA and 1\%Pd-nZVI@MisiC-MA but the difference was 
insignificant (Figure 3.35). After 5 minutes, nitrate- $\mathrm{N}$ concentrations were approximately 9 ppm for all types of Pd-doped nZVI@Misi materials prepared via the co-reduction method (MA). After 30 minutes, nitrate- $\mathrm{N}$ concentrations were $4.5 \pm 0.6 \mathrm{ppm}$ ( $55 \%$ nitrate conversion) for 0.1\%Pd-nZVI@MisiC-MA, $3.3 \pm 0.6$ ppm (67\% nitrate conversion) for 0.5\%Pd-nZVI@MisiCMA, and $3.1 \pm 0.6$ ppm (69\% nitrate conversion) for 1\%Pd-nZVI@MisiC-MA.

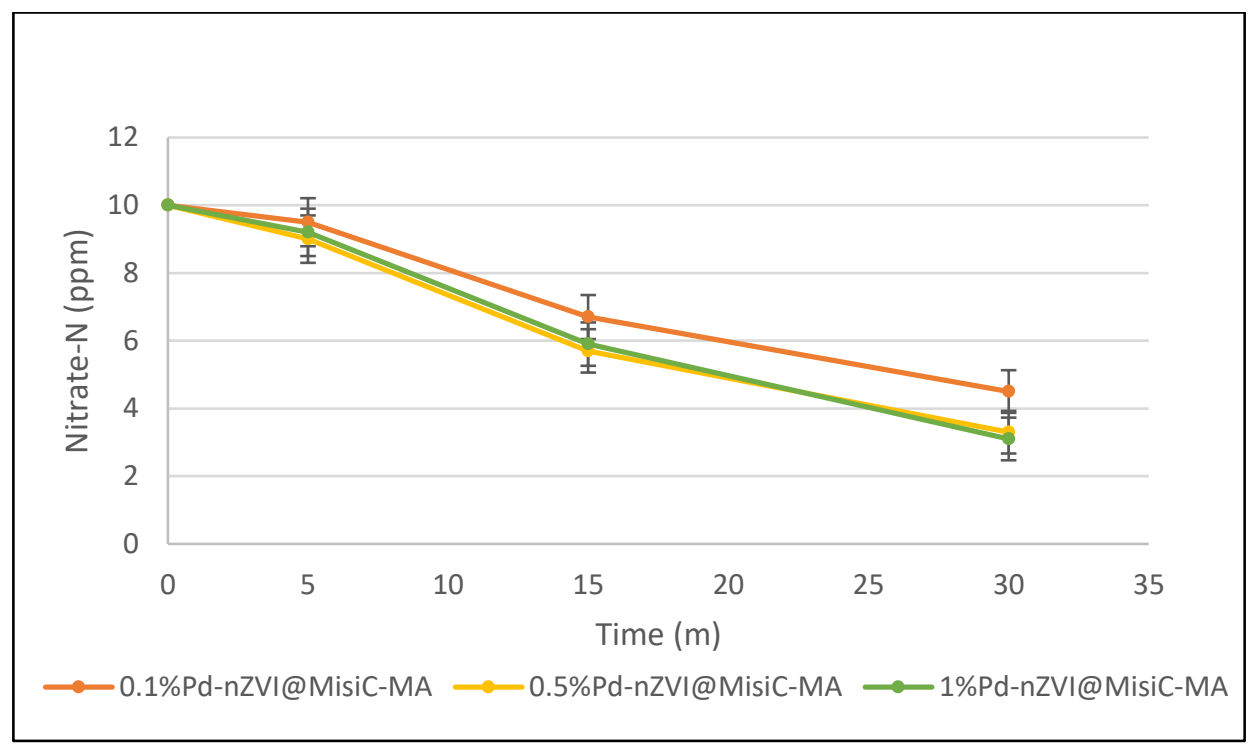

Figure 3.35. Reduction characterisation of Pd-nZVI@MisiC-MA on different Pd contents (0.150 g of material, $25.0 \mathrm{~mL}$ of $10 \mathrm{ppm}$ nitrate-N solution, under anaerobic conditions).

For MisiCFeOOH-based materials, the amount of Pd loading influenced on nitrate reduction. After 5 minutes, nitrate- $\mathrm{N}$ concentrations were approximately $10 \mathrm{ppm}$ using all materials (Figure 3.36). However, after 30 minutes, 1\%Pd-doped material reduced nitrate fastest while the reactivity of $0.1 \% \mathrm{Pd}$-doped material was lowest. Nitrate- $\mathrm{N}$ concentrations were $1.9 \pm 0.6$ ppm ( $81 \%$ nitrate conversion), $6.5 \pm 0.7 \mathrm{ppm}$ (35\% nitrate conversion) and $4.5 \pm 0.6 \mathrm{ppm}$ (55\% nitrate conversion) when either 1\%Pd-nZVI@MisiCFeOOH-MA, 0.5\%Pd-nZVI@MisiCFeOOHMA or $0.1 \% \mathrm{Pd}-\mathrm{nZVI@MisiCFeOOH-MA}$, respectively. The higher reactivity of $1 \% \mathrm{Pd}$-doped materials could be explained by the larger amount of $\mathrm{Pd}$ on the surface, resulting in more catalytic sites. The presence of $1 \% \mathrm{Pd}$ might start activating the catalytic function of palladium while both $0.1 \% \mathrm{Pd}$ and $0.5 \% \mathrm{Pd}$ was not enough. In some cases (e.g. $0.5 \% \mathrm{Pd}$ nZVI@MisiCFeOOH-MA), the nitrate reduction was not fast as nondoped nZVI@Misi. This could be due to the random errors during the experimental process. 


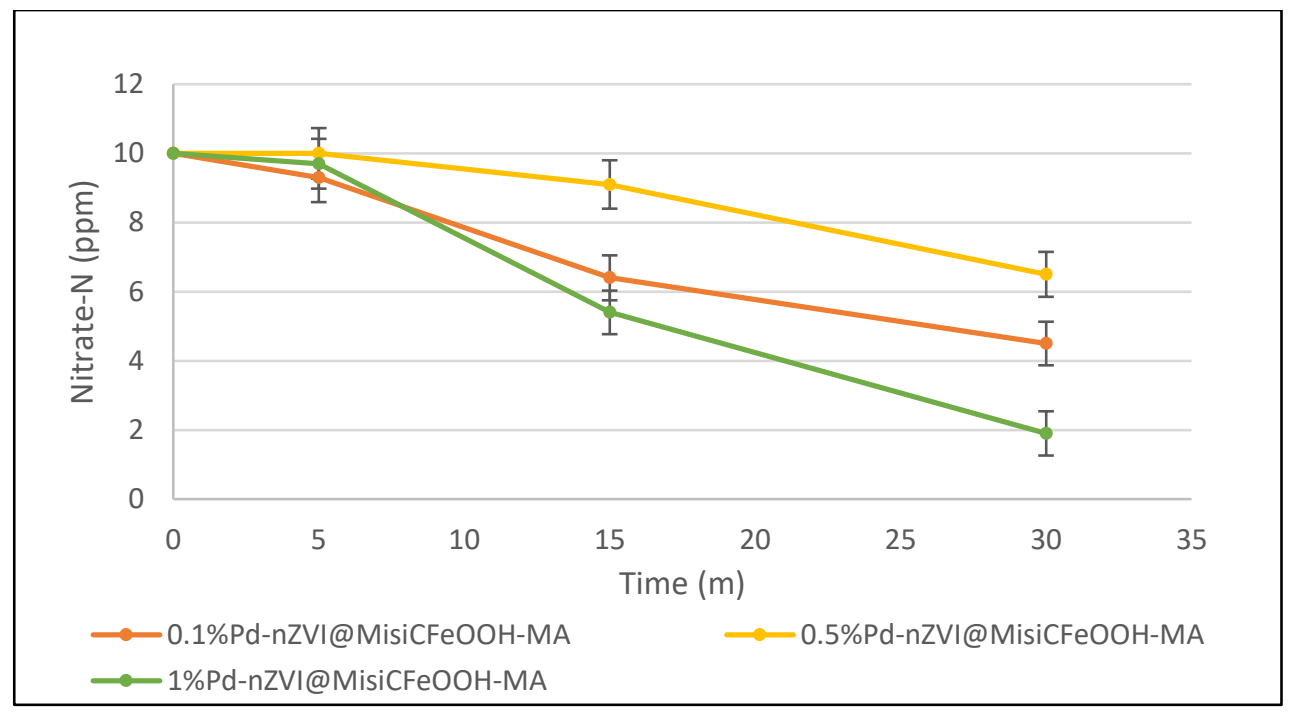

Figure 3.36. Reduction characterisation of Pd-nZVI@MisiCFeOOH-MA on different Pd contents $(0.150 \mathrm{~g}$ of material, $25.0 \mathrm{~mL}$ of $10 \mathrm{ppm}$ nitrate- $N$ solution, under anaerobic conditions).

\subsubsection{Variations on synthetic methods}

The effect of synthetic methods on the reactivity of Pd-nZVI on Misi was examined. For this, 0.5\%Pd-nZVI was doped on two different preparations of Misi, including calcined Misi (MisiC) and calcined/FeOOH-coated Misi (MisiCFeOOH), using two different methods, MA and MB1. The results showed that after 5 minutes, nitrate- $\mathrm{N}$ concentrations were more than $9 \mathrm{ppm}$ for all materials examined. After 30 minutes, materials synthesised using deposition method (MB1) had higher reactivity than those synthesised using co-reduction method (MA) and the difference between these materials is dependent upon preparations of Misi (Figure 3.37). For MisiC, the difference in the reactivity of materials prepared via co-reduction method (MA) and deposition method (MB1) was minimal. Nitrate-N concentrations were $2.7 \pm 0.6 \mathrm{ppm}$ (73\% nitrate conversion) and $3.3 \pm 0.6 \mathrm{ppm}$ (67\% nitrate conversion) using either $0.5 \% \mathrm{Pd}-$ nZVI@MisiC-MB1 or 0.5\%Pd-nZVI@MisiC-MA. For MisiCFeOOH, 0.5\%Pd-nZVI@MisiCFeOOHMB1 reduced nitrate two times faster than 0.5\%Pd-nZVI@MisiCFeOOH-MA. Nitrate-N concentrations were $3.1 \pm 0.6 \mathrm{ppm}$ (69\% nitrate conversion) and $6.5 \pm 0.7 \mathrm{ppm}$ (35\% nitrate conversion), respectively. 


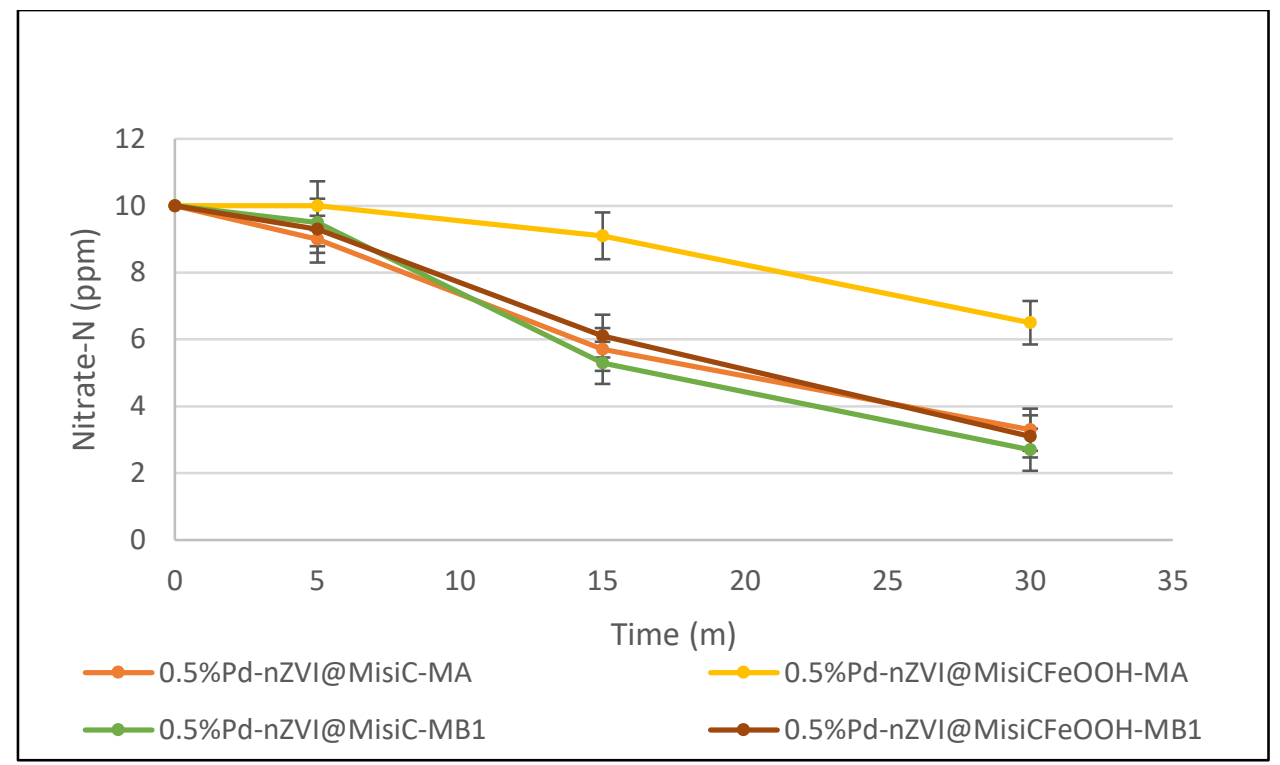

Figure 3.37. Reduction characterisation of Pd-nZVI@Misi prepared via different methods

(0.150 g of material, $25.0 \mathrm{~mL}$ of $10 \mathrm{ppm}$ nitrate-N solution, under anaerobic conditions).

In order to examine the products, two best materials, including 1\%Pd-nZVI@MisiCFeOOH-MA and 1\%Pd-nZVI@MisiCFeOOH-MB1 were used to do nitrate reduction and the ammonium concentrations of the resultant solutions were determined using the Nessler method. After 5 minutes, ammonium- $\mathrm{N}$ concentrations were $2.2 \pm 0.2 \mathrm{ppm}$ and $3.3 \pm 0.2 \mathrm{ppm}$ using either 1\%Pd-nZVI@MisiCFeOOH-MA or 1\%Pd-nZVI@MisiCFeOOH-MB1 (Figure 3.38). After 30 minutes, ammonium- $\mathrm{N}$ concentrations were $8.0 \pm 0.2 \mathrm{ppm}$ ( $80 \%$ ammonium production) and $7.8 \pm 0.2 \mathrm{ppm}$, respectively. This indicated that ammonium was also the predominant final product in the nitrate reduction using Pd-doped nZVI@Misi. 

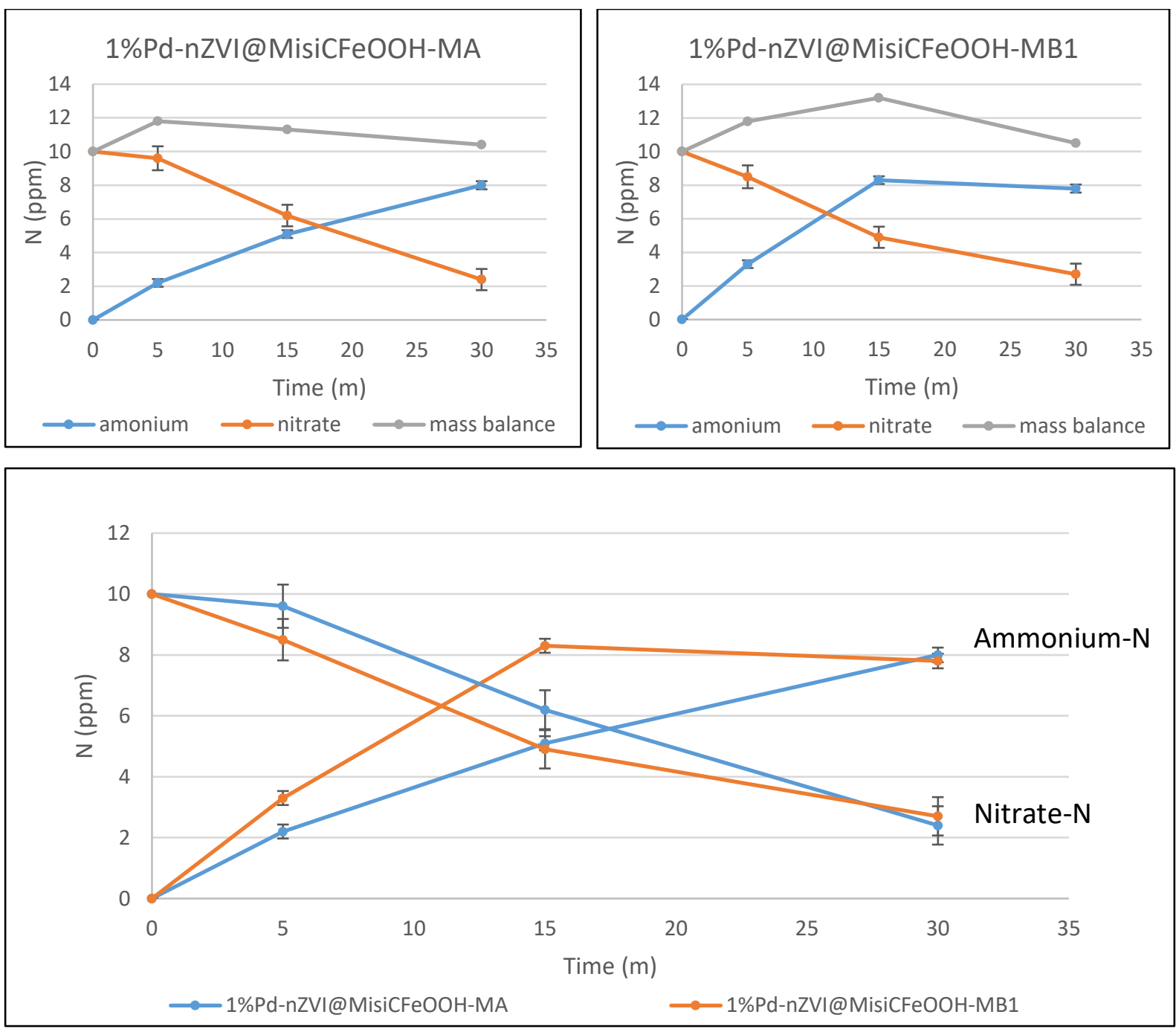

Figure 3.38. Nitrate reduction, ammonium production and mass balance by 1\%Pd-nZVI@Misi

( $0.150 \mathrm{~g}$ of materials, $25.0 \mathrm{~mL}$ of $10 \mathrm{ppm}$ nitrate- $N$ solution, under anaerobic conditions).

\subsubsection{Conclusion}

In comparison with Ni-doped and Cu-doped nZVI@Misi, Pd-doped nZVI@Misi had lower reactivity towards nitrate reduction. After 30 minutes, no nitrate was found when either $\mathrm{Ni}$ nZVI@Misi or CU-nZVI@Misi was used whereas more than 2 ppm nitrate-N was obtained for all types of Pd-nZVI@Misi systems. The higher Pd levels are, the more reactive these materials are. However, due to the cost of palladium source and the attempt finding the efficient materials for environmental application, bimetallic Pd-nZVI@Misi was not chosen for further study. 


\subsection{Trimetallic Pd-Cu-nZVI@Misi}

Unsupported trimetallic Pd-Cu-nZVI has previously been examined to reduce nitrate..$^{4-5}$ In these studies, trimetallic systems were synthesised using three-step procedures. The investigation of supported Pd-nZVI has not been reported yet. In this current study, trimetallic $\mathrm{Pd}-\mathrm{Cu}-\mathrm{nZVI}$ on Misi systems were synthesised using four synthetic methods, including $M A_{\text {tri, }}$ MC1, MC2, and MD (Section 3.1). Two different preparations of Misi were used, including calcined Misi (MisiC) and calcined/FeOOH-coated Misi (MisiFeOOH). In all synthetic experiments, the mass ratio of Fe to Misi is $1: 10$, the mass ratio of $\mathrm{Cu}$ to $\mathrm{nZVI}$ is $0.05: 1$ and the mass ratio of $\mathrm{Pd}$ to $\mathrm{nZVI}$ were varied from $0.5 \%$ to $2 \%$ with $\mathrm{CuCl}_{2}$ and $\mathrm{Pd}\left(\mathrm{CH}_{3} \mathrm{COO}\right)_{2}$ as the copper and palladium sources. Table 3.7 summarises Pd-Cu-nZVI@Misi examined. They include $0.5 \% \mathrm{Pd}-5 \% \mathrm{Cu}-\mathrm{nZVI}$ coated on MisiC and MisiCFeOOH using four different methods (co-reduction ( $\mathrm{MA}_{\text {tri }}$ ), deposition (MC1, MC2, and MD)), 1\%Pd-5\%Cu-nZVI coated on MisiC using co-reduction ( $\left.\mathrm{MA}_{\text {tri }}\right)$, and $2 \% \mathrm{Pd}-5 \% \mathrm{Cu}-\mathrm{nZVI}$ coated on MisiC using co-reduction ( $\left.\mathrm{MA}_{\text {tri }}\right)$. In these trimetallic systems, two variables were examined: variations on Pd loading and variations on synthetic method.

Table 3.7. List of Pd-nZVI@Misi systems synthesised

\begin{tabular}{|c|c|c|c|}
\hline \multirow{2}{*}{ Materials } & \multicolumn{2}{|c|}{ Misi preparation } & \multirow{2}{*}{ Method } \\
\hline & MisiC & MisiCFeOOH & \\
\hline \multirow{4}{*}{ 0.5\%Pd-5\%Cu-nZVI@ } & \multirow{4}{*}{$x$} & \multirow{4}{*}{$\mathrm{x}$} & Co-reduction $\left(\mathrm{MA}_{\text {tri }}\right)$ \\
\hline & & & Deposition (MC1) \\
\hline & & & Deposition (MC2) \\
\hline & & & Deposition (MD) \\
\hline 1\%Pd-5\%Cu-nZVI@ & $x$ & & Co-reduction $\left(\mathrm{MA}_{\text {tri }}\right)$ \\
\hline 2\%Pd-5\%Cu-nZVI@ & $x$ & & Co-reduction $\left(\mathrm{MA}_{\text {tri }}\right)$ \\
\hline
\end{tabular}

\subsubsection{Variations on Pd loading}

The effect of Pd loading on both the adsorption of Pd-Cu-nZVI onto Misi and the subsequent reactivity towards nitrate was examined. To investigate the effect of $\mathrm{Pd}$ loading on the 
morphology, Pd-nZVI was synthesised on MisiCFeOOH using method MC1 with two different $\mathrm{Pd}$ concentrations, including $0.5 \%$ and $1 \%$. COMPO images revealed that $\mathrm{nZVI}$ particles disperse on the surface of Misi with a relatively high degree of agglomeration (Figure 3.39). In some areas (red circle), the dispersion of nanoparticles on Misi is even whereas nanoparticles are observed in the form of aggregates in other areas (blue circle). There is no difference in the degree of agglomeration when $0.5 \% \mathrm{Pd}$ and $1 \% \mathrm{Pd}$ were deposited on $\mathrm{Cu}-$ nZVI@MisiCFeOOH (Figure 3.39a and 3.39b)

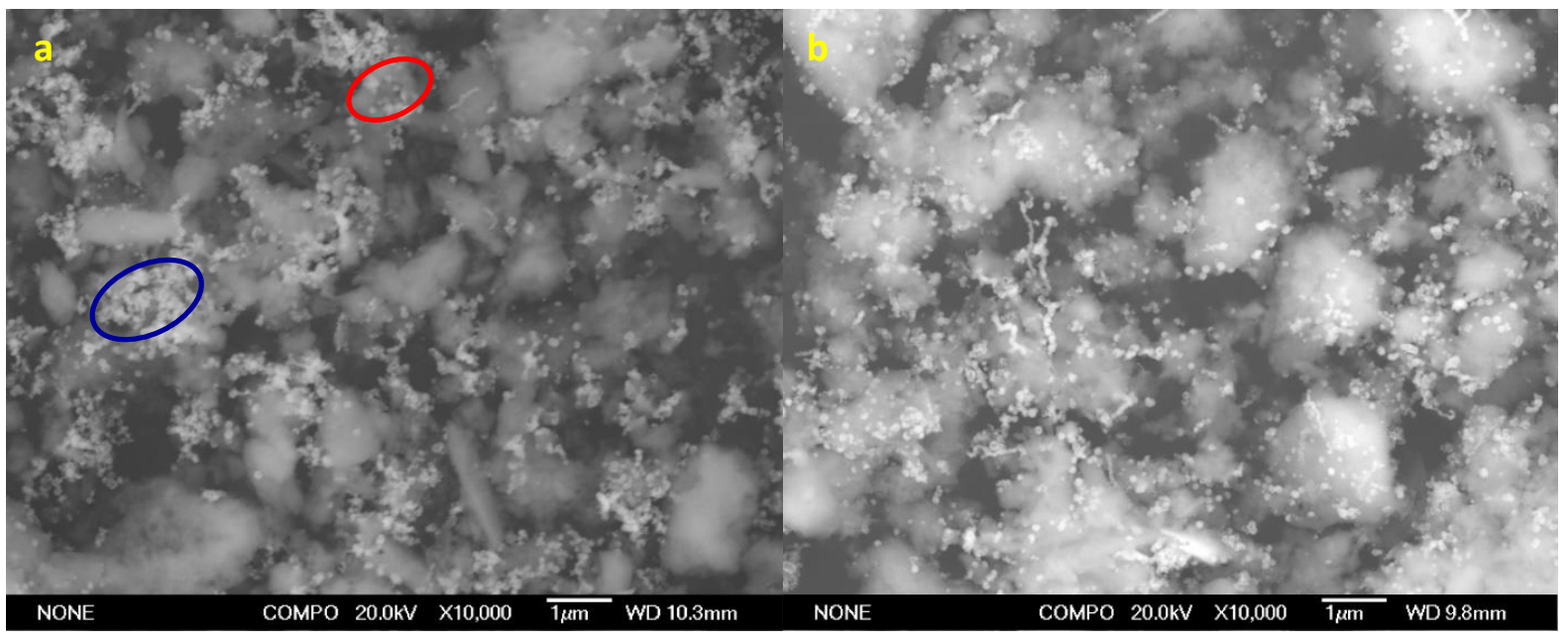

Figure 3.39. COMPO images of (a) 0.5\%Pd-nZVI@MisiCFeOOH-MC1 and (b) 1\%PdnZVI@MisiCFeOOH-MC1. The lighter component is attributed to Pd-Cu-nZVI and silicate is grey.

The reactivity of $\mathrm{Pd}-\mathrm{Cu}-\mathrm{nZVI}$ towards nitrate reduction on different $\mathrm{Pd}$ loadings was investigated. For this, Pd-Cu-nZVI was prepared on MisiC using method MC1 with different Pd loadings, including $0.5 \%, 1 \%$, and $2 \%$. The nitrate reaction was carried out with the molar ratio of Fe to nitrate at 15:1. After 5 minutes, the nitrate conversion increased with the increasing $\mathrm{Pd}$ contents, nitrate- $\mathrm{N}$ concentrations were $4.2 \pm 0.7 \mathrm{ppm}, 2.8 \pm 0.7 \mathrm{ppm}$, and $2.1 \pm 0.7 \mathrm{ppm}$ when either 0.5\%Pd-5\%Cu-nZVI@Misic, 1\%Pd-5\%Cu-nZVI@MisiC or 2\%Pd-5\%CunZVI@MisiC was used, respectively (Figure 3.40). However, after 15 minutes, nitrate was not detected for all materials examined. As such, $0.5 \% \mathrm{Pd}$ was chosen for further investigations due to the cost of Pd. 


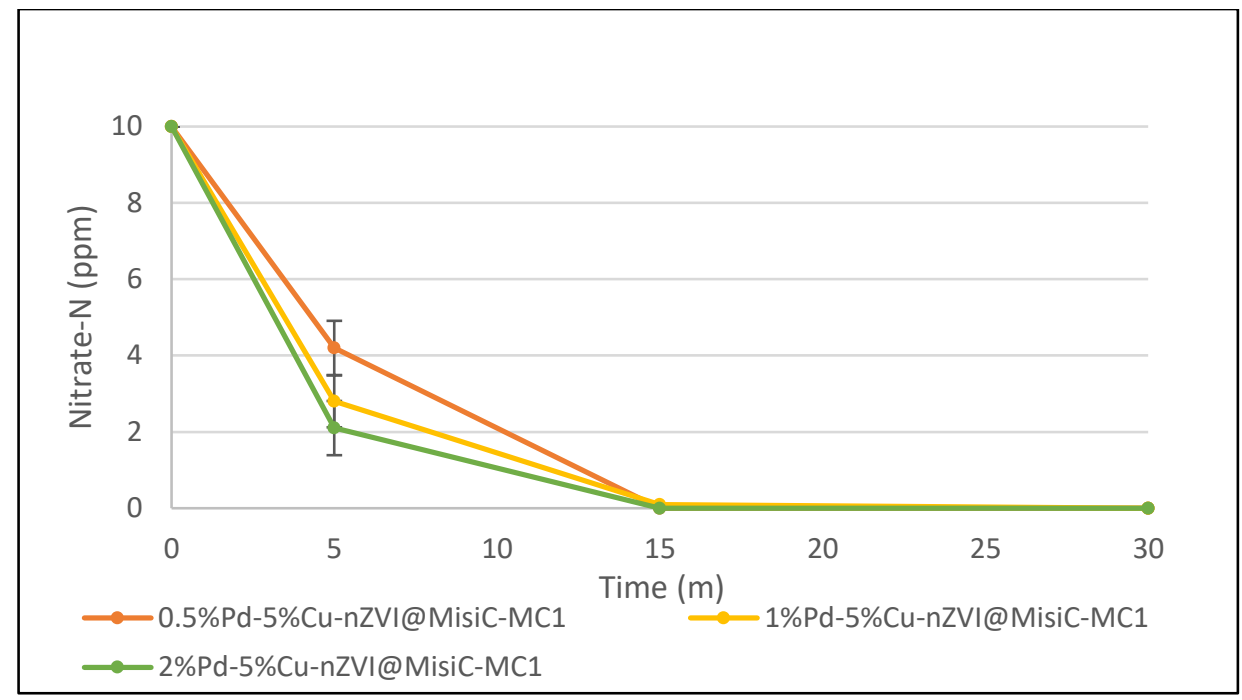

Figure 3.40. Reduction characterisation of Pd-Cu-nZVI@MisiC-MC1 on different Pd loadings

( $0.150 \mathrm{~g}$ of material, $25.0 \mathrm{~mL}$ of $10 \mathrm{ppm}$ nitrate-N solution, under anaerobic conditions).

\subsubsection{Variations on synthetic methods}

There have been two reported methods for synthesising trimetallic Pd-CU-nZVI. ${ }^{4-5}$ They were three step process with and without addition of $\mathrm{NaBH}_{4}$ after depositing $\mathrm{Cu}$ and $\mathrm{Pd}$. In this study, the effect of synthetic methods on both the distribution of nanoparticles on Misi and reactivity of Pd-Cu-nZVI@Misi was examined. For this, trimetallic Pd-Cu-nZVI@Misi was synthesised using four different methods, including $M A_{t r i}, M C 1, M C 2$, and $M D$ (Section 2.1.3). Two different preparations of Misi, including calcined Misi (MisiC) and calcined/FeOOHcoated Misi (MisiCFeOOH), were used. The mass ratios of Fe to Misi, Cu to nZVI and Pd to nZVI were 1:10, 0.05:1 and 0.005:1, respectively.

The morphology of the resultant materials was characterised via SEM. COMPO images revealed that the dispersion of $\mathrm{nZVI}$ on the surface of Misi is dependent upon synthetic methods. Materials prepared via method $\mathrm{MA}_{\text {tri }}$ has a good dispersion of nanoparticles on Misi with the lowest degree of agglomeration (Figure 3.41a). However, more agglomeration is observed in materials that had been prepared via method MC1, MC2, and MD (Figure 3.41b, $3.41 \mathrm{c}$, and 3.41d). The difference in the distribution of particles prepared via MC1, MC2, and MD is minimal and cannot be distinguished via SEM. 

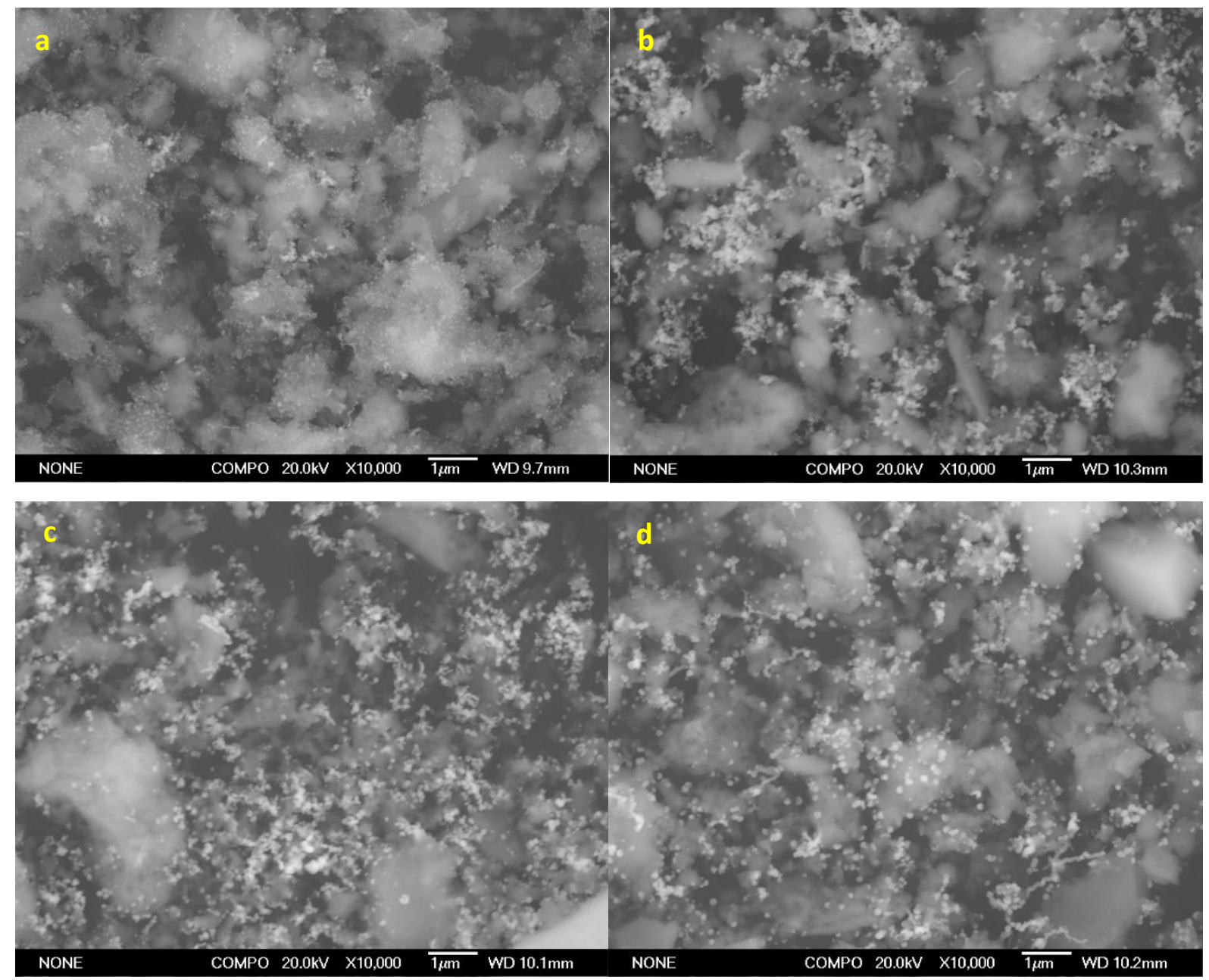

Figure 3.41. COMPO images of 0.5\%Pd-5\%Cu-nZVI@MisiCFeOOH synthesised via different methods (a) $M A_{t r i}$ (b) MC1, (c) MC2 and (d) MD. The lighter component is attributed to Pd$\mathrm{CU}-\mathrm{nZVI}$ and silicate is grey.

The composition and distribution of elements in trimetallic Pd-Cu-nZVI on Misi was investigated using TEM. The 0.5\%Pd-5\%Cu-nZVI@MisiCFeOOH-MC1 was initially examined. In TEM-EDS spectrum, a significant peak corresponding to $\mathrm{Fe}$ is observed while peaks corresponding to $\mathrm{Cu}$ and $\mathrm{Pd}$ are not detected. Other areas of this sample were also undertaken EDS spectra and the same results were obtained. For example, in this examined area (Figure 3.42), the mass percentage for $\mathrm{Fe}, \mathrm{Cu}$, and $\mathrm{Pd}$ was $84.7 \%, 0.4 \%$ and $0.04 \%$ and the atom percentage was $59.2 \%, 0.2 \%$ and $0.02 \%$, respectively. There is a difference in the $\mathrm{Cu}$ signal between trimetallic 0.5\%Pd-5\%Cu-nZVI@MisiCFeOOH-MC1 and bimetallic 5\%CunZVI@MisiCFeOOH-MB1. No Cu peak is found in trimetallic material while the strong peak of $\mathrm{Cu}$ is observed in bimetallic material (Figure 3.26) even though the same method and the same amount of Cu was used. 


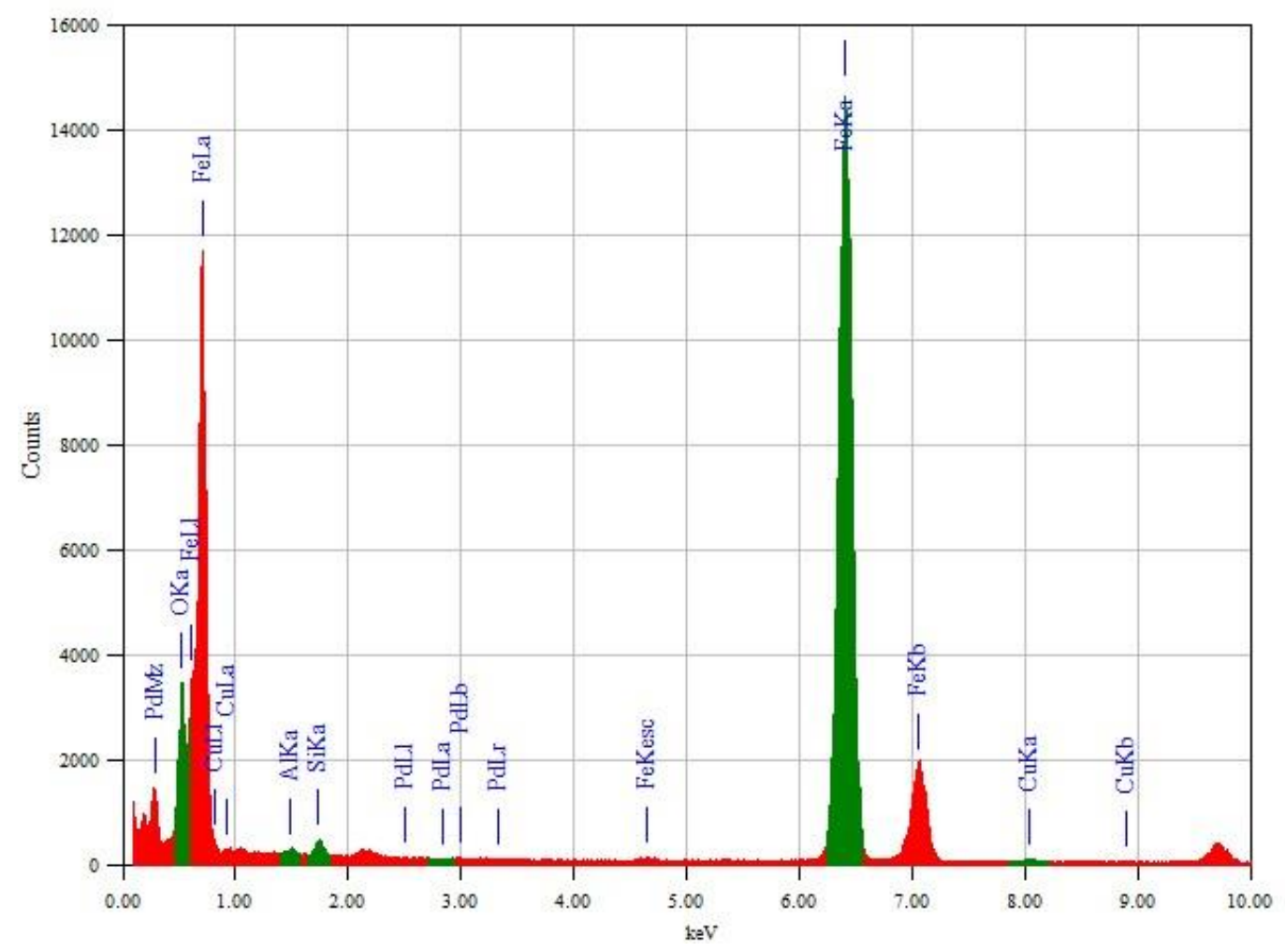

Figure 3.42. TEM-EDS spectrum of 0.5\%Pd-5\%CU-nZVI@MisiCFeOOH-MC1. Note: labels have been modified to highlight peaks relevant to this research. Please see appendix B.6 for complete spectrum.

The TEM-EDS mapping revealed the presence and distribution of elements in this material. Strong signals corresponding to the elements found in Misi (Si, O, Al, and $\mathrm{Na}$ ) are observed (Figure 3.43). The Fe signal is also significant, consistent with the strong peak found in the TEM-EDS spectrum. Even though the signals of $\mathrm{Cu}$ and Pd are observed, they are not reliable, especially Pd due to the lack of Pd signal in the TEM-EDS spectrum. When the signals of Fe and $\mathrm{Cu}$ are overlaid (Figure 3.43a), the density of $\mathrm{Cu}$ on the surface of Fe is slightly greater than in the core but the difference is not as great as that of 5\%Cu-nZVI@MisiCFeOOH-MB1 (Figure 3.27a). This can be explained in that the Pd deposition step may remove the Cu layer from 5\%Cu-nZVI@MisiCFeOOH-MB1 due to the reaction of $\mathrm{Cu}\left(\mathrm{E}^{0}=+0.34\right)$ with $\mathrm{Pd}(\mathrm{II})\left(\mathrm{E}^{0}=\right.$ +0.92 ). There is also an overlap of Fe and $\mathrm{O}$ with the $\mathrm{O}$ layer on the surface of Fe, similar to bimetallic Ni-nZVI@Misi and Cu-nZVI@Misi. There have been some studies investigating on unsupported trimetallic Pd-Cu-nZVI. ${ }^{4-5}$ In these studies, only SEM-EDS spectra was reported and the information about the distribution of these elements was unclear. Due to the lack of 
information about the elemental distribution in trimetallic Pd-Cu-nZVI@MisiCFeOOH-MC1, the TEM examination of other trimetallic materials was not undertaken.
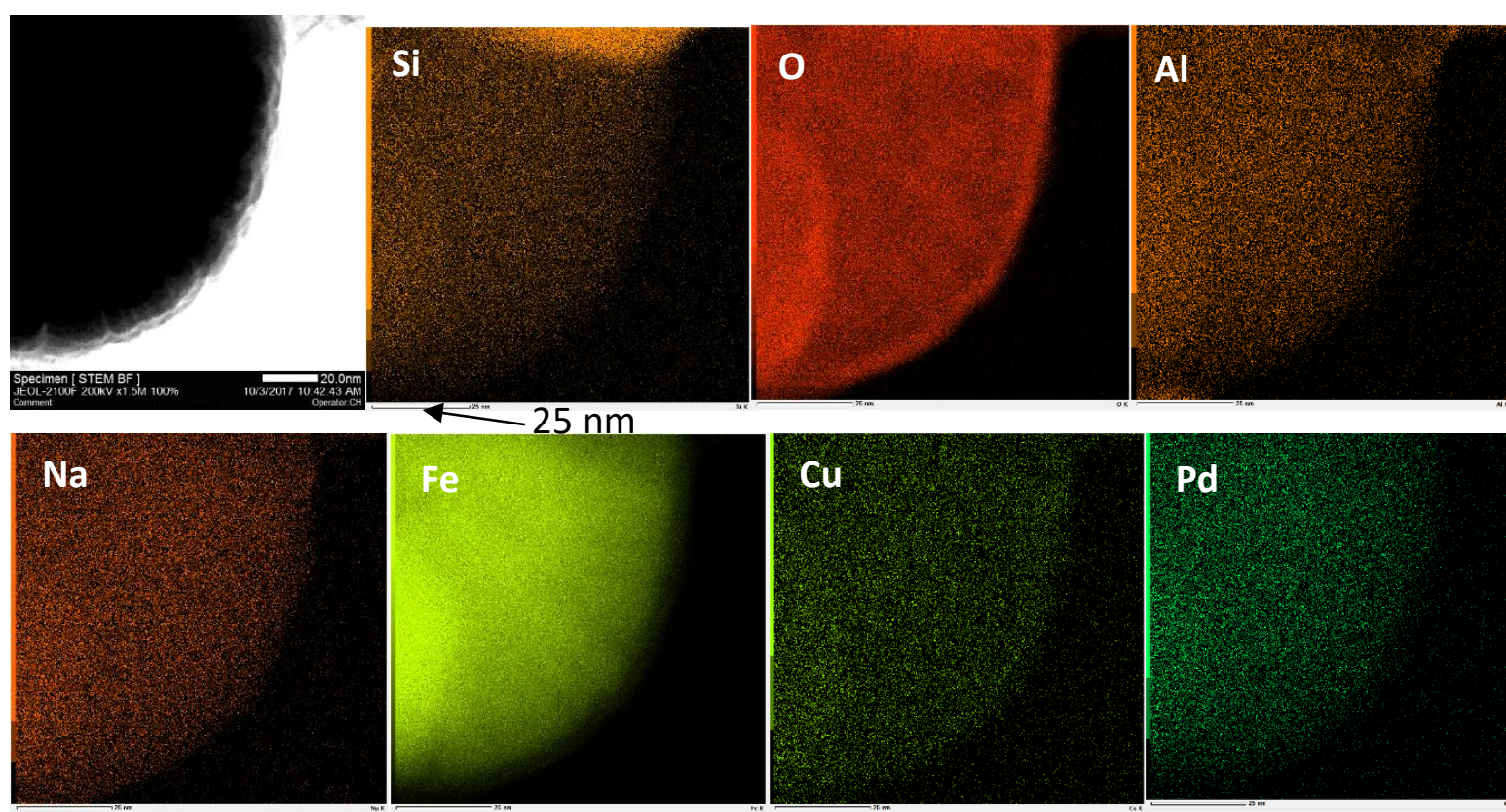

Pd

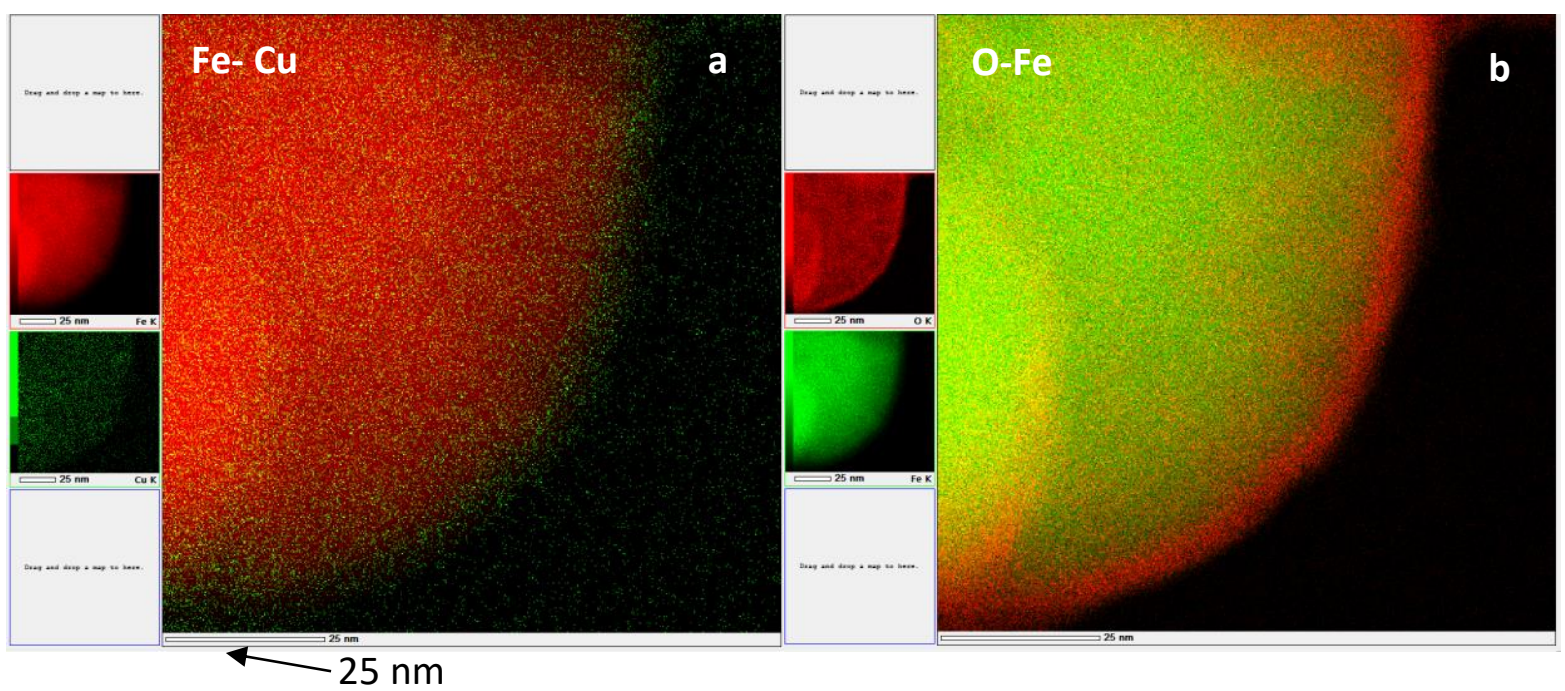

Figure 3.43. TEM-EDS mapping of 0.5\%Pd-5\%Cu-nZVI@MisiCFeOOH-MC1.

The elemental composition of trimetallic Pd-Cu-nZVI@Misi was also quantified using AAS. For this, trimetallic materials prepared via co-reduction method ( $\left.\mathrm{MA}_{\text {tri }}\right)$ and deposition method (MC1) were digested in the concentrated nitric acid and the concentrations of copper and iron in the resultant solutions were measured. The mass of MisiCFeOOH was subtracted from the initial amount of material. The results showed that the $\mathrm{Cu}: \mathrm{Fe}: \mathrm{MisiCFeOOH}$ mass ratio is $0.53: 12.65: 100$ (molar ratio Cu:Fe is 1:27.28) and 0.54:11.52:100 (molar ratio Cu:Fe is 1:24.38) for 0.5\%Pd-5\%Cu-nZVI@MisiCFeOOH-MA tri and 0.5\%Pd-5\%Cu-nZVI@MisiCFeOOH-MC1, respectively (Table 3.8). The mass ratios of $\mathrm{Cu}$ to MisiCFeOOH between two samples are 
similar and similar to the ratio of amount of chemicals used (Cu:MisiCFeOOH is $0.5: 100$ ). In contrast but consistent with other doped materials, the mass ratios of Fe to MisiCFeOOH are higher than the theoretical ratio (Fe:MisiCFeOOH is 10:100). The higher amount of Fe in these materials may be explained by the existence of Fe in Misi that had been coated by a FeOOH layer during Misi pre-treatment. Fe accounts for 0.94\% in MisiCFeOOH.

In addition, the mass ratio of Fe to Misi in material prepared via deposition method (MC1) is lower than that of in material prepared via co-reduction method $\left(\mathrm{MA}_{\text {tri }}\right)$. One of the possible reasons is the loss of Fe during the three-step procedure (MC1). In method MC1, nZVI reacts with $\mathrm{Cu}(\mathrm{II})$ and possibly with $\mathrm{Pd}(\mathrm{II})$ to form deposition layers. Another reason is that the number of washing steps in deposition method (MC1) is greater than that of co-reduction method ( $\left.M A_{\text {tri }}\right)$. This leads to more Fe removed from the suspension during the synthesis. In terms of palladium, it was not detected using AAS due to the low palladium concentration in solutions that resulted from these materials. Its detection should be done by an instrument with lower detection limit such as inductively coupled plasma or inductively coupled plasma mass spectrometry.

Table 3.8. Analysis of Pd-Cu-doped materials ( $0.250 \mathrm{~g}$ material, $5.0 \mathrm{~mL}$ concentrated $\mathrm{HNO}_{3}$ ). Note: Misi $=$ MisiCFeOOH .

\begin{tabular}{|c|c|c|c|c|}
\hline Material & $\mathrm{Cu}(\mathrm{mg})$ & $\mathrm{Fe}(\mathrm{mg})$ & Misi (mg) & $\begin{array}{c}\text { Mass ratio of } \\
\text { Cu:Fe:Misi }\end{array}$ \\
\hline $0.5 \%$ Pd-5\%Cu-nZVI@MisiCFeOOH-MAtri & $1.16 \pm 0.02$ & $28.0 \pm 0.1$ & $220.9 \pm 0.1$ & $0.53: 12.65: 100$ \\
\hline $0.5 \%$ Pd-5\%Cu-nZVI@MisiCFeOOH-MC1 & $1.20 \pm 0.02$ & $25.7 \pm 0.1$ & $223.1 \pm 0.1$ & $0.54: 11.52: 100$ \\
\hline MisiCFeOOH & 0 & 2.32 & 247.68 & $0: 0.94: 100$ \\
\hline
\end{tabular}

The reactivity of the supported $0.5 \% \mathrm{Pd}-5 \% \mathrm{Cu}-\mathrm{nZVI}$ on Misi prepared via different methods towards nitrate reduction was examined. In these studies, the molar ratio of Fe to nitrate is 15:1. All Pd-Cu-doped materials had higher reactivity than non-doped nZVI@Misi (Section 3.2). After 5 minutes, materials prepared via co-reduction method $\left(\mathrm{MA}_{\text {tri }}\right)$ had the lowest reactivity while materials prepared via deposition method (MC1) reduced nitrate fastest, regardless of Misi preparation. For MisiC-based materials, nitrate-N concentrations were 9.5 \pm 0.7 ppm and $4.2 \pm 0.6$ ppm when either 0.5\%Pd-5\%Cu-nZVI@MisiC-MAtri $\left(\mathrm{MA}_{\text {tri }}\right.$ referred as 
MA in figures) or 0.5\%Pd-5\%Cu-nZVI@MisiC-MC1 was used, respectively (Figure 3.44). For MisiCFeOOH-based materials, nitrate-N concentrations were $7.5 \pm 0.7 \mathrm{ppm}$ and $1.1 \pm 0.7 \mathrm{ppm}$ when either 0.5\%Pd-5\%Cu-nZVI@MisiCFeOOH-MAtri or 0.5\%Pd-5\%Cu-nZVI@MisiCFeOOHMC1 was used, respectively (Figure 3.45).

These results are similar to supported bimetallic nZVI@Misi. Materials prepared using sequential method were more reactive than those prepared using one step method. After 30 minutes, nitrate-N concentration was $2.9 \pm 0.6 \mathrm{ppm}$ (71\% nitrate conversion) using $0.5 \% \mathrm{Pd}$ 5\%Cu-nZVI@MisiC-MA tri while nitrate-N levels were below the detection limit using either other material, regardless Misi preparation and synthetic method. Based on these results, materials synthesised via deposition methods including MC1 and MD had higher reactivity than those synthesised via co-reduction method. Of these, materials synthesised via deposition method (MC1) had the highest reactivity. Furthermore, MisiCFeOOH was the better support than MisiC, regardless the synthetic method. For example, nitrate- $\mathrm{N}$ concentrations were $4.2 \pm 0.6 \mathrm{ppm}$ and $1.1 \pm 0.7 \mathrm{ppm}$ when either 0.5\%Pd-5\%Cu-nZVI@MisiCMC1 or 0.5\%Pd-5\%Cu-nZVI@MisiCFeOOH-MC1 was used. Therefore, trimetallic Pd-CunZVI@MisiCFeOOH prepared using deposition method (MC1) was chosen for further investigations. In addition, although trimetallic Pd-Cu-nZVI@MisiCFeOOH prepared using coreduction method $\left(\mathrm{MA}_{\text {tri }}\right)$ is slightly less reactive, it will also be examined and compared due to the ease of synthesis.

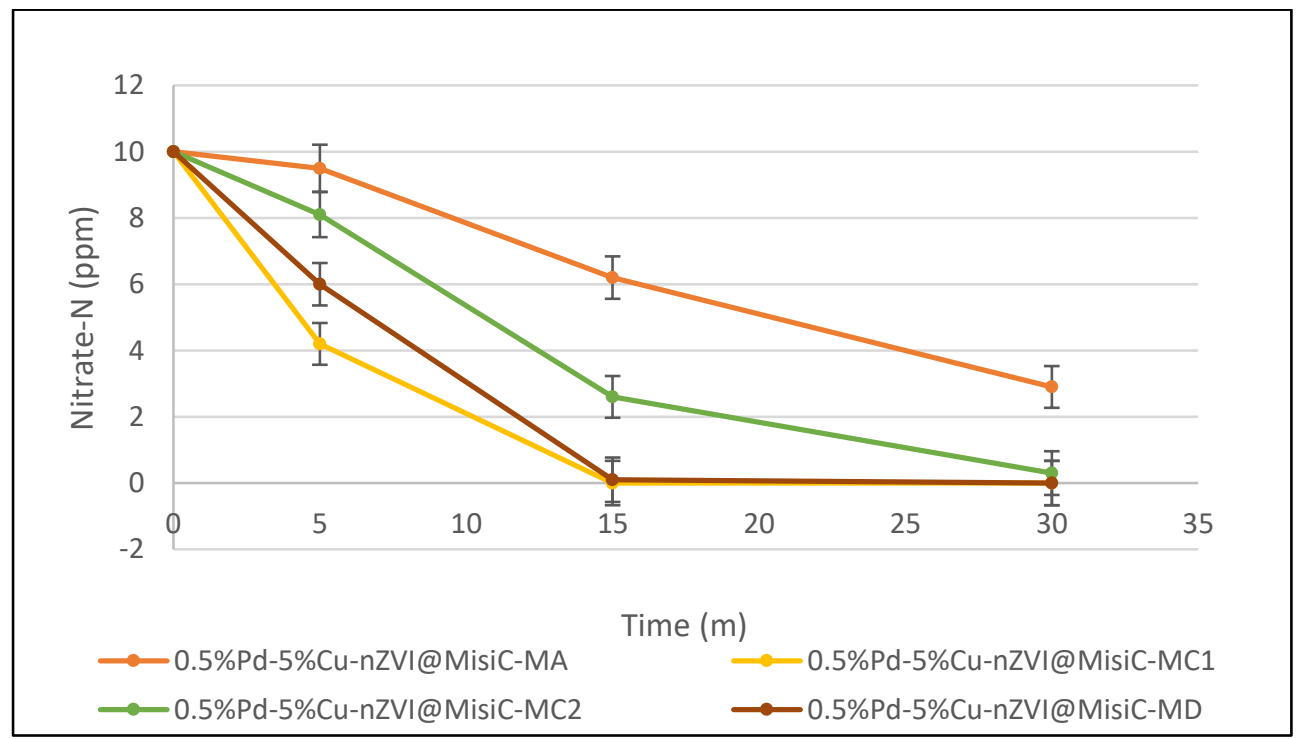

Figure 3.44. Reduction characterisation of Pd-Cu-nZVI@MisiC prepared via different methods (0.150 g of material, $25.0 \mathrm{~mL}$ of $10 \mathrm{ppm}$ nitrate-N solution, under anaerobic conditions). 


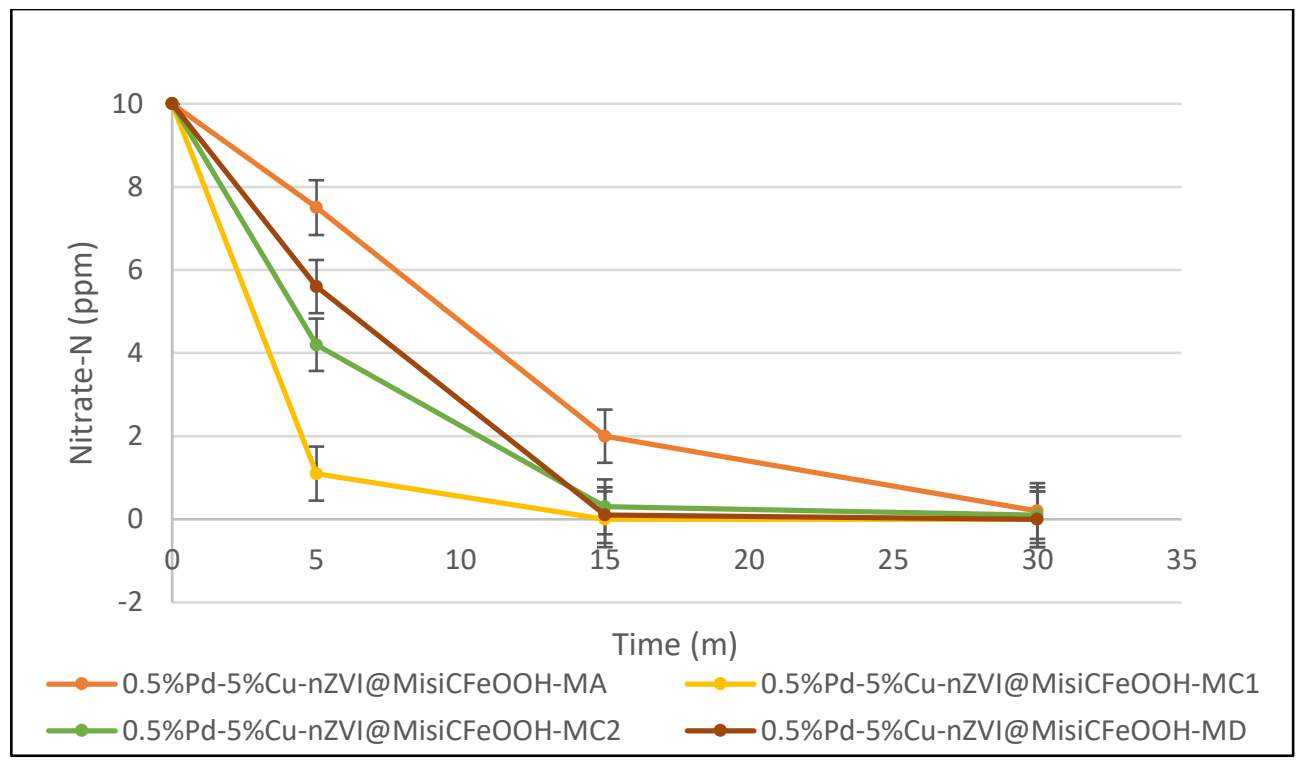

Figure 3.45. Reduction characterisation of Pd-Cu-nZVI@MisiCFeOOH prepared via different methods (0.150 g of material, $25.0 \mathrm{~mL}$ of $10 \mathrm{ppm}$ nitrate- $N$ solution, under anaerobic conditions).

It was reported that the introduction of Pd in unsupported trimetallic Pd-Cu-nZVI increased the nitrogen gas selectivity in nitrate reduction and decreased ammonium generation. ${ }^{4-5}$ To examine final $\mathrm{N}$-containing products, trimetallic $\mathrm{Pd}-\mathrm{Cu}-\mathrm{nZVI}$ on Misi was used for nitrate reduction and ammonium concentration in the resulting solutions was measured using the Nessler method. The $0.5 \% \mathrm{Pd}-5 \% \mathrm{Cu}-\mathrm{nZVI}$ on two different preparations of Misi, MisiC and MisiCFeOOH, were synthesised using two different synthetic methods, including $\mathrm{MA}_{\text {tri }}$ and MC1. For MisiC-based materials (Figure 3.46), after 5 minutes, ammonium-N concentrations were $2.6 \pm 0.2 \mathrm{ppm}$ and $5.2 \pm 0.2 \mathrm{ppm}$ when either 0.5\%Pd-5\%Cu-nZVI@MisiC-MA tri or 0.5\%Pd-5\%Cu-nZVI@MisiC-MC1 was used. After 30 minutes, ammonium-N concentrations were $8.8 \pm 0.3 \mathrm{ppm}$ ( $88 \%$ ammonium production) and $9.8 \pm 0.3 \mathrm{ppm}$ (98\% ammonium production). For MisiCFeOOH-based materials, after 5 minutes, ammonium- $\mathrm{N}$ concentrations were $3.0 \pm 0.2$ ppm and $9.8 \pm 0.3$ ppm when either 0.5\%Pd-5\%Cu-nZVI@MisiCFeOOH-MA tri or 0.5\%Pd-5\%Cu-nZVI@MisiCFeOOH-MC1 was used. After 30 minutes, ammonium-N concentrations were approximately $10 \mathrm{ppm}$ (100\% ammonium production). Interestingly, the ammonium generation using 0.5\%Pd-5\%Cu-nZVI@MisiCFeOOH-MC1 was fastest, maximum ammonium was formed after only 5 minutes. This is consistent with the fastest nitrate removal of this material. These observations suggested that ammonium is the prominent final product in nitrate reduction using trimetallic Pd-Cu-nZVI@Misi, which is inconsistent with 
reported research on unsupported Pd-Cu-nZVI. The presence of $\mathrm{Pd}-\mathrm{Cu}$ increased the selectivity of nitrogen gas and the nitrogen gas generation was up to $50 \% .^{4-5}$ However, in these reported studies, the percentage of the total mass of $\mathrm{Cu}$ and $\mathrm{Pd}$ was $10 \%$ and the mass ratio of $\mathrm{Pd}$ to Cu was 2:1.

In terms of nitrogen mass balance, total nitrate- $\mathrm{N}$ and ammonium- $\mathrm{N}$ was generally equal to the initial nitrogen used. In somes points, the mass balance values were higher. This can be explained by the errors during the measurement of nitrate and ammonium. Nitrate and ammonium were not always determined in the same batches and the same time. However, the aim of this chapter is to screen the reactivity of the materials synthesised as well as the main final products. Therefore, the exact concentrations of nitrate and ammonium should be done as the recovery of nitrogen is investigated. 

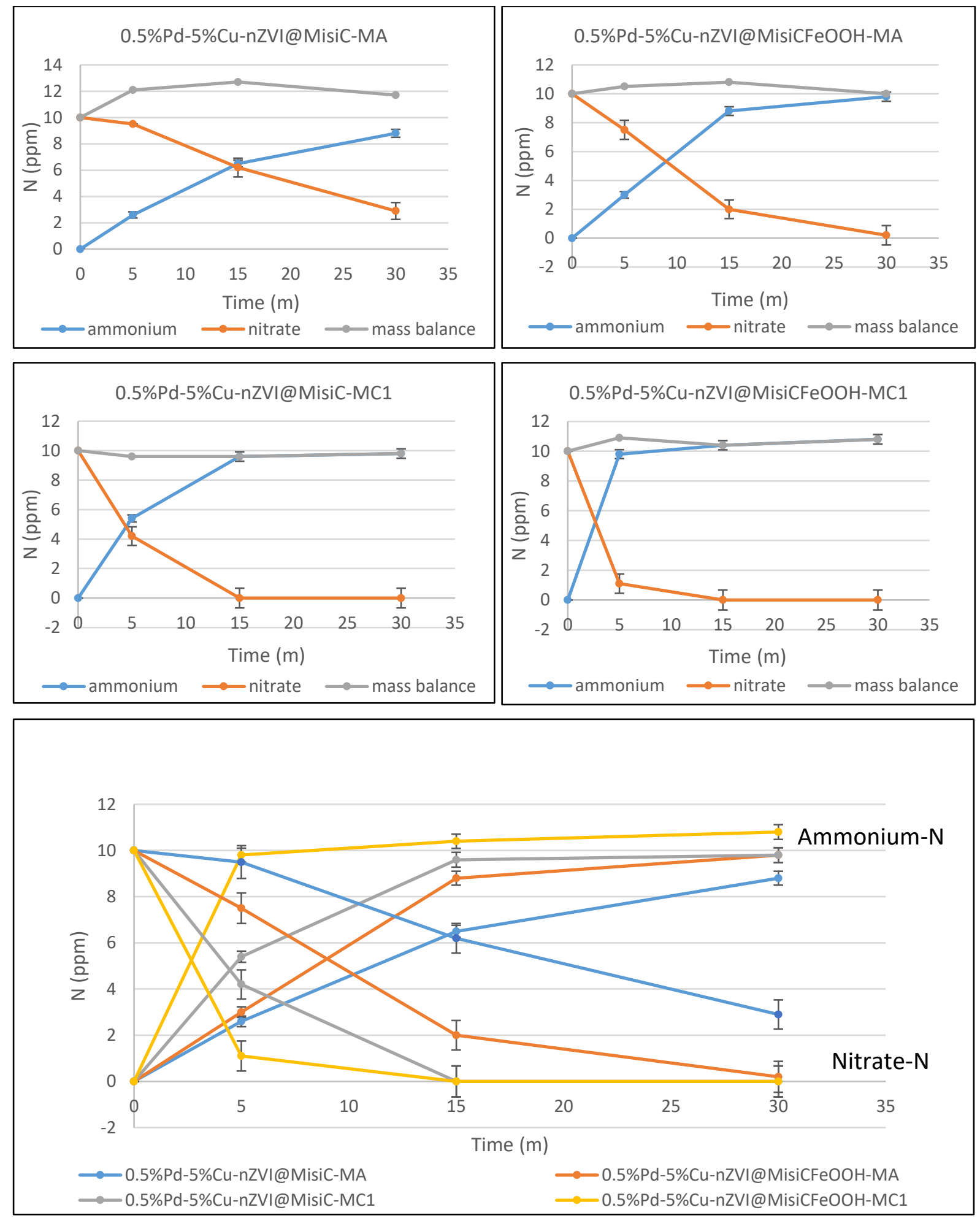

Figure 3.46. Nitrate reduction, ammonium production and mass balance by $0.5 \% \mathrm{Pd}-5 \% \mathrm{Cu}$ nZVI@MisiC $(0.150 \mathrm{~g}$ of materials, $25.0 \mathrm{~mL}$ of 10 ppm nitrate- $N$ solution, under anaerobic conditions). 


\subsubsection{Conclusion}

Trimetallic Pd-Cu-nZVI@Misi had higher reactivity than non-doped nZVI@Misi due to the potentially catalytic function of both copper and palladium. The difference in the reactivity of trimetallic Pd-Cu-nZVI@Misi and bimetallic Cu-nZVI@Misi was not evident. Trimetallic material was less reactive than bimetallic Cu-nZVI@Misi when they were prepared via the coreduction method. However, in this chapter, the nitrate concentration was measured using the UV-Vis; hence, the impact of nitrite on nitrate concentration might occur. The influence of palladium in greater detail will be discussed in Chapter 4 where nitrite analysis is examined using the IC.

\subsection{Conclusion}

In Chapter 3, several materials were examined their reactivity towards nitrate reduction with different doping metals. The addition of another metal or other metals had a positive effect on increasing the reduction. All bimetallic $\mathrm{nZVI}$ on Misi doped with $\mathrm{Ni}, \mathrm{Cu}, \mathrm{Zn}$, and $\mathrm{Pd}$ as well as trimetallic Pd-Cu-nZVI on Misi reduced nitrate faster than non-doped nZVI on Misi. After 30 minutes, nitrate conversion was more than 55\% and 35\% for any doped nZVI@Misi materials prepared using MisiC and MisiCFeOOH, respectively regardless of synthetic method. In contrast, after 30 minutes, nitrate conversion was 45\% and 21\% using either nZVI@MisiC or nZVI@MisiCFeOOH, respectively. This is consistent with previously reported papers about the catalytic role of the secondary metal. ${ }^{2,167}$ In these studies, the secondary metal gained the number of active sites, leading more interactions of nitrate and electrons. Moreover, the addition of the metal with the more negative reduction potential than Fe (for example, $\mathrm{Zn}$ ) may provide another reducing agent, accompanied with nZVI. As such, doping metal(s) on nZVI@Misi is an effective way to enhance the reactivity nZVI@Misi.

The differences in the amount of nitrate reduced were dependent upon dopant metals. Nidoped, Cu-doped, and Pd-Cu-doped materials reduced the most nitrate. After 30 minutes, more than 90\% of nitrate was reduced using either bimetallic nZVI@Misi. Bimetallic ZnnZVI@Misi and Pd-nZVI@Misi increased nitrate reduction compared to non-doped nZVI@Misi but the increase was minimal.Therefore, we chose bimetallic Ni-doped nZVI@Misi and Cu-doped nZVI@Misi as well as trimetallic Pd-Cu-nZVI on Misi for further investigation. 


\section{Nitrate reduction with optimised-doped nZVI@Misi}

\subsection{Introduction}

The aim of this chapter is to examine the reactivity towards nitrate reduction in greater detail using the most promising materials described in Chapter 3. The efficiency of these materials, nitrate conversion and nitrite accumulation, is investigated under anaerobic conditions. The change in the morphology and elemental distribution of these materials after nitrate reduction is also examined. In addition, how the preparation of the nZVI nanoparticles influencing the resultant reactivity was examined. For instance, as there is significant difference in the surface the nanoparticles prepared via co-reduction method (MA) and deposition method (MB), the change in the surface properties that might influence the overall reactivity of the nanoparticles was examined to ascertain. To date, this type of analysis has never been performed.

$\mathrm{Fe}(0)$ typically reacts with nitrate in a two-step process. The first step is the conversion of nitrate to nitrite, a two-electron reduction (eq. 4.1). The second step is the reaction of further Fe with nitrite to form ammonium, a six-electron process (eq. 4.2). As such, from nitrate to ammonium, requires eight electrons with nitrite, an intermediate product (eq. 4.3). To prevent the building of nitrite, the conversion of nitrite into other final products must be faster than the conversion of nitrate to nitrite.

$$
\begin{gathered}
\mathrm{Fe}_{(\mathrm{s})}^{0}+\mathrm{NO}_{3}^{-}+2 \mathrm{H}^{+} \longrightarrow \mathrm{Fe}^{2+}+\mathrm{NO}_{2}^{-}+\mathrm{H}_{2} \mathrm{O} \\
3 \mathrm{Fe}_{(\mathrm{s})}^{0}+\mathrm{NO}_{2}^{-}+8 \mathrm{H}^{+} \\
4 \mathrm{Fe}_{(\mathrm{s})}^{0}+\mathrm{NO}_{3}^{-}+10 \mathrm{H}^{+}
\end{gathered}
$$

Other gaseous $\mathrm{N}$-containing products such as $\mathrm{NO}_{2}, \mathrm{NO}, \mathrm{N}_{2} \mathrm{O}$ and $\mathrm{N}_{2}$ are also possible products in the reduction of nitrate, but only the production of nitrogen gas has been examined. A popular method for nitrogen gas estimation is $\mathrm{N}$-mass balance in which nitrogen gas is calculated by the subtraction of the remaining nitrate, nitrite, and ammonium from initial nitrate concentration. ${ }^{4-5,190}$ The reports of nitrogen gas selectivity are not highly reliable due to the different sensitivity for nitrate, nitrite and ammonium determination; in addition, these calculations assume that nitrogen gas is the only gaseous $\mathrm{N}$-containing product. Directly determining the amount of nitrogen gas produced has been conducted using residual gas 
analysis, which is a method used for testing the variation of gas components as a function of time in vacuum systems. ${ }^{238}$ In order to measure nitrogen gas, the reaction of nitrate with material was carried out in the reactor to form gaseous compounds. ${ }^{190}$ These gases then were employed in high vacuum systems and detected by quadrupole mass spectrometer in residual gas analyser. The results of this study showed that the amount of nitrogen gas that is directly measured is comparable to that being estimated by $\mathrm{N}$-mass balance; as such, estimating the $\mathrm{N}$-mass balance is semi-reliable.

The reaction between $\mathrm{Fe}(0)$ and nitrate occurs under a range of $\mathrm{pH}$, from slightly alkaline to acidic media, depending on the size of particles. Several studies have shown that $\mathrm{Fe}(0)$ powder only removes nitrate in acidic conditions where the reactivity of iron is not inhibited in the presence of an Fe oxide layer. For example, nitrate is readily removed at low $\mathrm{pH}(\mathrm{pH}<$ 3) and only negligible nitrate is reduced at a $\mathrm{pH}$ higher than $4 .{ }^{239}$ When the initial concentration was $32.9 \mathrm{ppm}$ nitrate- $\mathrm{N}(2.35 \mathrm{mM} / \mathrm{L})$, all nitrate was reduced at $\mathrm{pH}<3$ after 15 minutes and at $\mathrm{pH}$ of 3 after 25 minutes. Less than $15 \%$ of nitrate was reduced at a $\mathrm{pH}$ of 4.5 after 45 minutes. In a separate study, when the initial nitrate- $N$ concentration was $11.3 \mathrm{ppm}$ $(0.81 \mathrm{mM} / \mathrm{L}), 95 \%$ of nitrate was reduced at $\mathrm{pH}$ of 2 after 30 minutes and a small amount of nitrate was removed at $\mathrm{pH}>4 .{ }^{10}$ In contrast, Zhang et al. showed that $\mathrm{Fe}(0)$ powder can react with nitrate at neutral $\mathrm{pH}$ although the amount nitrate removed is minimal. ${ }^{240}$ For instance, when the initial nitrate- $\mathrm{N}$ concentration was $30 \mathrm{ppm}(2.14 \mathrm{mM} / \mathrm{L}), 98 \%$ of nitrate was reduced at a pH of 2.3 after 16 hours and less than $10 \%$ of nitrate was removed at a $\mathrm{pH}$ of 7.1 after 48 hours.

Nanosize $\mathrm{Fe}(0)(\mathrm{nZVI})$ is more reactive than $\mathrm{Fe}(0)$ powder, thus it can react with nitrate at higher $\mathrm{pH}$. For example, when the initial nitrate-N concentration was $100 \mathrm{ppm}$ and the molar ratio of Fe to nitrate was 3:1 (nZVI dosage was $1.25 \mathrm{~g} / \mathrm{L}$ ), all nitrate was removed at $\mathrm{pH}$ of 7 after 90 minutes. ${ }^{41}$ The effect of the initial $\mathrm{pH}$ of the solution on nitrate reduction has also been investigated in several studies. Zang et al. showed that the nitrate reduction decreased with increasing $\mathrm{pH}^{241}$ In their study, the initial nitrate-N concentration was $18 \mathrm{ppm}$ (80 ppm nitrate) and the molar ratio of Fe to nitrate was 23:1 (nZVI dosage was $1.67 \mathrm{~g} / \mathrm{L}$ ) using nZVI@graphite. All nitrate was reduced at a pH of 3 after 15 minutes, at a pH of 4 after 30 minutes and at either $\mathrm{pH}$ of 5 or $\mathrm{pH}$ of 6.7 after 50 minutes. A similar result has been reported in Kim et al.'s study on nZVI. ${ }^{242}$ Nitrate reduction slightly decreased when $\mathrm{pH}$ was from 3 to 7 
but the decrease was significant at pH 9 - 11. In their study, the initial nitrate-N concentration was $50 \mathrm{ppm}$, the molar ratio of Fe to nitrate was 2.5:1 (nZVI dosage of $0.5 \mathrm{~g} / \mathrm{L}$ ). Using this system, the rate constant decreased from $7.5 \times 10^{-2} /$ minute $(\mathrm{pH}$ of 3$)$ to $4.7 \times 10^{-2} /$ minute $(\mathrm{pH}$ of 7) while the rate constant was $6.7 \times 10^{-3} /$ minute at $\mathrm{pH}$ of 11 .

In this chapter, the reactivity of bimetallic Ni-nZVI@Misi and Cu-nZVI@Misi as well as trimetallic Pd-Cu-nZVI@Misi systems towards nitrate reduction was examined under anaerobic conditions. Bimetallic materials were synthesised via co-reduction method (MA) (Section 2.1.2.1) and deposition method (MB1) (Section 2.1.2.2). Trimetallic materials were prepared via co-reduction method ( $\mathrm{MA}_{\text {tri }}$ ) (Section 2.1.3.1) and deposition method (MC1) (Section 2.1.3.2). The mass ratio of elements added in bimetallic materials M:Fe:Misi is 0.5:10:100 and the mass ratio of element added in trimetallic materials Pd:Cu:Fe:Misi is 0.05:0.5:10:100. Two different preparations of Misi were utilised, including calcined Misi (MisiC) and calcined/FeOOH-coated Misi (MisiCFeOOH).

Three different molar ratios of Fe to nitrate were used in these studies, including 15:1 (10 ppm nitrate-N), 3:1 (50 ppm nitrate-N) and 1.5:1 (100 ppm nitrate-N). We initially focused on $10 \mathrm{ppm}$ nitrate- $\mathrm{N}$ as it is the maximum contaminant level in drinking water. As the nitrate concentrations were less than $1 \mathrm{ppm}$ after reduction when $10 \mathrm{ppm}$ of nitrate- $\mathrm{N}$ was reduced using many of our materials, the nitrate-N levels were increased to $50 \mathrm{ppm}$ and $100 \mathrm{ppm}$ in order to help differentiate any differences in reactivity for our doped nZVI@Misi materials. The $\mathrm{pH}$ of the solutions was not specifically altered as we wanted to ensure that our materials could work under "real-world" conditions. Initial attempts at using buffers (for example, HEPES) were carried out. However, the addition of HEPES buffer resulted in yellow solutions. Although the origin of the colour was not determined, concerns were raised that the buffers could damage the column of the IC. As such, the original plan of not specifically controlling the $\mathrm{pH}$ of the solutions was followed.

The concentrations of nitrate and nitrite were measured using IC. The concentrations of soluble metal ions, such as ion and dopant metals, were determined using AAS. The morphology of materials after nitrate reduction was also characterised via SEM and TEM. 


\subsection{Nitrate reduction with bimetallic Ni-nZVI@Misi under anaerobic conditions}

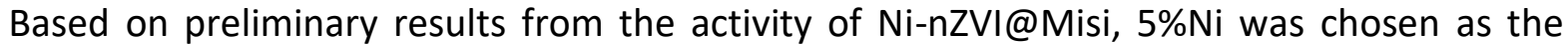
optimal concentration. Both calcined Misi (MisiC) and calcined/FeOOH-coated Misi (MisiCFeOOH) were well-behaved in adsorbing nanoparticles and the resulting materials reduced nitrate quickly. However, nitrate was measured using UV-Vis and nitrite was not monitored (Section 3.3), which did not give accurate results about reducing capacity of $5 \% \mathrm{Ni}$ nZVI@Misi. In this section, the reactivity of supported Ni-nZVI on Misi towards nitrate reduction, as determined by nitrate conversion and nitrite accumulation, was examined. Four materials, including 5\%Ni-nZVI@MisiC-MA, 5\%Ni-nZVI@MisiCFeOOH-MA, 5\% NinZVI@MisiC-MB1 and 5\%Ni-nZVI@MisiCFeOOH-MB1, were used. Two nitrate-N concentrations, 10 ppm and 50 ppm, were examined under anaerobic conditions.

The removal of 10 ppm of nitrate-N with Ni-nZVI@Misi was rapid, regardless of either Misi preparation or doping method. Within 5 minutes, the nitrate-N levels were below 3 ppm, and after 15 minutes, the nitrate- $\mathrm{N}$ levels were below the detection limit of the IC $(0.06 \mathrm{ppm})$ (Figure 4.1). The material, 5\%Ni-nZVI@MisiC-MB1, performed particularly well, removing more than $90 \%$ of nitrate after 5 minutes. As a control, the reactivity of the $\mathrm{Ni}$-doped systems was compared to that of non-doped nZVI@Misi. After 5 minutes, the nitrate-N levels were around $7 \mathrm{ppm}$ and after 15 minutes, the nitrate- $\mathrm{N}$ levels were more than $4 \mathrm{ppm}$ for both types of non-doped materials. After 30 minutes, the nitrate- $N$ levels were 6 ppm (40\% nitrate conversion) and 2.6 ppm (74\% nitrate conversion) when either nZVI@MisiC or nZVI@MisiCFeOOH was used, respectively. These results suggested that nickel plays a catalytic role in nitrate reduction. However, the difference in the reactivity among $\mathrm{Ni}$ nZVI@Misi systems is minimal and cannot be distinguished due to the rapid nitrate reduction, especially when the uncertainties of these values are considered. 


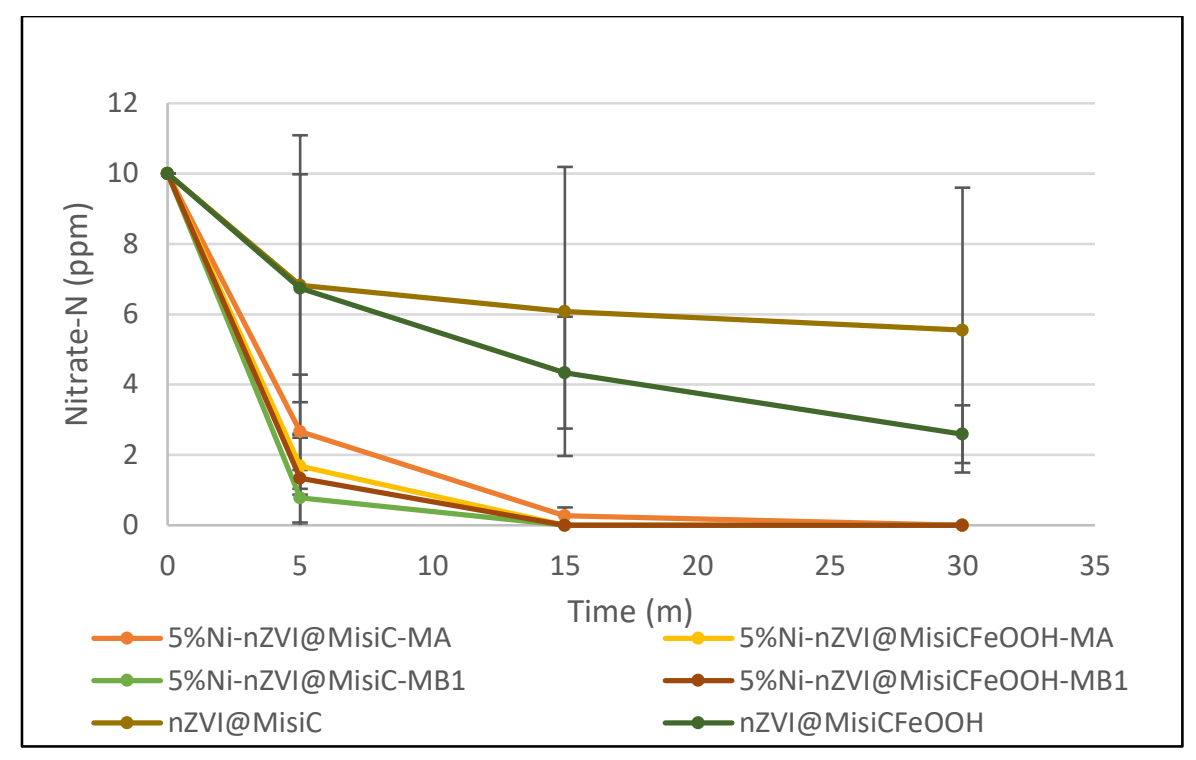

Figure 4.1. Reduction characterisation of 5\%Ni-nZVI@Misi towards nitrate- N 10 ppm under anaerobic conditions ( $0.150 \mathrm{~g}$ of materials and $25.0 \mathrm{~mL}$ of solution).

The problem of investigating the reduction using $10 \mathrm{ppm}$ of nitrate- $\mathrm{N}$ solution is the inconsistency in the nitrate concentrations when nitrate removal using samples of either the same batch or different batches of materials was examined in triplicate. Thus, high uncertainties in the results were observed (Figure 4.2). For example, after 5 minutes, nitrate$\mathrm{N}$ concentrations were $0.93 \mathrm{ppm}, 2.88 \mathrm{ppm}$, and $4.15 \mathrm{ppm}$ when samples of three different batches of 5\%Ni-nZVI@MisiC-MA were utilised. Combining these results would give an average nitrate- $\mathrm{N}$ concentration of $3 \pm 2 \mathrm{ppm}$ ( $3 \mathrm{ppm} \pm 66 \%$ ). Similarly, average nitrate- $\mathrm{N}$ concentrations were $1.7 \pm 0.8 \mathrm{ppm}(1.7 \mathrm{ppm} \pm 47 \%), 0.8 \pm 0.8 \mathrm{ppm}(0.8 \mathrm{ppm} \pm 100 \%)$ and $1 \pm$ 1 ppm (1 ppm \pm 100\%) using either 5\%Ni-nZVI@MisiCFeOOH-MA, 5\%Ni-nZVI@MisiC-MB1 or 5\%Ni-nZVI@MisiCFeOOH-MB1, respectively. These high uncertainties do not allow for correlation between materials used and nitrate-removal capacity. 


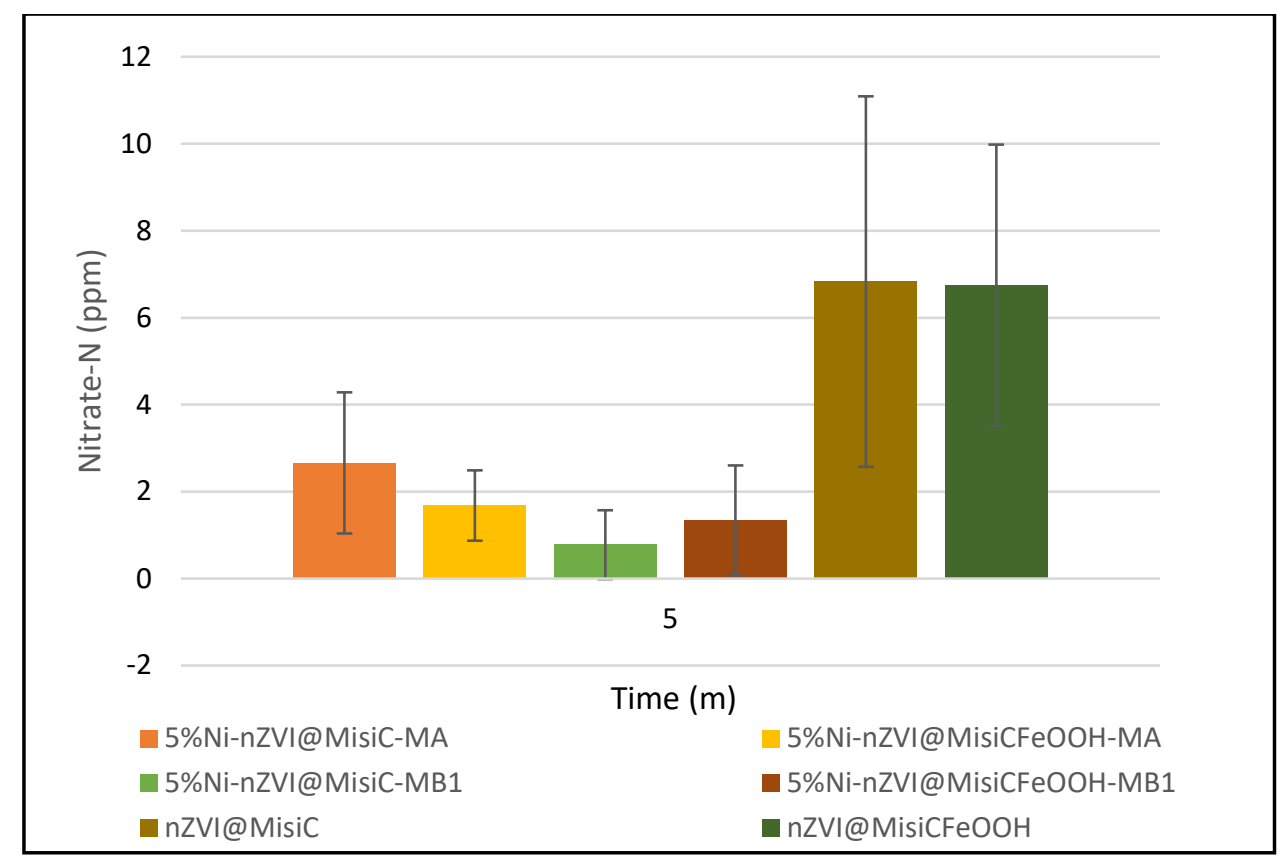

Figure 4.2. Reduction characterisation of 5\%Ni-nZVI@Misi towards nitrate-N 10 ppm under anaerobic conditions with high uncertainty after 5 minutes $(0.150 \mathrm{~g}$ of materials and $25.0 \mathrm{~mL}$ of solution).

The reaction intermediate nitrite was detected in these solutions, but the levels were very low when using Ni-nZVI@Misi materials. For instance, after 5 minutes, the nitrite- $\mathrm{N}$ concentrations were below $0.5 \mathrm{ppm}$ and after 15 minutes, the nitrite- $\mathrm{N}$ levels were below the detection limit of the IC (0.04 ppm) (Figure 4.3). Nitrite was also detected using non-doped nZVI@Misi as a control material. After 5 minutes, nitrite- $N$ levels were about 0.3 ppm, a minimal difference compared to Ni-doped systems. From 15 minutes, the nitrite-N level was still 0.2 ppm when eithernZVI@MisiC or nZVI@MisiCFeOOH was used. Based on these results, the proposed mechanism of nitrate reduction is that $n Z V I$ reacts with nitrate to form nitrite and then nitrite is reduced to form ammonium as the main final product, regardless of $\mathrm{Ni}$ doped or non-doped nZVI@Misi. However, Ni-doped nZVI@Misi converted nitrate to nitrite as well as nitrite to final products faster than non-doped nZVI@Misi. Interestingly, after 5 minutes, the concentrations of nitrate and nitrite using 5\%Ni-nZVI@MisiC-MA were higher than those using any other types of Ni-doped nZVI@Misi, highlighting this material was less reactive than the other $\mathrm{Ni}$-doped materials. 


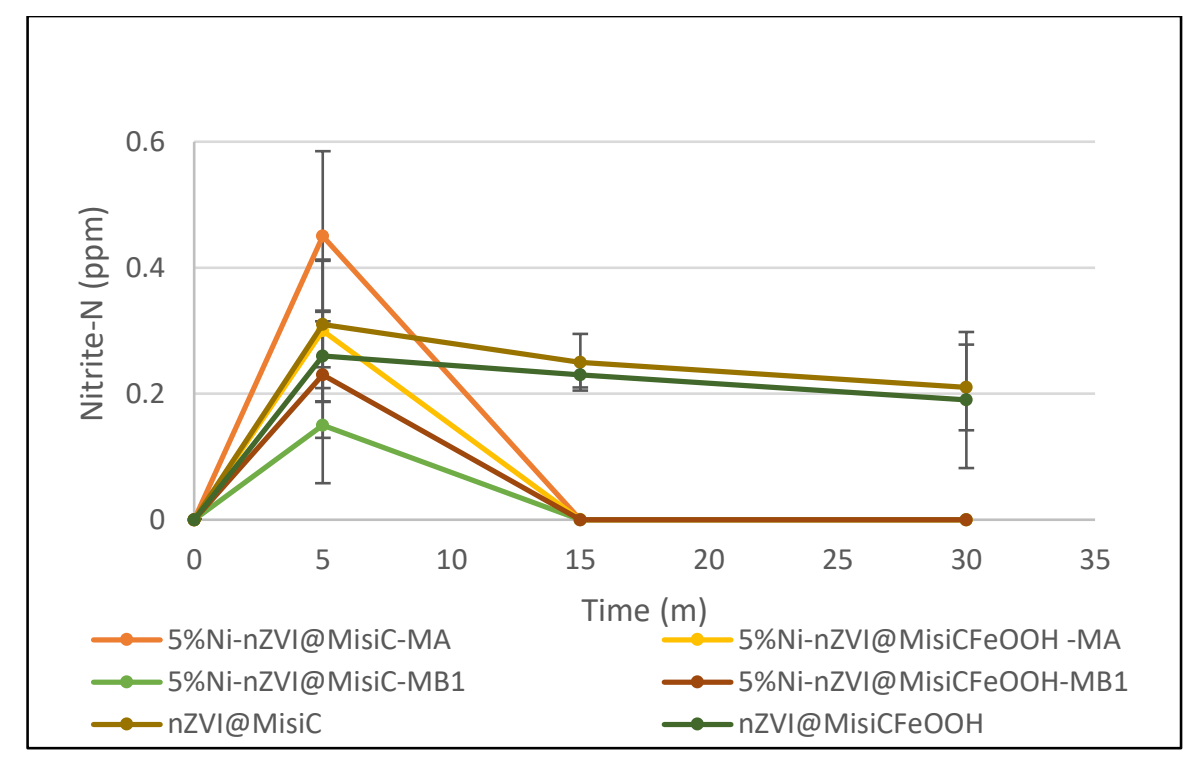

Figure 4.3. Nitrite accumulation in 5\%Ni-nZVI@Misi towards nitrate-N 10 ppm reduction under anaerobic conditions ( $0.150 \mathrm{~g}$ of materials and $25.0 \mathrm{~mL}$ of solution).

The nitrite concentrations were also inconsistent when samples of different batches were used for the reduction of $10 \mathrm{ppm}$ nitrate- $\mathrm{N}$ after 5 minutes (Figure 4.4). However, this inconsistency was less than in nitrate concentrations. For example, nitrite-N levels were 0.6 ppm, 0.34 ppm, and 0.42 ppm when samples of 5\%Ni-nZVI@MisiC-MA were used. Combining these values, the average nitrite- $N$ concentration was $0.5 \pm 0.1 \mathrm{ppm}(0.5 \mathrm{ppm} \pm 20 \%$ ). Similarly, the average nitrite- $\mathrm{N}$ concentrations were $0.3 \pm 0.1(0.3 \mathrm{ppm} \pm 33 \%)$ and $0.2 \pm 0.1$ (0.2 ppm $\pm 50 \%$ ) for 5\%Ni-nZVI@MisiCFeOOH-MA and 5\%Ni-nZVI@MisiC-MB1, respectively. 


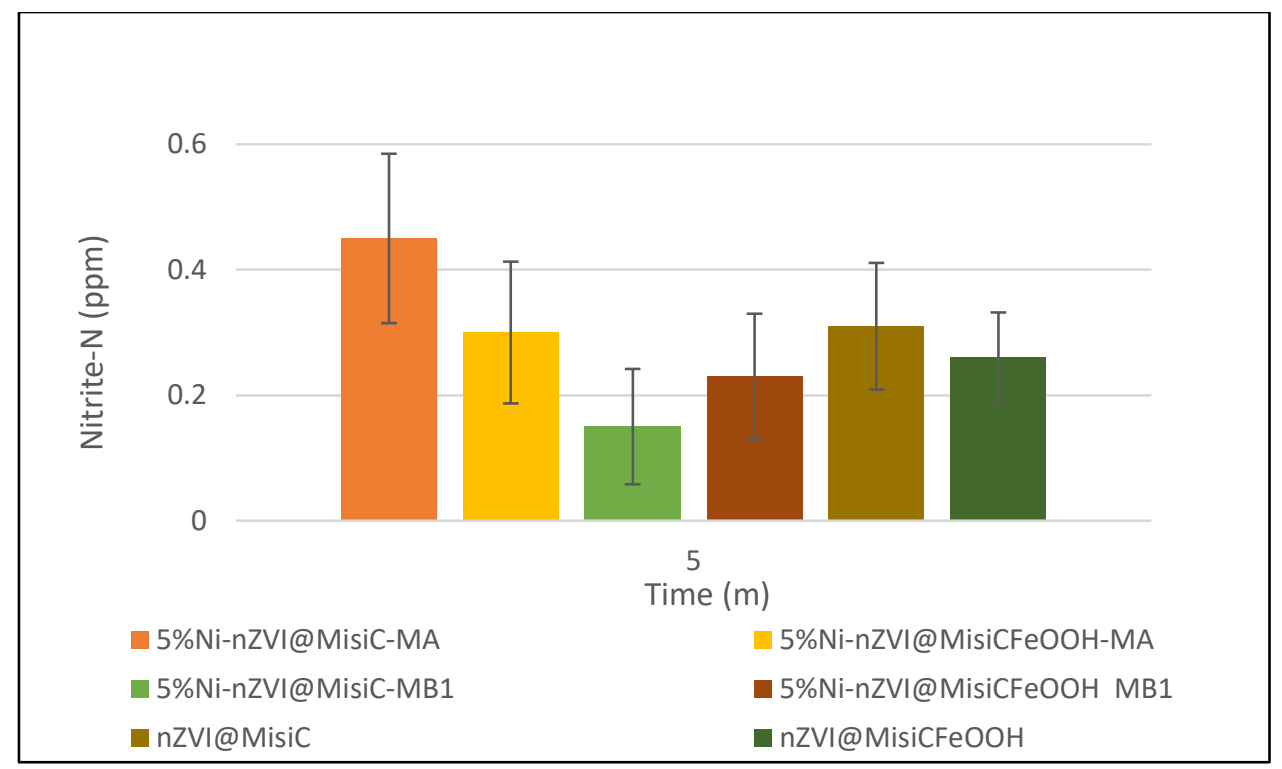

Figure 4.4. Nitrite accumulation in 5\%Ni-nZVI@Misi towards nitrate-N 10 ppm reduction under anaerobic conditions with high uncertainty after 5 minutes $(0.150 \mathrm{~g}$ of materials and

\section{$25.0 \mathrm{~mL}$ of solution).}

The high levels of uncertainty did not allow for correlation between materials used and their ability to reduce nitrate. There are some possible reasons for these results. The first and most important reason is from the preparation of the aliquots for the IC. Samples were manually withdrawn using syringes and then filtered, the number of particles in suspension that were taken may be slightly different at every set time interval. As such, during the filtration process, nitrate is still reacting, but now with an unknown quantity of nanoparticles. Secondly, the heterogeneous composition of Misi may cause the different adsorption capacity of nanoparticles on the surface of Misi, leading to the difference in the number of active sites in each batch. These reasons lead to the high uncertainty in the average nitrate concentrations; as such, we decided to examine higher nitrate concentrations to determine if the reactivity of the materials could be differential.

Increasing the concentration of nitrate- $\mathrm{N}$ to $50 \mathrm{ppm}$ allowed us to examine the differences in the reactivity of the materials with greater accuracy. The $50 \mathrm{ppm}$ of nitrate solution exceeded the reducing capacity of our materials (molar ratio of Fe to nitrate at 3:1 instead of the idealised 4:1 (eq. 4.3)); as such, we did not expect to see all the nitrate removed from the solutions. However, it did allow us to obtain more reliable data, especially with regards to the first time point. For all Ni-doped nZVI@Misi examined, more than 40\% of nitrate was reduced after 5 minutes. (Figure 4.5). After 5 minutes, the nitrate levels decreased gradually, 
with only nominal nitrate decrease between 15 and 30 minutes. For example, the nitrate-N concentrations were $32 \pm 4$ ppm, $24 \pm 3$ ppm, and $22 \pm 3$ ppm after 5 minutes, 15 minutes and 30 minutes, respectively when 5\%Ni-nZVI@MisiC-MA was utilised.

After 30 minutes, materials prepared using MisiC had lower nitrate conversion than those using MisiCFeOOH. Nitrate-N levels were $22 \pm 3$ ppm (56\% nitrate conversion) and $18 \pm 4$ ppm (64\% nitrate conversion) when either 5\%Ni-nZVI@MisiC-MA or 5\%Ni-nZVI@MisiCFeOOH-MA was used, respectively. Nitrate-N levels were $20 \pm 1$ ppm (60\% nitrate conversion) and $14 \pm 1$ ppm (72\% nitrate conversion) when either 5\%Ni-nZVI@MisiC-MB1 or 5\%NinZVI@MisiCFeOOH-MB1 was used, respectively.

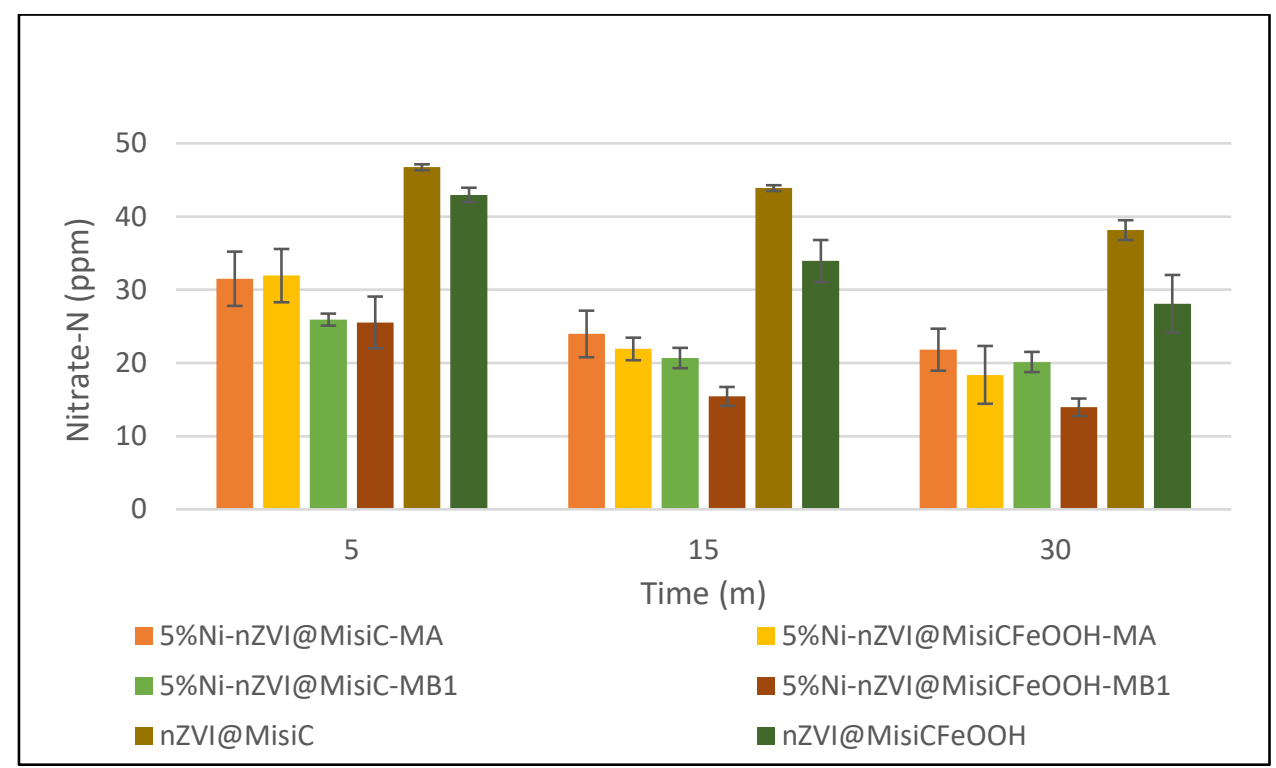

Figure 4.5. Reduction characterisation of 5\%Ni-nZVI@Misi towards nitrate-N 50 ppm under anaerobic conditions ( $0.15 \mathrm{~g}$ of materials and $25 \mathrm{~mL}$ of solution).

The reactivity of materials prepared via co-reduction method (MA) was relatively similar to that of materials prepared via deposition method (MB1) over the course of 30 minutes. For example, after 30 minutes, nitrate- $\mathrm{N}$ levels were $18 \pm 4 \mathrm{ppm}$ and $20 \pm 1 \mathrm{ppm}$ when either 5\%Ni-nZVI@MisiCFeOOH-MA or 5\%Ni-nZVI@MisiC-MB1 was used, respectively. However, of these $\mathrm{Ni}$-doped materials, 5\%Ni-nZVI@MisiCFeOOH-MB1 is the best performing material in terms of nitrate conversion from 15 minutes. This can be explained by the distribution of $\mathrm{Ni}$ and Fe in the nanoparticles. In 5\%Ni-nZVI@MisiCFeOOH-MB1, Ni is located on the surface of Fe (Figure 3.16), resulting in a higher number of catalytic sites for both nitrate adsorption and conversion into nitrite. 
The relative uncertainty of nitrate concentration was in a range of $5 \%-22 \%$ for the reactions with an initial nitrate- $\mathrm{N}$ concentration of $50 \mathrm{ppm}$. These uncertainties were much smaller than those being observed in the reaction of $10 \mathrm{ppm}$ nitrate- $\mathrm{N}$. Thus, a better correlation between materials and their reactivity towards nitrate reduction was observed when higher nitrate concentrations were used.

The results of nitrate reduction using Ni-doped nZVI@Misi were compared with those observed when the reactivity of non-doped $\mathrm{nZVI}$ on Misi was examined as the control. Nidoped nZVI@Misi reduced nitrate faster than non-doped nZVI@Misi. After 5 minutes, nitrate$\mathrm{N}$ levels were $46.7 \pm 0.4$ ppm and $43 \pm 1$ ppm when either nZVI@MisC or nZVI@MisiCFeOOH was used, respectively. After 30 minutes, nitrate- $\mathrm{N}$ concentrations were $38 \pm 1 \mathrm{ppm}$ nitrate ( $24 \%$ nitrate conversion) and $28 \pm 4$ ppm (44\% nitrate conversion), respectively.

The enhancement in the reactivity of Ni-doped systems is consistent with a report by Shi et at. on the investigation of supported Ni-nZVI on kaolinite ${ }^{192}$ and a report by Li et al. on the enhancement of $\mathrm{Ni}$ in supported $\mathrm{Ni}-\mathrm{nZVI}$ on biochar. ${ }^{243}$ Supported doped Ni-nZVI reduced nitrate faster than supported $\mathrm{nZVI}$, indicating the significant catalytic role of $\mathrm{Ni}$ in improving the reduction rate of nZVI. The total nitrate conversions using Ni-nZVI@kaolinite (86.3\%), and Ni-nZVI@biochar (over 93\%) were higher than observed with our materials. This difference in nitrate conversion is due to the different reaction conditions. In these studies, the reactions were in optimised conditions, including initial nitrate concentration, nZVI dosage, medium, and temperature. In the study on Ni-nZVI@kaolinite, the initial nitrate-N concentration was 41 ppm (180 ppm nitrate), molar ratio of Fe to nitrate was 3:1 (nZVI dosage of $0.5 \mathrm{~g} / \mathrm{L}$ ), initial $\mathrm{pH}$ of solution was 4 , and reaction was were run at $35^{\circ} \mathrm{C}$. Possibly, the lower initial nitrate concentration was the main reason for the high nitrate removal. In the study on $\mathrm{Ni}$ nZVI@biochar, the initial nitrate-N concentration was less than 50 ppm, and the molar ratio of Fe to nitrate was more than 8.6:1 (Ni-nZVI@biochar dosage was 4 g/L with 42.8\% nZVI). Additionally, Ni-nZVI@biochar was found to remove nitrate via two different pathways, including the oxidation of nanoparticles with nitrate and the adsorption of approximately about $7 \%$ of nitrate on the surface of biochar.

However, Ni-doped nZVI@Misi materials had higher reactivity compared to Ni-nZVI@zeolite with mass ratio at 1:10:20 reported by He et al.. ${ }^{193}$ The mass ratio of Ni:Fe:silicates in these materials was 1:10:20 and the initial nitrate- $\mathrm{N}$ was $50 \mathrm{ppm}$. He et al. reported that when the 
molar ratio of Fe of nitrate was 2.5:1 (Ni-nZVI dosage of $0.5 \mathrm{~g} / \mathrm{L}$ ) and $\mathrm{pH}$ was 5 , the nitrate-N level was 35 ppm (30\% nitrate conversion) after 30 minutes. In contrast, in our study, when the molar ratio of Fe of nitrate of $3: 1$ (nZVI dosage of $0.6 \mathrm{~g} / \mathrm{L}$ ), more than $56 \%$ nitrate conversion was observed using any types of Ni-nZVI@Misi. Additionally, the reaction of Nidoped nZVI@Misi with nitrate were occurred under neutral conditions without pH adjustment.

In addition to nitrate, the concentration of nitrite was monitored for our systems. The nitrite levels initially increased dramatically and then decreased gradually (Figure 4.6). These results were consistent with the two-step process of nitrate reduction in which nitrate is reduced to nitrite and then nitrite is converted into other products. When materials prepared via coreduction method (MA) were used, the nitrite accumulation in these two systems were similar for the duration of the reaction. After 5 minutes, nitrite-N levels were $4.2 \pm 0.8 \mathrm{ppm}$ and 3.7 $\pm 0.6 \mathrm{ppm}$ and after 30 minutes, nitrite-N concentrations were $4 \pm 1 \mathrm{ppm}(8.0 \%$ nitrite accumulation) and $2.8 \pm 0.9$ ppm (5.6\% nitrite accumulation) when either 5\%Ni-nZVI@MisiCMA or 5\%Ni-nZVI@MisiCFeOOH-MA was used, respectively. In Ni-doped materials, the rate of nitrate and nitrite conversion correlated; that is, when nitrate conversion was fast, nitrite conversion was also fast; however, when nitrate conversion was slow, nitrite accumulation was observed due to concurrent slow nitrite conversion.

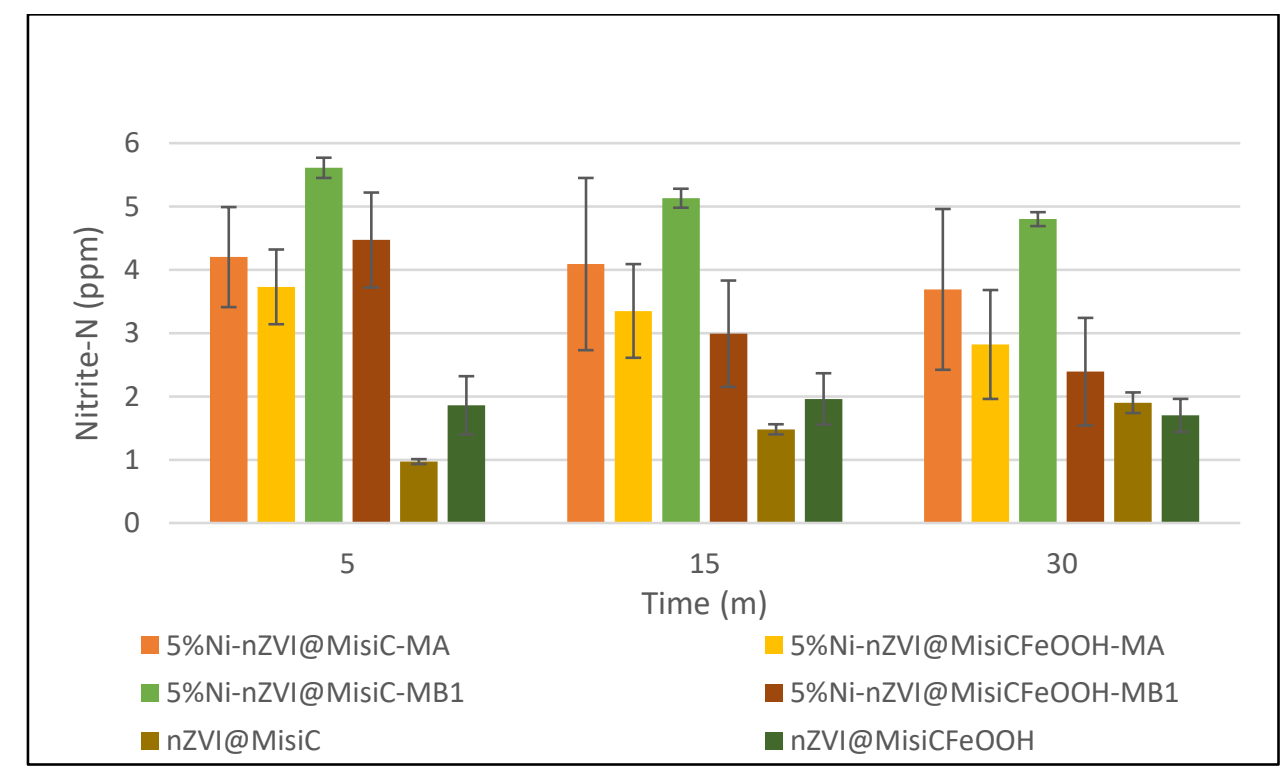

Figure 4.6. Nitrite accumulation in 5\%Ni-nZVI@Misi towards nitrate-N reduction of 50 ppm under anaerobic conditions ( $0.150 \mathrm{~g}$ of materials and $25.0 \mathrm{~mL}$ solution). 
When materials synthesised via deposition method (MB1) was used, the role of Misi preparation in supporting nanoparticles was more significant. The 5\%Ni-nZVI@MisiC-MB1 resulted in more nitrite build-up than 5\%Ni-nZVI@MisiCFeOOH-MB1. After 5 minutes, nitrite$\mathrm{N}$ levels were $5.6 \pm 0.2 \mathrm{ppm}$ and $4.5 \pm 0.7$ ppm using 5\%Ni-nZVI@MisiC-MB1 and 5\%NinZVI@MisiCFeOOH-MB1. After 30 minutes, nitrite-N concentrations were $4.8 \pm 0.1$ ppm (9.6\% nitrite accumulation) and $2.4 \pm 0.8 \mathrm{ppm}$ (4.8\% nitrite accumulation), respectively. Nitrite levels produced by all types of $\mathrm{Ni}$-doped materials were higher than those generated using non-doped nZVI@Misi. After 5 minutes, nitrite-N levels were $0.97 \pm 0.04$ ppm and $1.9 \pm$ $0.5 \mathrm{ppm}$, after 30 minutes, nitrite-N levels were $1.9 \pm 0.2 \mathrm{ppm}$ (3.8\% nitrite accumulation) and $1.7 \pm 0.3$ ppm (3.4\% nitrite accumulation) when either nZVI@MisiC or nZVI@MisiCFeOOH was used, respectively.

The formation of nitrite in the nitrate reduction using supported Ni-doped nZVI has not received much attention, with the nitrite levels either not reported or, if the levels were reported, the evidence was not always solid. For instance, in investigations of either $\mathrm{Ni}$ nZVI@kaolinite or Ni-nZVI@kaolin particles on the simultaneous removal of nitrate and heavy metal ions, no information about nitrite was published. ${ }^{192,}{ }^{199}$ In other studies, the authors used what was described as UV-Vis methodologies to determine the concentrations of nitrate, nitrite, and ammonium. One of the studies determined that nitrite was not formed in the nitrate reduction with bimetallic Ni-nZVI even though the initial nitrate- $\mathrm{N}$ concentration was $50 \mathrm{ppm} .{ }^{244}$ In another study, when the reduction of $20 \mathrm{ppm}$ nitrate- $\mathrm{N}$ was reduced using Ni-nZVI@biochar, the nitrite level was only 0.024 ppm. ${ }^{243}$ However, their UV-Vis methodologies were based on China EPA standards that we are unable to access in order to understand their methodologies.

The nitrite concentrations monitored using IC have only been reported in some studies using unsupported bimetallic Ni-nZVI. The amount of nitrite accumulated depended on reaction conditions. In a study, the initial nitrate-N concentration was 80 ppm (354 ppm nitrate), molar ratio of Fe to nitrate was 4.7:1 (nZVI dosage was $1.5 \mathrm{~g} / \mathrm{L}$ ) and the initial $\mathrm{pH}$ of the solution was 6.5. ${ }^{2}$ After 5 minutes, only $2.9 \mathrm{ppm}$ nitrite- $\mathrm{N}$ was found and after 40 minutes, no nitrite was detected. In a separate study, when the initial nitrate- $N$ concentration was 22.6 ppm (100 $\mathrm{ppm}$ nitrate) and molar ratio of Fe to nitrate was $6.6: 1$ (nZVI dosage of $0.6 \mathrm{~g} / \mathrm{L}$ ), nitrite- $\mathrm{N}$ was 3 ppm after 60 minutes. ${ }^{245}$ 
In summary, the introduction of Ni promoted nitrate reduction. The role of Ni was highlighted in several studies on both supported and unsupported Ni-doped nZVI. Similar to the conclusion by Ryu et al. that $\mathrm{Ni}$ catalyses for the formation of hydrogen in the nitrate reduction using Ni-nZVI, ${ }^{167}$ the mechanism in which nitrate reacts with Ni-nZVI@Misi can be postulated. Nitrate is reduced by electrons that are directly transferred from Fe to the $\mathrm{Ni}$ surface. Simultaneously, Fe reacts with water on the surface of $\mathrm{Ni}$ to form an adsorbed hydride ( $\left.\mathrm{Ni}-\mathrm{H}_{\text {ads }}\right)$. After adsorption of nitrate onto the $\mathrm{Ni}$ surface $\left(\mathrm{Ni}-\mathrm{NO}_{3}{ }^{-}\right.$ads $)$, the adsorbed hydrides will react with the adsorbed nitrate, forming nitrite and then final products.

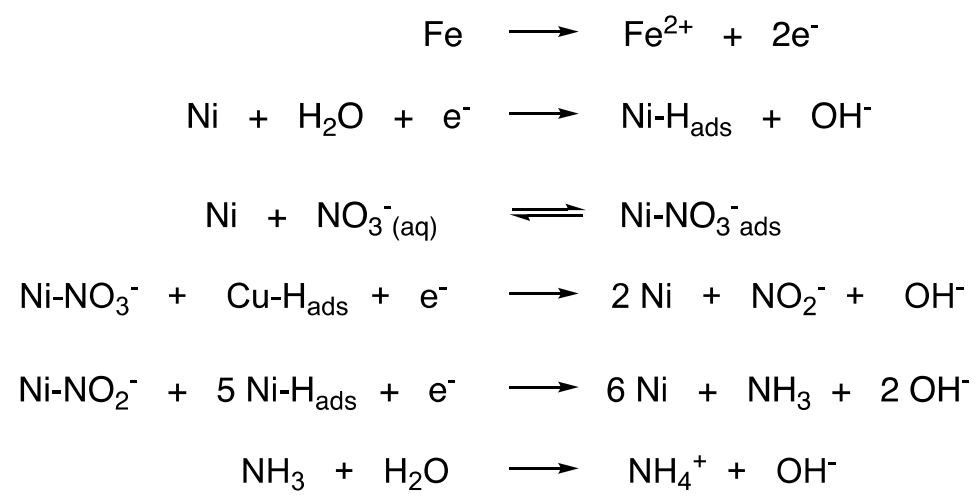

\section{Kinetics of nitrate reduction:}

The kinetics of nitrate reduction was reported in several studies. However, they were unclear and inconsistent. Nitrate was reduced by iron via different kinetic coefficients such as zero-

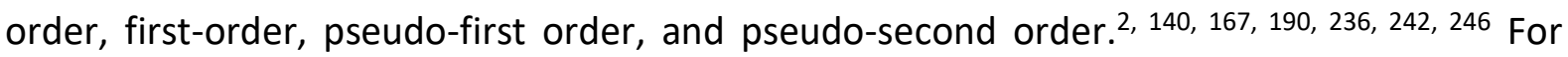
example, Chen et al. reported nitrate was reduced by nZVI via first-order reaction ${ }^{140}$ but Kim et al. reported that both pseudo-first order and pseudo-second order were a good fit for this reaction. ${ }^{242}$ Regarding bimetallic materials, nitrate was reduced by Ni-nZVI via zero-order ${ }^{2}$ or pseudo-first order reaction. ${ }^{167,246}$ In the investigation of trimetallic materials, nitrate reduction followed pseudo-first order when either Pd-Cu-ZVI, Au-Cu-nZVI or Pt-Cu-nZVI was used. ${ }^{190}$ In this study, the kinetics of nitrate reduction using 5\%Ni-nZVI@MisiCFeOOH-MB1 was examined. In the first 8 minutes, a significant amount of nitrate was removed, from 50 ppm to $24.6 \mathrm{ppm}$ nitrate-N. After the initial 8 minutes, the amount of nitrate increased to around $28.6 \mathrm{ppm}$. This indicated that nitrate de-adsorbed into solution from the surface of the materials. It should be noted that this behaviour has been observed, not commented on, in another study of nitrate reduction by unsupported Ni-nZVI. ${ }^{167}$ After 10 minutes, the rate of nitrate removal was significantly slower than the initial 8 minutes. The data was examined 
to determine if it fitted either standard first order (Figure 4.7b) or second order (Figure 4.7c) kinetics, but no linear relationship was observed. Therefore, the kinetics of nitrate reduction using 5\%Ni-nZVI@MisiCFeOOH-MB1 did not follow either zero-order, first-order or secondorder reaction. These studies were performed in duplicate to ensure reproducibility.
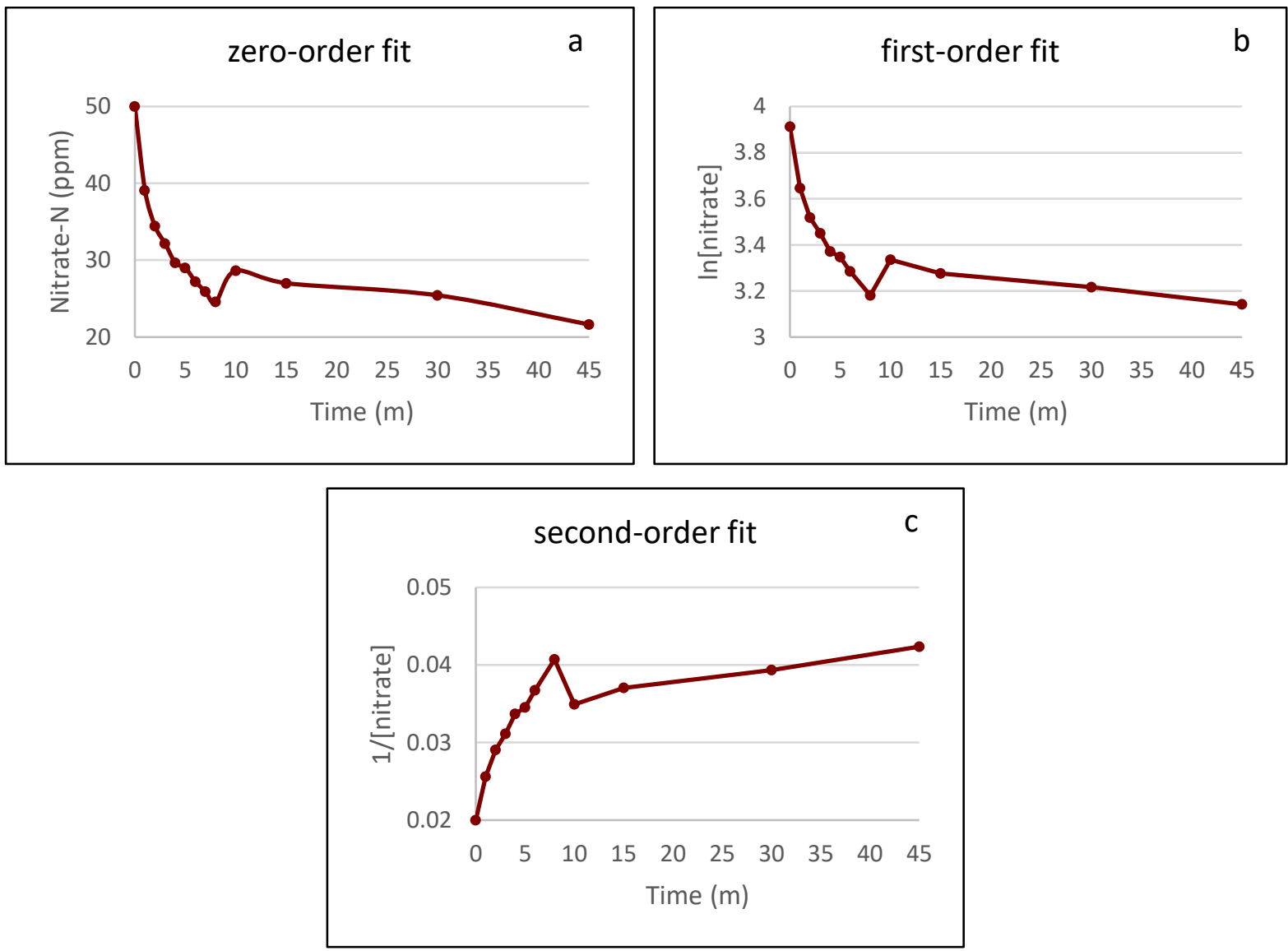

Figure 4.7. Kinetics of nitrate reduction using 5\%Ni-nZVI@MisiCFeOOH-MB1 under anaerobic conditions ( $0.150 \mathrm{~g}$ of materials and $25.0 \mathrm{~mL}$ of solution).

The kinetics of nitrate reduction was investigated for both two different stages. In the first 8 minutes, it follows either a first-order process with $\mathrm{k}=0.08$ minute ${ }^{-1}$ and $\mathrm{R}^{2}=0.88$ or a secondorder kinetics with $\mathrm{k}=0.02 \mathrm{ppm}^{-1}$ minute ${ }^{-1}$ and $\mathrm{R}^{2}=0.95$ (Figure 4.8a and $4.8 \mathrm{~b}$ ). unfortunately, the data does not allow us to confirm either process. After 10 minutes, the change in nitrate concentration was too small to be able to determine the reaction order. As such, the rate of nitrate reduction cannot be determined; however, it does follow a two-stage process presumably due to the change in the nature of the iron nanoparticle over time (see next section). 

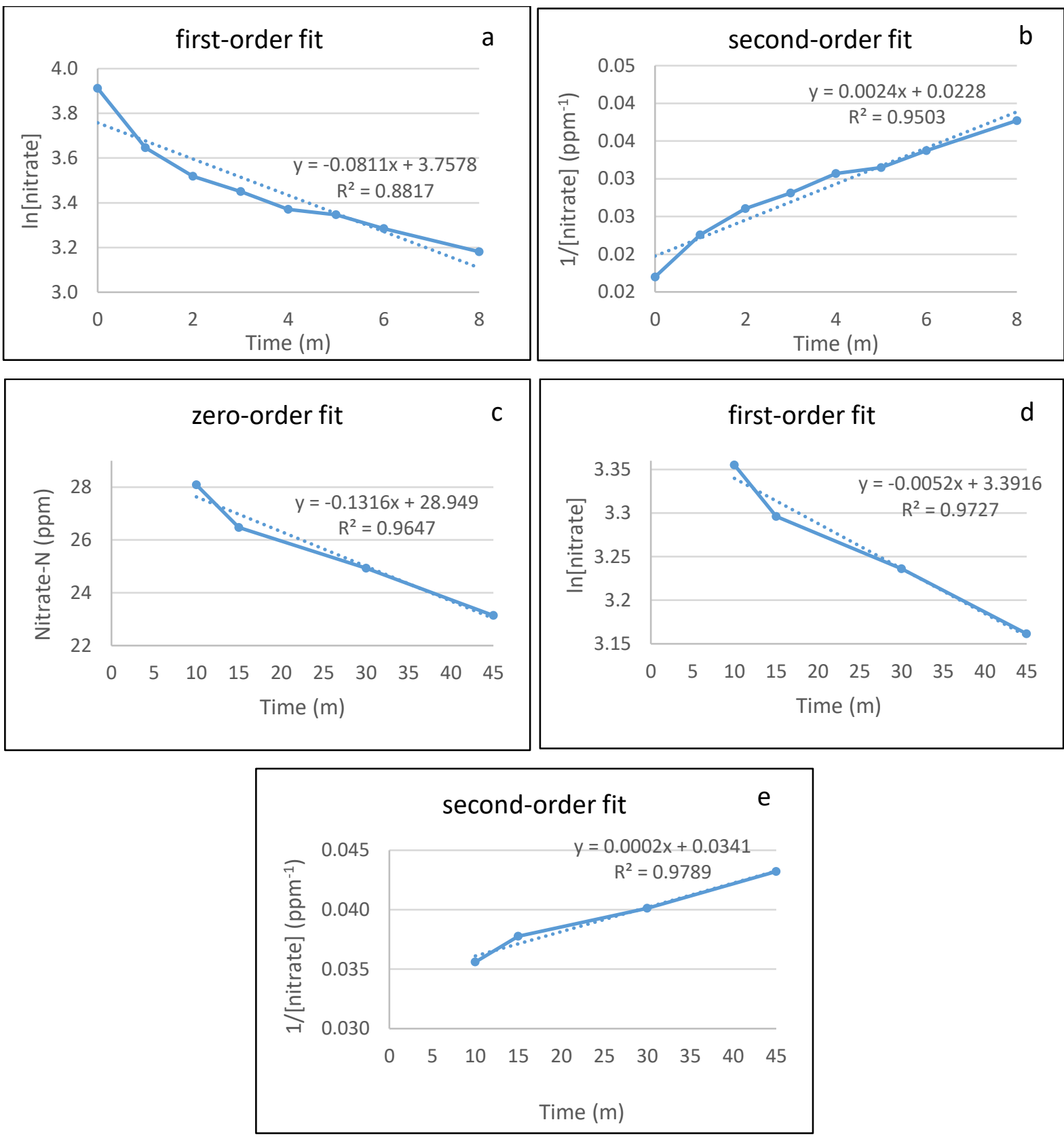

Figure 4.8. Kinetics of nitrate reduction using 5\%Ni-nZVI@MisiCFeOOH-MB1 under anaerobic conditions in different stages (0.150 $\mathrm{g}$ of materials and $25.0 \mathrm{~mL}$ of solution).

The change in the morphology and elemental distribution of materials during the reduction of nitrate was investigated via SEM and TEM analyses. We focused on $5 \% \mathrm{Ni}$ nZVI@MisiCFeOOH prepared via deposition method (MB1) and monitored changes in its morphology under two different reaction conditions: after reduction of $10 \mathrm{ppm}$ nitrate- $\mathrm{N}$ for 15 minutes and after reduction of $50 \mathrm{ppm}$ nitrate- $\mathrm{N}$ for 120 minutes. After nitrate reduction, each material was washed with deoxygenated deionised water, followed by deoxygenated ethanol, and then dried under vacuum. COMPO images showed the presence of silicate and 
nanoparticles in both samples with some nanoparticles unevenly dispersed on the surface of silicate. The composition of materials after nitrate reduction is primarily silicate with significantly fewer nanoparticles (Figure 4.9) than prior to the reaction (Figure $3.7 \mathrm{~d}$ ). The decrease in the number of nanoparticles is presumably due to the loss of nZVI over the course of the reaction. This observation was consistent with Shi et al.'s finding that the chain structure disappeared. ${ }^{192}$ However, the difference in the number of nanoparticles after between 10 ppm nitrate reduction and 50 ppm nitrate reduction was not determined via SEM.

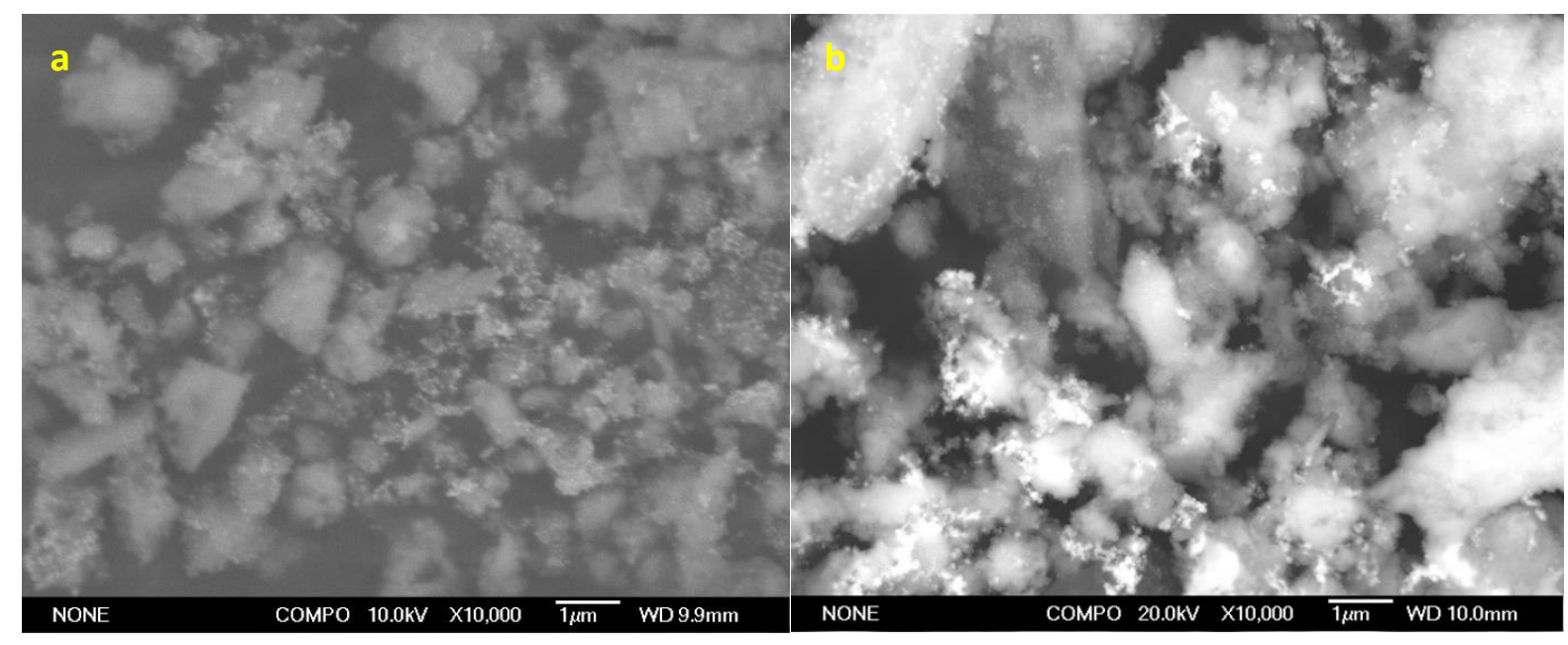

Figure 4.9. COMPO images of 5\%Ni-nZVI@MisiCFeOOH-MB1 after nitrate-N reduction of: (a) 10 ppm and (b) 50 ppm.

TEM analysis showed a significant change in the shape of materials before and after nitrate reduction. Before nitrate reduction, the Ni-nZVI particles of 5\%Ni-nZVI@MisiCFeOOH-MB1 (Figure 3.16b) comprises of a spherical core with an encompassing shell of $\mathrm{Ni}(0)$ and iron nickel oxides. These particles are aggregated into clusters. After reducing either $10 \mathrm{ppm}$ nitrate-N for 15 minutes (Figure 4.10a and 4.10b) or 50 ppm nitrate-N for 120 minutes (Figure 4.10c and 4.10d), the core-shell structure is diminished. This is consistent with Kang et al.'s report in which the shape of iron changed from spherule to sheet after nitrate reduction using Ni-nZVI. ${ }^{2}$ 

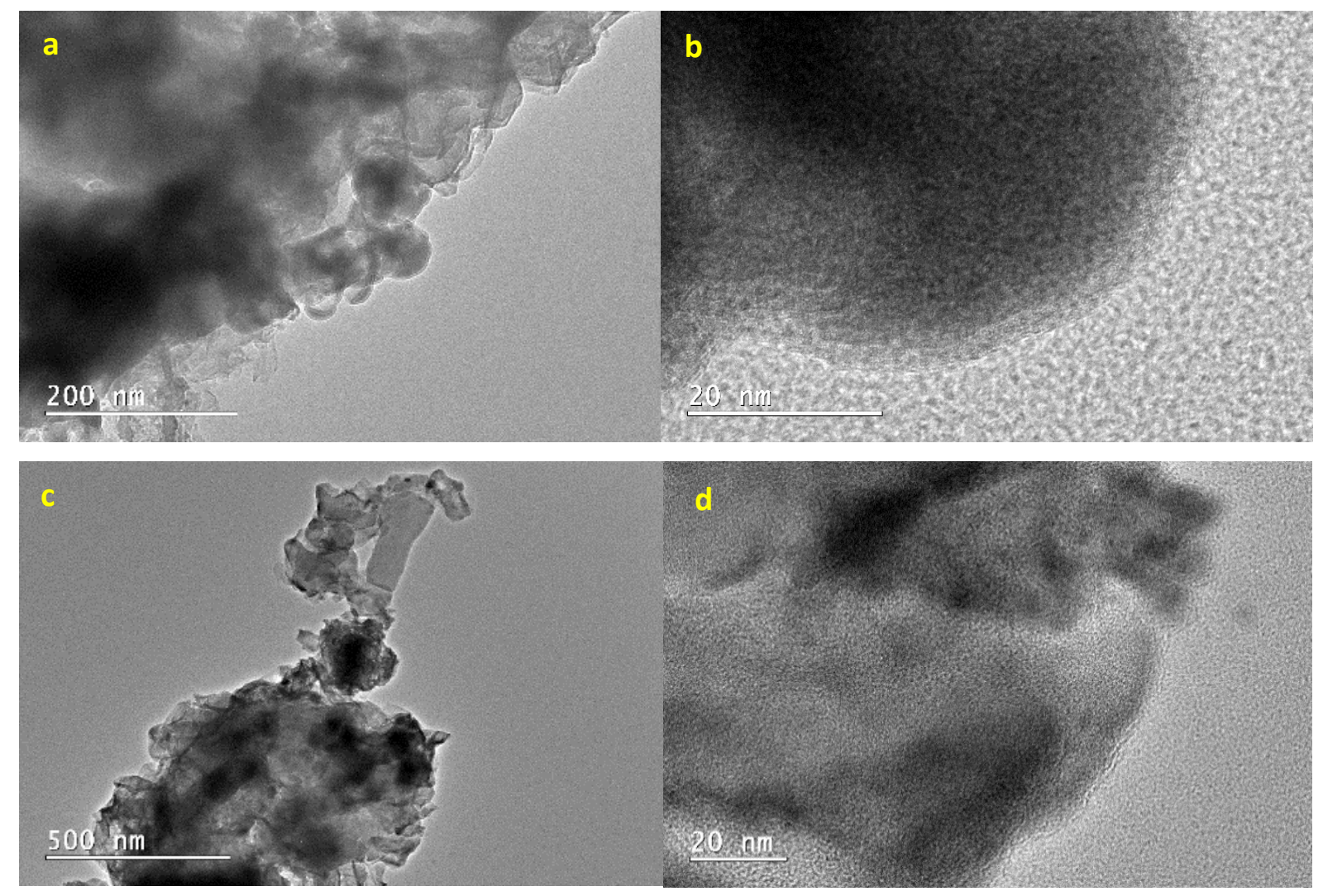

Figure 4.10. TEM images of 5\%Ni-nZVI@MisiCFeOOH-MB1 after nitrate-N reduction of (a),

(b) 10 ppm for 15 minutes and (c), (d) 50 ppm for 120 minutes.

There have been studies on the structure of $\mathrm{Ni}-\mathrm{nZVI}$ before and after reaction using TEM. ${ }^{2,167}$ However, these investigations focused on TEM images that reveal the shape of particles and the change in agglomeration. In this study, based on TEM images, only ill-defined materials are observed; as such, we performed TEM-EDS analysis to determine further information about the composition of the material. Figure 4.11 revealed the elemental composition of 5\%Ni-nZVI@MisiCFeOOH-MB1 via TEM-EDS spectrum after reducing 10 ppm nitrate-N for 15 minutes. Peaks corresponding to $\mathrm{Si}, \mathrm{O}, \mathrm{S}$, and $\mathrm{Al}$ are observed, indicating the presence of Misi. Peaks corresponding to $\mathrm{Fe}$ and $\mathrm{Ni}$ are also observed, confirming the existence of $\mathrm{Fe}$ and $\mathrm{Ni}$ after nitrate reduction. The mass percentage for Fe and $\mathrm{Ni}$ was $48.3 \%$ and $2.6 \%$ and the atom percentage was $23.2 \%$ and $1.2 \%$, respectively. This Fe:Ni mass ratio (19:1) was lower than that of before nitrate reduction (22:1) (Section 3.3.3, mass percentage for Fe and Ni was $40.4 \%$ and $1.8 \%$ ). This indicated that iron was removed from the material due to its reaction with nitrate. 


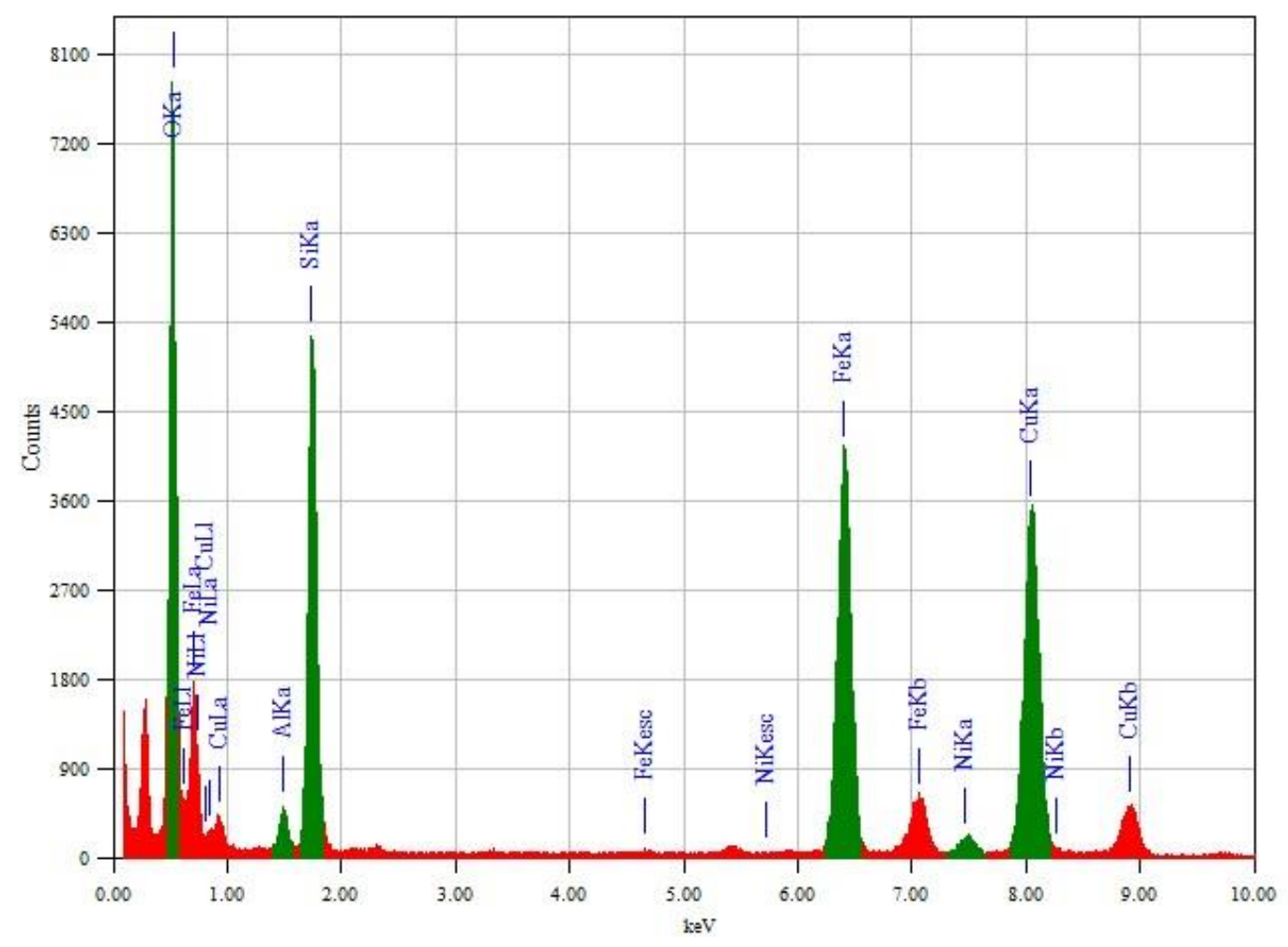

Figure 4.11. TEM-EDS spectrum of 5\%Ni-nZVI@MisiCFeOOH-MB1 after reduction of 10 ppm nitrate- $N(0.150 \mathrm{~g}$ of material, $25.0 \mathrm{~mL}$ of solution, under anaerobic conditions, 15 minutes).

Note: labels have been modified to highlight peaks relevant to this research. Please see appendix B.7 for complete spectrum.

In addition to the TEM-EDS spectra, TEM-EDS mapping of 5\%Ni-nZVI@MisiCFeOOH-MB1 after reducing $10 \mathrm{ppm}$ nitrate for 15 minutes confirmed the presence of both Misi and Ni-nZVI particles. Signals corresponding to elements such as $\mathrm{Si}, \mathrm{O}, \mathrm{Al}, \mathrm{S}$, and $\mathrm{Ti}$ are observed, consistent with the presence of Misi (Figure 4.11). The signals corresponding to Fe and Ni take the form of agglomerated nanoparticles. When the signals of $\mathrm{Fe}$ and $\mathrm{Ni}$ are overlaid, the significant difference in the elemental distribution between 5\%Ni-nZVI@MisiCFeOOH-MB1 before and after nitrate reduction is observed. Before reaction, there are core-shell particles with a well-dispersed layer of $\mathrm{Ni}(0)$ and iron nickel oxides on the surface of the $\mathrm{Fe}(0)$ core (Figure 3.16). After reduction, the $\mathrm{Ni}$ signal appears slightly enhanced on the surface of $\mathrm{Fe}$ than on $\mathrm{Fe}(0)$ core; however, the defined core-shell structure is no longer present (Figure 4.12a). Regarding to the signals of $\mathrm{O}, \mathrm{Fe}$ and $\mathrm{Ni}$, there is also a significant change in the distribution of these elements. Before reaction, a layer containing $\mathrm{O}$ and $\mathrm{Ni}$ encapsulates $\mathrm{Fe}$ (Figure 3.16). After reaction, little O presents in the Fe and $\mathrm{Ni}$ (Figure 4.12b, 4.12c and 4.12d). 
Interestingly, the agglomeration of nanoparticles appears to have increased. This could be due to the migration and agglomeration of the remaining $\mathrm{Fe}(0)$, although we are unable to determine the origin of the remaining $\mathrm{Fe}(0)$; for example, whether it is from unreacted nanoparticles, or from the migration of $\mathrm{Fe}(0)$ from partially reacted nanoparticles to form new Fe nanoparticles. 

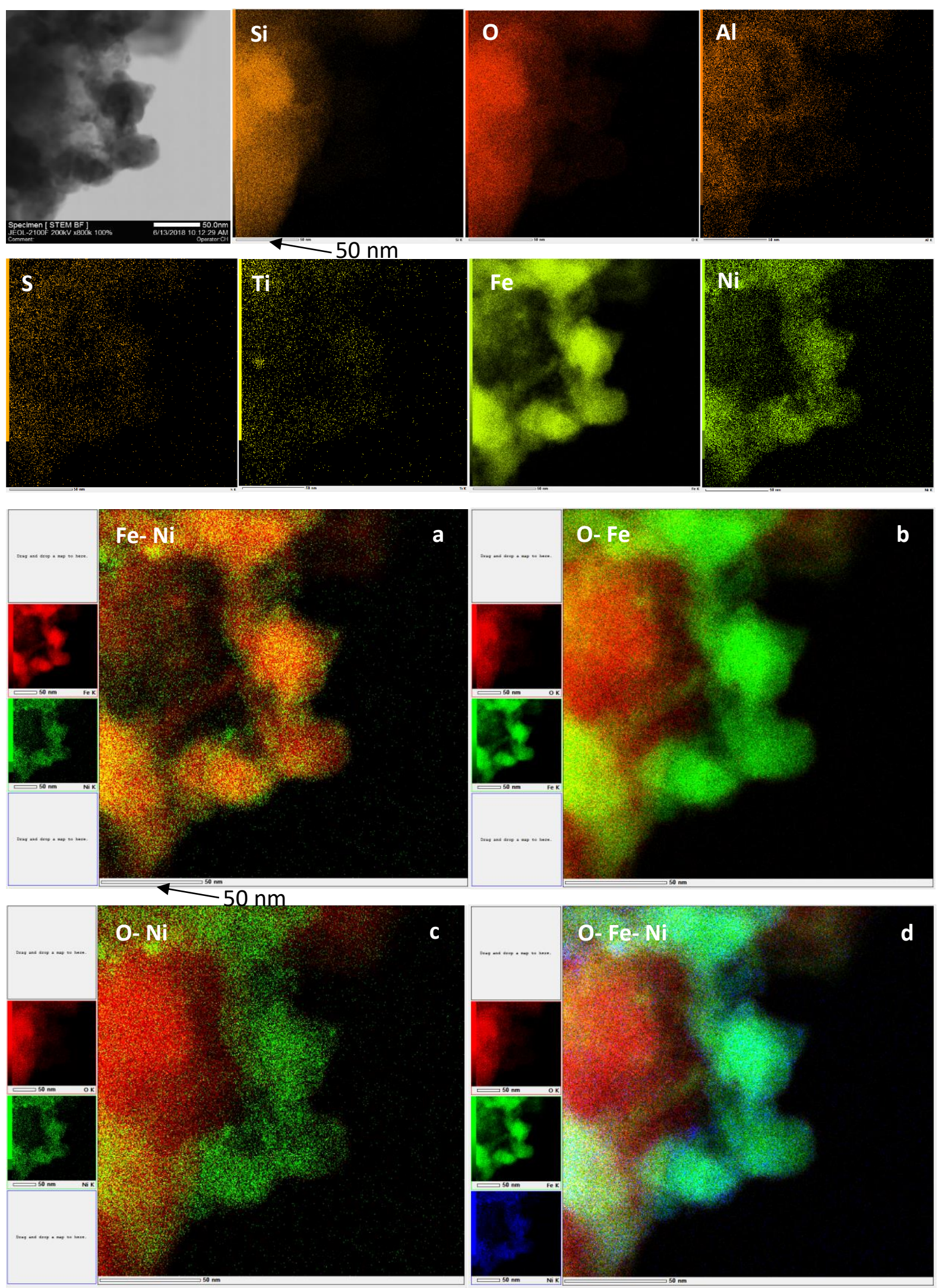

Figure 4.12. TEM-EDS mapping of 5\%Ni-nZVI@MisiCFeOOH-MB1 after reduction of 10 ppm nitrate- $N$ (0.150 g of material, $25.0 \mathrm{~mL}$ of solution, under anaerobic conditions, 15 minutes). 
TEM-EDS spectrum the material generated after reduction of $50 \mathrm{ppm}$ nitrate- $\mathrm{N}$ for 120 minutes revealed peaks corresponding to $\mathrm{Si}, \mathrm{O}, \mathrm{S}$, and $\mathrm{Al}$, indicating the presence of Misi. Peaks corresponding to Fe and $\mathrm{Ni}$ are also observed (Figure 4.13), confirming the presence of $\mathrm{Fe}$ and Ni. Ni percentage was significantly lower than Fe. The mass percentage for Fe and Ni was $40.31 \%$ and $2.67 \%$ and the atom percentage was $22.14 \%$ and $1.44 \%$, respectively. The mass ratio of $\mathrm{Fe}$ to $\mathrm{Ni}$ in this specific area of 15:1 that was lower than those of this material before the nitrate reduction (22:1) and after reduction of $10 \mathrm{ppm}$ of nitrate (19:1). This confirmed that the higher nitrate concentration removed more iron from the material.

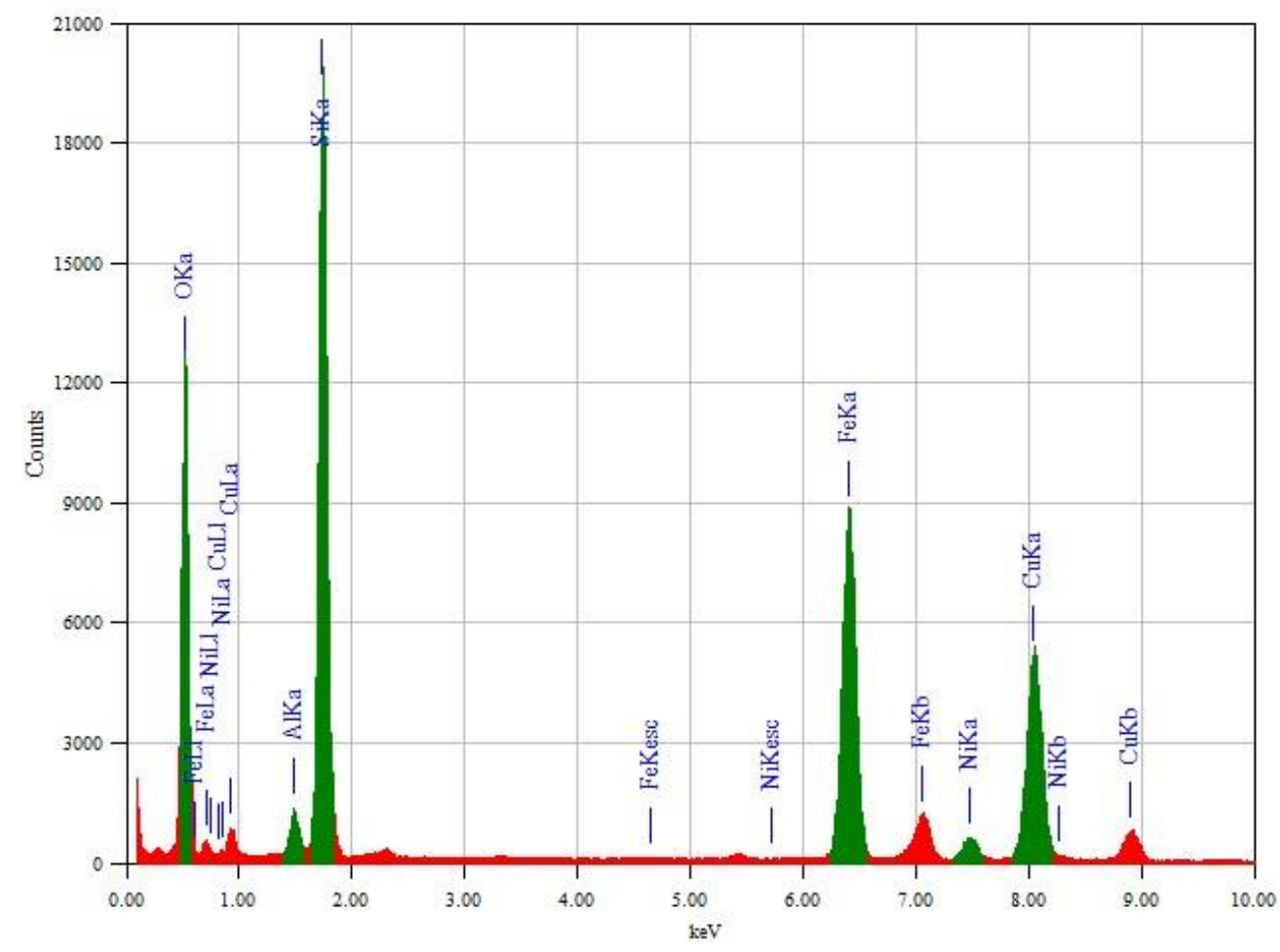

Figure 4.13. TEM-EDS spectrum of 5\%Ni-nZVI@MisiCFeOOH-MB1 after reduction of 50 ppm nitrate- $N$ ( $0.150 \mathrm{~g}$ of material, $25.0 \mathrm{~mL}$ of solution, under anaerobic conditions, 120 minutes). Note: labels have been modified to highlight peaks relevant to this research. Please see appendix B.8 for complete spectrum.

Similar to TEM-EDS spectrum, in TEM-EDS mapping of 5\%Ni-nZVI@MisiCFeOOH-MB1 after reduction of $50 \mathrm{ppm}$ nitrate- $\mathrm{N}$ for 120 minutes, signals corresponding to $\mathrm{Si}, \mathrm{O}, \mathrm{Al}, \mathrm{S}$, and $\mathrm{Ti}$ are observed, consistent with the presence of Misi (Figure 4.14). A few nanoparticles are also found, and they do not appear to be adsorbed directly onto the silicate. In these particles, the Fe and Ni signals are strong, and an overlap between these two elements is observed (Figure 4.14a). Additionally, the $\mathrm{O}$ signal does not relate to signals of $\mathrm{Fe}$ or $\mathrm{Ni}$, it is only observed 
overlaid with Si (Figure 4.14b, 4.14c, and 4.14d). These results indicate that the core-shell structure of $\mathrm{Ni}-\mathrm{nZVI}$ particles in which the combination of $\mathrm{Ni}(0)$ and potentially iron nickel oxides deposited on the surface of $\mathrm{Fe}(0)$ core before reaction has been properly destroyed. The remaining nanoparticles appear to be the mixture of Fe and $\mathrm{Ni}$ (Fe-Ni alloy). Interestingly, the presence of $\mathrm{Fe}(0)$ and potentially $\mathrm{Ni}(0)$ is noted, even though all of the zero-valent metal centers should have reacted with the excess nitrate added. The used ratio of Fe to nitrate is 3:1 compared to the theoretically idealised ratio of 4:1 (Section 4.1). 

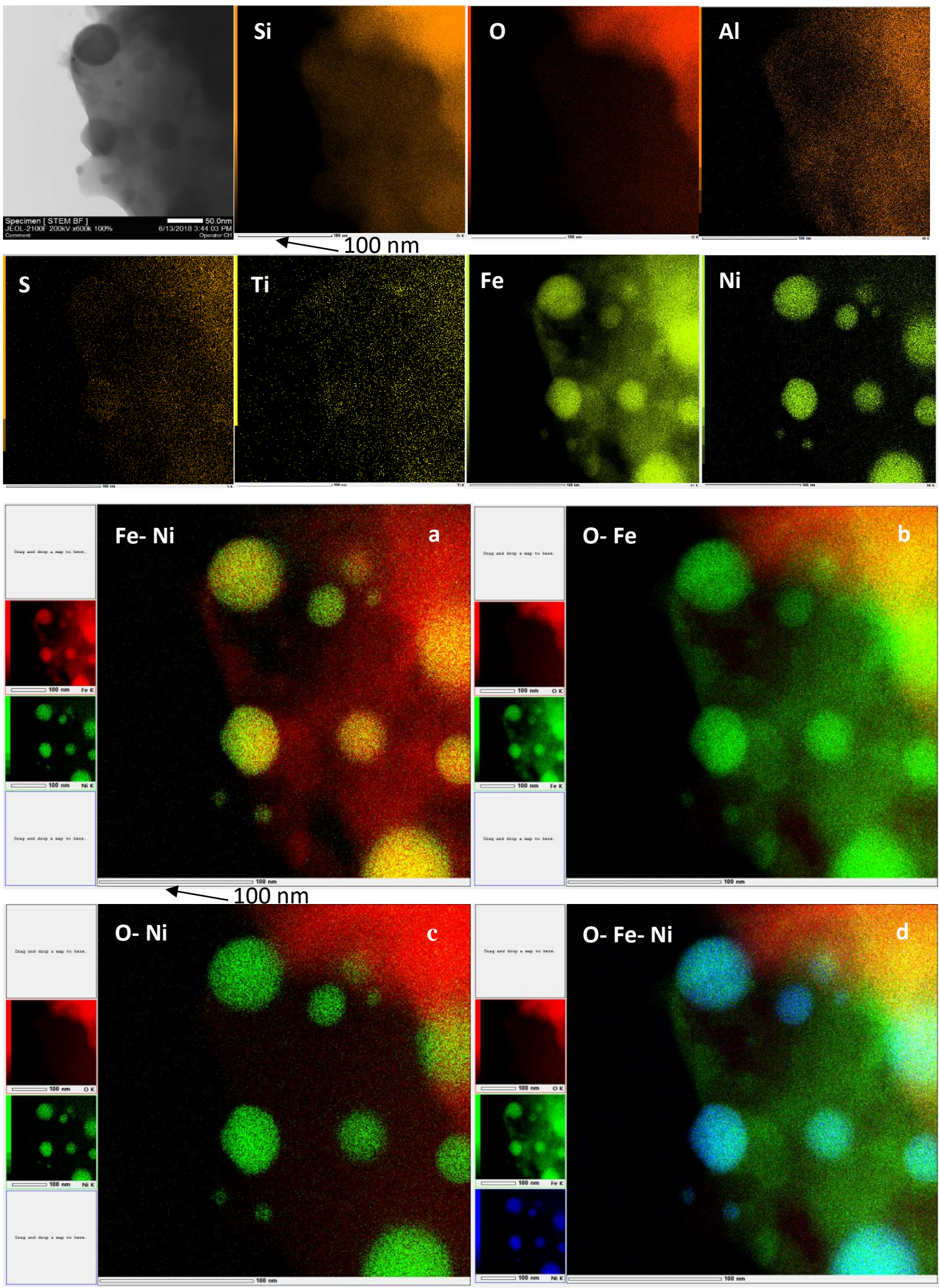

Figure 4.14. TEM-EDS mapping of 5\%Ni-nZVI@MisiCFeOOH-MB1 after reduction of 50 ppm nitrate-N (0.150 g material, $25.0 \mathrm{~mL}$ of solution, under anaerobic conditions, 120 minutes). 


\subsection{Nitrate reduction with bimetallic Cu-nZVI@Misi under anaerobic conditions}

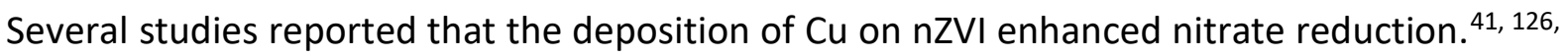
178, 181 In addition, Cu-nZVI also produces much more nitrite than non-doped nZVI. ${ }^{126}$ However, most of these studies have focused on unsupported Cu-nZVI. In this chapter, the reactivity of supported $\mathrm{CU}-\mathrm{nZVI}$ on Misi towards nitrate, as determined by nitrate conversion and nitrite accumulation, were examined under anaerobic conditions. Four materials, including $5 \% \mathrm{Cu}-$ nZVI@MisiC-MA, 5\%Cu-nZVI@MisiCFeOOH-MA, 5\%Cu-nZVI@MisiC-MB1 and 5\%CunZVI@MisiCFeOOH-MB1 were used. Two different nitrate-N concentrations, including 10 ppm and 50 ppm, were examined.

The removal of 10 ppm of nitrate-N using Cu-nZVI@Misi was rapid, regardless of either Misi preparation or synthetic method. Within 5 minutes, nitrate-N levels were below $6.5 \mathrm{ppm}$ and after 15 minutes, nitrate-N levels were below 1 ppm (Figure 4.15). Compared to non-doped nZVI@Misi as a control, all Cu-doped nZVI@Misi systems had higher reactivity. These results highlighted the role of copper as a catalyst in improving nitrate conversion. However, the difference in the reactivity among Cu-nZVI@Misi systems is minimal and cannot be distinguished due to the rapid nitrate reduction, especially when the uncertainties of these values are considered.

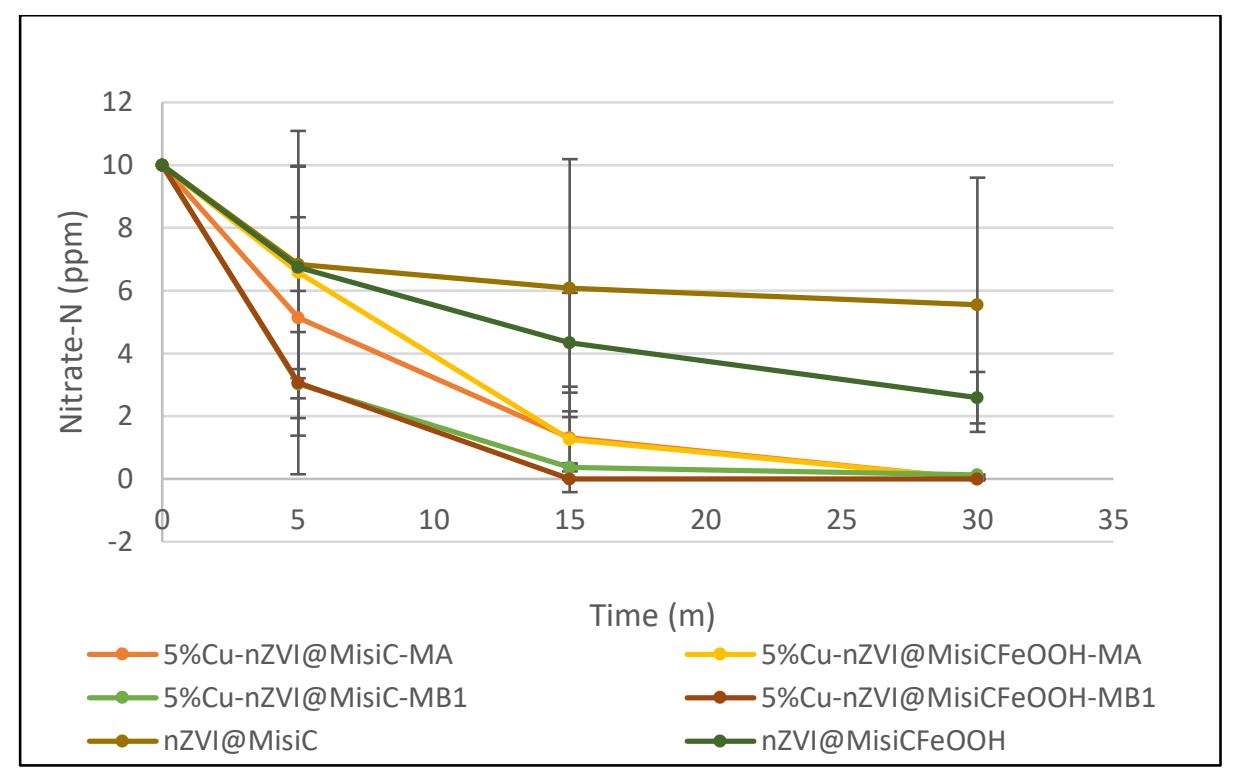

Figure 4.15. Reduction characterisation of 5\%CU-nZVI@Misi towards nitrate-N 10 ppm under anaerobic conditions (0.150 g of materials, $25.0 \mathrm{~mL}$ of solution). 
The inconsistency in the results of nitrate concentrations was observed for the reduction with an initial nitrate- $\mathrm{N}$ concentration of $10 \mathrm{ppm}$ when nitrate reduction using samples of either the same batch and or different batches of materials were examined in triplicate, particularly after 5 minutes (Figure 4.16). For example, after 5 minutes, the nitrate- $\mathrm{N}$ concentrations were $1.46 \mathrm{ppm}, 6.76 \mathrm{ppm}$, and $7.20 \mathrm{ppm}$ when samples of three different batches of $5 \% \mathrm{Cu}$ nZVI@MisiC-MA were used. Combining these results would give an average nitrate-N concentration of $5 \pm 3 \mathrm{ppm}$ ( $5 \mathrm{ppm} \pm 60 \%$ ). Similarly, the average nitrate-N concentrations were $7 \pm 3$ ppm ( $7 \mathrm{ppm} \pm 43 \%), 3 \pm 2 \mathrm{ppm}(3 \mathrm{ppm} \pm 67 \%)$ and $3 \pm 3$ ppm ( $3 \mathrm{ppm} \pm 100 \%)$ when either 5\%Cu-nZVI@MisiCFeOOH-MA, 5\%Cu-nZVI@MisiC-MB1 or 5\%Cu-nZVI@MisiCFeOOHMB1 was used, respectively. The high levels of uncertainty did not allow for correlation between materials used and their ability to reduce nitrate. As such, higher nitrate concentrations were examined to determine if the reactivity of the materials could be differential.

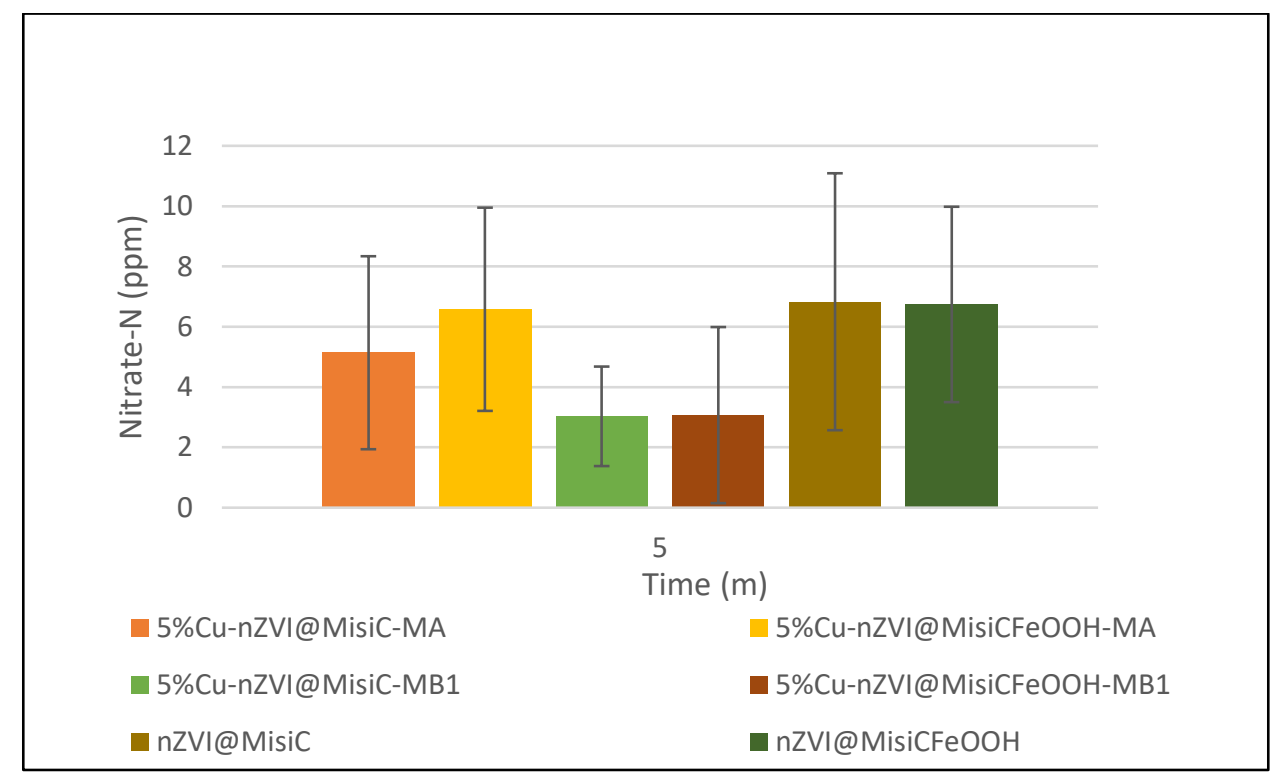

Figure 4.16. Reduction characterisation of 5\%Cu-nZVI@Misi towards nitrate-N 10 ppm under anaerobic conditions with high uncertainty after 5 minutes $(0.150 \mathrm{~g}$ of materials, 25.0 $m L$ of solution).

The reaction intermediate nitrite was also examined in the reduction of $10 \mathrm{ppm}$ nitrate- $\mathrm{N}$. The nitrite- $\mathrm{N}$ concentration initially increased (up to $4 \mathrm{ppm}$ after 5 minutes) and then decreased after 30 minutes (Figure 4.17). The amount of nitrite build-up depended on doping method for synthesising materials. After 5 minutes, materials prepared via deposition 
method (MB1) accumulated much more nitrite than materials prepared via co-reduction method (MA). Nitrite-N concentrations were 3 - 4 ppm when either 5\%Cu-nZVI@MisiC-MB1 or 5\%Cu-nZVI@MisiFeOOH-MB1 was used while nitrite-N concentration was around 1 ppm using either 5\%Cu-nZVI@MisiC-MA or 5\%Cu-nZVI@MisiCFeOOH-MA. However, after 15 minutes, nitrite- $\mathrm{N}$ concentrations were below $1 \mathrm{ppm}$ and after 30 minutes, nitrite- $\mathrm{N}$ concentrations were below the detection limit of the IC $(0.04 \mathrm{ppm})$ when any Cu-doped nZVI@Misi systems were used. As a control, nitrite production in Cu-doped nZVI@Misi was compared to that of non-doped nZVI@Misi. After 5 minutes, nitrite levels using Cu-doped systems were higher than those using non-doped systems. In contrast, after 30 minutes, nitrite levels using Cu-doped systems were lower than those using non-doped systems, but the difference was minimal.

In Cu-doped materials, after 5 minutes, the faster nitrate conversion was, the more nitrite accumulated. For example, Cu-doped materials prepared via deposition method (MB1) reduced nitrate faster than $\mathrm{Cu}$-doped materials prepared via co-reduction method (MA), therefore, more nitrite was present in $\mathrm{Cu}$-doped materials prepared via deposition method (MB1). This is different from Ni-doped systems in which the nitrate concentrations were directly proportional to nitrite concentrations.

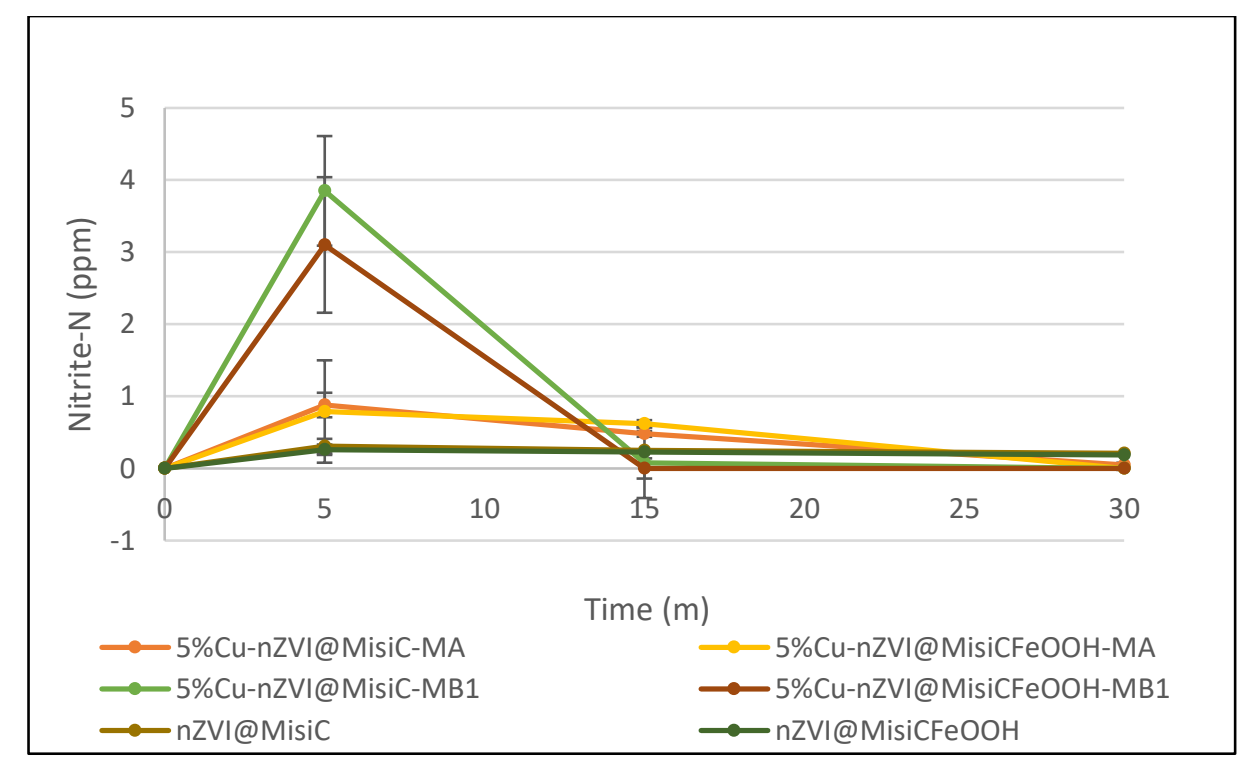

Figure 4.17. Nitrite accumulation using 5\%Cu-nZVI@Misi towards nitrate-N 10 ppm reduction under anaerobic conditions (0.15 $\mathrm{g}$ of materials, $25 \mathrm{~mL}$ of solution). 
Nitrite levels were also inconsistent when nitrate reduction of $10 \mathrm{ppm}$ was triplicated using samples of either the same batch or different batches. After 5 minutes, the average nitrite levels were $0.9 \pm 0.2 \mathrm{ppm}(0.9 \mathrm{ppm} \pm 22 \%), 0.8 \pm 0.7 \mathrm{ppm}(0.8 \mathrm{ppm} \pm 88 \%), 3 \pm 2 \mathrm{ppm}(3 \mathrm{ppm}$ $\pm 67 \%)$ and $3.1 \pm 0.9$ ppm (3.1 ppm \pm 29\%) when either 5\%Cu-nZVI@MisiC-MA, 5\%CunZVI@MisiCFeOOH-MA, 5\%Cu-nZVI@MisiC-MB1 or 5\%Cu-nZVI@MisiCFeOOH-MB1 was used, respectively (Figure 4.18). The low nitrite levels and the high uncertainties did not allow for the correlation between materials used and their ability in nitrate reduction. Therefore, more information about nitrite generation needs examining, for example, in the reduction of higher initial nitrate concentration.

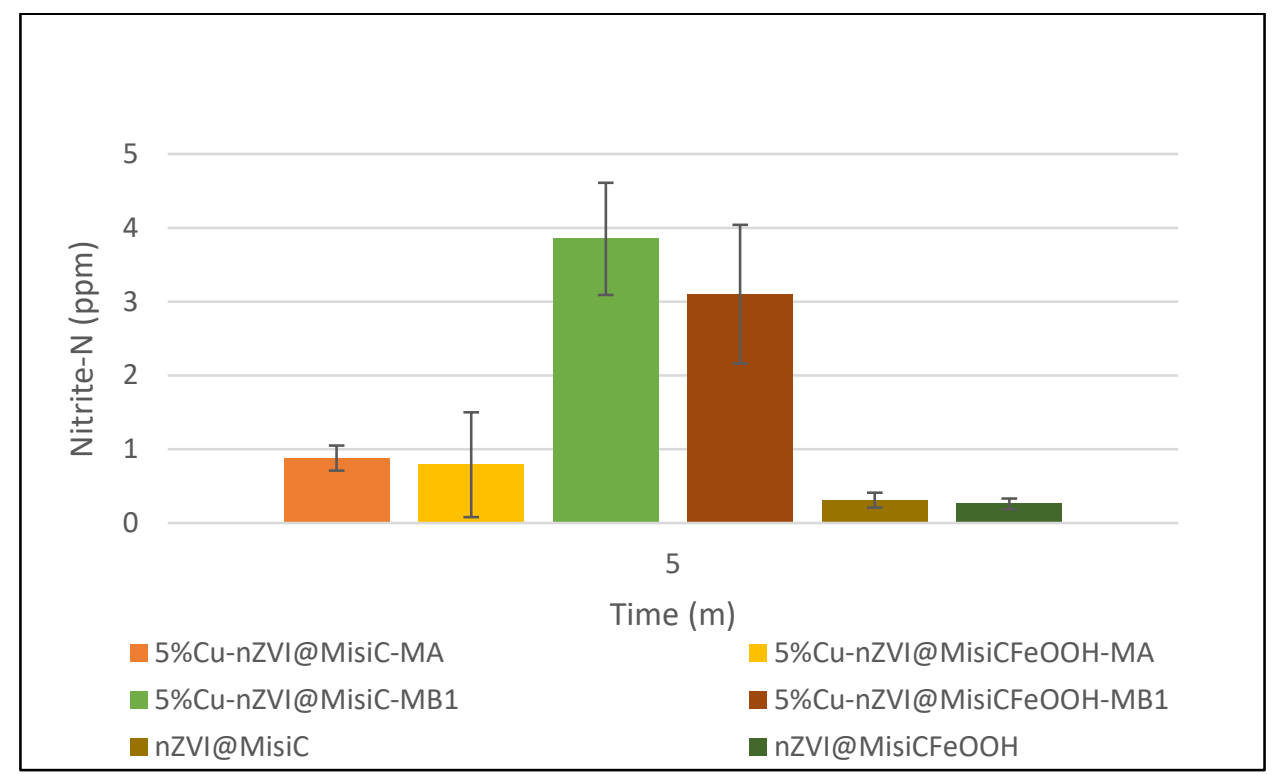

Figure 4.18. Nitrite accumulation using 5\%Cu-nZVI@Misi towards nitrate-N 10 ppm reduction under anaerobic conditions with high uncertainty $(0.150 \mathrm{~g}$ of materials, $25.0 \mathrm{~mL}$ of solution).

The capacity of nitrate removal and the difference in the reactivity of Cu-doped nZVI@Misi systems were examined when these materials were tested in a higher initial concentration. Similar to the results from Ni-doped nZVI@Misi systems, the 50 ppm of nitrate-N exceeds the reducing capacity of our Cu-nZVI@Misi materials (molar ratio of Fe to nitrate at 3:1 instead of the idealised ratio at 4:1); hence, all nitrate was not expected to be removed from the solutions. However, more reliable data can be collected, especially with regards to the initial time point as well as to compare the reactivity of these materials. 
The effect of synthetic methods on the reactivity of Cu-doped nZVI@Misi materials was evident. Cu-doped nZVI@Misi prepared via deposition method (MB1) exhibited significantly greater reactivity than those prepared via co-reduction method (MA), regardless of Misi preparation (Figure 4.19). For example, after 5 minutes, nitrate- $\mathrm{N}$ levels were $40 \pm 2 \mathrm{ppm}$ and $28 \pm 2$ ppm when either 5\%Cu-nZVI@MisiC-MA or 5\%Cu-nZVI@MisiC-MB1 was used. Nitrate$\mathrm{N}$ levels were $34 \pm 2$ ppm and $17 \pm 2$ ppm when either 5\%Cu-nZVI@MisiCFeOOH-MA or 5\%CunZVI@MisiCFeOOH-MB1 was used. After 30 minutes, nitrate-N levels in both Cu-doped materials prepared via deposition method (MB1) were three times lower than those in both $\mathrm{Cu}$-doped materials prepared via co-reduction method (MA). The nitrate- $\mathrm{N}$ concentrations were $27 \pm 2$ ppm (46\% nitrate conversion) and $10.0 \pm 0.7 \mathrm{ppm}$ (80\% nitrate conversion) when either 5\%Cu-nZVI@MisiC-MA or 5\%Cu-nZVI@MisiC-MB1 was used, respectively. The nitrate$\mathrm{N}$ concentrations were $17 \pm 1 \mathrm{ppm}$ (66\% nitrate conversion) and $5 \pm 1 \mathrm{ppm}$ (90\% nitrate conversion) when either 5\%Cu-nZVI@MisiCFeOOH-MA or 5\%Cu-nZVI@MisiCFeOOH-MB1 was used, respectively. These results confirmed the important role of the copper layer on the surface of nZVI particles that was generated via deposition method (MB1). There is a Cu layer on the surface of nZVI in 5\%Cu-nZVI@MisiCFeOOH-MB1 while Cu is only present as a small island on Fe in 5\%Cu-nZVI@MisiCFeOOH-MA (Section 3.4.2).

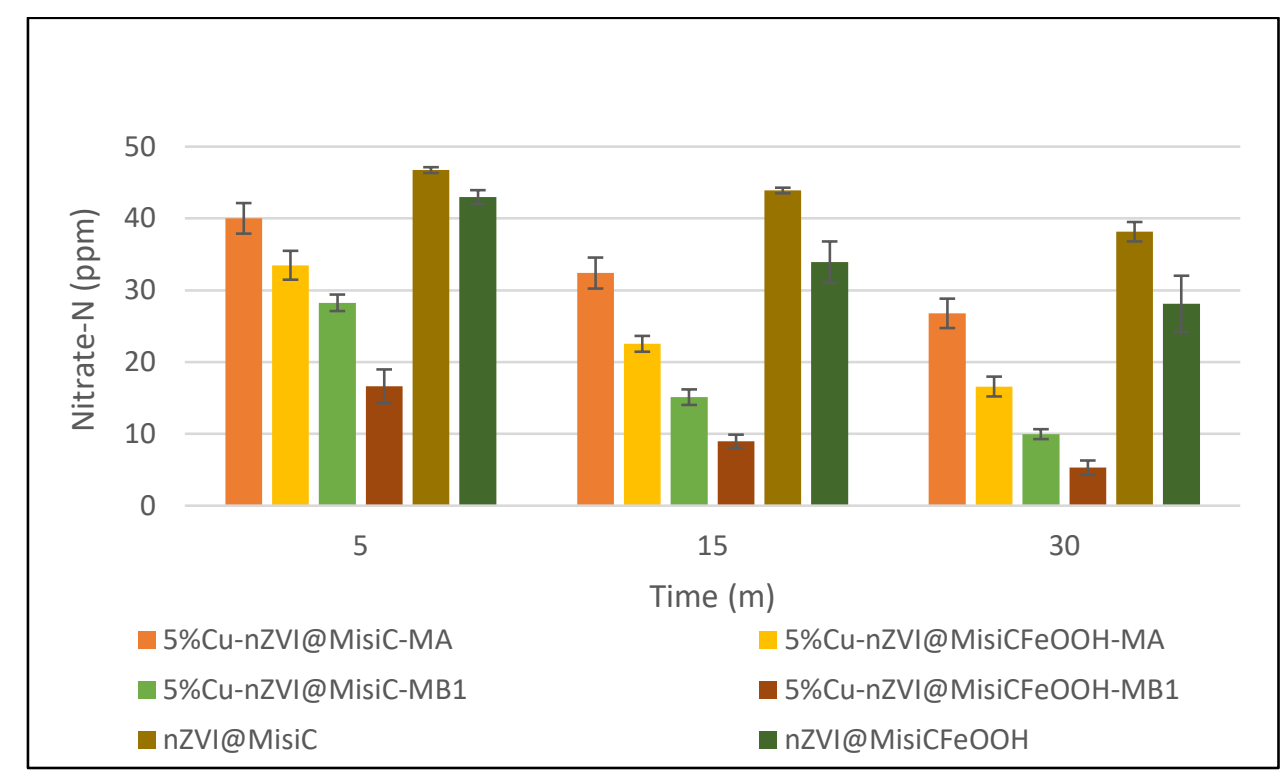

Figure 4.19. Reduction characterisation of 5\%Cu-nZVI@Misi towards nitrate-N 50 ppm under anaerobic conditions ( $0.150 \mathrm{~g}$ of materials, $25.0 \mathrm{~mL}$ solution).

Interestingly, the relative uncertainty levels of nitrate concentrations were in a range of $6 \%$ $20 \%$ for the reaction with an initial nitrate- $\mathrm{N}$ concentration of $50 \mathrm{ppm}$. These values were 
much smaller than the uncertainties for the reaction with an initial nitrate- $\mathrm{N}$ concentration of $10 \mathrm{ppm}$. As such, there is a better correlation of materials used and their reactivity towards nitrate reduction of $50 \mathrm{ppm}$.

The Misi preparation effected the reactivity of Cu-doped nZVI@Misi materials as MisiCFeOOH was more successful than MisiC, regardless of synthetic method (Figure 4.19). After 5 minutes, materials using MisiCFeOOH reduced nitrate faster than those using MisiC. For example, nitrate- $\mathrm{N}$ levels were $34 \pm 2$ ppm and $40 \pm 2$ when either 5\%Cu-nZVI@MisiCFeOOHMA or 5\%Cu-nZVI@MisiC-MA was used, respectively. Moreover, nitrate conversions in materials prepared using $\mathrm{MisiCFeOOH}$ were higher than those in materials prepared using MisiC. For example, after 30 minutes, the nitrate conversions were $66 \%$ and $46 \%$ when either 5\%Cu-nZVI@MisiCFeOOH-MA or 5\%Cu-nZVI@MisiC-MA was used, respectively. Although the difference in the distribution of nanoparticles on the surface of different preparations of Misi was not visually observed via SEM analysis, the importance of coating FeOOH layer in enhancing the reactivity of nanoparticles was indicated. This finding is consistent to that observed by Fraser in which the support preparation of non-doped nZVI@Misi had a significant effect on the reactivity of the nanoparticles. ${ }^{1}$ Although factors behind this were not determined, this does have implications on understanding the reactivity of nanoparticles in different media and how the chemistry of the surrounding media (eg. soils, etc) might affect the reactivity of the nanoparticles.

Compared to non-doped nZVI@Misi, bimetallic 5\%Cu-nZVI on either MisiC or MisiCFeOOH had greater reactivity towards nitrate reduction. Cu-doped material that had the lowest reactivity (5\%Cu-nZVI@MisiC-MA) still reduced nitrate much faster than non-doped nZVI@Misi that had the higher reactivity (nZVI@MisiCFeOOH). For example, after 30 minutes, the nitrate conversion values were 66\% and 44\% when either 5\%Cu-nZVI@MisiC-MA or nZVI@MisiCFeOOH was used, respectively. These results suggested that $\mathrm{Cu}$ is catalysing the reduction of nitrate by $\mathrm{nZVI}$. This has been noted by other researchers. The reactivity of $\mathrm{Cu}-$ $\mathrm{nZVI}$ was significantly increased when nitrate was reduced from solutions. For instance, $\mathrm{Cu}$ promoted nitrate reduction when $2.5 \% \mathrm{Cu}-\mathrm{nZVI}$ was used either acidic or neutral conditions. ${ }^{178}$ Nitrate removal increased by $37 \%$ and $36 \%$ when $\mathrm{Cu}-\mathrm{nZVI}$ was used as $\mathrm{pH}$ of 3 and $\mathrm{pH}$ of 7 compared to nZVI. In Hwang et al.'s study, nitrate conversion was $98 \%$ using CunZVI (5\% mol/mol) compared to 50\% using non-doped nZVI. ${ }^{41}$ Moreover, in Liou et al.'s study, 
the same finding was observed when Cu-nZVI $(0.5 \%-20 \% \mathrm{w} / \mathrm{w})$ was used to remove nitrate. ${ }^{126}$ Using 5\%CU-nZVI, nitrate conversion increased 3.6 times compared to nZVI. The reactivity of Cu-nZVI also increased when nitrate was reduced through a packed sand column. Hosseini et al. reported that the reducing performance of Cu-nZVI (2.5\% Cu w/w) was higher than that of $\mathrm{nZVI}$ but the increase was minimal (only 7\%). ${ }^{181}$ Nitrate conversion was $82 \%$ and $89 \%$ using either $\mathrm{nZVI}$ or $2.5 \% \mathrm{Cu}-\mathrm{nZVI}$, respectively. In contrast, this is inconsistent with the study in which Cu-nZVI@zeolite and nZVI@zeolite were examined. The researchers reported that there was no difference in the reactivity between using Cu-nZVI@zeolite and nZVI@zeolite in nitrate reduction, 100\% of nitrate (100 ppm) was reduced using both materials after 6 hours. ${ }^{194}$

However, supported Cu-nZVI@Misi materials were more active than reported unsupported Cu-nZVI. For example, when the same mass ratio of Fe and nitrate (15:1) was used, all nitrate$\mathrm{N}$ (13 ppm) was removed at pH 5 after 60 minutes using $5 \% \mathrm{Cu}-\mathrm{nZVI}{ }^{180}$ compared to all nitrate$\mathrm{N}$ (10 ppm) was removed at neutral pH after less than 30 minutes using 5\%Cu-nZVI@Misi. In comparison with reported supported Cu-nZVI, Cu-nZVI@Misi materials were more active. When the initial nitrate- $\mathrm{N}$ concentration was $50 \mathrm{ppm}$, the molar ratio of Fe to nitrate was 3:1 and the reaction was conducted in unadjusted $\mathrm{pH}$, nitrate conversion was from $46 \%$ to $90 \%$ after 30 minutes. In contrast, in the investigation of Cu-nZVI@zeolite, the initial nitrate-N concentration was $22.6 \mathrm{ppm}$ (100 ppm nitrate), molar ratio of Fe to nitrate was 27:1 (nZVI dosage of $2.46 \mathrm{~g} / \mathrm{L}$ ), the initial solution $\mathrm{pH}$ was 5.7, nitrate removal took 6 hours. ${ }^{194}$ As such, these supported 5\%Cu-nZVI@Misi systems, especially 5\%Cu-nZVI@MisiCFeOOH-MB1 will attribute to the new list of effective materials towards nitrate reduction.

Liou et al. proposed the mechanism of nitrate reduction using bimetallic $\mathrm{Cu}-\mathrm{ZVI}{ }^{236}$ In this study, Fe donates electrons $\left(\mathrm{E}^{0}=-0.44 \mathrm{~V}\right)$ to the catalytic $\mathrm{Cu}$ and water. Water accepts electrons $\left(E^{0}=-0.41 \mathrm{~V}\right)$ to produce $\mathrm{H}_{\mathrm{ads}}$ that adsorbs on the surface of $\mathrm{Cu}$ in the form of $\mathrm{Cu}$ -

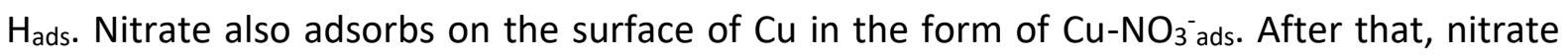
reacts with $\mathrm{H}_{\mathrm{ads}}$ on the $\mathrm{Cu}$ surface to generate nitrite and then final products such as ammonium. In materials prepared via deposition method (MB1), $\mathrm{Cu}$ is well-dispersed on the surface of nZVI particles (Figure 3.27), which offers more Cu sites for nitrate to adsorb on the surface. This is beneficial for the conversion step from nitrate into nitrite because $\mathrm{Cu}$ surfaces are the ideal substrate for nitrate adsorption and then reduction to nitrite. ${ }^{190}$ 


$$
\begin{aligned}
& \mathrm{Fe} \longrightarrow \mathrm{Fe}^{2+}+2 \mathrm{e}^{-} \\
& \mathrm{Cu}+\mathrm{H}_{2} \mathrm{O}+\mathrm{e}^{-} \longrightarrow \mathrm{Cu}-\mathrm{H}_{\mathrm{ads}}+\mathrm{OH}^{-} \\
& \mathrm{Cu}+\mathrm{NO}_{3}^{-}(\mathrm{aq}) \rightleftharpoons \mathrm{Cu}-\mathrm{NO}_{3}^{-} \text {ads } \\
& \mathrm{Cu}-\mathrm{NO}_{3}^{-}+\mathrm{Cu}-\mathrm{H}_{\mathrm{ads}}+\mathrm{e}^{-} \longrightarrow 2 \mathrm{Cu}+\mathrm{NO}_{2}^{-}+\mathrm{OH}^{-} \\
& \mathrm{Cu}-\mathrm{NO}_{2}^{-}+5 \mathrm{Cu}-\mathrm{H}_{\mathrm{ads}}+\mathrm{e}^{-} \longrightarrow 6 \mathrm{Cu}+\mathrm{NH}_{3}+2 \mathrm{OH}^{-} \\
& \mathrm{NH}_{3}+\mathrm{H}_{2} \mathrm{O} \longrightarrow \mathrm{NH}_{4}^{+}+\mathrm{OH}^{-}
\end{aligned}
$$

In addition to nitrate, the nitrite concentrations were also monitored for our Cu-doped nZVI systems. The accumulation of nitrite was dependent upon synthetic methods to prepare materials. Nitrite levels were 2 - 5 times greater when materials prepared via deposition method (MB1) rather than co-reduction method (MA) were used (Figure 4.20). After 5 minutes, nitrite- $\mathrm{N}$ concentrations were $14.6 \pm 0.8 \mathrm{ppm}$ and $18 \pm 3 \mathrm{ppm}$ using either $5 \% \mathrm{Cu}$ nZVI@MisiC-MB1 or 5\%Cu-nZVI@MisiCFeOOH-MB1. After 30 minutes, nitrite-N concentrations decreased, $15.84 \pm 0.09$ ppm (32\% nitrite accumulation) and $9 \pm 1$ ppm (18\% nitrite accumulation) using either 5\%Cu-nZVI@MisiC-MB1 or 5\%Cu-nZVI@MisiCFeOOH-MB1, respectively. In contrast, nitrite-N levels were about 3 - 4 ppm when either $5 \% \mathrm{Cu}$ nZVI@MisiC-MA or 5\%Cu-nZVI@MisiCFeOOH-MA was used. The higher nitrite concentrations observed in Cu-doped nZVI@Misi-MB1 systems can be explained by the faster nitrate reduction and potentially slower nitrite reduction of these materials compared to those using materials prepared via co-reduction method (MA). Furthermore, in Cu-doped materials, the conversion of nitrate and nitrite had different behaviour. The faster nitrate reduction was, the slower nitrite conversion was, which results in the higher nitrite accumulation. This is inconsistent with $\mathrm{Ni}$-doped materials. 


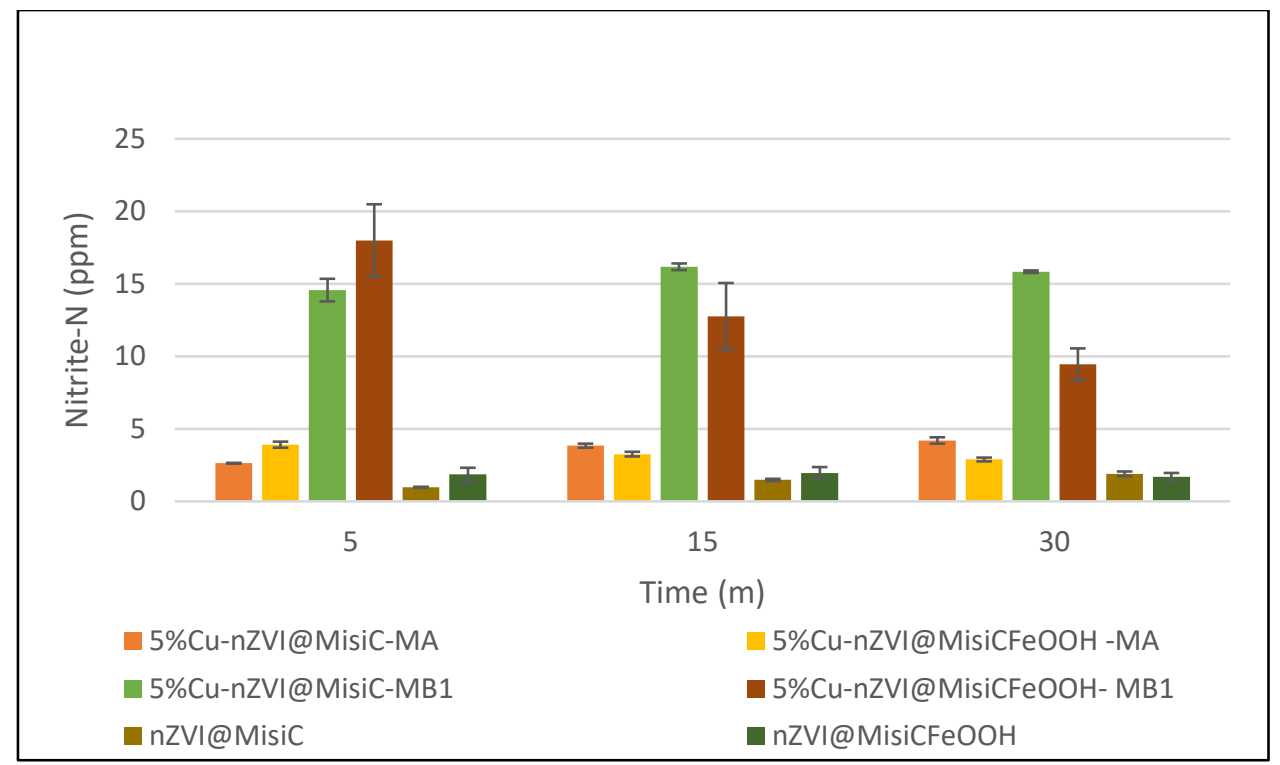

Figure 4.20. Nitrite accumulation using 5\%Cu-nZVI@Misi towards nitrate-N 50 ppm reduction under anaerobic conditions $(0.150 \mathrm{~g}$ of materials, $25.0 \mathrm{~mL}$ of solution).

There is also a difference in the nitrite accumulation when different preparations of Misi were used, with nitrite concentrations observed in materials using MisiCFeOOH were less than those using MisiC. The difference was minimal, regarding materials prepared via co-reduction method (MA) while it was more significant with regards to materials prepared via deposition method (MB1).

Nitrite accumulated in Cu-doped systems was higher than that of non-doped nZVI@MisiCFeOOH. Particularly, nitrite in Cu-doped systems prepared via method deposition (MB1) was up to eight times greater than that of non-doped systems. This can be partially explained by the difference in the nitrate conversion and then nitrite reduction. $\mathrm{Cu}$-doped nZVI@Misi reduced nitrate significantly faster than non-doped nZVI@Misi, therefore much more nitrite was formed and accumulated. The higher nitrite build-up in Cu-doped systems was consistent with that reported by Liou et al..126 However, in their study, nitrite accumulation was significantly higher than that observed in our systems. When the initial nitrate- $\mathrm{N}$ concentration was $40 \mathrm{ppm}$ and the molar ratio of Fe to nitrate was 2.1:1 using $\mathrm{Cu}$ nZVI, after 120 minutes, nitrite level-N was around 16 ppm (40\% nitrite accumulation). Therefore, a new finding observed in supported 5\%Cu-nZVI@Misi is that the presence of Misi helped speed up nitrite conversion, potentially due to the higher reactivity of supported materials. 
In summary, coating a FeOOH layer in the pre-treatment procedure of Misi plays an important role in enhancing the reactivity of $\mathrm{Cu}-\mathrm{nZVI}$ nanoparticles as well as minimising the build-up of nitrite. Using the deposition method (MB1) is successful in doping a secondary metal on the surface of $\mathrm{nZVI}$. Consequently, the materials prepared via deposition method (MB1) are more active than those prepared via co-reduction method (MA) towards nitrate reduction.

The change in the morphology of materials during the reduction of nitrate was investigated via SEM. We focused on 5\%Cu-nZVI@MisiCFeOOH prepared via deposition method (MB1) and monitored changes in its morphology under two different reaction conditions: after reduction of $10 \mathrm{ppm}$ nitrate for 15 minutes and after reduction of $50 \mathrm{ppm}$ nitrate for 120 minutes. After nitrate reduction, each material was washed with deoxygenated deionised water, followed by deoxygenated ethanol, and then dried under vacuum. COMPO images revealed the presence of silicate and nanoparticles in both samples, with some nanoparticles unevenly dispersed on the surface of the silicate. After nitrate reduction, the composition of materials is primarily silicate with significantly fewer nanoparticles (Figure 4.21) than prior to reaction (Figure 3.23d). Additionally, there is a significant level of agglomeration of nanoparticles in 5\%Cu-nZVI@MisiCFeOOH-MB1 after reduction of 10 ppm nitrate for 15 minutes whereas only a few islands of particles are observed in the material after reduction of 50 ppm nitrate for 120 minutes.

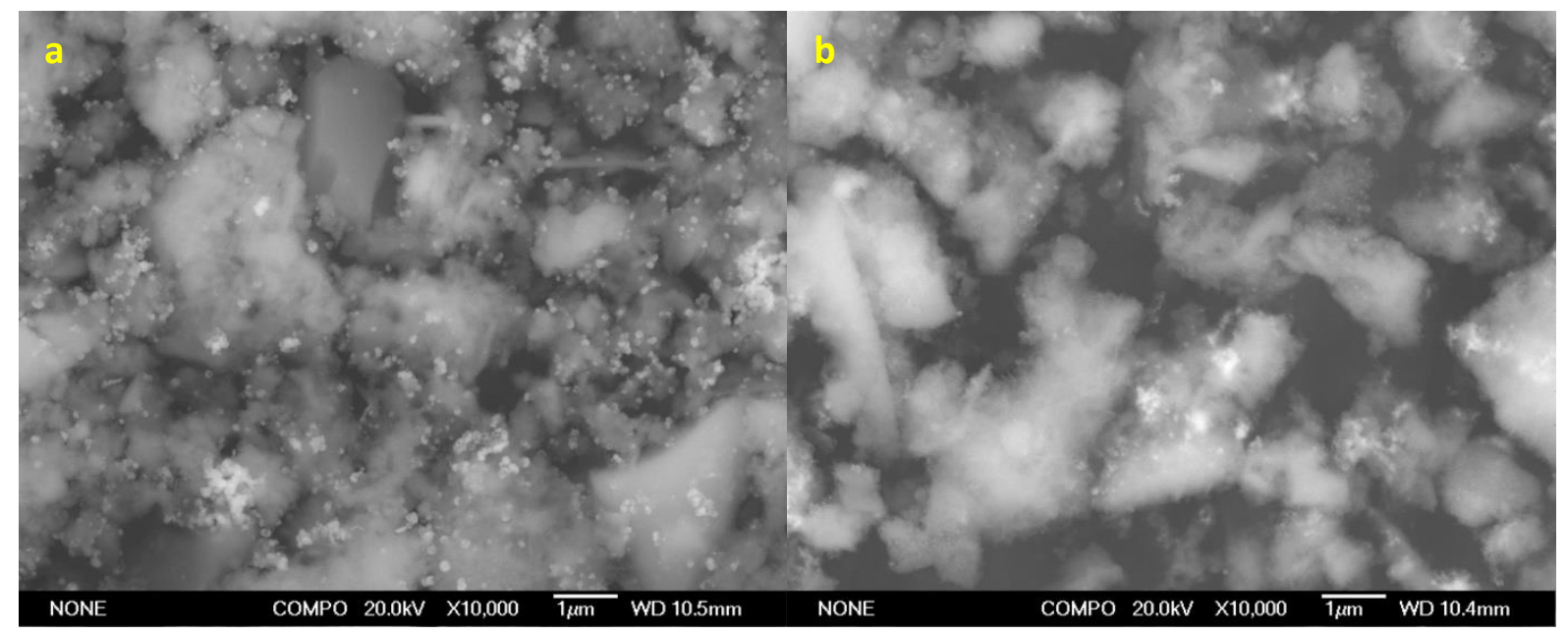

Figure 4.21. COMPO images of 5\%Cu-nZVI@MisiCFeOOH-MB1 after nitrate reduction of (a) $10 \mathrm{ppm}$ and (b) $50 \mathrm{ppm}$. 


\subsection{Nitrate reduction with trimetallic Pd-Cu-nZVI@Misi under anaerobic conditions}

There have several investigations on the effect of both $\mathrm{Pd}$ and $\mathrm{Cu}$ on the reactivity of $\mathrm{nZVI}$ materials. However, these trimetallic Pd-Cu-nZVI were unsupported materials with high concentration of total dopant metals (around 9\% - 10\%) and high ratio of $\mathrm{Pd}$ to $\mathrm{Cu}(2: 1)$. ${ }^{4-5 \text {, }}$ 247 In this study, the reactivity of $0.5 \% \mathrm{Pd}-5 \% \mathrm{Cu}-\mathrm{nZVI}$ supported on Misi towards nitrate, as determined by nitrate conversion and nitrite accumulation, was examined. To do this, four

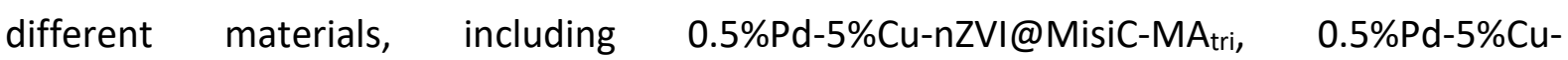
nZVI@MisiCFeOOH-MAtri, $\quad 0.5 \% P d-5 \% C u-n Z V I @ M i s i C-M C 1 \quad$ and 0.5\%Pd-5\%CunZVI@MisiCFeOOH-MC1 were used. Two different nitrate-N concentrations, including 10 ppm and 50 ppm, were examined.

The reactivity of trimetallic Pd-Cu-doped materials was a function of the synthetic method when 10 ppm nitrate-N was used. Materials prepared via deposition method (MC1) reduced nitrate much faster than materials prepared via co-reduction method ( $M A_{\text {tri }}$ ) (Figure 4.22). In addition, materials on MisiCFeOOH were more reactive than those on MisiC, regardless of the synthetic method. After 5 minutes, nitrate- $\mathrm{N}$ concentrations were more than $7.5 \mathrm{ppm}$ and after 15 minutes, nitrate- $\mathrm{N}$ concentrations were more than $2 \mathrm{ppm}$ when either material prepared via method co-reduction (MA) was used. In contrast, after 5 minutes, nitrate-N concentrations were below $1.3 \mathrm{ppm}$ and after 15 minutes, nitrate- $\mathrm{N}$ concentrations were below the detection limit of the IC $(0.06 \mathrm{ppm})$ when either material prepared via deposition method (MC1) was used. However, after 30 minutes, nitrate levels were below the detection limit for all types of trimetallic Pd-Cu-nZVI@Misi systems except for 0.5\%Pd-5\%CunZVI@MisiC-MA tri, where the nitrate-N concentration was about 2.6 ppm. These results suggested that $0.5 \% \mathrm{Pd}-5 \% \mathrm{Cu}-\mathrm{nZVI@MisiCFeOOH-MC1}$ was the best trimetallic material. The effectiveness of coating $\mathrm{FeOOH}$ layer on Misi and using the deposition method was highlighted in improving the reactivity of trimetallic systems. 


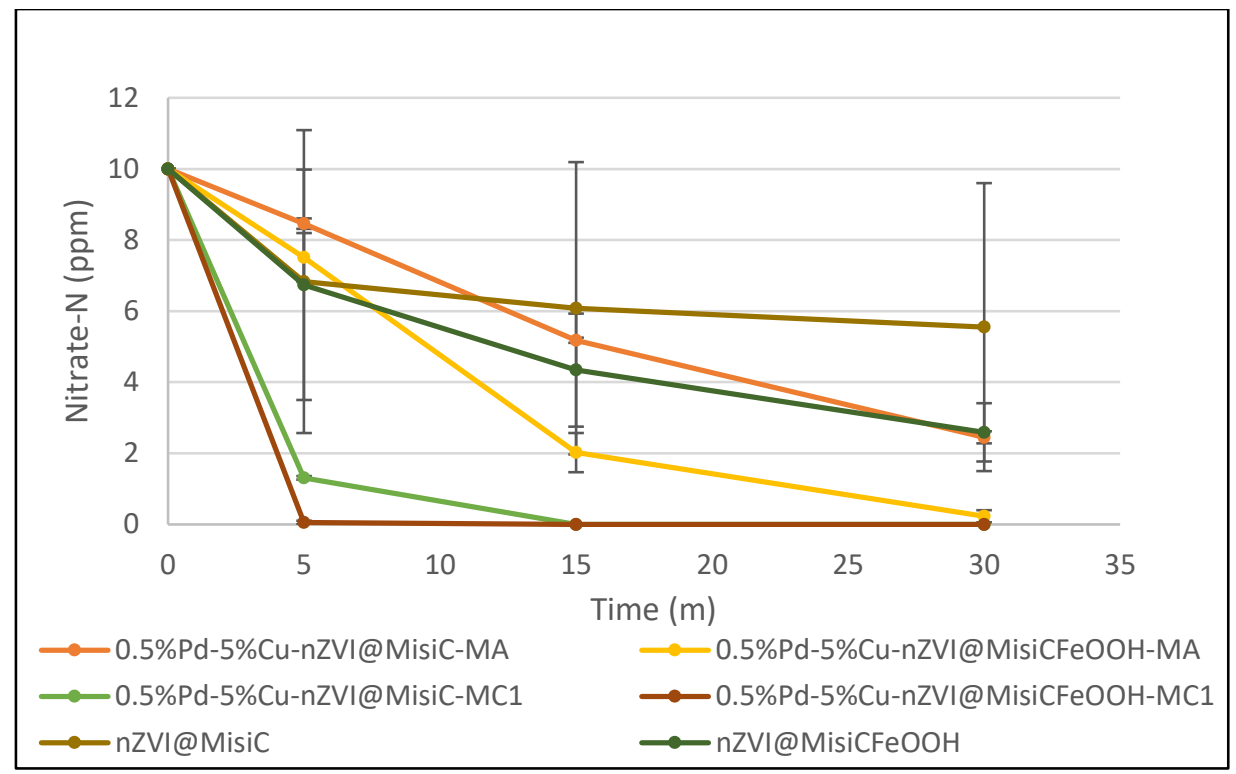

Figure 4.22. Reduction characterisation of 0.5\%Pd-5\%CU-nZVI@Misi towards nitrate-N 10 ppm under anaerobic conditions (0.150 $\mathrm{g}$ of materials, $25.0 \mathrm{~mL}$ of solution).

The reactivity of Pd-Cu-nZVI@Misi was compared to that of non-doped nZVI@Misi as the control material. Pd-Cu-doped materials prepared via deposition method (MC1) reduced nitrate faster than non-doped nZVI@Misi, regarless of Misi preparation over the course of 30 minutes. However, Pd-Cu-doped materials prepared via co-reduction method (MA) reduced nitrate more slowly than non-doped nZVI@Misi after 5 minutes and the contrast was observed after 15 minutes.

Nitrite was also detected in the reduction of $10 \mathrm{ppm}$ nitrate-N (Figure 4.23). After 5 minutes, nitrite- $\mathrm{N}$ concentrations were about 1 ppm using either 0.5\%Pd-5\%Cu-nZVI@MisiC-MA tri, 0.5\%Pd-5\%Cu-nZVI@MisiCFeOOH-MA tri or 0.5\%Pd-5\%Cu-nZVI@MisiC-MC1. In contrast, nitrite-N concentration was 3 ppm when 0.5\%Pd-5\%Cu-nZVI@MisiCFeOOH-MC1 was used. However, after 15 minutes, nitrite- $\mathrm{N}$ was only detected in materials prepared via co-reduction method $\left(\mathrm{MA}_{\text {tri }}\right)$, where the nitrite- $\mathrm{N}$ concentrations were less than $1 \mathrm{ppm}$. The conversion of nitrite depended on Misi preparation. Materials based on MisiCFeOOH reduced nitrite more slowly than materials based on MisiC, leading to the lower nitrite accumulation. Combining with the results of nitrate reduction, it can be concluded that $\mathrm{Pd}$-Cu-doped nZVI@MisiCFeOOH had higher reactivity in both nitrate and nitrite conversion. 


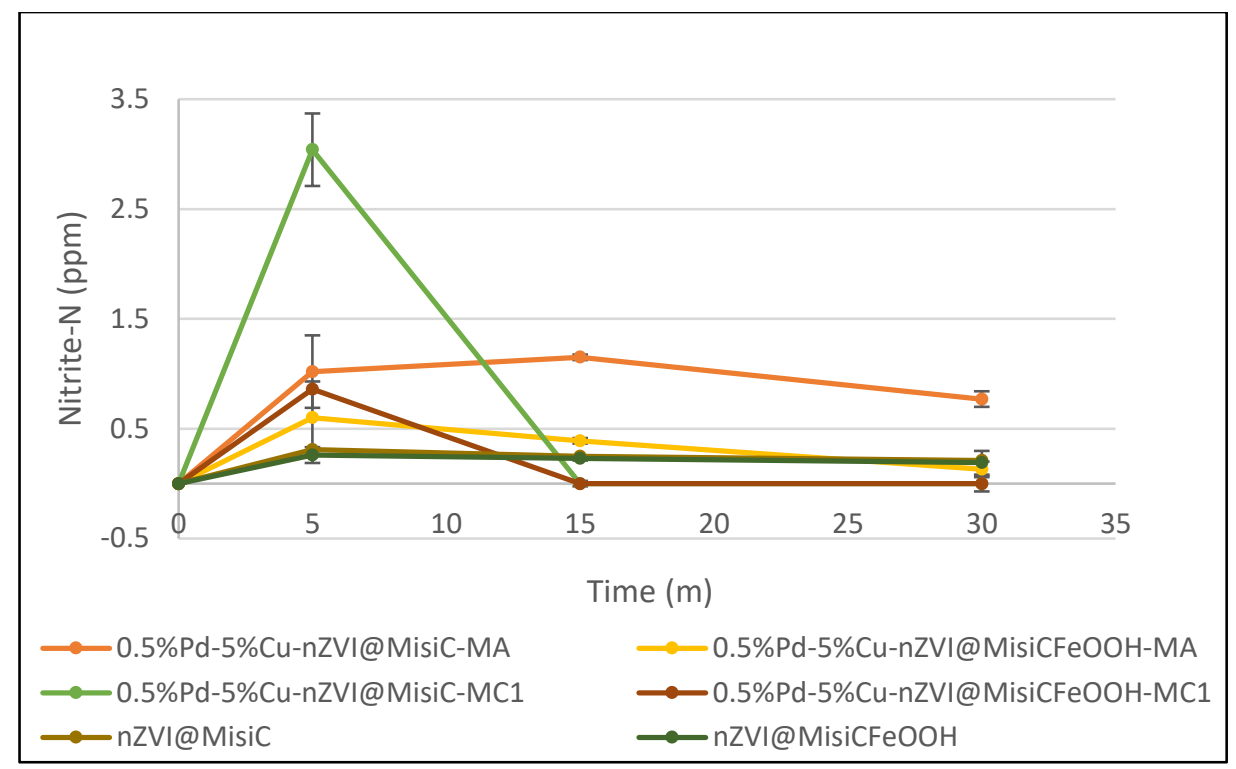

Figure 4.23. Nitrite accumulation using 0.5\%Pd-5\%Cu-nZVI@Misi towards nitrate-N 10 ppm reduction under anaerobic conditions ( $0.150 \mathrm{~g}$ of materials, $25.0 \mathrm{~mL}$ of solution).

As a control material, the nitrite production using $\mathrm{Pd}$-Cu-doped materials was compared to that of non-doped nZVI@Misi. After 5 minutes, nitrite levels accumulated by Pd-Cu-doped materials were higher than that produced by non-doped nZVI@Misi, especially 0.5\%Pd-5\%CunZVI@MisiC-MC1 where nitrite level was three times greater. After 15 minutes, 0.5\%Pd5\%Cu-nZVI@Misi prepared via co-reduction method (MA tri $\left._{1}\right)$ accumulated more nitrite while 0.5\%Pd-5\%Cu-nZVI@Misi prepared via deposition method (MC1) accumulated less nitrite than non-doped nZVI@Misi; however, this difference was minimal.

The difference in the reactivity of trimetallic materials was exemplified when 50 ppm nitrate$\mathrm{N}$ was used. Materials prepared via deposition method (MC1) had significantly greater reactivity than those prepared via co-reduction method ( $\left.M A_{\text {tri }}\right)$ (Figure 4.24). When either MisiC or MisiCFeOOH was used as a support, materials prepared via deposition method (MC1) reduced nitrate 2 - 4 times faster than materials prepared co-reduction method $\left(\mathrm{MA}_{\text {tri }}\right)$. For example, after 5 minutes, nitrate- $\mathrm{N}$ levels were $39 \pm 2 \mathrm{ppm}$ and $17 \pm 3 \mathrm{ppm}$ using either 0.5\%Pd-5\%Cu-nZVI@MisiCFeOOH-MA or 0.5\%Pd-5\%Cu-nZVI@MisiCFeOOH-MC1, respectively. After 30 minutes, nitrate- $\mathrm{N}$ levels were $19.3 \pm 0.8$ ppm (61\% nitrate conversion) and $5 \pm 2$ ppm (90\% nitrate conversion), respectively. 


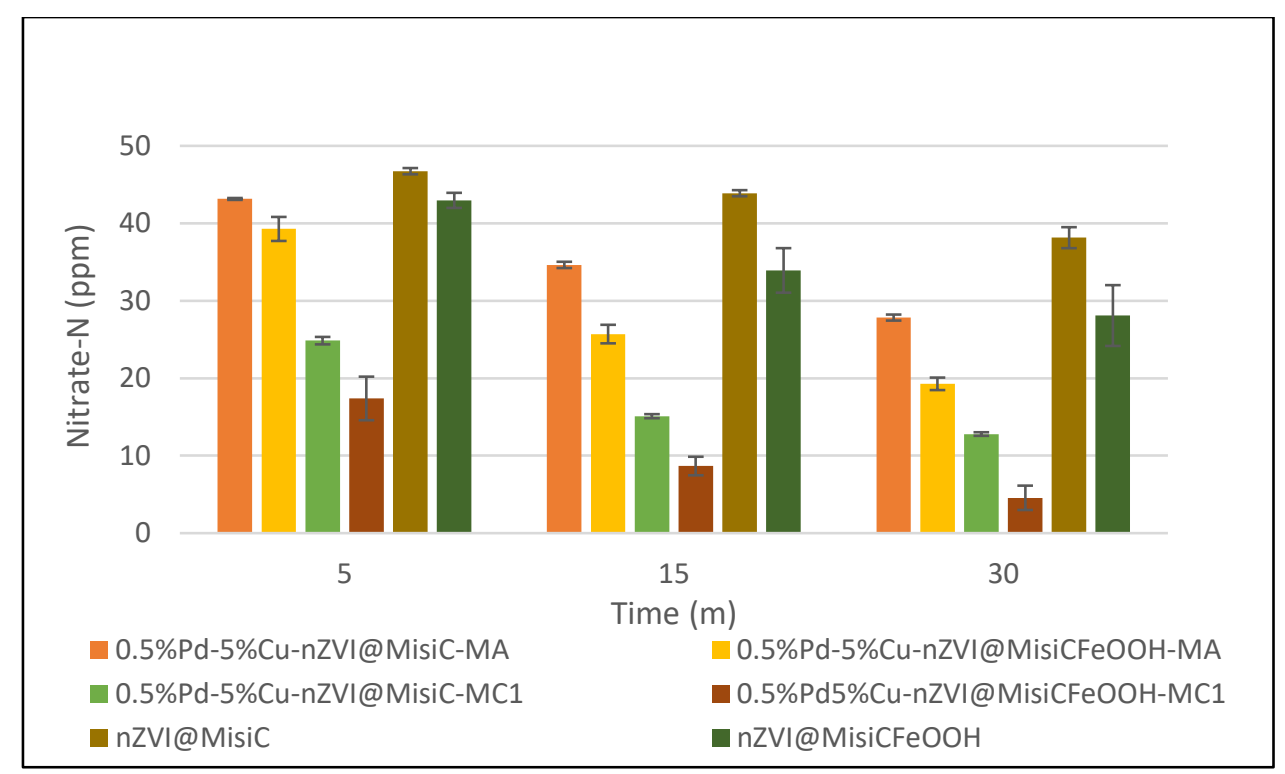

Figure 4.24. Reduction characterisation of 0.5\%Pd-5\%Cu-nZVI@Misi towards nitrate-N 50 ppm under anaerobic conditions $(0.150 \mathrm{~g}$ of materials, $25.0 \mathrm{~mL}$ of solution).

Moreover, materials on $\mathrm{MisiCFeOOH}$ were more active than those on MisiC, regardless of synthetic method. The nitrate reduction using Pd-Cu-nZVI on MisiCFeOOH was up to 3 times faster than Pd-CU-nZVI on MisiC. For example, after 5 minutes, nitrate-N levels were $24.9 \pm$ 0.5 ppm and $17 \pm 3$ ppm when either 0.5\%Pd-5\%Cu-nZVI@MisiC-MC1 or 0.5\%Pd-5\%CunZVI@MisiCFeOOH-MC1 was used. After 30 minutes, nitrate-N levels were $12.8 \pm 0.2$ ppm (74\% nitrate conversion), and $5 \pm 2 \mathrm{ppm}$ (90\% nitrate conversion), respectively. Based on these results, 0.5\%Pd-5\%Cu-nZVI@MisiCFeOOH-MC1 performed the highest reactivity while the lowest nitrate conversion was obtained by 0.5\%Pd-5\%Cu-nZVI@MisiC-MA tri.

In comparison with non-doped nZVI@Misi, the reactivity of all types of Pd-Cu-doped systems was higher, regardless of the synthetic method and Misi preparation. After 5 minutes, nitrate levels using non-doped nZVI@Misi were up to three times greater than that observed in PdCU-nZVI@Misi systems. After 30 minutes, nitrate conversion observed in Pd-Cu-doped systems was 2 - 3 times greater than that observed in non-doped nZVI@Misi. The difference in the reactivity between $\mathrm{Pd}$-Cu-doped materials prepared via co-reduction method $\left(\mathrm{MA}_{\text {tri }}\right)$ and non-doped nZVI@Misi in nitrate reduction of 50 ppm was inconsistent with that in nitrate reduction of $10 \mathrm{ppm}$. The reason for this phenomenon is not certain but it could be due to the low concentration of nitrate $(10 \mathrm{ppm})$, resulting in data that were less reliable than data collected when a higher concentration of nitrate-N (50 ppm) was used. 
In the reduction of $50 \mathrm{ppm}$ nitrate, the catalytic role of $\mathrm{Pd}-\mathrm{Cu}$ was evident. Our results are partly consistent with an investigation that was reported by Lubphoo et al. ${ }^{4-5}$ In these studies, unsupported trimetallic Pd-Cu-nZVI was synthesised by the deposition method (MC1). The ratio of $\mathrm{Pd}$ to $\mathrm{Cu}$ was either 1:1 or 2:1 and the total mass ratio of dopant metals to Fe was 1:10. In nitrate reduction, the initial nitrate- $\mathrm{N}$ concentration was $22.6 \mathrm{ppm}$ and molar ratio of Fe to nitrate was 2.8:1. Using this system, nitrate reduction was higher than $\mathrm{nZVI}$ after 30 minutes. The postulated reason for the improvement in reactivity was due to $\mathrm{Cu}$ and $\mathrm{Pd}$ acting as the catalysts and helping facilitate electron transfer. However, in their studies, the introduction of Pd increased the selectivity of nitrogen gas that was not found in our study. In contrast, our results are also inconsistent with their results as nZVI reduced nitrate faster than trimetallic systems after 20 minutes. They postulated that $\mathrm{nZVI}$ reacted with nitrate via direct electron transfer from Fe while trimetallic materials reduced nitrate via $\mathrm{H}$ atom that was generated from Fe corrosion with water and then adsorbed on the surface of $\mathrm{Cu}$ after which, nitrate and $\mathrm{H}_{\text {ads }}$ reacts on the surface of $\mathrm{Cu}$.

In addition to nitrate, the nitrite accumulated in Pd-Cu-doped materials was also monitored. Nitrite accumulation depended on the synthetic methods. Materials prepared via coreduction method $\left(\mathrm{MA}_{\text {tri }}\right)$ accumulated less nitrite than those being prepared via deposition method (MC1) (Figure 4.25). For materials prepared via co-reduction method ( $\left.M A_{\text {tri }}\right)$, nitrite$\mathrm{N}$ levels were approximately $4 \mathrm{ppm}$ over the course of 30 minutes. These levels were slightly higher than those observed in non-doped nZVI@Misi. In contrast, for materials prepared via deposition method (MC1), nitrite-N concentrations were in a range of $8-16 \mathrm{ppm}$. These levels were up to 9 times greater than those observed in non-doped nZVI@Misi. Specifically, after 5 minutes, nitrite- $\mathrm{N}$ concentration was around $15 \mathrm{ppm}$ for both types of $\mathrm{Pd}$-Cu-doped prepared via deposition method (MC1). After 30 minutes, nitrite- $\mathrm{N}$ concentrations were 16.3 $\pm 0.7 \mathrm{ppm}$ ( $33 \%$ nitrite accumulation) and $8 \pm 2 \mathrm{ppm}$ (16\% nitrite accumulation) when either 0.5\%Pd-5\%Cu-nZVI@MisiC-MC1 or 0.5\%Pd-5\%Cu-nZVI@MisiCFeOOH-MC1 was used, respectively. Combining the results of nitrate conversion and nitrite build-up, $0.5 \% \mathrm{Pd}-5 \% \mathrm{Cu}-$ nZVI@MisiCFeOOH-MC1 is the most effective trimetallic material in reducing nitrate and minimising the toxic nitrite formation. 


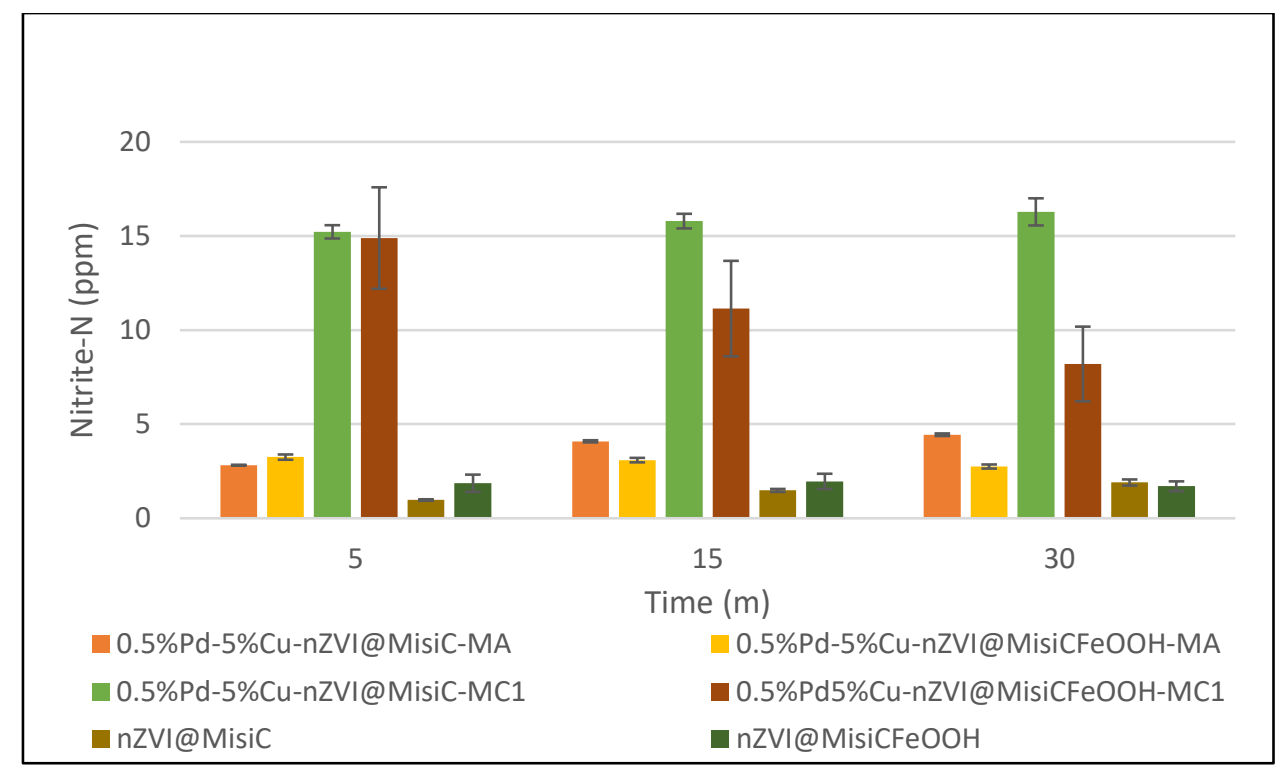

Figure 4.25. Nitrite accumulation using 0.5\%Pd-5\%Cu-nZVI@Misi towards nitrate-N 50 ppm reduction under anaerobic conditions ( $0.150 \mathrm{~g}$ of materials, $25.0 \mathrm{~mL}$ of solution).

The nitrate conversion and nitrite accumulation using supported trimetallic materials had opposite behaviours. The faster nitrate was converted, the more nitrite was built up. For example, 0.5\%Pd-5\%Cu-nZVI@Misi prepared via deposition method (MC1) removed nitrate faster than 0.5\%Pd-5\%Cu-nZVI@Misi prepared via co-reduction method ( $\left.M A_{\text {tri }}\right)$. Therefore, the higher nitrite accumulation was observed in 0.5\%Pd-5\%Cu-nZVI@Misi prepared via deposition method (MC1).This relation was similar to that of Cu-doped nZVI@Misi.

To determine the effect of $\mathrm{Pd}$ in $\mathrm{Pd}-\mathrm{Cu}$-doped materials towards nitrate reduction, the reactivity of $\mathrm{Pd}-\mathrm{Cu}$-doped and $\mathrm{Cu}$-doped materials was compared. When materials were synthesised via co-reduction method (MA), regardless Misi preparation, Pd-Cu-doped materials had similar reactivity to $\mathrm{Cu}$-doped materials. For example, after 30 minutes, the nitrate- $\mathrm{N}$ concentrations were $27 \pm 2$ ppm and $27.8 \pm 0.4$ ppm using either 5\%Cu-nZVI@MisiC$M A_{\text {tri }}$ or $0.5 \% \mathrm{Pd}-5 \% \mathrm{Cu}-\mathrm{nZVI@MisiC-MA}$ tri, respectively. When materials were prepared via the deposition method (MB1 and MC1), the effectiveness of the introduction of Pd was unclear. For example, after 5 minutes, 0.5\%Pd-5\%Cu-nZVI@MisiC-MB1 was more reactive than 5\%Cu-nZVI@MisiC-MB1, the nitrate-N concentrations were $24.9 \pm 0.5$ ppm and $28 \pm 1$ ppm, respectively. In contrast, after 30 minutes, 5\%Cu-nZVI@MisiC-MB1 was more reactive than $0.5 \% \mathrm{Pd}-5 \% \mathrm{Cu}-\mathrm{nZVI@MisiC-MB1}$, the nitrate-N concentrations were $10.0 \pm 0.7 \mathrm{ppm}$ and $12.8 \pm 0.2 \mathrm{ppm}$, respectively. In systems prepared using MisiCFeOOH, the reactivity of bimetallic $\mathrm{Cu}$-doped and trimetallic Pd-Cu-doped was similar. For example, after 30 minutes, 
the nitrate- $\mathrm{N}$ concentrations were $5 \pm 1 \mathrm{ppm}$ and $5 \pm 2 \mathrm{ppm}$ using either $5 \% \mathrm{Cu}$ nZVI@MisiCFeOOH-MB1 or 0.5\%Pd-5\%Cu-nZVI@MisiCFeOOH-MC1, respectively. As such, the addition of Pd was not significantly effective in the step converting nitrate to nitrite. This can be explained by the small amount of Pd in material (mass ratio of Pd to Misi is 0.5:100), presumably is not enough to activate its catalytic role. In addition, the Pd deposition changed the elemental distribution of Cu-doped material. In 5\%Cu-nZVI@MisiFeOOH-MB1, there is a good dispersion of $\mathrm{Cu}$ on the surface of nZVI (Section 3.5). However, the addition of Pd decreases the Cu signal on the surface of nZVI (Section 3.7).

Other authors reported that $\mathrm{Pd}$ is a place for nitrite adsorption prior to being reduced ${ }^{5}$ or a catalyst for nitrite reduction to final products. ${ }^{248}$ In order to examine the effect of $\mathrm{Pd}$, the reactivity of 5\%Cu-nZVI@MisiCFeOOH-MB1 and 0.5\%Pd-5\%Cu-nZVI@MisiCFeOOH-MC1 in reducing $20 \mathrm{ppm}$ of nitrite- $\mathrm{N}$ was tested. The results showed that the presence of Pd increased the nitrite conversion to final products (Figure 4.26) but the increase was minimal. After 5 minutes, the nitrite-N levels were $5.7 \pm 0.6 \mathrm{ppm}$ and $3.7 \pm 0.4 \mathrm{ppm}$ and after 15 minutes, the nitrite- $\mathrm{N}$ levels were $0.75 \pm 0.08 \mathrm{ppm}$ and $0.18 \pm 0.02 \mathrm{ppm}$ when either $5 \% \mathrm{Cu}$ nZVI@MisiCFeOOH-MB1 or 0.5\%Pd-5\%Cu-nZVI@MisiCFeOOH-MC1 was used. After 30 minutes, nitrite-N levels were less than $0.1 \mathrm{ppm}$ for both materials.

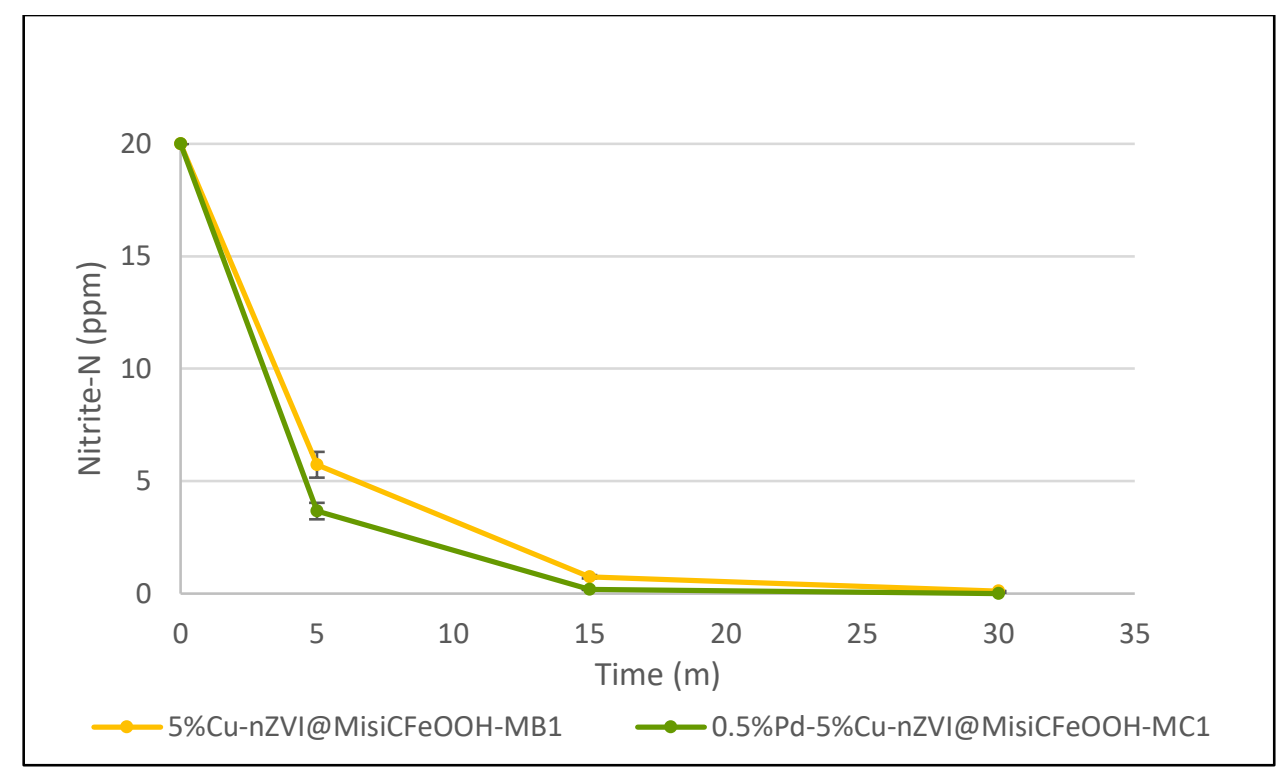

Figure 4.26. Reduction characterisation of 5\%CU-nZVI@MisiCFeOOH-MB1 and 0.5\%Pd5\%CU-nZVI@MisiCFeOOH-MC1 towards nitrite-N 20 ppm under anaerobic conditions $(0.150$ $g$ of materials, $25.0 \mathrm{~mL}$ of solution). 
According to Lubphoo et al., the mechanism of nitrate removal proceeds via both reduction of nitrate by electrons, directly transferred from Fe and by an adsorbed hydride, formed from the reduction of Fe with water. ${ }^{5}$ Fe plays a role of donating electrons to catalytic metals (Cu and $\mathrm{Pd}$ ) and then these electrons react with nitrate. In addition, Fe also donates electrons to water to produce $\mathrm{H}_{\mathrm{ads}}$ attaching on the surface of $\mathrm{Cu}$ and $\mathrm{Pd}$ in the form of $\mathrm{Cu}-\mathrm{H}_{\mathrm{ads}}$ and $\mathrm{Pd}$ $\mathrm{H}_{\mathrm{ads}}$, respectively. Nitrate in the solution adsorbs on the surface of $\mathrm{Cu}$ and then $\mathrm{Cu}-\mathrm{NO}_{3}{ }^{-}$ads reacts with $\mathrm{Cu}-\mathrm{H}_{\text {ads }}$ to form nitrite that adsorbs on the surface of Pd. Afterwards, nitrite is reduced by $\mathrm{Pd}-\mathrm{H}_{\mathrm{ads}}$ to form final products.

$$
\begin{aligned}
& \mathrm{Fe} \longrightarrow \mathrm{Fe}^{2+}+2 \mathrm{e}^{-} \\
& \mathrm{Fe}+2 \mathrm{H}_{2} \mathrm{O} \longrightarrow \mathrm{Fe}^{2+}+\mathrm{H}_{2}+2 \mathrm{OH}^{-} \\
& 2 \mathrm{Cu}+\mathrm{H}_{2} \longrightarrow 2 \mathrm{Cu}-\mathrm{H}_{\mathrm{ads}} \\
& 2 \mathrm{Pd}+\mathrm{H}_{2} \longrightarrow 2 \mathrm{Pd}-\mathrm{H}_{\mathrm{ads}} \\
& \mathrm{Cu}+\mathrm{NO}_{3}^{-}(\mathrm{aq}) \rightleftharpoons \mathrm{Cu}-\mathrm{NO}_{3}^{-}{ }^{-}{ }^{-} \\
& \mathrm{Cu}-\mathrm{NO}_{3}^{-}+\mathrm{Cu}-\mathrm{H}_{\mathrm{ads}}+\mathrm{e}^{-} \longrightarrow 2 \mathrm{Cu}+\mathrm{NO}_{2}^{-}+\mathrm{OH}^{-} \\
& \mathrm{Pd}+\mathrm{NO}_{2}^{-}(\mathrm{aq}) \rightleftharpoons \mathrm{Pd}-\mathrm{NO}_{2}^{-} \text {ads } \\
& \text { Pd-NO }{ }_{2}^{-}+5 \mathrm{Pd}-\mathrm{H}_{\mathrm{ads}}+\mathrm{e}^{-} \longrightarrow 6 \mathrm{Pd}+\mathrm{NH}_{3}+2 \mathrm{OH}^{-} \\
& \mathrm{NH}_{3}+\mathrm{H}_{2} \mathrm{O} \longrightarrow \mathrm{NH}_{4}^{+}+\mathrm{OH}^{-}
\end{aligned}
$$

There has been no investigation on the morphology of trimetallic nZVI after nitrate reduction. In this study, the change in morphology of Pd-Cu-doped materials was examined via SEM analysis. We focused on 0.5\%Pd-5\%Cu-nZVI@MisiCFeOOH-MC1 and monitored changes in its morphology under two different reaction conditions: after reduction of $10 \mathrm{ppm}$ nitrate- $\mathrm{N}$ at 15 minutes and after reduction of $50 \mathrm{ppm}$ nitrate- $\mathrm{N}$ at 120 minutes. After nitrate reduction, each material was washed with deoxygenated deionised water, followed by deoxygenated ethanol and then dried under vacuum to get resulting material. COMPO images also revealed the presence of silicate and nanoparticles in both samples but the density of nanoparticles changes. Significantly fewer nanoparticles are observed (Figure 4.27) than prior to reaction (Figure 3.42b). After reduction of $10 \mathrm{ppm}$ nitrate- $\mathrm{N}$ at 15 minutes, Fe particles are observed on Misi and they are still in the form of agglomeration. In contrast, after reduction of $50 \mathrm{ppm}$ nitrate- $\mathrm{N}$ at 120 minutes, there are few agglomerates on some islands of Misi. 


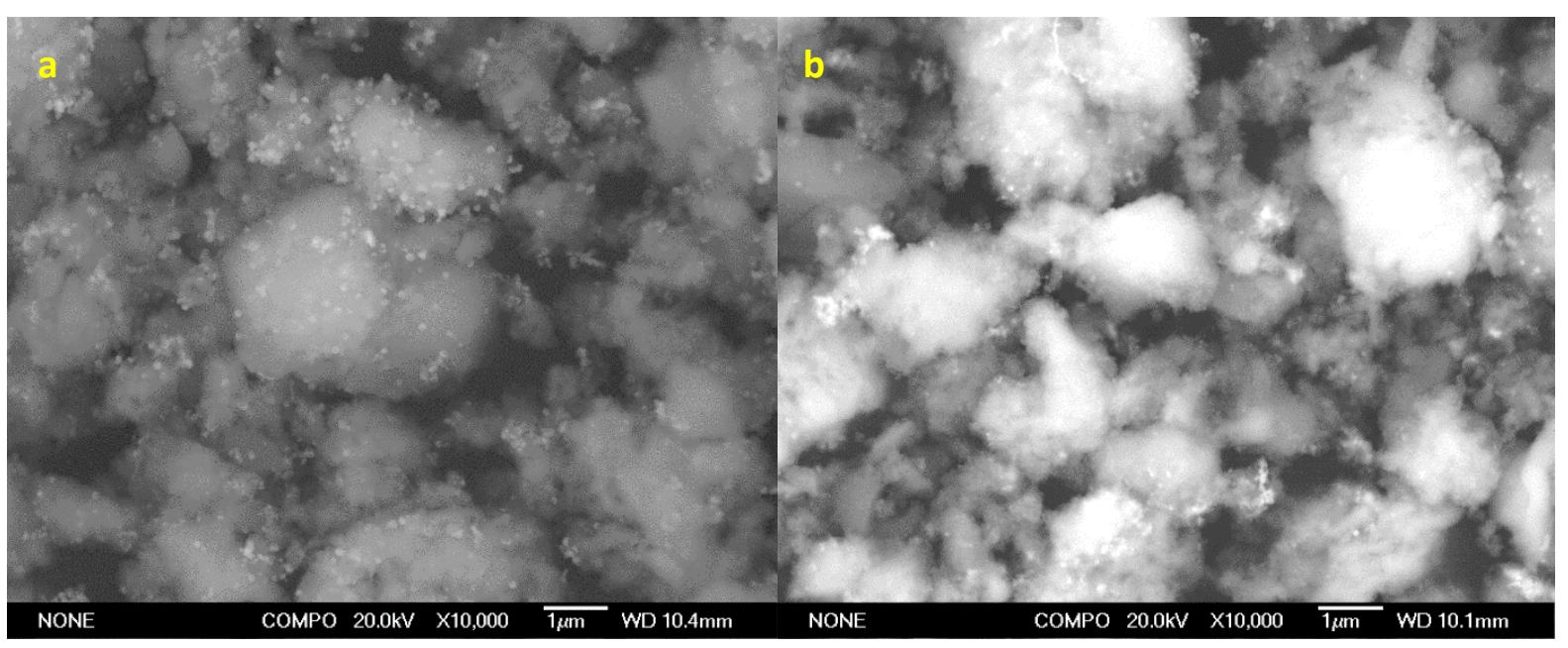

Figure 4.27. COMPO images of 0.5\%Pd-5\%Cu-nZVI@MisiCFeOOH-MC1 after nitrate- $N$ reduction of (a) $10 \mathrm{ppm}$ and (b) $50 \mathrm{ppm}$.

\subsection{Effect of reaction time and initial nitrate amount on nitrate reduction under} anaerobic conditions

\subsubsection{Effect of reaction time}

For the above examples, the maximum reaction time was 30 minutes. This arbitrary time limit was chosen to allow us to screen more materials. For the three most promising materials in each class, 5\%Ni-nZVI@MisiCFeOOH-MB1, 5\%Cu-nZVI@MisiCFeOOH-MB1 and 0.5\%Pd5\%Cu-nZVI@MisiCFeOOH-MC1, as well as non-doped nZVI@MisiCFeOOH, longer reaction periods were examined in order to determine the maximum nitrate removal capacity for each of these systems. To do this, the materials were added to $50 \mathrm{ppm}$ nitrate- $\mathrm{N}$ solution and the reactions were monitored in 180 minutes.

The nitrate removal was initially fast (in 30 minutes) and then gradually to the end of the test period (Figure 4.28) when either M-doped material or Pd-Cu-doped material was used. For example, when 5\%Ni-nZVI@MisiCFeOOH-MB1 was used, nitrate conversion was 72\% and $87 \%$ after 30 minutes and 180 minutes, respectively. The similar tendency was observed using either $\mathrm{Cu}$-doped material or Pd-Cu-doped material. The nitrate conversion was $91 \%$ and $97 \%$ when 5\%Cu-nZVI@MisiCFeOOH-MB1 was used, respectively. The nitrate conversion was 94\% and 99\% when 0.5\%Pd-5\%Cu-nZVI@MisiCFeOOH-MC1 was used, respectively. Considering the difference of nitrate conversion between 30 minutes and 180 minutes in each material, choosing 30 minutes as the course of the reaction in our research is reasonable because less than $14 \%$ of nitrate was reduced during a prolonged time of 150 minutes. 
In contrast, nZVI@MisiCFeOOH reduced nitrate gradually during the course of reaction time (180 minutes). After 30 minutes, nitrate conversion was 51\% and after 180 minutes, nitrate conversion was $86 \%$. Despite the same total nitrate conversion when either $5 \% \mathrm{Ni}$ nZVI@MisiCFeOOH-MB1 or nZVI@MisiCFeOOH was used, Ni-doped system reduced nitrate faster than the non-doped system in short-time period. These results confirmed the catalytic role of $\mathrm{Ni}$ as well as $\mathrm{Cu}$ in enhancing the reactivity of doped $\mathrm{nZVI}$ on Misi.

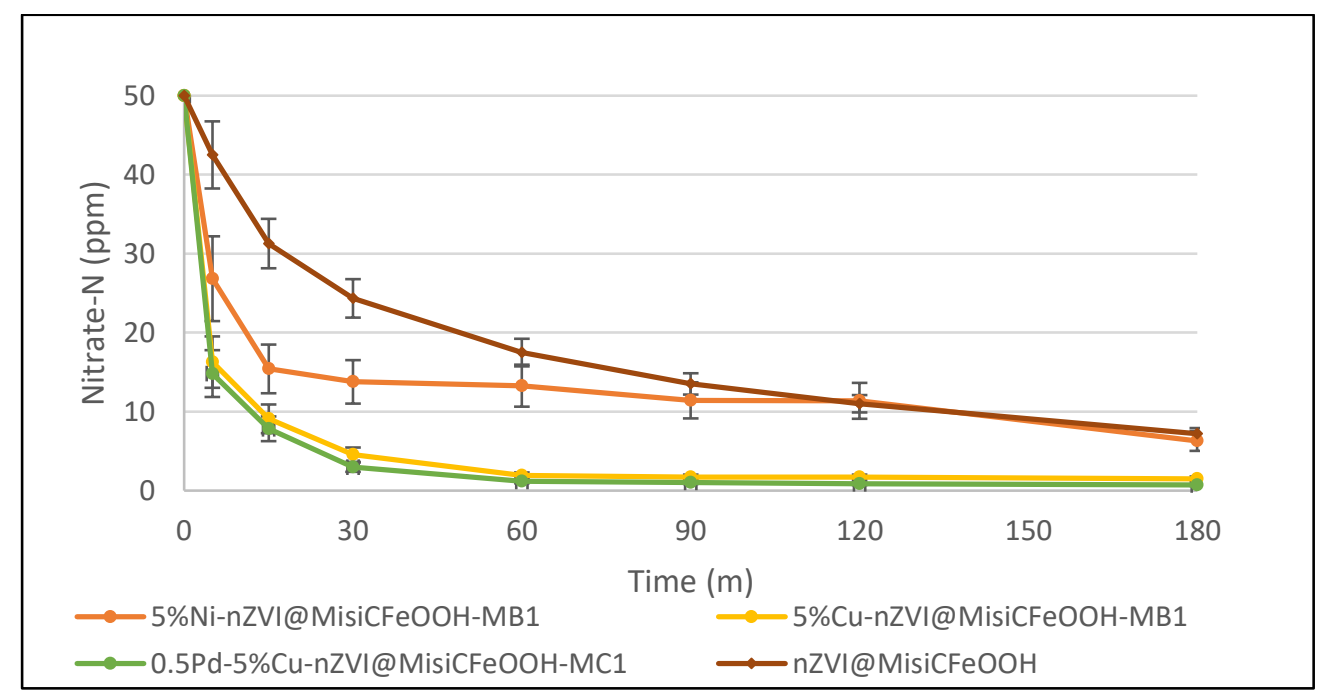

Figure 4.28. Effect of reaction time on nitrate reduction under anaerobic conditions $(0.150 \mathrm{~g}$ of materials, $25.0 \mathrm{~mL}$ of $50 \mathrm{ppm}$ nitrate- $N$ solution).

\subsubsection{Effect of initial nitrate amount}

The difference in the reactivity of metal-doped nZVI@ Misi and non-dop nZVI@Misi materials towards nitrate reduction was again observed when $100 \mathrm{ppm}$ of nitrate- $\mathrm{N}$ was used. For this, the four representative materials, including 5\%Ni-nZVI@MisiCFeOOH-MB1, 5\%CunZVI@MisiCFeOOH-MB1, 0.5\%Pd-5\%Cu-nZVI@MisiCFeOOH-MC1 and nZVI@MisiCFeOOH were used. Doped nZVI@Misi had higher reactivity than non-doped nZVI@Misi and the significant catalytic role towards nitrate conversion was observed in materials containing $\mathrm{Cu}$ (Figure 4.29). In addition, both Cu-doped and Pd-Cu-doped materials had the same reactivity in nitrate conversion. After 5 minutes, half of the initial nitrate (50 ppm) was removed using either $\mathrm{Cu}$-doped material or $\mathrm{Pd}-\mathrm{Cu}$-doped material and $32 \mathrm{ppm}$ nitrate-N was reduced using 5\%Ni-nZVI@MisiCFeOOH-MB1. In contrast, only 6 ppm of nitrate was reduced using nZVI@MisiCFeOOH. After 30 minutes, the nitrate conversion was 65\% for either Cu-doped material or Pd-Cu-doped material and 43\% for 5\%Ni-nZVI@MisiCFeOOH-MB1 while only 35\% 
was observed in nZVI@MisiCFeOOH. These results confirmed the important role of metaldoped in promoting nitrate reduction.

Comparing with the reduction of the $50-\mathrm{ppm}$ nitrate- $\mathrm{N}$ solution, a higher rate of nitrate removal was observed. For example, when 5\%Ni-nZVI@MisiCFeOOH-MB1 was used, after 5 minutes, nitrate- $\mathrm{N}$ concentration was from $100 \mathrm{ppm}$ to $68 \mathrm{ppm}$ ( $32 \mathrm{ppm}$ nitrate-N removed) while nitrate- $\mathrm{N}$ concentration decreased from $50 \mathrm{ppm}$ to $27 \mathrm{ppm}$ ( $23 \mathrm{ppm}$ nitrate-N removed) in the reduction of $100 \mathrm{ppm}$ and $50 \mathrm{ppm}$, respectively.

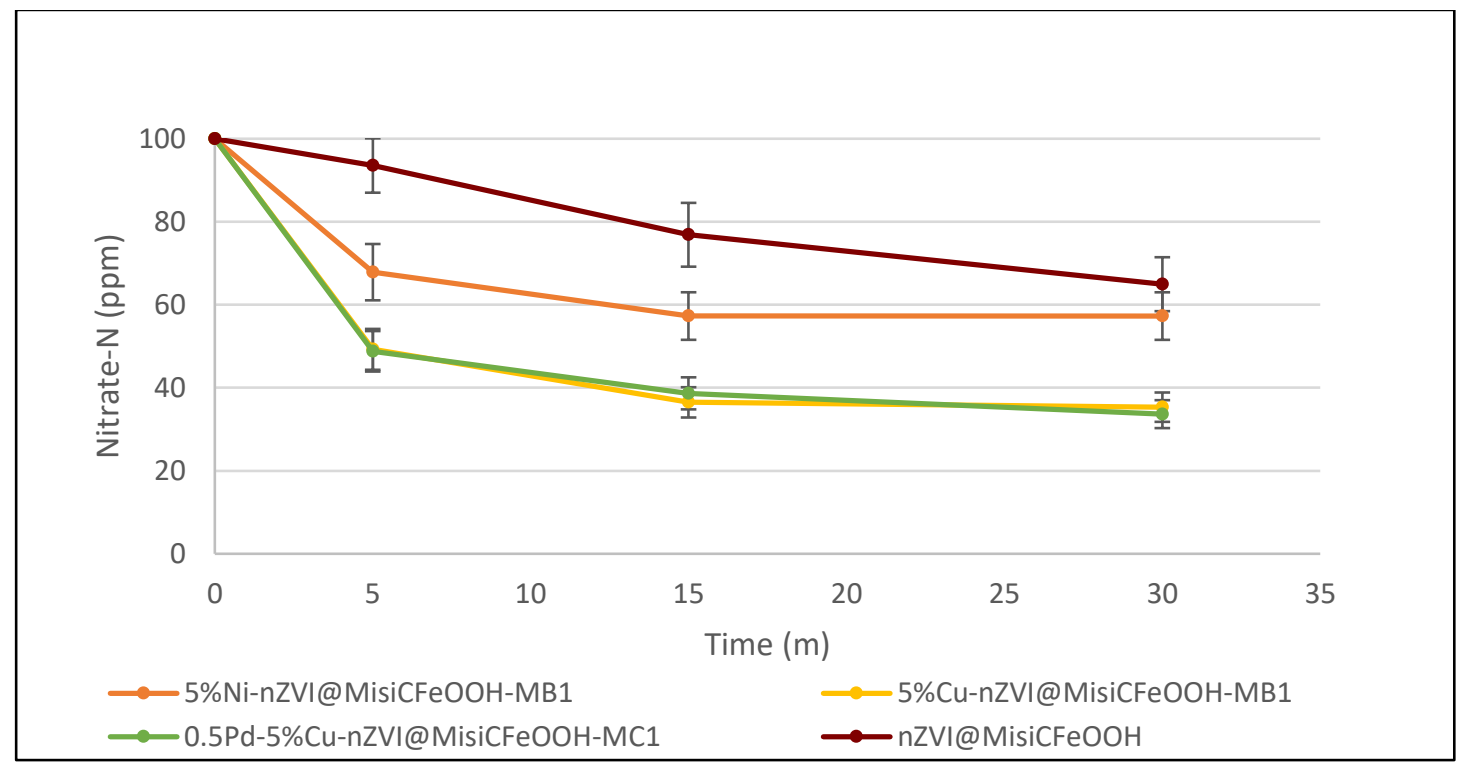

Figure 4.29. Reduction characterisation of representative materials towards nitrate-N 100 ppm under anaerobic conditions ( $0.150 \mathrm{~g}$ of materials and $25.0 \mathrm{~mL}$ of solution).

In terms of nitrite production, non-doped nZVI@MisiCFeOOH accumulated nitrite with the lowest level although it reduced nitrate the most slowly (Figure 4.30). Over the course of 30 minutes, nitrite levels were around $3.5 \mathrm{ppm}$. In contrast, both $\mathrm{Cu}$-doped and $\mathrm{Pd}$-Cu-doped materials reduced nitrate fastest, but they accumulated a significant amount of nitrite of around $25 \mathrm{ppm}$. In addition, after 5 minutes, significant amount of nitrite remained in solution (Figure 4.30). This could be due to the lack of the reductants. Potentially, most of the $\mathrm{Fe}(0)$ was consumed in the first step from nitrate to nitrite leading to less Fe participating the second step from nitrite to final products. As such, the nitrite concentrations were constant. 


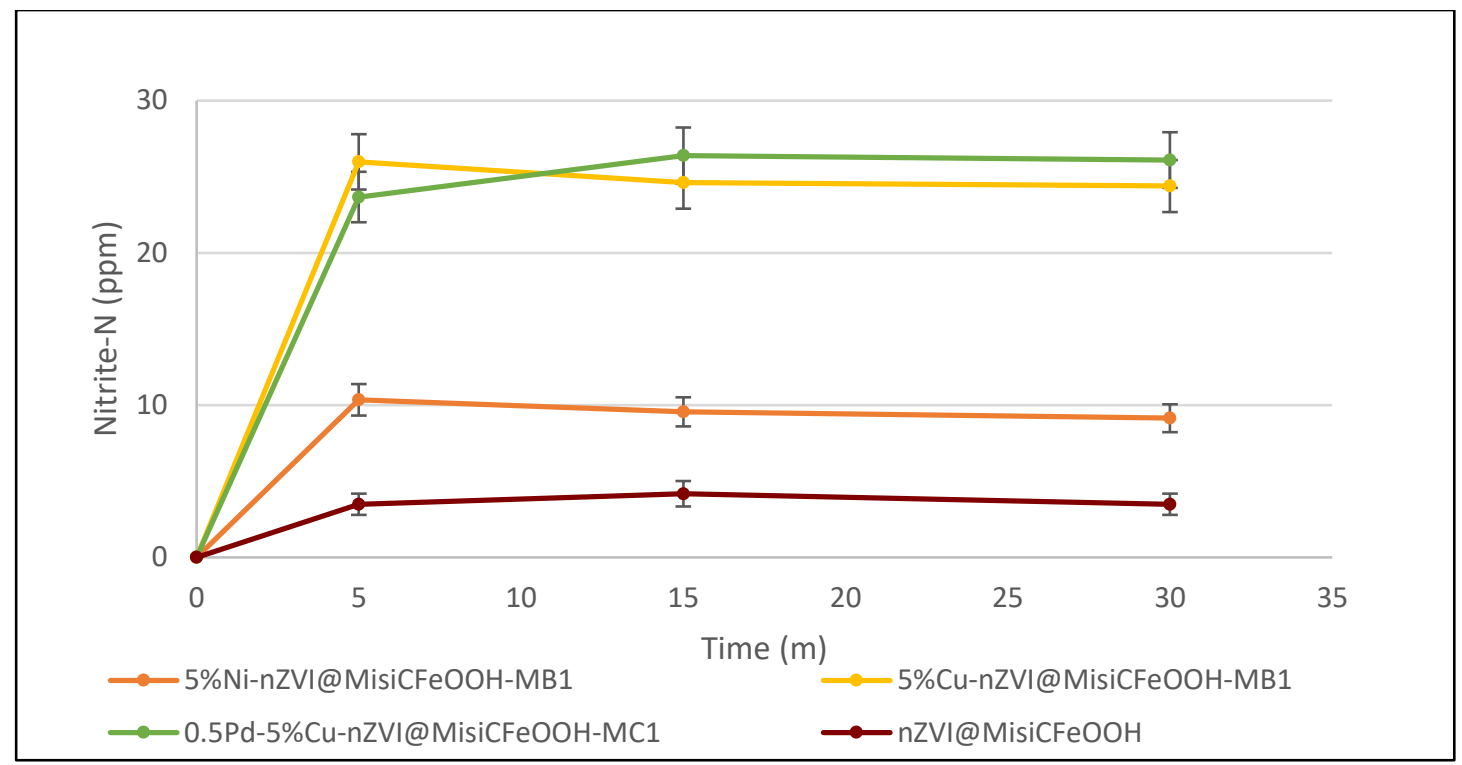

Figure 4.30. Nitrite accumulation using representative materials towards nitrate- $N 100$ ppm reduction under anaerobic conditions ( $0.150 \mathrm{~g}$ of materials and $25.0 \mathrm{~mL}$ of solution).

\subsection{Conclusion}

Doping metals on the surface of nZVI@Misi to generate supported bimetallic and trimetallic systems was an effective method in improving nitrate reduction. Misi that was calcined and coated with $\mathrm{FeOOH}$ was an efficient support in enhancing the reactivity of bimetallic nanoparticles. Additionally, doping metals on the surface of nZVI@Misi using deposition method (MB1 or MC1) increased the reactivity of the materials more significantly than using co-reduction method (MA or MAtri). As such, 5\%Ni-nZVI@MisiCFeOOH-MB1, 5\%CunZVI@MisiCFeOOH-MB1, and 0.5\%Pd-5\%Ni-nZVI@MisiCFeOOH-MC1 have potential to be used in nitrate remediation of "real" waters.

Among these doped materials, both 5\%Cu-nZVI@MisiCFeOOH-MB1 and 0.5\%Pd-5\%CunZVI@MisiCFeOOH-MC1 had a higher reactivity in nitrate conversion than 5\% NinZVI@MisiCFeOOH-MB1. However, Ni-doped material reduced nitrite to final products faster than Cu-doped and Pd-Cu-doped materials. As such, less nitrite was accumulated in $5 \% \mathrm{Ni}$ nZVI@MisiCFeOOH-MB1. Although the catalytic role of Pd in nitrate conversion was insignificant, the presence of $\mathrm{Pd}$ slightly increased nitrite reduction to final products relative to 5\%Cu-nZVI@MisiCFeOOH-MB1. 


\section{Effect of reaction conditions on nitrate reduction}

\subsection{Introduction}

The aim of this chapter is to examine the reactivity of our doped nZVI@Misi materials towards nitrate reduction when they are exposed to oxygen and naturally available ions. This examination undertakes two variables: (1) the efficiency of doped nZVI@Misi materials as determined by nitrate conversion and nitrite accumulation under aerobic conditions, and (2) the effect of coexisting ions on nitrate reduction using these materials under both anaerobic and aerobic conditions. In addition, the generation of soluble iron and other dopant metals is also examined.

There have been numerous studies on nitrate reduction using nZVI, ${ }^{156,165} \mathrm{Ni}$-doped nZVI, Cudoped nZVI, nZVI@chitosan, nZVI@sodium oleate, ${ }^{244}$ or Ni-nZVI@zeolite. ${ }^{193}$ However, these reactions are commonly carried out in anaerobic conditions such as in a sealed reaction vessel or under a flow of inert gas (nitrogen or argon). Therefore, the reactivity of nZVI particles was not affected by any oxidising factors in the open environment.

The synthetic methods used to generate nZVI results in it having a core-shell structure. ${ }^{48}$ The composition and the thickness of shell vary, depending on the synthetic methods and conditions (vide infra). ${ }^{48,249}$ In addition, the longer the particles are in contact with oxidising media, the thicker the oxide/hydroxide layer becomes, which slows down electron transfer from the $\mathrm{Fe}(0)$ core to the surface. ${ }^{250}$ As such, when materials containing $\mathrm{nZVI}$ are exposed to aerobic conditions, the presence of oxygen might affect the reactivity of these materials.

In "real" waters, there are numerous available ions which have an impact on nitrate reduction. Some ions can speed up nitrate reduction while other ions inhibit the reactivity of $\mathrm{nZVI}$ towards nitrate reduction. For example, nitrate reduction is significantly increased in the presence of either $\mathrm{Cd}(\mathrm{II}),{ }^{251} \mathrm{Cu}(\mathrm{II}),{ }^{199}$ or $\mathrm{Pb}(\mathrm{II}) .{ }^{192}$ In contrast, some ions can compete with nitrate in the adsorption onto the nZVI surface, and essentially inhibit nitrate reduction. For instance, at $\mathrm{pH}$ 's less than 8 , the surface of the iron nanoparticles becomes positively charged, and anions, such as sulfate or phosphate, adsorb onto this positively charged surface of nZVI which decreases the number of available nZVI active sites for nitrate reduction. ${ }^{48,252}$

There have been some studies in which the effect of chloride, sulfate, bicarbonate, carbonate, and phosphate were investigated. Chloride is reported to passivate nZVI when nZVI was used 
to remove TCE. ${ }^{150}$ This was attributed to the formation of complexes on the surface of nZVI. Sulfate also had negative effect on the reactivity of both nZVI and bimetallic nZVI. For example, the removal of TCE using nZVI decreased in the presence of sulfate. ${ }^{150}$ In addition, sulfate decreased both the removal rate and degradation yield when Pd-nZVI was used to remove pentachlorophenol. ${ }^{184}$ The inhibition of sulfate was due to the formation of complexes on the surface of $\mathrm{nZVI}$, leading to the blockage of the active sites on the surface of nanoparticles. ${ }^{150,} 184$ Bicarbonate and carbonate also affected the reactivity of nZVI composites. For example, bicarbonate decreased the removal of TCE. ${ }^{150}$ Carbonate inhibited the decolourisation of MO by Pd-nZVI@bentonite. ${ }^{191}$ The negative effect of carbonate/ bicarbonate was reported due to the formation of complexes or precipitation of substances such as siderite $\left(\mathrm{FeCO}_{3}\right)^{150,191}$ and iron-carbonate hydroxides $\left(\mathrm{Fe}(\mathrm{OH}) \mathrm{CO}_{3}\right)^{191}$ on the surface of nanoparticles. Similar to other ions, phosphate inhibited nZVI in removing TCE due to the precipitation of vivianite $\left(\mathrm{Fe}_{3}\left(\mathrm{PO}_{4}\right)_{2} \cdot 8 \mathrm{H}_{2} \mathrm{O}\right) \cdot{ }^{150}$

In this study, three representative materials which are described in Chapter 4 are used to study the effect of both aerobic conditions as well as the effect of coexisting ions. These are 5\%Ni-nZVI@MisiCFeOOH-MB1， 5\%Cu-nZVI@MisiCFeOOH-MB1， and 0.5\%Pd-5\%CunZVI@MisiCFeOOH-MC1. These materials were chosen as they not only readily reduce nitrate but also span the range of the different types of materials examined. Non-doped nZVI@MisiCFeOOH was used as a control material. Two different molar ratios of Fe to nitrate 15:1 (10 ppm nitrate-N) and 3:1 (50 ppm nitrate-N) were used to investigate nitrate reduction under anaerobic conditions (see Chapter 4) versus aerobic conditions. During nitrate reduction, the concentrations of soluble metals such as iron and doped metal ions were also measured.

Decisions on the appropriate concentrations of potential inhibitory ions were based on preliminary studies on the reduction of 10 ppm nitrate- $\mathrm{N}$ using 5\%Ni-nZVI@MisiCFeOOHMB1. The amount of chloride, sulfate, carbonate, and phosphate was increased stepwise until either an inhibitory effect was observed, or $100 \mathrm{ppm}$ of the ion was reached. The concentration ranges included chloride and sulfate-S (10 - 100 ppm each), carbonate (5 - 20 ppm), and phosphate (1 - $10 \mathrm{ppm}$ ). Neither chloride nor sulfate significantly influenced nitrate reduction when their concentrations were up to $100 \mathrm{ppm}$. Nitrate conversion started being inhibited when the concentrations of carbonate- $C$ and phosphate-P were 20 ppm and 10 ppm, 
respectively. As such, we decided to examine the effect of these ions, chloride (100 ppm), sulfate-S (100 ppm), carbonate-C (20 ppm) and phosphate-P (10 ppm) on nitrate reduction of $10 \mathrm{ppm}$. The concentrations of nitrate, nitrite, and phosphate were measured using IC. The concentrations of metal ions, such as iron and doped metal ions, were determined using AAS.

\subsection{Nitrate reduction under aerobic conditions}

nZVI rapidly reacts with oxygen, water, and natural occurring constituents in the environment. The aging of $\mathrm{nZVI}$ can be oxidation and transformation of the shell composition. Sarathy et al. reported that when $\mathrm{nZVI}$ is exposed to deionised water and dissolved oxygen, the original shell of nZVI was broken down and the oxide shell was transformed to a more protective layer. ${ }^{253}$ In another study, when $\mathrm{nZVI}$ was exposed to an oxygen atmosphere, the ageing was due to the recovery and recrystallisation of amorphous structure to the crystalline phase. ${ }^{254}$ Regardless of mechanism, ageing nZVI results in the loss of the active $\mathrm{Fe}(0)$ sites available, decreasing its reactivity towards TCE. ${ }^{131,255}$ Another study examined the effect of reused bimetallic Pd-nZVI on the removal of chlorobenzenes. ${ }^{256}$ The reactivity of aged particles decreased due to Pd dislodgment from Pd-nZVI and the encapsulation of Pd islets by the iron oxides. However, the effect of ageing due to exposure to atmosphere has not been investigated. In this current study, the reactivity of doped and non-doped nZVI@Misi towards nitrate reduction was examined in the presence of oxygen gas.

The nitrate removal of 10 ppm nitrate in aerobic conditions was rapid when doped nZVI@Misi materials were used (Figure 5.1). After 5 minutes, both 5\%Ni-nZVI@MisiCFeOOH-MB1 and 5\%Cu-nZVI@MisiCFeOOH-MB1 reduced nitrate to the detection limit of the IC (0.06 ppm). The trimetallic material, 0.5\%Pd-5\%Cu-nZVI@MisiCFeOOH-MC1, reduced nitrate more slowly than bimetallic materials with the nitrate- $\mathrm{N}$ level at $1.8 \mathrm{ppm}$ after 5 minutes. However, after 15 minutes, nitrate-N concentrations were below the detection limit for all types of doped materials. Thus, the reactivity of these materials was not significantly different. 


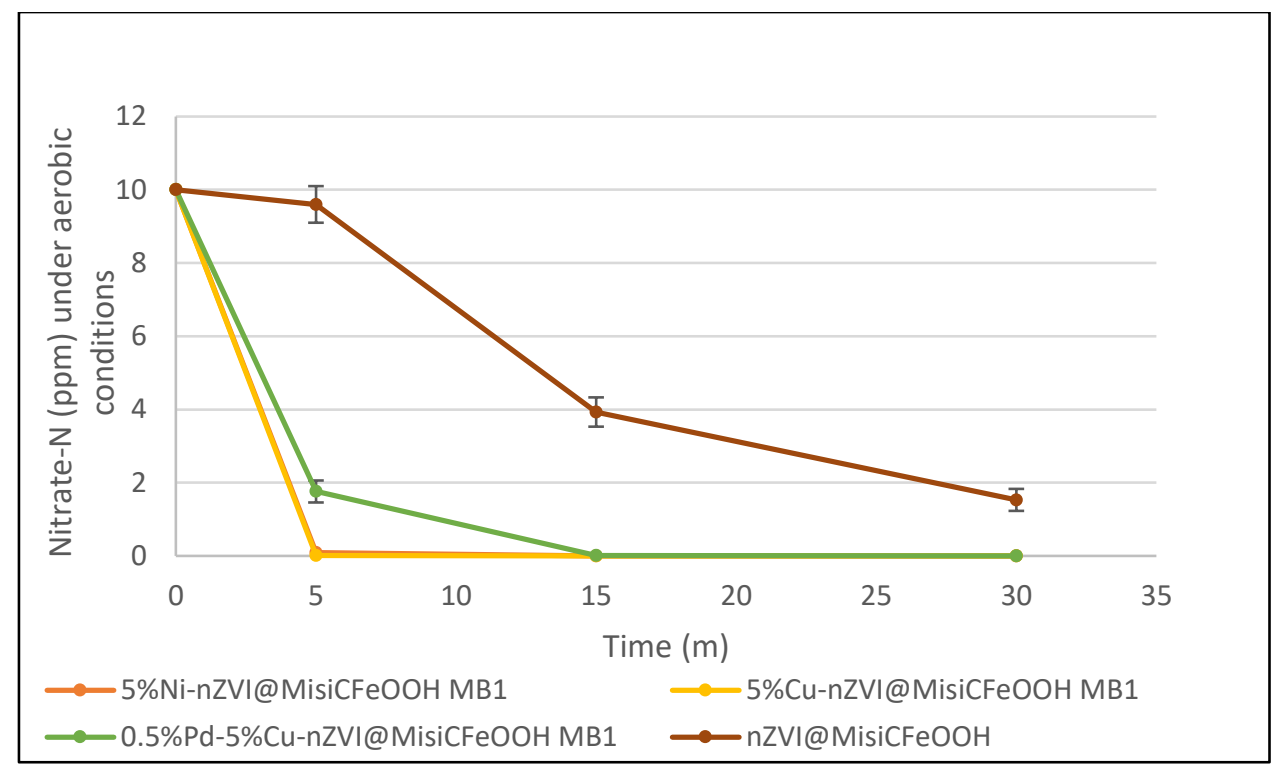

Figure 5.1. Nitrate reduction of $10 \mathrm{ppm}$ nitrate- $\mathrm{N}$ under aerobic conditions $(0.150 \mathrm{~g}$ of materials, $25.0 \mathrm{~mL}$ of solution).

Although the difference in the reactivity of doped nZVI@Misi was minimal, the role of doping metal(s) on nZVI@Misi was evident. Doped materials were much better in nitrate reduction than non-doped nZVI@Misi. After 5 minutes, the nitrate-N level was 9.6 ppm and after 15 minutes, the nitrate-N level was 4.0 ppm when nZVI@MisiCFeOOH was used.

Nitrite was not detected using Ni-doped material while nitrite accumulation was observed using Cu-doped, Pd-Cu-doped, and nZVI@MisiCFeOOH materials during the reaction. After 5 minutes, nitrite- $\mathrm{N}$ concentration was the most significant at $2.4 \mathrm{ppm}$ using $0.5 \% \mathrm{Pd}-5 \% \mathrm{Cu}-$ nZVI@MisiCFeOOH-MC1 whereas nitrite-N concentration was 0.3 ppm for either $5 \% \mathrm{Cu}$ nZVI@MisiCFeOOH-MB1 or nZVI@MisiCFeOOH-MC1. After 15 minutes, nitrite-N concentrations were below the detection limit of the IC $(0.04 \mathrm{ppm})$ for all materials examined. As the reactivity difference between the doped materials was minimal when $10 \mathrm{ppm}$ nitrate$\mathrm{N}$ was examined, more concentrated nitrate solutions were investigated to help magnify any differences. A significant amount of nitrate was reduced by doped materials when $50 \mathrm{ppm}$ of nitrate- $\mathrm{N}$ was used under aerobic conditions. After 5 minutes, about $50 \%$ of nitrate was removed by $\mathrm{Ni}$-doped material and approximately two-thirds of nitrate was removed by either $\mathrm{Cu}$-doped or Pd-Cu-doped material (Figure 5.2). The nitrate- $\mathrm{N}$ levels were $27 \pm 4 \mathrm{ppm}$, $14 \pm 2$ ppm, and $19 \pm 2$ ppm when either 5\%Ni-nZVI@MisiCFeOOH-MB1, 5\%CunZVI@MisiCFeOOH-MB1 or 0.5\%Pd-5\%Cu-nZVI@MisiCFeOOH-MC1 was used, respectively. 
After 30 minutes, the nitrate- $\mathrm{N}$ levels were $18 \pm 4 \mathrm{ppm}$ (64\% nitrate conversion), $6.9 \pm 0.5$ ppm (86\% nitrate conversion), and $10 \pm 4$ ppm (80\% nitrate conversion), respectively. Interestingly, 5\%Cu-nZVI@MisiCFeOOH-MB1 had slightly higher reactivity than $0.5 \% \mathrm{Pd}$ 5\%Cu-nZVI@MisiCFeOOH-MC1 during the course of the reaction. The difference in the reactivity of $\mathrm{Cu}$-doped and $\mathrm{Pd}$-Cu-doped materials towards nitrate conversion under aerobic conditions is different from that observed in anaerobic conditions (Section 4.3 and 4.4).

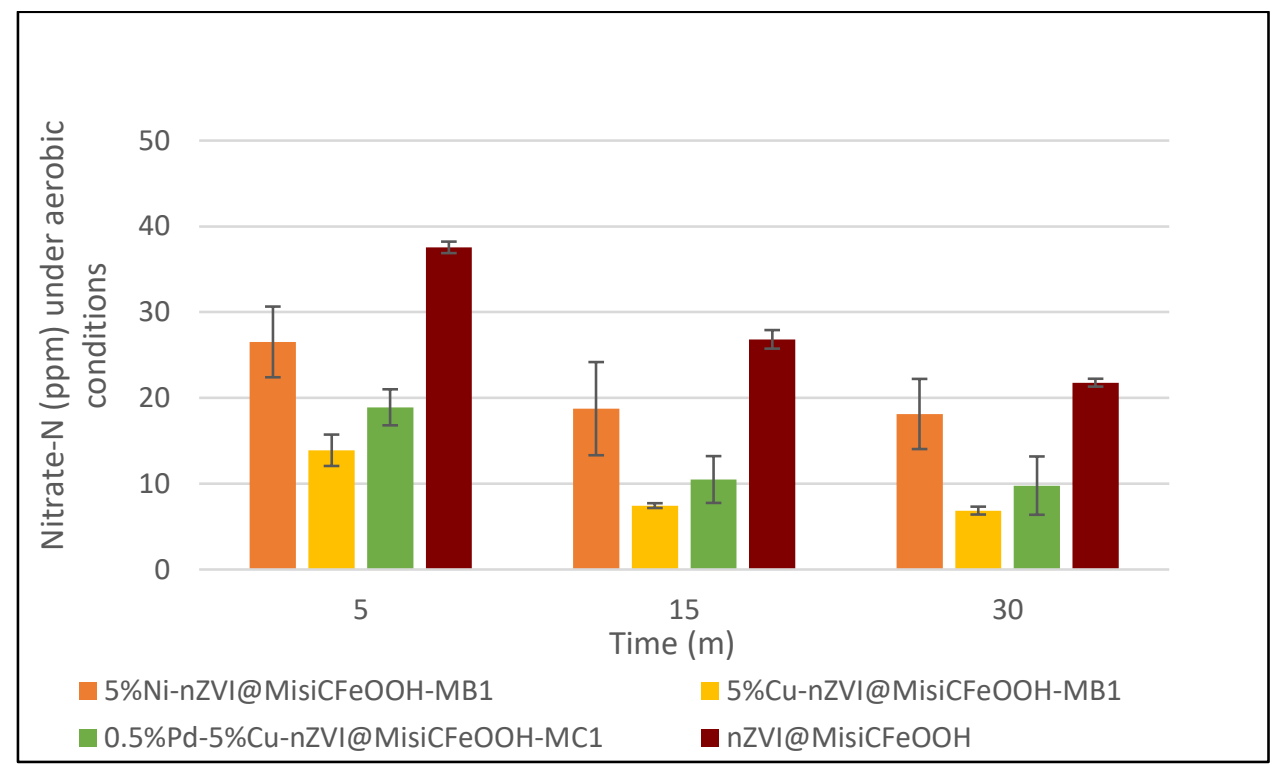

Figure 5.2. Nitrate reduction of $50 \mathrm{ppm}$ nitrate- $\mathrm{N}$ under aerobic conditions $(0.150 \mathrm{~g}$ of materials, $25.0 \mathrm{~mL}$ of solution).

As a control material, the reactivity of non-doped nZVI@MisiCFeOOH was also examined. nZVI@MisiCFeOOH reduced nitrate much more slowly than doped materials. After 5 minutes, the nitrate-N level was $37.6 \pm 0.7 \mathrm{ppm}$ and after 30 minutes, the nitrate- $\mathrm{N}$ concentration was $21.8 \pm 0.5 \mathrm{ppm}$ (56\% nitrate conversion).

Both $\mathrm{Cu}$-doped and $\mathrm{Pd}$-Cu-doped produced more nitrite compared to $\mathrm{Ni}$-doped and nondoped nZVI@Misi (Figure 5.3). After 5 minutes, the nitrite-N levels were $16 \pm 2$ ppm and 12.2 \pm 0.8 ppm using Cu-doped or Pd-Cu-doped material while nitrite-N levels were $3.6 \pm 0.9$ ppm and $1.72 \pm 0.06$ ppm using Ni-doped material and non-doped nZVI@MisiCFeOOH, respectively. After 30 minutes, the nitrite levels in all materials decreased but these decreases were minimal. Nitrite-N levels were $2.6 \pm 0.8 \mathrm{ppm}$ ( $5 \%$ nitrite accumulation), $14 \pm 2$ ppm (28\% nitrite accumulation), $11 \pm 1 \mathrm{ppm}$ (22\% nitrite accumulation) and $1.0 \pm 0.1 \mathrm{ppm}$ (2\% nitrite accumulation) when either 5\%Ni-nZVI@MisiCFeOOH-MB1, 5\%Cu-nZVI@MisiCFeOOH-MB1, 
0.5\%Pd-5\%Cu-nZVI@MisiCFeOOH-MC1 or nZVI@MisiCFeOOH was used, respectively. Although 5\%Cu-nZVI@MisiCFeOOH-MB1 reduced nitrate faster than 0.5\%Pd-5\%CunZVI@MisiCFeOOH-MC1, the nitrite conversion in the presence of Pd in the material was faster, leading to a lower nitrite accumulation using Pd-Cu-doped material. This is similar to the mechanism of nitrate reduction using these materials under anaerobic condition. Pd catalysed the nitrite reduction step due to the supply of the surface for nitrite adsorption before nitrite was reduced to final products.

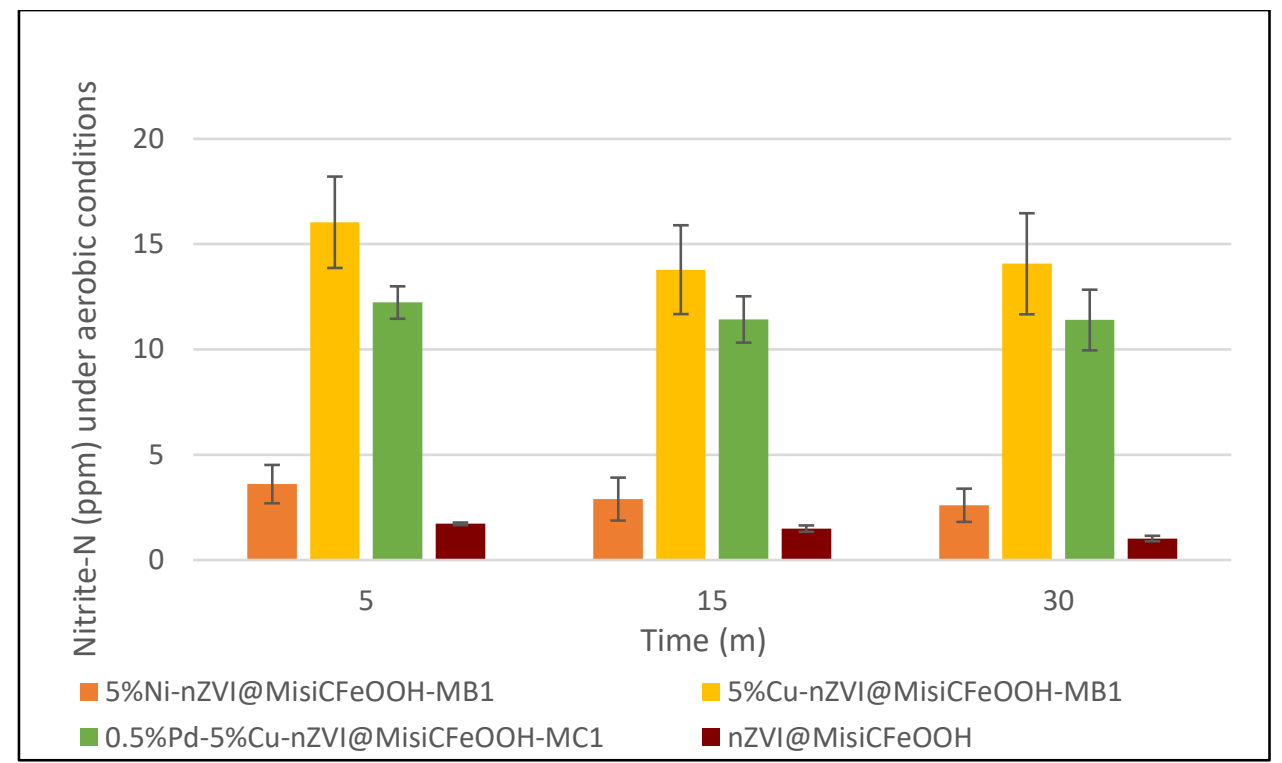

Figure 5.3. Nitrite accumulation in the reduction of $50 \mathrm{ppm}$ nitrate- $\mathrm{N}$ under aerobic conditions ( $0.150 \mathrm{~g}$ of materials, $25.0 \mathrm{~mL}$ of solution).

\subsection{Comparison of the reactivity of materials under anaerobic and aerobic conditions}

The reactivity of doped nZVI@Misi materials under aerobic conditions was lower than that observed under anaerobic conditions. Oxygen affected the reactivity of trimetallic materials while its effect on bimetallic materials was insignificant. The decrease in their reactivity in the presence of oxygen could be due to the passivation of the outer layer of nanoparticles which diminishes electron transfer from the $\mathrm{Fe}(0)$ core to nitrate. ${ }^{253,255}$ For example, after 30 minutes, the nitrate- $\mathrm{N}$ concentrations were $14 \pm 1 \mathrm{ppm}$ and $18 \pm 4 \mathrm{ppm}$ when $5 \% \mathrm{Ni}$ nZVI@MisiCFeOOH-MB1 was used either under anaerobic or aerobic condition, respectively (Figure 5.4). As such, these doped systems are promising materials for nitrate removal. 


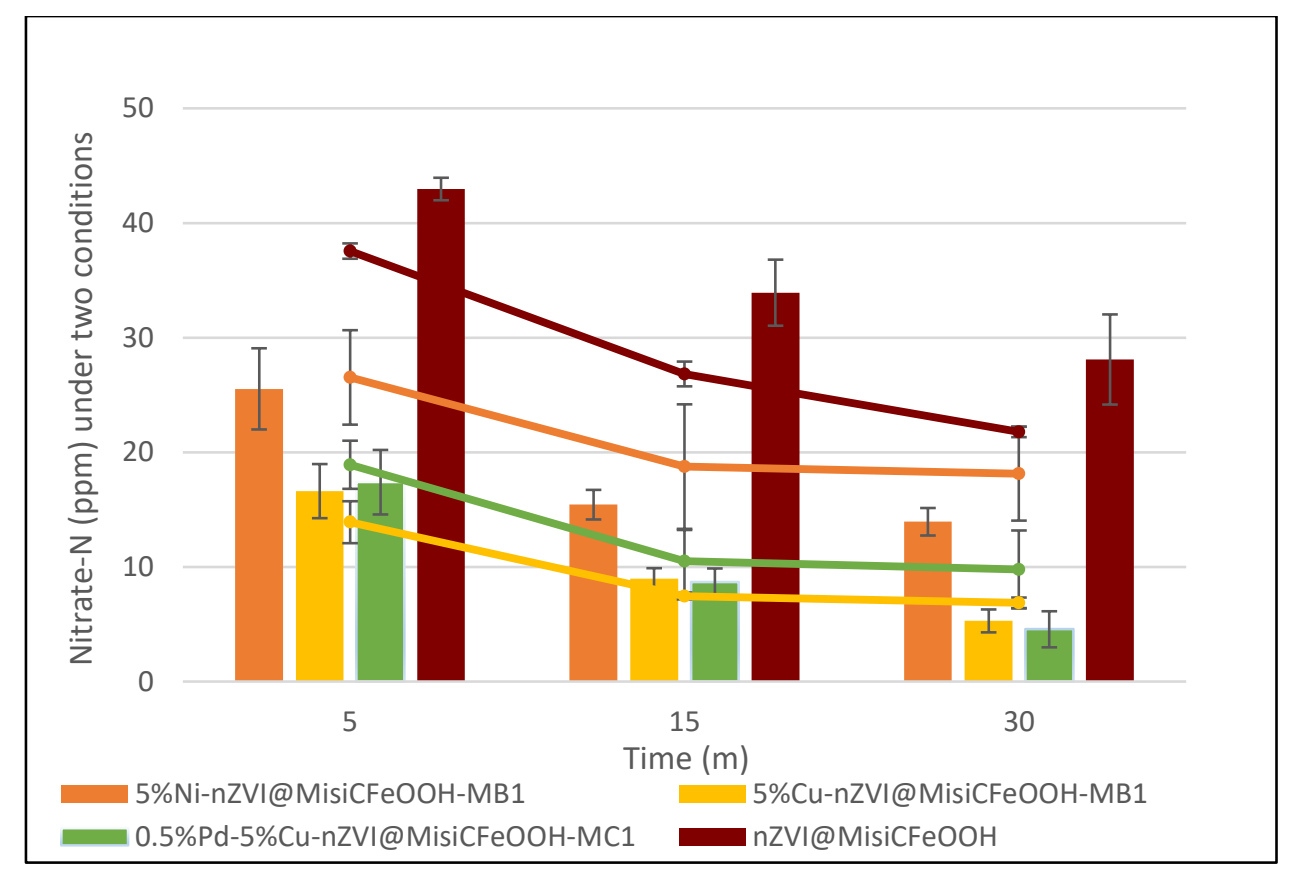

Column: anaerobic conditions; Line: aerobic conditions

Figure 5.4. Nitrate reduction of $50 \mathrm{ppm}$ nitrate- $N$ under anaerobic and aerobic conditions (0.150 $\mathrm{g}$ of materials, $25.0 \mathrm{~mL}$ of solution).

The effect of oxygen on the reactivity of doped $\mathrm{nZVI}$ materials has not received much attention. However, the insignificant decrease in the reactivity of $0.5 \% \mathrm{Pd}-5 \% \mathrm{Cu}$ nZVI@MisiCFeOOH-MC1 is inconsistent with Liu et al.'s report. ${ }^{247}$ In their study, 3\%Cu-6\%Pd$\mathrm{nZVI}$ was synthesised by simultaneous deposition of both $\mathrm{Pd}$ and $\mathrm{Cu}$ on $\mathrm{nZVI}$. The initial nitrate- $\mathrm{N}$ concentration was $22.6 \mathrm{ppm}$ and the molar ratio of Fe to nitrate was $2.8: 1$. In this reported system, the reactivity of trimetallic significantly decreased with increasing dissolved oxygen. After 60 minutes, nitrate conversion was $82.3 \%, 38.1 \%$, and $20.4 \%$ in the presence of $0.42 \mathrm{mg} / \mathrm{L}, 6.05 \mathrm{mg} / \mathrm{L}$, and $10.41 \mathrm{mg} / \mathrm{L}$ of dissolved oxygen. Although dissolved oxygen was never measured for our systems, as the reactions were performed in air, a maximum dissolved oxygen level of $9 \mathrm{mg} / \mathrm{L}$ can be assumed at $20^{\circ} \mathrm{C} .{ }^{257}$

In related research investigating the removal of nitrate with ZVI under aerobic conditions, nitrate was only reduced in buffered conditions. ${ }^{45}$ In our study, nZVI@MisiCFeOOH reduced nitrate under aerobic conditions without $\mathrm{pH}$ control. This can be explained by the important role of both nanosized Fe and Misi support. Nanosized Fe increases the reducing capacity of Fe as well as the introduction of Misi decreases agglomeration. Therefore, the reactivity of nZVI@Misi increases. Additionally,nZVI@MisiCFeOOH under aerobic conditions was more 
active than under anaerobic conditions. After 30 minutes, the nitrate- $\mathrm{N}$ levels were $28 \pm 4$ ppm (44\% nitrate conversion) and $21.8 \pm 0.5 \mathrm{ppm}$ (56\% nitrate conversion) when nZVI@MisiCFeOOH was used either under anaerobic or aerobic conditions, respectively. This is inconsistent with a study in which the chemical transformation of nZVI was examined when nZVI was exposed in oxygen-saturated water for 24 hours. ${ }^{258}$ Both the reducing capacity and reactive lifetime of $n Z V I$ decreased. Thus, more $n Z V I$ was required to treat a similar mass of contaminants than when used under anaerobic conditions.

In our study, the reactivity of nZVI@MisiCFeOOH is higher under aerobic conditions than under aerobic conditions. This can be explained by the formation of oxidants when $\mathrm{nZVI}$ is exposed to oxygen. Fe reacted with water in the presence of oxygen to form Fe(II) and $\mathrm{H}_{2} \mathrm{O}_{2} \cdot{ }^{246}$ Subsequently, Fe reacted with $\mathrm{H}_{2} \mathrm{O}_{2}$ to form Fe(II). These oxidants can be $\mathrm{Fe}(\mathrm{II}), \mathrm{H}_{2} \mathrm{O}_{2}, \mathrm{O}_{2}{ }^{\bullet-}$ (eq. 5.1 - 5.4) which may provide active components for nitrate reduction.

$$
\begin{gathered}
\mathrm{Fe}_{(\mathrm{s})}^{\mathrm{s}}+\mathrm{O}_{2} \underset{\left(\mathrm{H}_{2} \mathrm{O}\right)}{\stackrel{2 \mathrm{H}^{+}}{\longrightarrow}} \mathrm{Fe}^{2+}+\mathrm{H}_{2} \mathrm{O}_{2} \\
\mathrm{Fe}^{\mathrm{O}}{ }_{\mathrm{s})}+\mathrm{H}_{2} \mathrm{O}_{2} \stackrel{\text { (eq. 5.1) }}{\stackrel{2 \mathrm{H}^{+}}{\longrightarrow}} \mathrm{Fe}^{2+}+2 \mathrm{H}_{2} \mathrm{O} \\
\mathrm{Fe}^{2+}+\mathrm{O}_{2} \stackrel{\text { (eq. 5.2) }}{\longrightarrow} \mathrm{Fe}^{3+}+\mathrm{O}_{2}^{\cdot-} \\
\mathrm{Fe}^{2+}+\mathrm{O}_{2}^{\cdot-} \stackrel{\text { (eq. 5.3) }}{\stackrel{2 \mathrm{H}^{+}}{\left(\mathrm{H}_{2} \mathrm{O}\right)}} \mathrm{Fe}^{3+}+\mathrm{H}_{2} \mathrm{O}_{2}
\end{gathered}
$$

Nitrite accumulation under the two conditions was also compared. When $5 \% \mathrm{Ni}$ nZVI@MisiCFeOOH-MB1 was used, the nitrite build-up under anaerobic and aerobic conditions was similar (Figure 5.5). After 30 minutes, the nitrite- $\mathrm{N}$ levels were $2.4 \pm 0.8 \mathrm{ppm}$ and $2.6 \pm 0.8$ ppm when 5\%Ni-nZVI@MisiCFeOOH-MB1 was used under either anaerobic conditions or aerobic conditions, respectively. This indicated that Ni-doped material $(5 \% \mathrm{Ni}-$ nZVI@MisiCFeOOH-MB1) was not affected by oxygen gas in both steps of nitrate reduction including nitrate conversion and nitrite reduction. When $\mathrm{Cu}$-doped and $\mathrm{Pd}$-Cu-doped systems were used, the difference in nitrite accumulation in two reaction conditions was dependent upon the reaction time (Figure 5.5). After 5 minutes, nitrite accumulation under anaerobic conditions was more than that aerobic conditions. For instance, nitrite- $\mathrm{N}$ levels were $18 \pm 2$ ppm and $16 \pm 2$ ppm when 5\%Cu-nZVI@MisiCFeOOH-MB1 was used, respectively. This suggested that the step from nitrate to nitrite under anaerobic conditions was faster than 
that under aerobic condition. In contrast, after 30 minutes, nitrite accumulation under anaerobic conditions was less than that under aerobic conditions. For instance, nitrite-N levels were $9 \pm 1$ ppm and $14 \pm 2$ ppm when 5\%Cu-nZVI@MisiCFeOOH-MB1 was used, respectively. This meant that nitrite reduction under anaerobic conditions was faster than under aerobic conditions. In other words, during the course of the reaction, these materials were more reactive under anaerobic conditions than under aerobic conditions.

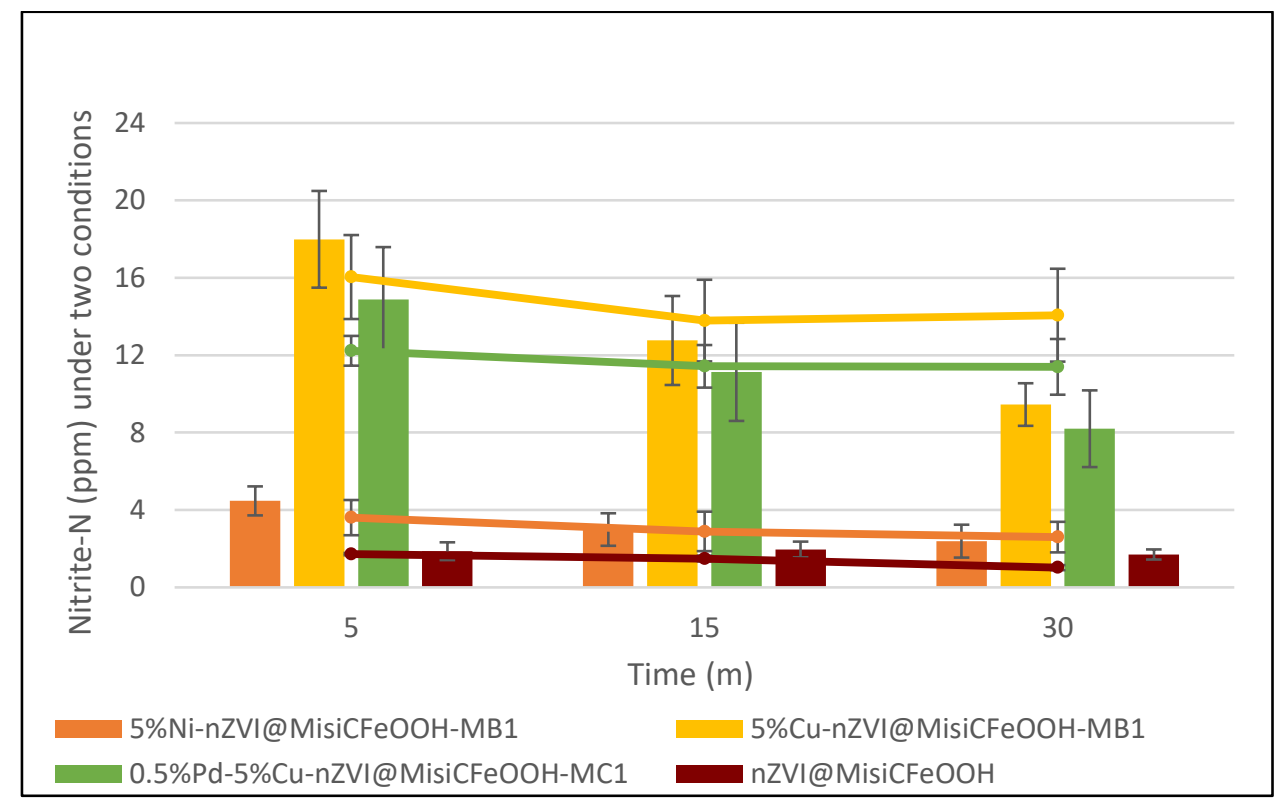

\section{Column: anaerobic conditions; Line: aerobic conditions}

Figure 5.5. Nitrite accumulation in the reduction of $50 \mathrm{ppm}$ nitrate- $N$ under anaerobic and aerobic conditions ( $0.15 \mathrm{~g}$ of materials, $25 \mathrm{~mL}$ of solution).

Due to the slightly lower reactivity and higher accumulation of nitrite of doped materials when the nitrate reduction was conducted under aerobic conditions, we examined whether nitrite as the intermediate might react with oxygen gas or dissolved oxygen to form nitrate. For this, the reduction of $10 \mathrm{ppm}$ nitrite- $\mathrm{N}$ was examined under anaerobic and aerobic conditions. Two materials, 5\%Ni-nZVI@MisiCFeOOH-MB1 and 0.5\%Pd-5\%CunZVI@MisiCFeOOH-MC1 were used. The results showed that the decrease in the reactivity is not due to the re-formation of nitrate. Using 0.5\%Pd-5\%Cu-nZVI@MisiCFeOOH-MC1, nitrate$\mathrm{N}$ levels were below the detection limit of the IC $(0.06 \mathrm{ppm})$ during the course of the reaction in both conditions. Using 5\%Ni-nZVI@MisiCFeOOH-MB1, only a small amount of nitrate was detected after 5 minutes, $0.13 \mathrm{ppm}$ and $0.09 \mathrm{ppm}$ under either anaerobic condition or aerobic conditions, respectively. In addition, the higher nitrite accumulation under aerobic conditions 
is due to the slower nitrite conversion. For example, after 5 minutes, nitrite- $\mathrm{N}$ concentrations were 0.43 ppm and 2.15 ppm using 0.5\%Pd-5\%Cu-nZVI@MisiCFeOOH-MC1 either under anaerobic conditions or aerobic conditions, respectively.

\subsection{Determination of soluble metals during nitrate reduction}

One of the concerns of the real-life application of our materials is the generation of the byproducts after the reaction. In nitrate reduction using doped and non-doped nZVI@Misi, byproducts are not only $\mathrm{N}$-containing substances but also metal ions. We aimed to examine if the metal ions could accumulate during the reaction. The mobility and transport of iron and other metal ions after nitrate removal using materials containing these metals have not received much attention. In order to determine the generation of the metal ions during the course of the reaction, we first examined the stability of our system under aerobic conditions. For this, we examined the conversion of Fe and other doped metals by measuring the concentrations of metal ions including soluble iron, nickel, and copper in the solutions during the reduction of $10 \mathrm{ppm}$ and $50 \mathrm{ppm}$ nitrate- $\mathrm{N}$. Our four standard materials, $5 \% \mathrm{Ni}-$ nZVI@MisiCFeOOH-MB1， ５\%Cu-nZVI@MisiCFeOOH-MB1， 0.5\%Pd-5\%CunZVI@MisiCFeOOH-MC1, and nZVI@MisiCFeOOH were utilised.

The formation of soluble iron was a function of reaction time and initial nitrate concentrations. In the reduction of $10 \mathrm{ppm}$ nitrate-N using doped materials, the soluble iron levels gradually increased over the course of the reaction (Figure 5.6). After 30 minutes, the iron levels were 1.75 ppm using the Ni-doped system and around 1 ppm using Cu-doped and Pd-Cu-doped materials. In contrast, for the non-doped nZVI@MisiCFeOOH, the iron level reached its maximum after 5 minutes $(1.0 \mathrm{ppm})$ and then decreased back down to below 0.5 ppm at both the 15- and 30-minute marks. The difference in the trend generating iron using nZVI@MisiCFeOOH can be explained by the very slow nitrate removal. In the first 5 minutes, very little nitrate was reduced (Figure 5.1). There might be a process of nitrate adsorption on the active sites and the corrosion of $\mathrm{Fe}(0)$ to form $\mathrm{Fe}(\mathrm{II})$, leading to the higher level of iron. After 15 minutes, a significant amount of nitrate was reduced, indicating the production of hydroxide. This resulted in the decrease in iron concentration. 


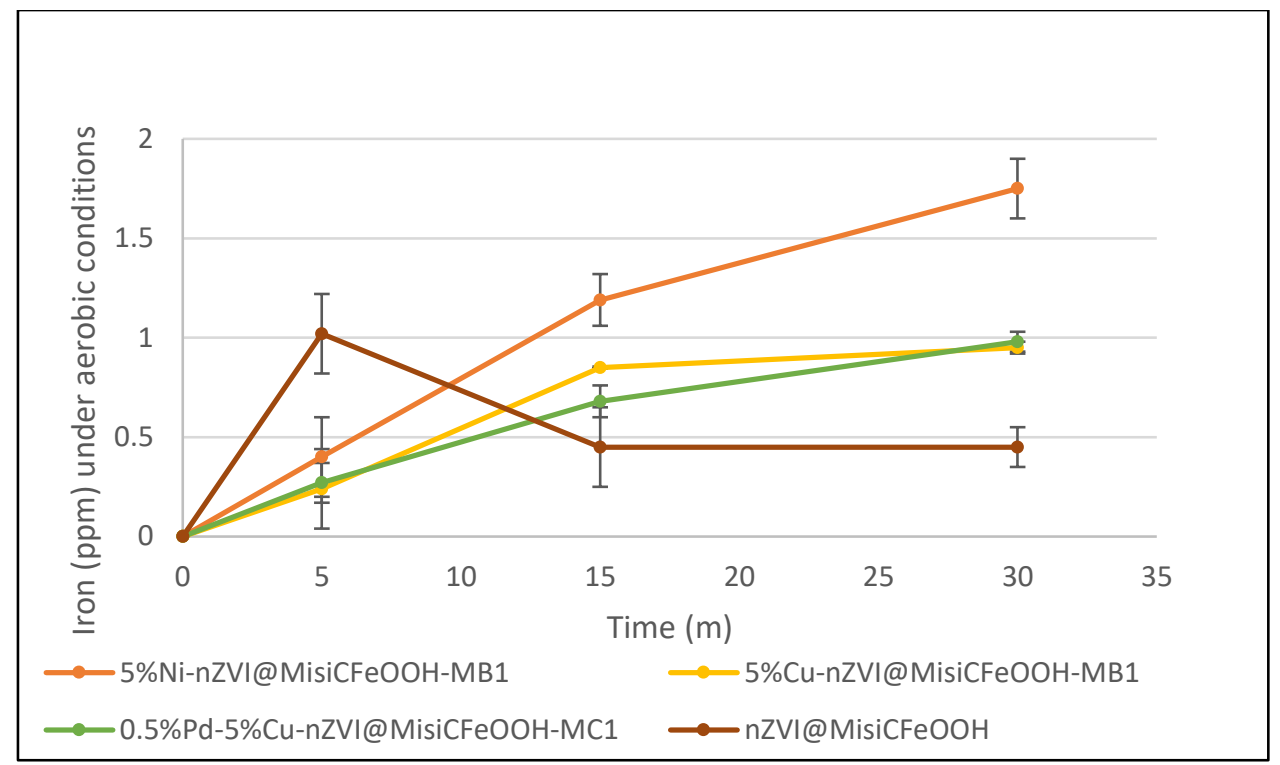

Figure 5.6. Iron concentration in nitrate reduction of $10 \mathrm{ppm}$ nitrate- $\mathrm{N}$ under aerobic conditions ( $0.150 \mathrm{~g}$ of materials, $25.0 \mathrm{~mL}$ of solution).

Interestingly, the levels of soluble iron after reducing $50 \mathrm{ppm}$ nitrate- $\mathrm{N}$ were less than those observed after reducing $10 \mathrm{ppm}$ nitrate-N. After 5 minutes, iron concentrations were less than $0.5 \mathrm{ppm}$ (Figure 5.7) and after 30 minutes, iron concentrations were around the detection limit of the AAS (0.09 pm) for all types of doped materials. For nZVI@MisiCFeOOH, the iron level was slightly higher at $0.2 \mathrm{ppm}$.

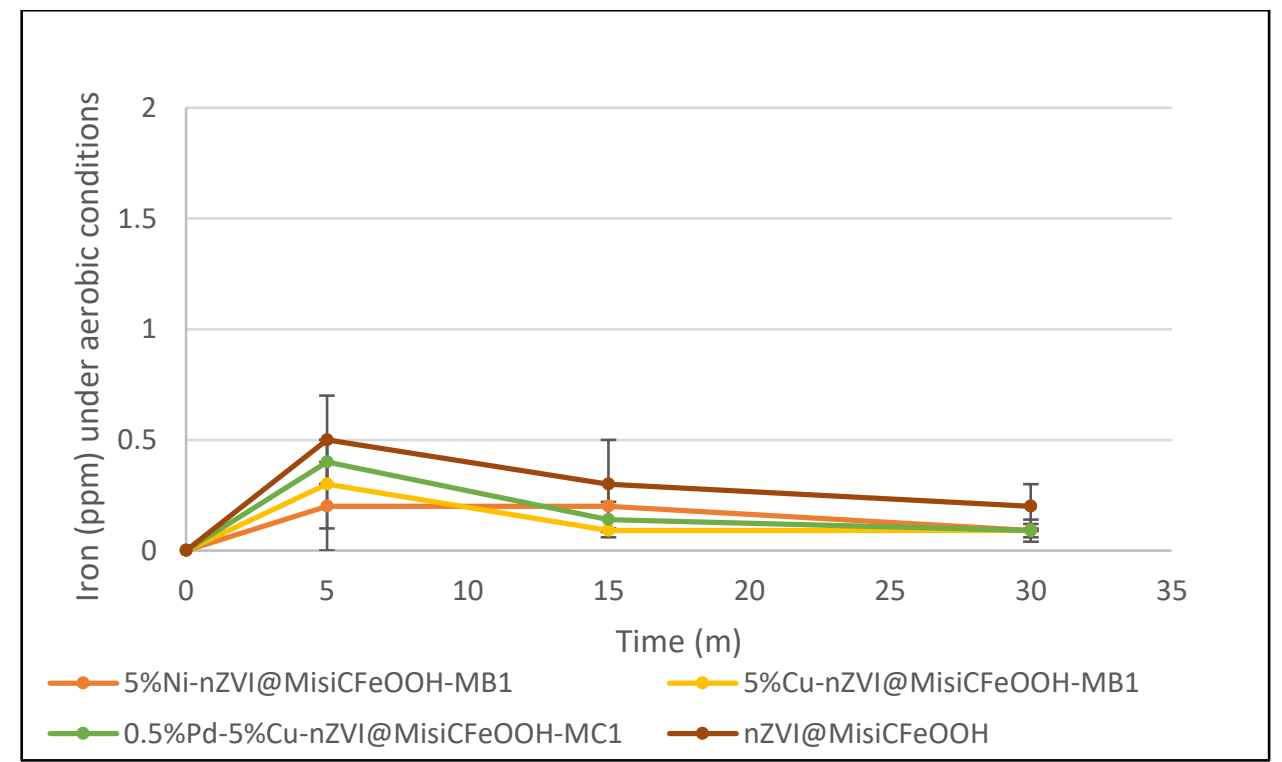

Figure 5.7. Iron concentration in nitrate reduction of $50 \mathrm{ppm}$ nitrate- $\mathrm{N}$ under aerobic conditions ( $0.150 \mathrm{~g}$ of materials, $25.0 \mathrm{~mL}$ of solution). 
The iron generation is inconsistent with a study in which nitrate was reduced using $\mathrm{Ni}$ nZVI@zeolite with the mass ratio of Ni:Fe:zeolite at 1:10:20 reported by He al et.. ${ }^{193}$ In the supporting information of this study, iron concentrations increased with increasing nitrate concentrations and were significantly higher than those observed in our research. The molar ratio of Fe to nitrate was 2.5 and the initial $\mathrm{pH}$ was 5 without $\mathrm{pH}$ control in the presence of phosphate-P $10 \mathrm{ppm}$. In this reported system, after 30 minutes, iron levels increased from 8 $\mathrm{ppm}$ to more than $20 \mathrm{ppm}$ with increasing nitrate- $\mathrm{N}$ concentration from $20 \mathrm{ppm}$ to $100 \mathrm{ppm}$ but after 180 minutes, iron levels were around $2 \mathrm{ppm}$. They explained that the higher the nitrate concentration, the higher the concentration of soluble iron. However, they also postulated that the generation of soluble iron was faster than the formation of $\mathrm{Fe}(\mathrm{OH})_{2}$ and $\mathrm{Fe}(\mathrm{OH})_{3}$, thus more soluble iron were found.

According to Cheng et al., the solution pH was 8.8 after reduction of $175 \mathrm{ppm}$ of nitrate- $\mathrm{N}$ using $\mathrm{ZVI}$ in $\mathrm{pH}$ neither control nor buffer. At this $\mathrm{pH}, \mathrm{Fe}(\mathrm{OH})_{3}$ and a complex of $\mathrm{Fe}(\mathrm{II}), \mathrm{Fe}(\mathrm{III})$ (from iron corrosion) with hydroxide was formed. ${ }^{45}$ In our studies, after nitrate reduction, the concentrations of the metal ions were low. A possible reason for this phenomenon is the removal of insoluble by-products. The reduction of nitrate generates hydroxide ions, leading to an increase in the solution $\mathrm{pH}$. The high $\mathrm{pH}$ results in the precipitation of oxides/hydroxides of iron, nickel, and copper. ${ }^{143}$ Therefore, as a consequence of no $\mathrm{pH}$ control, the production of hydroxide ions is the key requirement for the removal of metal ions as the by-products.

In our study, the reasons for the lower iron concentration in the reduction of $50 \mathrm{ppm}$ nitrate$\mathrm{N}$ are unclear. During the reactions, the difference in the $\mathrm{pH}$ of solutions between $10 \mathrm{ppm}$ and $50 \mathrm{ppm}$ studies are insignificant. Unfortunately, $\mathrm{pH}$ values are not consistent in different batches of experiments, ranging from 8.9 to 9.5 after 30 minutes. However, a possible reason for lower ion concentrations is due to the corrosion of nanoparticles with water after finishing nitrate reduction. For the $10 \mathrm{ppm}$ nitrate- $\mathrm{N}$ samples, the iron levels continued to increase after all nitrate was removed from solution. Therefore, despite no nitrate reduction, iron still reacted with water to form soluble iron that was observed. This theory is supported when the corrosion of these materials with deionised water was examined. After 30 minutes, soluble iron concentrations were more than $7 \mathrm{ppm}$ using doped materials (Figure 5.8). Additionally, in the reaction of $10 \mathrm{ppm}$ nitrate- $\mathrm{N}$, after 30 minutes, the iron concentration was lower using 
nZVI@MisiCFeOOH than using doped materials potentially due to the gradual nitrate reduction over the course of 30 minutes.

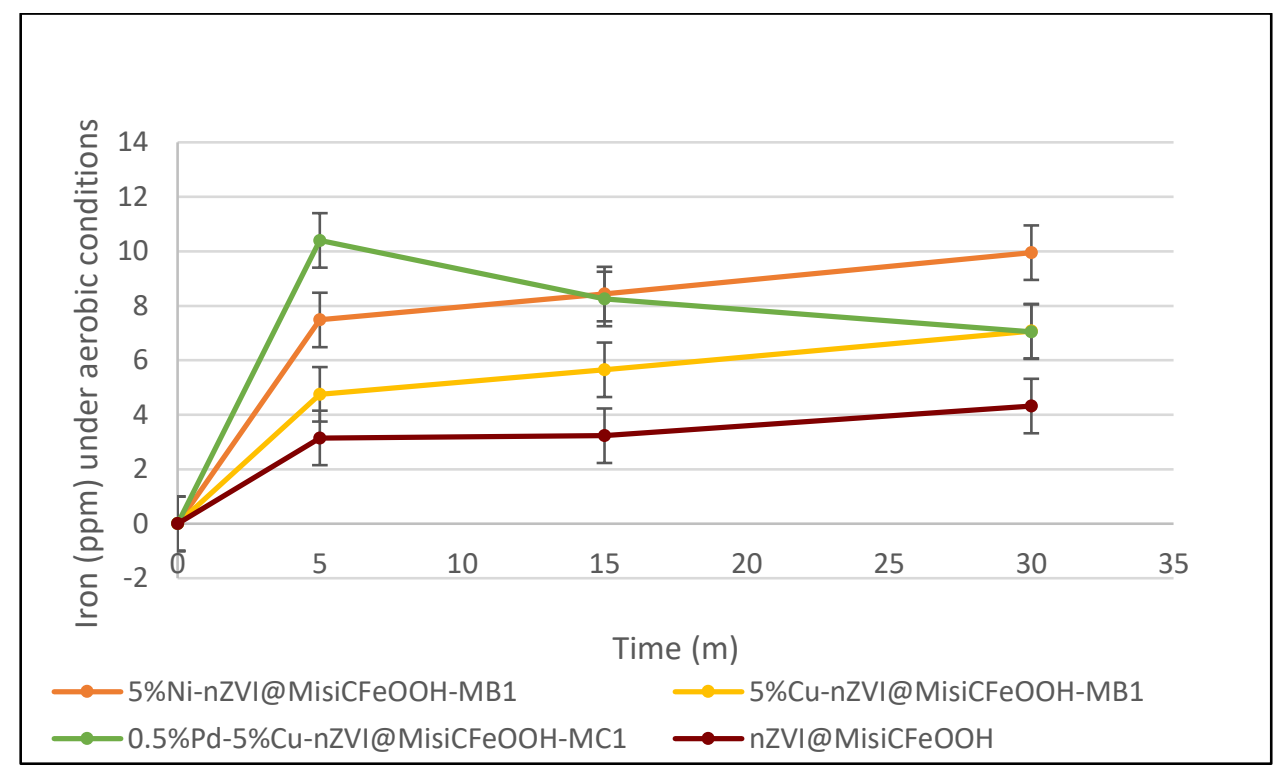

Figure 5.8. Corrosion of materials under aerobic conditions $(0.150 \mathrm{~g}$ of materials, $25.0 \mathrm{~mL}$ of deionised water).

Soluble nickel was also observed during the reaction between $\mathrm{Ni}$-doped material and nitrate when both 10 ppm and 50 ppm nitrate-N were used. In 10 ppm nitrate reduction, nickel level reached a maximum of $0.1 \mathrm{ppm}$ after 30 minutes. In 50 ppm nitrate reduction, nickel levels were around the detection limit of the AAS $(0.04 \mathrm{ppm})$ during the course of 30 minutes. In nitrate reduction using $\mathrm{Cu}$-doped material, soluble copper concentrations were below the detection limit of the AAS $(0.66 \mathrm{ppm})$. Therefore, the leaching of nickel was insignificant while the leaching of $\mathrm{Cu}$ needs further examination using instruments with higher sensitivity.

\subsection{Effect of coexisting anions on nitrate reduction}

The reactivity of doped and non-doped materials towards nitrate reduction in the presence of common ions was examined. Three representative doped materials, $5 \% \mathrm{Ni}$ nZVI@MisiCFeOOH-MB1, 5\%Cu-nZVI@MisiCFeOOH-MB1, and 0.5\%Pd-5\%CunZVI@MisiCFeOOH-MC1 and nZVI@MisiCFeOOH as a control material were used. Ions, chloride (100 ppm), sulfate-S (100ppm), carbonate-C (20 ppm), and phosphate-P (10 ppm), were used to examine their influence on nitrate reduction of reducing $10 \mathrm{ppm}$ nitrate- $\mathrm{N}$. The molar ratio of Fe to nitrate was 15:1 and the reactions were conducted under both anaerobic and aerobic conditions. 


\subsubsection{Effect of chloride on nitrate reduction}

Chloride is one of the most common inorganic anions in waters and wastewaters. It is nontoxic to aquatic life. According to EPA, the criterion of continuous chloride concentration is up to $230 \mathrm{ppm}$ in freshwater and unlimited in saltwater. ${ }^{259}$ In chemistry, it is assumed to be an inert anion in that it is unable to be reduced by $\mathrm{Fe}(0) .{ }^{150}$ However, chloride could affect contaminant removal in the adsorbing step. Although there have been some studies on nitrate reduction, most of them have focused on the importance of $\mathrm{H}^{+}$in changing the solution $\mathrm{pH}^{43}, 234,239$ Chloride was introduced to solution by adding $\mathrm{HCl}$ as a $\mathrm{pH}$ controllable agent. In Hwang et al.'s study, the effect of chloride on nitrate reduction was examined; however, they investigated simple nZVI. ${ }^{260}$ In our study, we used chloride in the form of $\mathrm{KCl}$ to examine if this anion could affect nitrate reduction using doped and non-doped nZVI@MisiCFeOOH.

Chloride did not inhibit nitrate reduction when either Cu-doped or Pd-Cu-doped material was used under both anaerobic and aerobic conditions (Figure 5.9 and 5.10). After 5 minutes, nitrate- $\mathrm{N}$ concentration was below the detection limit of the IC $(0.06 \mathrm{ppm})$ using either $\mathrm{Cu}$ doped or Pd-Cu-doped. In contrast, chloride inhibited nitrate reduction using $\mathrm{Ni-doped}$ material. After 5 minutes, nitrate-N levels were $5.7 \mathrm{pm}$ and $3.2 \mathrm{ppm}$ either under anaerobic conditions or aerobic conditions, respectively. These nitrate levels were significantly higher than when in the absence of chloride. However, after 30 minutes, nitrate- $\mathrm{N}$ concentrations were below the detection limit for all types of doped materials. 

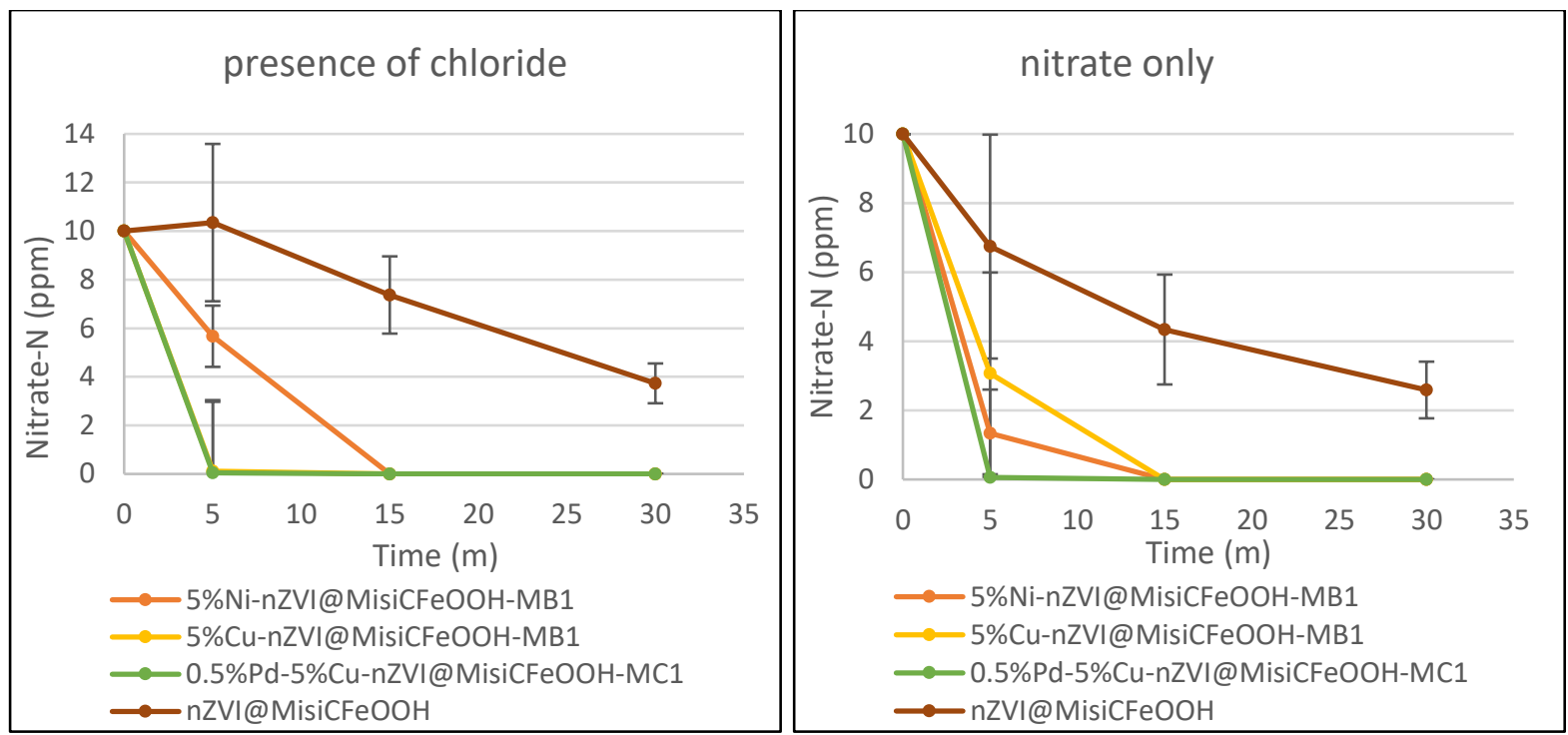

Figure 5.9. Effect of chloride on nitrate reduction and nitrate reduction in the absence of ions under anaerobic conditions $(0.150 \mathrm{~g}$ of material, $25.0 \mathrm{~mL}$ of a solution containing $10 \mathrm{ppm}$ nitrate- $N$ and 100 ppm chloride).
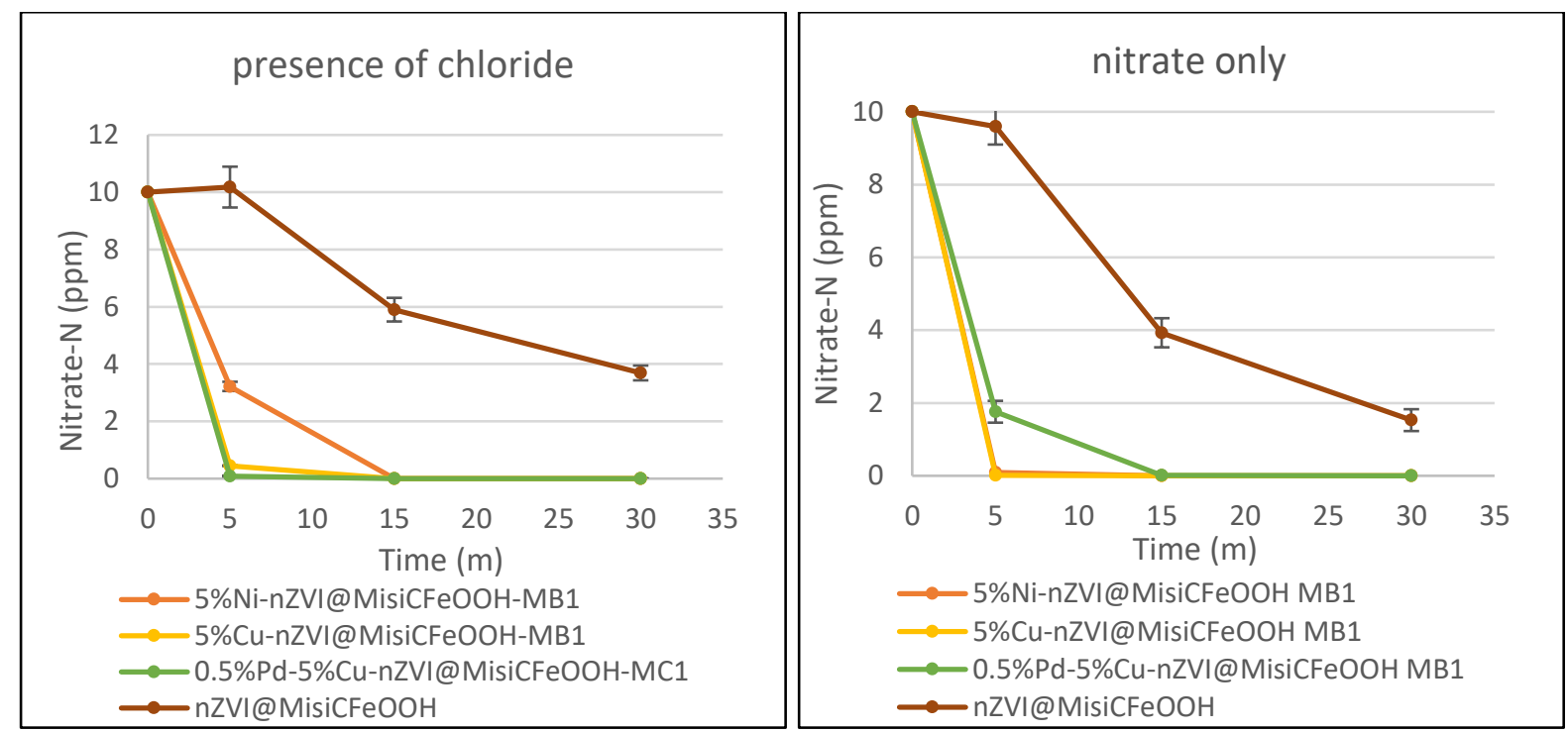

Figure 5.10. Effect of chloride on nitrate reduction and nitrate reduction in the absence of ions under aerobic conditions $(0.150 \mathrm{~g}$ of material, $25.0 \mathrm{~mL}$ of a solution containing $10 \mathrm{ppm}$ nitrate-N and 100 ppm chloride).

Nitrite levels after 5 minutes were slightly higher under anaerobic conditions compared to aerobic conditions. After 30 minutes, no nitrite was detected for all types of materials under either condition. The nitrite levels were insignificantly different from those observed when 
chloride is absent. Thus, chloride did not inhibit nitrate reduction using doped materials except for the Ni-doped system with minimal inhibition.

In contrast to the doped systems, the non-doped system was significantly inhibited by chloride, with no nitrate removed after 5 minutes and the nitrate- $\mathrm{N}$ levels at $3.7 \mathrm{ppm}$ after 30 minutes under both anaerobic and aerobic conditions, higher than the $2.6 \mathrm{ppm}$ and $1.5 \mathrm{ppm}$ observed in the absence of chloride. The effect is consistent with Hwang et al.'s study in which $\mathrm{nZVI}$ was used to remove nitrate. ${ }^{260}$ The initial nitrate- $\mathrm{N}$ was $100 \mathrm{ppm}$, the molar ratio of Fe to nitrate was 3.1:1 and an initial pH of the unbuffered solution was 7. In this reported system, nitrate removal decreased in the presence of $1,800 \mathrm{ppm}$ chloride. Hwang et al.'s explained that at low concentration of chloride, nZVI was completely oxidised to form magnetite as the main product of $\mathrm{nZVI}$ corrosion. In contrast, at a high concentration of chloride, amorphous iron hydroxide was observed. Therefore, chloride could be a competitive anion with nitrate onto the surface of nZVI particles.

In our doped materials, iron nanoparticles are protected by an outer layer which is potentially a combination of either $\mathrm{Ni}(0)$ and iron-nickel oxides in $\mathrm{Ni}$-doped material or $\mathrm{Cu}(0)$ and copper oxides in Cu-doped material (Section 3.3 and 3.4). This layer limits the corrosion of nZVI. Additionally, dopant metals function as the catalyst, enhancing electron transfer from Fe core to nitrate. As such, the reactivity of the doped materials in nitrate reduction was maintained in the presence of chloride (100 ppm).

\subsubsection{Effect of sulfate on nitrate reduction}

Similar to chloride, sulfate is considered to be an inert anion because it is unable to be reduced by $\mathrm{Fe}(0) .{ }^{150}$ However, sulfate is able to be a ligand and form complexes with iron, ${ }^{48}$ which can prevent contaminants from adsorbing on the surface of composites. Typically, sulfate is introduced to nitrate reaction when $\mathrm{H}_{2} \mathrm{SO}_{4}$ was added as a pH controllable agent. In Liou et al.'s study, sulfate in $\mathrm{Na}_{2} \mathrm{SO}_{4}$ inhibited nitrate reduction using $\mathrm{nZVI}$ and $\mathrm{Cu}-\mathrm{nZVI} .^{252}$ In this study, we investigated the reactivity of materials in removing nitrate in the presence of sulfate using $\mathrm{K}_{2} \mathrm{SO}_{4}$.

The removal of $10 \mathrm{ppm}$ nitrate- $\mathrm{N}$ in the presence of sulfate was rapid when any of the doped nZVI@MisiCFeOOH materials were used under both anaerobic and aerobic conditions (Figure 5.11 and 5.12). The levels of nitrate and nitrite when sulfate was present were not significantly 
different from those observed when sulfate is absent. Therefore, sulfate did not inhibit the reactivity of doped nZVI@MisiCFeOOH materials. This finding is inconsistent with a study in which nitrate was removed by Cu-nZVI in the presence of sulfate. ${ }^{252}$ When the initial nitrate$\mathrm{N}$ concentration was $20 \mathrm{ppm}$ and the molar ratio of Fe to nitrate was $4.7: 1$, nitrate removal was slower and more nitrite accumulated in the presence of $3.2 \mathrm{ppm}$ sulfate-S. The lack of the inhibition of sulfate using our doped materials could be due to the high reactivity of doped nZVI nanoparticles when these particles were adsorbed on the surface of Misi and higher Fe:nitrate ratio.
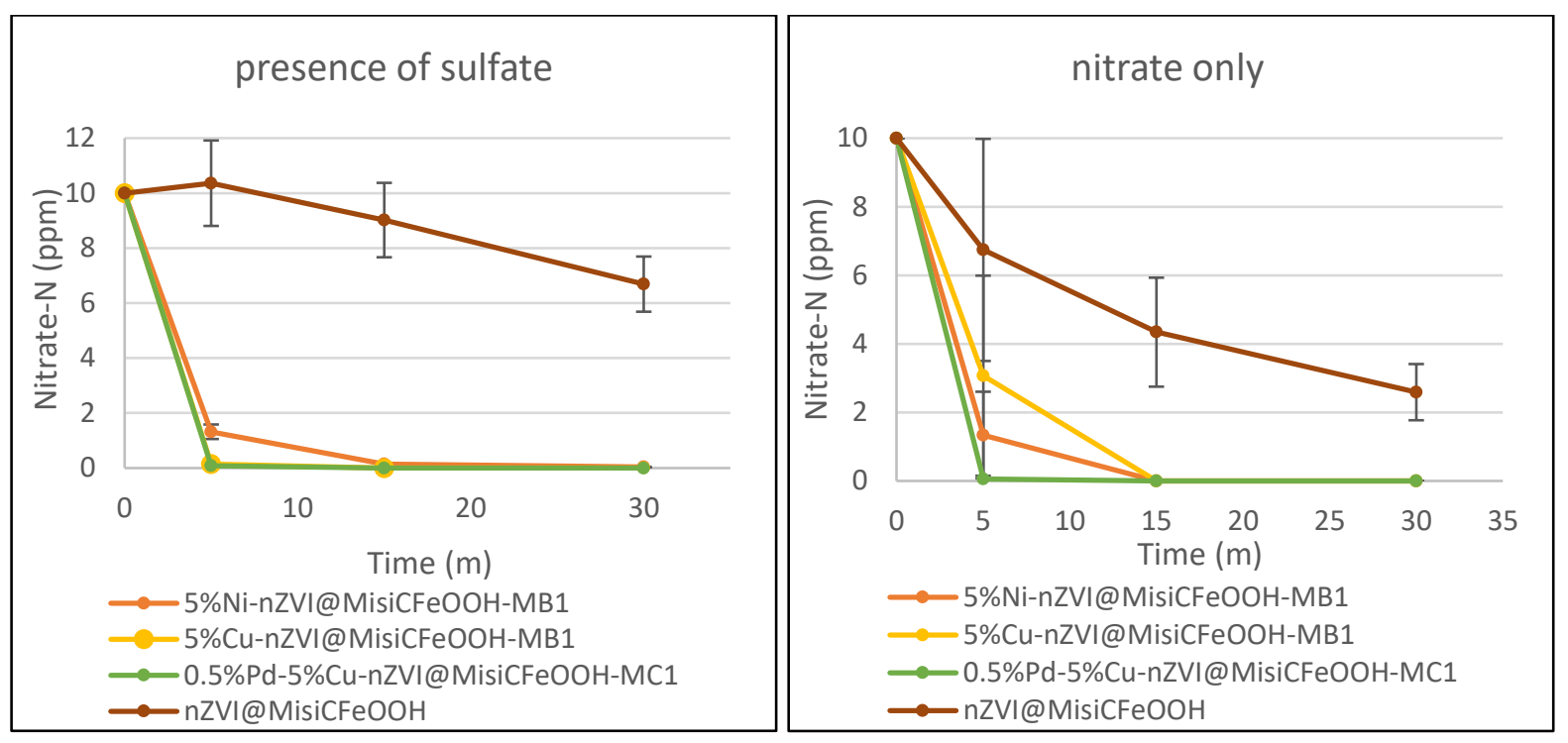

Figure 5.11. Effect of sulfate on nitrate reduction and nitrate reduction in the absence of ions under anaerobic conditions $(0.150 \mathrm{~g}$ of material, $25.0 \mathrm{~mL}$ of a solution containing $10 \mathrm{ppm}$ nitrate-N and 100 ppm sulfate-S). 

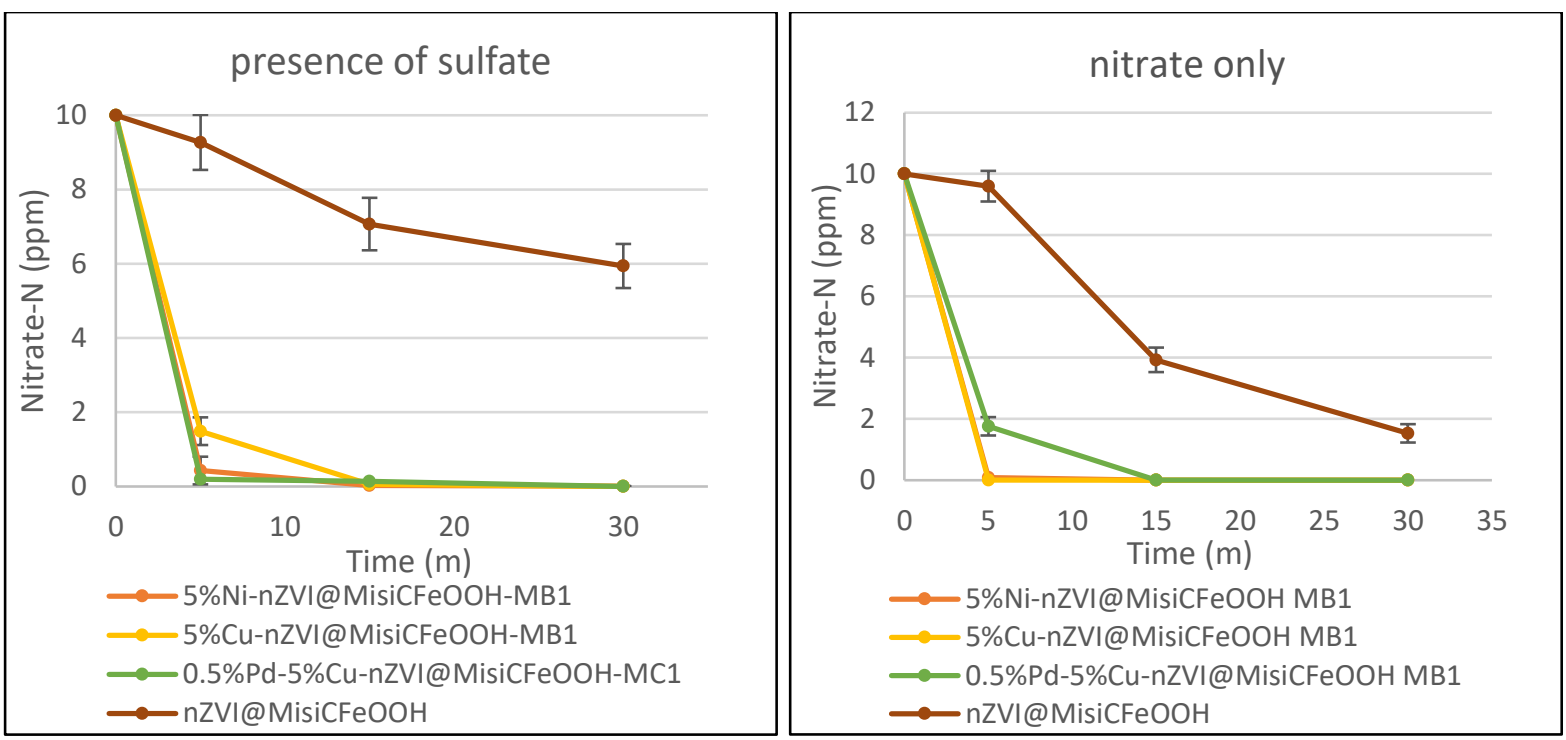

Figure 5.12. Effect of sulfate on nitrate reduction and nitrate reduction in the absence of ions under aerobic conditions $(0.150 \mathrm{~g}$ of material, $25.0 \mathrm{~mL}$ of a solution containing $10 \mathrm{ppm}$ nitrate-N and 100 ppm sulfate-S).

In contrast, sulfate affected nitrate reduction using nZVI@MisiCFeOOH (Figure 5.11 and 5.12). After 5 minutes, nitrate- $\mathrm{N}$ concentrations were greater than $9.3 \mathrm{ppm}$ under either condition. After 30 minutes, nitrate- $\mathrm{N}$ levels were $6.7 \mathrm{ppm}$ and $5.9 \mathrm{ppm}$ under either anaerobic or aerobic conditions, significantly higher than the $2.6 \mathrm{ppm}$ and $1.5 \mathrm{ppm}$ observed in the absence of sulfate. The deactivation of sulfate on nZVI@MisiCFeOOH is consistent with that found by Liou et al. ${ }^{252}$ In their study, the initial nitrate- $\mathrm{N}$ concentration was $20 \mathrm{ppm}$ and the molar ratio of Fe to nitrate was 4.7:1. Using this system, nitrate removal was slower in the presence of sulfate-S $(3.2 \mathrm{ppm})$. The decrease in nitrate conversion in the presence of sulfate can be explained by the adsorption of sulfate on the surface layer $\mathrm{FeOOH}^{261}$ and the formation of iron-sulfate complexes. ${ }^{234}$ These substances may block active Fe(0) sites, preventing electron transfer from $\mathrm{Fe}(0)$ to nitrate.

In summary, similar to chloride, sulfate is a friendly anion in nitrate reduction when doped nZVI@MisiCFeOOH was used. It did not influence the reactivity of doped nZVI@MisiCFeOOH materials due to the protection of an outer layer $(\mathrm{Ni}(\mathrm{O})$ and iron-nickel oxides in $\mathrm{Ni}$-doped material or $\mathrm{Cu}(0)$ and copper oxides in Cu-doped material) and the catalytic role of the dopants. In contrast, sulfate lowered the reactivity of nZVI@MisiCFeOOH. Thus, doped materials are promising materials for nitrate reduction in the presence of sulfate under both anaerobic and aerobic conditions. 


\subsubsection{Effect of carbonate on nitrate reduction}

Natural waters contain a variety of weak acid and bases that are the main sources of buffering. One of them is a carbon source in the form of carbonic, bicarbonate, and carbonate that always occurs together in equilibrium. Both carbonate and bicarbonate are considered to be inhibitory ions towards contaminant removal using nZVI composites. For example, bicarbonate affects the reactivity of $\mathrm{nZVI}$ in removing $\mathrm{TCE}^{150}$ or uranium ions. ${ }^{262}$ Carbonate also inhibited the removal of MO when Pd-nZVI@bentonite was used. ${ }^{191}$ In another study, both bicarbonate and carbonate decreased $\mathrm{Cr}(\mathrm{VI})$ removal when Ni-nZVI was used, with carbonate having more significant inhibition than bicarbonate. ${ }^{263}$ In a study on the effect of carbonate on nitrate reduction, carbonate interfered with the reactivity of both $\mathrm{nZVI}$ and $\mathrm{Cu}$ $\mathrm{nZVI}{ }^{252}$ In our study, the influence of carbonate in $\mathrm{K}_{2} \mathrm{CO}_{3}$ on nitrate reduction was examined to determine whether carbonate affects doped and non-doped nZVI@Misi materials.

\subsubsection{Effect of carbonate on nitrate reduction in anaerobic conditions}

Carbonate affected the reactivity of all doped nZVI@Misi materials examined under anaerobic conditions. After 5 minutes, nitrate- $\mathrm{N}$ concentrations were significantly higher in the presence of carbonate than in the absence of carbonate in anaerobic conditions (Figure 5.13). After 30 minutes, when 5\%Ni-nZVI@MisiCFeOOH-MB1 was used, nitrate-N concentration was higher than when carbonate was absent. In contrast, when either $\mathrm{Cu}$ doped or Pd-Cu-doped nZVI@MisiCFeOOH was used, the nitrate concentrations were similar to those found in the absence of carbonate. Nitrite build-up was also monitored in these reactions. The nitrite levels were relatively similar in the presence or absence of carbonate when any of doped materials were used. 

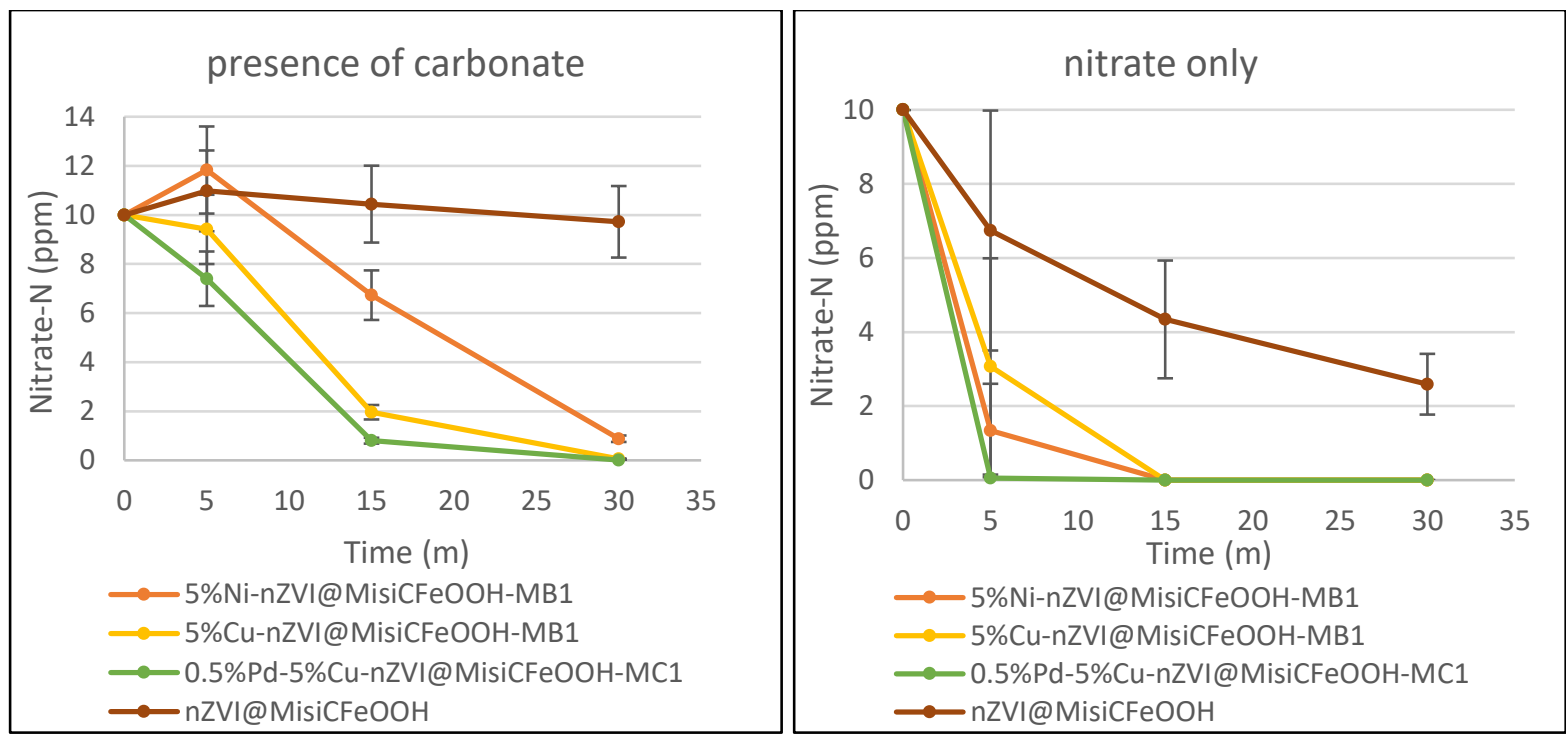

Figure 5.13. Effect of carbonate on nitrate reduction and nitrate reduction in the absence of ions under anaerobic conditions $(0.150 \mathrm{~g}$ of material, $25.0 \mathrm{~mL}$ of a solution containing 10 ppm nitrate- $N$ and 20 ppm carbonate-C).

In contrast, carbonate deactivated nZVI@MisiCFeOOH in removing nitrate under anaerobic conditions. After 30 minutes, the nitrate- $\mathrm{N}$ concentrations were unchanged compared to the initial concentration of $10 \mathrm{ppm}$ nitrate-N. At some points over the course of the reaction, the nitrate- $\mathrm{N}$ levels were slightly greater than $10 \mathrm{ppm}$. This can be explained by the random errors that have made in the preparation step of the initial solutions. Additionally, the eluent for running the $\mathrm{IC}$ is a mixture of carbonate and bicarbonate. Thus, this may introduce uncertainty in nitrate concentrations.

Our results of the effect of carbonate are consistent with those found by Liou et al.. ${ }^{252}$ In their study, the effect of carbonate on nitrate reduction using nZVI or Cu-nZVI was examined. The initial nitrate- $\mathrm{N}$ concentration was $20 \mathrm{ppm}$ and the initial molar ratio of Fe to nitrate was 4.7:1. In this reported system, the nitrate reduction was slower in the presence of carbonate $(1.2$ ppm).

\subsubsection{Effect of carbonate on nitrate reduction in aerobic conditions}

Carbonate also affected the reactivity of doped nZVI@Misi materials under aerobic conditions although the effect was not as strong as under anaerobic. For instance, after 5 minutes, nitrate levels were significantly higher than in the absence of added carbonate (Figure 5.14). However, after 30 minutes, the nitrate concentrations were similar to those found in the 
absence of carbonate. Nitrite build-up was dependent upon the nature of materials. After 5 minutes, when either Ni-doped or Pd-Cu-doped material was used, nitrite concentrations were similar to those observed in the absence of carbonate. In contrast, when Cu-doped material was used, the nitrite level was significantly higher than in the absence of carbonate. However, after 30 minutes, nitrite levels were slightly higher than when carbonate was absent for all types of doped materials.
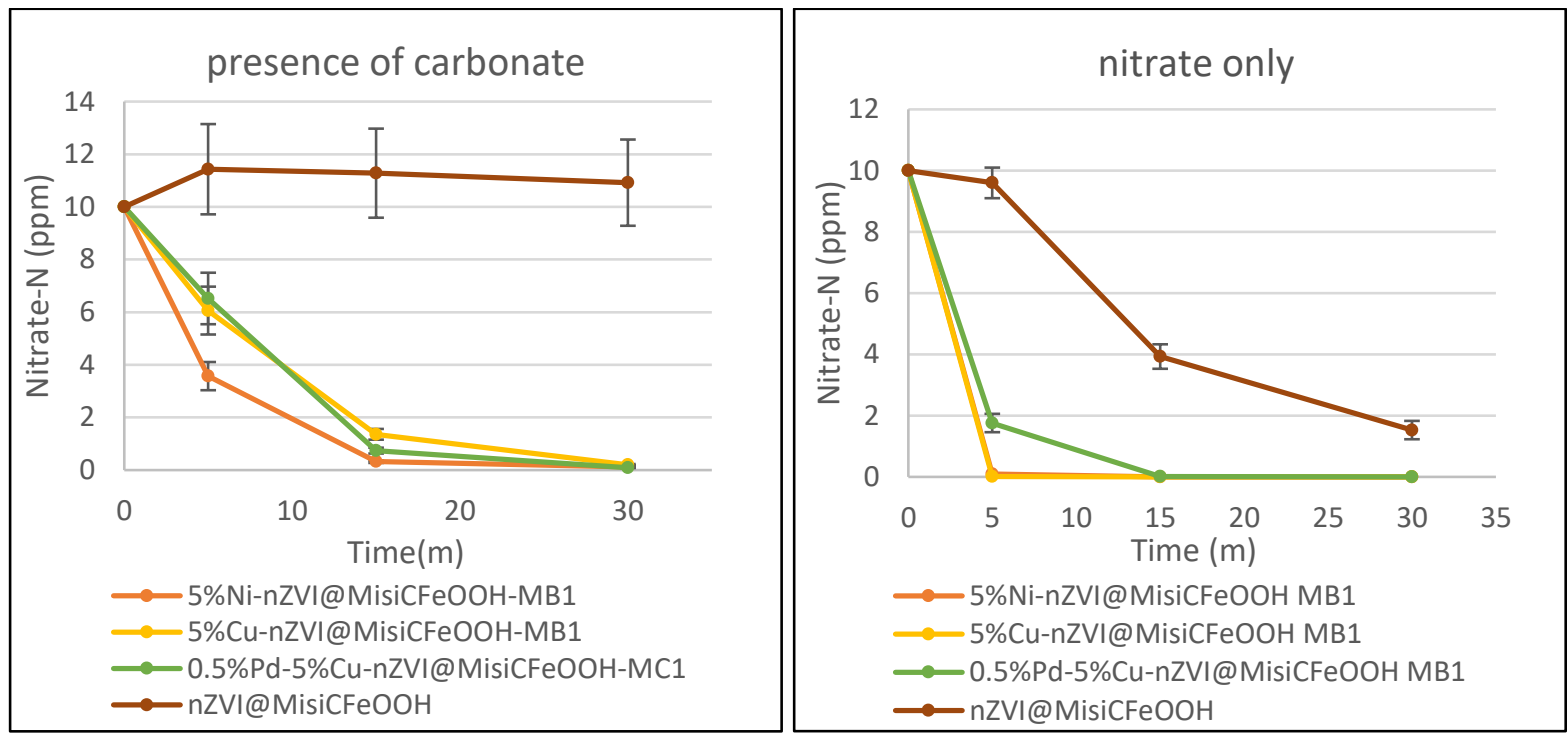

Figure 5.14. Effect of carbonate on nitrate reduction and nitrate reduction in the absence of ions under aerobic conditions $(0.150 \mathrm{~g}$ of material, $25.0 \mathrm{~mL}$ of a solution containing $10 \mathrm{ppm}$ nitrate- $N$ and 20 ppm carbonate-C).

In contrast to doped materials but similar to under anaerobic conditions, carbonate deactivated nZVI@MisiCFeOOH in removing nitrate. After 30 minutes, the nitrate-N concentrations remained at 10 ppm. Therefore, nZVI@MisiCFeOOH should not be used in nitrate removal in the presence of carbonate regardless of either condition.

The inhibition of carbonate on nitrate reduction could be due to the corrosion of iron with a carbonate solution, resulting in the formation of complexes and precipitates. ${ }^{191}$ For example, carbonate may generate precipitate $\mathrm{FeCO}_{3}{ }^{150}$ or both $\mathrm{FeCO}_{3}$ and $\mathrm{Fe}(\mathrm{OH})_{2}$ on the surface of particles. ${ }^{252}$ In addition, carbonate can form a combination of magnetite/maghemite and carbonate green rust $\left(\mathrm{Fe}^{(\mathrm{III})} \mathrm{Fe}_{4} \mathrm{IIII}_{2}(\mathrm{OH})_{12} \cdot \mathrm{CO}_{3}\right){ }^{262}$ The production of these substances onto the surface of particles prevents the electron transfer from $\mathrm{Fe}(0)$ core to nitrate using doped materials and deactivates using non-doped nZVI@MisiCFeOOH. Moreover, carbonate with 
negatively charged can adsorb on the surface of nZVI particles, which competes with nitrate. As a result, a decrease in nitrate reduction was observed.

In summary, carbonate inhibits nitrate reduction using doped materials. The inhibition is under aerobic conditions less significant than under anaerobic conditions. As such, doped materials are able to remove nitrate from "real" waters in the presence of carbonate, especially under aerobic conditions.

\subsubsection{Effect of phosphate on nitrate reduction}

Phosphate has been reported as an inhibitory ion towards Fe reactivity. At $\mathrm{pH}$ less than 8 , phosphate combines with positively charged components on the surface of Fe to form complexes. ${ }^{48}$ In several studies, phosphate affects the removal of contaminants. For example, hydrogen phosphate $\left(\mathrm{HPO}_{4}{ }^{2-}\right)$ decreased the removal of TCE when $\mathrm{nZVI}$ was used. ${ }^{150}$ Dihydrogen phosphate $\left(\mathrm{H}_{2} \mathrm{PO}_{4}^{-}\right)$inhibited the removal of tetracycline when $\mathrm{nZVI}$ on polyvinylpyrrolidone was used. ${ }^{264}$ Phosphate $\left(\mathrm{PO}_{4}{ }^{3-}\right)$ also affected nitrate reduction when $\mathrm{nZVI}$ or CU-nZVI was used and it had the most significant passivation compared to sulfate or carbonate. ${ }^{252}$ In this study, the effect of phosphate $\left(\mathrm{KH}_{2} \mathrm{PO}_{4}\right)$ on nitrate reduction was examined to investigate if phosphate could inhibit doped and non-doped nZVI@Misi.

\subsubsection{Effect of phosphate on nitrate reduction in anaerobic conditions}

Phosphate passivated doped nZVI@MisiCFeOOH in reducing nitrate under anaerobic conditions. Both nitrate and nitrite concentrations were higher in the presence of phosphate (Figure 5.15). These differences were significant when all types of doped materials were used. Of these materials examined, $\mathrm{Cu}$-doped reduced nitrate with the slowest rate while both $\mathrm{Ni}$ doped and $\mathrm{Pd}$-Cu-doped materials had the same nitrate conversion. In contrast, phosphate deactivated nitrate reduction when nZVI@MisiCFeOOH was used. The nitrate level not only did not decrease but also was higher than the initial concentration over the course of 30 minutes. The reason for this result was not evident; however, this could be due to the impact of phosphate on the determination of nitrate using the IC, leading to positive errors in nitrate concentrations. 

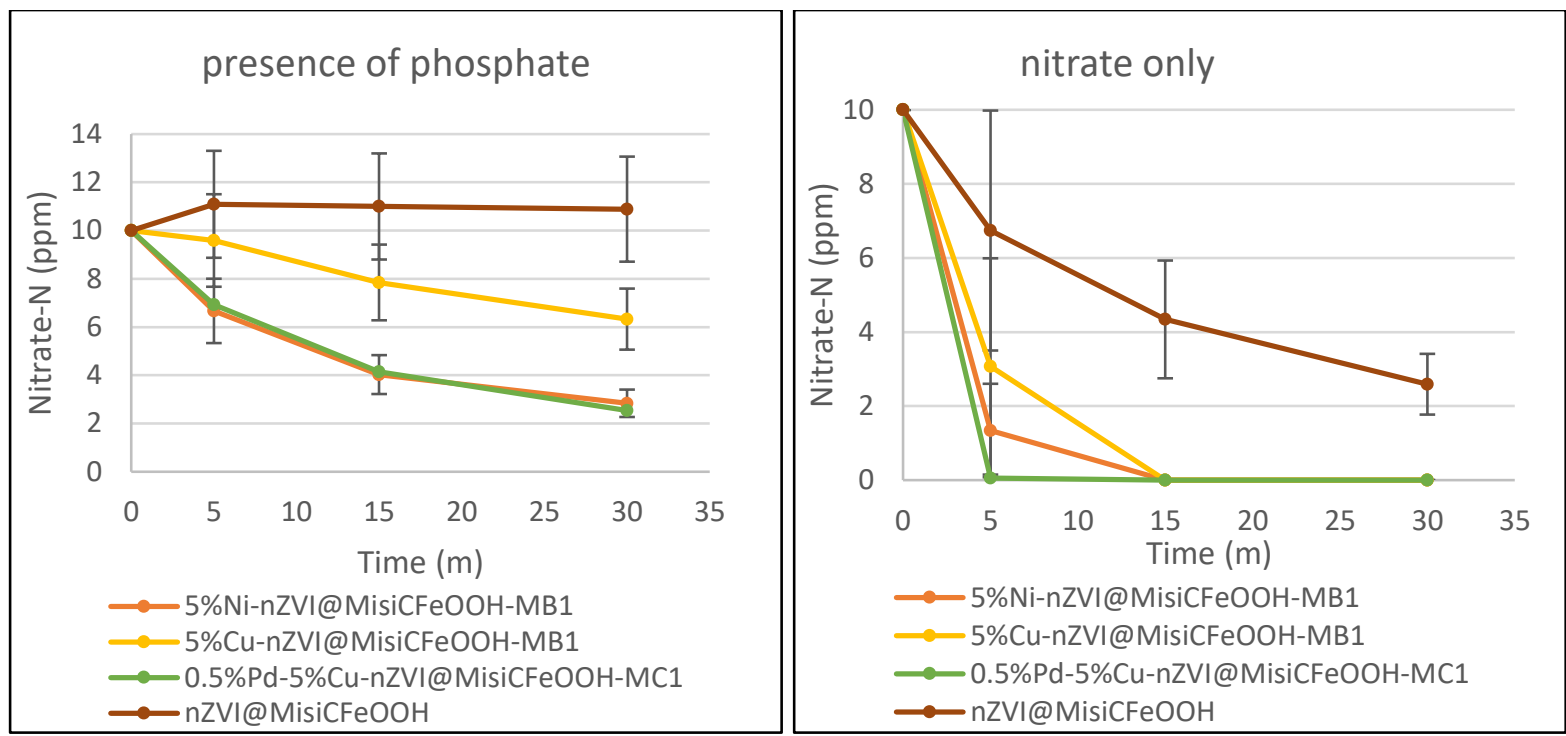

Figure 5.15. Effect of phosphate on nitrate reduction and nitrate reduction in the absence of ions under anaerobic conditions $(0.150 \mathrm{~g}$ of material, $25.0 \mathrm{~mL}$ of a solution containing 10 ppm nitrate-N and 10 ppm phosphate-P).

The inhibition of phosphate under anaerobic conditions is consistent with some studies in which the influence of phosphate on nitrate reduction was examined. Liou et al. investigated the effect of phosphate $\left(\mathrm{PO}_{4}{ }^{3-}\right)$ in nitrate reduction using Cu-nZVI or Ni-nZVI@zeolite. ${ }^{252}$ The initial nitrate- $\mathrm{N}$ concentration was $20 \mathrm{ppm}$ and the molar ratio of Fe to nitrate was 4.7:1. In this reported system, nitrate removal was slower in the presence of $\mathrm{PO}_{4}{ }^{3-}$ at $3.1 \mathrm{ppm}$ phosphate-P. In He et al.'s study, phosphate inhibited nitrate removal using supported NinZVI@zeolite (the mass ratio is 1:10:20). ${ }^{193}$ The nitrate- $N$ concentration was 20 ppm, the molar ratio of Fe to nitrate was $6.3: 1$ and the solution $\mathrm{pH}$ was 5 . Nitrate reduction was inhibited in the presence of 5 ppm phosphate-P.

\subsubsection{Effect of phosphate on nitrate reduction in aerobic conditions}

Phosphate also affected the reactivity of doped nZVI@Misi materials under aerobic conditions although the effect was not as strong as under anaerobic conditions (Figure 5.16). Additionally, nitrite build-up was higher in the presence of phosphate, but the difference was insignificant. The less significant influence of phosphate under aerobic conditions leaves open the possibility of using these doped materials to remove nitrate from "real" waters in the presence of phosphate. Similar to those under anaerobic conditions, Cu-doped materials had the highest level of inhibition. 

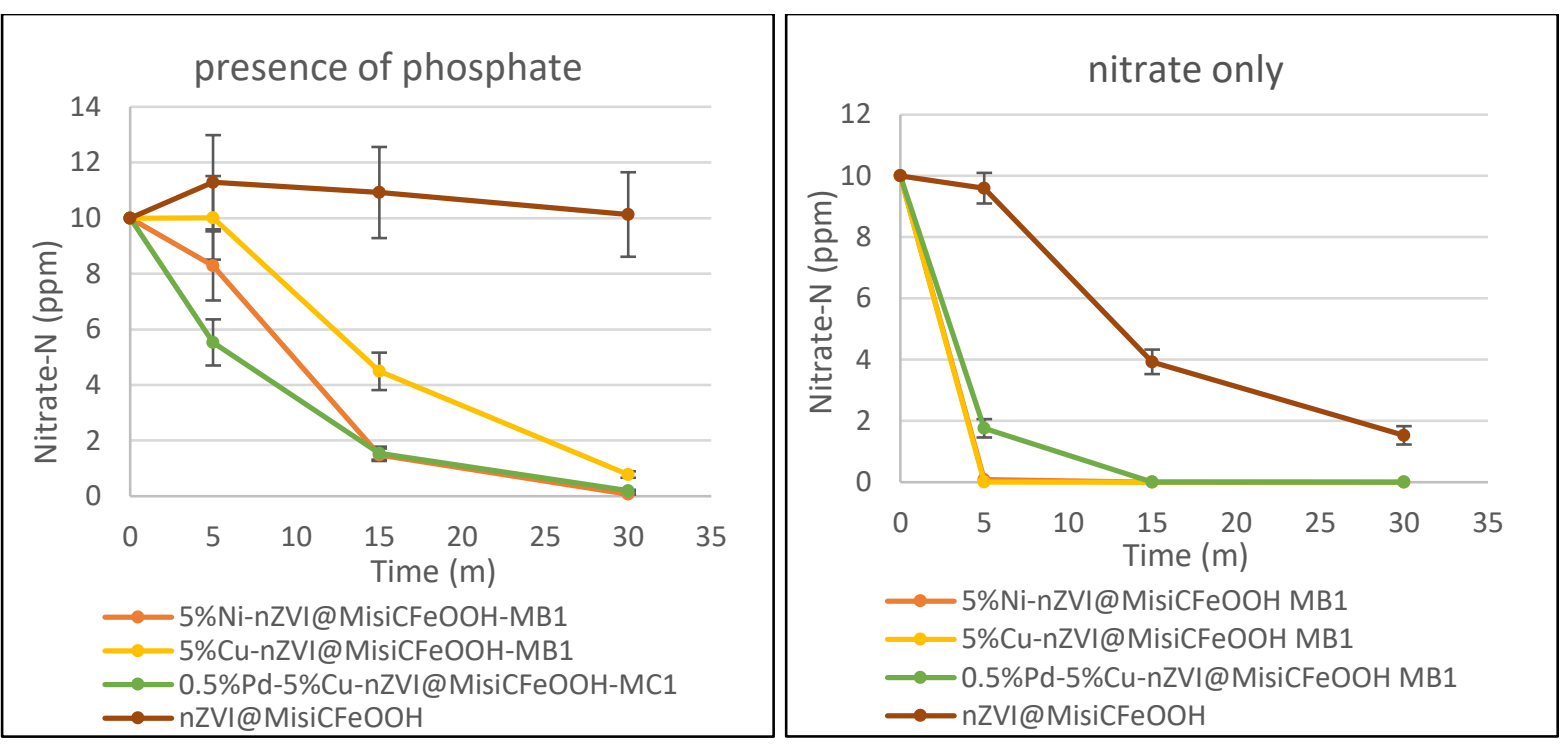

Figure 5.16. Effect of phosphate on nitrate reduction and nitrate reduction in the absence of ions under aerobic conditions $(0.150 \mathrm{~g}$ of material, $25.0 \mathrm{~mL}$ of a solution containing $10 \mathrm{ppm}$ nitrate- $N$ and 10 ppm phosphate-P).

Interestingly, all materials, 5\%Ni-nZVI@MisiCFeOOH-MB1, 5\%Cu-nZVI@MisiCFeOOH-MB1, 0.5\%Pd-5\%Cu-nZVI@MisiCFeOOH-MC1, and nZVI@MisiCFeOOH, had high capacity in removing phosphate in the presence of nitrate under both anaerobic and aerobic conditions (Table 5.1). Under anaerobic conditions, after 5 minutes, phosphate-P levels decreased from $10 \mathrm{ppm}$ to less than $2 \mathrm{ppm}$ and after 30 minutes, phosphate-P levels were less than $0.5 \mathrm{ppm}$ (95\% phosphate removal). Phosphate removal was faster under aerobic conditions. After 5 minutes, phosphate-P levels were less than $0.2 \mathrm{ppm}$ ( $98 \%$ phosphate removal). Thereby, supported doped $\mathrm{nZVI}$ on MisiCFeOOH can simultaneously remove nitrate and phosphate effectively. These findings are consistent with a previous investigation on simultaneous removal of nitrate and phosphate using Ni-nZVI@zeolite. ${ }^{193}$ 
Table 5.1. Phosphate concentrations during nitrate reduction in the presence of phosphate (10 ppm as the initial phosphate-P).

\begin{tabular}{|l|c|c|c|c|c|c|}
\hline Phosphate-P (ppm)/ Time & \multicolumn{2}{|c|}{5 minutes } & \multicolumn{2}{|c|}{15 minutes } & \multicolumn{2}{|c|}{30 minutes } \\
\hline Conditions & $\mathrm{N}_{2}$ & Air & $\mathrm{N}_{2}$ & Air & $\mathrm{N}_{2}$ & Air \\
\hline 5\%Ni-nZVI@MisiCFeOOH-MB1 & 0.98 & 0.05 & 0.87 & 0.08 & 0.23 & 0.23 \\
\hline 5\%Cu-nZVI@MisiCFeOOH-MB1 & 0.67 & 0.12 & 0.30 & 0.07 & 0.31 & 0.15 \\
\hline 0.5\%Pd-5\%Cu-nZVI@MisiCFeOOH-MC1 & 0.10 & 0.09 & 0.17 & 0.10 & 0.19 & 0.10 \\
\hline nZVI@MisiCFeOOH & 2.02 & 0.18 & 0.96 & 0.07 & 0.54 & 0.04 \\
\hline MisiCFeOOH & 2.06 & & 0.79 & & 0.45 & \\
\hline MisiC & 10.05 & & 9.86 & & 9.71 & \\
\hline
\end{tabular}

$\mathrm{N}_{2}$ : anaerobic conditions; Air: aerobic conditions

The inhibition of phosphate on nitrate reduction as well as the removal of phosphate by our materials can be explained via three reasons. Firstly, the initial pH of the solution containing nitrate and phosphate $\left(\mathrm{H}_{2} \mathrm{PO}_{4}{ }^{-}\right)$was less than 6.3 at which phosphate may directly adsorb on the positively charged surface of nZVI. ${ }^{48,264}$ Secondly, phosphate may form complexes with products from iron corrosion, for example, the complexes of phosphate on iron oxides, ${ }^{265}$ the complexes of phosphate and $\mathrm{Fe}(\mathrm{II}) / \mathrm{Fe}$ (III) on the surface of materials ${ }^{252}$ or iron-phosphate (vivianite- $\left.\mathrm{Fe}_{3}\left(\mathrm{PO}_{4}\right)_{2} .8 \mathrm{H}_{2} \mathrm{O}\right) .{ }^{150}$ Finally, during the nitrate reduction, hydroxide is produced, therefore, phosphate may be removed from the solutions by co-precipitation. ${ }^{193}$ As such, these forms of phosphate diminish the active sites for the adsorption and then removal of contaminants.

\subsubsection{Simultaneous effect of coexisting anions on nitrate reduction}

In the previous sections, the effect of individual ions was examined. Natural water typically contains all these ions. The effect on nitrate reduction may be accumulative, or independent in the presence of all ions. There have been several studies on the effect of anions on the reactivity of iron materials. However, typically, contaminants were examined in the presence 
of individual ion. In this study, the simultaneous effect of all four coexisting ions on nitrate reduction was examined.

\subsubsection{Simultaneous effect of coexisting anions on nitrate reduction under anaerobic} conditions

The presence of these four ions affected nitrate reduction using doped materials under anaerobic conditions. Nitrate reduction was significantly slower than that observed in both the absence of ions and the presence of individual ion (Figure 5.17). The positive effect of Pd in trimetallic nZVI@MisiCFeOOH was observed. Among doped materials, 5\% NinZVI@MisiCFeOOH-MB1 reduced nitrate fastest (65\% nitrate conversion) with the lowest nitrite accumulation. As such, Ni-doped material was selected as the most effective material for nitrate removal in the presence of chloride, sulfate, carbonate, and phosphate under anaerobic conditions. In contrast, the presence of four coexisting ions deactivated nZVI@MisiCFeOOH. No nitrate was reduced over the course of 30 minutes.
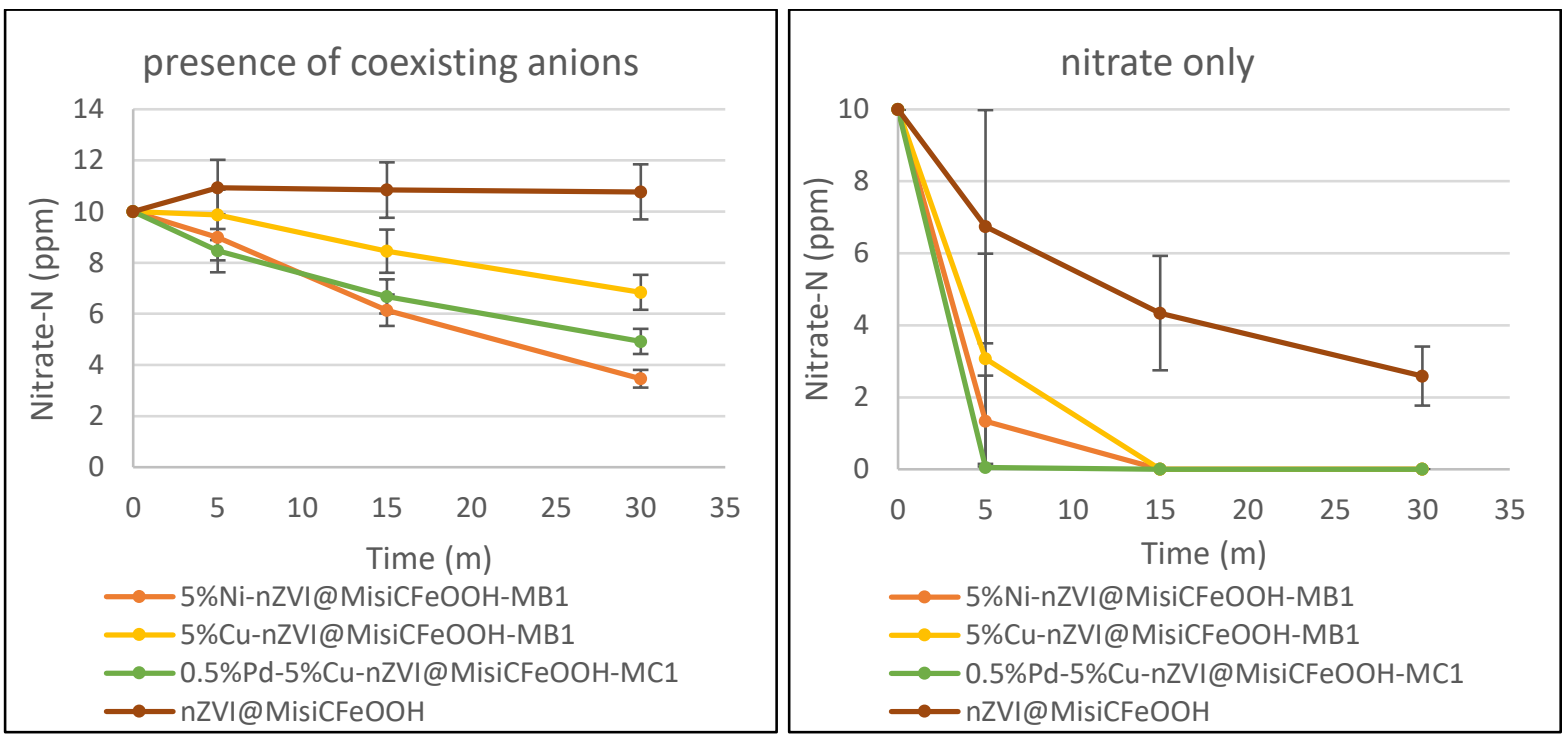

Figure 5.17. Effect of coexisting anions on nitrate reduction and nitrate reduction in the absence of ions under anaerobic conditions $(0.150 \mathrm{~g}$ of material, $25.0 \mathrm{~mL}$ of a solution containing 10 ppm nitrate-N, 100 ppm chloride, 100 ppm sulfate-S, 20 ppm carbonate-C and 10 ppm phosphate-P).

\subsubsection{Simultaneous effect of coexisting anions on nitrate reduction under aerobic conditions}

These ions simultaneously affected nitrate reduction using doped materials under aerobic conditions, but the effect was not as strong as under anaerobic conditions. Nitrate reduction 
was significantly slower than that observed in both the absence of ions and the presence of individual ion (Figure 5.18). Although the nitrate reduction was slightly slower using Ni-doped material ( $75 \%$ nitrate conversion) than that using Pd-Cu-doped systems ( $84 \%$ nitrate conversion), no nitrite was detected using $\mathrm{Ni}$-doped system. In contrast, but similar to the results under anaerobic conditions, four anions deactivated nZVI@MisiCFeOOH. No nitrate was reduced over the course of 30 minutes.
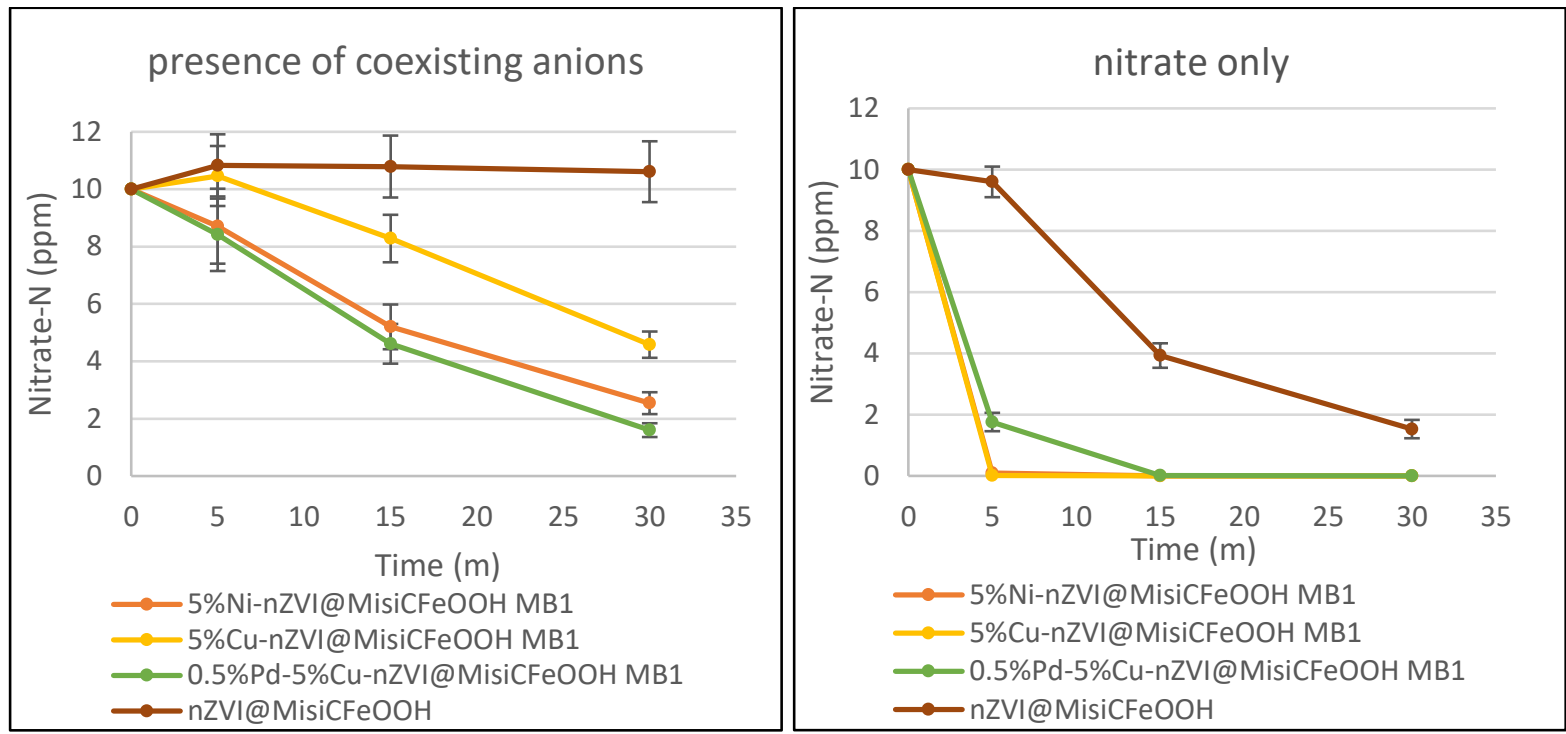

Figure 5.18. Effect of coexisting anions on nitrate reduction and nitrate reduction in the absence of ions under aerobic conditions $(0.150 \mathrm{~g}$ of material, $25.0 \mathrm{~mL}$ of a solution containing 10 ppm nitrate-N, 100 ppm chloride, 100 ppm sulfate-S, 20 ppm carbonate-C and 10 ppm phosphate-P).

Doped nZVI@MisiCFeOOH and non-doped nZVI@MisiCFeOOH materials had the capacity of removing phosphate in the presence of nitrate- $\mathrm{N}(10 \mathrm{ppm})$, chloride (100 ppm), sulfate-S (100 ppm), and carbonate-C (20 ppm) under both anaerobic and aerobic conditions (Table 5.2). In anaerobic, after 30 minutes, phosphate-P levels were approximately $2 \mathrm{ppm}(80 \%$ of phosphate removal). In aerobic conditions, phosphate was removed faster. After 30 minutes, phosphate-P were below 0.5 ppm (95\% phosphate removal). 
Table 5.2. Phosphate concentrations during nitrate reduction in the presence of coexisting anions (10 ppm as the initial phosphate-P).

\begin{tabular}{|l|c|c|c|c|c|c|}
\hline Phosphate-P (ppm)/ Time & \multicolumn{2}{|c|}{5 minutes } & \multicolumn{2}{|c|}{15 minutes } & \multicolumn{2}{c|}{30 minutes } \\
\hline Conditions & $\mathrm{N}_{2}$ & Air & $\mathrm{N}_{2}$ & Air & $\mathrm{N}_{2}$ & Air \\
\hline 5\%Ni-nZVI@MisiCFeOOH-MB1 & 1.94 & 1.14 & 1.47 & 0.54 & 1.56 & 0.52 \\
\hline 5\%Cu-nZVI@MisiCFeOOH-MB1 & 4.28 & 1.95 & 2.35 & 0.40 & 1.89 & 0.42 \\
\hline 0.5\%Pd-5\%Cu-nZVI@MisiCFeOOH-MC1 & 1.96 & 0.09 & 1.19 & 0.10 & 1.12 & 0.10 \\
\hline nZVI@MisiCFeOOH & 4.60 & 3.94 & 3.14 & 0.66 & 2.42 & 0.43 \\
\hline
\end{tabular}

$\mathrm{N}_{2}$ : anaerobic conditions; Air: aerobic conditions

In nitrate reduction without other ions, the reactivity of doped materials in removing $10 \mathrm{ppm}$ nitrate- $\mathrm{N}$ was not distinguishable due to the very fast reaction. However, with higher nitrate$\mathrm{N}$ concentrations (50 ppm), Cu-doped material converted nitrate to nitrite faster than the other materials. The reduction produced Fe(II) that could possibly combine with phosphate to form solid substances including $\mathrm{Fe}_{3}\left(\mathrm{PO}_{4}\right)_{2}$ in anaerobic conditions or $\mathrm{FePO}_{4}$ in aerobic conditions. These substances might deposit on the surface of nZVI fastest, leading to the prevention of electron transfer from the Fe core to nitrate.

In summary, all doped materials had high reactivity in co-decontamination of nitrate and phosphate when coexisting anions are present, especially in aerobic conditions. Of these, 5\%Ni-nZVI@MisiCFeOOH-MB1 not only reduced contaminants effectively but also eliminated nitrite build-up. As such, it will be a promising material to apply in natural waters.

\subsection{Conclusion}

Doped materials, including Ni-doped, Cu-doped, and Pd-Cu-doped on Misi, had high reactivity towards nitrate reduction under aerobic conditions. Although these materials were affected by the presence of oxygen, the inhibition was minimal. In addition, during reactions, the generation of soluble iron and dopant metals was insignificant. Interestingly, both chloride and sulfate did not interfere with nitrate reduction. In contrast, carbonate and phosphate 
were inhibitory coexisting ions towards nitrate reduction. This could be due to the competition and formation of precipitates that prevent the electron transfer. The inhibition was less under aerobic conditions than anaerobic conditions. The reason for this phenomenon was not fully understood. However, it can be partly explained by the formation of radical ions, similar to that when nZVI@MisiCFeOOH was exposed to oxygen (eq.5.1 - 5.4). Compared to previously reported materials, our doped materials were passivated less significantly when they were exposed to aerobic conditions. The reactivity of our doped materials maintained in the presence of chloride and sulfate. In addition, although interference was observed with both carbonate and phosphate, the interference of these ions only occurs at concentrations that are typically not observed in ecosystems. Encouragingly, the concentrations in which we did observe an inhibitory effect were significantly higher than that observed with other reported systems. For instance, both nZVI and Cu-nZVI were inhibited when they were used to remove nitrate in the presence of either carbonate-C (1.2 ppm) or phosphate-P (3.1 ppm). ${ }^{252}$ As such, these doped material in this current study, especially, 5\%Ni-nZVI@MisiCFeOOH is the ideal material to apply to "real" environments. 


\section{Nitrate reduction from lake water}

\subsection{Introduction}

In this chapter, the investigations of doped nZVI@Misi materials towards nitrate reduction from lake water are discussed. This includes both the reactivity of doped nZVI@Misi and the generation of soluble metal by-products. The reactivity of these materials was examined through nitrate conversion and nitrite accumulation.

Natural waters consist of numerous elements, including not only contaminants that need removing but also common ions that often inhibit the materials designed to remove contaminants. For example, phosphate can influence nZVI when nZVI was used for nitrate reduction. ${ }^{48}$ Carbonate passivates both $n Z V I$ and $\mathrm{Cu}-\mathrm{nZVI}$ when these materials were used to reduce nitrate. ${ }^{252}$ Humic acid, a common natural organic substance, can activate and passivate the nitrate reduction using $\mathrm{nZVI}$, depending on its state ${ }^{266}$ or its concentration. ${ }^{267}$ When humic acid is in bulk phase, it serves as an electron transfer mediator, consequently, accelerating nitrate reduction. However, when humic acid absorbs on the surface of nZVI, it acts as an inhibitory factor, decreasing nitrate reduction. In contrast to inhibitory ions, several ions are promoting factors that speed up nitrate removal. Metal ions such as $\mathrm{Cd}(\mathrm{II}), \mathrm{Cu}(\mathrm{II})$, and $\mathrm{Pb}(\mathrm{II})$ were useful in enhancing nitrate reduction when either nZVI, ${ }^{251} \mathrm{Ni}$-nZVI@kaoline, ${ }^{199}$ or Ni-nZVI@kaolinite ${ }^{192}$ was used, respectively. Finally, some major ions do not significantly interact with the materials; for instance, $\mathrm{Na}(\mathrm{I})$ had a slightly inhibitory effect towards nitrate reduction when $\mathrm{ZVI}$ was used in the presence of $\mathrm{CO}_{2} \cdot{ }^{268}$ Similarly, chloride also did not significantly affect nitrate reduction when nZVI@MisiCFeOOH was used. ${ }^{1}$

Due to the variety of components and their interactions, natural waters are the best place to evaluate the effectiveness of materials in removing contaminant or co-contaminants. In this research, the reactivity of our most promising materials towards nitrate reduction from Lake Horowhenua (New Zealand) was examined. Firstly, the reactivity of doped materials towards nitrate reduction was screened using samples collected on April 27, 2017. The investigations included nitrate reduction from lake water and lake water that was spiked with $10 \mathrm{ppm}$ nitrate-N with and without $\mathrm{pH}$ adjustment. Secondly, the reactivity was studied in greater details with water collected on April 23, 2018. The investigations included nitrate reduction from lake water spiked with $10 \mathrm{ppm}$ and $50 \mathrm{ppm}$ nitrate- $\mathrm{N}$ in order to examine the reactivity 
of these materials under more extreme conditions as well as the determination of the soluble iron. The iron was measured to not only to monitor its production during the reaction, but to also ascertain the viability of our materials for environmental remediation. That is, if the amount of soluble iron is greater than the maximum allowable in drinking water, then these materials might not be viable. All investigations were conducted under both anaerobic and aerobic conditions.

\subsection{Lake Horowhenua}

Lake Horowhenua is a small shallow lake with the surface area of $2.9 \mathrm{~km}^{2}$ and the mean depth of $1.3 \mathrm{~m}^{269}$ It is located in Horowhenua district, Southern Manawatu-Wanganui, the west coast of the North Island of New Zealand. It currently receives the runoffs from intensive agriculture and received the treated sewage water from the town of Levin for 25 years, between 1962 and 1987. ${ }^{269-270}$ Nutrients from sewage discharge have accumulated in the sediment and are the predominant reason for the current hypertrophic condition. ${ }^{270}$ Lake Horowhenua has high nitrogen and low phosphorus in winter while low nitrogen and high phosphorus in summer. The source of nitrogen is fertilizer leaching from the horticulture, market gardening, and intensive dairy farming with the most nitrogen arriving via the inflow of the Arawhata Stream. From 1989 to 2008, the total nitrogen concentration in this inflow increased from $10.5 \mathrm{ppm}$ to $13.6 \mathrm{ppm}$. In contrast, the main source of phosphorus is from sediment.

The water quality of Lake Horowhenua has decreased since 1997 and it was ranked as one of the most polluted lakes in New Zealand. ${ }^{271}$ Considering the trophic level index that concludes water clarity, chlorophyll content, total phosphorous and nitrogen, Lake Horowhenua is considered to be super trophic. Due to the poor quality, the lake is only suitable for some activities such as boating, rowing, and sailing. However, in summer, these activities are not allowed due to toxic algal blooms containing cyanobacteria.

The lake water that was collected has a yellowish colour. It has sediments and green components that is assumed algae (Figure 6.1). $\mathrm{pH}$ value of lake water was around 7. The concentrations of anions in these samples were measured using IC (Table 6.1). The nitrate- $\mathrm{N}$ concentrations were approximately $1 \mathrm{ppm}$. The levels of nitrite and phosphate were not detected using the IC. The levels of chloride and sulfate were detected, approximately $19 \mathrm{ppm}$ 
and $4 \mathrm{ppm}$, respectively. However, they were not high enough to influence the nitrate reduction (see Chapter 5).

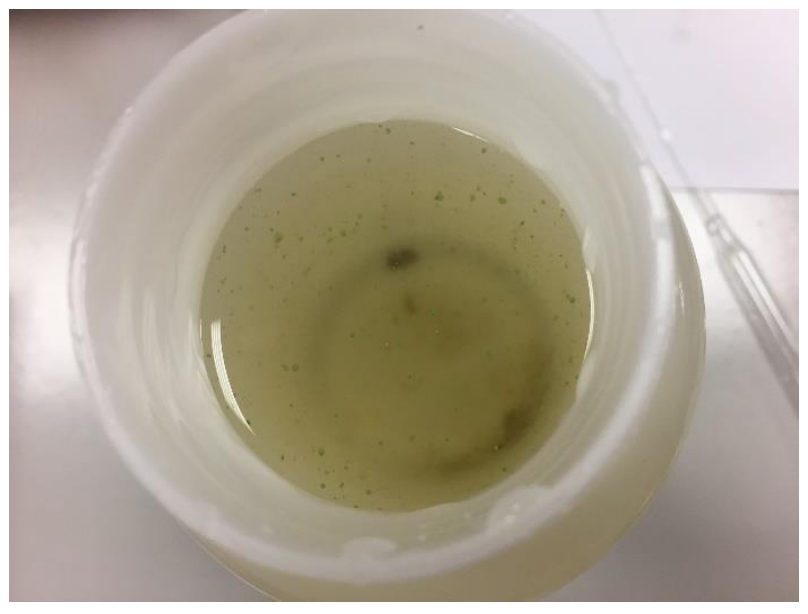

Figure 6.1. Water sample in Lake Horowhenua.

Table 6.1. The components of the sample collected from Lake Horowhenua.

\begin{tabular}{|l|c|c|c|c|c|c|}
\hline Sample & $\mathrm{pH}$ & chloride & Nitrite-N & Nitrate-N & Sulfate-S & Phosphate-P \\
\hline April 27, 2017 & 6.8 & 19.2 & ND & 0.7 & 4.2 & ND \\
\hline April 23, 2018 & 7.1 & 19.7 & ND & 1.3 & 3.4 & ND \\
\hline
\end{tabular}

ND*: no detection

\subsection{Preliminary results of nitrate reduction from lake water}

There have been numerous studies on the effect of coexisting ions on the reactivity of nZVI materials. The effect of these ions was discussed in Section 5.5. In addition to inorganic ions, naturally organic substances, typically humic acid, are always present in natural waters. In most of the studies, the influence of humic acid on the reactivity of nZVI and doped nZVI were examined towards organic compound removal. For example, when chloroform was dechlorinated by $\mathrm{nZVI}$, humic acid at the concentration of less than $100 \mathrm{ppm}$ promoted electron transfer. ${ }^{272}$ However, humic acid was commonly reported to inhibit nZVI even though its concentration was low (less than $50 \mathrm{ppm}$ ). For instance, humic acid slowed down the removal of carbon tetrachloride, ${ }^{272}$ decabromodiphenyl ${ }^{273}$ using nZVI. Moreover, humic acid affected $\mathrm{Ni}-\mathrm{nZVI} \mathrm{I}^{274}$ and $\mathrm{Pd}-\mathrm{nZVI}{ }^{186}$ when they were used to remove 2,4-dichlorophenol. 
The effect of humic acid on nitrate reduction was also reported by Kim et al.. ${ }^{266-267}$ In their study, nitrate removal using nZVI decreased when humic acid exceeded 50 ppm. ${ }^{266}$

In this research, the ability of materials in removing nitrate from lake water was screened. The initial nitrate-N concentration taken on April 27, 2017 was 0.7 ppm. This lake water and this lake water spiked with $10 \mathrm{ppm}$ nitrate- $\mathrm{N}$ were investigated. The effect of $\mathrm{pH}$ adjustment on nitrate reduction was also examined. Three representative materials, $5 \% \mathrm{Ni}$ nZVI@MisiCFeOOH-MB1, 5\%Cu-nZVI@MisiCFeOOH-MB1, and 0.5\%Pd-5\%CunZVI@MisiCFeOOH-MC1 were used.nZVI@MisiCFeOOH was also used as a control material.

\subsubsection{Nitrate reduction from lake water}

Doped materials had the capacity of reducing nitrate from lake water under both anaerobic and aerobic conditions. After 30 minutes, nitrate- $\mathrm{N}$ levels were below the detection limit of the IC (0.06 ppm) for all types of doped materials (Figure 6.2 and 6.3). Moreover, nitrite was only observed at less than $0.1 \mathrm{ppm}$ when $\mathrm{Cu}$-doped system was used under anaerobic conditions. In contrast, nZVI@MisiCFeOOH reduced nitrate from lake water very slowly. Nitrate-N levels were 0.7 ppm and 0.6 ppm when nZVI@MisiCFeOOH was used under either anaerobic or aerobic conditions, respectively. As such, doped nZVI@MisiCFeOOH reduces nitrate significantly faster than non-doped nZVI@MisiCFeOOH. Of these doped materials, trimetallic nZVI@MisiCFeOOH reduced nitrate with the fastest rate while Ni-doped material appeared to have lowest activity. However, the small level of initial nitrate in lake water (0.7 ppm nitrate-N) was not enough to differentiate the reactivity of these doped materials. Therefore, further investigations were carried out using lake water spiked with nitrate. 


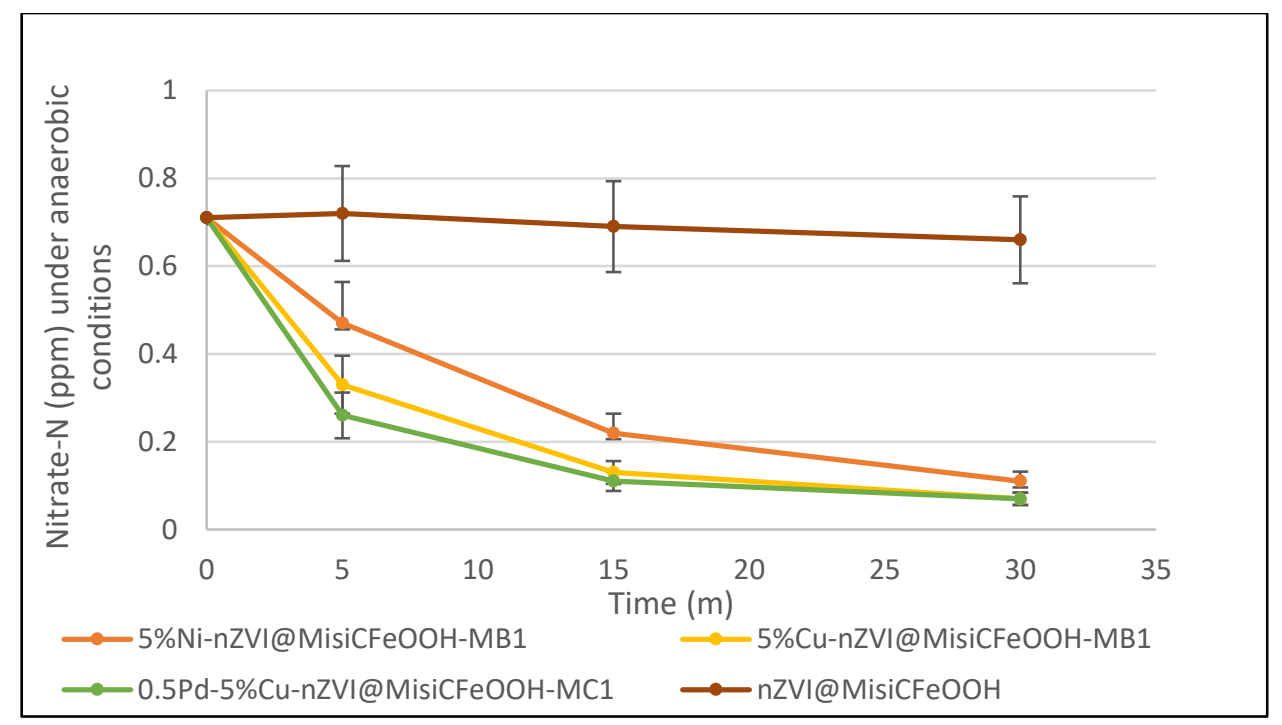

Figure 6.2. Nitrate reduction in lake water under anaerobic conditions (0.150 g of material, $25.0 \mathrm{~mL}$ of sample).

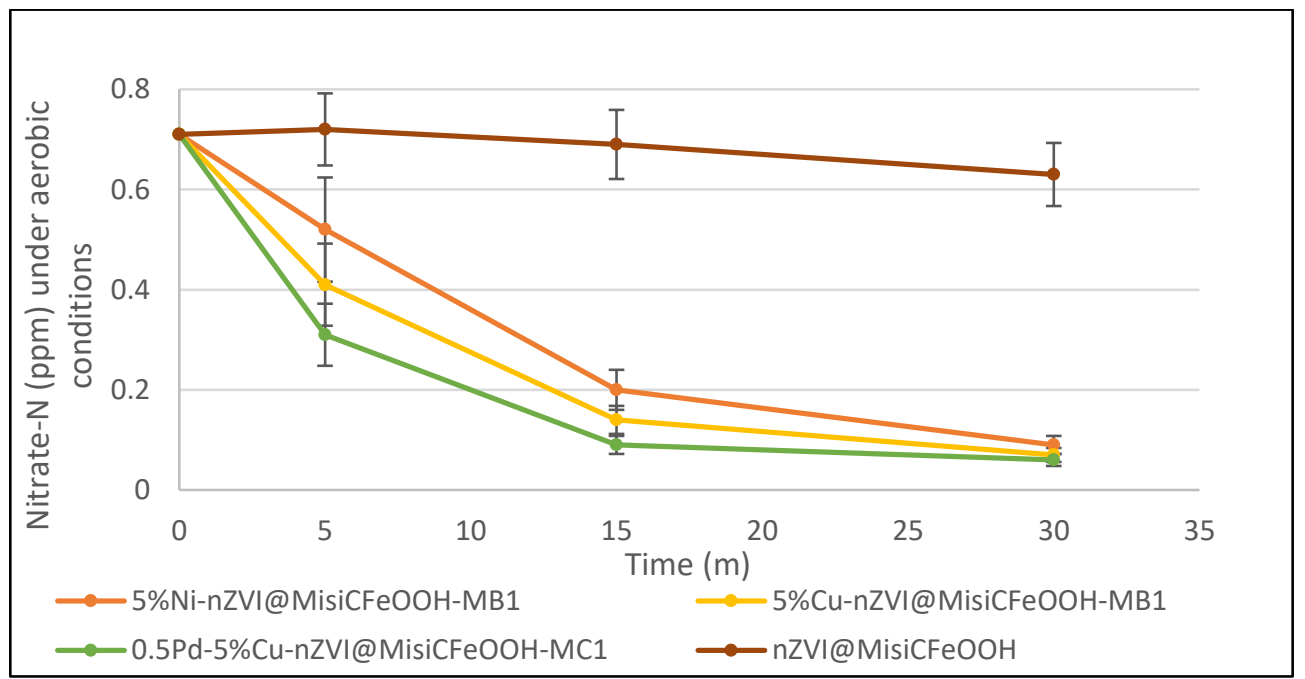

Figure 6.3. Nitrate reduction in lake water under aerobic conditions $(0.150 \mathrm{~g}$ of material, $25.0 \mathrm{~mL}$ of sample).

\subsubsection{Nitrate reduction from lake water spiked with $10 \mathrm{ppm}$ nitrate-N}

\subsubsection{Nitrate reduction from lake water spiked with $10 \mathrm{ppm}$ nitrate-N at neutral pH}

In order to examine the reactivity of doped materials in "real" water, the lake water was spiked with $10 \mathrm{ppm}$ nitrate- $\mathrm{N}$ to give a final nitrate- $\mathrm{N}$ concentration of $10.7 \mathrm{ppm}$. The resulting solution was used for nitrate reduction to determine if these materials could reduce nitrate in the presence of numerous factors in natural water. In addition, nitrite accumulation and 
the change in the $\mathrm{pH}$ of the solutions were also monitored. These experiments were carried out without $\mathrm{pH}$ adjustment in anaerobic and aerobic conditions.

a. Nitrate reduction from lake water spiked with $10 \mathrm{ppm}$ nitrate- $\mathrm{N}$ without $\mathrm{pH}$ adjustement under anaerobic conditions

Three representative materials reduced nitrate from lake water spiked with $10 \mathrm{ppm}$ nitrate$\mathrm{N}$ under anaerobic conditions, the degree of which was dependent upon the nature of materials. Ni-doped system reduced nitrate fastest while Cu-doped system had the lowest reactivity (Figure 6.4). However, the difference in their reactivity was minimal. After 30 minutes, the nitrate conversion was 99\%, 94\%, and $97 \%$ when either $5 \% \mathrm{Ni}$ nZVI@MisiCFeOOH-MB1, $\quad$ 5\%Cu-nZVI@MisiCFeOOH-MB1 $\quad$ or $\quad$ 0.5\%Pd-5\%CunZVI@MisiCFeOOH-MC1 was used. In contrast to doped materials, nZVI@MisiCFeOOH as a control material reduced nitrate very slowly. After 30 minutes, only $7 \%$ of the initial nitrate was reduced.

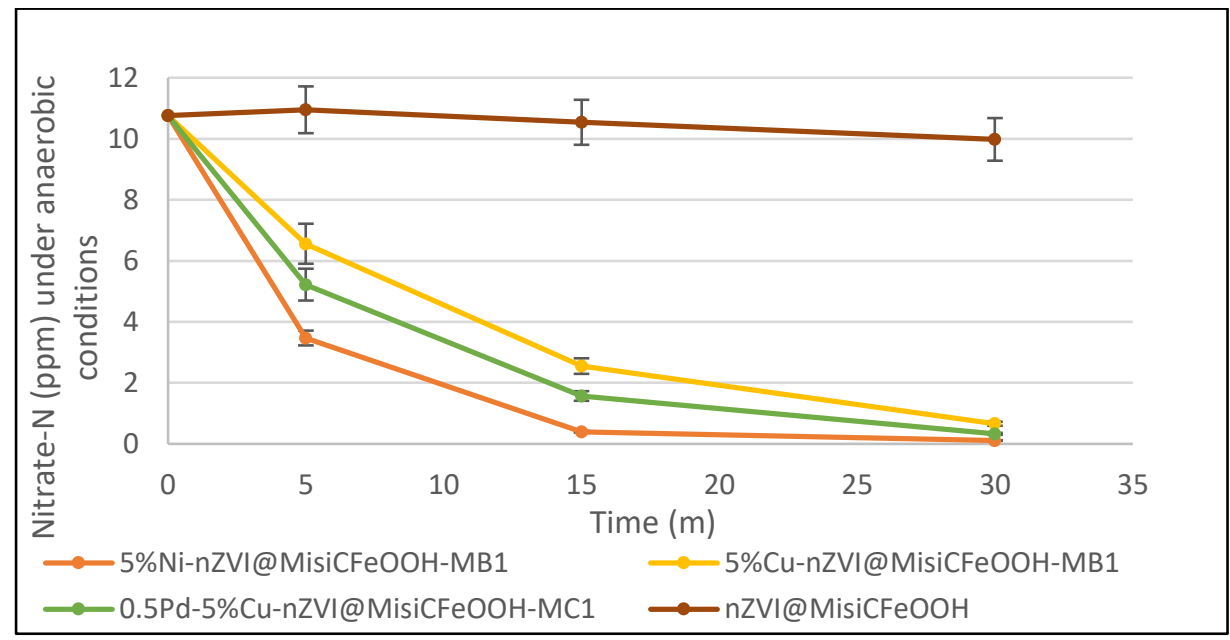

Figure 6.4. Nitrate reduction in lake water spiked with $10 \mathrm{ppm}$ nitrate-N without $\mathrm{pH}$ adjustment under anaerobic conditions (0.150 g of material, $25.0 \mathrm{~mL}$ of sample).

Nitrite was also monitored during the nitrate reduction from lake water (Figure 6.5). Nitrite build-up using $\mathrm{Ni}$-doped and non-doped materials was lower than both $\mathrm{Cu}$-doped and Pd-Cudoped systems. After 30 minutes, nitrite build-up was less than $2 \%$ for $5 \% \mathrm{Ni}$ nZVI@MisiCFeOOH-MB1, 5\%Cu-nZVI@MisiCFeOOH-MB1, and nZVI@MisiCFeOOH. In contrast, the nitrite accumulation was 12\% when 5\%Cu-nZVI@MisiCFeOOH-MB1 was utilised. 
The accumulation of nitrite in lake water was consistent with the nitrite generation in labprepared samples using the same materials.

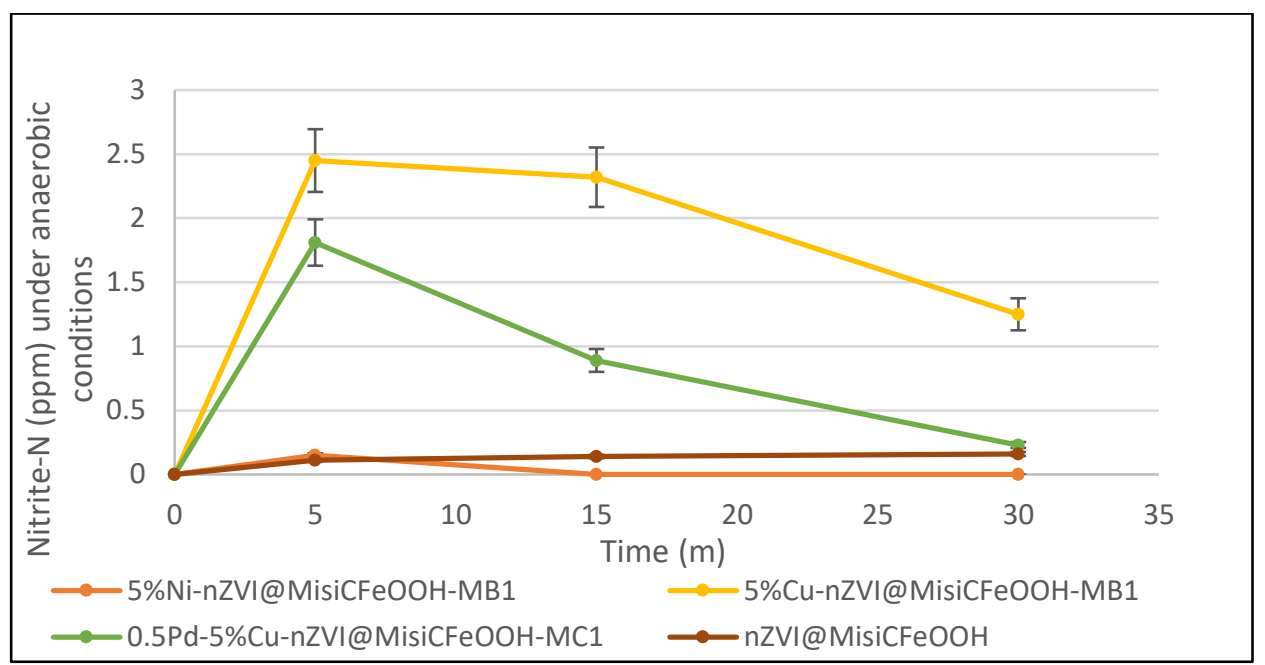

Figure 6.5. Nitrite accumulation in nitrate reduction from lake water spiked with $10 \mathrm{ppm}$ without $\mathrm{pH}$ adjustment under anaerobic conditions $(0.150 \mathrm{~g}$ of materials, $25.0 \mathrm{~mL}$ of sample).

The reduction of iron with nitrate generates hydroxide ions that may affect the generation of by-products. To investigate the change in hydroxide production, the $\mathrm{pH}$ of the solutions was monitored during the reaction (Figure 6.6). After 5 minutes, the $\mathrm{pH}$ notably increased to more than 8.3 using both doped and non-doped materials. Of these, the $\mathrm{pH}$ was highest when $\mathrm{Ni}$ doped material was used, which is consistent with the faster nitrate reduction. Between 15 minutes and 30 minutes, the $\mathrm{pH}$ slightly increased to 9.5 with the doped materials while it decreased to 7.6 with the non-doped nZVI@MisiCFeOOH. The change in the pH in doped systems can be explained by the reduction of nitrate over the course of 30 minutes. In contrast, the pH changes of nZVI@MisiCFeOOH can be attributed to the corrosion of iron in solution. 


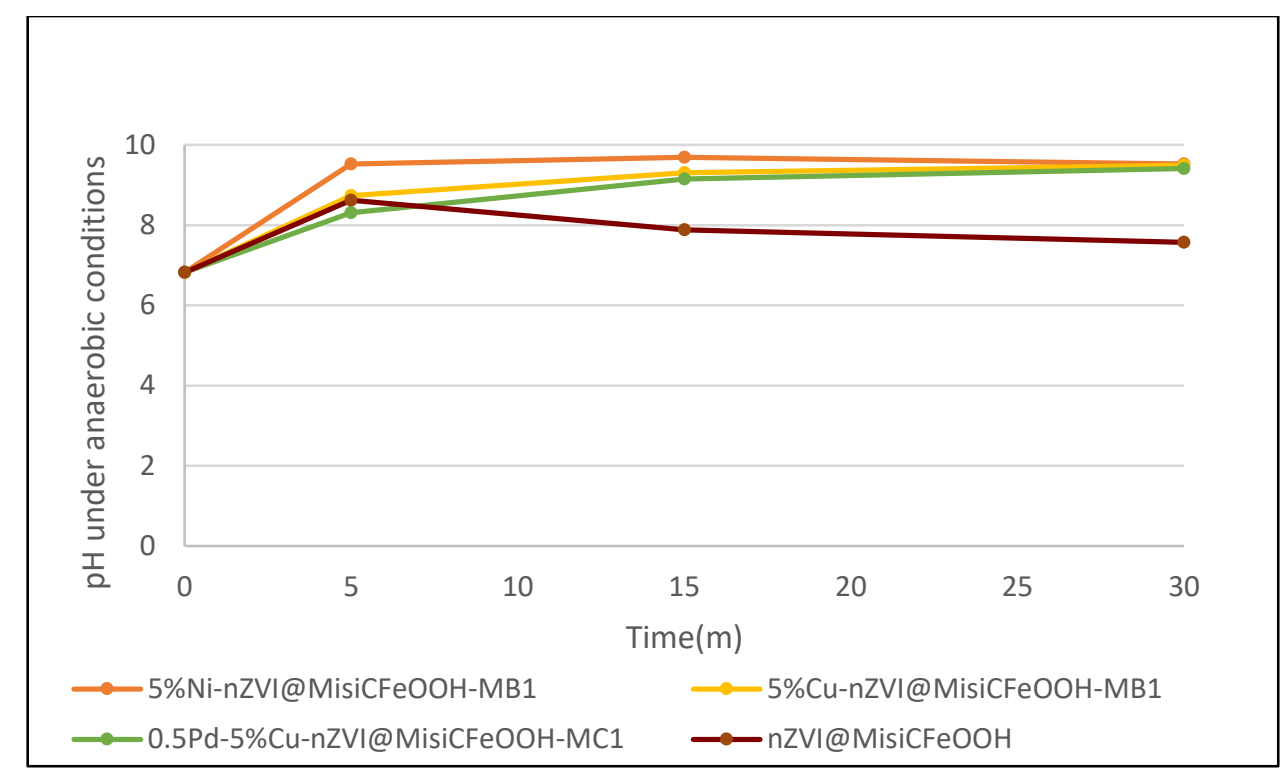

Figure 6.6. $\mathrm{pH}$ change in nitrate reduction from lake water spiked with $10 \mathrm{ppm}$ without $\mathrm{pH}$ adjustment under anaerobic conditions ( $0.150 \mathrm{~g}$ material, $25.0 \mathrm{~mL}$ of sample).

From these preliminary experiments of nitrate reduction from lake water, it is noted that these doped materials were not significantly inhibited by undetermined factors in natural water under anaerobic conditions. These doped materials were still active even though they reduced nitrate from lake water more slowly than lab-prepared samples. In contrast, nondoped nZVI@Misi was almost deactivated. More details about the activity of these materials as well as the difference in their reactivity undergone further examination.

\section{b. Nitrate reduction from lake water spiked with $10 \mathrm{ppm}$ nitrate- $\mathrm{N}$ at neutral $\mathrm{pH}$ under aerobic} conditions

The ability of the materials to reduce nitrate under aerobic conditions was examined. Doped materials reduced nitrate from lake water spiked with $10 \mathrm{ppm}$ under aerobic conditions (Figure 6.7) and the reduction amount was similar to anaerobic conditions. The difference in the reactivity of these doped materials under aerobic conditions was insignificant. After 30 minutes, the nitrate conversion was 99\%, 97\%, and $96 \%$ when either $5 \% \mathrm{Ni}$ nZVI@MisiCFeOOH-MB1, $\quad$ 5\%Cu-nZVI@MisiCFeOOH-MB1 $\quad$ or $\quad$ 0.5Pd-5\%CunZVI@MisiCFeOOH-MC1 was used, respectively. In contrast, the non-doped material reduced nitrate slowly; after 30 minutes, only $13 \%$ of the initial nitrate was removed. 


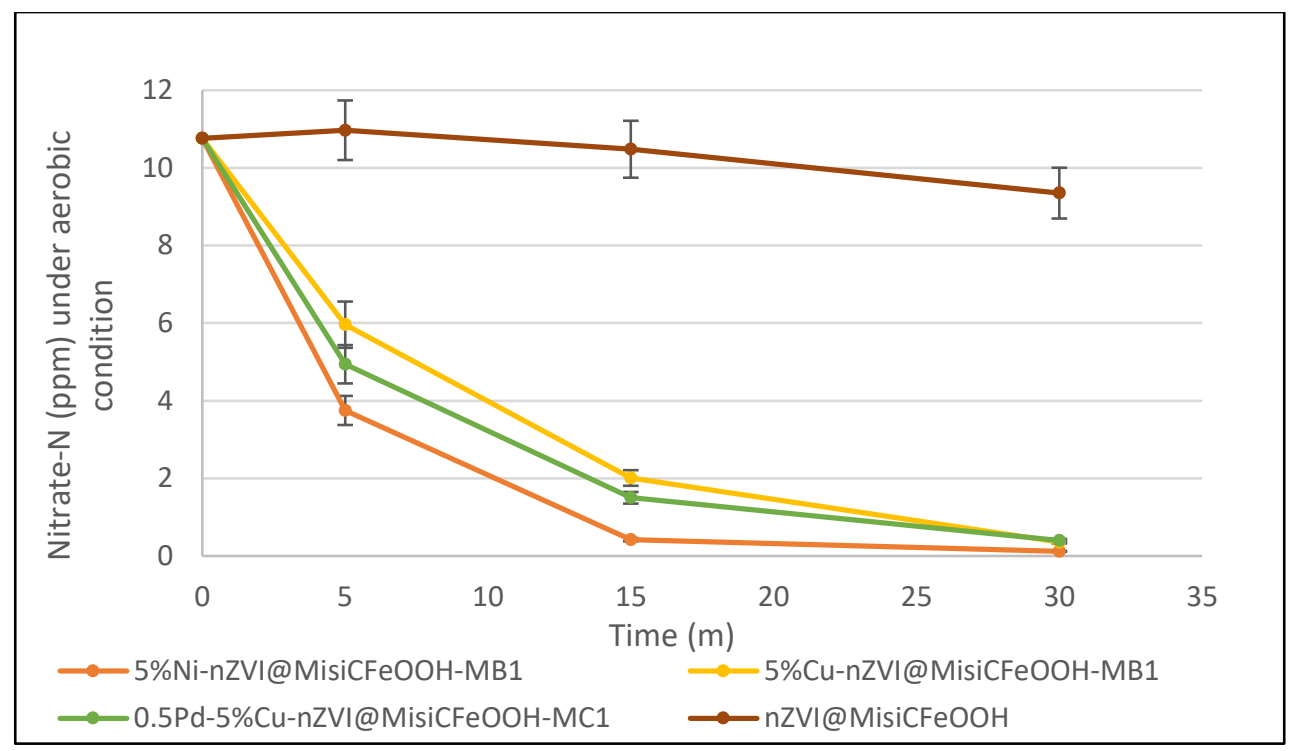

Figure 6.7. Nitrate reduction in lake water spiked with $10 \mathrm{ppm}$ nitrate- $\mathrm{N}$ without $\mathrm{pH}$ adjustment under aerobic conditions $(0.150 \mathrm{~g}$ of material, $25.0 \mathrm{~mL}$ of sample).

Nitrite was also monitored during the reaction. The degree of the nitrite accumulation depended on the nature of materials, which was similar to anaerobic conditions (Figure 6.8). Interestingly, nitrite accumulation in Cu-doped materials was only $3 \%$ compared to $12 \%$ under anaerobic conditions.

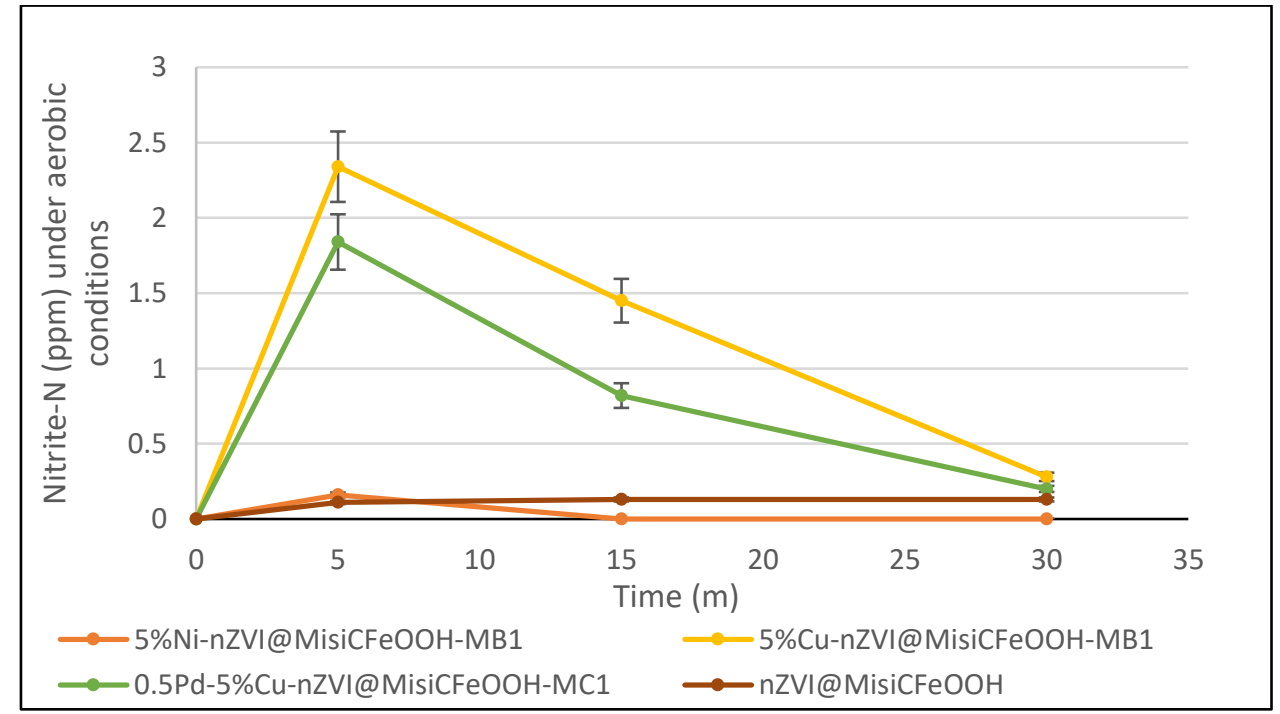

Figure 6.8. Nitrite accumulation in nitrate reduction from lake water spiked with $10 \mathrm{ppm}$ without $\mathrm{pH}$ adjustment under aerobic conditions ( $0.150 \mathrm{~g}$ of materials, $25.0 \mathrm{~mL}$ of sample). The change in the solution $\mathrm{pH}$ under aerobic conditions was similar to that under anaerobic conditions (Figure 6.9). This confirmed the similarity of nitrate reduction under two 
conditions. It was encouraging that nitrate reduction should be examined under both anaerobic and aerobic conditions.

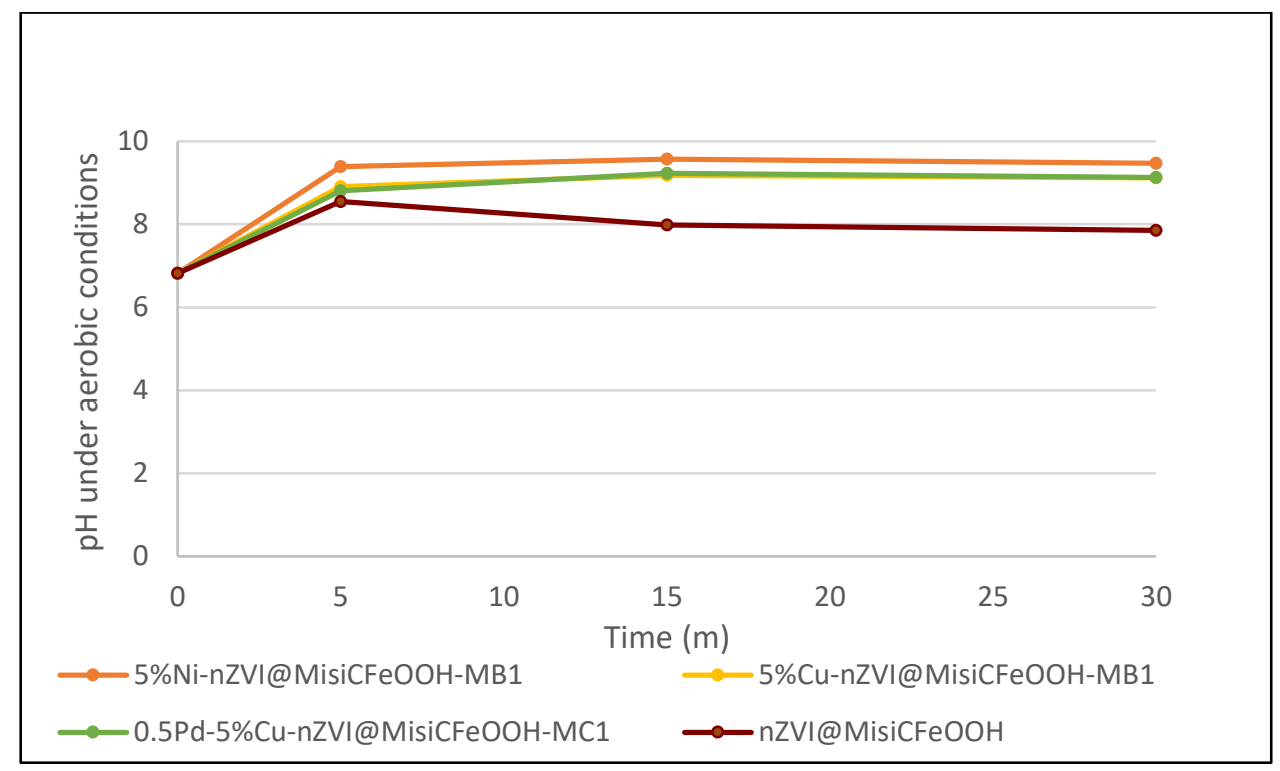

Figure 6.9. $\mathrm{pH}$ change in nitrate reduction from lake water spiked with $10 \mathrm{ppm}$ without $\mathrm{pH}$ adjustment under aerobic conditions (0.150 g materials, $25.0 \mathrm{~mL}$ of sample).

In conclusion, doped materials had high reactivity in nitrate reduction from lake water spiked with 10 ppm nitrate- $\mathrm{N}$ without $\mathrm{pH}$ adjustment under anaerobic and aerobic conditions. There was no difference in their reactivity between these two conditions. Of these, $\mathrm{Ni}$-doped system reduced nitrate fastest with no nitrite accumulation. After the reaction, the $\mathrm{pH}$ in doped materials was greater than non-doped material. This can be explained by the hydroxide generation from the interaction of doped materials with nitrate.

\subsubsection{Nitrate reduction from lake water spiked with $10 \mathrm{ppm}$ nitrate- $\mathrm{N}$ at $\mathrm{pH}$ of 3}

Acidic conditions are favourable for the reaction of $n Z V I$ with nitrate. For instance, a significant rate enhancement was observed at pH's less than 3 when nZVI@graphite reacts with nitrate. ${ }^{241}$ In another study, when nitrate was reduced using nZVI, the reaction was faster at $\mathrm{pH}$ 's less than 7; however, at $\mathrm{pH}$ 's between 3 and 7, this effect was insignificant. ${ }^{242}$ At $\mathrm{pH}^{\prime} \mathrm{s}$ higher than 9, nitrate reduction was significantly decreased. Acidic conditions also affected the reactivity of bimetallic nZVI. For example, nitrate removal using Ni-nZVI@biochar decreased when the initial $\mathrm{pH}$ increased from 3 to 9 but the decrease was minimal. ${ }^{243}$ However, these investigations were conducted in lab samples. In this current study, the effect of acidic conditions on the activity of these representative materials in removing nitrate from 
lake water was examined. For this, lake water was spiked with $10 \mathrm{ppm}$ nitrate-N (final nitrate$\mathrm{N}$ concentration of $10.7 \mathrm{ppm}$ ) and the $\mathrm{pH}$ was adjusted to 3 prior to the addition of our materials.

At $\mathrm{pH} 3$, nitrate reduction was rapid when all types of doped materials were used under both anaerobic and aerobic conditions. After 30 minutes, nitrate- $\mathrm{N}$ levels were around the detection limit of the IC (0.06 ppm) (Figure 6.10 and 6.11). Interestingly, there was no difference in their reactivity under two conditions. Nitrite was only observed in both $\mathrm{Cu}$ doped nZVI@Misi and Pd-Cu-doped nZVI@Misi at 1.4\%.

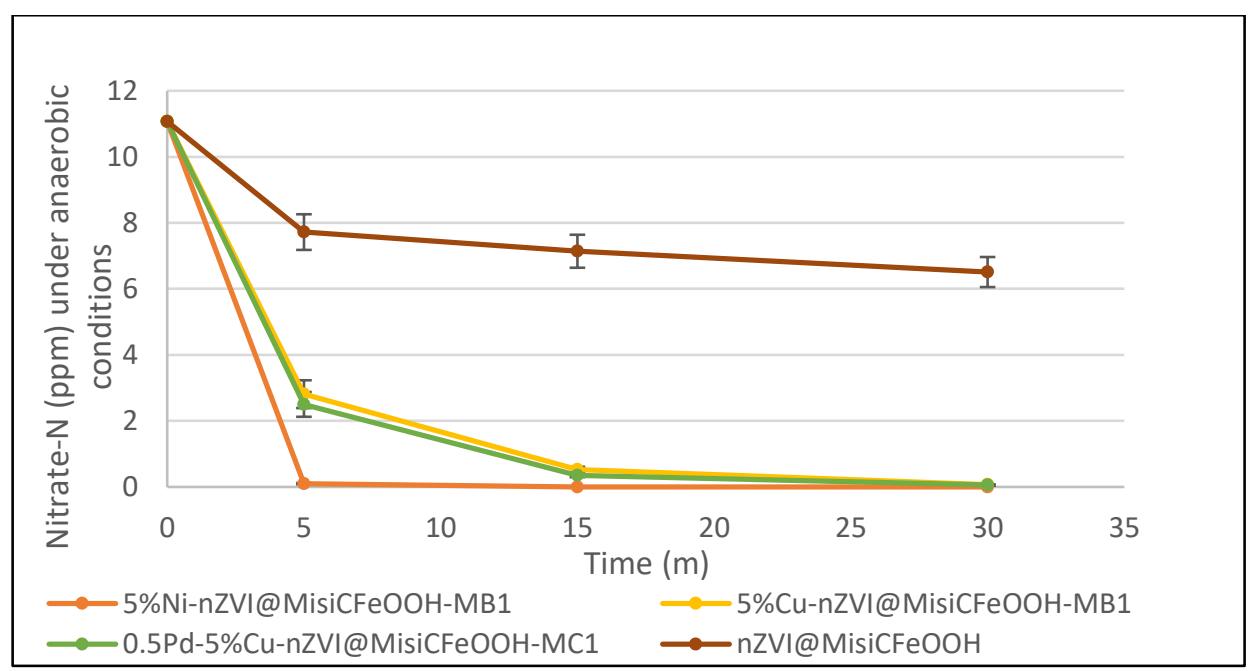

Figure 6.10. Nitrate reduction in lake water spiked with 10 ppm at initial pH 3 under anaerobic conditions ( $0.150 \mathrm{~g}$ of material, $25.0 \mathrm{~mL}$ of sample).

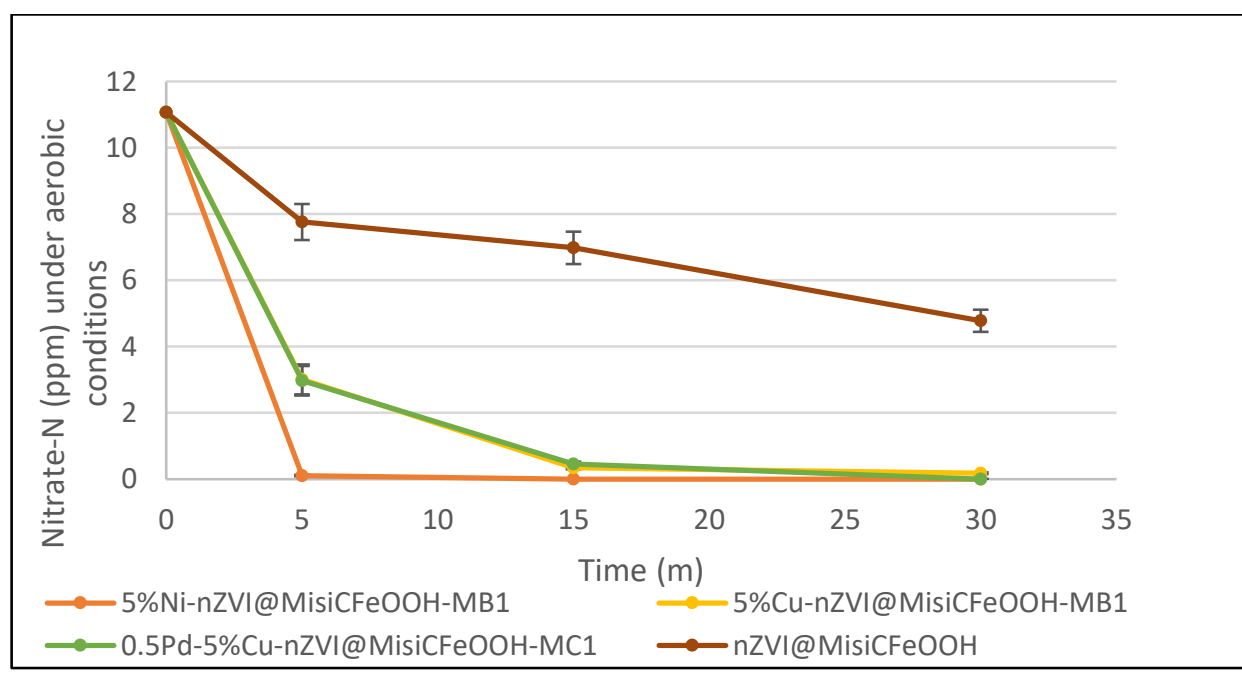

Figure 6.11. Nitrate reduction in lake water spiked with $10 \mathrm{ppm}$ at initial pH 3 under aerobic conditions ( $0.150 \mathrm{~g}$ of material, $25.0 \mathrm{~mL}$ of sample). 
The initial pH significantly influenced the reactivity of nZVI@MisiCFeOOH. After 30 minutes, at neutral $\mathrm{pH}$, nitrate conversion was $7 \%$ and $13 \%$ under anaerobic and aerobic conditions, respectively. In contrast, at low initial pH, nitrate conversion was $39 \%$ and $56 \%$, respectively. The faster nitrate reduction at $\mathrm{pH}$ of 3 can be explained by the elevation of Fe corrosion. $\mathrm{H}^{+}$ ions may dissolve the protective layer (ion oxides) on the surface of $n Z \mathrm{VI},{ }^{263}$ leading to more Fe active sites to react with nitrate. These results were inconsistent to those reported by Kim et al., who studied the effect of $\mathrm{pH}$ on nitrate reduction using $\mathrm{nZVI}{ }^{242}$ At initial $\mathrm{pH}^{\prime} \mathrm{s}$ between 3 and 7 , only a small change in the rate of nitrate reduction was observed, presumably due to a rapid rise of $\mathrm{pH}$ to 10 , regardless of the initial $\mathrm{pH}$ of the solution. However, in their study, a higher concentration (50 ppm) of nitrate- $\mathrm{N}$ was used.

The solution $\mathrm{pH}$ was monitored over the course of our reactions. After 5 minutes, the $\mathrm{pH}$ increased significantly to range from 5.3 to 6.0 and stabilised at a pH of 6 between 15 minutes and 30 minutes under both anaerobic and aerobic conditions, (Figure 6.12 and 6.13). Of particular interest is that $\mathrm{pH}$ in $\mathrm{nZVI@MisiCFeOOH}$ was similar to doped materials even though nZVI@MisiCFeOOH reduced nitrate significantly more slowly.

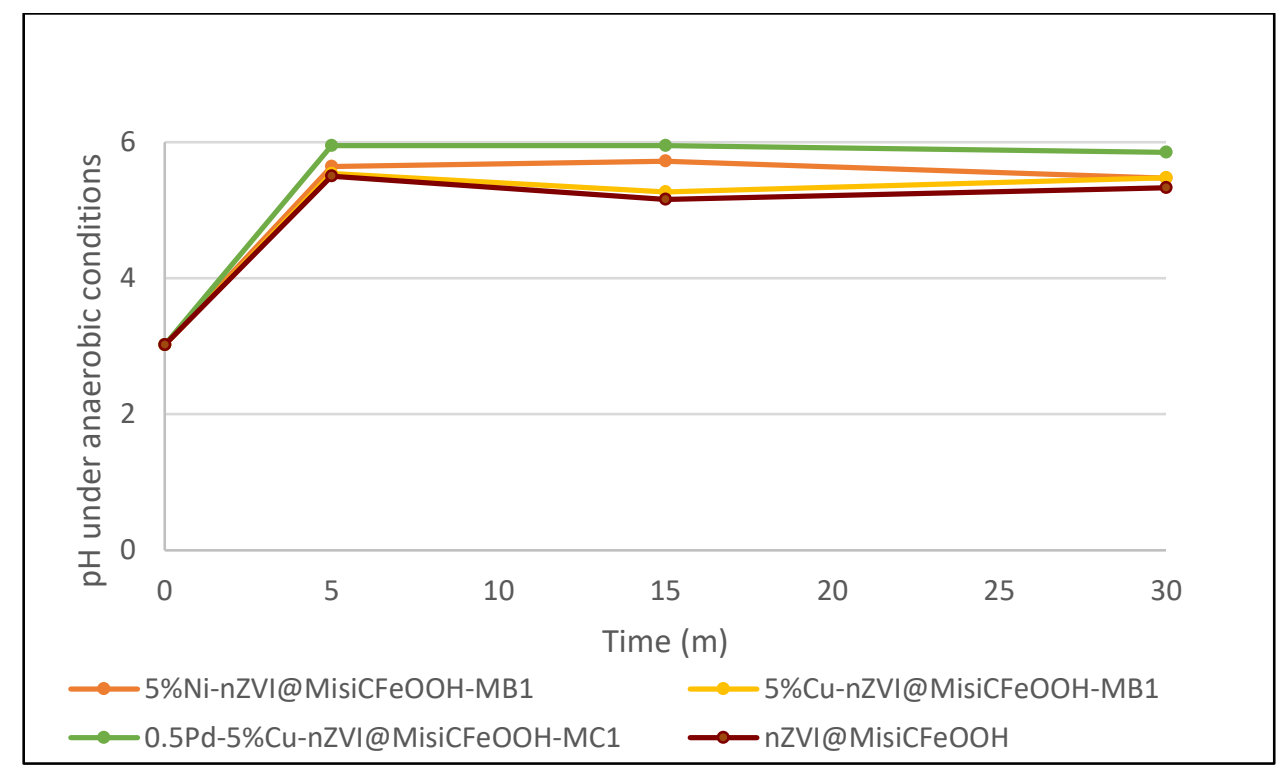

Figure 6.12. $\mathrm{pH}$ change in nitrate reduction from lake water spiked with $10 \mathrm{ppm}$ at initial pH 3 under anaerobic conditions ( $0.150 \mathrm{~g}$ of material, $25.0 \mathrm{~mL}$ of sample). 


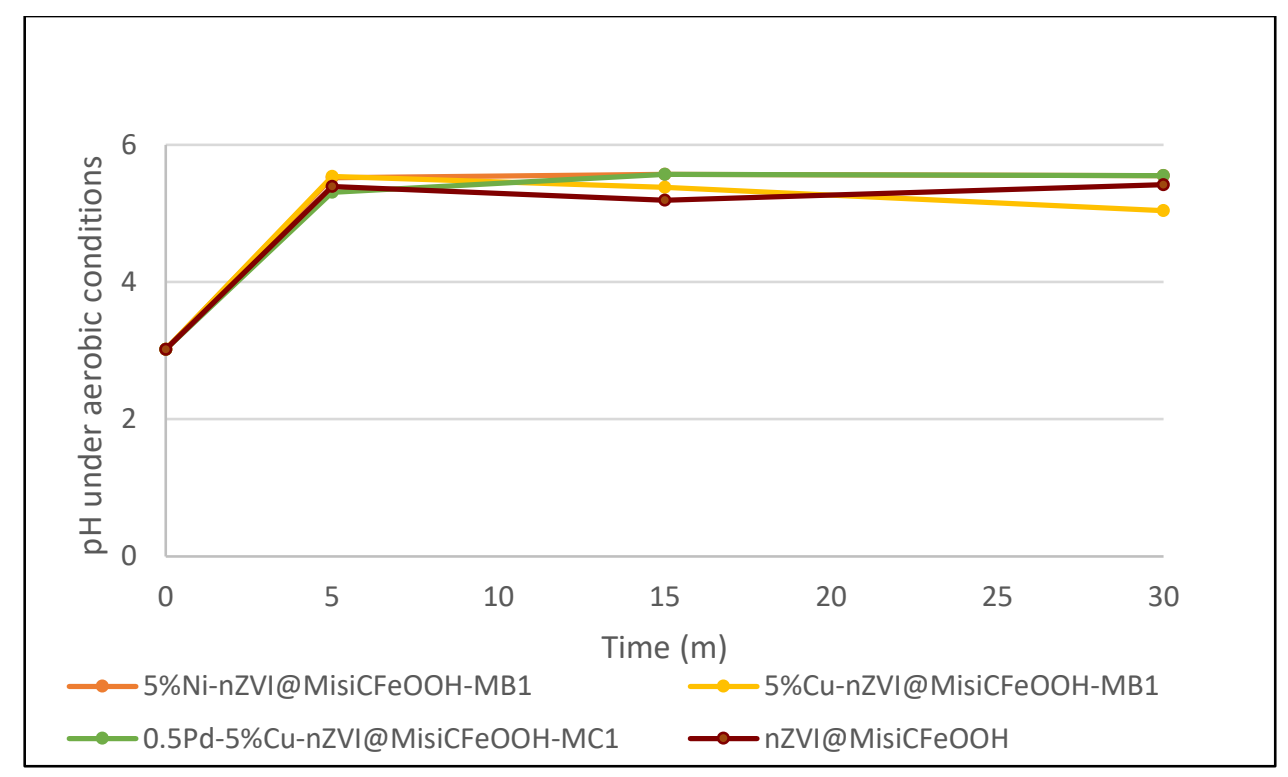

Figure 6.13. $\mathrm{pH}$ change in nitrate reduction from lake water spiked with $10 \mathrm{ppm}$ at initial $\mathrm{pH}$ 3 under aerobic conditions ( $0.150 \mathrm{~g}$ of material, $25.0 \mathrm{~mL}$ of sample).

These results show the significance of $\mathrm{pH}$ adjustment on nitrate conversion. Although these are important observations, it is encouraging that doped materials in this current study are still reactive at higher $\mathrm{pH}^{\prime} \mathrm{s}$ in contrast to the non-doped system. This confirms that these doped systems are more applicable to "normal" conditions. As such, the reactivity of doped materials will be examined in greater detail at only neutral $\mathrm{pH}$.

\subsection{Nitrate reduction from lake water}

The reactivity of doped materials towards nitrate reduction from lake water was examined in greater detail using lake water collected on April 23, 2018. The initial nitrate-N concentration was $1.3 \mathrm{ppm}$. To understand the reactivity of our materials in greater detail, and to ascertain any differences in their reactivity, the lake water was spiked with $10 \mathrm{ppm}$ and $50 \mathrm{ppm}$ nitrate$\mathrm{N}$ to give the solutions with the final nitrate- $\mathrm{N}$ concentrations of $11.3 \mathrm{ppm}$ and $51.3 \mathrm{ppm}$, respectively. The nitrate reactions were conducted under both anaerobic and aerobic conditions. Four different materials, including 5\%Ni-nZVI@MisiCFeOOH-MB1, 5\%CunZVI@MisiCFeOOH-MB1, 0.5\%Pd-5\%Cu-nZVI@MisiCFeOOH-MC1, and nZVI@MisiCFeOOH, were used.

\subsubsection{Nitrate reduction from lake water spiked with $10 \mathrm{ppm}$ nitrate- $\mathrm{N}$}

The addition of $10 \mathrm{ppm}$ of nitrate- $\mathrm{N}$ to the lake water was chosen in order to compare the activity of these representative materials in removing this amount of nitrate from lake water 
and lab samples. In the lab sample, when nitrate was reduced using doped materials, all nitrate was reduced to below the detection limit and no nitrite accumulation was observed in less than 15 minutes under both anaerobic and aerobic conditions.nZVI@MisiCFeOOH had lower activity; however, after 30 minutes, nitrate conversion was $74 \%$ and $85 \%$ under anaerobic and aerobic conditions, respectively.

\subsubsection{Nitrate reduction from lake water spiked with $10 \mathrm{ppm}$ nitrate-N under anaerobic} conditions

All doped materials had the capacity of reducing nitrate from lake water spiked with $10 \mathrm{ppm}$ under anaerobic conditions (Figure 6.14). The difference in their reactivity was insignificant. After 30 minutes, nitrate-N levels were $4.9 \pm 0.1 \mathrm{ppm}$ ( $57 \%$ nitrate conversion), $4 \pm 2 \mathrm{ppm}$ (65\% nitrate conversion), and $3.9 \pm 0.4 \mathrm{ppm}$ (65\% nitrate conversion) when either $5 \% \mathrm{Ni}$ nZVI@MisiCFeOOH-MB1, 5\%Cu-nZVI@MisiCFeOOH-MB1 or $\quad 0.5 \% \mathrm{Pd}-5 \% \mathrm{Cu}-$ nZVI@MisiCFeOOH-MC1 was used, respectively. As a control material, nitrate reduction using nZVI@MisiCFeOOH was also investigated. Nitrate-N levels were $10.3 \pm 0.3$ ppm ( $9 \%$ nitrate conversion). Comparing to the results from the lab sample, nitrate reduction was significantly slower.

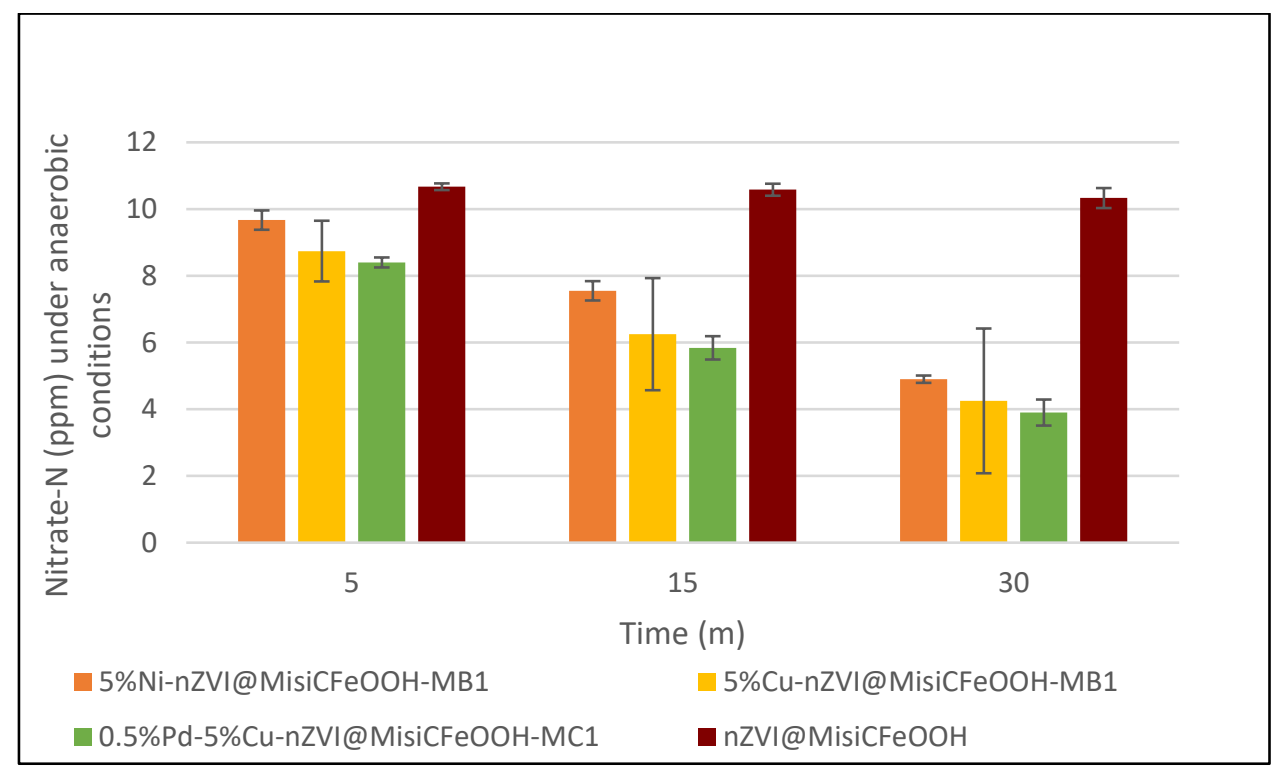

Figure 6.14. Nitrate reduction in lake water spiked with 10 ppm under anaerobic conditions (0.150 g of material, $25.0 \mathrm{~mL}$ of sample).

Nitrite was also monitored in nitrate reduction from lake water. Over the course of 30 minutes, no nitrite was detected using either Ni-doped or non-doped materials (Table 6.2). In 
contrast, after 30 minutes, nitrite- $N$ concentrations were $3 \pm 1$ ppm (27\% nitrite accumulation) and $2.3 \pm 0.2$ (20\% nitrite accumulation) using either $5 \% \mathrm{Cu}$ nZVI@MisiCFeOOH-MB1 or 0.5\%Pd-5\%Cu-nZVI@MisiCFeOOH-MC1, respectively. The nitrite accumulation in both $\mathrm{Cu}$-doped and Pd-Cu-doped materials was significantly higher than that observed in the lab samples. These results indicated that both nitrate and nitrite conversion were affected by coexisting substances in lake water under anaerobic conditions.

Table. 6.2. Nitrite accumulation in nitrate reduction from lake water spiked with $10 \mathrm{ppm}$ nitrate- $\mathrm{N}$ under anaerobic conditions ( $0.150 \mathrm{~g}$ of material, $25.0 \mathrm{~mL}$ of sample).

\begin{tabular}{|l|c|c|c|}
\hline Nitrite-N (ppm)/Time & 5 minutes & 15 minutes & 30 minutes \\
\hline 5\%Ni-nZVI@MisiCFeOOH-MB1 & ND & ND & ND \\
\hline 5\%Cu-nZVI@MisiCFeOOH-MB1 & $1 \pm 1$ & $2 \pm 1$ & $3 \pm 1$ \\
\hline 0.5\%Pd-5\%Cu-nZVI@MisiCFeOOH-MC1 & $1.44 \pm 0.09$ & $2.3 \pm 0.2$ & $2.3 \pm 0.2$ \\
\hline nZVI@MisiCFeOOH & ND & ND & ND \\
\hline
\end{tabular}

\subsubsection{Nitrate reduction from lake water spiked with $10 \mathrm{ppm}$ nitrate-N under aerobic} conditions

Doped materials removed nitrate from lake water spiked with $10 \mathrm{ppm}$ of nitrate-N faster under aerobic conditions than anaerobic conditions (Figure 6.15). The difference in the reactivity of the doped materials under aerobic conditions was minimal. After 30 minutes, nitrate- $\mathrm{N}$ levels were $1.6 \pm 0.5 \mathrm{ppm}$ (86\% nitrate conversion), $2 \pm 2 \mathrm{ppm}$ ( $82 \%$ nitrate conversion), and $2.1 \pm 0.9 \mathrm{ppm}$ (81\% nitrate conversion) when either $5 \% \mathrm{Ni}$ nZVI@MisiCFeOOH-MB1，5\%Cu-nZVI@MisiCFeOOH-MB1 $\quad$ or $\quad 0.5 \% \mathrm{Pd}-5 \% \mathrm{Cu}-$ nZVI@MisiCFeOOH-MC1 was used, respectively. In contrast, only $12 \%$ of the initial nitrate was removed using non-doped nZVI@MisiCFeOOH. Similar to under anaerobic conditions, the nitrate reduction was considerably slower than that observed in the lab samples. However, these doped materials had higher reactivity under aerobic conditions than under anaerobic conditions. 


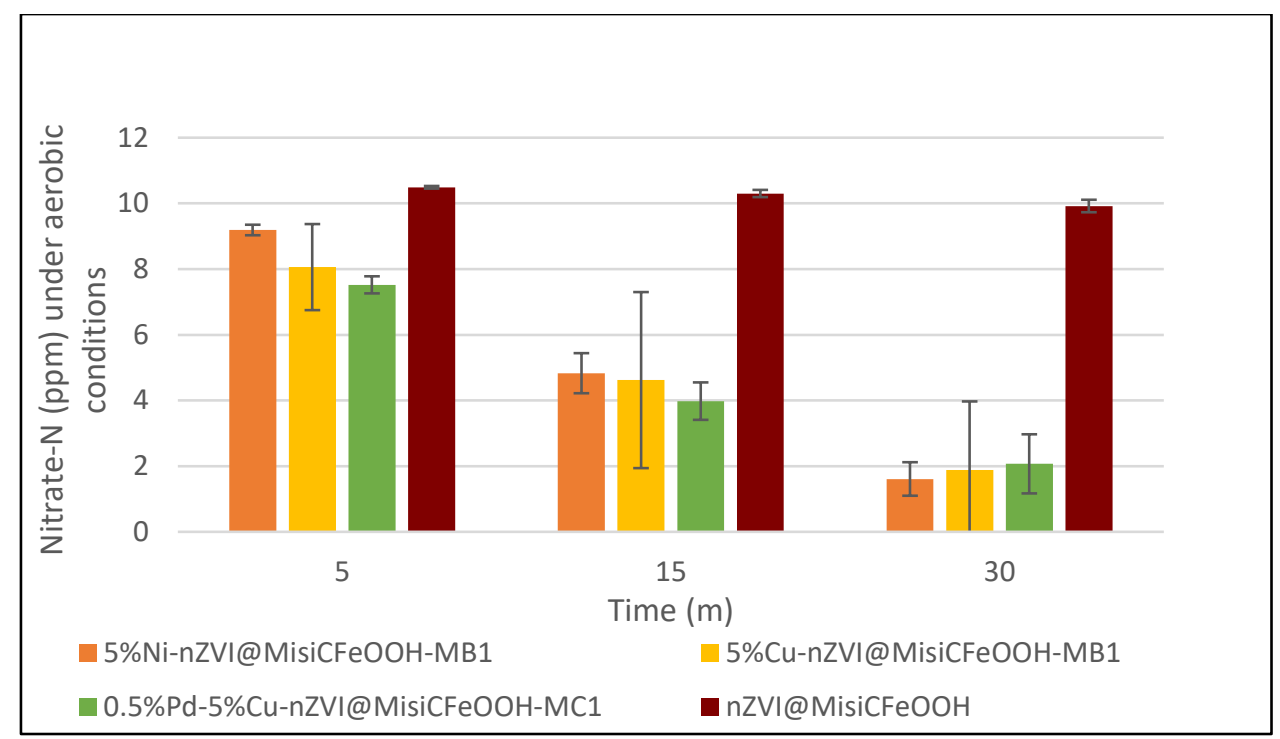

Figure 6.15. Nitrate reduction in lake water spiked with 10 ppm under aerobic conditions (0.150 g of material, $25.0 \mathrm{~mL}$ of sample).

The nitrite- $\mathrm{N}$ concentrations using $\mathrm{Ni}$-doped and non-doped materials were below the detection limit over the course of 30 minutes (Table 6.3). Although nitrite was detected using both $\mathrm{Cu}$-doped and Pd-Cu-doped materials after 5 minutes and 15 minutes, no nitrite was detected after 30 minutes. Nitrite accumulation was lower than that observed under anaerobic conditions. This indicated that oxygen elevated both nitrate and nitrite conversion. Although the reasons for this phenomenon are unknown, these results would be helpful in applying these materials to "real" conditions. In addition, nitrite accumulation was relatively similar to that in the lab sample. These results suggested that coexisting substances in lake water significantly affected nitrate conversion whereas their effect on nitrite conversion was minimal under aerobic conditions.

Table. 6.3. Nitrite accumulation in nitrate reduction from lake water spiked with $10 \mathrm{ppm}$ nitrate- $\mathrm{N}$ under aerobic conditions ( $0.150 \mathrm{~g}$ of material, $25.0 \mathrm{~mL}$ of sample).

\begin{tabular}{|c|c|c|c|}
\hline Nitrite-N (ppm)/ Time & 5 minutes & 15 minutes & 30 minutes \\
\hline 5\%Ni-nZVI@MisiCFeOOH-MB1 & ND & ND & ND \\
\hline 5\%Cu-nZVI@MisiCFeOOH-MB1 & $1 \pm 1$ & $2 \pm 2$ & ND \\
\hline 0.5\%Pd-5\%Cu-nZVI@MisiCFeOOH-MC1 & $1.6 \pm 0.3$ & $1.3 \pm 0.3$ & ND \\
\hline nZVI@MisiCFeOOH & ND & ND & ND \\
\hline
\end{tabular}


In conclusion, doped materials were able to reduce nitrate from lake water spiked with 10 ppm nitrate- $\mathrm{N}$ under both anaerobic and aerobic conditions. Both nitrate and nitrite conversion were faster under aerobic conditions than under anaerobic conditions. In contrast, non-doped nZVI@MisiCFeOOH only reduced a small amount of nitrate (less than $12 \%)$ under both conditions.

\subsubsection{Nitrate reduction from lake water spiked with $50 \mathrm{ppm}$ nitrate-N}

Although these representative materials did not reduce all nitrate in lake water spiked with $10 \mathrm{ppm}$, the difference in their reactivity was not really clear. We decided to carry out nitrate reduction from lake water spiked with $50 \mathrm{ppm}$ nitrate-N. Results from lake water were also compared with those in lab samples to determine if naturally occurring ions could affect the reduction of high nitrate concentration.

\subsubsection{Nitrate reduction from lake water spiked with $50 \mathrm{ppm}$ nitrate-N under anaerobic} conditions

In anaerobic conditions, all types of doped materials reduced nitrate from lake water spiked with 50 ppm nitrate-N but nitrate removal was significantly slower than in lab samples (Figure 6.16). After 30 minutes, $\mathrm{Ni}$-doped and $\mathrm{Cu}$-doped materials reduced nitrate faster than trimetallic Pd-Cu-doped material. Nitrate-N concentrations were $24.4 \pm 0.7$ ppm (52\% nitrate conversion), $24 \pm 1 \mathrm{ppm}$ (53\% nitrate conversion) and $28 \pm 3$ ppm (45\% nitrate conversion) when either 5\%Ni-nZVI@MisiCFeOOH-MB1, 5\%Cu-nZVI@MisiCFeOOH-MB1 or 0.5\%Pd5\%Cu-nZVI@MisiCFeOOH-MC1 was used, respectively. In contrast, nitrate-N concentrations were $47.3 \pm 0.5$ ppm ( $8 \%$ nitrate conversion) when nZVI@MisiCFeOOH was used as a control material. 


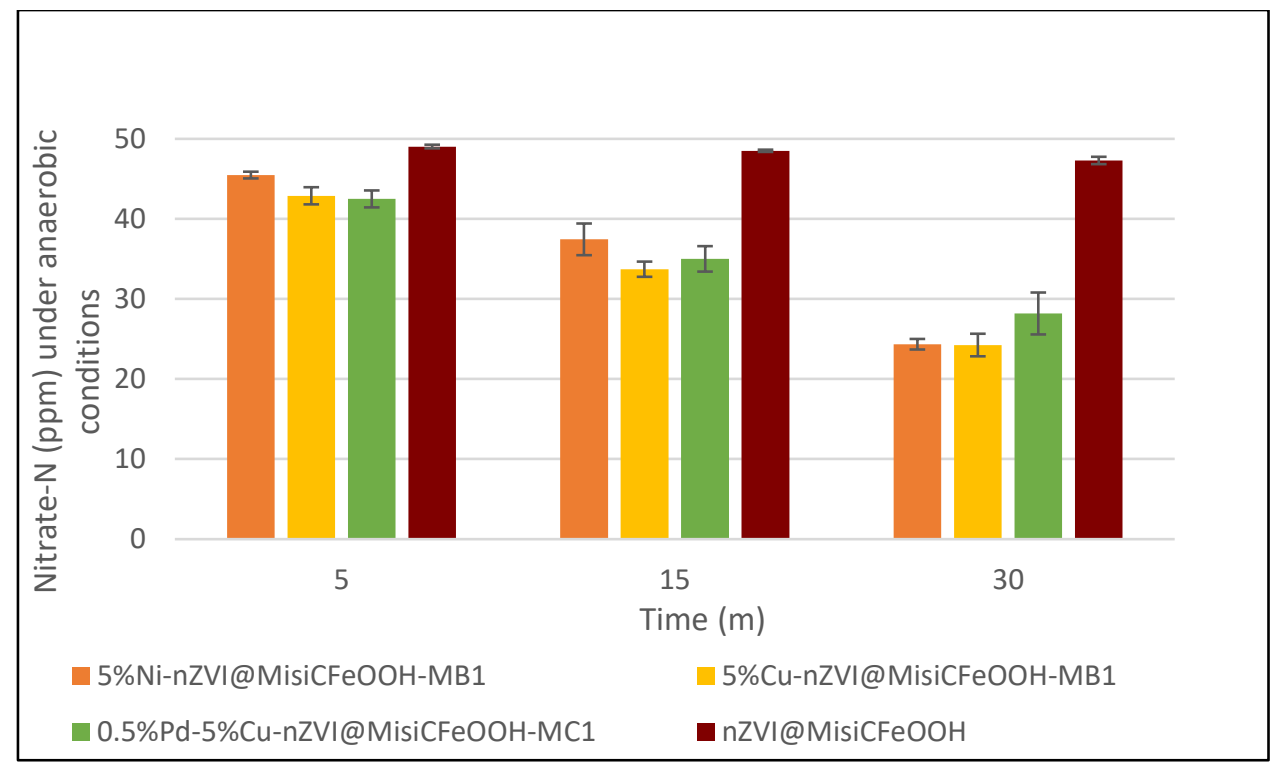

Figure 6.16. Nitrate reduction in lake water spiked with 50 ppm under anaerobic conditions (0.150 g of material, $25.0 \mathrm{~mL}$ of sample).

Nitrite was also monitored in these reactions (Table 6.4). Nitrite accumulation using Ni-doped material was significantly lower than both $\mathrm{Cu}$-doped and Pd-Cu-doped materials. After 30 minutes, the nitrite-N levels were $1.4 \pm 0.2 \mathrm{ppm}$ ( $3 \%$ nitrite accumulation), $15 \pm 2 \mathrm{ppm}$ (29\% nitrite accumulation) and $9.9 \pm 0.9 \mathrm{ppm}(19 \%$ nitrite accumulation) when either $5 \% \mathrm{Ni}-$ nZVI@MisiCFeOOH-MB1, 5\%Cu-nZVI@MisiCFeOOH-MB1 or $\quad$ 0.5\%Pd-5\%CunZVI@MisiCFeOOH-MC1 was used, respectively. Nitrite accumulation in both Cu-doped and $\mathrm{Pd}-\mathrm{Cu}$-doped materials was higher in lake water than in lab samples. In contrast, $\mathrm{Ni}$-doped material accumulated was less nitrite in lake water. The presence of $\mathrm{Pd}$ in $\mathrm{Pd}-\mathrm{Cu}$ doped material promoted the nitrite reduction, which is similar to nitrite accumulation in nitrate reduction from lab-prepared samples. In contrast to doped materials but consistent with nitrate reduction, nitrite- $\mathrm{N}$ concentrations using non-doped nZVI@MisiCFeOOH were below the detection of the IC over the course of 30 minutes. 
Table. 6.4. Nitrite accumulation in nitrate reduction from lake water spiked with $50 \mathrm{ppm}$ nitrate-N under anaerobic conditions ( $0.150 \mathrm{~g}$ of material, $25.0 \mathrm{~mL}$ of sample).

\begin{tabular}{|c|c|c|c|}
\hline Nitrite-N (ppm)/ Time & 5 minutes & 15 minutes & 30 minutes \\
\hline 5\%Ni-nZVI@MisiCFeOOH-MB1 & ND & $1.8 \pm 0.2$ & $1.4 \pm 0.2$ \\
\hline 5\%Cu-nZVI@MisiCFeOOH-MB1 & $6 \pm 1$ & $11 \pm 1$ & $15 \pm 2$ \\
\hline 0.5\%Pd-5\%Cu-nZVI@MisiCFeOOH-MC1 & $4.5 \pm 0.2$ & $8.2 \pm 0.3$ & $9.9 \pm 0.9$ \\
\hline nZVI@MisiCFeOOH & ND & ND & ND \\
\hline
\end{tabular}

In anaerobic conditions, the difference in reactivity of these materials towards nitrate reduction from lab-prepared samples against lake water is the outstanding reducing capacity of $\mathrm{Ni}$-doped material. Using this material resulted in the fastest nitrate conversion and no nitrite build-up. As such, 5\%Ni-nZVI@MisiCFeOOH-MB1 is the most promising material.

\subsubsection{Nitrate reduction from lake water spiked with $50 \mathrm{ppm}$ nitrate-N under aerobic conditions}

As with the $10 \mathrm{ppm}$ samples, doped materials reduced nitrate from lake water spiked with 50 ppm faster under aerobic conditions than under anaerobic conditions. The reactivity of these three materials was similar over the course of 30 minutes (Figure 6.17). For example, after 30 minutes, the nitrate- $\mathrm{N}$ concentrations were $20 \pm 4 \mathrm{ppm}$ (61\% nitrate conversion), $19 \pm 3 \mathrm{ppm}$ (63\% nitrate conversion), and $22 \pm 2 \mathrm{ppm}$ (57\% nitrate conversion) when either 5\% NinZVI@MisiCFeOOH-MB1, 5\%Cu-nZVI@MisiCFeOOH-MB1 or $\quad$ 0.5\%Pd-5\%CunZVI@MisiCFeOOH-MC1 was used, respectively. The difference in the reactivity of these materials was minimal, which is inconsistent with that was observed in lab-prepared samples. In contrast, but consistent with our other results, nitrate-N concentration was $46 \pm 2 \mathrm{ppm}$ (10\% nitrate conversion) when nZVI@MisiCFeOOH was used. 


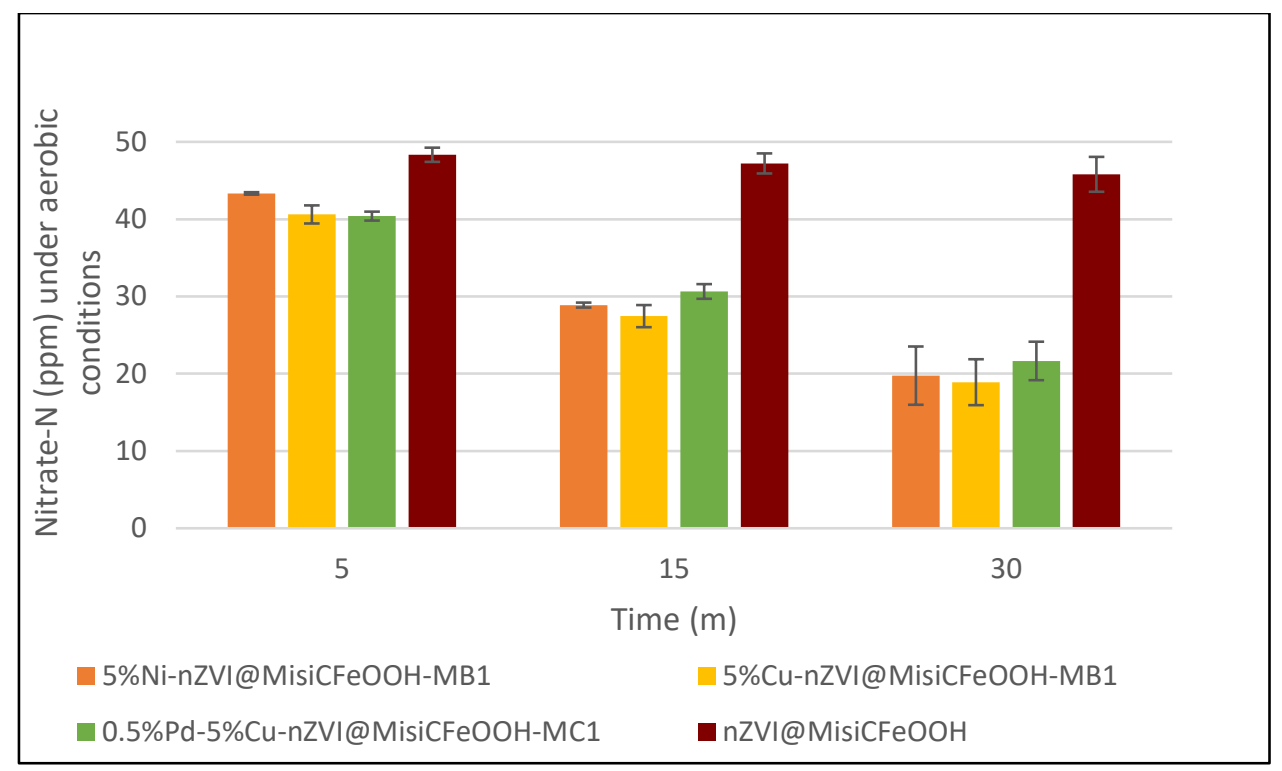

Figure 6.17. Nitrate reduction in lake water spiked with 50 ppm under aerobic conditions (0.150 g of material, $25.0 \mathrm{~mL}$ of sample).

Nitrite was not detected in $\mathrm{Ni}$-doped system while nitrite levels were significant in either $\mathrm{Cu}$ doped or Pd-Cu-doped system during the course of the reaction (Table 6.5). However, nitrite accumulation was lower than that observed under anaerobic conditions. After 30 minutes, nitrite- $\mathrm{N}$ levels were $13 \pm 1 \mathrm{ppm}$ (25\% nitrite accumulation) and $9 \pm 2 \mathrm{ppm}$ (18\% nitrite accumulation) when either 5\%Cu-nZVI@MisiCFeOOH-MB1 or 0.5\%Pd-5\%CunZVI@MisiCFeOOH-MC1 was used, respectively. Nitrite accumulation using these three materials was lower in lake water than in lab samples under aerobic conditions. Although PdCu-doped nZVI@Misi did not reduce nitrate faster than Cu-doped nZVI@Misi, the introduction of Pd increased the nitrite reduction, leading to less nitrite build-up.

Table. 6.5. Nitrite accumulation in nitrate reduction from lake water spiked with $50 \mathrm{ppm}$ nitrate- $\mathrm{N}$ under aerobic conditions ( $0.150 \mathrm{~g}$ of material, $25.0 \mathrm{~mL}$ of sample).

\begin{tabular}{|c|c|c|c|}
\hline Nitrite-N (ppm)/ Time & 5 minutes & 15 minutes & 30 minutes \\
\hline 5\%Ni-nZVI@MisiCFeOOH-MB1 & ND & ND & ND \\
\hline 5\%Cu-nZVI@MisiCFeOOH-MB1 & $6.6 \pm 0.9$ & $12.4 \pm 0.4$ & $13 \pm 1$ \\
\hline 0.5\%Pd-5\%Cu-nZVI@MisiCFeOOH-MC1 & $5.4 \pm 0.4$ & $8 \pm 1$ & $9 \pm 2$ \\
\hline nZVI@MisiCFeOOH & ND & ND & ND \\
\hline
\end{tabular}


In summary, nitrate reduction from lake water spiked was considerably slower when both doped and non-doped materials were used under both anaerobic and aerobic conditions. It could be due to the presence of organic matters in natural water, which was investigated in several studies about the effect of humic acid on iron composite. For example, humic acid passivated $\mathrm{nZVI},{ }^{272-273} \mathrm{Ni}-\left.\mathrm{nZVI}\right|^{274}$ or $\mathrm{Pd}-\mathrm{nZVI}{ }^{186}$ when these particles were used to remove organic substances. The inhibition of these substances was explained by the competition on the surface of active sites. ${ }^{186,272-274}$ In a separate study, humic acid inhibited Ni-nZVI in $\mathrm{Cr}(\mathrm{VI})$ removal due to the formation of chelate Fe-humic acid and Ni-humic acid in which both iron and nickel acted as coordination centers. ${ }^{263}$ As a result, the number of active sites of both iron and nickel decreased. Another possible reason for the lower reducing performance of these materials in lake water is the effect of ionic strength. The higher ionic strength, the slower reduction of $\mathrm{nZVI}$ with nitrate. ${ }^{242}$ In this case, lake water consists of numerous ions, potentially resulting in higher ionic strength compared to lab samples.

In lake water, all types of doped materials reduced nitrate faster under aerobic conditions than under anaerobic conditions. This is inconsistent with the results when these materials were examined in lab samples. Although the reason for the differences under both conditions is unclear, it could be due to the interaction between coexisting factors in lake water with the surface of nanoparticles in the presence of oxygen. In addition, nitrite accumulation was lower in lake water than in lab samples, except lake water spiked with $10 \mathrm{ppm}$ under aerobic conditions.

\subsection{Determination of soluble iron during nitrate reduction from lake water}

During the nitrate reduction from lab samples (10 ppm and $50 \mathrm{ppm})$ under aerobic conditions, the concentrations of soluble iron were less than $2 \mathrm{ppm}$ and $0.5 \mathrm{ppm}$, respectively. In this study, the generation of soluble iron from lake water was also examined in order to determine if the amount of iron could change. For this, after removing nitrate from lake water spiked 10 ppm nitrate- $\mathrm{N}$ and $50 \mathrm{ppm}$ nitrate- $\mathrm{N}$, solutions were filtered via syringe filter $0.22 \mu \mathrm{m}$ and then measured iron concentrations using the AAS. The initial iron level in lake water before the reaction was $0.3 \mathrm{ppm}$.

For lake water spiked with $10 \mathrm{ppm}$ nitrate- $\mathrm{N}$, after 5 minutes, the soluble iron concentrations were around $4 \mathrm{ppm}$ for all doped systems tested (Figure 6.18). These were more than eight 
times greater than those observed in the lab sample. However, after 30 minutes, the iron levels were around $0.5 \mathrm{ppm}$. The decrease in the soluble iron concentrations at 30 minutes can be explained by the formation of insoluble substances (iron oxides/hydroxides). ${ }^{143}$

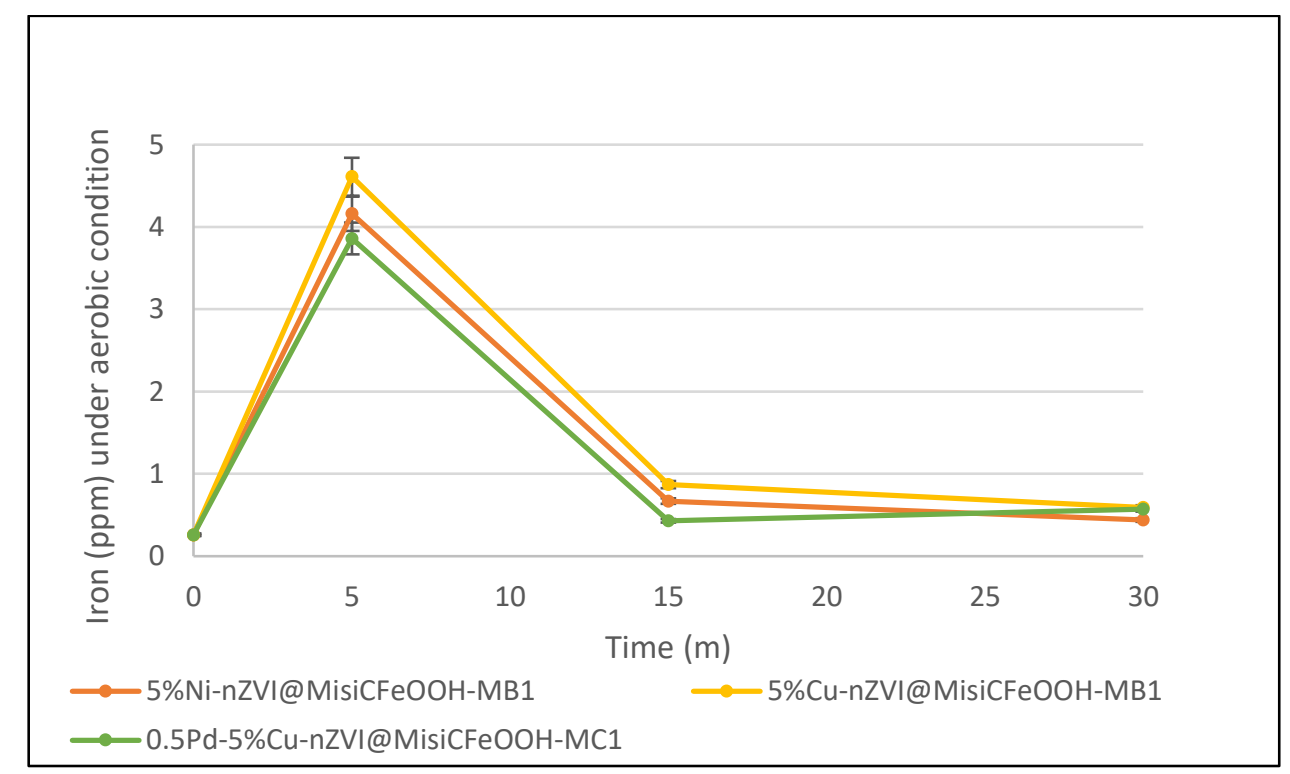

Figure 6.18. Iron concentration in nitrate reduction from lake water spiked with 10 ppm under aerobic conditions ( $0.150 \mathrm{~g}$ of material, $25.0 \mathrm{~mL}$ of sample).

Iron concentrations were lower than those in the lab samples after 30 minutes. This could be due to the slow nitrate reduction in lake water. As discussed in Section 5.4, our materials released a significant amount of iron into solution due to the dissolution of the particles. However, significantly less iron was produced upon reaction with nitrate. This is attributed to the rise in $\mathrm{pH}$ of the solution which results in the precipitation of soluble iron. In the lake samples, nitrate reduction was gradual, the increased iron levels at 5 minutes could be due to the corrosion of nanoparticles. Another possible reason for lower iron concentration in lake water is that soluble iron can be taken by components in lake water such as microalgae ${ }^{275}$ and phytoplankton. ${ }^{276}$

For lake water spiked with 50 ppm nitrate-N, after 5 minutes, the iron levels ranged from 1.6 ppm to $3.9 \mathrm{ppm}$ for all doped materials examined (Figure 6.19). The iron concentration using Ni-doped was lowest while 0.5\%Pd-5\%Cu-nZVI@MisiCFeOOH-MC1 produced iron with the highest level. These iron concentrations were more than three times greater than in lab samples. However, after 30 minutes, the iron levels were around the detection limit of the AAS (0.09 ppm) using Ni-doped material. Iron levels were $0.4 \mathrm{ppm}$ and $0.2 \mathrm{ppm}$ when either 
5\%Cu-nZVI@MisiCFeOOH-MB1 or 0.5\%Pd-5\%Cu-nZVI@MisiCFeOOH-MC1 was used, respectively. These concentrations were similar to those in the lab samples and close to the maximum iron level for drinking water $(0.3 \mathrm{ppm}) .{ }^{277}$ Therefore, iron levels produced using these doped nZVI@MisiCFeOOH systems should not affect drinking water supply.

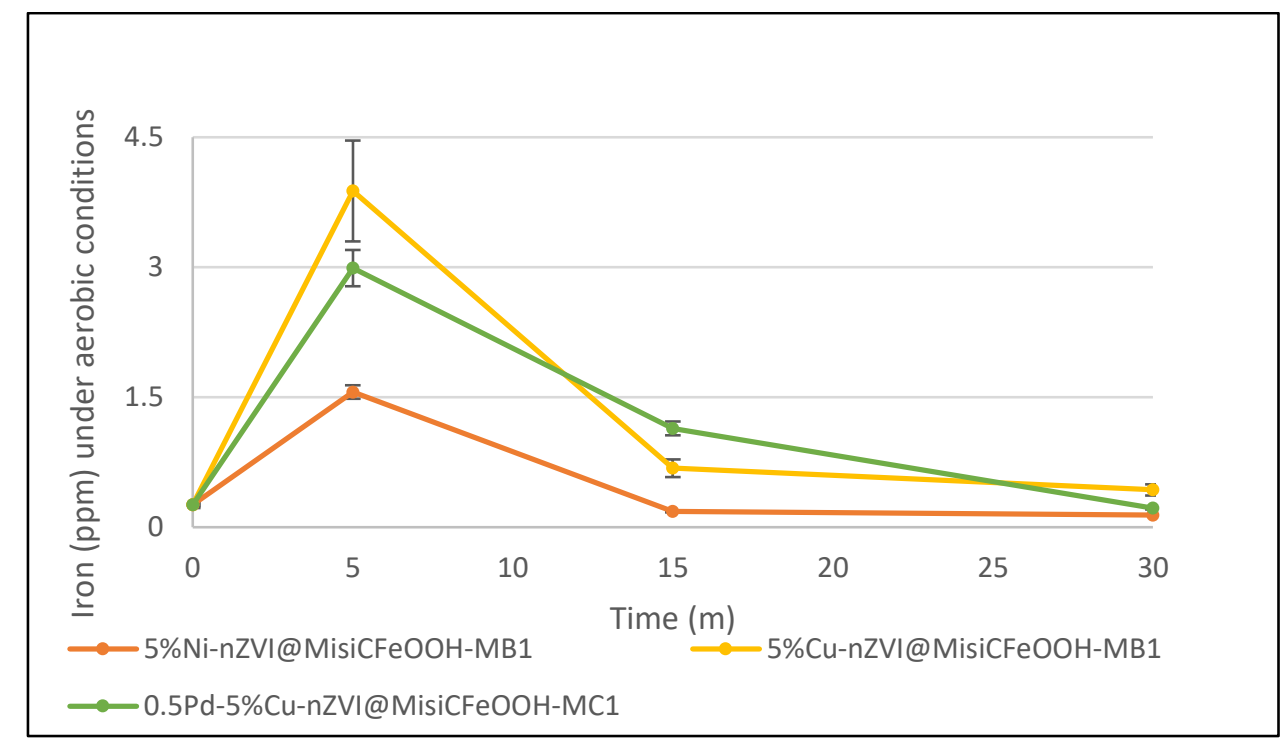

Figure 6.19. Iron concentration in nitrate reduction from lake water spiked with 50 ppm under aerobic conditions ( $0.150 \mathrm{~g}$ of material, $25.0 \mathrm{~mL}$ of sample).

\subsection{Conclusion}

Doped nZVI@MisiCFeOOH materials reduced nitrate from lake water spiked in both neutral $\mathrm{pH}$ and $\mathrm{pH}$ of 3 . Their reactivity was more significant under aerobic conditions than under anaerobic conditions. In contrast, non-doped nZVI@MisiCFeOOH only effectively reduced nitrate from lake water at a pH of 3. Nitrite was detected using $\mathrm{Cu}$-doped and $\mathrm{Pd}$-Cu-doped materials while no nitrite was observed in $\mathrm{Ni}$-doped material. The reducing capacity of these materials in lake water was lower than that in lab-prepared samples, potentially due to the presence of several factors in natural water. Interestingly, of these studied materials, $\mathrm{Ni}$ doped system was the most active while the fastest reduction was observed when Cu-doped material was used to remove nitrate from lab-prepared samples. Additionally, the by-product in post-treatment solutions was not concerns because the concentration of soluble iron was insignificant (below $0.5 \mathrm{ppm}$ ). Therefore, these doped materials are potentially promising materials in removing nitrate from "real" waters without attention about their ageing when exposed to the air. Of these, 5\% Ni-nZVI@MisiCFeOOH is the best performing material due to high nitrate conversion and no detectable nitrite build-up. 


\section{Heavy metal removal}

\subsection{Introduction}

In this chapter, investigations on the reactivity of doped and non-doped materials towards toxic heavy metals are discussed. This includes the removal of heavy metals, generation of soluble metal by-products and elemental distribution on the materials after removal. In addition, the removal of toxic heavy metals from natural water is also examined.

Toxic heavy metals such as cadmium, lead, chromium (VI), mercury, to name a few can be persistent and non-biodegradable. They damage human health and are harmful to other organisms. Due to their danger, several methods have been used to remove them. Of these, $\mathrm{nZVI}$ has widely been used as an effective method.

Heavy metals are removed from waters by $\mathrm{nZVI}$ in different pathways, depending on their standard reduction potential and their ability to form oxides. The pathways include adsorption, complex formation, co-precipitation, reduction, or a combination of these. ${ }^{116}$ The adsorption, complex formation, and co-precipitation are caused by electrostatic interaction and surface bonding without electron transfer. These are main pathways of the removal of metals with standard reduction potential close or more negative than that of iron. In the reduction pathway, metals with standard reduction potential more positive than that of iron are reduced by iron. However, typically, the removal of metal ions involves more than one pathway. For example, the reduction potential of $\mathrm{Cd}(\mathrm{II}) / \mathrm{Cd}$ is $-0.40 \mathrm{~V}$, very close to that of $\mathrm{Fe}(\mathrm{II}) / \mathrm{Fe}(-0.44 \mathrm{~V})$, so $\mathrm{Cd}(\mathrm{II})$ can be removed by $\mathrm{nZVI}$ via adsorption ${ }^{136,154,278-280}$ or complex formation of $\mathrm{Cd}(\mathrm{II})$ on the surface of $\mathrm{nZVI}$ with no apparent reduction. ${ }^{116,}{ }^{279-280}$ Techniques including X-ray photoelectron spectroscopy (XPS), energy dispersive X-ray pectroscopy in conjunction with scanning electron microscope (SEM-EDS) and energy dispersive X-ray spectroscopy in conjunction with transmission electron microscopy (TEM-EDS) have provided evidence of the presence of Cd(II) on the surface of nZVI. For example, the peak corresponding to $\mathrm{Cd}(\mathrm{II})$ in XPS ${ }^{116,136,154,280}$ or in SEM-EDS ${ }^{278}$ confirmed the presence of Cd(II) on the surface of $\mathrm{nZVI}$ when $\mathrm{Cd}(\mathrm{II})$ was removed by $\mathrm{nZVI}$. TEM-EDS analysis of $\mathrm{nZVI}$ after $\mathrm{Cd}(\mathrm{II})$ removal showed the formation of nanofiber which consists of iron oxides and cadmium ferrite $\left(\mathrm{CdFe}_{2} \mathrm{O}_{4}\right){ }^{279}$ However, in Liu et al.'s study, the mechanism of the $\mathrm{Cd}(\mathrm{II})$ removal was via a combination of adsorption (predominant) and reduction (auxiliary) when $\mathrm{Cd}(\mathrm{II})$ was removed 
using nZVI@chitosan beads. ${ }^{281}$ In this study, both $\mathrm{Cd}(\mathrm{II})$ and $\mathrm{Cd}(0)$ peaks were observed in XPS.

Although the reduction potential of $\mathrm{Pb}(\mathrm{II}) / \mathrm{Pb}(-0.13 \mathrm{~V})$ is more positive than that of $\mathrm{Fe}(\mathrm{II}) / \mathrm{Fe}$, $\mathrm{Fe}(0)$ has removed $\mathrm{Pb}(\mathrm{II})$ via different pathways. For example, Huang et al. reported that $\mathrm{Pb}(\mathrm{II})$ was removed by $\mathrm{nZVI}$ via both adsorption and reduction (eq. 7.1). ${ }^{154}$ In their study, both peaks of $\mathrm{Pb}(\mathrm{II})$ and $\mathrm{Pb}(0)$ were observed in XPS analysis. In Zhang et al.'s study, $\mathrm{Pb}(\mathrm{II})$ was removed by $\mathrm{nZVI}$ by the combination of adsorption, co-precipitation, and reduction (small part). ${ }^{169}$ In this study, XPS showed peaks of $\mathrm{Pb}(\mathrm{II})$ (high intensity) and $\mathrm{Pb}(0)$ (low intensity). In addition, XPS also showed a decrease in the percentage of $\mathrm{OH}^{-}$and an increase in the percentage of $\mathrm{O}^{2-}$ after removal, confirming the presence of $\mathrm{Pb}(\mathrm{OH})_{2}$ that bonded with $\mathrm{Fe}(\mathrm{OH})_{2}$ and $\mathrm{Fe}(\mathrm{OH})_{3}$. In contrast, in Ponder et al.'s study, Pb(II) was removed using nZVI@PolyFlo resin via reduction and potentially adsorption. ${ }^{117}$ In their study, both $\mathrm{Pb}(0)$ and $\mathrm{Pb}(\mathrm{II})$ were detected in XPS analysis but they were not sure about the origin of $\mathrm{Pb}(\mathrm{II})$ which can be from the direct adsorption or the oxidation during the reaction.

The reduction potential of $\mathrm{Cr}_{2} \mathrm{O}_{7}^{2-} / \mathrm{Cr}(\mathrm{II})(+1.33 \mathrm{~V})$ is significantly more positive than Fe. The fully oxidised chromium, dichromate $\left(\mathrm{Cr}_{2} \mathrm{O}_{7}{ }^{2-}\right)$ referred to in this thesis as $\mathrm{Cr}(\mathrm{VI}) . \mathrm{Cr}(\mathrm{VI})$ is removed via reduction, ${ }^{117}$ or a combination of reduction and precipitation. ${ }^{154}$ In Huang et al.'s study, $\mathrm{Cr}(\mathrm{VI})$ was removed via a combination of reduction and precipitation (eq. 7.2 - 7.5)..$^{154}$ In this study, signals corresponding to $\mathrm{Cr}_{2} \mathrm{O}_{3}$ and $\mathrm{FeCr}_{2} \mathrm{O}_{4}$ was observed using XPS. However, in a separate study, Li et al. reported that $\mathrm{Cr}(\mathrm{VI})$ was removed by $\mathrm{nZVI}$ via reduction to form $\mathrm{Cr}$ (III) and then $\mathrm{Cr}$ (III) incorporated into $\mathrm{FeOOH}$ of the shell to form hydroxide of iron and chromium. ${ }^{171}$ XPS analysis suggested the presence of $\mathrm{Cr}_{2} \mathrm{O}_{3}$ and $\mathrm{Cr}(\mathrm{OH})_{3}$.

$$
\begin{gathered}
\mathrm{Fe}^{(0)}{ }_{(\mathrm{s})}+\mathrm{Pb}^{2+}{ }_{(\mathrm{aq})} \longrightarrow \mathrm{Fe}^{2+}{ }_{(\mathrm{aq})}+\mathrm{Pb}_{(\mathrm{s})} \\
3 \mathrm{Fe}^{(0)}{ }_{(\mathrm{s})}+\mathrm{Cr}_{2} \mathrm{O}_{7}{ }^{2-}{ }_{(\mathrm{aq})}+14 \mathrm{H}^{+} \longrightarrow \\
\mathrm{Fe}^{2+}{ }_{(\mathrm{s})}+\mathrm{Cr}_{2} \mathrm{O}_{7}{ }^{2-}{ }_{(\mathrm{aq})}+14 \mathrm{H}^{+} \longrightarrow \mathrm{Fe}^{2+}{ }_{(\mathrm{aq})}+2 \mathrm{Cr}^{3+}{ }_{(\mathrm{aq})}+7 \mathrm{H}_{2} \mathrm{O} \\
(1-\mathrm{x}) \mathrm{Fe}^{3+}{ }_{(\mathrm{s})}+\mathrm{x} \mathrm{Cr}^{3+}{ }_{(\mathrm{aq})}+3 \mathrm{H}_{2} \mathrm{O} \longrightarrow 6 \mathrm{Fe}^{3+}{ }_{(\mathrm{aq})}+2 \mathrm{Cr}^{3+}{ }_{(\mathrm{aq})}+7 \mathrm{H}_{2} \mathrm{O} \\
(1-\mathrm{x}) \mathrm{Fe}^{3+}{ }_{(\mathrm{s})}+\mathrm{x} \mathrm{Cr}^{3+}{ }_{(\mathrm{aq})}+2 \mathrm{H}_{2} \mathrm{O} \longrightarrow \mathrm{Cr}_{\mathrm{x}} \mathrm{Fe}_{(1-\mathrm{x})}(\mathrm{OH})_{3(\mathrm{~s})}+3 \mathrm{H}^{+}{ }_{(\mathrm{aq})} \\
\end{gathered}
$$


We wanted to investigate the ability of our materials to remove heavy metals from solution. As the surface of the material might be a key in the removal of heavy metals, we decided to investigate if the method of preparation of the doped-nZVI could have an effect. For instance, materials prepared via co-reduction method (MA) typically have the dopant metals found within the $\mathrm{nZVI}$, whereas materials prepared via deposition method (MB1) have the dopant metals on the surface of the nZVI. In this study, supported materials including Ni-doped, Cudoped and non-doped nZVI@Misi were used. Bimetallic materials were prepared via both coreduction (MA) and deposition (MB1). Misi that had been calcined and then coated with FeOOH layer was used as the support. Three toxic heavy metals, $\mathrm{Cd}(\mathrm{II}), \mathrm{Pb}(\mathrm{II})$, and $\mathrm{Cr}(\mathrm{VI})$, were investigated. The reactivity of these materials towards heavy metals was examined in both lab samples and lake water spiked with the heavy metals. The concentrations of soluble heavy metals including iron, nickel, and copper were measured using AAS. The elemental distribution of nZVI@Misi after heavy metal removal was also characterised via TEM.

\subsection{Cadmium removal by doped and non-doped nZVI supported on Misi}

Cadmium is a potential carcinogen. If an area is contaminated with cadmium, then some type remediation is necessary, depending upon if it is a waterway or soil. The WHO has set the maximum allowable concentration for cadmium at $0.003 \mathrm{ppm}$ in drinking water. ${ }^{19}$ Various methods have been used to remove it from waters. Of these, $\mathrm{nZVI}$ is considered as an effective agent, which was reported in numerous studies. ${ }^{136,154-155,278-279}$ Supported nZVI was also used to remove Cd(II). For example, nZVI@fly ash removed Cd(II) effectively282 or nZVI@bentonite removed Cd(II) faster than bentonite. ${ }^{283}$ In addition, bimetallic nZVI was investigated. For example, Su et al. used Au-nZVI for simultaneous removal of nitrate and Cd(II); however, Au did not increase the removal of $\mathrm{Cd}(\mathrm{II}) .{ }^{251}$ Therefore, in this current study, the effectiveness of dopant metals and synthetic methods on the reactivity of supported nZVI materials towards Cd(II) removal were examined.

In cadmium studies, the molar ratio of Fe to $\mathrm{Cd}(\mathrm{II})$ was 40:1 (20 ppm Cd(II)). The reactions were carried out under anaerobic conditions. The concentrations of cadmium, iron, nickel, and copper were measured over the course of the reaction. In addition, the elemental distribution of nZVI@MisiCFeOOH after Cd(II) removal was also examined if cadmium could precipitate on the surface of $\mathrm{nZVI}$. 


\subsubsection{Reactivity of doped and non-doped nZVI@Misi towards cadmium removal}

More than $10 \mathrm{ppm}$ of $\mathrm{Cd}(\mathrm{II})$ was removed by all types of materials examined after 5 minutes, (Figure 7.1). The Ni-doped material prepared via co-reduction method (MA) removed Cd(II) fastest, with the other materials showing similar reactivity. The cadmium concentration was 3 ppm for 5\%Ni-nZVI@MisiCFeOOH-MA while more than 6 ppm was observed for other materials. However, after 30 minutes, the difference in their reactivity was more obvious. $\mathrm{Ni}$ doped materials reduced Cd(II) fastest while non-doped nZVI@MisiCFeOOH was the slowest performing material. For example, the cadmium concentrations were $0.50 \pm 0.09$ ppm (98\% Cd(II) removal) and $4.7 \pm 0.3$ ppm (77\% Cd(II) removal) when either 5\%Ni-nZVI@MisiCFeOOHMA or nZVI@MisiCFeOOH was used, respectively. These results suggested that the introduction of dopant metals $(\mathrm{Ni}, \mathrm{Cu}$ ) helped catalyse the removal of $\mathrm{Cd}(\mathrm{II})$. This was inconsistent with a report by Su et al. in which the removal of Cd(II) using Au-nZVI was similar to that observed using nZVI. ${ }^{251}$ However, in their study, unsupported nZVI was used, the molar ratio of Fe to $\mathrm{Cd}(\mathrm{II})$ ranged from $10: 1$ to $100: 1$ and the solution contained both $\mathrm{Cd}$ (II) (10 - 100 ppm) and nitrate-N (15 ppm). In a separate study, the initial $\mathrm{Cd}(\mathrm{II})$ concentration was $20 \mathrm{ppm}$ and the molar ratio of Fe to Cd(II) was 50:1 using nZVI@fly ash. ${ }^{282}$ After 5 minutes, all Cd(II) was removed, indicating that their material removed $\mathrm{Cd}(\mathrm{II})$ faster than our materials. However, in their study, a higher ratio of Fe to $\mathrm{Cd}$ (II) was used compared to our ratio (40:1). 


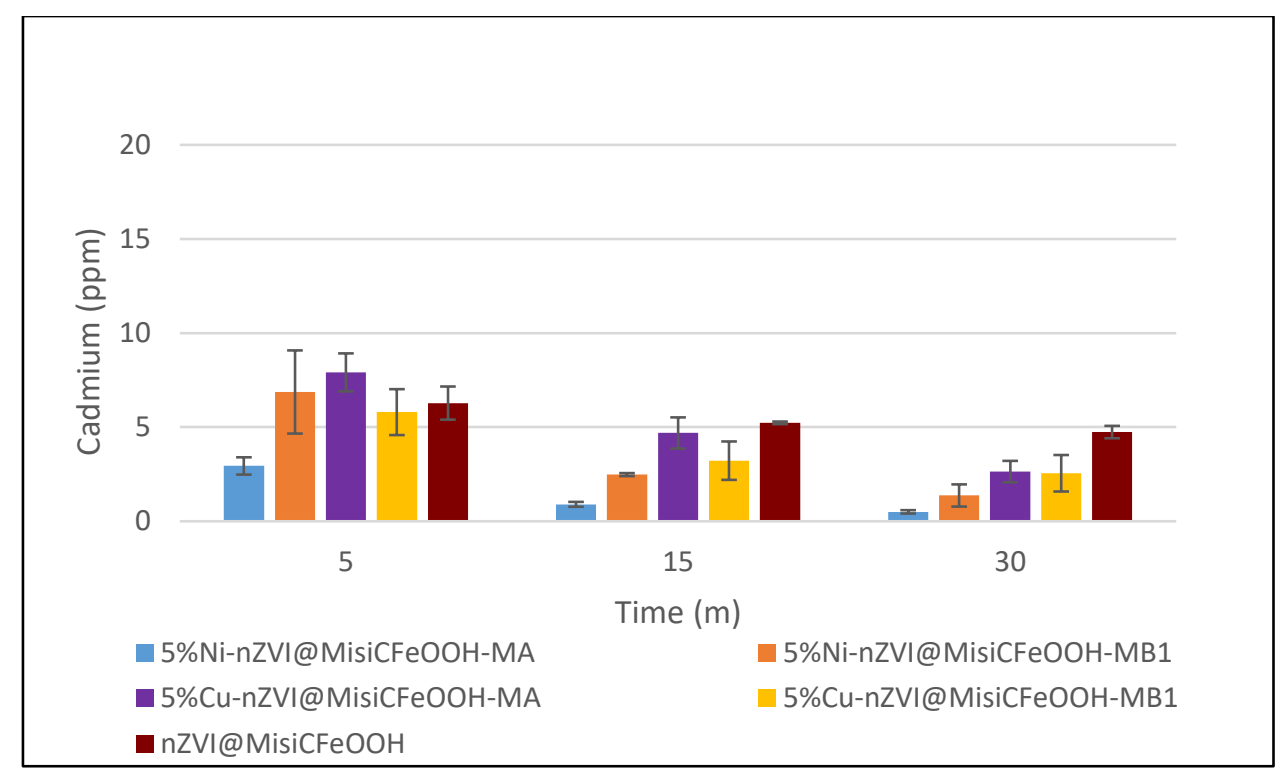

Figure 7.1. Removal characterisation towards Cd(II) $(0.10 \mathrm{~g}$ of material, $25.0 \mathrm{~mL}$ of $20 \mathrm{ppm}$ solution).

In addition, Ni-doped material prepared via co-reduction method (MA) had significantly higher reactivity in 15 minutes than that prepared via deposition method (MB1). However, the difference in their reactivity was minimal after 30 minutes. For example, after 5 minutes, cadmium concentrations were $2.9 \pm 0.5 \mathrm{ppm}$ and $7 \pm 2 \mathrm{ppm}$ using either $5 \% \mathrm{Ni}-$ nZVI@MisiCFeOOH-MA or 5\%Ni-nZVI@MisiCFeOOH-MB1, respectively. After 30 minutes, cadmium concentrations were $0.50 \pm 0.09 \mathrm{ppm}$ and $1.4 \pm 0.6 \mathrm{ppm}$ for $5 \% \mathrm{Ni}-$ nZVI@MisiCFeOOH-MA and 5\%Ni-nZVI@MisiCFeOOH-MB1, respectively. The difference in the kinetics of $\mathrm{Cd}(\mathrm{II})$ removal using materials prepared via co-reduction and deposition methods is in contrast to that of nitrate reduction. The higher reactivity of Ni-doped material prepared via co-reduction method (MA) could be due to its surface properties in which might promote the removal of $\mathrm{Cd}(\mathrm{II})$ via adsorption in which $\mathrm{Cd}(\mathrm{II})$ adsorbed on the surface of $\mathrm{nZVI}$ by combining with the iron oxides layer to form $\mathrm{CdFeO}_{4} \cdot{ }^{279}$ In 5\%Ni-nZVI@MisiCFeOOH-MA, $\mathrm{Ni}$ is found inside nZVI particles; as such, it would not affect the oxidation of iron during the synthesis (Section 3.3). In contrast, in 5\%Ni-nZVI@MisiCFeOOH-MB1, Ni is on the surface of nZVI (Section 3.3), slowing down iron not only from oxidising at the surface, but also minimising the surface area of the iron oxide shell. ${ }^{172}$ As such, the number of iron oxide sites for $\mathrm{Cd}(\mathrm{II})$ adsorption decreases.

Regarding Cu-doped materials, within 15 minutes, Cu-doped material prepared via deposition method (MB1) removed $\mathrm{Cd}(\mathrm{II})$ faster than that prepared via co-reduction method (MA). For 
example, after 5 minutes, cadmium concentrations were $8 \pm 1 \mathrm{ppm}$ and $6 \pm 1 \mathrm{ppm}$ using either 5\%Cu-nZVI@MisiCFeOOH-MA or 5\%Cu-nZVI@MisiCFeOOH-MB1, respectively. The reason for this was unclear, but there might be reduction of $\mathrm{Cd}(\mathrm{II})$ accompanied with adsorption of $\mathrm{Cd}(\mathrm{II})$ at the start of removal process. Surface Cu of nZVI (5\%Cu-nZVI@MisiCFeOOH-MB1) promotes the electron transfer more effectively than $\mathrm{Cu}$ within the nZVI (5\%Cu-nZVI@MisiCFeOOHMA). However, after 30 minutes, there was no difference in these Cu-doped materials.

Although the mechanism of the $\mathrm{Cd}(\mathrm{II})$ removal using supported doped and non-doped nZVI@Misi was not determined, its removal was due to nanoparticles, including original nZVI and other substances produced by nZVI. Control experiments to investigate the role of the support (MisiCFeOOH) in removing $\mathrm{Cd}(\mathrm{II})$ were performed and showed no decrease in the amount of $\mathrm{Cd}(\mathrm{II})$ upon addition of a $20 \mathrm{ppm}$. This indicated that $\mathrm{Cd}(\mathrm{II})$ is not adsorbed by Misi. These results are in contrast to previously reported supports such as kaolinite ${ }^{89-90,92}$ and goethite. ${ }^{92,94}$

\subsubsection{Determination of soluble metals in cadmium removal}

Several investigations on $\mathrm{Cd}(\mathrm{II})$ removal using supported $\mathrm{nZVI}$ composites have been reported. However, there was no discussion about the soluble iron, including leaching. In this current study, the concentrations of iron, nickel, and copper were monitored during the reaction. The results showed that a significant amount of iron was produced when all types of the materials were used. Iron levels ranged from $6 \mathrm{ppm}$ to $11 \mathrm{ppm}$ over the course of the monitored reaction (Figure 7.2). Of these, non-doped nZVI@MisiCFeOOH produced the least amount of iron. Materials prepared via co-reduction method (MA) generated less soluble iron than those prepared via deposition method (MB1). For example, after 30 minutes, iron levels were $8.8 \pm 0.1$ ppm, $11.2 \pm 0.4$ ppm when either 5\%Ni-nZVI@MisiCFeOOH-MA or 5\% NinZVI@MisiCFeOOH-MB1 was used, respectively. The iron detected in the solutions could be due to the reduction of $\mathrm{nZVI}$ with $\mathrm{Cd}(\mathrm{II})$ or the corrosion of $\mathrm{nZVI}$ with water. After 30 minutes, the corrosion of iron with water produced more than 4 ppm soluble iron (Section 5.4). 


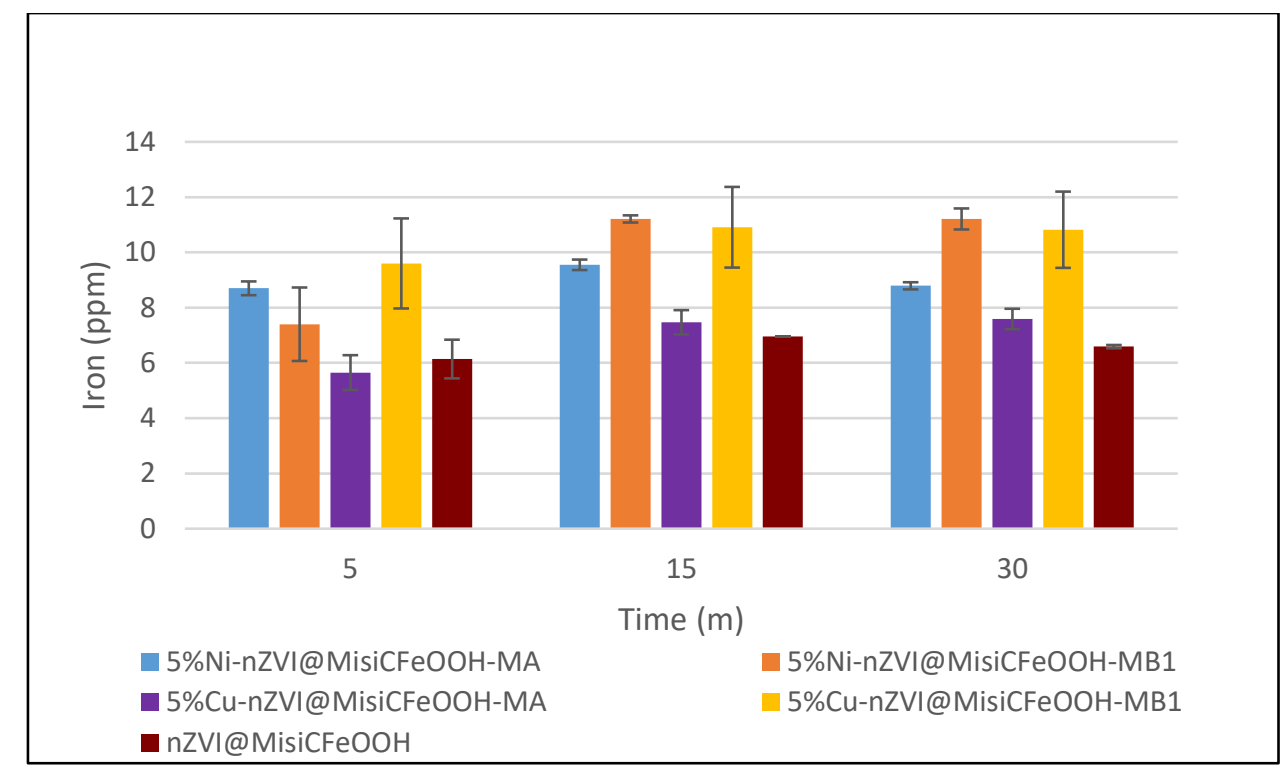

Figure 7.2. Iron concentration in the removal of Cd(II) 20 ppm (0.10 g material, $25.0 \mathrm{~mL} \mathrm{of}$ solution).

In a related study, Cd(II) was removed using unsupported nZVI. ${ }^{251}$ The initial Cd(II) concentration was $20 \mathrm{ppm}$ and the molar ratio of Fe to $\mathrm{Cd}(\mathrm{II})$ was 50:1. After 90 minutes, 60\% of $\mathrm{Cd}(\mathrm{II})$ was removed and the iron concentration was more than $9 \mathrm{ppm}$. Comparing to our results, nZVI@MisiCFeOOH removed Cd(II) faster, more effectively and produced less soluble iron.

Nickel was also measured during the reaction between Ni-doped nZVI@Misi and Cd(II) (Table 7.1). Nickel concentrations using 5\%Ni-nZVI@MisiCFeOOH-MB1 were higher than those using 5\%Ni-nZVI@MisiCFeOOH-MA. In contrast, in the removal of Cd(II) using Cu-doped nZVI@Misi, the concentration of $\mathrm{Cu}(\mathrm{II})$ never exceeded the detection limit of the AAS (0.66 ppm).

Table 7.1. Nickel concentration in Cd(II) removal.

\begin{tabular}{|c|c|c|c|}
\hline Nickel $(\mathrm{ppm}) /$ time & 5 minutes & 15 minutes & 30 minutes \\
\hline 5\%Ni-nZVI@MisiCFeOOH-MA & $0.60 \pm 0.03$ & $0.34 \pm 0.03$ & $0.21 \pm 0.02$ \\
\hline 5\%Ni-nZVI@MisiCFeOOH-MB1 & $1.0 \pm 0.2$ & $0.8 \pm 0.1$ & $0.51 \pm 0.07$ \\
\hline
\end{tabular}

The predominant mechanism of $\mathrm{Cd}(\mathrm{II})$ removal has been reported as adsorption or complex formation of $\mathrm{Cd}(\mathrm{II})$ on the surface of nZVI. ${ }^{116,154,278-279}$ Our findings indicated that the mechanism of $\mathrm{Cd}(\mathrm{II})$ removal might be adsorption/complexation and potentially reduction. 
The adsorption/complexation is caused by the relative similarity of the reduction potential of iron $(-0.44 \mathrm{~V})$ and cadmium $(-0.40 \mathrm{~V})$. An example for these pathways is obtained when the concentrations of cadmium and iron were calculated in $\mathrm{mM}$ using the most effective material (5\%Ni-nZVI@MisiCFeOOH-MA) (Figure 7.3). The ratio of cadmium concentration removed $(174 \mathrm{mM})$ to iron concentration produced $(157 \mathrm{mM})$ was 1.1:1 that was higher than expected ratio 1:1 (eq 7.1). The cadmium reduction might occur due to the presence of soluble Fe(II) in solutions. If only adsorption/complexation occurred, consistent with the removal of Cd(II) by $\mathrm{nZVI}, 116,136,154,278-280$ the reactivity of doped materials, especially those prepared via deposition method (MB1), would be lower than non-doped nZVI@MisiCFeOOH due to the limit of adsorption sites. If $\mathrm{Cd}(\mathrm{II})$ removal is resulted by a combination of adsorption/complexation as well as reduction, this is consistent with a report in which $\mathrm{Cd}(\mathrm{II})$ was removed using nZVI@chitosan beads.281

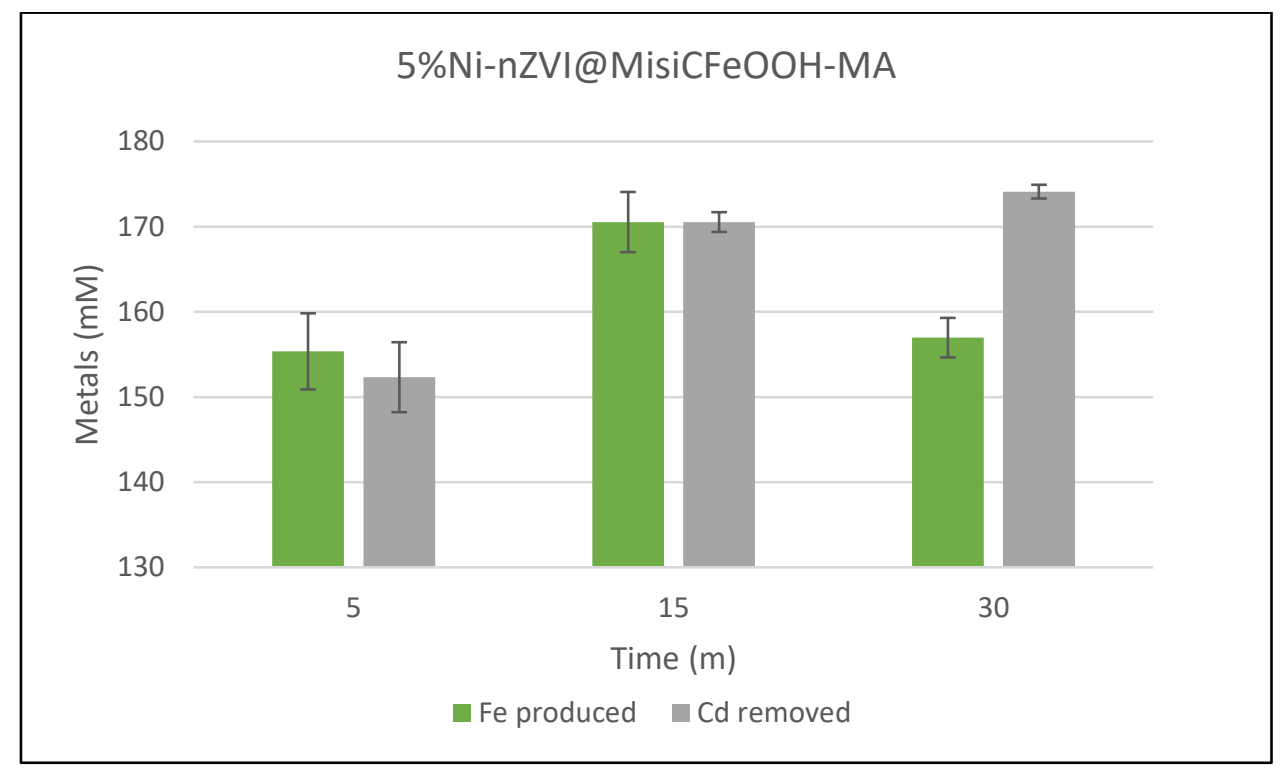

Figure 7.3. Cadmium and iron concentrations using 5\%Ni-nZVI@MisiCFeOOH-MA.

\subsubsection{Elemental distribution of material after cadmium removal}

In order to examine the fate of cadmium after removal, the elemental distribution of nZVI@MisiCFeOOH after 30-minute reaction was examined via TEM. The spectra of this specific area showed peaks corresponding to $\mathrm{Si}, \mathrm{O}$, and $\mathrm{Al}$, consistent with the presence of Misi (Figure 7.4). A strong Fe signal and a weak Cd signal are observed, indicating the presence of both iron and cadmium in the material. 


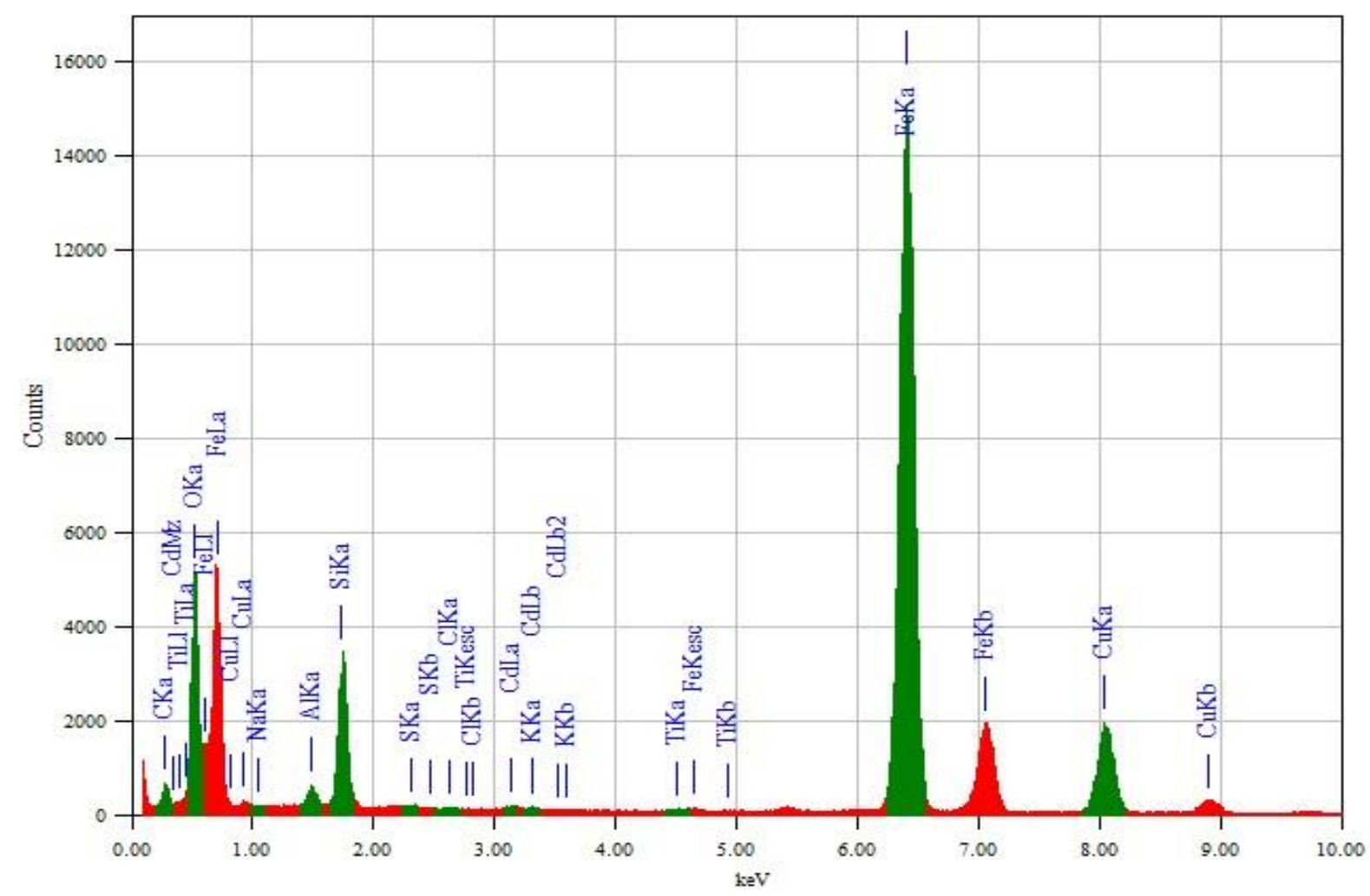

Figure 7.4. TEM-EDS spectrum of nZVI@MisiCFeOOH after Cd(II) removal (0.10 g of material, $25.0 \mathrm{~mL}$ of $20 \mathrm{ppm}$ solution).

EDS mapping of nZVI@MisiCFeOOH after Cd(II) removal confirmed the presence of both Misi and $\mathrm{nZVI}$ particles. The overlay of $\mathrm{Si}, \mathrm{O}, \mathrm{Al}, \mathrm{S}$, and Ti corresponds to Misi (Figure 7.5). When the signals of $\mathrm{Fe}$ and $\mathrm{Cd}$ were overlaid, $\mathrm{Cd}$ is observed on wherever $\mathrm{Fe}$ is present; however, it appears as if there is a layer of $\mathrm{Cd}$ on top of Fe (Figure 7.5a). The signals of $\mathrm{O}$ and $\mathrm{Cd}$ appear to overlap on the $\mathrm{nZVI}$ particles (Figure 7.5c). When the signals of $\mathrm{Fe}, \mathrm{O}$, and $\mathrm{Cd}$ are combined (Figure 7.5b and 7.5d), it becomes evident that there is a core-shell structure in which there is a $\mathrm{Cd}(0)$ or $\mathrm{CdO}$ shell encapsulating an $\mathrm{Fe}(0)$ core, although we cannot distinguish between the two oxidation states. $\mathrm{Cd}(\mathrm{II})$ can be present from the direct adsorption of $\mathrm{Cd}(\mathrm{II})$ on the surface of material whereas $\mathrm{Cd}(0)$ can be generated from the reduction of $\mathrm{Cd}(\mathrm{II})$ with $\mathrm{Fe}$ nanoparticles, which then adsorbs on the surface of nZVI. 

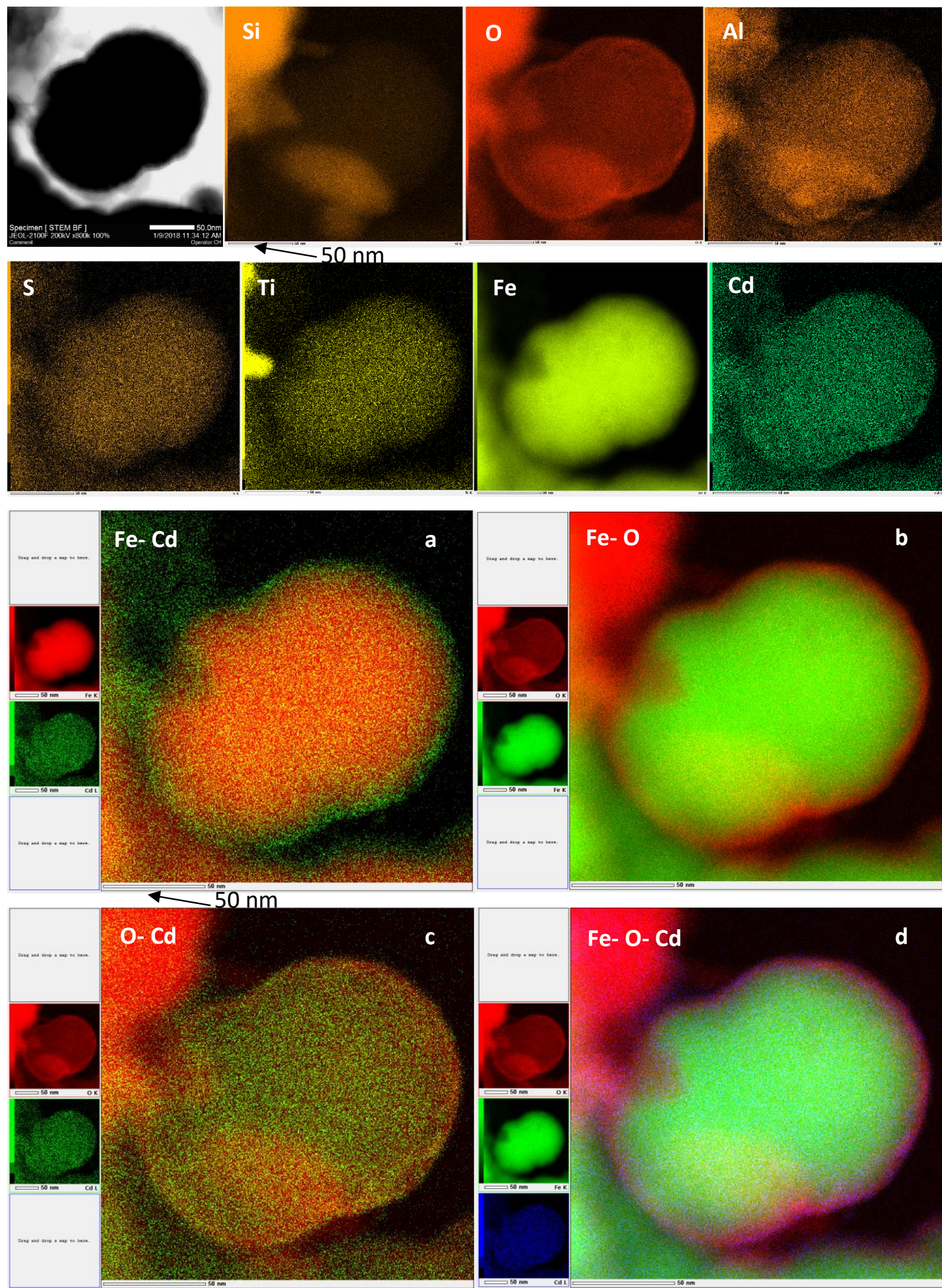

Figure 7.5. TEM-EDS mapping of nZVI@MisiCFeOOH after Cd(II) removal (0.10 g material, $25.0 \mathrm{~mL}$ solution of $20 \mathrm{ppm}$ ). 


\subsubsection{Conclusion}

In summary, all of the materials examined removed $\mathrm{Cd}$ (II) from aqueous solution. Cd(II) was removed via adsorption/complexation and potentially reduction. The addition of dopant metals such as $\mathrm{Ni}$ and $\mathrm{Cu}$ increased the reactivity of these materials. During the reaction, iron and nickel were detected in solutions while copper concentrations were below the detection limit of the AAS. Cadmium was observed on the surface of Fe nanoparticles, but its form was not determined. Among the doped nZVI@Misi, materials prepared via co-reduction method (MA) were more effective than those via deposition method (MB1) due to their higher or similar reactivity but lower iron production. Therefore, 5\%Ni-nZVI@MisiCFeOOH-MA and 5\%Cu-nZVI@MisiCFeOOH-MA were chosen for Cd(II) removal from lake water.

\subsection{Lead removal by doped and non-doped nZVI supported on Misi}

Lead is a highly poisonous metal, with multiple pathways in biological systems, including interfering with heme biosynthesis and causing neurological problem. ${ }^{63}$ The WHO has set the maximum allowable concentration for lead at $0.01 \mathrm{ppm}$ in drinking water. ${ }^{19}$ Due to its toxicity to human health, the removal of lead is a significant concern. There have been several methods for $\mathrm{Pb}(\mathrm{II})$ removal, including $\mathrm{nZVI}$ composites such as unsupported $\mathrm{nZVI}{ }^{116,154,169}$ as well as supported nZVI such as nZVI@PolyFlo resin,, ${ }^{117}$ nZVI@bentonite,,283 nZVI@chitosan beads, ${ }^{281}$ and nZVI@sepiolite. ${ }^{284}$ In addition, bimetallic and supported bimetallic Ni-nZVI were also examined. However, conflicting results have been reported with regards to the catalytic role of $\mathrm{Ni}$. For example, in a study, the addition of $\mathrm{Ni}$ as a dopant did not influence the reactivity of $\mathrm{nZVI}$ when $\mathrm{Ni}-\mathrm{nZVI}$ was used to remove $\mathrm{Pb}(\mathrm{II}) .{ }^{177}$ In contrast, in another study, more Pb(II) was removed using Ni-nZVI@kaolinite than nZVI@kaolinite.192

In this study, the reactivity of doped and non-doped nZVI@Misi towards Pb(II) removal was examined. The molar ratios of Fe to $\mathrm{Pb}(\mathrm{II})$ of 74:1 (20 ppm $\mathrm{Pb}(\mathrm{II})$ ) and $37: 1$ (40 ppm $\mathrm{Pb}(\mathrm{II})$ ) were tested. The reactions were carried out under anaerobic conditions. During the reactions, the concentrations of soluble metals in solutions were also monitored. After the reaction, the elemental distribution of nZVI@MisiCFeOOH was also examined via TEM.

\subsubsection{Reactivity of doped and non-doped nZVI@Misi towards lead removal}

The reactivity of these materials towards $\mathrm{Pb}(\mathrm{II})$ was initially examined when $\mathrm{Pb}\left(\mathrm{NO}_{3}\right)_{2}(20$ ppm) was used. After 5 minutes, the lead levels were around the detection limit of the AAS 
(0.05 ppm) for all materials used. The rapid removal of $\mathrm{Pb}(\mathrm{II})$ might be partly due to the presence of nitrate as nitrate reduction would result in an elevation of $\mathrm{pH}$, thus promoting $\mathrm{Pb}$ (II) removal by precipitation. This is consistent with a study in which $\mathrm{Pb}$ (II) removal increased when the solution $\mathrm{pH}$ increased from 5 to 9.285

The removal of $\mathrm{Pb}$ (II) (40 ppm) using $\mathrm{PbCl}_{2}$ was examined in order to examine to remove any influence of nitrate. Only three representative materials, 5\%Ni-nZVI@MisiCFeOOH-MA, 5\%Ni-nZVI@MisiCFeOOH-MB1 and nZVI@MisiCFeOOH were trialled. Consistent with the 20 ppm study, the removal of $\mathrm{Pb}(\mathrm{II})$ was rapid and the lead concentrations were below the detection limit of the AAS (0.05 ppm) after 5 minutes when any of the doped and non-doped materials were used. These results indicated that the presence of nitrate did not help enhance $\mathrm{Pb}(\mathrm{II})$ removal.

There has been a report in which Pb(II) was removed using Ni-nZVI@kaolinite. ${ }^{192}$ However, in this study, the $\mathrm{Pb}$ (II) removal was investigated in the presence of nitrate. In addition, the initial concentrations of $\mathrm{Pb}(\mathrm{II})(300 \mathrm{ppm})$ and nitrate $(180 \mathrm{ppm})$ were significantly higher than levels we examined. Although this system was able to remove, the conditions are comparable with ours.

Fu et al. reported a similar study in which Pb(II) was removed using nZVI@sepiolite. ${ }^{284}$ In their study, the molar ratio of Fe to $\mathrm{Pb}(\mathrm{II})$ was 15:1 (nZVI dosage was $0.16 \mathrm{~g} / \mathrm{L}$ ) and $\mathrm{pH}$ of solution was 4 - 6. All $\mathrm{Pb}(\mathrm{II})(40 \mathrm{ppm})$ was removed after 5 minutes; however, the removal of $\mathrm{Pb}(\mathrm{II})$ decreased at $\mathrm{Pb}$ (II) concentration higher than $50 \mathrm{ppm}$. Although their ratio of $\mathrm{Fe}$ to $\mathrm{Pb}$ (II) was lower, acidic condition is favourable for Fe corrosion. ${ }^{241}$

There have been several supports such as bentonite, ${ }^{283,286}$ graphene oxide, ${ }^{286}$ zeolite $^{287}$ that can adsorb $\mathrm{Pb}(\mathrm{II})$. To evaluate if MisiCFeOOH can absorb $\mathrm{Pb}(\mathrm{II})$, a control experiment was carried out. The results showed that $\mathrm{Pb}$ (II) concentration was $18.4 \pm 0.4$ ( $8 \% \mathrm{~Pb}$ (II) removal) after 30 minutes (Table 7.2). This indicated that the contribution of $\mathrm{MisiCFeOOH}$ in removing $\mathrm{Pb}(\mathrm{II})$ was insignificant and the majority of $\mathrm{Pb}(\mathrm{II})$ removal was due to the interaction between $\mathrm{Pb}(\mathrm{II})$ and nanoparticles. These results are in contrast to an investigation in which $\mathrm{Pb}$ (II) was removed using nZVI@bentonite. In this study, the $\mathrm{Pb}(\mathrm{II})$ removal using bentonite was greater than one-half of that using nZVI@bentonite. ${ }^{283}$ 
Table 7.2. Lead concentration using MisiCFeOOH.

\begin{tabular}{|c|c|c|c|c|}
\hline Lead $(\mathrm{ppm}) /$ time & 0 minute & 5 minutes & 15 minutes & 30 minutes \\
\hline MisiCFeOOH & 20 & $19.1 \pm 0.8$ & $18.9 \pm 0.2$ & $18.4 \pm 0.4$ \\
\hline
\end{tabular}

\subsubsection{Determination of soluble metals in lead removal}

\subsubsection{Determination of soluble metals in the removal of lead 20 ppm}

During the studies involving the removal of $20 \mathrm{ppm}$ of $\mathrm{Pb}(\mathrm{II})$ in $\mathrm{Pb}\left(\mathrm{NO}_{3}\right)_{2}$, the concentrations of iron, nickel, and copper were measured. The iron concentrations decreased over the course of the reaction (Figure 7.6). This can be explained by the positive effect of nitrate. The coremoval of nitrate by iron produced hydroxides, leading to the decrease in iron in the form of iron hydroxides. A significant decrease in iron concentration was not observed for $\mathrm{Ni}$-doped systems whereas iron concentrations significantly decreased with the use of Cu-doped and non-doped nZVI@Misi. This phenomenon could be due to the higher reactivity of Ni-doped towards $\mathrm{Pb}\left(\mathrm{NO}_{3}\right)_{2}$ over other materials. The $\mathrm{Ni}$-doped material prepared via co-reduction method (MA) produced less iron than the Ni-doped material prepared via deposition method (MB1) although the reverse was observed in Cu-doped systems. For example, after 5 minutes, the iron concentrations were $2.7 \pm 0.4$ ppm and $4 \pm 1$ ppm for 5\% Ni-nZVI@MisiCFeOOH-MA and 5\%Ni-nZVI@MisiCFeOOH-MB1, respectively. The 5\%Cu-nZVI@MisiCFeOOH-MA and 5\%Cu-nZVI@MisiCFeOOH-MB1 produced 6.7 \pm 0.7 ppm and $3.9 \pm 0.6$ ppm, respectively. The reason for this difference should be examined in further studies. Interestingly, after 5 minutes, the iron concentrations using both 5\%Cu-nZVI@MisiCFeOOH-MB1 (6.7 \pm 0.7 ppm) and nZVI@MisiCFeOOH $(5.9 \pm 0.4$ ppm) were higher than 5.4 ppm that is expected iron concentration if all $\mathrm{Pb}(\mathrm{II})$ is removed via reduction. This indicates the corrosion of iron during $\mathrm{Pb}(\mathrm{II})$ removal. However, after 30 minutes, the soluble iron levels were relatively similar, ranging from 1.9 to $3.0 \mathrm{ppm}$ for all materials examined. 


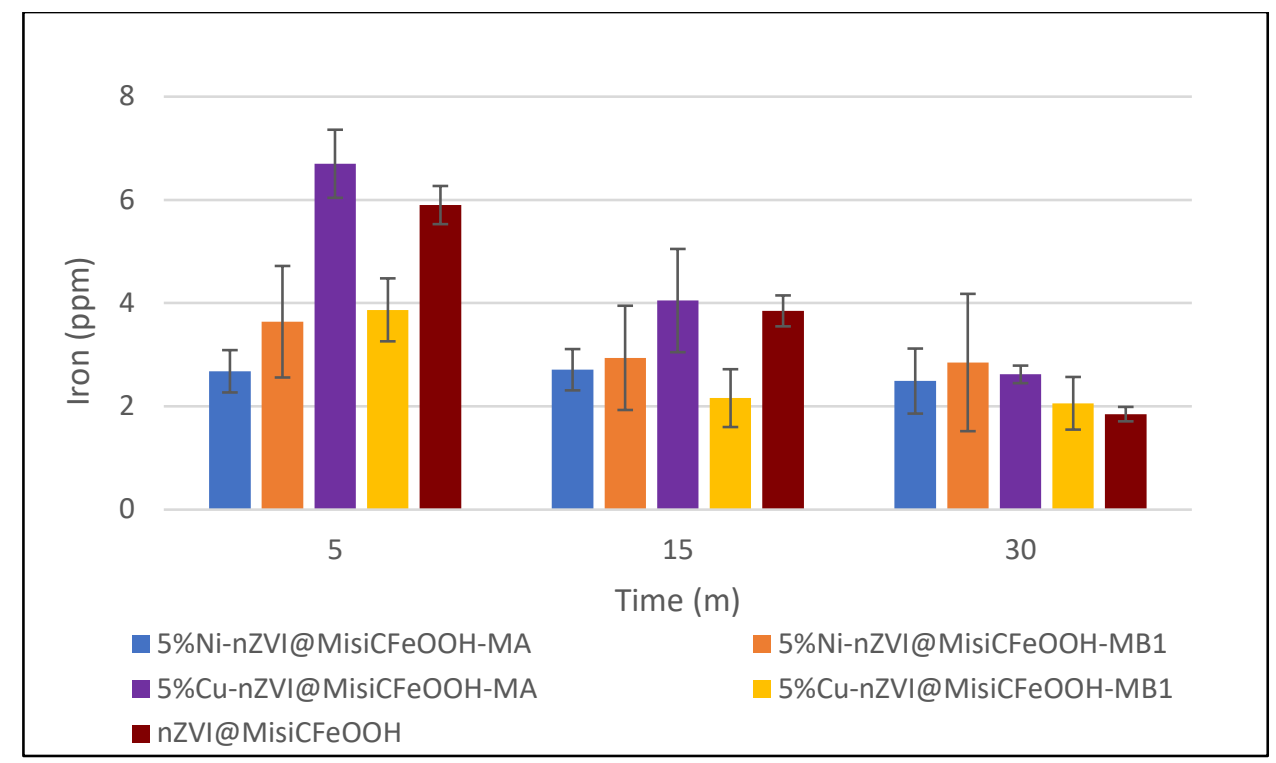

Figure 7.6. Iron concentration in the removal of $20 \mathrm{ppm} \mathrm{Pb(II)} \mathrm{(} 0.10 \mathrm{~g}$ of material, $25.0 \mathrm{~mL}$ of solution).

Nickel was also detected during the reaction between Ni-doped nZVI@MisiCFeOOH and Pb(II) (Table 7.3). After 30 minutes, nickel levels were less than $0.14 \mathrm{ppm}$. In contrast, in the removal of $\mathrm{Pb}$ (II) using Cu-doped nZVI@MisiCFeOOH, the concentrations of copper were below the detection limit of the AAS (0.66 ppm).

Table 7.3. Nickel concentration in the removal of $\mathrm{Pb}(\mathrm{II}) 20 \mathrm{ppm}$.

\begin{tabular}{|c|c|c|c|}
\hline Nickel $(\mathrm{ppm}) /$ time & 5 minutes & 15 minutes & 30 minutes \\
\hline 5\%Ni-nZVI@MisiCFeOOH-MA & $0.23 \pm 0.02$ & $0.17 \pm 0.03$ & $0.14 \pm 0.04$ \\
\hline 5\%Ni-nZVI@MisiCFeOOH-MB1 & $0.29 \pm 0.07$ & $0.3 \pm 0.1$ & $0.13 \pm 0.02$ \\
\hline
\end{tabular}

\subsubsection{Determination of soluble metals in the removal of lead $40 \mathrm{ppm}$}

A significant amount of iron was generated during the removal of $40 \mathrm{ppm} \mathrm{Pb}(\mathrm{II})$. After 30 minutes, the iron concentrations ranged from $5.7 \mathrm{ppm}$ to $14.1 \mathrm{ppm}$ for all types of materials used (Figure 7.7). Despite no difference in the reactivity of these materials in removing 40 $\mathrm{ppm} \mathrm{Pb}(\mathrm{II})$, the generation of iron was significantly different. The amount of iron that nZVI@MisiCFeOOH produced was lower than other materials. Additionally, 5\%NinZVI@MisiCFeOOH-MB1 produced considerably more iron than 5\%Ni-nZVI@MisiCFeOOHMA. 


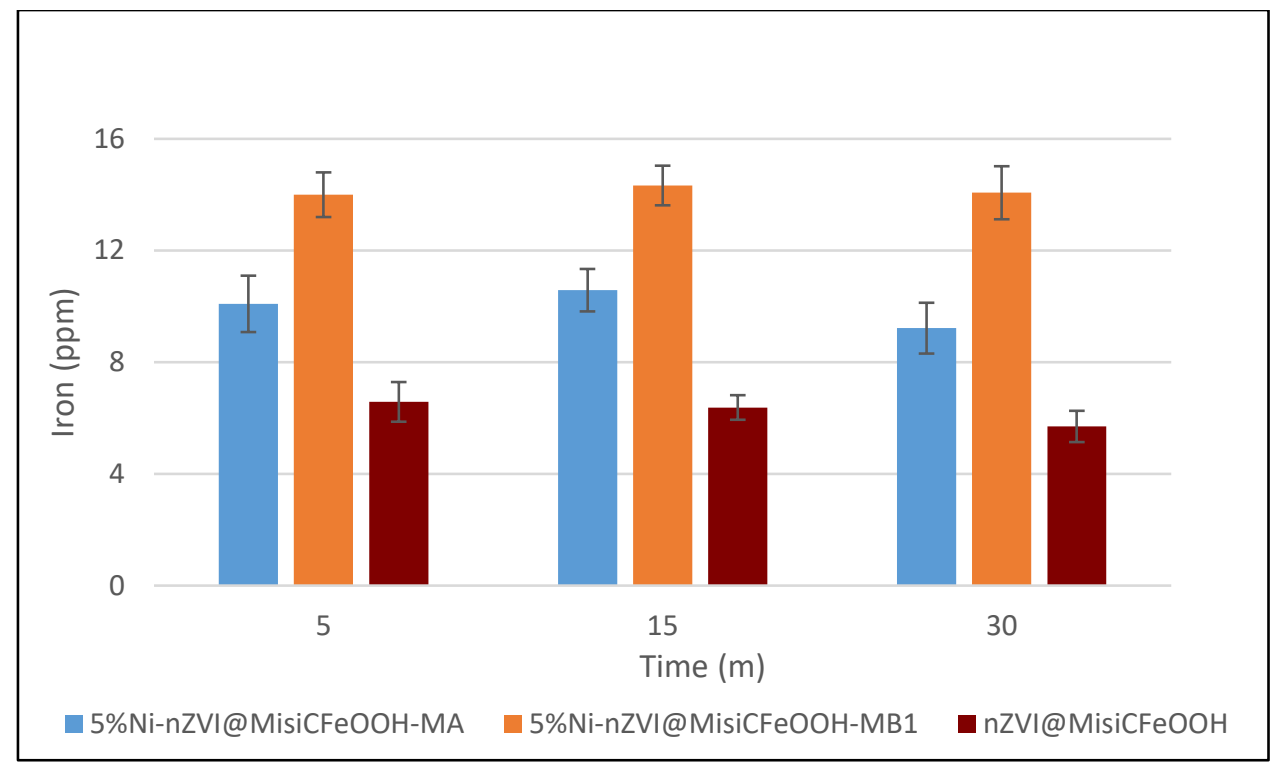

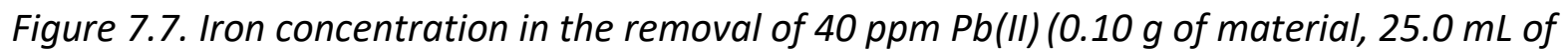
solution).

Although the initial $\mathrm{Pb}(\mathrm{II})$ concentrations doubled, from $20 \mathrm{ppm}$ to $40 \mathrm{ppm}$, the iron levels increased more than three times. This might be due to the difference in $\mathrm{Pb}(\mathrm{II})$ sources. When $\mathrm{Pb}(\mathrm{II})(20 \mathrm{ppm})$ using $\mathrm{Pb}\left(\mathrm{NO}_{3}\right)_{2}$ was used, nitrate- $\mathrm{N}(2.7 \mathrm{ppm})$ was also reduced, leading to an increase in the $\mathrm{pH}$ of the solution. This may enhance the precipitation of iron to form iron hydroxides.

During the removal of 40 ppm Pb(II) using Ni-doped nZVI@MisiCFeOOH, nickel was also detected (Table 7.4). These nickel levels were higher than those being produced in the removal of $20 \mathrm{ppm} \mathrm{Pb}(\mathrm{II})$. After 30 minutes, nickel levels were $0.3 \mathrm{ppm}$. The copper concentrations were still below the detection limit $(0.66 \mathrm{ppm})$ during the reaction between CU-doped nZVI@MisiCFeOOH and Pb(II). This is consistent with the copper results in the removal of $20 \mathrm{ppm} \mathrm{Pb}(\mathrm{II})$.

Table 7.4. Nickel concentration in the removal of $\mathrm{Pb}(\mathrm{II}) 40 \mathrm{ppm}$.

\begin{tabular}{|c|c|c|c|}
\hline Nickel $(\mathrm{ppm}) /$ time & 5 minutes & 15 minutes & 30 minutes \\
\hline 5\%Ni-nZVI@MisiCFeOOH-MA & $0.7 \pm 0.2$ & $0.47 \pm 0.2$ & $0.35 \pm 0.04$ \\
\hline 5\%Ni-nZVI@MisiCFeOOH-MB1 & $1.03 \pm 0.07$ & $0.48 \pm 0.07$ & $0.30 \pm 0.04$ \\
\hline
\end{tabular}


There are two possible reasons for the formation of iron. Firstly, iron can be generated in the oxidation of $\mathrm{nZVI}$ by $\mathrm{Pb}$ (II) because $\mathrm{Pb}$ (II) is removed via reduction. ${ }^{116,154,169}$ Secondly, iron can be produced from the corrosion of nZVI with water (Section 5.4) after nZVI reacts with $\mathrm{Pb}(\mathrm{II})$ completely. This can be evident if the soluble iron concentrations are higher than that estimated when all $\mathrm{Pb}$ (II) was reduced by $\mathrm{nZVI}$, in this case $10.8 \mathrm{ppm}$ of iron for the removal of $40 \mathrm{ppm} \mathrm{Pb}(\mathrm{II})$.

\subsubsection{Elemental distribution of nZVI@MisiCFeOOH after lead removal}

The elemental distribution of nZVI@MisiCFeOOH after removing 20 ppm Pb(II) for 30 minutes was examined via TEM. In the TEM-EDS spectrum, peaks corresponding to $\mathrm{Si}, \mathrm{O}$, and Al were observed, consistent with the presence of Misi (Figure 7.8). A peak corresponding to Fe was strong. The mass percentage and atom percentage of Fe were $83.67 \%$ or $54.89 \%$, respectively. The peak corresponding to $\mathrm{Pb}$ is coincidental with the peak corresponding to $\mathrm{S}$. As $\mathrm{S}$ is present in Misi, we could not determine if this signal is due to either $\mathrm{Pb}$ or $\mathrm{S}$.

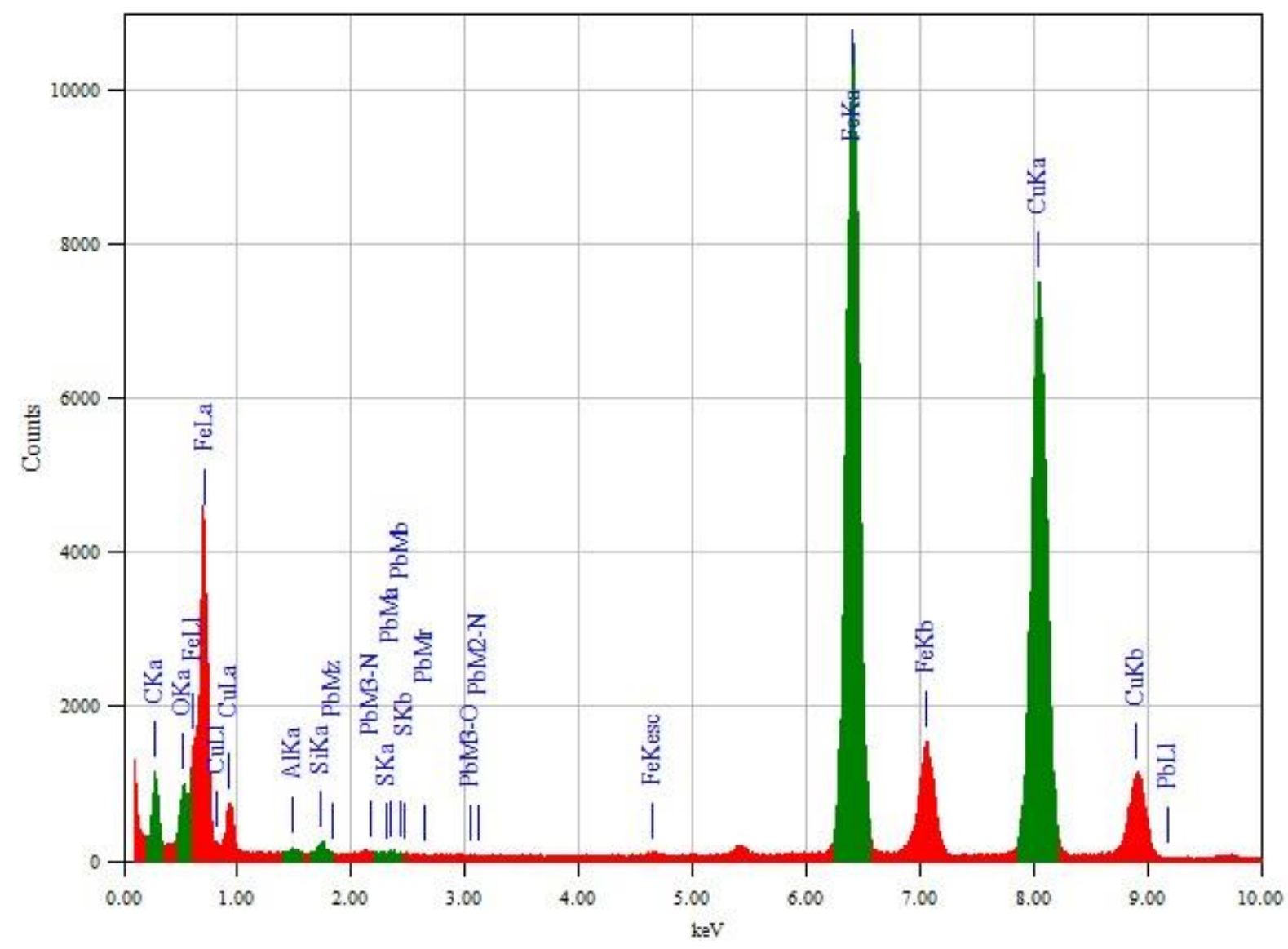

Figure 7.8. TEM-EDS spectrum of nZVI@MisiCFeOOH after Pb(II) removal (0.1 g of material, $25 \mathrm{~mL}$ of $\mathrm{Pb}(\mathrm{II}) 20$ ppm solution). 
The EDS mapping of this material was unable to confirm the fate of lead as the signals of $\mathrm{Si}$, $\mathrm{O}, \mathrm{Al}$, attributed to Misi, overlaid those of $\mathrm{Fe}, \mathrm{S}$ and $\mathrm{Pb}$ (Figure 7.9). Thus, although the STEM image appeared to be a core-shell structure, it is unable to comment further on this material.
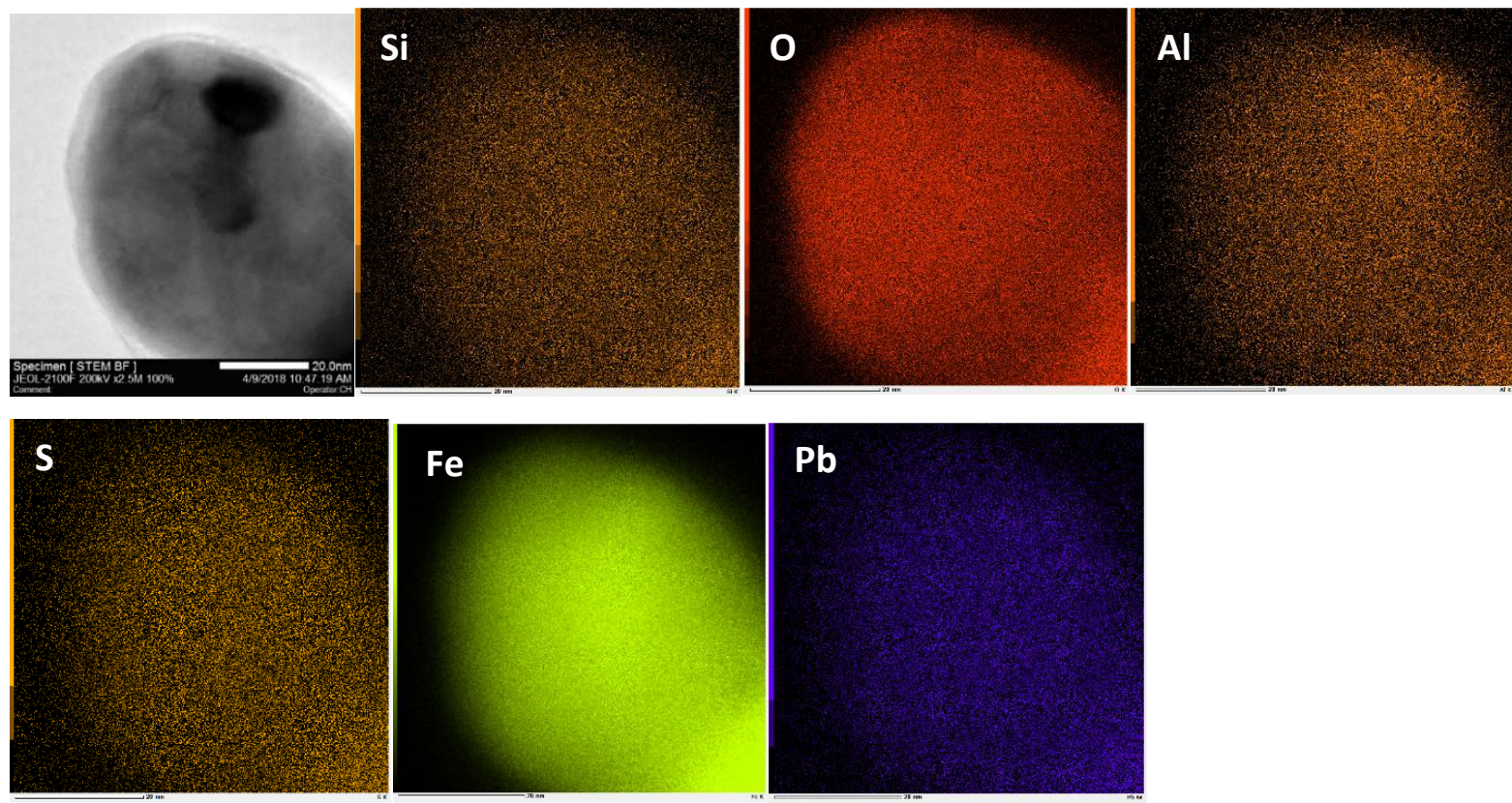

Figure 7.9. TEM-EDS mapping of nZVI@MisiCFeOOH after the removal of $\mathrm{Pb}(\mathrm{II})(0.10 \mathrm{~g}$ material, $25.0 \mathrm{~mL}$ of 20 ppm $\mathrm{Pb}$ (II) solution).

\subsubsection{Conclusion}

In summary, the role of dopant metals could not be evaluated in the $\mathrm{Pb}$ (II) removal due to the rapid reaction of both supported doped and non-doped nZVI@MisiCFeOOH. During the reaction, iron and nickel were detected in solutions whereas copper concentrations were below the detection limit of the AAS. There was no clear evidence for the presence of $\mathrm{Pb}$ on the surface of $\mathrm{nZVI}$ particles due to the weak signal and the overlap of $\mathrm{Pb}$ and $\mathrm{S}$.

\subsection{Chromium removal by doped and non-doped nZVI supported on Misi}

Chromium (VI) is a potential carcinogen and highly toxic to humans, animals and plants. ${ }^{65-66}$ The WHO has set the maximum allowable concentration for chromium total at $0.05 \mathrm{ppm}$ in drinking water. ${ }^{19}$ Due to its toxicity, the removal of chromium has received a lot of attention. Typically, $\mathrm{Cr}(\mathrm{VI})$ is reduced into $\mathrm{Cr}(\mathrm{III})$ that precipitates with other ions. Several studies have reported the use of $n Z V I$ to remove $\mathrm{Cr}(\mathrm{VI}) .{ }^{117,154,288}$ Supported $\mathrm{nZVI}$ have been also used to remove Cr(VI). For example, nZVI@PolyFlo resin, ${ }^{117}$ nZVI@graphene, ${ }^{289}$ nZVI@SS, 198 nZVI@sepiolite, ${ }^{284}$ and nZVI@multiwalled carbon nanotubes have all been examined for their 
ability to remove $\mathrm{Cr}(\mathrm{VI}){ }^{290}$ In addition, supported bimetallic systems were also used to remove $\mathrm{Cr}(\mathrm{VI})$. For instance, Ag-nZVI@activated carbon was used to remove $\mathrm{Cr}(\mathrm{VI})$; however, the effect of $\mathrm{Ag}$ was not examined. ${ }^{204}$ In other studies, both Ni-nZVI@SS198 and NinZVI@montmorillonite ${ }^{291}$ removed Cr(VI) faster and more effectively than nZVI@SS and nZVI@montmorillonite, respectively. In this current research, the reactivity of doped and non-doped materials towards $\mathrm{Cr}(\mathrm{VI})$ was examined. Five representative materials, $5 \% \mathrm{Ni}$ nZVI@MisiCFeOOH-MA, 5\%Ni-nZVI@MisiCFeOOH-MB1, 5\%Cu-nZVI@MisiCFeOOH-MA, 5\%Cu-nZVI@MisiCFeOOH-MB1, and nZVI@MisiCFeOOH were used. The molar ratio of Fe to $\mathrm{Cr}(\mathrm{VI})$ was 19:1 (20 ppm $\mathrm{Cr}(\mathrm{VI}))$. The reactions were carried out under anaerobic conditions. During the reaction, the concentrations of total chromium, soluble by-products such as iron, nickel, and copper, were measured.

\subsubsection{Reactivity of materials towards chromium removal}

Both doped and non-doped materials removed $\mathrm{Cr}(\mathrm{VI})$, the degree of which was dependent upon dopant metals (Figure 7.10). Ni-doped and Cu-doped materials removed $\mathrm{Cr}(\mathrm{VI})$ faster than non-doped nZVI@MisiCFeOOH, confirming the role of dopant metals. For example, after 30 minutes, more than $40 \%$ of initial $\mathrm{Cr}(\mathrm{VI})$ was removed while $30 \%$ of $\mathrm{Cr}(\mathrm{VI})$ was removed using non-doped nZVI@MisiCFeOOH. Moreover, both types of Ni-doped materials had higher reactivity than both types of $\mathrm{Cu}$-doped materials. After 30 minutes, $\mathrm{Cr}(\mathrm{VI})$ removal was greater than $60 \%$ using both types of $\mathrm{Ni}$-doped materials while less than $45 \%$ was observed using both types of Cu-doped materials. The enhancement in the rate of $\mathrm{Cr}(\mathrm{VI})$ removal using both $\mathrm{Ni}$ doped and $\mathrm{Cu}$-doped could be potentially due to the catalytic role of $\mathrm{Ni}$ and $\mathrm{Cu}$. These dopants could promote the electron transfer from $\mathrm{nZVI}$, leading to a faster removal of $\mathrm{Cr}(\mathrm{VI})$ compared to non-doped nZVI@Misi. Additionally, Ni-doped materials had higher reactivity than Cudoped systems, potentially due to the more negative reduction potential of nickel compared to copper. 


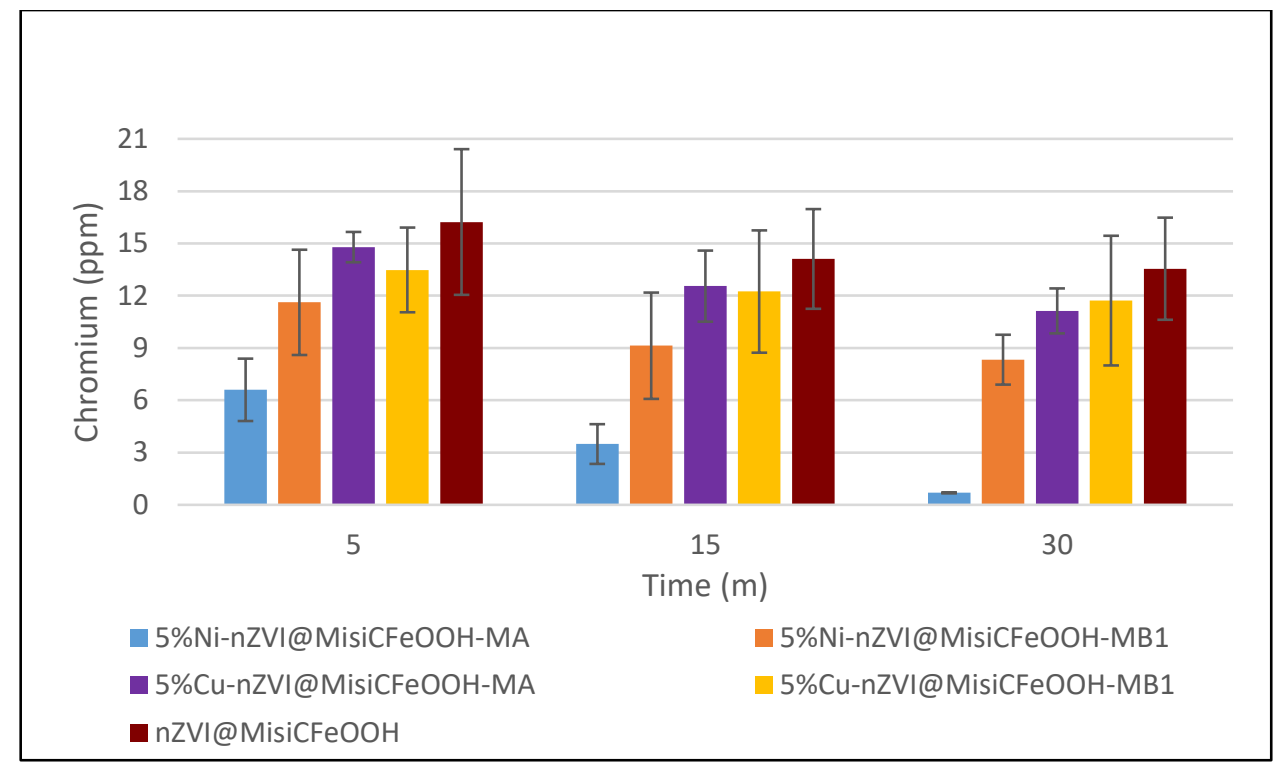

Figure 7.10. Removal characterisation towards $\mathrm{Cr}(\mathrm{VI})(0.10 \mathrm{~g}$ of material, $25.0 \mathrm{~mL}$ of $20 \mathrm{ppm}$ solution).

$\mathrm{Ni}$-doped material prepared via co-reduction method $(\mathrm{MA})$ removed $\mathrm{Cr}(\mathrm{VI})$ significantly faster than that prepared via deposition method (MB1). For example, after 30 minutes, the concentrations of chromium were $0.69 \pm 0.03(97 \% \mathrm{Cr}(\mathrm{VI})$ removal) and $8 \pm 1(60 \% \mathrm{Cr}(\mathrm{VI})$ removal) when either 5\%Ni-nZVI@MisiCFeOOH-MA or 5\%Ni-nZVI@MisiCFeOOH-MB1 was used, respectively. In contrast, there was no difference in the reactivity of Cu-doped materials. For example, after 30 minutes, the concentration of chromium was $11 \pm 1$ (45\% $\mathrm{Cr}(\mathrm{VI})$ removal) and $12 \pm 4$ (40\% Cr(VI) removal) when either 5\%Cu-nZVI@MisiCFeOOH-MA or 5\%Cu-nZVI@MisiCFeOOH-MB1 was used, respectively. The reason for the difference in the reactivity of these materials were uncertain and will need further investigation.

The enhancement in the reactivity of doped materials was consistent with Lu et al.'s study in which Ni-nZVI@montmorillonite was used to remove $\mathrm{Cr}(\mathrm{VI}) .{ }^{291}$ In this study, the initial $\mathrm{Cr}(\mathrm{VI})$ was $50 \mathrm{ppm}$, the material dosage was $0.8 \mathrm{~g} / \mathrm{L}$ (unpublished percentage of $\mathrm{Fe}$ ) and $\mathrm{pH}$ was 3 . The presence of $\mathrm{Ni}$ significantly increased the amount of $\mathrm{Cr}(\mathrm{VI})$ removed. After 60 minutes, all Cr(VI) was removed using Ni-nZVI@montmorillonite whereas 70\% $\mathrm{Cr}(\mathrm{VI})$ was removed using nZVI@montmorillonite. Our results are also consistent with a study in which Ni-nZVI@SS was used to remove $\mathrm{Cr}(\mathrm{VI})(0.2 \mathrm{ppm}-1 \mathrm{ppm})$ at pH 2. ${ }^{198}$ After 120 minutes, the amount of $\mathrm{Cr}(\mathrm{VI})$ removed using Ni-nZVI@SS doubled that being removed by nZVI@SS. However, Ni-nZVI 
coated on Misi systems exhibited faster reactivity as they reacted with $\mathrm{Cr}(\mathrm{VI})$ without $\mathrm{pH}$ adjustment and resulted in higher $\mathrm{Cr}(\mathrm{VI})$ removal.

One of the methods for chromium removal reported is adsorption using kaolinite and its modified forms, ${ }^{292-293}$ or bentonite as absorbents. ${ }^{294}$ We investigated if MisiCFeOOH can remove any $\mathrm{Cr}(\mathrm{VI})$. After 30 minutes, only 3.5\% of $\mathrm{Cr}(\mathrm{VI})$ was removed (Table 7.5$)$; as such, the removal of $\mathrm{Cr}(\mathrm{VI})$ in our systems is primarily due to its interactions with nanoparticles.

Table 7.5. Chromium concentration using MisiCFeOOH.

\begin{tabular}{|c|c|c|c|c|}
\hline Chromium $(\mathrm{ppm}) /$ time & 0 minute & 5 minutes & 15 minutes & 30 minutes \\
\hline MisiCFeOOH & 20 & $19.3 \pm 0.2$ & $19.3 \pm 0.3$ & $19.3 \pm 0.4$ \\
\hline
\end{tabular}

\subsubsection{Determination of soluble metals in chromium removal}

The determination of ions during $\mathrm{Cr}(\mathrm{VI})$ removal has been not received much attention. In this current study, the concentrations of soluble by-products were monitored during the reaction. The results showed that iron concentrations were below the detection limit of the AAS $(0.09$ $\mathrm{ppm}$ ) over the course of 30 minutes when all types of doped and non-doped materials were used. The low iron could be due to the formation of insoluble iron compounds. In $\mathrm{Cr}(\mathrm{VI})$ removal, $\mathrm{Cr}(\mathrm{VI})$ is converted into $\mathrm{Cr}(\mathrm{III})$ and then $\mathrm{Cr}(\mathrm{III})$ combines with $\mathrm{Fe}(\mathrm{III})$ to form $\mathrm{Cr}_{\mathrm{x}} \mathrm{Fe}_{1}$ ${ }_{x}(\mathrm{OH})_{3}$ and/or $\mathrm{Cr}_{x} \mathrm{Fe}_{1-x}(\mathrm{OOH})$ (eq. 7.4 and eq. 7.5$)^{171,295}$ or $\mathrm{Cr}_{0.67} \mathrm{Fe}_{0.33}(\mathrm{OH})_{3 .}{ }^{117}$ These insoluble substances could either precipitate out on the surface of the nanoparticles ot Misi, or as their own separated entity; however, in any case, these would be discarded during filtration.

Nickel was formed during the reduction of Ni-doped nZVI@MisiCFeOOH with $\mathrm{Cr}(\mathrm{VI})$. Nickel levels were 0.2 ppm and 0.4 ppm when either 5\%Ni-nZVI@MisiCFeOOH-MA or 5\% NinZVI@MisiCFeOOH-MB1 was used, respectively (Table 7.6). In contrast, the copper concentrations never exceeded the detection limit of the AAS $(0.66 \mathrm{ppm})$ when Cu-doped materials reacted with $\mathrm{Cr}(\mathrm{VI})$. Although there have been studies in which supported Ni-nZVI were used to remove $\mathrm{Cr}(\mathrm{VI})$, the determination of soluble ions, including iron and nickel, was not published. ${ }^{198,} 291$ 
Table 7.6. Nickel concentration in $\mathrm{Cr}(\mathrm{VI})$ removal.

\begin{tabular}{|c|c|c|c|}
\hline Nickel $(\mathrm{ppm}) /$ time & 5 minutes & 15 minutes & 30 minutes \\
\hline 5\%Ni-nZVI@MisiCFeOOH-MA & $0.22 \pm 0.08$ & $0.2 \pm 0.2$ & $0.2 \pm 0.2$ \\
\hline 5\%Ni-nZVI@MisiCFeOOH-MB1 & $0.4 \pm 0.3$ & $0.4 \pm 0.2$ & $0.4 \pm 0.2$ \\
\hline
\end{tabular}

The decrease of chromium concentration and the generation of by-products suggested a mechanism of $\mathrm{Cr}(\mathrm{VI})$ removal in which $\mathrm{Cr}(\mathrm{VI})$ is removed via a combination of reduction and adsorption. ${ }^{117,171} 171,204,289$ The former is caused by reducing $\mathrm{Cr}(\mathrm{VI})$ to form $\mathrm{Cr}$ (III) by nZVI as suggested by the difference in the reduction potential of $\mathrm{Fe}(\mathrm{II}) / \mathrm{Fe}(-0.44 \mathrm{~V})$ and $\mathrm{Cr}(\mathrm{VI}) / \mathrm{Cr}(\mathrm{III})$ $(+1.33 \mathrm{~V})$. The latter is due to the adsorption or co-precipitation of $\mathrm{Cr}(\mathrm{III})$ with other ions such as $\mathrm{OH}^{-}$and $\mathrm{Fe}(\mathrm{III})$ on the surface of materials. Evidence for this mechanism is from the lack of iron detected in solution.

\subsubsection{Conclusion}

In summary, doped and non-doped materials removed $\mathrm{Cr}(\mathrm{VI})$ from aqueous solution. The introduction of dopant metals $(\mathrm{Ni}$ and $\mathrm{Cu}$ ) increased the reactivity of doped nZVI@MisiCFeOOH and the catalytic role of $\mathrm{Ni}$ was more significant than $\mathrm{Cu}$. Among these materials, 5\%Ni-nZVI@MisiCFeOOH-MA was the best performing. In addition, during the reaction, the concentrations of both iron and copper were below the detection limit while nickel was detected but nickel concentrations were less than $0.4 \mathrm{ppm}$. Based on $\mathrm{Cr}(\mathrm{VI})$ removal and iron generation, it can be concluded that $\mathrm{Cr}(\mathrm{VI})$ was removed from the solution via both adsorption and reduction mechanisms.

\subsection{Heavy metal removal from lake water}

The reactivity of our doped and non-doped materials towards heavy metal removal from lake water was examined to determine if coexisting ions could affect the removal or if these materials could deactivate as exposed to the air. Lake water sample collected on 23 April 2018 was used to test $\mathrm{Cd}(\mathrm{II})$ and $\mathrm{Pb}(\mathrm{II})$. As these ions were not detected in the lake water, we spiked the water with 20 ppm of each ion. Three representative materials, 5\%Ni-nZVI@MisiCFeOOHMA, 5\%Cu-nZVI@MisiCFeOOH-MA and nZVI@MisiCFeOOH, were used to investigate their reactivity as well as the generation of soluble by-product during the reaction. All experiments were conducted under both anaerobic conditions and aerobic conditions. 


\subsubsection{Cadmium removal from lake water}

\subsubsection{Cadmium removal from lake water spiked with 20 ppm under anaerobic conditions}

The removal of $\mathrm{Cd}(\mathrm{II})$ from lake water spiked with $20 \mathrm{ppm}$ was faster than from the lab samples. After 30 minutes, more than $90 \%$ of $\mathrm{Cd}(\mathrm{II})$ was removed (Figure 7.11). Specifically, the soluble cadmium levels were $0.15 \pm 0.07$ ppm (99\% Cd(II) removal), $1 \pm 1$ ppm (95\% Cd(II) removal) and $2 \pm 1$ ppm (90\% Cd(II) removal) when 5\%Ni-nZVI@MisiCFeOOH-MA, 5\%CunZVI@MisiCFeOOH-MA and nZVI@MisiCFeOOH were used, respectively. This indicated that the $\mathrm{Cd}(\mathrm{II})$ removal was not inhibited by any coexisting factors in lake water. In contrast, these factors enhanced $\mathrm{Cd}(\mathrm{II})$ removal. This enhancement can be explained by the presence of biomass, for example, algae that can absorb Cd(II). ${ }^{61,296-297}$ This was evident in a controlling experiment. After spiking lake water with $20 \mathrm{ppm} \mathrm{Cd}^{2+}$ and stirring, the concentration of $\mathrm{Cd}(\mathrm{II})$ in the resulting solution was $15.7 \mathrm{ppm}$.

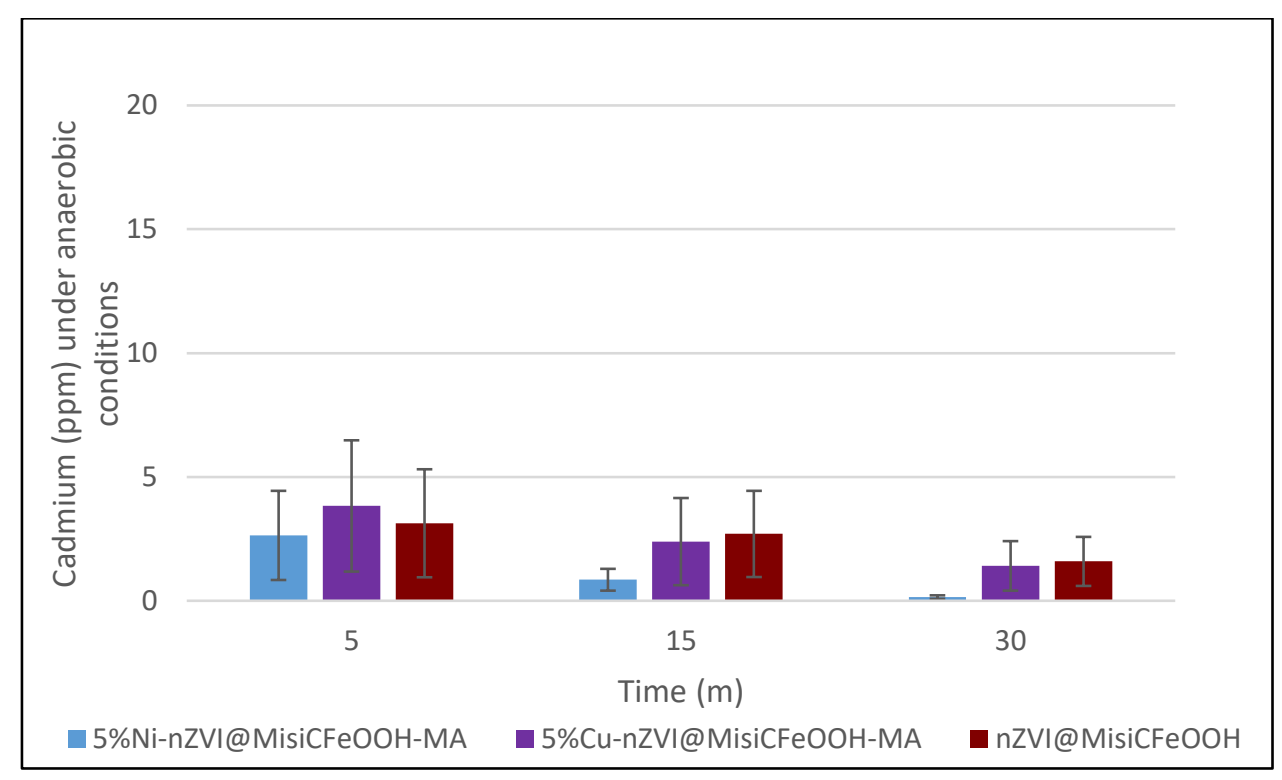

Figure 7.11. Cadmium concentration in the removal of $C d(I I)$ in lake water spiked with 20 ppm under anaerobic conditions ( $0.10 \mathrm{~g}$ of material, $25.0 \mathrm{~mL}$ of sample).

During the $\mathrm{Cd}(\mathrm{II})$ removal from lake water under anaerobic conditions, the production of soluble metals was monitored. After 30 minutes, soluble iron levels were $1.6 \pm 0.7 \mathrm{ppm}, 3 \pm 1$ ppm, and $2.8 \pm 0.5$ ppm when 5\%Ni-nZVI@MisiCFeOOH-MA, 5\%Cu-nZVI@MisiCFeOOH-MA, and nZVI@MisiCFeOOH were used, respectively (Figure 7.12). These iron concentrations were significantly lower than those observed in the study of lab samples. The lower levels of soluble iron may be explained by the absorption of iron ions by organic substances in lake water such 
as algae. ${ }^{275,298}$ The soluble nickel levels at $0.2 \mathrm{ppm}$ and copper levels at below the detection limit were similar to those observed in the study of lab samples.

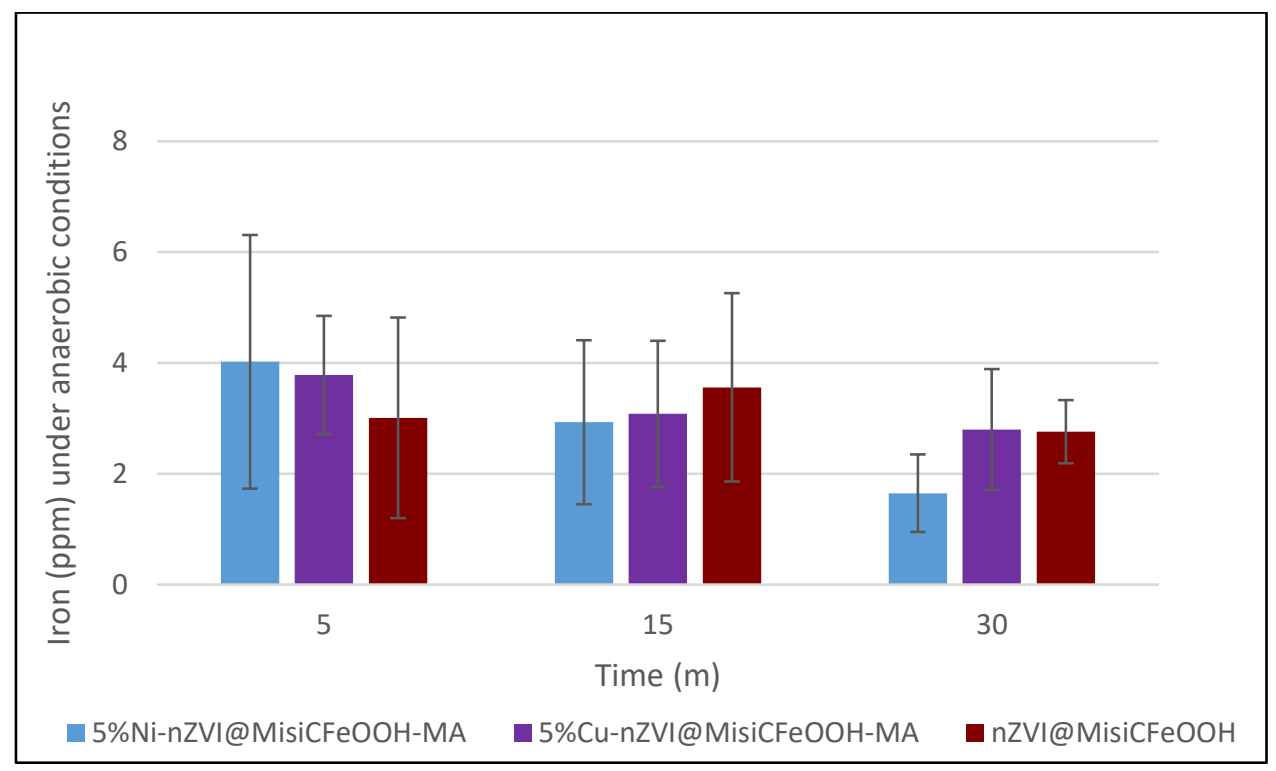

Figure 7.12. Iron concentration in the removal of Cd(II) in lake water spiked 20 ppm Cd(II) under anaerobic conditions ( $0.10 \mathrm{~g}$ of material, $25.0 \mathrm{~mL}$ of the sample).

\subsubsection{Cadmium removal from lake water spiked with 20 ppm under aerobic conditions}

The aging of $\mathrm{nZVI}$ is known to decrease the reactivity of materials. ${ }^{131,255}$ In this study, the removal of $\mathrm{Cd}(\mathrm{II})$ was examined when these materials are exposed to oxidising media. For this, the removal was conducted under aerobic conditions. When Ni-doped or Cu-doped materials was used, the $\mathrm{Cd}(\mathrm{II})$ removal was similar to that observed under anaerobic conditions (Figure 7.13). After 30 minutes, more than 93\% of $\mathrm{Cd}(\mathrm{II})$ was removed. In contrast, non-doped nZVI@MisiCFeOOH was slightly more active under aerobic conditions, which could be due to the change in the surface properties. When non-doped material interacts with the oxidising agents, there could be a change or formation of iron oxides/hydroxides on the surface of $\mathrm{nZVI}$, potentially promoting the adsorption of $\mathrm{Cd}(\mathrm{II})$. For example, in Wang et al.'s study, in fresh nZVI, there was a peak of Fe(0) in XPS analysis; however, when nZVI was aged in atmosphere, the oxidation caused a thicker shell, leading to no peak of $\mathrm{Fe}(0)$ detected by XPS. ${ }^{254}$ 


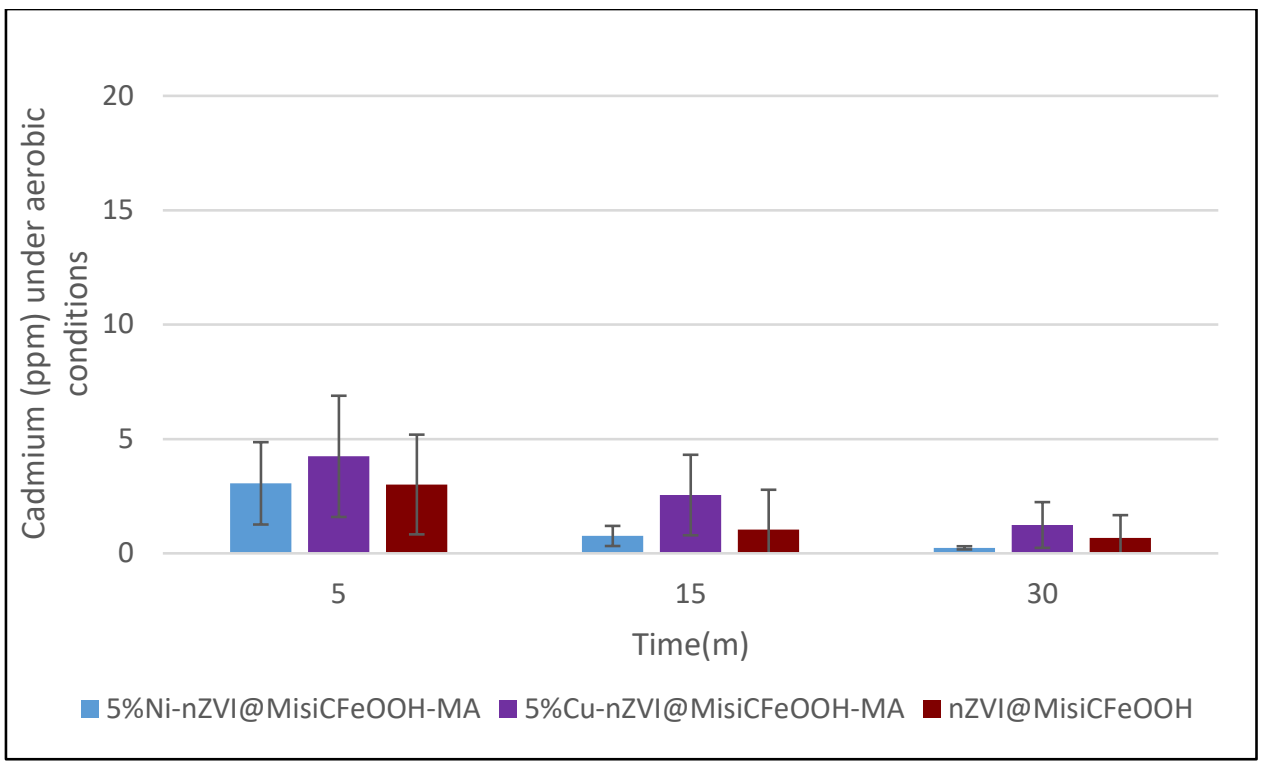

Figure 7.13. Cadmium concentration in the removal of Cd(II) in lake water spiked with 20 ppm under aerobic conditions ( $0.10 \mathrm{~g}$ of material, $25.0 \mathrm{~mL}$ of sample).

When both $\mathrm{Ni}$-doped and $\mathrm{Cu}$-doped materials were used, more iron was produced under aerobic conditions than under anaerobic conditions over the course of 30 minutes; however, these differences were minimal (Figure 7.14). When non-doped nZVI@MisiCFeOOH was used, iron levels were higher under aerobic conditions than that observed under anaerobic conditions within 15 minutes. The opposite trend was observed after 30 minutes. Nickel levels were also measured, ranging from $0.2 \mathrm{ppm}$ to $0.4 \mathrm{ppm}$ during the reaction of $5 \% \mathrm{Ni}$ nZVI@MisiCFeOOH-MA with Cd(II) while no copper was detected during the reaction of 5\%Cu-nZVI@MisiCFeOOH-MA with Cd(II). 


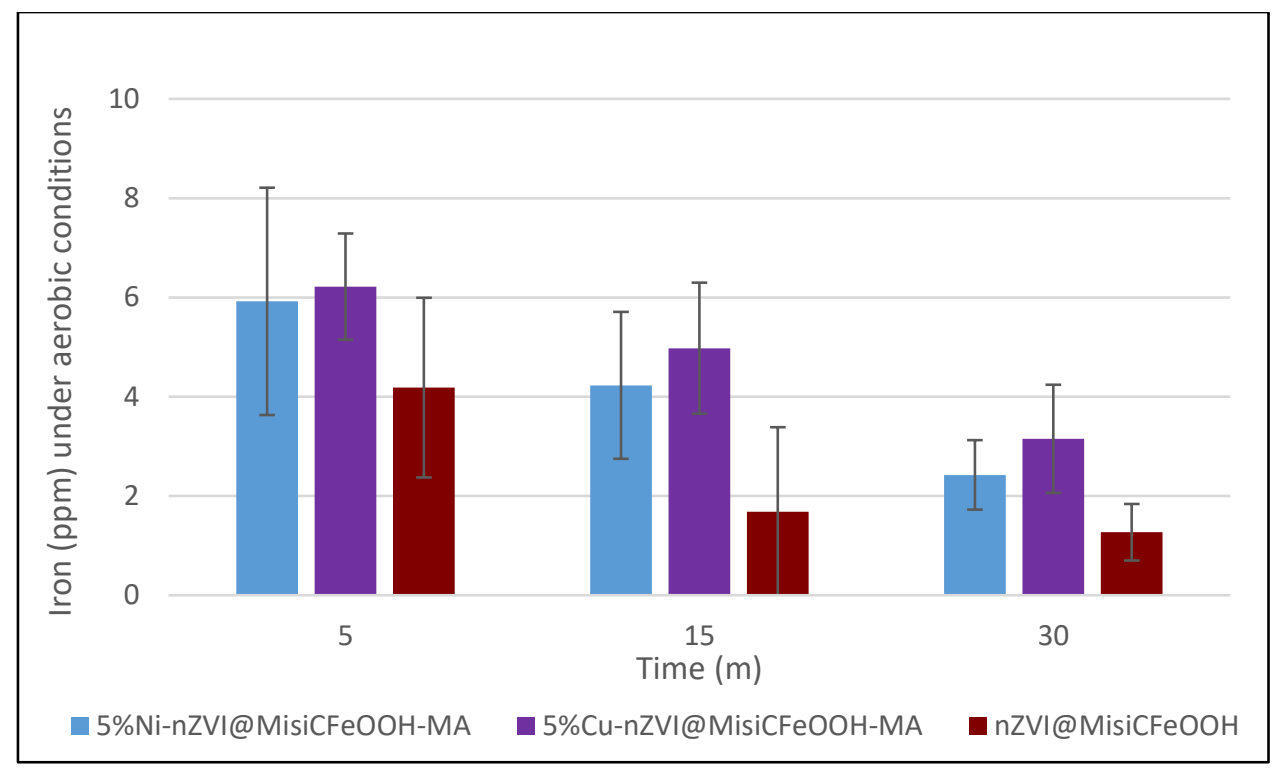

Figure 7.14. Iron concentration in the removal of $C d(I I)$ in lake water spiked 20 ppm under aerobic conditions ( $0.10 \mathrm{~g}$ of material, $25.0 \mathrm{~mL}$ of sample).

In summary, these representative doped and non-doped materials effectively removed Cd(II) from lake water spiked under both anaerobic and aerobic conditions. The $\mathrm{Cd}(\mathrm{II})$ removal from lake water was more effective than from lab samples, although the amount of $\mathrm{Cd}(\mathrm{II})$ absorbed by biomass was significant (22\%). The presence of oxygen did not affect $\mathrm{Cd}(\mathrm{II})$ removal; in contrast, enhanced the reactivity of non-doped material. Therefore, these materials have potential to be used in removing Cd(II) from "real" waters.

\subsubsection{Lead removal from lake water}

To determine if naturally occurring factors could affect, the removal of $\mathrm{Pb}(\mathrm{II})$ from lake water spiked with 20 ppm was examined under both anaerobic and aerobic conditions. After 5 minutes, lead concentrations were below $0.3 \mathrm{ppm}$ and after 15 minutes, lead levels were below the detection limit of the AAS $(0.05 \mathrm{ppm})$. This indicated that the effect of coexisting ions in lake water was not observed in the removal of $20 \mathrm{ppm} \mathrm{Pb}(\mathrm{II})$.

During the reactions under both anaerobic and aerobic conditions, the concentrations of iron and dopant metals were examined. The iron concentrations under aerobic conditions were relatively similar to those observed under anaerobic conditions (Figure 7.15 and 7.16). Additionally, these iron concentrations were similar to those observed in the lab samples. Nickel concentrations were less than $0.2 \mathrm{ppm}$ under anaerobic conditions, which is consistent with those in the lab samples while nickel concentrations were around $0.4 \mathrm{ppm}$, slightly higher 
under aerobic conditions. In summary, $\mathrm{Pb}$ (II) removal and iron generation were not affected by the coexisting ions in lake water under both anaerobic and aerobic conditions. Therefore, these materials have potential to remove $\mathrm{Pb}(\mathrm{II})$ from "real" waters.

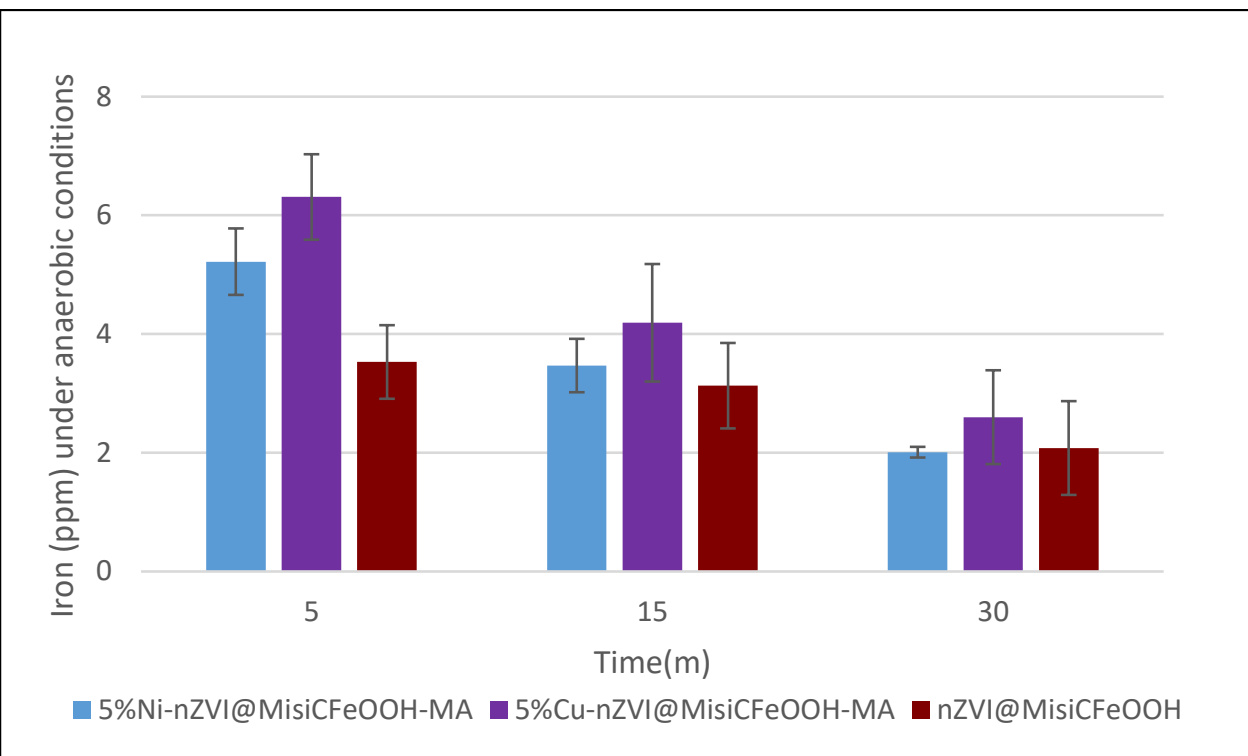

Figure 7.15. Iron concentration in the removal of $\mathrm{Pb}(\mathrm{II})$ in lake water spiked with $20 \mathrm{ppm}$ under anaerobic conditions ( $0.10 \mathrm{~g}$ of material, $25.0 \mathrm{~mL}$ of sample).

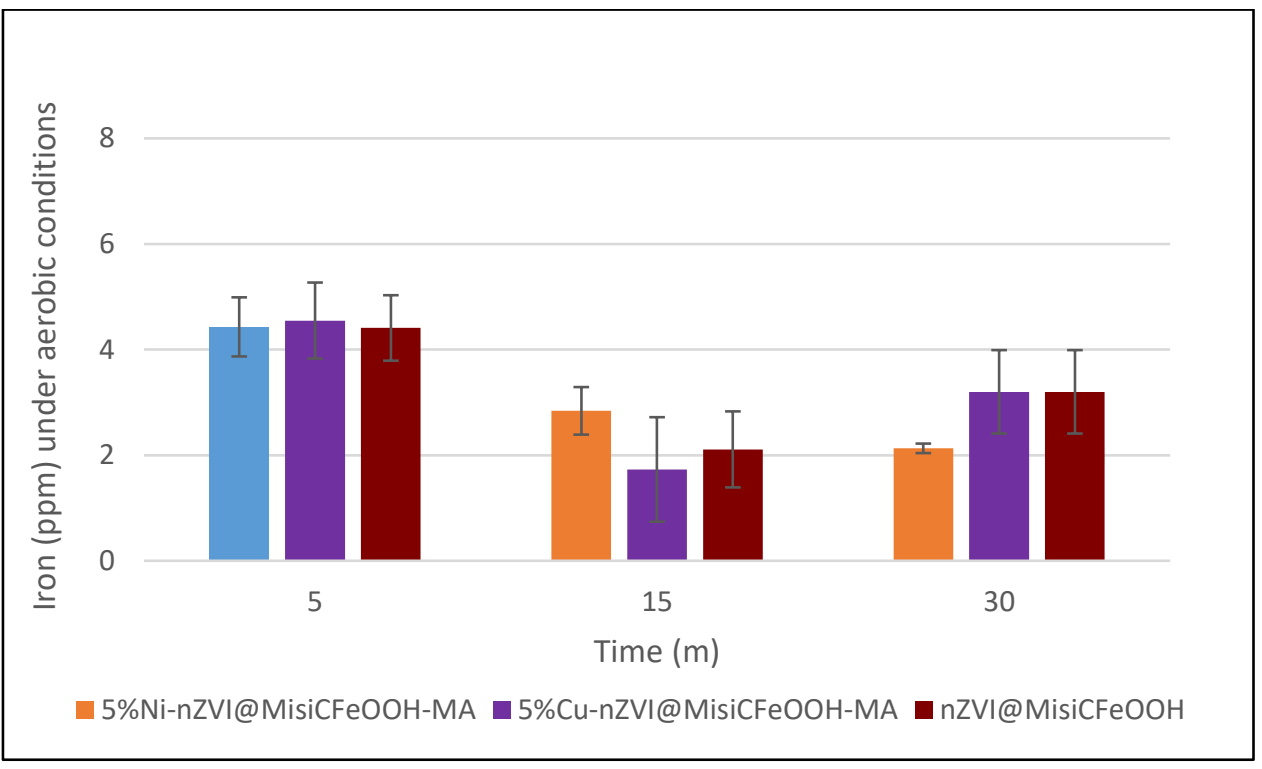

Figure 7.16. Iron concentration in the removal of $\mathrm{Pb}(\mathrm{II})$ in lake water spiked with $20 \mathrm{ppm}$ under aerobic conditions ( $0.10 \mathrm{~g}$ of material, $25.0 \mathrm{~mL}$ of sample). 


\subsection{Conclusion}

Our materials, including doped and non-doped nZVI@MisiCFeOOH, have the ability to remove toxic heavy metals. The dopant metals enhanced the removal of $\mathrm{Cd}(\mathrm{II})$ and $\mathrm{Cr}(\mathrm{VI})$ whereas the effect of these dopants on $\mathrm{Pb}(\mathrm{II})$ removal needs further examination, potentially using higher $\mathrm{Pb}(\mathrm{II})$ concentrations. Iron was found during removal of both $\mathrm{Cd}(\mathrm{II})$ and $\mathrm{Pb}(\mathrm{II})$ while iron concentrations were below the detection limit during the removal of $\operatorname{Cr}(\mathrm{VI})$. In addition, these materials were not influenced by coexisting ions in natural water. Therefore, using these systems to remove heavy metals from "real" waters is promising. 


\section{Summary and future work}

\subsection{Summary}

In this thesis, the reactivity of supported bimetallic and trimetallic nZVI@Misi towards nitrate and heavy metal removal had been studied.

Numerous dopant metals, $\mathrm{Ni}, \mathrm{Cu}, \mathrm{Zn}$, and $\mathrm{Pd}$, were used to synthesise supported bimetallic nZVI@Misi and Pd and Cu were used to generate supported trimetallic Pd-Cu-nZVI@Misi. These materials were prepared using different preparations of Misi and synthetic methods. All these materials had higher reactivity than non-doped nZVI@Misi, indicating that the dopant metals have catalytic role in terms of reactivity enhancement. Of these, $\mathrm{Ni}, \mathrm{Cu}$, and Pd-Cu were the most promising dopants. In addition, Misi that was calcined and coated with MisiCFeOOH was the most efficient support in improving the reactivity of bimetallic and trimetallic nanoparticles. Moreover, the two-step synthesis in which the dopant metal was deposited on the top of pre-formed nZVI@Misi (deposition method) was significantly more effective than co-reduction method in which the dopant and iron ions were simultaneously reduced to produce M-nZVI@Misi. The materials produced using the deposition method were more reactive regards to nitrate reduction.

The bimetallic nanoparticles Ni-nZVI@MisiCFeOOH or Cu-nZVI@MisiCFeOOH prepared via deposition method had a core-shell structure in which $\mathrm{Fe}(0)$ serves as a core and a combination of either $\mathrm{Ni}(0)$ and iron-nickel oxides or $\mathrm{Cu}(0)$ and copper oxides as a shell. Due to the elemental distribution of the main reducing agent $(\mathrm{Fe})$ and catalyst $(\mathrm{Ni}, \mathrm{Cu})$, the reducing capacity of supported bimetallic materials increased significantly. In contrast, in trimetallic Pd-Cu-nZVI@MisiCFeOOH prepared via deposition method, the Cu signal was slightly greater on the surface of Fe and Pd signal was not reliable. Due to this elemental distribution and the tiny amount of Pd in materials, the effectiveness of Pd addition was minimal. However, Pd slightly accelerated nitrite conversion, leading to less nitrite accumulation compared to bimetallic Cu-nZVI@Misi.

The reactivity of supported bimetallic and trimetallic nZVI@MisiCFeOOH was not significantly affected when these materials were exposed to oxygen. Even though the amount of iron used was less than that needed to remove all nitrate in the solutions, the nitrate removal was relatively similar under anaerobic and aerobic conditions. Additionally, chloride and sulfate 
did not influence their reactivity. Although carbonate and phosphate inhibited these materials, the interference of these ions only occurs at high concentrations. Interestingly, in the presence of inhibitory coexisting ions, the reactivity of these materials was higher under aerobic conditions than under anaerobic conditions. Moreover, during the reduction, the concentrations of soluble iron, nickel, and copper were low, which is beneficial for the application in terms of environmental remediation. As such, these new materials show promising in treating nitrate.

Supported bimetallic and trimetallic nZVI@MisiCFeOOH effectively reduced nitrate from natural water collected in Lake Horowhenua. The reactivity was diminished compared to lab samples. Encouragingly, their reactivity under aerobic conditions was higher than under anaerobic conditions. After nitrate removal, the concentrations of soluble iron were minimal, and were around the maximum allowable level for drinking water. It should be noted that the non-doped nZVI@Misi was unable to reduce a significant amount of nitrate from lake water. Supported bimetallic materials had the capacity of removing toxic heavy metals such as cadmium, lead, and chromium (VI) both from lab-prepared and lake water. Their reactivity was not affected by coexisting factors in lake water. During the removal of cadmium and lead, soluble iron was detected. In contrast, the concentrations of soluble iron were below the detection limit in the removal of chromium (VI).

In summary, this research synthesised new materials that easily dispersed in water and efficiently removed contaminants. Of the supported bimetallic and trimetallic nZVI@MisiCFeOOH, the one prepared by doping 5\%Ni-nZVI on Misi that was calcined and then coated with FeOOH (5\%Ni-nZVI@MisiCFeOOH) was the best towards nitrate reduction and heavy metal removal. In particular, the 5\%Ni-nZVI@MisiCFeOOH prepared using deposition method is evaluated as the most effective material in reducing nitrate. Even though it relatively reduced nitrate more slowly than both $\mathrm{Cu}$-doped and $\mathrm{Pd}$-Cu-doped, the generation of by-product (nitrite) was observed with the lowest levels. The new findings of the elemental distribution of $\mathrm{Ni}$ dopant and iron are useful in explaining the catalytic effectiveness of the dopant as well as the interaction between these two metals. The $5 \% \mathrm{Ni}$ nZVI@MisiCFeOOH prepared using co-reduction method is an outstanding material in removing heavy metals. For instance, the 5\%Ni-nZVI@MisiCFeOOH-MA reduced both Cd(II) and $\mathrm{Cr}(\mathrm{VI})$ faster than other materials. Unfortunately, due to the time constraints, we were 
unable to determine why this material showed such significantly higher activity. As such, 5\%Ni-nZVI@MisiCFeOOH will be a promising material that should be scaled for the remediation contaminants from the "real" environment.

\subsection{Future work}

As a result of this work presented, there are some directions for future work.

Bimetallic nZVI@Misi: Firstly, the capacity of these materials should be examined towards other heavy metals (mercury, arsenic) and organic substances. In addition, the reactivity of these materials in simultaneously removing contaminants should be investigated. After that, we can apply these materials in natural waters containing numerous pollutants. Secondly, these materials should be scaled with attention about optimising the ratio of $\mathrm{Fe}$ to borohydride to remain their reactivity but minimise the cost. Thirdly, other supports should be trialled to adsorb bimetallic nanoparticles to evaluate their supporting effectiveness.

Trimetallic Pd-Cu-nZVI@Misi: Previous studies reported the introduction of Pd increased nitrogen selectivity; however, this was not observed in our materials, potentially, due to the low concentration of Pd. Therefore, an increase in Pd concentration and an alternation in the ratio of $\mathrm{Cu}$ to $\mathrm{Pd}$ should be examined in order to determine the usefulness of these dopants. Doped materials prepared via deposition method (MB1) reduced nitrate faster than those prepared via co-reduction method (MA). However, the contrast was observed in the removal of cadmium and chromium. Therefore, the effect of synthetic method on the reactivity of materials towards heavy metals need further examination.

The non-doped nZVI@Misi was shut down whereas the doped systems remained active in removing nitrate from natural water. The factors might be due to humics and sediments which might form complexes with iron oxides on surface of nZVI. In contrast, bimetallic nanoparticles might be protected by a dopant layer, maintaining the number of electrons transferring from the $\mathrm{Fe}(0)$ core. This should be a further study area.

In contrast to the reactivity observed with nitrate, the non-doped nZVI@Misi was still active when it was used to remove cadmium and lead in lake water. This could be due to the removal mechanism of these metals by nZVI. One of the pathways of the removal of these ions is adsorption that depends on the surface property. Therefore, the removal of chromium and other metals that have significantly higher reduction potential than iron should be further examined. 


\section{References}

1. Fraser, H. P. Methods to improve nZVI adsorbance on silicates surface for nitrate reduction. Victoria University of Wellington, Library of Victoria University of Wellington, 2018.

2. Kang, H.; Xiu, Z.; Chen, J.; Cao, W.; Guo, Y.; Li, T.; Jin, Z., Environ Technol 2012, 33 (16-18), 2185-92.

3. Wang, Q.; Jeong, S. W.; Choi, H., Hazard Mater 2012, 213-214, 299-310.

4. Lubphoo, Y.; Chyan, J.-M.; Grisdanurak, N.; Liao, C.-H., Taiwan Institute of Chemical Engineers 2015, 57, 143-153.

5. Lubphoo, Y.; Chyan, J. M.; Grisdanurak, N.; Liao, C. H., Taiwan Institute of Chemical Engineers 2016, 59, 285-294.

6. Schrick, B.; Blough, J. L.; Jones, A. D.; Mallouk, T. E., Chemistry of Materials 2002, 14 (12), 51405147.

7. Nolan, B. T.; Ruddy, B. C.; Hitt, K. J.; Helsel, D. R., Environ. Sci. Technol. 1997, 31, 2229-2236.

8. Shrimali, M.; Singh, K. P., Environmental Pollution 2001, 112, 351- 359.

9. Samatya, S.; Kabay, N.; Yüksel, Ü.; Arda, M.; Yüksel, M., Reactive and Functional Polymers 2006, 66 (11), 1206-1214.

10. Huang, C.-P.; Wang, H.-W.; Chiu, P.-C., Water Res 1998, 32, 2257-2264.

11. Pan, J. R.; Huang, C.; Hsieh, W.-P.; Wu, B.-J., Separation and Purification Technology 2012, 84, 52-55.

12. Lohumi, N.; Gosain, S.; Jain, A.; Gupta, V. K.; Verma, K. K., Analytica Chimica Acta 2004, 505 (2), 231-237.

13. Holbrook, S. Farming communities face crisis over nitrate pollution, study says. https://thefern.org/2012/03/farming-communities-facing-crisis-over-nitrate-pollution-study-says/

(accessed May 2, 2019).

14. J Lin, C.; Lo, S. L., Water Res 2005, 39 (6), 1037-46.

15. Esmail, M. Y.; Astrofsky, K. M.; Lawrence, C.; Serluca, F. C., Chapter 20 - The Biology and Management of the Zebrafish. In Laboratory Animal Medicine (Third Edition), Fox, J. G.; Anderson, L. C.; Otto, G. M.; Pritchett-Corning, K. R.; Whary, M. T., Eds. Academic Press: Boston, 2015; pp 10151062.

16. Drozd, V. M.; Branovan, I.; Shiglik, N.; Lushchyk, M. L.; Platonova, T. Y.; Pashkevich, V. I.; Kudelsky, A. V.; Shimanskaya, I.; Danilova, L. I.; Biko, J.; Reiners, C., Cytology and Genetics 2016, 50 (6), 372-376.

17. Tajtakova, M.; Semanova, Z.; Tomkova, Z.; Szokeova, E.; Majoros, J.; Radikova, Z.; Sebokova, E.; Klimes, I.; Langer, P., Chemosphere 2006, 62 (4), 559-64.

18. Ward, M. H., Reviews on Environmental Health 2009, 24 (4), 357-363.

19. WHO A global overview of national regulations and standards for drinking-water quality. https://apps.who.int/iris/bitstream/handle/10665/272345/9789241513760-eng.pdf?ua=1 (accessed April 11, 2019).

20. Khan, F. A.; Ansari, A. A., The Botanical Review 2005, 71 (4), 449-482.

21. Khan, M. N.; Mohammad, F., Eutrophication: Challenges and Solutions. In Eutrophication: Causes, Consequences and Control, Ansari, A. A.; Gill, S. S., Eds. Springer, Dordrecht: 2014; Vol. 2, pp 1-15.

22. Dorgham, M. M., Effects of Eutrophication. In Eutrophication: Causes, Consequences and Control, Ansari, A. A.; Gill, S. S., Eds. Springer, Dordrecht: 2014; Vol. 2, pp 29-44.

23. Anderson, D. M.; Glibert, P. M.; M., B. J., Estuaries 2002, 25, 704-726.

24. European Commission: Nitrate pollution falling, but greater efforts still needed to meet water quality standards across the EU; European Commision, 2010.

25. Tirado, R. Nitrates in drinking water in the Philippines and Thailand; Greenpeace Research Laboratories, 2007. 
26. Trends in nitrogen leaching from agriculture; Statistics New Zealand, 2017.

27. Nitrate leaching from livestock; Statistics New Zealand, 2019.

28. $\quad$ Our fresh water; Statistics New Zealand, 2017; p 55.

29. Baea, B.-U.; Junga, Y.-H.; Hana, W.-W.; Shin, H.-S., Water Research 2002, 36, 3330-3340.

30. Boumediene, M.; Achour, D., Desalination 2004, 168, 187-194.

31. Clifford, D.; Lin, C.-C.; Horng, L.-L.; Boegel, J. Nitrate removal from drinking water in Glendale, Arizona; United States Environmental Protection Agency: 1987.

32. Schoeman, J. J.; Steyn, A., Desalination 2003, 155, 15-26.

33. Archna; Sharma, S. K.; Sobti, R. C., E-Journal of Chemistry 2012, 9 (4), 1667-1675.

34. Mizuta, K.; Matsumoto, T.; Hatate, Y.; Nishihara, K.; Nakanishi, T., Bioresour Technol 2004, 95 (3), 255-7.

35. Mohsenipour, M.; Shahid, S.; Ebrahimi, K., Asian Journal of Chemistry 2014, 26 (23), 78817886.

36. Warneke, S.; Schipper, L. A.; Bruesewitz, D. A.; McDonald, I.; Cameron, S., Ecological Engineering 2011, 37 (3), 511-522.

37. Aslan, S.; Türkman, A., Water Science and Technology 2003, 48 (11-12), 489-495.

38. Hashemi, S. E.; Heidarpour, M.; Mostafazadeh-Fard, B., Water Science and Technology 2011, 63 (11), 2700-2706.

39. Volokita, M.; Belkin, S.; Abeliovich, A.; Soares, M. I. M., Water Research 1996, 30 (4), 965-971.

40. Nurizzo, C.; Mezzanotte, V., Wal. Sci. Tech. Vol. 26 1992, 26 (3-4), 827-834.

41. Hwang, Y. H.; Kim, D. G.; Shin, H. S., Hazard Mater 2011, 185 (2-3), 1513-1521.

42. Alowitz, M. J.; Scherer, M. M., Environmental Science \& Technology 2002, 36 (3), 299-306.

43. Su, C.; Puls, R. W., Environ. Sci. Technol 2004, 38, 2715-2720.

44. Westerhoff, P.; James, J., Water Research 2003, 37 (8), 1818-1830.

45. Cheng, I. F.; Muftikian, R.; Fernando, Q.; Korte, N., Chemosphere, 1997, 35 (11), 2689-2695.

46. Jones, M. N., Water Res 1984, 18 (5), 643-646.

47. Kumar, M.; Chakraborty, S., Hazard Mater 2006, 135 (1-3), 112-21.

48. Li, X.-Q.; Elliott, D. W.; Zhang, W.-X., Critical Reviews in Solid State and Materials Sciences 2006, $31(4), 111-122$.

49. Wang, C.-B.; Zhang, W.-X., Environmental Science \& Technology 1997, 31, 2154-2156.

50. Sohn, K.; Kang, S. W.; Ahn, S.; Woo, M.; Yan, S.-K., Environ. Sci. Technol 2006, 40, 5514-5519.

51. Savic-Gajic, I.; Savic, I.; Gajic, D., The role and health risk of heavy metals in human organism. In Heavy metals and health, Castillo, L., Ed. Nova Science: 2016; pp 47-89.

52. Nacke, H.; Goncalves, A. C., Jr.; Schwantes, D.; Nava, I. A.; Strey, L.; Coelho, G. F., Arch Environ Contam Toxicol 2013, 64 (4), 537-44.

53. Bramley, R. G. V., New Zealand Journal of Agricultural Research 2012, 33 (4), 505-519.

54. Removing lead-based paint. https://www.health.govt.nz/your-health/healthyliving/environmental-health/hazardous-substances/removing-lead-based-paint (accessed June $25^{\text {th }}$, 2019).

55. Jaishankar, M.; Tseten, T.; Anbalagan, N.; Mathew, B. B.; Beeregowda, K. N., Interdiscip Toxicol 2014, 7 (2), 60-72.

56. Kouba, A.; Buřič, M.; Kozák, P., Water, Air, \& Soil Pollution 2009, 211 (1-4), 5-16.

57. Lindskog, S., Pharmacology \& Therapeutics 1997, 74 (1), 1-20.

58. Messerschmidt, A., 8.14 - Copper Metalloenzymes. In Comprehensive Natural Products II, Liu, H.-W.; Mander, L., Eds. Elsevier: Oxford, 2010; pp 489-545.

59. Gupta, C. P., Applied Chemistry 2014, 7 (11), 38-46.

60. Oyaro, N.; Juddy, O.; Murago, E. N. M.; Gitonga, E., Food, Agriculture \& Environment 2007, 5 (3-4), 119-121.

61. Sheng, P. X.; Ting, Y. P.; Chen, J. P.; Hong, L., J Colloid Interface Sci 2004, 275 (1), 131-41.

62. Goel, J.; Kadirvelu, K.; Rajagopal, C.; Kumar Garg, V., Hazard Mater 2005, 125 (1-3), 211-20. 
63. Moore, M.; Goldberg, A.; Yeung, A., Lead Effects on the Heme Biosynthetic Pathway Relationship to Toxicity. 2006; Vol. 514, p 191-203.

64. Lewicki, S.; Zdanowski, R.; Krzyzowska, M.; Lewicka, A.; Debski, B.; Niemcewicz, M.; Goniewicz, M., Ann Agric Environ Med 2014, 21 (2), 331-5.

65. Gheju, M., Water, Air, \& Soil Pollution 2011, 222 (1-4), 103-148.

66. Cohen, M. D.; Kargacin, B.; Klein, C. B.; Costa, M., Crit Rev Toxicol 1993, 23 (3), 255-81.

67. Kibria, G. Trace/heavy Metals and Its Impact on the Environment, Biodiversity and Human Health- A Short Review; 2016.

68. Ku, Y.; Jung, I.-L., Wat. Res. 2001, 35 (1), 135-142.

69. Baltpurvins, K. A.; Burns, R. C.; Lawrance, G. A.; Stuart, A. D., Wat. Res. 1997, 31 (5), 973-980.

70. Mirbagheri, S. A.; Hosseini, S. N., Desalination 2005, 171 (1), 85-93.

71. Ozverdi, A.; Erdem, M., Hazard Mater 2006, 137 (1), 626-32.

72. Chen, Q.; Luo, Z.; Hills, C.; Xue, G.; Tyrer, M., Water Res 2009, 43 (10), 2605-14.

73. Pang, F. M.; Kumar, P.; Teng, T. T.; Mohd Omar, A. K.; Wasewar, K. L., Taiwan Institute of Chemical Engineers 2011, 42 (5), 809-815.

74. Ying, X.; Fang, Z., Hazard Mater 2006, 137 (3), 1636-42.

75. Chang, Y.-K.; Chang, J.-E.; Lin, T.-T.; Hsu, Y.-M., Hazardous Materials 2002, B94, 89-99.

76. Abo-Farha, S. A.; Abdel-Aal, A. Y.; Ashour, I. A.; Garamon, S. E., J Hazard Mater 2009, 169 (13), 190-4.

77. Alyuz, B.; Veli, S., Hazard Mater 2009, 167 (1-3), 482-8.

78. Kang, S. Y.; Lee, J. U.; Moon, S. H.; Kim, K. W., Chemosphere 2004, 56 (2), 141-7.

79. Motsi, T.; Rowson, N. A.; Simmons, M. J. H., International Journal of Mineral Processing 2009, $92(1-2), 42-48$.

80. Ostroski, I. C.; Barros, M. A.; Silva, E. A.; Dantas, J. H.; Arroyo, P. A.; Lima, O. C., J Hazard Mater 2009, $161(2-3), 1404-12$.

81. Taffarel, S. R.; Rubio, J., Minerals Engineering 2009, 22 (4), 336-343.

82. Dąbrowski, A.; Hubicki, Z.; Podkościelny, P.; Robens, E., Chemosphere 2004, 56 (2), 91-106.

83. Shrivastava, P. K.; Gupta, S. K., Chemical Sciences and Applications 2015, 6 (1).

84. Fu, F.; Wang, Q., Environ Manage 2011, 92 (3), 407-18.

85. Jusoh, A.; Su Shiung, L.; Ali, N. a.; Noor, M. J. M. M., Desalination 2007, 206 (1-3), 9-16.

86. Kang, K. C.; Kim, S. S.; Choi, J. W.; Kwon, S. H., Industrial and Engineering Chemistry 2008, 14 (1), 131-135.

87. Wang, H.; Zhou, A.; Peng, F.; Yu, H.; Yang, J., Colloid Interface Sci 2007, 316 (2), 277-83.

88. Kabbashi, N. A.; Atieh, M. A.; Al-Mamun, A.; Mirghami, M. E. S.; Alam, M. D. Z.; Yahya, N., Environmental Sciences 2009, 21 (4), 539-544.

89. Arias, M.; Barral, M. T.; Mejuto, J. C., Chemosphere 2002, 48, 1081-1088.

90. Suraj, G.; Iyer, C. S. P.; Lalithambika, M., Applied Clay Science 1998, 13, 293-306.

91. Yavuza, O.; Altunkaynak, Y.; Guzel, F., Water Research 2003, 37, 948-952.

92. Angove, M. J.; Wells, J. D.; Johnson, B. B., Colloids and Surfaces 1999, 146, 243-251.

93. Wu, Z.; Gu, Z.; Wang, X.; Evans, L.; Guo, H., Environmental Pollution 2003, 121, 469-475.

94. Collins, C. R.; Ragnarsdottir, K. V.; Sherman, D. M., Geochimica et Cosmochimica Acta 1999, $63(19 / 20), 2989-3002$.

95. $\quad$ Naseem, R.; Tahir, S. S., Wat. Res. 2001, 35 (16), 3982-3986.

96. Erdem, M.; Altundoğan, H. S.; Tümen, F., Minerals Engineering 2004, 17 (9-10), 1045-1052.

97. Bayat, B., Hazardous Materials 2002, B95, 275-290.

98. Sheng, G.; Wang, S.; Hu, J.; Lu, Y.; Li, J.; Dong, Y.; Wang, X., Colloids and Surfaces A: Physicochemical and Engineering Aspects 2009, 339 (1-3), 159-166.

99. Apiratikul, R.; Pavasant, P., Chemical Engineering Journal 2008, 144 (2), 245-258.

100. Apiratikul, R.; Pavasant, P., Bioresour Technol 2008, 99 (8), 2766-77.

101. Ajjabi, L. C.; Chouba, L., Environ Manage 2009, 90 (11), 3485-9.

102. Pan, J.-h.; Liu, R.-x.; Tang, H.-x., Environmental Sciences 2007, 19 (4), 403-408. 
103. Quintelas, C.; Rocha, Z.; Silva, B.; Fonseca, B.; Figueiredo, H.; Tavares, T., Chemical Engineering Journal 2009, 152 (1), 110-115.

104. Amini, M.; Younesi, H.; Bahramifar, N., Colloids and Surfaces A: Physicochemical and Engineering Aspects 2009, 337 (1-3), 67-73.

105. Tsekova, K.; Todorova, D.; Dencheva, V.; Ganeva, S., Bioresour Technol 2010, 101 (6), 1727-

31.

106. Labanda, J.; Khaidar, M. S.; Llorens, J., Desalination 2009, 249 (2), 577-581.

107. Mohsen-Nia, M.; Montazeri, P.; Modarress, H., Desalination 2007, 217 (1-3), 276-281.

108. Murthy, Z. V.; Chaudhari, L. B., Hazard Mater 2008, 160 (1), 70-7.

109. Murthy, Z. V. P.; Chaudhari, L. B., Chemical Engineering Journal 2009, 150 (1), 181-187.

110. Figoli, A.; Cassano, A.; Criscuoli, A.; Mozumder, M. S.; Uddin, M. T.; Islam, M. A.; Drioli, E., Water Res 2010, 44 (1), 97-104.

111. Sun, Y.; Li, J.; Huang, T.; Guan, X., Water Research 2016, 100, 277-295.

112. Rangsivek, R.; Jekel, M. R., Water Research 2005, 39 (17), 4153-4163.

113. Feng, P.; Guan, X.; Sun, Y.; Choi, W.; Qin, H.; Wang, J.; Qiao, J.; Li, L., Journal of Environmental Sciences 2015, 31, 175-183.

114. Jassam, S. H.; Nasir, M. J., Kerbala University 2013, 11 (3).

115. Sheet, I.; Kabbani, A.; Holail, H., Energy Procedia 2014, 50, 130-138.

116. Li, X.-Q.; Zhang, W.-X., Phys. Chem. C 2007, 111, 6939-6946.

117. Ponder, S. M.; Darab, J. G.; Mallouk, T. E., Environ. Sci. Technol. 2000, 34, 2564-2569.

118. Agrawal, A.; Tratnyek, P. G., Environ. Sci. Technol. 1996, 30, 153-160.

119. Liou, Y. H.; Lo, S.-L.; Lin, C.-J.; Kuan, W. H.; Weng, S. C., Journal of Hazardous Materials 2005, 126 (1), 189-194.

120. Agrawal, A.; Ferguson, W. J.; Gardner, B. O.; Christ, J. A.; Bandstra, J. Z.; Tratnyek, P. G., Environmental Science \& Technology 2002, 36 (20), 4326-4333.

121. Tsai, Y. J.; Chou, F. C.; Cheng, T. C., Hazard Mater 2009, 163 (2-3), 743-7.

122. Stefaniuk, M.; Oleszczuk, P.; Ok, Y. S., Chemical Engineering Journal 2016, 287, 618-632.

123. Zhang, W.-x.; Wang, C.-B.; Lien, H.-L., Catalysis Today 1998, 40, 387-395.

124. Martin, J. E.; Herzing, A. A.; Yan, W.; Li, X.-q.; Koel, B. E.; Kiely, C. J.; Zhang, W.-x., Langmuir 2008, 24 (8), 4329-4334.

125. Tratnyek, P. G.; Johnson, R. L., Nano Today 2006, 1 (2), 44-48.

126. Liou, Y. H.; Lo, S. L.; Lin, C. J.; Kuan, W. H.; Weng, S. C., Hazard Mater 2005, 127 (1-3), 102-10.

127. Rosická, D.; Šembera, J., Nanoscale Research Letters 2011, 6 (1), 527.

128. O'Carroll, D.; Sleep, B.; Krol, M.; Boparai, H.; Kocur, C., Advances in Water Resources 2013, 51, 104-122.

129. Kuhn, L. T.; Bojesen, A.; Timmermann, L.; Nielsen, M. M.; Mørup, S., Journal of Physics: Condensed Matter 2002, 14 (49), 13551-13567.

130. Nurmi, J. T.; Tratnyek, P. G.; Sarathy, V.; Baer, D. R.; Amonette, J. E.; Pecher, K.; Wang, C.; Linehan, J. C.; Matson, D. W.; Penn, R. L.; Driessen, M. D., Environmental Science \& Technology 2005, 39 (5), 1221-1230.

131. Liu, Y.; Majetich, S. A.; Tilton, R. D.; Sholl, D. S.; Lowry, G. V., Environmental Science \& Technology 2005, 39 (5), 1338-1345.

132. Sun, Y.-P.; Li, X.-q.; Cao, J.; Zhang, W.-x.; Wang, H. P., Advances in Colloid and Interface Science 2006, 120 (1), 47-56.

133. Kim, H.-S.; Ahn, J.-Y.; Hwang, K.-Y.; Kim, I.-K.; Hwang, S., Environ. Sci. Technol. 2010, 44, 17601766.

134. Mu, Y.; Jia, F.; Ai, Z.; Zhang, L., Environ. Sci.: Nano 2017, 4, 27-45.

135. Olegario, J. T.; Yee, N.; Miller, M.; Sczepaniak, J.; Manning, B., Journal of Nanoparticle Research 2010, 12 (6), 2057-2068.

136. Boparai, H. K.; Joseph, M.; O'Carroll, D. M., Environ Sci Pollut Res Int 2013, 20 (9), 6210-21. 
137. Kržišnik, N.; Mladenovič, A.; Škapin, A. S.; Škrlep, L.; Ščančar, J.; Milačič, R., Science of The Total Environment 2014, 476-477, 20-28.

138. Suzuki, T.; Moribe, M.; Oyama, Y.; Niinae, M., Chemical Engineering Journal 2012, 183, 271 -

277.

139. Liu, H. B.; Chen, T. H.; Chang, D. Y.; Chen, D.; Liu, Y.; He, H. P.; Yuan, P.; Frost, R., Materials Chemistry and Physics 2012, 133 (1), 205-211.

140. Chen, S.-S.; Hsu, H.-D.; Li, C.-W., Nanoparticle Research 2004, 6 (6), 639-647.

141. Kassaee, M. Z.; Motamedi, E.; Mikhak, A.; Rahnemaie, R., Chemical Engineering Journal 2011, 166 (2), 490-495.

142. Machado, S.; Pinto, S. L.; Grosso, J. P.; Nouws, H. P.; Albergaria, J. T.; Delerue-Matos, C., Sci Total Environ 2013, 445-446, 1-8.

143. Sohn, K.; Kang, S. W.; Ahn, S.; Woo, M.; Yang, S.-K., Environ. Sci. Technol. 2006, 40, 5514-5519.

144. Li, X.-q.; Zhang, W.-X., Langmuir 2006, 22, 4638-4642.

145. Liou, Y. H.; Lo, S. L.; Kuan, W. H.; Lin, C. J.; Weng, S. C., Water Res 2006, 40 (13), 2485-92.

146. G. N. Glavee; K. J. Klabunde; Sorensen, C. M.; Hadjipanayis, G. C., Inorg. Chem 1995, 34, $28-$

35.

147. Park, H.; Park, Y. M.; Oh, S. K.; You, K. M.; Lee, S. H., Environ Technol 2009, 30 (3), 261-7.

148. Wang, W.; Jin, Z. H.; Li, T. L.; Zhang, H.; Gao, S., Chemosphere 2006, 65 (8), 1396-404.

149. Hwang, Y.-H.; Kim, D.-G.; Shin, H.-S., Applied Catalysis B: Environmental 2011, 105 (1-2), 144-

150.

150. Liu, Y.; Phenrat, T.; Lowry, G. V., Environmental Science \& Technology 2007, 41 (22), 7881-

7887.

151. Zhuang, Y.; Ahn, S.; Seyfferth, A. L.; Masue-Slowey, Y.; Fendorf, S.; Luthy, R. G., Environ Sci Technol 2011, 45 (11), 4896-903.

152. Fagerlund, F.; Illangasekare, T. H.; Phenrat, T.; Kim, H. J.; Lowry, G. V., Contam Hydrol 2012, 131 (1-4), 9-28.

153. Lien, H.-L.; Zhang, W.-x., Environmental Engineering 1999, 125 (11), 1042-1047.

154. Huang, P.; Ye, Z.; Xie, W.; Chen, Q.; Li, J.; Xu, Z.; Yao, M., Water Res 2013, 47 (12), 4050-8.

155. Calderon, B.; Fullana, A., Water Res 2015, 83, 1-9.

156. Choe, S.; Chang, Y.-Y.; Hwang, K.-Y.; Khim, J., Chemosphere 2000, 41, 1307-1311.

157. Yang, G. C.; Lee, H. L., Water Res 2005, 39 (5), 884-94.

158. Khalil, A. M. E.; Eljamal, O.; Jribi, S.; Matsunaga, N., Chemical Engineering Journal 2016, 287, 367-380.

159. Grieger, K. D.; Fjordboge, A.; Hartmann, N. B.; Eriksson, E.; Bjerg, P. L.; Baun, A., Contam Hydrol 2010, $118(3-4), 165-83$.

160. Xie, Y.; Dong, H.; Zeng, G.; Tang, L.; Jiang, Z.; Zhang, C.; Deng, J.; Zhang, L.; Zhang, Y., J Hazard Mater 2017, 321, 390-407.

161. El-Temsah, Y. S.; Joner, E. J., Environmental Toxicology 2012, 27 (1), 42-49.

162. Yan, W.; Herzing, A. A.; Kiely, C. J.; Zhang, W. X., Contam Hydrol 2010, 118 (3-4), 96-104.

163. Shand, M.; Anderson, J. A., Catalysis Science \& Technology 2013, 3 (4), 879-899.

164. Fanning, J. C., Coordination Chemistry Reviews 2000, 199, 159-179.

165. An, Y.; Li, T.; Jin, Z.; Dong, M.; Li, Q., Desalination 2010, 252 (1-3), 71-74.

166. Sohn, K.; Kang, S. W.; Ahn, S.; Woo, M.; Yang, S.-K., Environ Sci Technol 2006, 40 ( 17), $5514-$ 5519.

167. Ryu, A.; Jeong, S.-W.; Jang, A.; Choi, H., Applied Catalysis B: Environmental 2011, 105 (1-2), 128-135.

168. Kanel, S.; Manning, B.; Charlet, L.; Choi, H., Environ Sci Technol 2005, 39 (5), 1291-1298.

169. Zhang, Y.; Su, Y.; Zhou, X.; Dai, C.; Keller, A. A., Hazard Mater 2013, 263 Pt 2, 685-93.

170. Dai, C.; Zhou, Z.; Zhou, X.; Zhang, Y., Water, Air, \& Soil Pollution 2013, 225 (1).

171. Li, X.-Q.; Cao, J.; Zhang, W.-X., Ind. Eng. Chem. Res. 2008, 47, 2131-2139.

172. Zhou, S.; Li, Y.; Chen, J.; Liu, Z.; Wang, Z.; Na, P., RSC Advances 2014, 4 (92), 50699-50707. 
173. Lin, Y.; Chen, Z.; Megharaj, M.; Naidu, R., Journal of Colloid and Interface Science 2012, 381 (1), 30-35.

174. Gautam, R. K.; Rawat, V.; Banerjee, S.; Sanroman, M. A.; Soni, S.; Singh, S. K.; Chattopadhyaya, M. C., Journal of Molecular Liquids 2015, 212, 227-236.

175. Tehrani, M. R. F.; Vossoughi, M.; Shamsai, A., Environment Protection Engineering 2014, 40, 75-86.

176. Han, Y.; Yan, W., Water Res 2014, 66, 149-59.

177. Saberi, A., Iranica Journal of Energy \& Environment 2012, 3 (2), 189-196.

178. Krasae, N.; Wantala, K.; Tantriratna, P.; Grisdanurak, N., App. Envi. Res. 2014, 36 (2), 15-23.

179. Guo, J.; Guo, P.; Yu, M.; Sun, Z.; Li, P.; Yang, T.; Liu, J.; Zhang, L., Polish Journal of Environmental Studies 2018, 27 (5), 2023-2028.

180. Muradova, G. G.; Gadjieva, S. R.; Palma, L. D.; Vilardi, G., Chemical Engineering Transactions 2016, 47.

181. Hosseini, S. M.; Ataie-Ashtiani, B.; Kholghi, M., Nitrate reduction by nano-Fe/Cu particles in packed column. 2011; Vol. 276, p 214-221.

182. Muradova, G. G.; Gadjieva, S. R.; Palma, L. D.; Vilardi, G., Chemical Engineering Transactions 2016, 47, 205-210.

183. Lien, H.-L.; Zhang, W.-X., Applied Catalysis B: Environmental 2007, 77 (1-2), 110-116.

184. Shih, Y.-h.; Chen, M.-Y.; Su, Y.-F., Applied Catalysis B: Environmental 2011, 105 (1-2), 24-29.

185. Wang, X.; Chen, C.; Chang, Y.; Liu, H., Hazard Mater 2009, 161 (2-3), 815-23.

186. Zhang, Z.; Shen, Q.; Cissoko, N.; Wo, J.; Xu, X., J Hazard Mater 2010, 182 (1-3), 252-8.

187. Gong, J.; Lee, C.-S.; Chang, Y.-Y.; Chang, Y.-S., Journal of hazardous materials 2015, 286, 107-

117.

188. Su, Y.; Adeleye, A. S.; Huang, Y.; Sun, X.; Dai, C.; Zhou, X.; Zhang, Y.; Keller, A. A., Water research 2014, 63, 102-111.

189. Daud, M.; Khan, Z.; Ashgar, A.; Danish, M. I.; Qazi, I. A., Nanotechnology 2015, 2015, 1-7.

190. Liou, Y. H.; Lin, C. J.; Weng, S. C.; Ou, H. H.; Lo, S. L., Environ. Sci. Technol. 2009, 43, 2482-2488.

191. Wang, T.; Su, J.; Jin, X.; Chen, Z.; Megharaj, M.; Naidu, R., Hazard Mater 2013, 262, 819-25.

192. Shi, L.; Du, J.; Chen, Z.; Megharaj, M.; Naidu, R., Colloid Interface Sci 2014, 428, 302-307.

193. He, Y.; Lin, H.; Dong, Y.; Li, B.; Wang, L.; Chu, S.; Luo, M.; Liu, J., Chemical Engineering Journal 2018, 347, 669-681.

194. Zeng, Y.; Walker, H.; Zhu, Q., Hazard Mater 2017, 324 (Pt B), 605-616.

195. Zhang, Y.; Li, Y.; Li, J.; Hu, L.; Zheng, X., Chemical Engineering Journal 2011, 171 (2), 526-531.

196. Wu, L.; Ritchie, S. M., Chemosphere 2006, 63 (2), 285-92.

197. Lu H., T. B., Wang J., Hao H., Chemical Engineering Transactions 2017, 60, 169-174.

198. Wang, J.; Ji, B.; Shu, Y.; Chen, W.; Zhu, L.; Chen, F., Polish Journal of Environmental Studies 2018, 27 (6), 2785-2792.

199. Cai, X.; Gao, Y.; Sun, Q.; Chen, Z.; Megharaj, M.; Naidu, R., Chemical Engineering 2014, 244, $19-26$.

200. Doong, R.-A.; Saha, S.; Lee, C.-H.; Lin, H.-P., RSC Advances 2015, 5 (110), 90797-90805.

201. Zhou, Y.; Kuang, Y.; Li, W.; Chen, Z.; Megharaj, M.; Naidu, R., Chemical Engineering Journal 2013, 223, 68-75.

202. Wang, X.; Le, L.; Alvarez, P. J. J.; Li, F.; Liu, K., Taiwan Institute of Chemical Engineers 2015, 50, 297-305.

203. Bragança, L. F. F. P. G.; Avillez, R. R.; Moreira, C. R.; Pais da Silva, M. I., Materials Chemistry and Physics 2013, 138 (1), 17-28.

204. Kakavandi, B.; Kalantary, R. R.; Farzadkia, M.; Mahvi, A. H.; Esrafili, A.; Azari, A.; Yari, A. R.; Javid, A. B., Environmental Health Science \& Engineering 2014, 12.

205. General guide to Microsilica; Auckland.

206. Chisholm, D. H., Performance characteristics of concrete incorporating a natural amorphous silica. In The third CANMET/ACI International conference, Auckland, New Zealand, 1997. 
207. Walker, R.; Pavía, S., Materials and Structures 2010, 44 (6), 1139-1150.

208. Qiu, X.; Fang, Z.; Liang, B.; Gu, F.; Xu, Z., Hazard Mater 2011, 193, 70-81.

209. Ensie, B.; Samad, S., Desalination 2014, 347, 1-9.

210. American Public Health Association, A. W. W. A., Water Environment Federation, Standard Methods for the Examination of Water and Wastewater. In Nitrogen (nitrate), 1999.

211. American Public Health Association, A. W. W. A., Water Environment Federation, Standard Methods for the Examination of Water and Wastewater. In Determination of anions by ion chromatography, 1999.

212. Harris, D. C., Quantitative chemical analysis. $6^{\text {th }}$ ed.; New York, 2003.

213. Jeol, Invitation to the SEM World. Japan.

214. Wen, J. G., Transmission Electron Microscopy. In Practical Materials Characterization, Sardela, M., Ed. Springer: New York, 2014; pp 189-229.

215. Font-Bardia, M.; Alcobé, X., X-ray single crystal and powder difraction: posibilities and applications. Spain.

216. Dinnebier, R. E.; Billinge, S. J. L., Principles of Powder Diffraction. Royal Society of Chemistry, 2008.

217. Housecroft, C. E.; Sharpe, A. G., Inorganic Chemistry. Italy, 2012; p 121-124.

218. Liu, J.; Wang, C.; Shi, J.; Liu, H.; Tong, Y., Hazard Mater 2009, 163 (1), 370-5.

219. Haddad, P. R., Anal Bioanal Chem 2004, 379 (3), 341-3.

220. Weiss, J., Handbook of Ion Chromatography. $4^{\text {th }}$ ed.; Wiley-VCH: 2016.

221. Khan, H. U., The Role of Ion Exchange Chromatography in Purification and Characterization of Molecules. In Ion Exchange Technologies, Kilislioglu, A., Ed. IntechOpen, 2012.

222. Elkin, K.; Riviello, J.; Small, H., Journal of Chromatography A 2015, 1403, 63-69.

223. Karu, N.; Hutchinson, J. P.; Dicinoski, G. W.; Hanna-Brown, M.; Srinivasan, K.; Pohl, C. A.; Haddad, P. R., Journal of Chromatography A 2012, 1253, 44-51.

224. Wahab, M. F.; Anderson, J. K.; Abdelrady, M.; Lucy, C. A., Analytical chemistry 2014, 86 (1), 559-66.

225. Kapinus, E. N.; Revelsky, I. A.; Ulogov, V. O.; Lyalikov, Y. A., Journal of Chromatography B 2004, 800 (1-2), 321-323.

226. Ito, K.; Nomura, R.; Fujii, T.; Tanaka, M.; Tsumura, T.; Shibata, H.; Hirokawa, T., Analytical and bioanalytical chemistry 2012, 404 (8), 2513-7.

227. Tirumalesh, K., Talanta 2008, 74 (5), 1428-1434.

228. Connolly, D.; Paull, B., Analytica Chimica Acta 2001, 441 (1), 53-62.

229. Hu, W.; Haddad, P. R.; Hasebe, K.; Tanaka, K.; Tong, P.; Khoo, C., Analytical chemistry 1999, 71 (8), 1617-1620.

230. Burrows, A.; Holman, J.; Parsons, A.; Pilling, G.; Price, G., Chemistry ${ }^{3}$ introducing inorganic, organic and physical chemistry. Oxford University Press: New York, 2009.

231. Krug, F. J.; Růžička, J.; Hansen, E. H., Analyst 1979, 104, 47-54.

232. Elzea, J. M.; Rice, S. B., Clays and Clay Minerals 1996, 44 (4), 492-500.

233. Su, J.; Lin, S.; Chen, Z.; Megharaj, M.; Naidu, R., Desalination 2011, 280 (1-3), 167-173.

234. Choe, S.; Liljestrand, H. M.; Khim, J., Applied Geochemistry 2004, 19 (3), 335-342.

235. Sparis, D.; Mystrioti, C.; Xenidis, A.; Papassiopi, N., Desalination and Water Treatment 2013, 51 (13-15), 2926-2933.

236. Liou, Y. H.; Lo, S. L.; Lin, C. J.; Hu, C. Y.; Kuan, W. H.; Weng, S. C., Environ. Sci. Technol. 2005, 39, 9643-9648.

237. Keen, O. S.; Love, N. G.; Linden, K. G., 10 - Nitrate Photochemistry in the Context of Water Reclamation. 2014; p 229-246.

238. Liu, J.; Lei, D.; Li, Q., Renewable Energy 2016, 86, 949-954.

239. Huang, Y. H.; Zhang, T. C., Water Res 2004, 38 (11), 2631-2642.

240. Zhang, T. C.; Huang, Y. H., Environmental Engineering 2005, 131 (3), 461-470. 
241. Zhang, H.; Jin, Z.-H.; Han, L.; Qin, C.-H., Transactions of Nonferrous Metals Society of China 2006, 16, 345-349.

242. Kim, D.-G.; Hwang, Y.-H.; Shin, H.-S.; Ko, S.-O., KSCE Journal of Civil Engineering 2015, 20 (1), 175-187.

243. Li, P.; Lin, K.; Fang, Z.; Wang, K., Cleaner Production 2017, 151, 21-33.

244. An, Y.; Li, T.; Jin, Z.; Dong, M.; Xia, H.; Wang, X., Bioresour Technol 2010, 101 (24), 9825-8.

245. Bae, S.; Hamid, S.; Jung, J.; Sihn, Y.; Lee, W., Environmental Technology 2016, 37 (9), $1077-$ 1087.

246. Lee, C.; Sedlak, D. L., Environmental Science \& Technology 2008, 42 (22), 8528-8533.

247. Liu, H.; Guo, M.; Zhang, Y., Environmental Technology 2014, 35 (7), 917-924.

248. Jung, J.; Bae, S.; Lee, W., Applied Catalysis B: Environmental 2012, 127, 148-158.

249. John E. Martin; Andrew A. Herzing; Weile Yan; Xiao-Qin Li; Bruce E. Koel; Christopher J. Kiely; Zhang, W.-X., Langmuir 2008, 24, 4329-4334.

250. Wang, C. M.; Baer, D. R.; Thomas, L. E.; Amonette, J. E.; Antony, J.; Qiang, Y.; Duscher, G., Applied Physics 2005, 98 (9), 094308.

251. Su, Y.; Adeleye, A. S.; Huang, Y.; Sun, X.; Dai, C.; Zhou, X.; Zhang, Y.; Keller, A. A., Water Res 2014, 63, 102-111.

252. Liou, Y.-H.; Lo, S.-L.; Weng, S.-C., Western Pacific Earth Sciences 2010, 10, 41-56.

253. Sarathy, V.; Tratnyek, P. G.; Nurmi, J. T.; Baer, D. R.; Amonette, J. E.; Chun, C. L.; Penn, R. L.; Reardon, E. J., The Journal of Physical Chemistry C 2008, 112 (7), 2286-2293.

254. Wang, Q.; Lee, S.; Choi, H., The Journal of Physical Chemistry C 2010, 114 (5), 2027-2033.

255. Liu, Y.; Lowry, G. V., Environmental Science \& Technology 2006, 40 (19), 6085-6090.

256. Zhu, B.-W.; Lim, T.-T., Environmental Science \& Technology 2007, 41 (21), 7523-7529.

257. American Public Health Association, A. W. W. A., Water Environment Federation, Standard Methods for the Examination of Water and Wastewater. In 4500-O Oxygen (dissolved), 1999.

258. Reinsch, B. C.; Forsberg, B.; Penn, R. L.; Kim, C. S.; Lowry, G. V., Environ. Sci. Technol. 2010, 44, 3455-3461.

259.

260. Hwang, Y.; Kim, D.; Shin, H. S., Environ Technol 2015, 36 (9-12), 1178-87.

261. Ostergren, J. D.; Brown, G. E., Jr.; Parks, G. A.; Persson, P., Colloid Interface Sci 2000, 225 (2), 483-493.

262. Hua, Y.; Wang, W.; Huang, X.; Gu, T.; Ding, D.; Ling, L.; Zhang, W. X., Chemosphere 2018, 201, 603-611.

263. Zhu, F.; Li, L.; Ren, W.; Deng, X.; Liu, T., Environ Pollut 2017, 227, 444-450.

264. Chen, H.; Luo, H.; Lan, Y.; Dong, T.; Hu, B.; Wang, Y., J Hazard Mater 2011, 192 (1), 44-53.

265. Stumm, W.; Morgan, J. J., Aquatic Chemistry: Chemical Equilibria and Rates in Natural Waters. $3^{\text {rd }}$ ed.; John Wiley \& Sons: New York, Chichester, Brisbane, Toronto, Singapore, 1996; Vol. 126.

266. Kim, D.-G.; Hwang, Y.-H.; Shin, H.-S.; Ko, S.-O., Environmental Technology 2013, 34 (12), 16251635.

267. Kim, D.-G.; Hwang, Y.-H.; Shin, H.-S.; Ko, S.-O., Desalination and Water Treatment 2012, 49 (13), 147-156.

268. Ruangchainikom, C.; Liao, C. H.; Anotai, J.; Lee, M. T., Chemosphere 2006, 63 (2), 335-43.

269. Gibbs, M. M.; White, E., Hydrobiologia 1994, 275 (1), 467-477.

270. Gibbs, M. Lake Horowhenua Review: Assessment of Opportunities to Address Water Quality Issues in Lake Horowhenua; NIWA, 2011.

271. https://www.horizons.govt.nz/HRC/media/Media/Water/Lake-Horowhenua-Report122017.pdf?ext=.pdf (accessed 25 March, 2019).

272. Feng, J.; Zhu, B.-w.; Lim, T.-T., Chemosphere 2008, 73 (11), 1817-1823.

273. Tan, L.; Liang, B.; Fang, Z.; Xie, Y.; Tsang, E. P., Journal of Nanoparticle Research 2014, 16 (12), 2786.

274. Zhang, Z.; Cissoko, N.; Wo, J.; Xu, X., J Hazard Mater 2009, 165 (1-3), 78-86. 
275. Sutak, R.; Botebol, H.; Blaiseau, P. L.; Leger, T.; Bouget, F. Y.; Camadro, J. M.; Lesuisse, E., Plant Physiol 2012, 160 (4), 2271-84.

276. Sunda, W. G.; Huntsman, S. A., Marine Chemistry 1995, 50 (1), 189-206.

277. Iron in Drinking-water, Background document for development of WHO Guidelines for Drinking-water Quality; WHO, 1996.

278. Boparai, H. K.; Joseph, M.; O'Carroll, D. M., J Hazard Mater 2011, 186 (1), 458-65.

279. Soto Hidalgo, K. T.; Guzmán-Blas, R.; Ortiz-Quiles, E. O.; Fachini, E. R.; Corchado-García, J.; Larios, E.; Zayas, B.; José-Yacamán, M.; Cabrera, C. R., RSC Advances 2015, 5 (4), 2777-2784.

280. Zhang, Y.; Li, Y.; Dai, C.; Zhou, X.; Zhang, W., Chemical Engineering Journal 2014, 244, 218-226.

281. Liu, T.; Yang, X.; Wang, Z. L.; Yan, X., Water Res 2013, 47 (17), 6691-700.

282. Ma, L.; Wei, Q.; Chen, Y.; Song, Q.; Sun, C.; Wang, Z.; Wu, G., R Soc Open Sci 2018, 5 (2), 171051.

283. Zarime, N. A.; Yaacob, W. Z. W.; Jamil, H., 2018, 1940, 020029.

284. Fu, R.; Yang, Y.; Xu, Z.; Zhang, X.; Guo, X.; Bi, D., Chemosphere 2015, 138, 726-34.

285. Moazeni, M.; Ebrahimi, A.; Rafiei, N.; Pourzamani, H. R., Health Scope 2016, 6 (2).

286. Yu, C.; Shao, J.; Sun, W.; Yu, X., Arabian Journal of Chemistry 2018.

287. Dawagreh, A. K.; Hailat, M.; Hussam Alkhasawneh, A., Evaluation of natural zeolite as sorbent material for the removal of lead from waste water. 2017; Vol. 36, p 67-74.

288. Rahmani, A. R.; Samadi, M. T.; Noroozi, R., Environmental and Ecological Engineering 2011, 5

(2).

289. Jabeen, H.; Chandra, V.; Jung, S.; Lee, J. W.; Kim, K. S.; Kim, S. B., Nanoscale 2011, 3 (9), $3583-$ 5.

290. Lv, X.; Xu, J.; Jiang, G.; Xu, X., Chemosphere 2011, 85 (7), 1204-9.

291. Lu, H.; Tian, B.; Wang, J.; Hao, H., Chemical Engineering Transactions 2017, 60.

292. Bhattacharyya, K. G.; Sen Gupta, S., Industrial \& Engineering Chemistry Research 2006, 45 (21), $7232-7240$.

293. Li, Y.; Yue, Q.-Y.; Gao, B.-Y., Applied Clay Science 2010, 48 (3), 481-484.

294. Tewari, N.; Guha, B. K.; Vasudevan, P., Adsorption study of hexavalent chromium by bentonite clay. 2005; Vol. 17, p 2184-2190.

295. Fan, Z.; Zhang, Q.; Gao, B.; Li, M.; Liu, C.; Qiu, Y., Chemosphere 2019, 217, 85-94.

296. Imani, S.; Rezaei-Zarchi, S.; Hashemi, M.; Borna, H.; Javid, A.; Zand, A. m.; Abarghouei, H. B., Medicinal Plants Research 2011, 5 (13), 2775-2780.

297. Figueira, M. M., Biotechnology and Bioengineering 1997, 54 (4), 344-350.

298. García-Casal, M. N.; Pereira, A. C.; Leets, I.; Ramírez, J.; Quiroga, M. F., The Journal of Nutrition 2007, $137(12), 2691-2695$. 
Appendix

A. XRD spectra

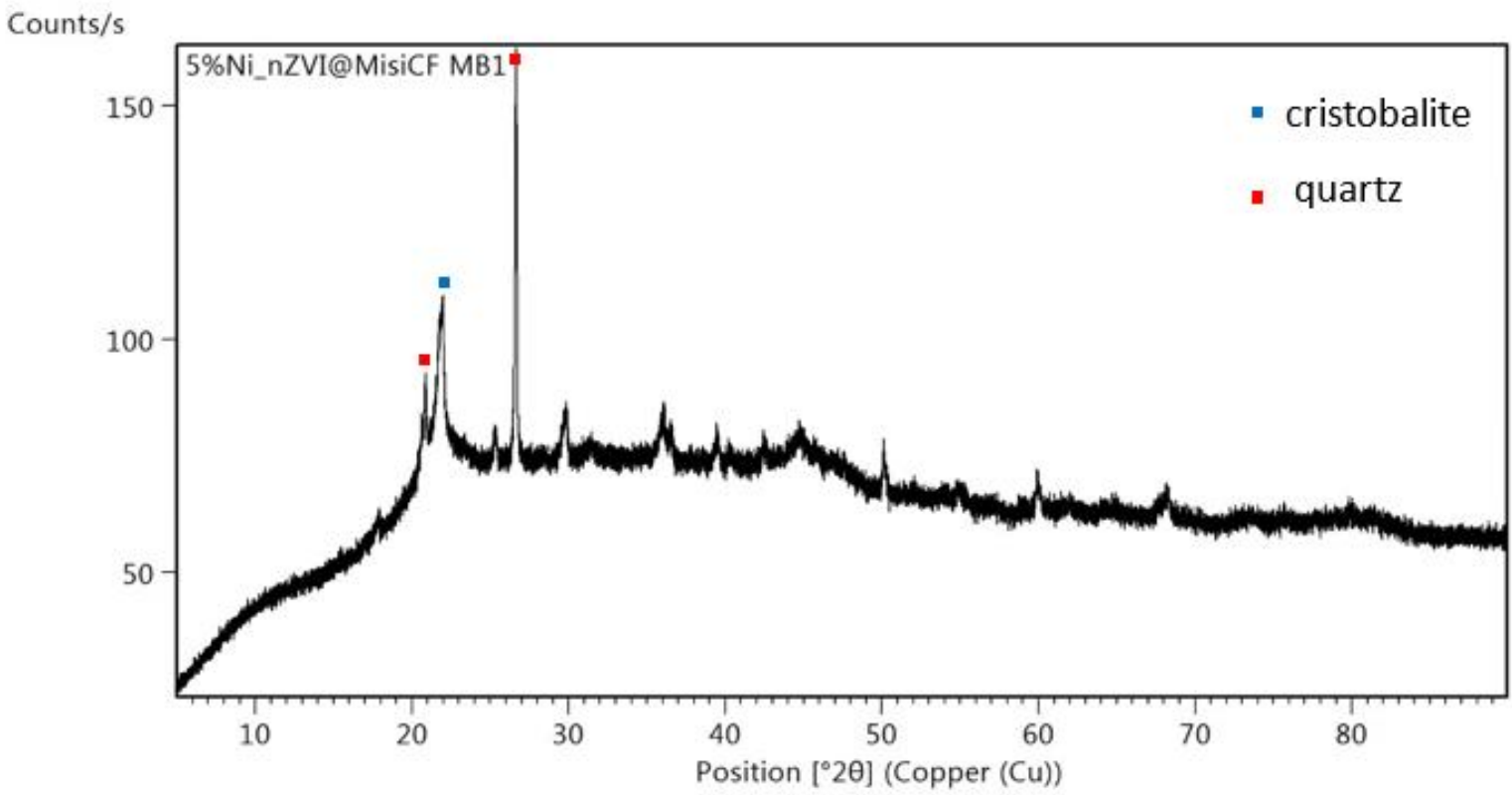

A.1. XRD spectrum of 5\%Ni-nZVI@MisiCFeOOH-MB1.

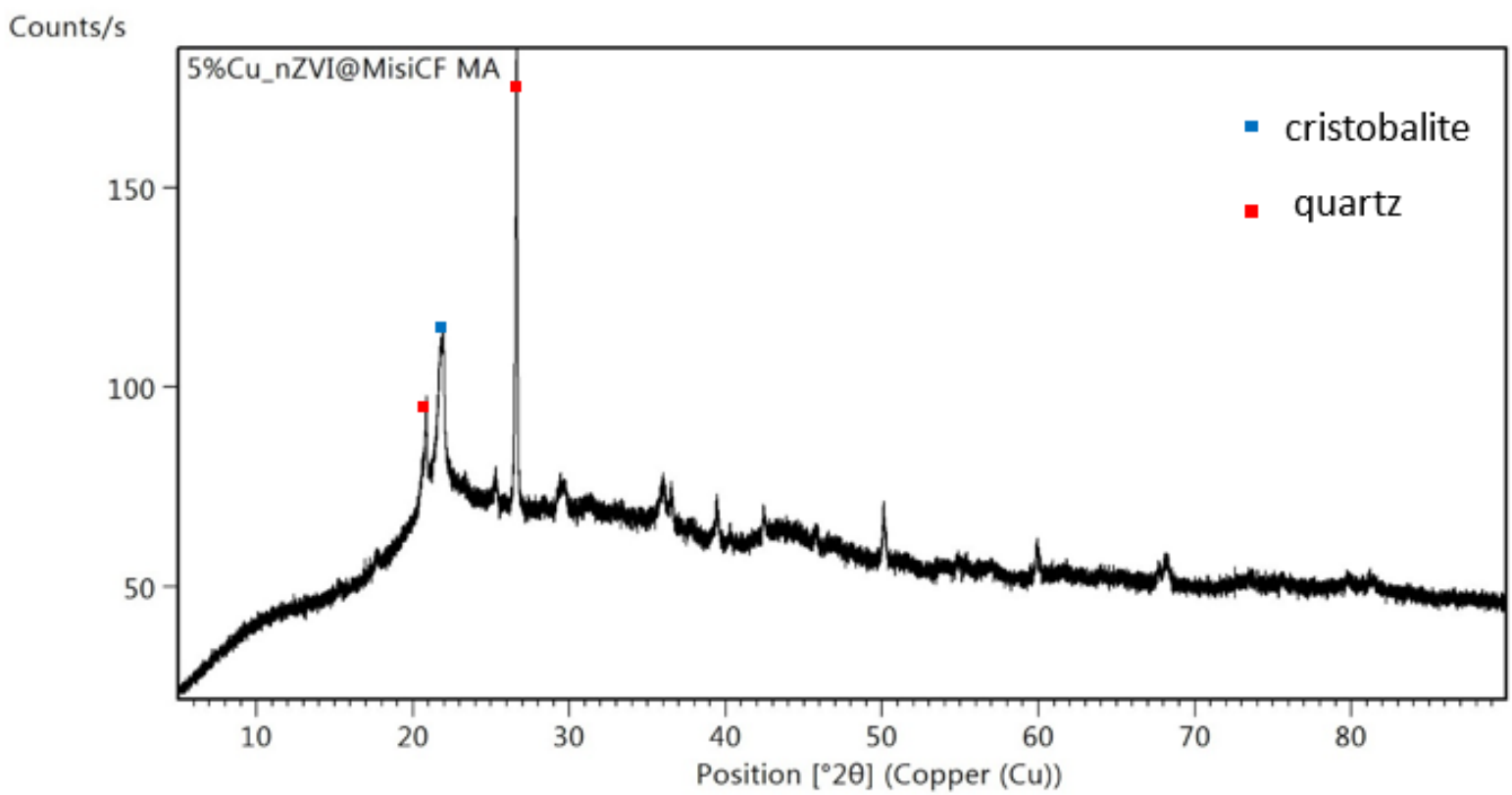

A.2. XRD spectrum of 5\%Cu-nZVI@MisiCFeOOH-MA. 


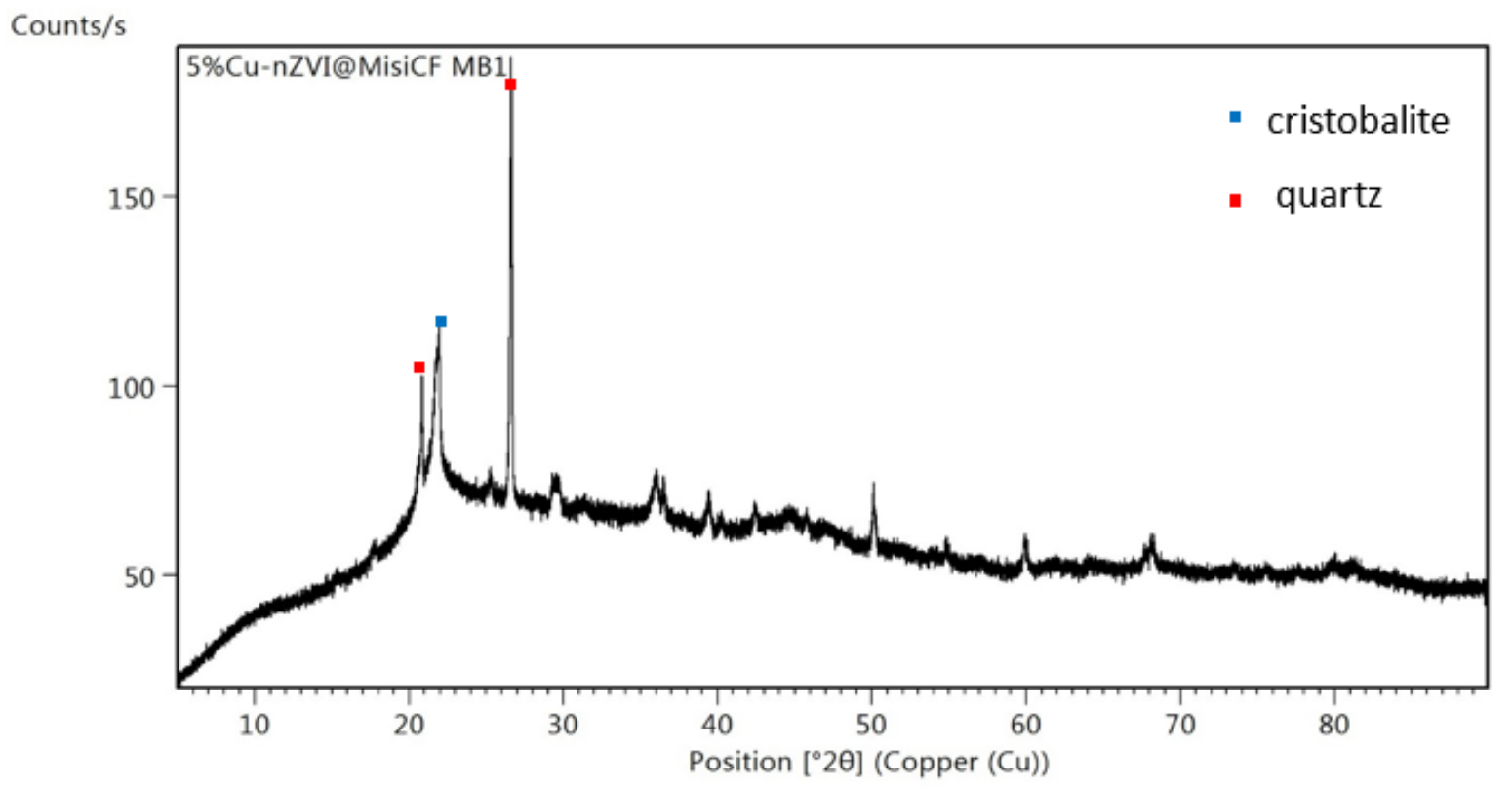

A.3. XRD spectrum of 5\%CU-nZVI@MisiCFeOOH-MB1.

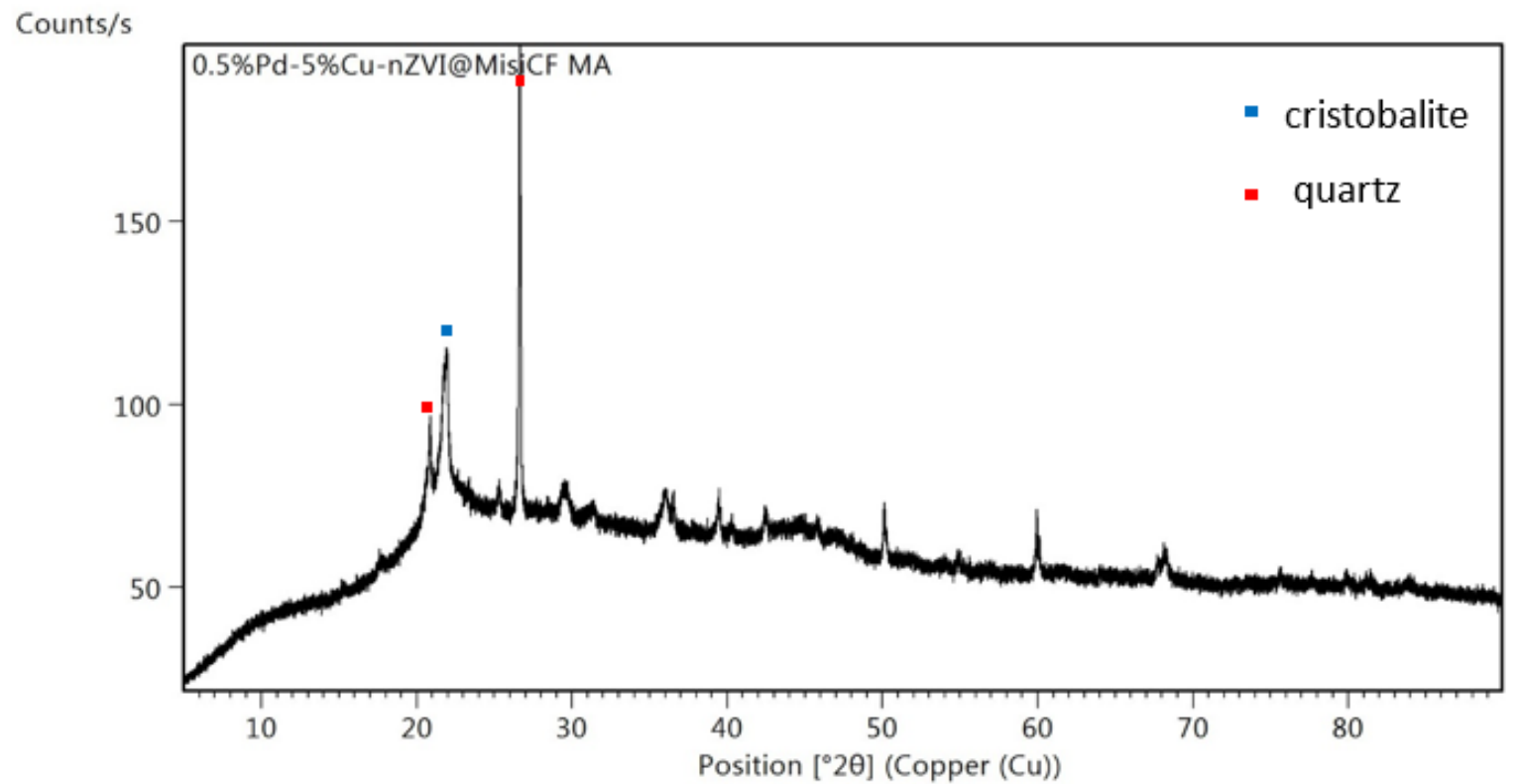

A.4. XRD spectrum of 0.5\%Pd-5\%Cu-nZVI@MisiCFeOOH-MA $A_{t r i}$ 


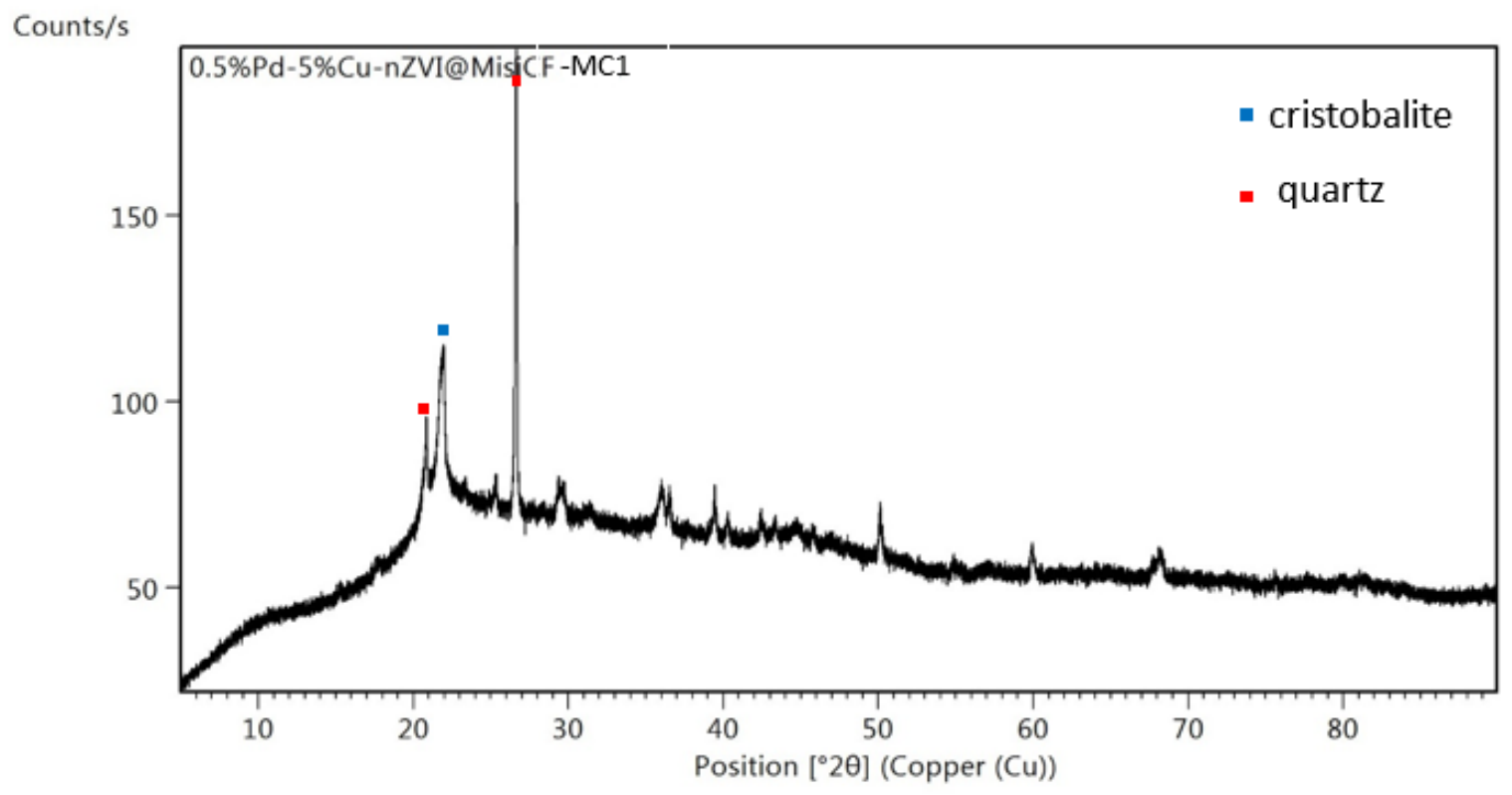

A.5. XRD spectrum of 0.5\%Pd-5\%Cu-nZVI@MisiCFeOOH-MC1.

B. Complete TEM-EDS spectra

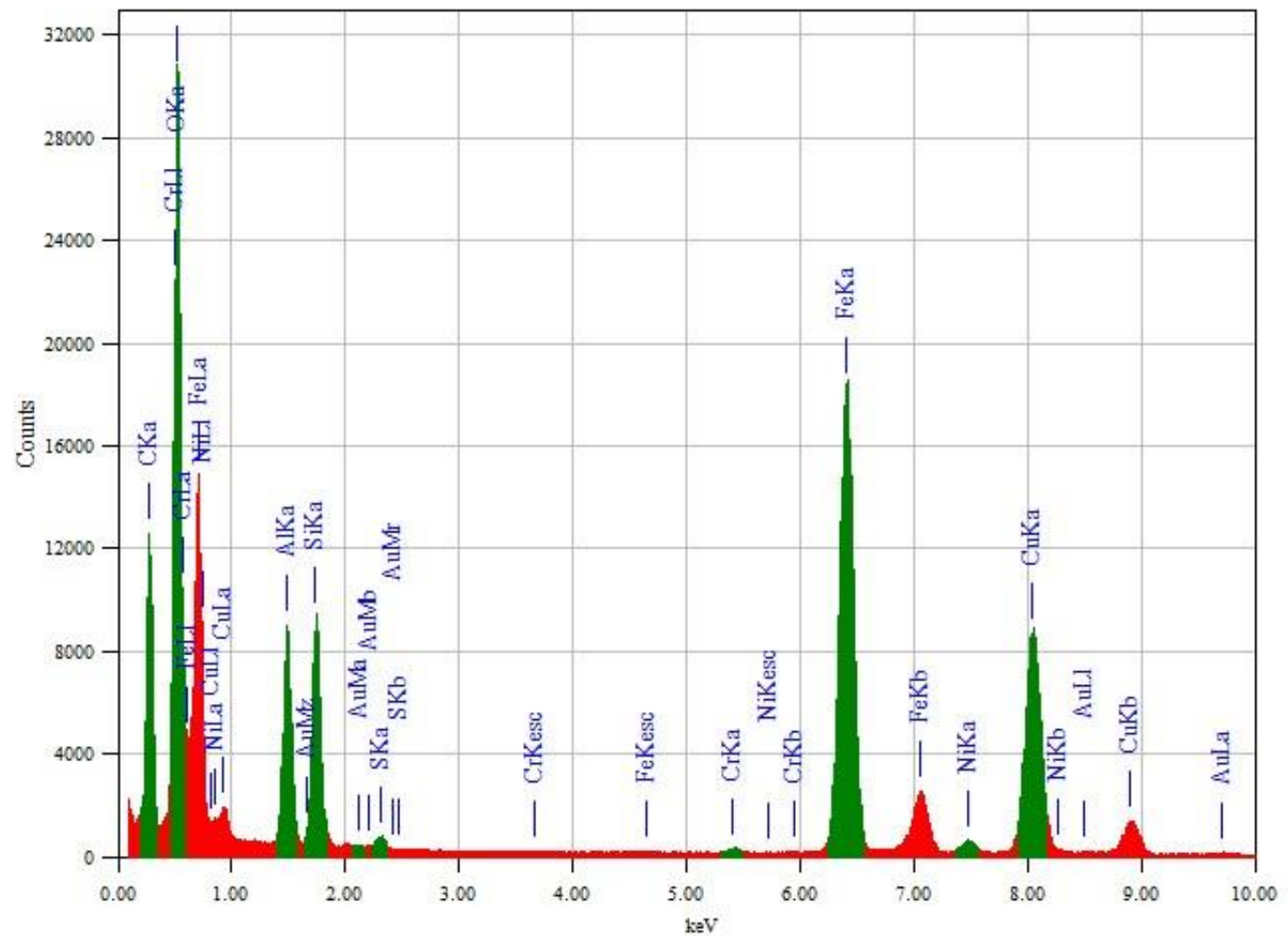

B.1. TEM-EDS spectrum of 5\%Ni-nZVI@MisiCFeOOH-MA. 


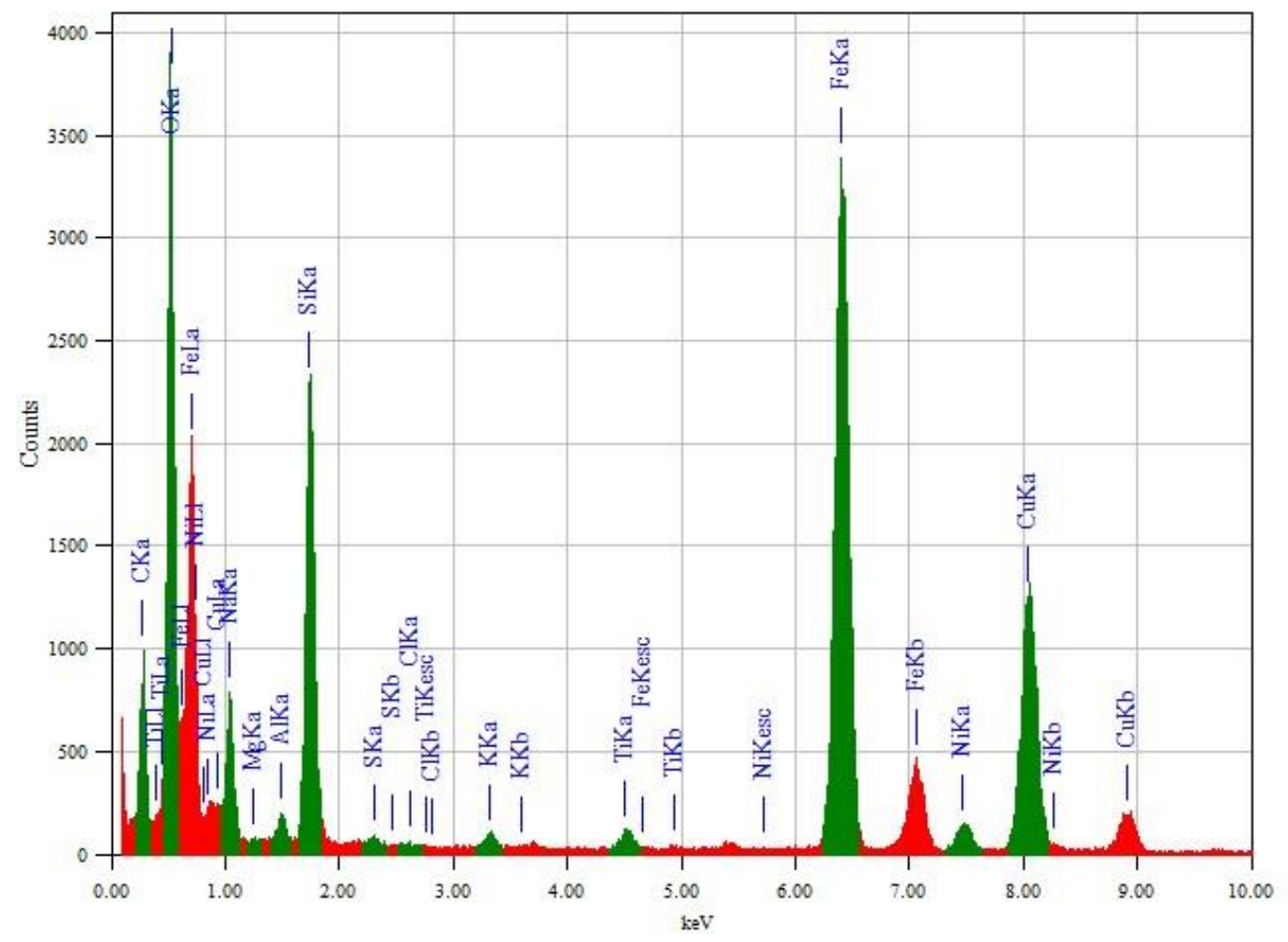

B.2. TEM-EDS spectrum of 5\%Ni-nZVI@MisiCFeOOH-MB1.

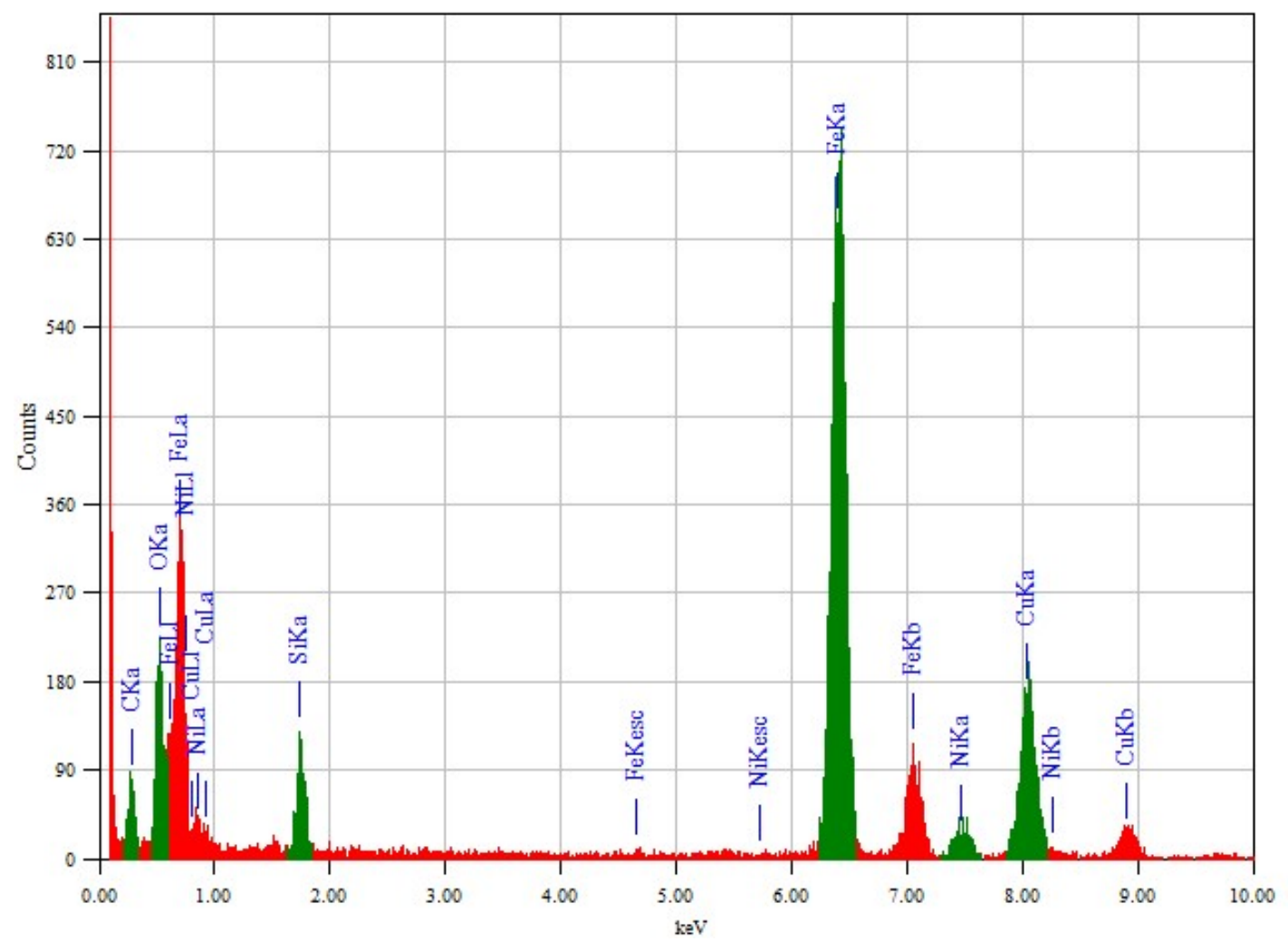

B.3. TEM-EDS spectrum of 5\%Ni-nZVI@MisiCFeOOH-MB2. 

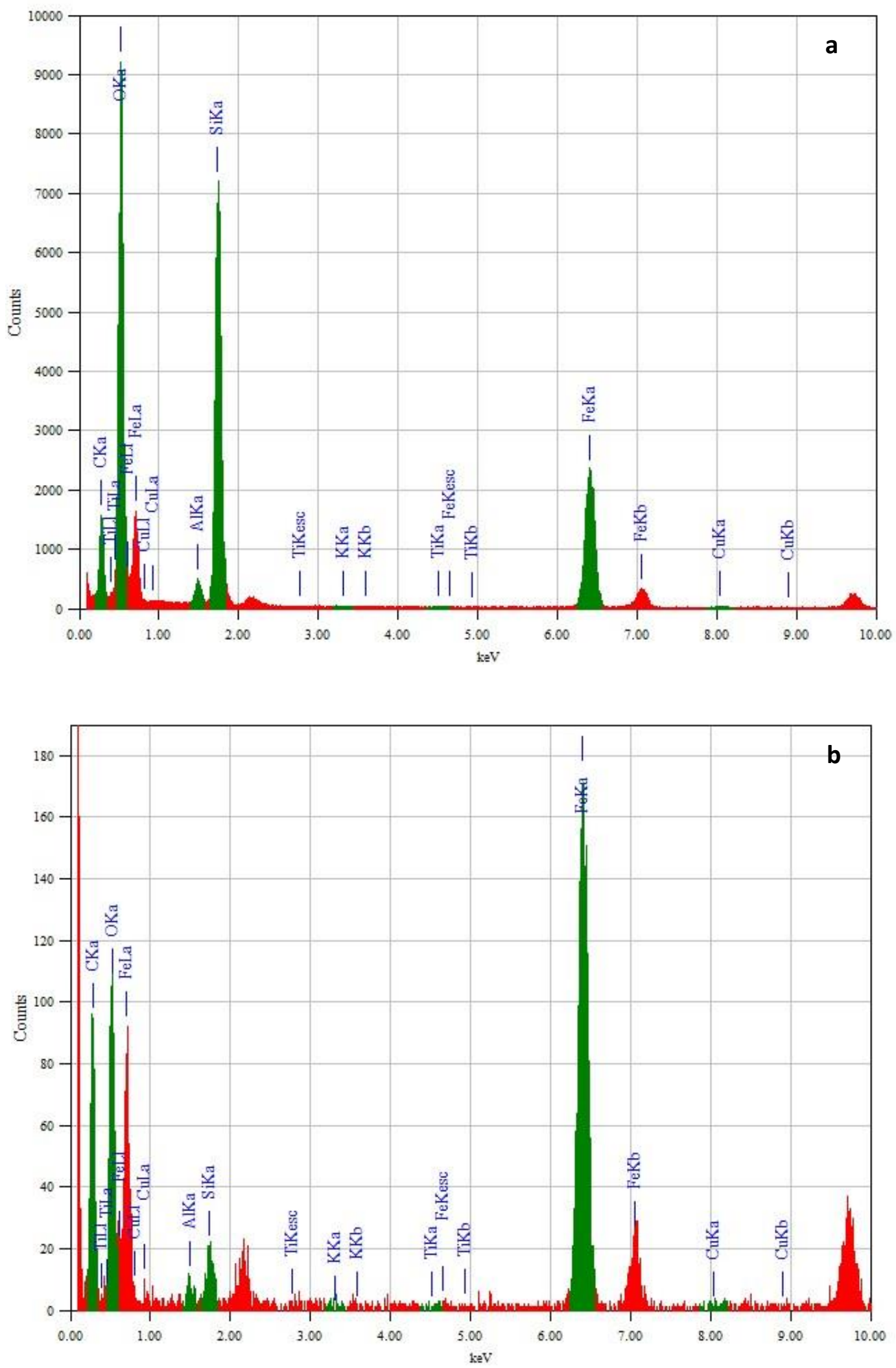

B.4. TEM-EDS spectra of 5\%CU-nZVI@MisiCFeOOH-MA in different areas $a, b$, and c. Area $c$ is in the next page. 


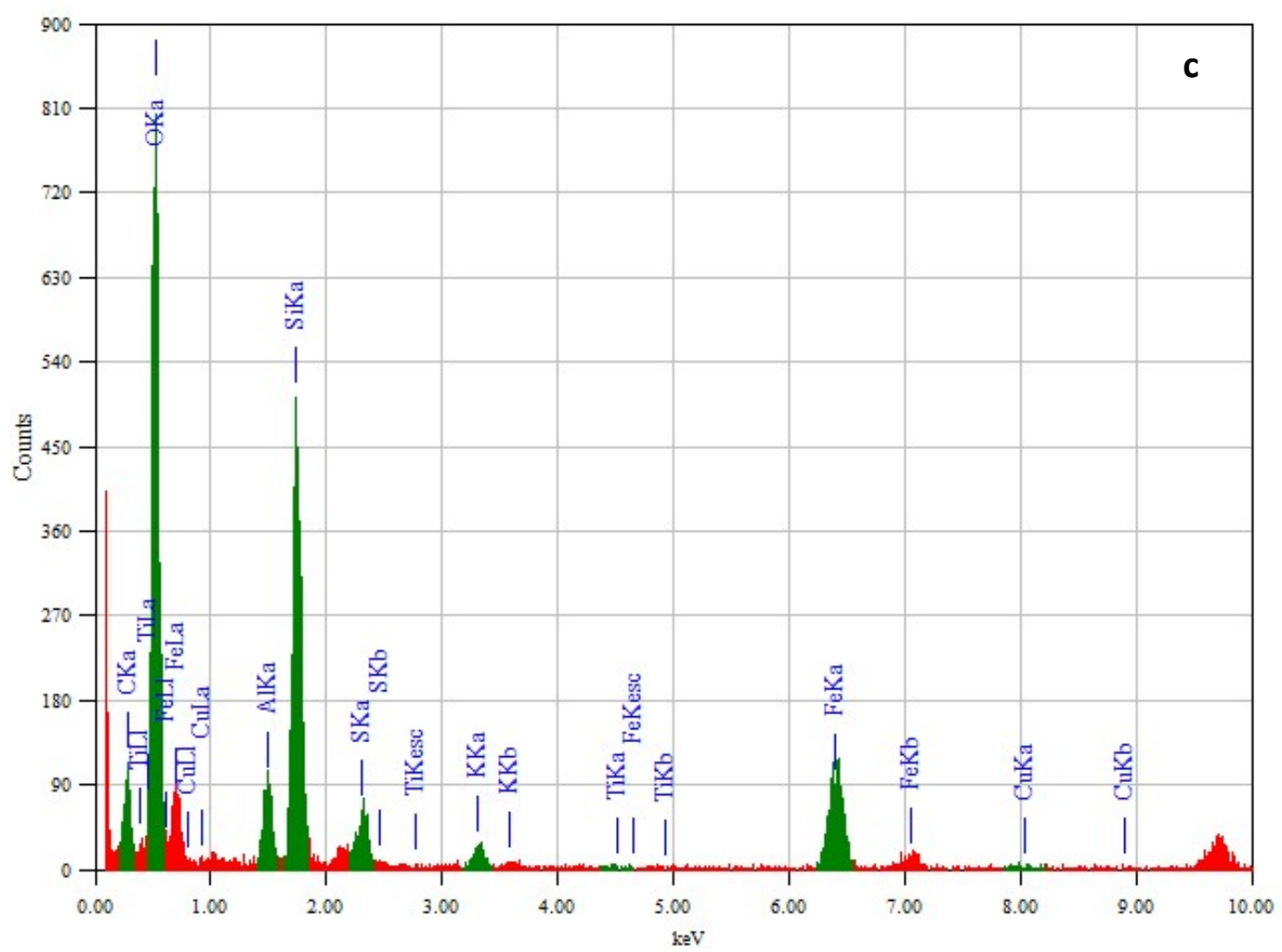

B.4. TEM-EDS spectra of 5\%CU-nZVI@MisiCFeOOH-MA in different areas $a, b$, and c. Areas $a$ and $b$ are in the previous page.

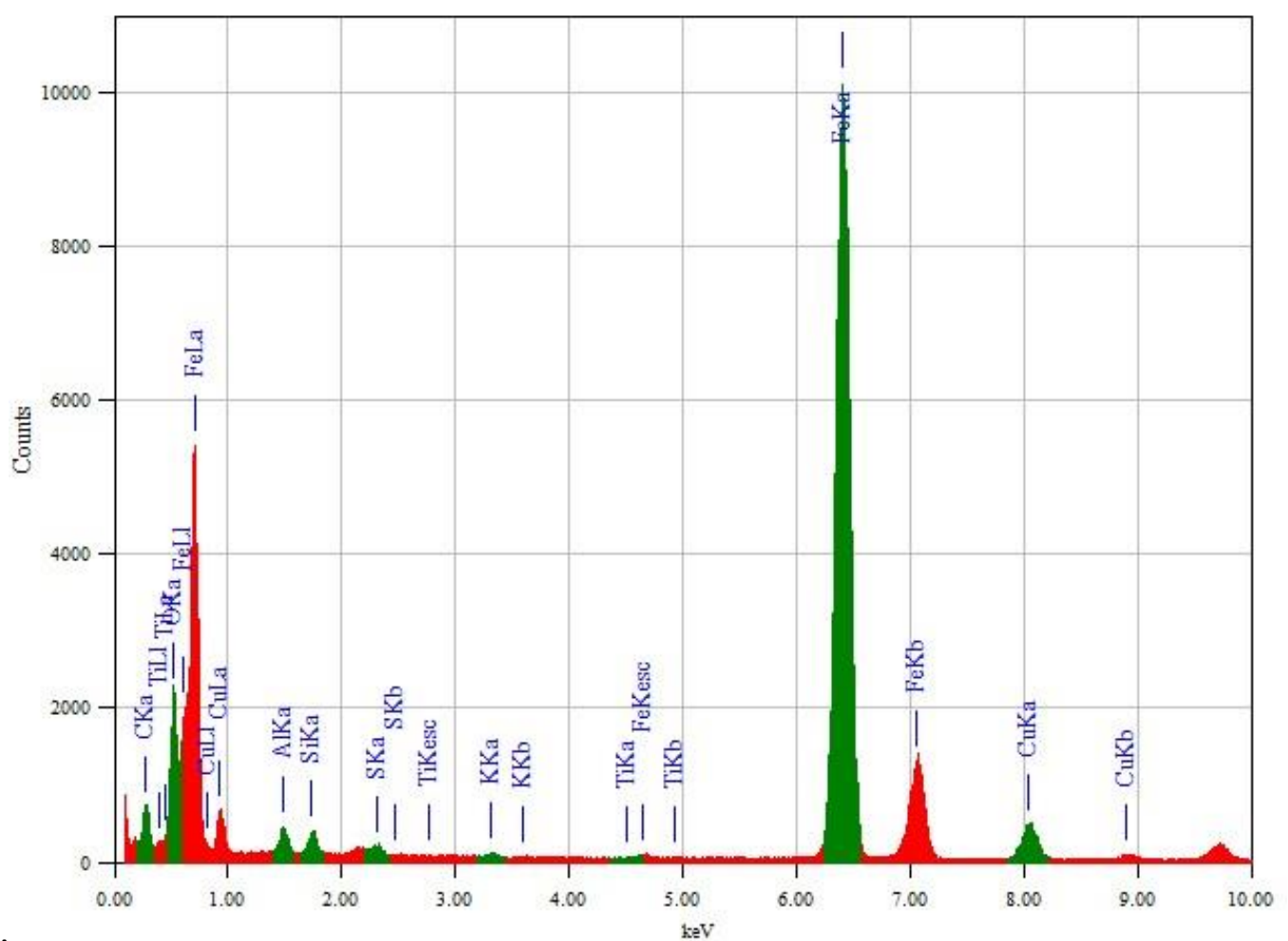

B.5. TEM-EDS spectrum of 5\%Cu-nZVI@MisiCFeOOH-MB1. 


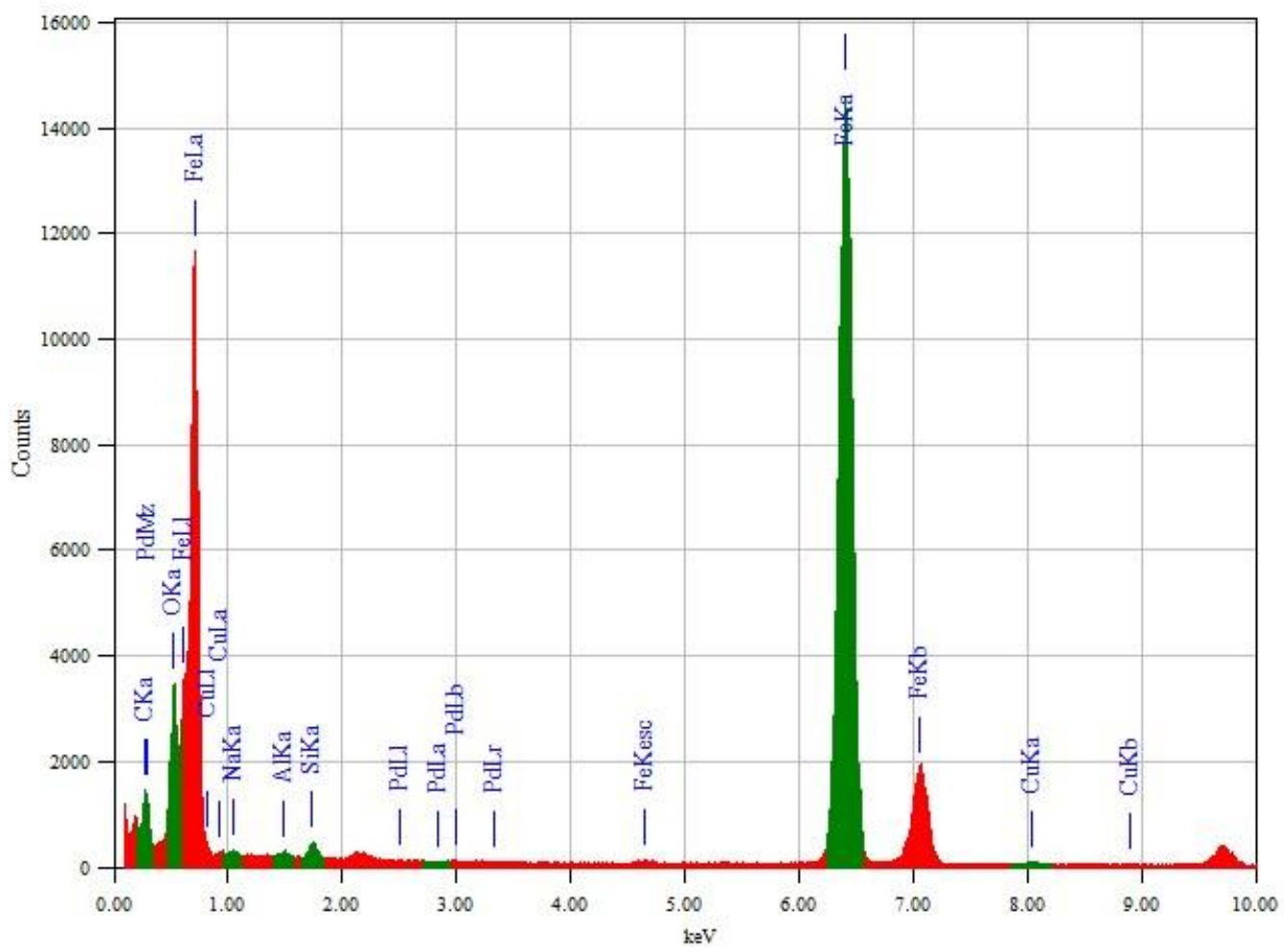

B.6. TEM-EDS spectrum of 0.5\%Pd-5\%Cu-nZVI@MisiCFeOOH-MC1.

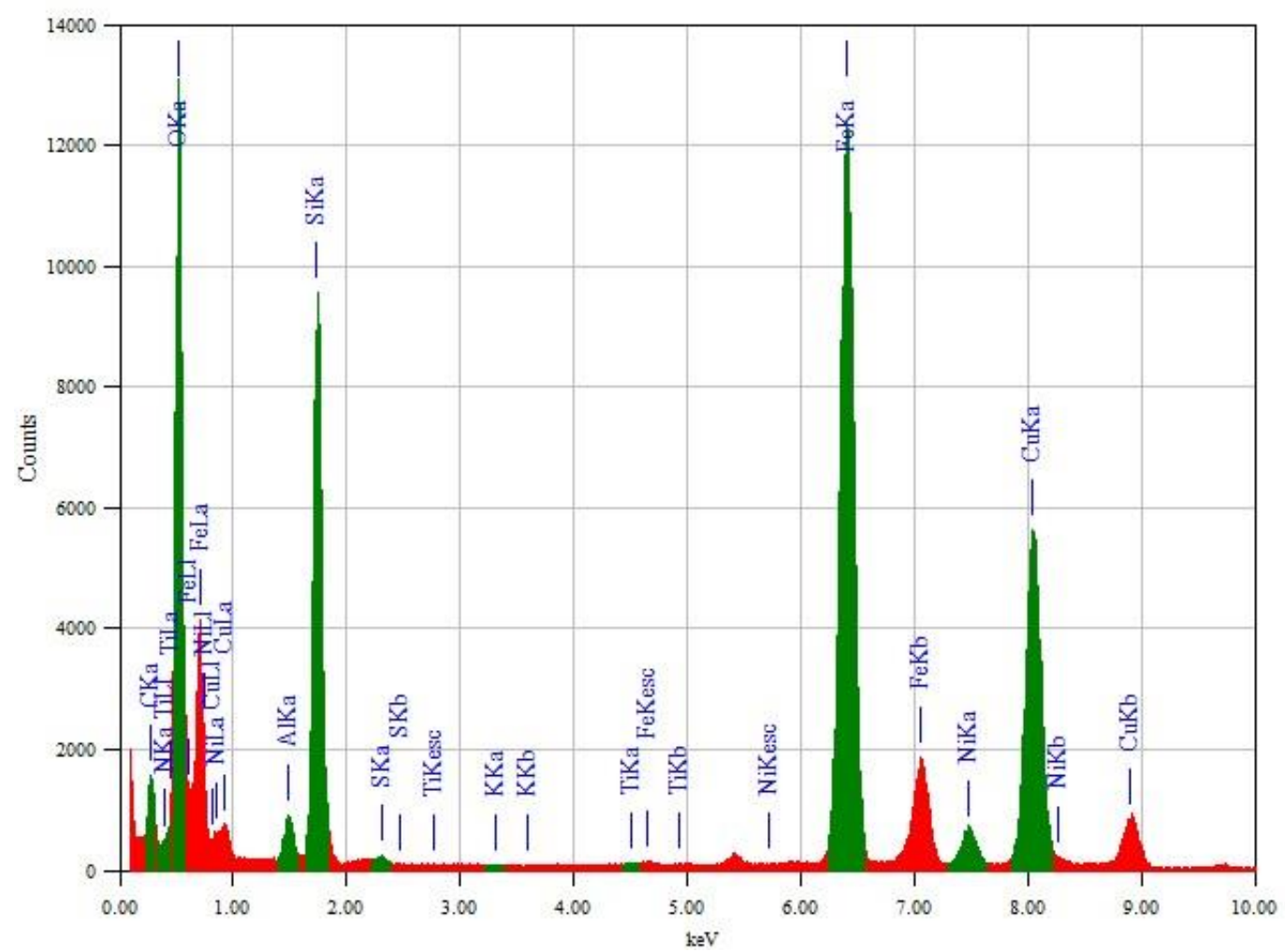

B.7. TEM-EDS spectrum of 5\%Ni-nZVI@MisiCFeOOH-MB1 after reduction of 10 ppm nitrate$N$ (0.150 g material, $25 \mathrm{~mL}$ of solution, under anaerobic conditions, 15 minutes). 


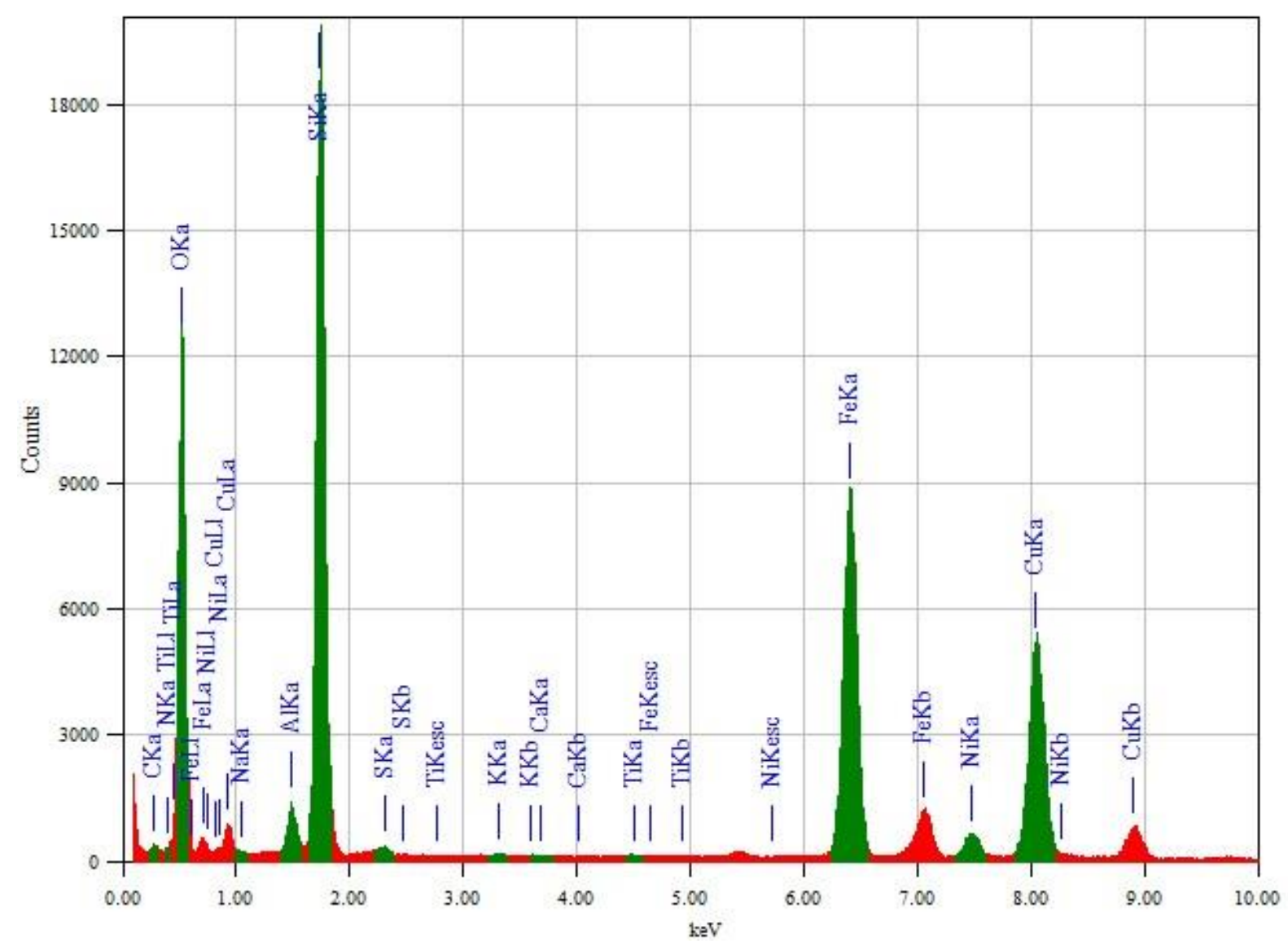

B.8. TEM-EDS spectrum of 5\%Ni-nZVI@MisiCFeOOH-MB1 after reduction of 50 ppm nitrate$N(0.150 \mathrm{~g}$ material, $25 \mathrm{~mL}$ of solution, under anaerobic conditions, 120 minutes). 
C. List of chemicals used

\begin{tabular}{|c|c|c|}
\hline Name & Source & Purity \\
\hline Cadmium standard for AAS & Sigma Aldrich & \\
\hline $\mathrm{CdCl}_{2}$ & Sigma Aldrich & $99.99 \%$ \\
\hline Chromium standard for AAS & Sigma Aldrich & \\
\hline Copper standard for AAS & Sigma Aldrich & \\
\hline Ethanol (absolute) & Fisher Chemical & AR Grade \\
\hline $\mathrm{FeSO}_{4} \cdot 7 \mathrm{H}_{2} \mathrm{O}$ & Riedel-deHaën & $99.5 \%$ \\
\hline $\mathrm{HNO}_{3}$ & Sigma Aldrich & $68-70 \%$ \\
\hline $\mathrm{Hgl}_{2}$ & Sigma Aldrich & $99.0 \%$ \\
\hline Iron standard for AAS & Sigma Aldrich & \\
\hline $\mathrm{KCl}$ & Sigma-Aldrich & $99.0 \%$ \\
\hline $\mathrm{K}_{2} \mathrm{Cr}_{2} \mathrm{O}_{7}$ & Sigma-Aldrich & $99.0 \%$ \\
\hline $\mathrm{K}_{2} \mathrm{SO}_{4}$ & Sigma-Aldrich & $99.0 \%$ \\
\hline $\mathrm{K}_{2} \mathrm{CO}_{3}$ & Sigma-Aldrich & $99.0 \%$ \\
\hline $\mathrm{KH}_{2} \mathrm{PO}_{4}$ & Sigma-Aldrich & $99.0 \%$ \\
\hline KI & Sigma Aldrich & $99 \%$ \\
\hline $\mathrm{KNO}_{3}$ & Vickers Lab Ltd & $99.5 \%$ \\
\hline Lead standard for AAS & Fluka & \\
\hline Microsilica 600 & Golden Bay Cement & \\
\hline $\mathrm{NaBH}_{4}$ (powder) & Sigma Aldrich & $96 \%$ \\
\hline $\mathrm{NaNO}_{2}$ & Sigma Aldrich & $97 \%$ \\
\hline $\mathrm{NaOH}$ & Pure Science & $90 \%$ \\
\hline $\mathrm{NH}_{4} \mathrm{Cl}$ & Sigma Aldrich & $99.5 \%$ \\
\hline Nickel standard for AAS & Sigma Aldrich & \\
\hline Nitrate standard for IC & Sigma-Aldrich & \\
\hline Nitrite standard for IC & Sigma-Aldrich & \\
\hline $\mathrm{Pb}\left(\mathrm{NO}_{3}\right)_{2}$ & Sigma Aldrich & $99.0 \%$ \\
\hline $\mathrm{PbCl}_{2}$ & Sigma Aldrich & $98 \%$ \\
\hline $\mathrm{Pd}\left(\mathrm{CH}_{3} \mathrm{COO}\right)_{2}$ & Sigma Aldrich & \\
\hline Phosphate standard for IC & Fluka & \\
\hline $\mathrm{ZnCl}_{2}$ & Sigma Aldrich & $>97 \%$ \\
\hline
\end{tabular}

